\title{
Chemical and Biological Aspects of Secondary Metabolites from Tongan Marine Sponges
}

\section{Victoria \\ UNIVERSITY OF WELLINGTON}

Te Whare Wānanga

o te Ūpoko o te Ika a Māui

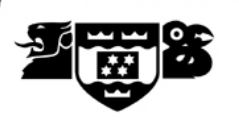

\author{
A thesis \\ submitted to Victoria University of Wellington \\ in fulfilment of the \\ requirements for the degree of \\ Doctor of Philosophy \\ in Chemistry.
}

Victoria University of Wellington

2012 


\section{Abstract}

This thesis describes the isolation and structural elucidation of 17 new secondary metabolites from Tongan marine sponges including examples of alkaloids, polyketides and terpenoids. In the process of this work, 19 sponge specimens were subjected to preliminary NMR-guided investigation. Nine organisms were selected for further analysis on the basis of the structural novelty perceived within the HMBC spectrum of crude fractions generated by the first chromatographic purification of their crude extracts, and the apparent rarity of the specimen.

Investigation of two different demosponge specimens afforded the $\gamma$-hydroxybutenolide sesterterpenes (23 and $\mathbf{2 4}$ ), and small quantities of the potently cytotoxic alkaloid 14-bromohomofascaplysin (29).

The analysis of two samples of a dictyoceratid sponge yielded the new labdane diterpenes luakuliides A-C (33-36), characterised by a bridging hemi-acetal function on the B-ring of the labdane bicycle. Luakuliide A (33) and its methyl acetal derivative $\mathbf{3 4}$ were found to display interesting immunomodulatory activity.

Seven new $\alpha$-pyrone polyketides, lehualides E-K (69-75), were isolated from a Plakortis sp. Lehualides $\mathrm{H}-\mathrm{K}(\mathbf{7 2}-\mathbf{7 5})$ display a range of sulfur functionalities, the natures of which were determined by spectroscopic comparison with synthesised model compounds.

Another plakinid sponge specimen contained four new polyketides (95-98), all of which possess different cyclic peroxide moieties. Cyclic peroxides 95, 97 and 98 displayed potent cytoxicity against human promyelocytic leukemia cells (HL60). Chemical genetic and phenoytypic profiling studies of 95 were undertaken in Saccharomyces cerevisiae yeast using the homozygous diploid and heterozygous diploid deletion libraries. These studies indicate that 95 acts to disrupt $\mathrm{Ca}^{2+}$ homeostasis, leading to elevation of intracellular $\mathrm{Ca}^{2+}$ levels.

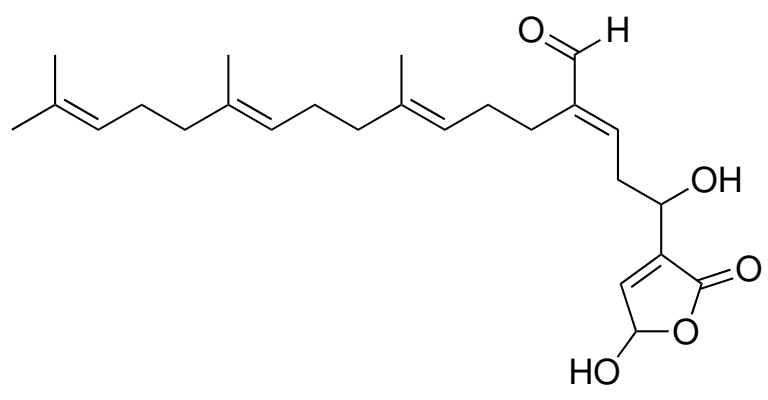




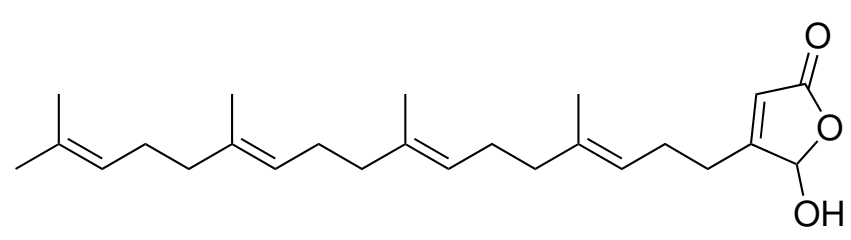

24

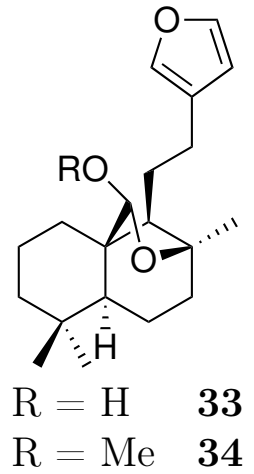<smiles>[R]Oc1c(C)c(C/C=C(\C)CCCCCCc2ccccc2)oc(=O)c1OC</smiles>

$69 \mathrm{R}=\mathrm{Me}$

$70 \mathrm{R}=\mathrm{H}$<smiles>[R]SCCCCCCCCCCc1oc(=O)c(OC)c(OC)c1C</smiles>

$72 \mathrm{R}=\mathrm{Ac}$

$73 \mathrm{R}=\mathrm{Me}$

35<smiles>CC(=O)CC1(O)c2ccccc2-[n+]2ccc3c([nH]c4cc(Br)ccc43)c21</smiles>

29

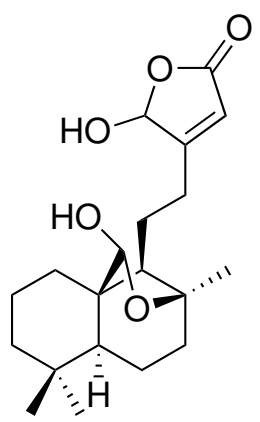

36<smiles>COc1c(O)c(C)c(C/C=C(\C)CCCCCCCCc2ccccc2)oc1=O</smiles>

71

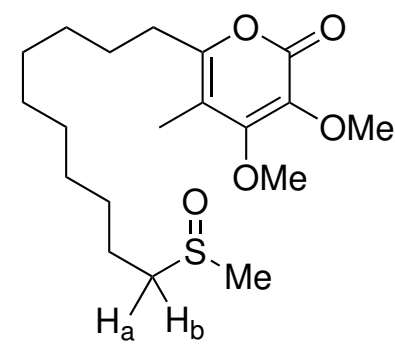

74<smiles>COc1c(C)c(C)oc(=O)c1OC</smiles><smiles>CCCCCCCCCCSS</smiles><smiles>CCc1oc(=O)c(OC)c(OC)c1C</smiles>

75<smiles>[R]CC(C)CCCCCCCCC[C@]1(C)C[C@@](C)(CC(=O)O[Na])OO1</smiles>

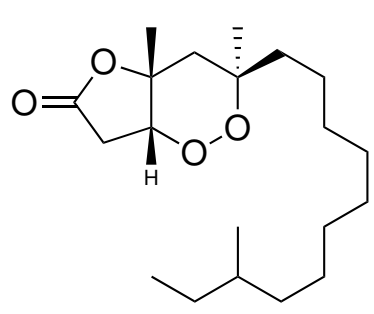

97<smiles>CCC(C)CCCCCCCCC[C@]1(C)C[C@@H](C)[C@](O)(CC(=O)O)OO1</smiles>

98 


\section{Acknowledgments}

Over the last three years I've been lucky enough to have tremendous support from family, friends and work-mates. Without your friendship and help I would not have been able to achieve this. Thank you very much.

My supervisor Peter, thank you for being such a fountain of knowledge and for your guidance throughout my studies. Your uncanny ability to solve the snarliest of problems and inspire me when even I was lacking in enthusiasm has more than made up for the difficulties I've encountered when trying to pin you down. To the Natural Products Groups and the occupants of LB212 and LB211 over the years; the Peter's Moore and Clark, Russel Hewitt and Nathaniel Dasyam. Particular thanks to Jono Singh, Helen Woolner, Rob Keyzers, Emma Aitken, and Taitusi Taufa for all the help and support you offered, and to John Ryan for all your help with NMR.

Everyone in the Chemical Genetics lab, thank you for all your help with my assays. I'm particularly grateful to David Bellows and Paul Atkinson for allowing me to work in your lab for so long, and to James Matthews for helping me with everything from assay design to proof-reading. Thank you also to Dora Leahy and Rosemary Heathcott for your technical guidance, and to Heather Best and Christina Roberts for help with writing and therapeutic hot chocolates in recent months. Ploi Yibmantasiri, I've loved finding someone who shares my enthusiasm for midafternoon cocktails.

Alec La Grow, Bryan O'Leary, Nick Monahan and Ash Dangerfield - we've had some good times over the years. Thank you for always believing in me and my abilities.

Ed Willis, Anne O'Driscoll, Graham Fairweather and Livvy Eaton - you all kept me sane at various points during my $\mathrm{PhD}$, and were always there for a cup of tea (or coffee) and to go for a run. Patricia Stein and Shona de Sain, you helped me cope at the very end and for that I will be eternally grateful.

Mum, dad, James and Felix, thank you for always having been there to help me through whatever life has thrown at me over the years.

Paul, you helped me through everything in the last couple of months, thanks for cooking me dinner, drying my tears and making me laugh when I needed to. I will always cherish the time we've spent together. 
For Florence,

I know you would have been proud and I miss you everyday. 


\section{Contents}

Abstract

Acknowledgments

$\mathbf{V}$

Dedication vii

Table of Contents $\quad$ ix

List of Figures $\quad$ XV

List of Schemes $\quad$ xxi

List of Tables $\quad$ xxiii

Glossary $\quad$ xxvii

1 Introduction 1

1.1 Natural products as therapeutic agents . . . . . . . . . . . . . . 1

1.2 Marine natural products . . . . . . . . . . . . . . . . 3

1.3 Sponges ....................... 5

1.3.1 Microbial symbiosis in marine sponges . . . . . . . . . . 7

1.4 The Tongan marine ecoregion . . . . . . . . . . . . . . . . 8

1.5 Organism selection and screening . . . . . . . . . . . . . . 10

1.5.1 Bioassay-guided isolation . . . . . . . . . . . . . . 10

1.5.2 Spectroscopy-guided isolation . . . . . . . . . . . . . 11

1.5.3 Advances in NMR screening . . . . . . . . . . . . . . . . 12

1.6 Assigning biological applications . . . . . . . . . . . . . . . . . . . . 14

1.6.1 Chemical genetic profiling . . . . . . . . . . . . 15

1.6.2 Gene expression profiling . . . . . . . . . . . . . . 15 
1.7 Research objectives . . . . . . . . . . . . . . . . 16

1.8 Summary . . . . . . . . . . . . . . . . . . 16

2 Organism Screening and Selection $\quad 19$

2.1 Collection areas . . . . . . . . . . . . . . . . . 19

2.2 Organism selection . . . . . . . . . . . . . . . . . . 19

2.3 The Vava'u group . . . . . . . . . . . . . . . . . 20

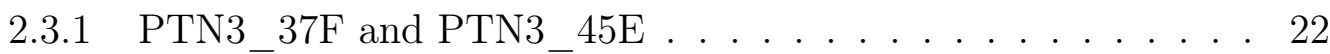

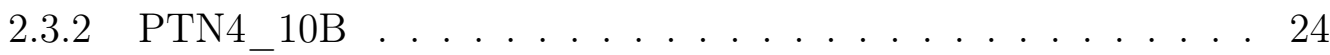

2.4 'Eua Island . . . . . . . . . . . . . . . . . . . . . . 26

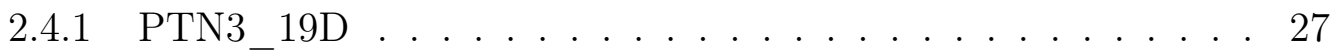

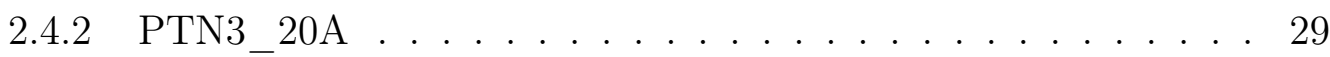

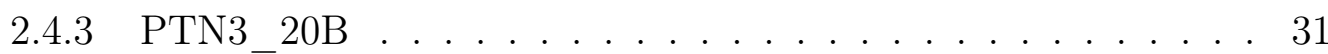

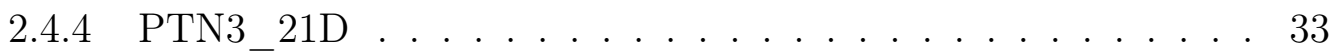

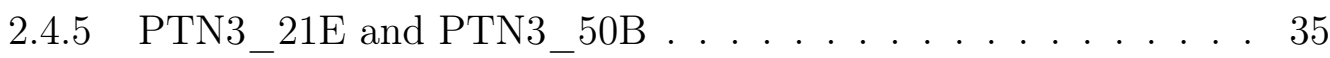

2.5 Concluding remarks . . . . . . . . . . . . . . 36

$\begin{array}{lll}3 & \text { Luakuliides A-C } & 37\end{array}$

3.1 The order Dictyoceratida . . . . . . . . . . . . . . . 37

3.2 Labdane diterpenoids from the dictyoceratid sponge "luakuli" . . . . . 38

3.2.1 Isolation and identification of luakuliides $\mathrm{A}-\mathrm{C}$. . . . . . . 38

3.2.2 Biological activity of the luakuliides . . . . . . . . . . . 57

3.2.3 Labdane diterpenes . . . . . . . . . . . . . . . . . 57

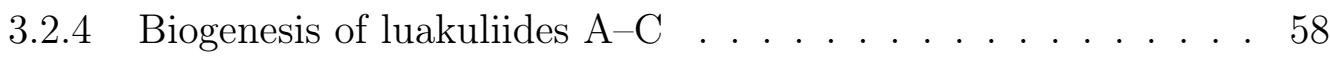

3.2.5 Concluding remarks . . . . . . . . . . . . . . 62

4 Chemical Analyses of Plakinid Sponges 63

4.1 The family Plakinidae . . . . . . . . . . . . . . 63 
4.1.1 The Plakortis and Plakinastrella genera . . . . . . . . . . . 64

$4.2 \quad \alpha$-Pyrone metabolites from Plakortis sp. . . . . . . . . . . . . . 68

4.2.1 Isolation and identification of lehualides E-K . . . . . . . . 69

4.2.2 Bioactivity of the lehualides . . . . . . . . . . . . 92

4.2.3 Determination of sulfur functionalities . . . . . . . . . . . . 94

4.2.4 $\alpha$ - and $\gamma$-Pyrone metabolites from marine invertebrates . . . . 98

4.2.5 Biogenesis of the lehualides . . . . . . . . . . . . 101

4.2 .6 Concluding remarks . . . . . . . . . . . . . . . . 104

4.3 Cyclic peroxides from a plakinid sponge . . . . . . . . . . . . 105

4.3.1 Isolation and identification of cyclic peroxides . . . . . . . 105

4.3.2 Growth inhibition of eukaryotes . . . . . . . . . . . 125

4.3 .3 Organism classification . . . . . . . . . . . . . 126

4.3.4 Cyclic peroxide natural products . . . . . . . . 126

4.3.5 Biogenesis of cyclic peroxides from plakinid sponges . . . . . . 130

4.3.6 Concluding remarks . . . . . . . . . . . . . . 136

5 Chemical Genetic Profiling of Marine Cyclic Peroxides 137

5.1 The biological effects of marine cyclic peroxides . . . . . . . . . 137

5.2 Biological assessment in S. cerevisiae . . . . . . . . . . . . 138

5.3 Chemical genetic profiling in S. cerevisiae. . . . . . . . . . 139

5.3.1 Homozygous profiling . . . . . . . . . . . . 143

5.3.2 Haploinsufficiency profiling . . . . . . . . . . . . . . 143

5.4 Preliminary studies in S. cerevisiae . . . . . . . . . . . . . 144

5.4.1 Phenotypic profiling of epiplakinic acid I and plakortolide X . 146

5.5 Chemical genetic profile of epiplakinic acid I . . . . . . . . . . . . 149

5.5.1 Homozygous profiling of epiplakinic acid I . . . . . . . . . . . 149

5.5.2 Haploinsufficiency profiling of epiplakinic acid I . . . . . . . 151 
5.5 .3 Plakortide F acid . . . . . . . . . . . . . . . . . 153

5.5.4 The $\mathrm{Ca}^{2+}$ second messenger system . . . . . . . . . . . 156

5.5.5 Epiplakinic acid I induces a $\mathrm{Ca}^{2+}$-related stress response . . . 156

5.5.6 Calcineurin signaling . . . . . . . . . . . . . 158

5.5.7 Interactions between calcineurin signaling and TOR . . . . . 160

5.6 Mechanistic insights from phenotypic and chemical genetic profiling . 162

5.6 .1 Future directions . . . . . . . . . . . . . . . . 162

5.6 .2 Concluding remarks . . . . . . . . . . . . . . . . 164

6 Concluding Remarks 165

$\begin{array}{lll}7 & \text { General Experimental Procedures } & 167\end{array}$

7.1 Isolation of new diterpenes from PTN3_45E . . . . . . . . . 168

7.1 .1 Animal material . . . . . . . . . . . . . . . 168

7.1.2 Extraction of PTN3_45E . . . . . . . . . 168

7.2 Isolation of known terpenes from PTN4_10B . . . . . . . . . 169

7.2.1 Animal material . . . . . . . . . . . . . . 169

7.2 .2 Initial extraction of PTN4_10B . . . . . . . . . 169

7.3 Isolation of 14-bromohomofascaplysin . . . . . . . . . . . . . . 170

7.3.1 Animal material . . . . . . . . . . . . . . . 170

7.3.2 Extraction of PTN3_20B ................ 170

7.3.3 $\mathrm{Me}_{2} \mathrm{CO}$-free extraction of PTN3_20B . . . . . . . 170

7.4 Isolation of halenaquinone . . . . . . . . . . . . . 171

7.4 .1 Animal material . . . . . . . . . . . . . . . 171

7.4.2 Extraction of PTN3_21D . . . . . . . . . . 171

7.5 Isolation of luakuliides $\mathrm{A}-\mathrm{C} \ldots \ldots$. . . . . . . . . . . . . . . . .

7.5.1 Animal material . . . . . . . . . . . . . . . 171

7.5.2 Screening extraction of PTN3_21E . . . . . . . . 172 
7.5.3 Extraction of PTN3_50B . . . . . . . . . . . 172

7.6 Isolation of lehualides $\mathrm{E}-\mathrm{K} \ldots \ldots$. . . . . . . . . . . . . 174

7.6 .1 Animal material . . . . . . . . . . . . . . . . . . 174

7.6.2 Initial extraction of Plakortis sp. PTN3_20A . . . . . . . 174

7.6.3 Second extraction of Plakortis sp. PTN3_20A . . . . . . 175

7.6.4 Preparation of spectroscopic model compounds . . . . . . 176

7.7 Isolation of new cyclic peroxides . . . . . . . . . . . . . 179

7.7 .1 Animal material . . . . . . . . . . . . . . 179

7.7.2 Initial extraction of PTN3_19D . . . . . . . . 179

7.7.3 Second extraction of PTN3_19D . . . . . . . . . 180

7.8 Biological evaluation of cyclic peroxides . . . . . . . . . . . . 182

7.8 .1 Yeast strains . . . . . . . . . . . . . . . . 182

7.8.2 Preliminary assay protocols . . . . . . . . . . . . 182

7.8.3 Chemical genetic profiling of epiplakinic acid I . . . . . . . 186

7.8 .4 Imaging studies . . . . . . . . . . . . . . . . . . 191

7.8.5 Transformation of 8025- $\alpha$ strain . . . . . . . . . . . . 192

7.8.6 Attempted transformation of $z r t 1 \Delta / z r t 1 \Delta$ and
cch1 $1 \Delta /$ ch $1 \Delta$ strains . . . . . . . . . . . . . . 193

A Sponge Screening and Cyclic Loading Protocol 195

A.1 Cyclic loading and backloading . . . . . . . . . . . . 195

A.2 Screening protocol . . . . . . . . . . . . . 196

A.3 Cyclic loading . . . . . . . . . . . . . . . . . . . 197

A.4 Processing and NMR analysis . . . . . . . . . . . . . . 198

A.5 Spicule analysis . . . . . . . . . . . . . . . . . . 200

B Yeast Growth Media $\quad 201$

C NMR Data for Known Compounds 202

xiii 
D ${ }^{1}$ H NMR Spectra of Known Compounds 208

$\begin{array}{ll}\text { E NMR Spectra of Luakuliides A-C } & 217\end{array}$

$\begin{array}{lll}\text { F NMR Spectra of Lehualides E-K } & 248\end{array}$

G NMR Spectra of New Cyclic Peroxides 284

H Phenotypic Profile of Epiplakinic Acid I 305

I Chemical Genetic Profile of Epiplakinic Acid I 311

$\begin{array}{ll}\text { Bibliography } & 317\end{array}$ 


\section{List of Figures}

1.1 Taxonomic classification within the phylum Porifera. . . . . . . . 6

1.2 The Kingdom of Tonga . . . . . . . . . . . . . . . . . . . . . . . . . . 9

1.3 A generic ${ }^{1} \mathrm{H}$ NMR spectrum. . . . . . . . . . . . . . . . . 12

1.4 A generic HMBC spectrum. . . . . . . . . . . . . . . . . . . 13

2.1 The Vava'u island group. . . . . . . . . . . . . . . . . . . . . 20

2.2 Surface photo of the sponge PTN3_45E . . . . . . . . . . . . 22

$2.3{ }^{1} \mathrm{H}$ NMR screen spectrum $\left(\mathrm{CD}_{3} \mathrm{OD}\right)$ of PTN3_45E . . . . . . . 23

2.4 HMBC screen spectrum $\left(\mathrm{CD}_{3} \mathrm{OD}\right)$ of PTN3_45E . . . . . . . 23

2.5 Surface photo of the sponge PTN4_10B . . . . . . . . . . . 24

$2.6{ }^{1} \mathrm{H}$ NMR screen spectrum $\left(\mathrm{CD}_{3} \mathrm{OD}\right)$ of PTN4_10B . . . . . . . 25

2.7 HMBC screen spectrum $\left(\mathrm{CD}_{3} \mathrm{OD}\right)$ of PTN4_10B . . . . . . . 25

2.8 The Tongatapu island group. . . . . . . . . . . . . . . . . 26

2.9 Surface photo of the plakinid specimen PTN3_19D . . . . . . . . 27

$2.10{ }^{1} \mathrm{H}$ NMR screen spectrum $\left(\mathrm{CD}_{3} \mathrm{OD}\right)$ of PTN3_19D . . . . . . . 27

2.11 HMBC screen spectrum $\left(\mathrm{CD}_{3} \mathrm{OD}\right)$ of PTN3_19D . . . . . . . . 28

2.12 Surface photo of the Plakortis specimen PTN3_20A . . . . . . . . 29

$2.13{ }^{1} \mathrm{H}$ NMR screen spectrum $\left(\mathrm{CD}_{3} \mathrm{OD}\right)$ of PTN3_20A $\ldots . . . . .30$

2.14 HMBC screen spectrum $\left(\mathrm{CD}_{3} \mathrm{OD}\right)$ of PTN3_20A . . . . . . . 30

2.15 Surface photo of the sponge PTN3_20B . . . . . . . . . . 31

$2.16{ }^{1} \mathrm{H}$ NMR spectrum $\left(\mathrm{CD}_{3} \mathrm{OD}\right)$ of PTN3_20B . . . . . . . . . 32

2.17 HMBC experiment $\left(\mathrm{CD}_{3} \mathrm{OD}\right)$ of PTN3_20B . . . . . . . . . 32

2.18 Surface photo of the sponge PTN3_21D . . . . . . . . . 33

$2.19{ }^{1} \mathrm{H}$ NMR spectrum $\left(\mathrm{CD}_{3} \mathrm{OD}\right)$ of PTN3_21D . . . . . . . . 34

2.20 HMBC experiment $\left(\mathrm{CD}_{3} \mathrm{OD}\right)$ of $\mathrm{PTN} 3 \_21 \mathrm{D} \ldots \ldots . \ldots . \ldots 34$ 
2.21 Vava'u collection of the dictyoceratid sponge "luakuli" . . . . . . . . . 35

$2.22{ }^{1} \mathrm{H}$ spectrum $\left(\mathrm{CD}_{3} \mathrm{OD}\right)$ of $\mathrm{PTN} 3 \_21 \mathrm{E} \ldots \ldots \ldots . \ldots . \ldots . \ldots 35$

2.23 HMBC experiment $\left(\mathrm{CD}_{3} \mathrm{OD}\right)$ of PTN3_50B $\ldots \ldots \ldots . . \ldots 36$

3.1 Taxonomic classification of the order Dictyoceratida. . . . . . . . . 37

3.2 Correlations evidencing substructures in the screen HMBC spectrum

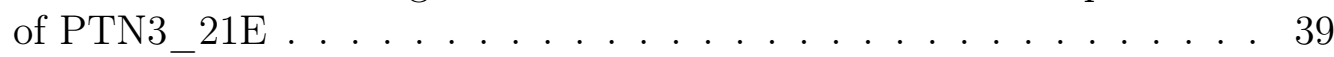

3.3 Purification strategy employed in the isolation of luakuliides A-C . . 40

3.4 The furan system of luakuliide A . . . . . . . . . . . . . . . . 42

3.5 NMR correlations establishing the C-9 to C-12, C-5 to C-7 and C1 to $\mathrm{C}-3$ alkyl segments in luakuliide A . . . . . . . . . . . . . . 42

3.6 The gem-dimethyl substructure of luakuliide A . . . . . . . . . . . . 43

3.7 COSY and HMBC correlations establishing the C-7 to C-9 connections in luakuliide $\mathrm{A} \ldots \ldots$. . . . . . . . . . . . . . . 43

3.8 Establishment of the cyclodecane system of luakuliide A . . . . . . . 44

3.9 COSY and HMBC correlations establishing the CH-20 hemi-acetal bridge in luakuliide A . . . . . . . . . . . . . . . 45

3.10 The trans-fused [4.4.0]-bicyclodecane system of luakuliide A . . . . . 45

3.11 Determination of relative stereochemistry in luakuliide A . . . . . . . 46

3.12 NMR correlations establishing the C-9 to C-12, C-5 to C-7 and C1 to $\mathrm{C}-3$ alkyl segments in luakuliide B . . . . . . . . . . . . . . 49

3.13 HMBC correlations establishing the connections from C-1 to C-5 in luakuliide B . . . . . . . . . . . . . . . . . 50

3.14 COSY and HMBC correlations completing the cyclodecane system of luakuliide B ..................... 50 50

3.15 The [4.4.0]-bicyclodecane system and bridging hemi-acetal moiety of luakuliide B . . . . . . . . . . . . . . . . . 51

3.16 The $\alpha$-substituted $\alpha, \beta$-unsaturated ester and $\gamma$ - hemi-acetal methine in luakuliide B . . . . . . . . . . . . . . . . . . . . . 52

3.17 The $\alpha$-substituted $\gamma$-hydroxybutenolide moiety of luakuliide B . . . . 52

3.18 The $\beta$-substituted- $\gamma$-hydroxybutenolide moiety of luakuliide $\mathrm{C}$. . . . 54 
4.1 Taxonomic classification of the order Homoscleophorida.

4.2 Depiction of the PKS1 propagation units used in the biosynthesis of plakortether B . . . . . . . . . . . . . 65

4.3 Diod spicules recovered from the Plakortis sponge PTN3_20A . . . . 69

4.4 Correlations evidencing substructures in the screen HMBC spectrum

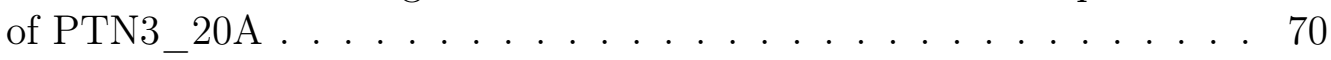

4.5 Purification strategy employed in the isolation of lehualides E-K. . . 71

4.6 The mono alkyl-substituted benzene ring of lehualide E . . . . . . . . 72

4.7 COSY, HMBC and 1D NOE correlations confirming the C-7 to C-11 segment in lehualide $\mathrm{E} \ldots \ldots . \ldots 73$

4.8 COSY and HMBC correlations establishing the C-5 to C-8 connections in lehualide $\mathrm{E} \ldots \ldots . \ldots 73$

4.9 HMBC and 1D NOE correlations establishing the C-3 to C-6 tetrasubstituted diene in lehualide $\mathrm{E}$. . . . . . . . . . . . . . . 74

4.10 1D TOCSY correlations confirming the linear connectivity in lehualide E ............................ 75

$4.11{ }^{1} \mathrm{H}$ NMR spectral comparison between of lehualides E and F . . . . 77

4.12 COSY and selected HMBC correlations establishing the C-17 to C-23 segment of lehualide $\mathrm{F} \ldots \ldots . \ldots 78$

4.13 COSY and HMBC correlations confirming the C-5 to C-6 and C8 to C-9 alkenes in lehualide F . . . . . . . . . . . . . . 78

4.14 HMBC and 1D NOE correlations establishing the C-4-C-5 bond and methoxy substitution of $\mathrm{C}-3$ in lehualide $\mathrm{F} \ldots \ldots . . .79$

4.15 The $\alpha$-pyrone system present in lehualide $\mathrm{F} \ldots \ldots$. . . . . . . . . . 79

$4.16{ }^{1} \mathrm{H}$ NMR spectral comparison of lehualides $\mathrm{F}$ and $\mathrm{G} \ldots \ldots$. . . . . . 81

4.17 1D TOCSY and bsHSQC-TOCSY correlations used to elucidate the apliphatic chain of lehualide G . . . . . . . . . . . . . . 82

4.18 The dimethoxy-substituted $\alpha$-pyrone segment of lehualide H $\ldots$. . . 84

4.19 The thioacetate moiety of lehualide H . . . . . . . . . . 85

4.20 Positive-ion mode HRESIMS spectra of lehualides I and J . . . . . . . 87

4.21 The methyl sulfide function of lehualide I . . . . . . . . . . . . . 88 
$4.22{ }^{1} \mathrm{H}$ NMR spectral study of lehualide I . . . . . . . . . . . . . . . 89

4.23 The sulfoxide centre of lehualide J. . . . . . . . . . . . . . . . . 89

$4.24{ }^{1} \mathrm{H}$ NMR spectral comparison of lehualide $\mathrm{H}$ and octanethioacetate. . 95

$4.25{ }^{1} \mathrm{H}$ NMR spectral comparison of lehualide I and methyloctylsulfide. . 95

$4.26{ }^{1} \mathrm{H}$ NMR spectral comparison of lehualide $\mathrm{J}$ and methyloctylsulfoxide. 95

4.27 A diod spicule recovered from the plakinid sponge PTN3_19D . . . . 105

4.28 Correlations evidencing substructures in the screen HMBC spectrum of PTN3_19D ......................... 106

4.29 Overall purification strategy employed during isolation of the cyclic peroxide metabolites . . . . . . . . . . . . . . 108

4.30 COSY and HMBC correlations establishing the C-1 to C-4 linkages of epiplakinic acid I . . . . . . . . . . . . . . . . . . . . . . 109

4.31 HMBC correlations establishing the C-3 to C-6, and C-19 linkages epiplakinic acid I . . . . . . . . . . . . . . . . . . . . . . 110

4.32 COSY and HMBC correlations establishing the C-14 to C-17 segment of epiplakinic acid I . . . . . . . . . . . . . . . . . . . 110

4.33 1D TOCSY correlations establishing the $\mathrm{C}_{9}$ methylene chain of epiplakinic acid I . . . . . . . . . . . . . . . . . . . . . 111

$4.341 \mathrm{D}$ NOE correlations proving relative stereochemistry of the 1,2dioxolane nucleus of epiplakinic acid I . . . . . . . . . . . . . . 111

4.35 The C-21 methyl ester moiety of epiplakinic acid I methyl ester. . . . 113

4.36 COSY and HMBC correlations establishing the C-1 to C-5 connections within plakortolide $\mathrm{X} \ldots \ldots . \ldots 115$

4.37 COSY and HMBC correlations confirming the C-3 to C-5 connections in plakortolide $\mathrm{X} \ldots \ldots . \ldots 116$

4.38 HMBC correlations establishing the C-5 to C-7 linkages in plakortolide $\mathrm{X} \ldots \ldots \ldots \ldots$. . . . . . . . . . . . . . . . . . . . . . . . .

4.39 1D TOCSY correlations comfirming linear connectivity in plakortolide X . . . . . . . . . . . . . . . . . . 117

4.40 Stereochemistry about the bicyclic nucleus of plakortolide X . . . . . 117

4.41 COSY and selected HMBC correlations evidencing the C-3 to C-5 connections in the 1 -hydroxy-1,2-dioxane metabolite . . . . . . . . 120 
4.42 The C-1 to C-4 and C-5 to C-7 connections in the 1-hydroxy-1,2dioxane metabolite . . . . . . . . . . . . . . . . 121

4.43 The completed linear structure of the 1-hydroxy-1,2-dioxane metabolite121

4.44 1D NOE correlations evidencing relative configuration in the 1hydroxy-1,2-dioxane metabolite . . . . . . . . . . . . . . 122

4.45 MTT-assays for cyclic peroxides against the HL-60 cell line . . . . . . 125

5.1 Open reading frame deletion within the S. cerevisiae YKO strains . . 140

5.2 Chemical genetic profiling revealing the importance of a deleted gene to strain fitness under assay conditions . . . . . . . . . . . . . . . . . 142

5.3 Synthetic genetic interactions uncovered by the homozygous profiling assay . . . . . . . . . . . . . . . . . . . . 143

5.4 Depiction of drug-induced haploinsufficiency . . . . . . . . . . . . . . 144

5.5 The kinetics of epiplakinic acid I inhibiting growth of S. cerevisiae cells 145

5.6 Epiplakinic acid I, plakortolide $\mathrm{X}$ and $\mathrm{CaCl}_{2}$ cause a similar decrease in cell size in S. cerevisiae strains . . . . . . . . . . . . . . . . . . 146

5.7 Cell cycle of $S$. cerevisiae . . . . . . . . . . . . . . . . . . . . . . 148

5.8 Bud index of S. cerevisiae cells when treated with epiplakinic acid I and plakortolide X. . . . . . . . . . . . . . . . . . . . . . 148

5.9 Depiction of the ion-channel and ATP-pump system for $\mathrm{Ca}^{2+}$ homeostasis in S. cerevisiae . . . . . . . . . . . . . . . . . . . . 154

5.10 Translocation studies in the Crz1p-GFP strain . . . . . . . . . . . . 157

5.11 Qualitative staining of WT cells with Calcium Orange dye . . . . . . 158

5.12 Flow cytometry imaging ROS formation with DCF-DA and PI . . . . 159

5.13 Calcineurin signaling pathway in S. cerevisiae . . . . . . . . . . 160

5.14 Antagonistic interactions between the calcineurin and TORC2 signaling pathways . . . . . . . . . . . . . . . . . . 161

A.1 A depiction of the cyclic loading process. . . . . . . . . . . . . 196

H.1 Dose-response curves for epiplakinic acid I and plakortolide X against WT and $\Delta p d r$ yeast strains . . . . . . . . . . . . . . . . . 305

H.2 Activity of epiplakinic acid I and plakortolide $\mathrm{X}$ is enhanced in the presence of high $\mathrm{Ca}^{2+} \ldots \ldots$. . . . . . . . . . . . . 306 
H.3 Dose-response curves for epiplakinic acid I and plakortolide $\mathrm{X}$ in the presence of EGTA . . . . . . . . . . . . . . . . . . . . 307

H.4 Epiplakinic acid I causes an increase in S-phase population in $S$. cerevisiae cells. . . . . . . . . . . . . . . . . . . 307

H.5 Epiplakinic acid I affects a decrease in cell size in S. cerevisiae. . . . . 308

H.6 Hypersensitivity of cytoskeletal mutants to epiplakinic acid I . . . . . 308

H.7 Dose-response curves for epiplakinic acid I against $\mathrm{Ca}^{2+}$-responsive deletions strains . . . . . . . . . . . . . . . 309

H.8 Translocation studies in the Zrt1p-GFP strain . . . . . . . . . . . . . 310 


\section{List of Schemes}

3.1 Formation of the labdane, halimane and clerodane skeletons . . . . . 60

3.2 Proposed generation of the hemi-acetal functions, furan and $\gamma$ hydroxybutenolide moieties of luakuliides A-C . . . . . . . . . . . 61

4.1 Conversion of plakortolides to plakortones via seco-plakortolides. . . . 66

4.2 Synthesis of the spectroscopic model compounds of lehualides H-J. . 94

4.3 Mechanism for $\alpha$-pyrone degradation as proposed by Kong et al. . . . 101

4.4 Formation of coenzyme M from L-cysteine and sulfoactaldehyde. . . . 102

4.5 Proposed biogenesis for lehualides $\mathrm{H}-\mathrm{K}$. . . . . . . . . . . . . . . . 103

4.6 Biosynthesis of cyclic peroxides via Diels-Alder cycloaddition of molecular oxygen . . . . . . . . . . . . . . . . 130

4.7 Proposed biosynthesis of 1,2-dioxolane cyclic peroxides . . . . . . . . 132

4.8 Proposed biosynthesis of 1,2 -dioxane cyclic peroxides . . . . . . . 133

4.9 Proposed biosynthesis of the plakortolide bicycle by intramolecular cyclisation . . . . . . . . . . . . . . . . . 134

4.10 Proposed biosynthesis of the 1-hydroxy-1,2-dioxane metabolite . . . . 135 


\section{List of Tables}

2.1 Sponge Specimens Analysed During This Study. . . . . . . . . . . . . 21

3.1 NMR Spectroscopic Data for the Acetal Moieties of Luakuliide A and Luakuliide A Methyl Acetal . . . . . . . . . . . . . . . . . . . 46

3.2 NMR Spectroscopic Data of Luakuliide A . . . . . . . . . . . . . . 47

3.3 NMR Spectroscopic Data of Luakuliide A Methyl Acetal . . . . . . . 48

3.4 NMR Spectroscopic Data of Luakuliide B . . . . . . . . . . . . . . . 53

3.5 NMR Spectroscopic Data of $\gamma$-Hydroxybutenolide Moieties . . . . . . 55

3.6 NMR Spectroscopic Data of Luakuliide C . . . . . . . . . . . . . . 56

4.1 NMR Spectroscopic Data of Lehualide E . . . . . . . . . . . 76

4.2 NMR Spectroscopic Data of Lehualide F . . . . . . . . . . . . . . 80

4.3 NMR Spectroscopic Data of Lehualide G . . . . . . . . . . . . . . 83

4.4 NMR Spectroscopic Data of Lehualide H . . . . . . . . . . . . 86

4.5 NMR Spectroscopic Data of Lehualide I . . . . . . . . . . . . . . 90

4.6 NMR Spectroscopic Data of Lehualide J . . . . . . . . . . . . . . . 91

4.7 Inhibition of HL-60 Cells by Lehualides F-I . . . . . . . . . . . . . . 92

4.8 NMR Spectroscopic Data of Lehualide K . . . . . . . . . . . . . . . . 93

4.9 Chemical Shift Analysis of the Sulfur Moieties in Lehualides H-J . . . 97

4.10 NMR Spectroscopic Data of Epiplakinic Acid I . . . . . . . . . . . . . 112

4.11 NMR Spectroscopic Data of Epiplakinic Acid I Methyl Ester . . . . . 114

4.12 Comparison of Salient NMR Chemical Shifts in Plakortolides T and X 118

4.13 NMR Spectroscopic Data of Plakortolide X . . . . . . . . . . . . . . 119

4.14 Comparison of Salient NMR Chemical Shifts of 1-hydroxy-1,2-dioxane Metabolites . . . . . . . . . . . . . . . . 123

4.15 NMR Spectroscopic Data of the 1-Hydroxy-1,2-Dioxane Metabolite 98124

5.1 Synergistic Inhibitory Effects of Cyclic Peroxides and $\mathrm{CaCl}_{2} \ldots \ldots$ 
5.2 Decrease in Cell Size Caused by Cyclic Peroxides and $\mathrm{CaCl}_{2}$. . . . 147

5.3 Effects of Epiplakinic Acid I on the S. cerevisiae Cell Cycle . . . . . . 148

5.4 Functional Categories of Deletion Strains Sensitive to Epiplakinic Acid I . . . . . . . . . . . . . . . . . . . . . . . . 150

5.5 Growth Inhibitory Effects of Epiplakinic Acid I on Cytoskeletal Deletion Mutant Strains . . . . . . . . . . . . . . . . . . 151

5.6 Functional Categories of Deletion Strains Haploinsufficient to Epiplakinic Acid I . . . . . . . . . . . . . . . . . . . . . . . . 152

5.7 Functional Categories of Genes Differentially Regulated in the Presence of Plakortide F Acid . . . . . . . . . . . . . . . . . . . . 153

5.8 Growth Inhibitory Effects of Epiplakinic Acid I on $\mathrm{Ca}^{2+}$-Responsive Deletion Strains . . . . . . . . . . . . . . . . . . . 155

5.9 Growth Inhibitory Effects of Epiplakinic Acid I on $\mathrm{Ca}^{2+}$-Pump and Channel Deletion Strains . . . . . . . . . . . . . . . 155

5.10 Proposed Ion Pump Deletion-GFP Strains . . . . . . . . . . . . . 163

7.1 Yeast Strains Used During this Study . . . . . . . . . . . . . . . 183

7.2 PCR Master Mix . . . . . . . . . . . . . . . . . . . 188

C.1 NMR Spectroscopic Data of iso-Secothorectolide . . . . . . . . . . . . 202

C.2 NMR Spectroscopic Data of 1-Hydroxyluffarin Q . . . . . . . . . 203

C.3 NMR Spectroscopic Data of Ambliol B . . . . . . . . . . . . . . . 203

C.4 NMR Spectroscopic Data of Luffariellolide . . . . . . . . . . . . . . . 204

C.5 NMR Spectroscopic Data of $5 \alpha, 8 \alpha$-Epidioxysterol 27 . . . . . . . . 205

C.6 NMR Spectroscopic Data of $5 \alpha, 8 \alpha$-Epidioxysterol 28 . . . . . . . . 206

C.7 NMR Spectroscopic Data of 14-Bromohomofascaplysin . . . . . . . . 207

C.8 NMR Spectroscopic Data of Halenaquinone . . . . . . . . . . . . . . . 207

I.1 Homozygous Profile of Epiplakinic Acid I . . . . . . . . . . . . . . . . 311

I.2 Haploinsufficiency Profile of Epiplakinic Acid I . . . . . . . . . . . . . 312

I.3 Haploinsufficiency Profile of Epiplakinic Acid I Continued . . . . . . . 313 
I.4 Functional Categories of Deletion Strains Resistant to Epiplakinic Acid I . . . . . . . . . . . . . . . . . . . . . . . . . . 314

I.5 Functional Categories of Heterozygous Deletion Strains Resistant to Epiplakinic Acid I . . . . . . . . . . . . . . . . . . . . . 315 


\section{Glossary}

\begin{tabular}{|c|c|}
\hline Ac & Acetyl \\
\hline br & Broad \\
\hline $\mathbf{C}_{18}$ & Octadecyl derivatised silica gel \\
\hline COSY & $\begin{array}{l}\text { Correlation spectroscopy }\left({ }^{1} \mathrm{H} \text { to }{ }^{1} \mathrm{H} \text { correlations depicted }\right. \\
\text { by } \longrightarrow \text { and }-)\end{array}$ \\
\hline d & Doublet \\
\hline$\Delta$ & Indicates deletion of the preceding gene \\
\hline$\Delta p d r$ & Drug-sensitised yeast strain \\
\hline$\delta$ & Chemical shift (ppm) \\
\hline DAD & Diode array detector \\
\hline DCF-DA & Dichlorofluorescein diacetate \\
\hline$d_{6}$-DMSO & Deuterated DMSO \\
\hline DIOL & 2,3-Dihydroxy-1-propoxypropyl-derivatized silica gel \\
\hline DMSO & Dimethyl sulfoxide \\
\hline EGTA & Ethylene glycol tetraacetic acid \\
\hline Et & Ethyl \\
\hline EU & European Union \\
\hline FACS & Fluorescence-activated cell sorting \\
\hline FDA & United States Food and Drug Administration \\
\hline GC & Gas chromatography \\
\hline GFP & Green fluorescent protein \\
\hline HIP & Haploinsufficiency profiling \\
\hline HL-60 & Human promyelocytic leukemia cell line \\
\hline HMBC & $\begin{array}{l}\text { Heteronuclear multiple-bond correlation }\left({ }^{1} \mathrm{H} \text { to }{ }^{13} \mathrm{C} \text { correlations }\right. \\
\text { depicted by } \longrightarrow)\end{array}$ \\
\hline HOP & Homozygous profiling \\
\hline HPLC & High pressure (performance) liquid chromatography \\
\hline HP20/HP20SS & PSDVB stationary support \\
\hline HRESIMS & High resolution electrospray ionization mass spectrometry \\
\hline HSQC & Heteronuclear single-quantum correlation \\
\hline HTS & High-throughput screening \\
\hline $\mathrm{IC}_{50}$ & Dose that is inhibitory to $50 \%$ of test subjects \\
\hline ICP-MS & Inductively coupled plasma mass spectrometry \\
\hline IPA & Isopropyl alcohol (2-propanol) \\
\hline$J$ & Scalar coupling constant \\
\hline LB & Lysogeny broth, bacterial growth media \\
\hline LH-20 & Crosslinked dextran-based size exclusion resin \\
\hline $\mathbf{m}$ & Multiplet (NMR coupling pattern) \\
\hline$m / z$ & Mass to charge ratio \\
\hline
\end{tabular}


MALDI-TOF Matrix-assisted laser desorption/ionisation time of flight

MDR Multiple drug resistance

Me Methyl

MIC Minimum inhibitory concentration

mult. Multiplicity

MS Mass spectrometry

MTT assay Colormetric cytotoxicity assay using

3-(4,5-dimethyl-thiazol-2-yl)-2,5-diphenyltetrazolium bromide

NMR Nuclear magnetic resonance

NOE Nuclear Overhauser effect $\left({ }^{1} \mathrm{H}\right.$ to ${ }^{1} \mathrm{H}$ enhancements depicted by $\longrightarrow)$

NOESY Nuclear Overhauser enhancement spectroscopy

PCR Polymerase chain reaction

PDR Pleiotropic drug resistance

Pet. ether Petroleum ether (hexanes)

PI Propidium iodide

pos. Position

ppm Parts per million

PSDVB Poly(styrene-divinylbenzene)

q Quartet

quin Quintet

RFP Red fluorescent protein

$\mathrm{S}$

Singlet (NMR coupling pattern)

SAM S-adenosylmethionine

$\mathrm{SC}$

Synthetic complete yeast growth media

SD Synthetic deficient yeast growth media

SEM Scanning electron microscope

sept Septet

SCUBA Self contained underwater breathing apparatus

t Triplet

TLC Thin layer chromatography

TOCSY Total correlation spectroscopy $\left({ }^{1} \mathrm{H}\right.$ to ${ }^{1} \mathrm{H}$ correlations depicted by $\longrightarrow$ and $\longrightarrow$ )

VUW Victoria University of Wellington

WT Wild type yeast

YPD Yeast extract peptone dextrose, complete medium for yeast growth 


\section{Chapter 1}

\section{Introduction}

\subsection{Natural products as therapeutic agents}

For millennia human societies have relied upon nature for their fundamental needs, and for medicines in particular. ${ }^{1}$ Egyptian documentation of this relationship dates back to $4000 \mathrm{BC},{ }^{1}$ however, the treatment of human disease is still a pressing and costly concern. Care and support of cancer patients alone in the US totaled more than 124 billion USD in $2010 .^{2}$ As the leading cause of death in New Zealand, cancers account for $29.4 \%$ of all deaths at an estimated cost of 511 million NZD per year. ${ }^{3}$ As such, the development of new therapeutic agents is beneficial to society, if only to lessen these financial burdens.

Naturally-derived compounds continue to play an integral role in the treatment of human disease, and the pharmaceutical landscape is heavily populated by nonsynthetic compounds. ${ }^{4}$ Over the past 30 years roughly $60 \%$ of the introduced small molecule pharmaceutical leads have been natural products, derivatives thereof, or synthetic mimics of natural product pharmacophores. ${ }^{4-7}$ One such entity is the microtubule stabiliser paclitaxel (1, TAXOL ${ }^{\circledR}$, Bristol-Myers Squibb). Isolated from the Pacific yew tree, Taxus brevifolia in 1967, paclitaxel has been marketed from the early 1990s and is estimated to have extended the lives of several hundred thousands of lung, ovarian and breast cancer patients. ${ }^{8}$ Annual sales of TAXOL ${ }^{\circledR}$ peaked at 1.6 billion USD in 2000. ${ }^{9}$ Another significant example is the immunosuppressive macrolide rapamycin $\left(\mathbf{2}\right.$, Rapamune ${ }^{\circledR}$, Wyeth). First developed as an antifungal agent, rapamycin has been used widely since 1999 for the prevention of organ transplant rejection. ${ }^{4}$ In 2011 a total of 94,493 life-saving organ transplants were carried out in the US alone, with each patient then requiring immunosuppressive treatment for an average cost of more than 24,000 USD per annum. ${ }^{10}$

These two structurally different and important therapeutic agents are both products of a living organism's secondary metabolism. Secondary metabolites are nonessential to the immediate survival of an organism as they are not involved in 
normal growth, development or reproduction. ${ }^{11}$ Significant biochemical input is required during their synthesis, justified by the physiological advantages they confer upon the producing organism. ${ }^{12}$ Organisms devoid of formal cellular immune systems and/or adequate forms of physical protection are thought to rely upon secondary metabolites to deliver an alternative chemical defence system against assault. ${ }^{13}$ Consequentially, a chemical arsenal of metabolites have been isolated from such organisms and display activities including antifungal, antimicrobial, and cytotoxicity. ${ }^{14}$ While these metabolites may not have co-evolved with human receptors they have been evolved to interact with biomolecules in general. ${ }^{6}$ It is this innate and varied biological activity that has made secondary metabolites and their derivatives integral to the development of medicinal chemistry to the point where they are estimated to have more than doubled the average life-span of human beings in the 20 th century alone. ${ }^{15}$

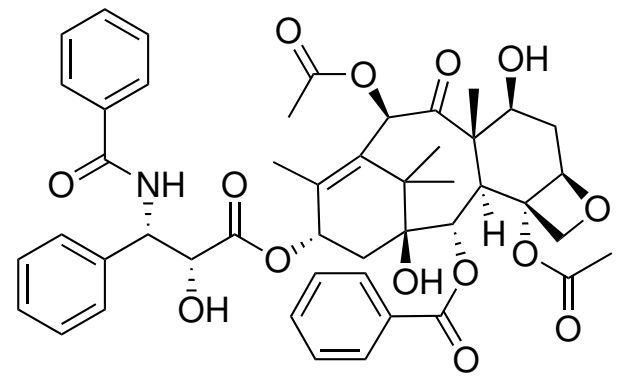

1

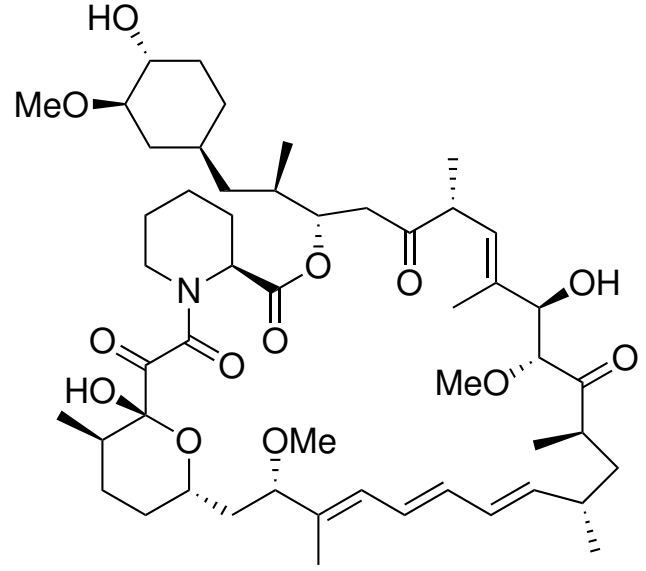

2

In response to growing demand for new therapeutic agents, the 1990s saw the advent of high-throughput screening (HTS) methods and combinatorial chemistry techniques. These new HTS techniques made screening entire compound libraries faster than it was to isolate and characterise a single natural product. However, the use of molecular scaffolds and combinatorial techniques has resulted in libraries of topographically similar molecules, ${ }^{16}$ many of which have not demonstrated biological characteristics appropriate for clinical trials. Sorafenib (3, Nexavar, Bayer) is the only compound developed by these methods to have gained FDA approval, ${ }^{4,17}$ marketed since 2005 for the treatment of renal carcinomas.<smiles>CNC(=O)c1cc(Oc2ccc(NC(=O)Nc3ccc(Cl)c(C(F)(F)F)c3)cc2)ccn1</smiles> 
Structural trends have been observed between artificially designed and naturally occurring molecules, and although subtle, these differences between the compound classes appear to induce drastic differences in target specificity and biological potency. ${ }^{5,18,19}$ Compounds of natural derivation generally exhibit a greater number of chiral centres, and corresponding steric complexity. Oxygen-based functions predominate those of nitrogen, sulfur and the halogens, providing a high number of hydrogen-bond donors. There is also a trend towards greater aromaticity within the structures. $^{20-23}$ As many of these aspects are readily incorporated into synthetic strategies, synthetic chemistry will maintain an integral role in pharmaceutical development. Although, our improving understanding of the genetic basis to the production of some natural products may offer new possibilities for large-scale production of such compounds in the future. It is clear, however, that the unmatched structural diversity and complexity evident within natural products will continue to inspire synthetic chemists in years to come. Natural products chemistry will remain essential to drug discovery, ${ }^{18,24}$ in the continued search for new and unexplored areas of chemical space. ${ }^{25,26}$

\subsection{Marine natural products}

Oceans encompass $70 \%$ of the Earth's surface ${ }^{27}$ forming a myriad of ecosystems that are home to an estimated $90 \%$ of all species. ${ }^{27}$ Of the 38 identified animal phyla, 26 are exclusively aquatic and 19 of these are exclusively marine. In comparison, Onchyophoria (the velvet worms) is the only living, solely terrestrial phylum. It is therefore unsurprising that environments such as coral reefs and the deep sea floor are estimated to house a greater diversity of flora and fauna than tropical rainforests, ${ }^{14}$ and are believed to contain a similar degree of chemical diversity.

As of 2011 more than 24,000 novel marine natural products have been reported, ${ }^{28}$ displaying a diverse array of structures and chemical functionality. ${ }^{24}$ Owing to the availability of chlorine and bromine in seawater, halogen atoms are frequently observed in marine metabolites, in comparison to their terrestrial counterparts. ${ }^{24}$ Cyanate (4), isothiocyanate (5) functions have appeared primarily in the marine environment, while the dichloro-imine function of compound $\mathbf{6}$ has only been observed in sponge metabolites. ${ }^{24}$ Structural singularity in marine natural products also extends to carbon skeletons. The ladder-like cyclic polyether structure is characteristic of the marine toxins, exemplified by maitotoxin $(\mathbf{7})$, the largest nonproteinaceous toxin known. ${ }^{29}$ 


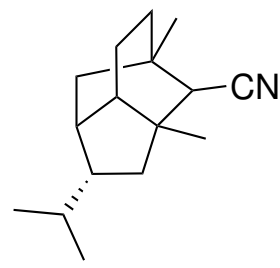

4

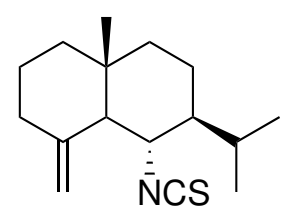

5<smiles>CC(C)=CCCC1CC(=C(Cl)CN=C(Cl)Cl)CC(O)C1Cl</smiles>

6

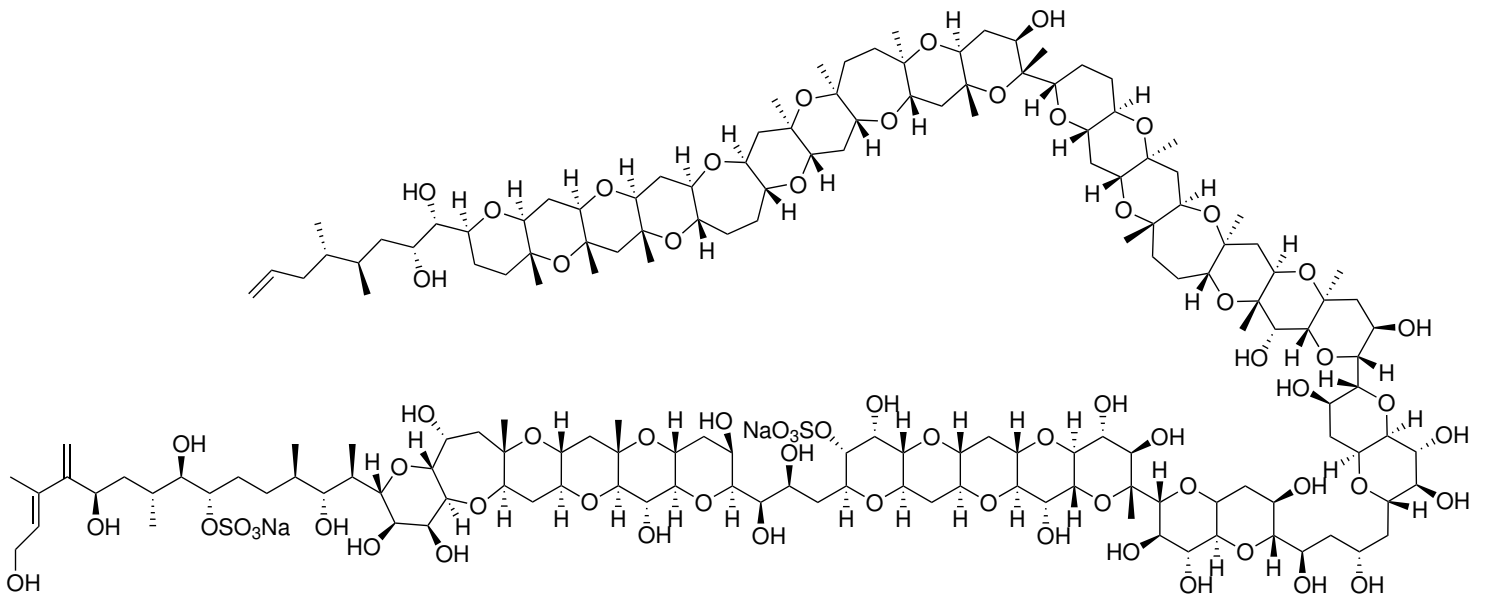

7

Many of the organisms investigated from the marine environment are sessile invertebrates, most of which lack both physical defences and formal cellular immune systems and require potent and diverse chemical defences. ${ }^{13}$ Owing to the intrinsic dilution factor of the marine environment, compounds for chemical defence must be highly potent to elicit an effect upon release into the surroundings. ${ }^{4}$

The isolation of potent toxins, particularly those known to humans, initially dominated the natural products field. Tetrodotoxin (8) was widely utilised in Voodoo practices in the creation of the 'living-dead'. ${ }^{30}$ The neurotoxin acts by binding to site 1 of the fast voltage-gated $\mathrm{Na}^{+}$channels found in most forms of muscle tissue detrimentally affecting muscle contraction and silencing neural activity. ${ }^{31}$ Tetrodotoxin (8) is produced by the bacteria Vibrio alginolyticus, which is harboured by Taricha sp. newts, Atelopus sp. toads, and the blue-ringed octopus Hapalochlaena sp. ${ }^{32}$ Occurrence of the bacteria within species of puffer fish led to the infamy of $\mathbf{8}$ as a source of lethal food poisoning. ${ }^{31,32}$

The release of toxic chemical entities by sponges and other marine organisms is not solely restricted to defence. Pheromones are released for communication between organisms of many species, for instance quorum sensing by dinoflagellates preceding algal blooms. ${ }^{33}$ Another dinoflagellate isolate, saxitoxin (9), has gained notoriety as the source of human paralytic shellfish poisoning (PSP) in tainted shellfish. The molecule shares the tetrodotoxin binding site, and has also been isolated from some species of puffer fish. ${ }^{34}$ 


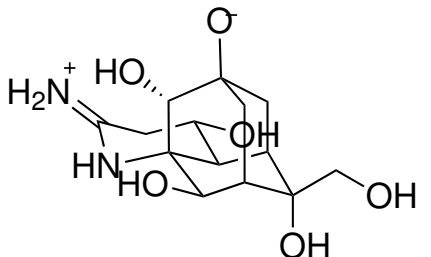

8

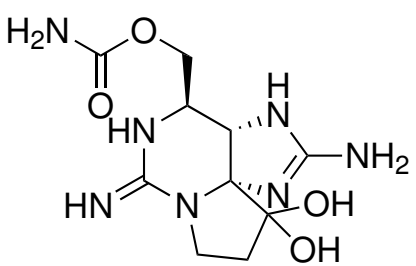

9

Investigation of marine cone snails of the genus Conus from the west and south-western Pacific eventually led to development of ziconotide (10, Prialt ${ }^{\circledR}$, Neurex/Elan), the first true marine natural product to gain FDA approval. ${ }^{35}$ The fish-hunting cone snails spear their prey with harpoon-like hollow teeth filled with incapacitating venom. The peptidic toxins are highly potent and selective, acting as both agonists and antagonists of the ligand- and voltage-gated ion channels, and G-protein-coupled-receptors. ${ }^{36}$ Intrathecal delivery of the synthetic $\omega$-conotoxin 10 was approved by the FDA and EU in 2004 and 2005 respectively. ${ }^{35}$ As the most potent non-opioid analglesic, the development of ziconotide has been a particularly important success in the marine natural products field.

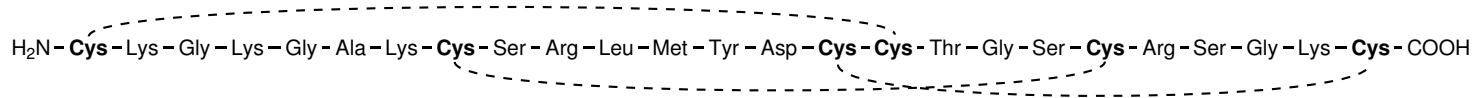

\subsection{Sponges}

Sponges (phylum Porifera), are the oldest metazoans (multicellular organisms) ${ }^{37}$ and have been in wide-spread existence since the pre-Cambrian era, ca. 560 million years ago. ${ }^{38}$ Taxonomy within the Porifera phylum is uncertain, with reclassifications frequently taking place even at class level. Environmental factors exert a dramatic influence over physical characteristics of sponges such as size, colour and shape, preventing the use of macro-morphological and -physical characteristics as tools in sponge description. ${ }^{38}$ Instead, skeletal networks form the basis of classification, dividing phylum Porifera into three classes. The class Demospongiae encompasses 80-90\% of all sponge species, and the organisms contain siliceous and/or spongian fibres. Calcarea sponges exhibit calcite $\mathrm{CaCO}_{3}$ spicules, while Hexactinellida sponges have a siliceous spicule network that comprises most of the mass of the organism (see Figure 1.1).

Sponges are devoid of features commonly found in other animals including developed circulatory, digestive, nervous and muscular systems. ${ }^{38}$ In combination with a low level of cellular organisation and vegetative appearance, these absences prevented the classification of the phylum into Animalia until the 18th century. ${ }^{38}$ These 
sessile filter feeders are also devoid of a formal cellular immune system, and rely upon their secretion of toxic secondary metabolites to ward off unwanted bacterial, fungal, and parasitic infections. ${ }^{13,37}$ These metabolites also offer protection against encroachment, ${ }^{13}$ and surface encrustation resulting in the disruption of their waterpumping systems. ${ }^{38}$ Perhaps unsurprisingly, sponges are the source of over $30 \%$ of the marine natural products isolated over the last few decades. ${ }^{28,39,40}$

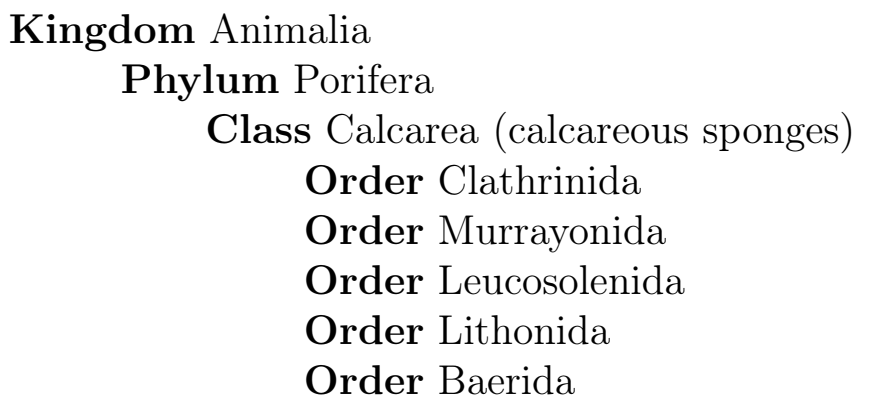

Class Hexactinellida (glass sponges)

Order Amphodiscosida

Order Hexactinosida

Order Aulocalycoida

Order Lychnicosida

Order Lyssacinosida

Class Demospongiae (spongin fibres)

Order Homosclerophida

Order Spirophorida

Order Astrophorida

Order Hadromerida

Order Chondrosida

Order Poecilosclerida

Order Halichondrida

Order Agelasida

Order Haplosclerida

Order Dictyoceratida

Order Dendroceratida

Order Halisarcida

Order Verongida

Order Verticillitida

Figure 1.1 Taxonomic classification within the phylum Porifera to order level, after Hooper and van Soest. ${ }^{38}$

An early foray into the metabolite profile of the sponge Cryptotethia crypta by Bergmann, Feeney and Burke yielded the unusual arabinosyl nucleosides spongothymidine (11) and spongouridine (12). ${ }^{41-43}$ Subsequent development of synthetic analogues generated the anticancer agent Ara-C (13) and the antiviral agent azidothymidine or AZT (14, Retrovir ${ }^{\circledR}$, Glaxo-SmithKline). ${ }^{44,45}$ AZT provided a major break-through in the AIDS therapy in 1990s, helping to dispel the notion that HIV/AIDS was a death sentence, ${ }^{45}$ and is still in use today. Recent efforts have seen a synthetic reproduction of the pharmacophore of the macrocyclic- 
polyether halichondrin B, isolated from Halichondria okadai, gained FDA approval in November 2010. The microtubule-targeting qualities of halichondrin B were recognised shortly after its reported isolation in 1986, and administration of the derivative Halaven (15, Eisai Co.) for the treatment of non-small cell lung cancers, prostate cancer, sarcoma and breast cancers was approved 24 years later. ${ }^{46,47}$

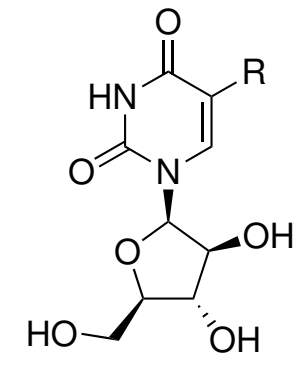

$11 \mathrm{R}=\mathrm{Me}$

$12 \mathrm{R}=\mathrm{H}$<smiles>Nc1ccn([C@@H]2O[C@H](CO)[C@@H](O)[C@H]2O)c(=O)n1</smiles>

13<smiles>Cc1cn([C@H]2CC([N+]#N)[C@@H](CO)O2)c(=O)[nH]c1=O</smiles>

14

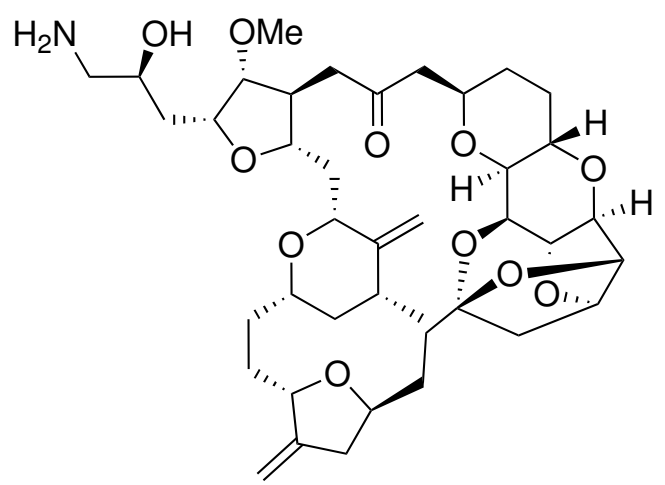

15

\subsubsection{Microbial symbiosis in marine sponges}

Many sponges are known to host an array of microbial symbionts, which can account for up to $60 \%$ of the living tissue in some sponges. ${ }^{48,49}$ The true extent of microbial contribution to the secondary metabolite profile of sponges is not known, primarily due the difficulties associated with ex-host culturing of many of these microbial symbionts. ${ }^{24,49}$ Microbial origins are often inferred when isolates from a single organism display unusual structural diversity, or conversely, metabolites classes are isolated from several unrelated sponges. Structural motifs typical of bacterial metabolism are also indicative of microbial origin, such as the polyketide cyclic peroxides frequently encountered in Indo-Pacific Plakortis and Plakinastrella sponges. Cellular localisation of many typical Plakortis simplex metabolites in cells of the Sphingomonas strain PS193 has been demonstrated, although polyketide cyclic peroxides were not among these. ${ }^{50}$ Physical separation of sponge cells from their associated symbiotic cyanobacteria has also been successful. Such work has indicated cyanobacterial origins (Oscillatoria spongelia) for 13-dimethylisodysodenin 
(16), which was previously classified as a metabolite of the sponge Dysidea herbacea. ${ }^{51}$<smiles>CC(CC(=O)N(C)C(CCC(Cl)(Cl)Cl)C(=O)NCc1nccs1)C(Cl)(Cl)Cl</smiles>

16

Investigations of Svenzea, ${ }^{52}$ Mycale, ${ }^{53}$ Myxilla and Haliclona sponges ${ }^{54}$ by Lee et al. have shown that up to $75 \%$ of sponge-associated bacteria are vertically transferred during embryogenesis, as opposed to horizontal transfer from the environment. ${ }^{52}$ This demonstrates a species-specific relationships between sponges and their bacterial communities. ${ }^{54}$

\subsection{The Tongan marine ecoregion}

While the VUW marine natural products program has traditionally investigated organisms from New Zealand, the past few years have seen frequent recollection of the same organisms and the isolation of similar metabolites. Therefore in this study new collection areas were selected to provide new organisms for investigation. The Kingdom of Tonga was chosen as such a site (Figure 1.2).

The Tongan archipelago is located in the south-west Pacific Ocean, bordered to the west by the Fijian islands, and to the north by the Samoan territories. The Territorial Sea with and Exclusive Economic Zone of the kingdom comprises of $660,000 \mathrm{~km}^{2}$ encompassing 171 islands, 52 of them inhabited, ${ }^{56}$ which are divided into three main groups - Vava'u, Ha'apai and Tongatapu. The subtropical Tongan marine environment offers a range of habitats, which coupled with geographical isolation intrinsic to the island nation and a number of major ocean currents in the region create diverse marine communities.

Although Tonga is home to an abundance of marine life, there have been very few reports of secondary metabolites isolated from Tongan marine organisms. The last compound reported from Tongan waters was in 2001, although a number of polyketide and terpenoid structures were reported during the $1980 \mathrm{~s} .{ }^{57,58}$ However, environmental similarity across the Indo-Pacific region should allow parallels to be drawn with metabolites isolated from organisms collected in the neighbouring Fijian islands, while the change in geographical location may induce subtle chemical 


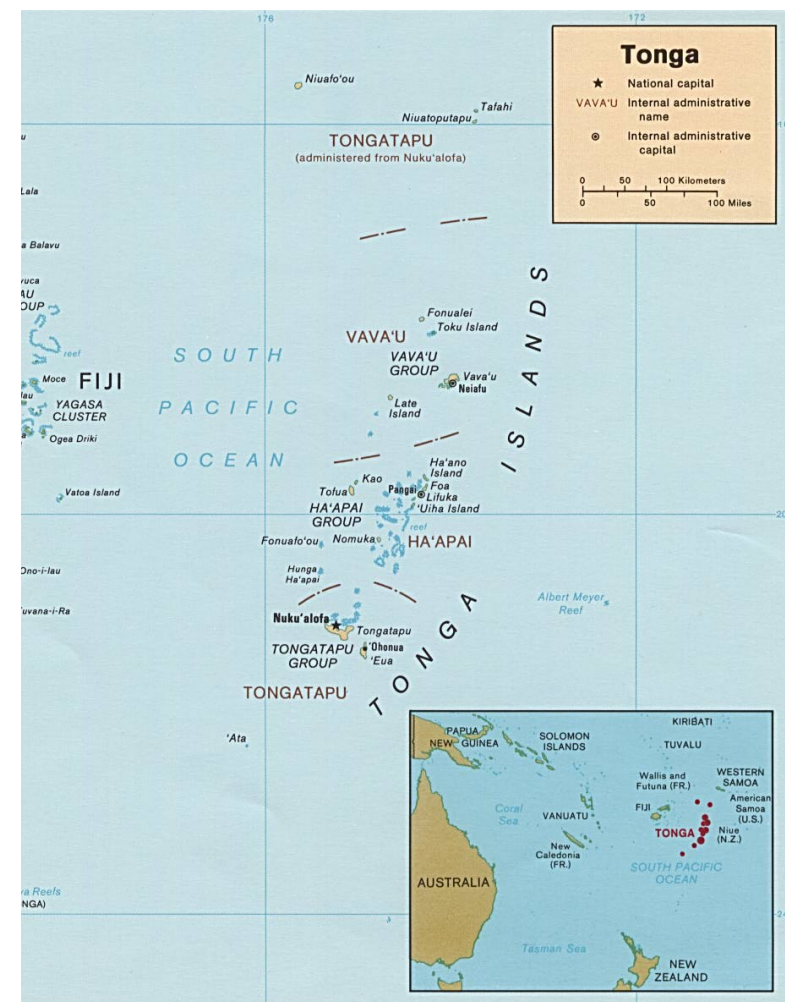

Figure 1.2 The Kingdom of Tonga. ${ }^{55}$

differences. Chemical analysis of a Fijian Cacospongia mycofijiensis specimen during the 1980s yielded the macrolides laulimalide (17), latrunculin A (18) and mycothiazole (19). ${ }^{57-59}$ The hypothesis of chemical similarity across the Indo-Pacific region was proven by analysis of an 'Euan C. mycofijiensis specimen within this laboratory, which afforded both 19, 18, and isomers of 17. However, the 'Euan specimen was also found to contain small quantities of zampanolide (20), ${ }^{60}$ which had not been reported since its initial isolation in $1996 .{ }^{61}$ The potent microtubule stabilising activity of $\mathbf{2 0}$ was subsequently uncovered by Field et al. ${ }^{62}$

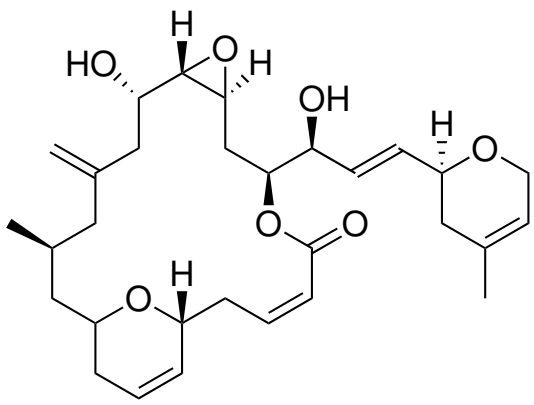

17<smiles></smiles>

18 
<smiles>C=CC/C=C\Cc1csc(C(C)(C)[C@@H](O)C/C=C/C(=C)CCNC(=O)OC)n1</smiles>

19

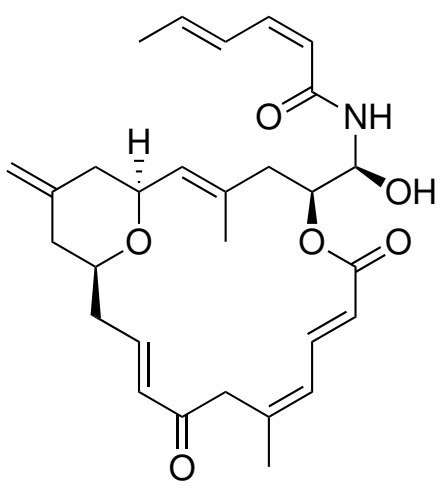

20

\subsection{Organism selection and screening}

There are several approaches to the selection of organisms to screen. Organisms with ethnopharmacological* history are frequently investigated as probable sources of potently bioactive compounds, albeit without structural novelty. Taxonomy is also considered; organisms closely related to species proven to produce unique and bioactive molecules are frequently collected. ${ }^{63}$ Random sampling of organisms from several locations is a fruitful approach in discovering new metabolites, although laborious and expensive. ${ }^{63}$ As the microbial populations within sponge specimens and their metabolites profiles vary in response to changes in environment stresses, ${ }^{39,60}$ the investigation of well-known species from different environments should culminate in isolation of new compounds.

The Indo-Pacific in general has been well examined by natural product research programs, ${ }^{57-59,61}$ and similar species diversity is observed across the region. Hence, this study will focus on the investigation of encrusting organisms that are difficult to collect, and those with cryptic colouration and sparse distribution. Such organisms are less likely to have been subject to prior investigation in Tonga and more likely to yield structural novelty. Analysis of organisms from unexplored or geographically isolated areas will also be undertaken, although logistical difficulties may hinder the recollection of organisms collected from remote areas.

\subsubsection{Bioassay-guided isolation}

Bioassay-guided isolation has been the governing paradigm in natural products research since the early 1980s. Used successfully for decades, the approach involves screening crude biological extracts from target organisms against assays for specific modes of activity. Although the method guarantees a biologically useful endproduct, it is not without flaws. Such studies culminate in structural elucidation only

\footnotetext{
*Ethnopharmacology entails the study of plants used in traditional medications and their active constituents.
} 
after iterative cycles of chromatography and bioassay assessment, hence frequently result in isolation of known compounds. ${ }^{24}$ The monetary value of a bioactive compound is significantly lessened without structural novelty: one can claim rights to a specific use, but not governing 'ownership' rights to the structure. Assessing crude extracts also precludes the use of more sophisticated biological assays. Assay bias may also disregard a wide range of metabolites present in an organic extract, and risk an incomplete understanding of a given compound's full biological potential. Pharmaceuticals such as rapamycin (2) are currently marketed for a use unrelated to the application for which they were initially intended. Furthermore, in vitro testing cannot adequately assess a compound's in vivo activity.

From a practical perspective, the wide range of polarities present in a crude extract can render a compound insoluble in the assay media, further excluding potentially bioactive compounds. Potent activity of a low-level compound can also be masked by the mild activities of compounds present at high-levels, while synergistic interactions between compounds within an active fraction can smear a positive biological activity across many fractions during purification. ${ }^{64}$

\subsubsection{Spectroscopy-guided isolation}

Spectroscopy-guided isolation uses chemistry to narrow the isolation focus. As opposed to a positive bioactivity, interesting and uncommon structural motifs are tracked throughout chromatographic cycles. Such focus on unusual structural motifs facilitates structural dereplication at an early stage, increasing the chances of discovering a novel structure free from prior patent claim.

Nuclear magnetic resonance (NMR) spectroscopy is an ideal tool for organism screening and monitoring expedient isolation procedures. Sample preparation and spectral acquisition is quick to perform, and the non-destructive technique allows sample recovery. The ability to discern chemical functionality from ${ }^{1} \mathrm{H}$ NMR spectra is useful in optimisation of chromatographic conditions; from the selection of an appropriate stationary-phase through to the use of ion-exchange media and $\mathrm{pH}$ buffers for acidic and basic chemical moieties. Comparative insensitivity of the technique relative to other forms of spectroscopic analysis is a significant disadvantage. However, new powerful instruments fitted with cryogenic electronics and micro-probes can accommodate small sample sizes $(<1 \mathrm{mg})$ and have dramatically improved the detection of minor compounds. The use of such technologies in 2D correlation experiments allows facile observation of comparatively insensitive nuclei such as ${ }^{13} \mathrm{C}$ and ${ }^{15} \mathrm{~N} .{ }^{5,65}$

In comparison to NMR, greater sensitivity is offered by mass spectrometry (MS), and 
by MS-coupled liquid chromatography and gas chromatography systems (LC-MS and GC-MS). However, ionisation susceptibility can differ widely between molecular classes, hence assessment by NMR can prove more all-encompassing. Additionally, sub-structure identification offered by $2 \mathrm{D}$ NMR spectra can be more informative than that obtained from MS fragmentation patterns.

\subsubsection{Advances in NMR screening}

The use of NMR screening within the VUW marine natural products program has been well developed over the past decade. Expansion from analysis of ${ }^{1} \mathrm{H}$ NMR spectra (Figure 1.3) to digital compilation of 2D heteronuclear single quantum coherence (HSQC) spectra has led to the isolation of many structurally unique biologically active secondary metabolites from New Zealand sponges and red algae. ${ }^{48,60,66}$ The spectral 'masks' of compiled HSQC spectra proved highly useful in organism selection. ${ }^{48,60,66}$ By masking out the common correlations is it relatively simple to determine the degree of structural novelty present within a given spectrum. However, the HSQC spectrum is fundamentally limited to the observation of protonated centres and the application of New Zealand spectral masks to Tongan organisms is questionable. Drastic changes in the metabolite composition of crude extracts may circumvent the 'masking' function of the HSQC screen technologies. ${ }^{60}$

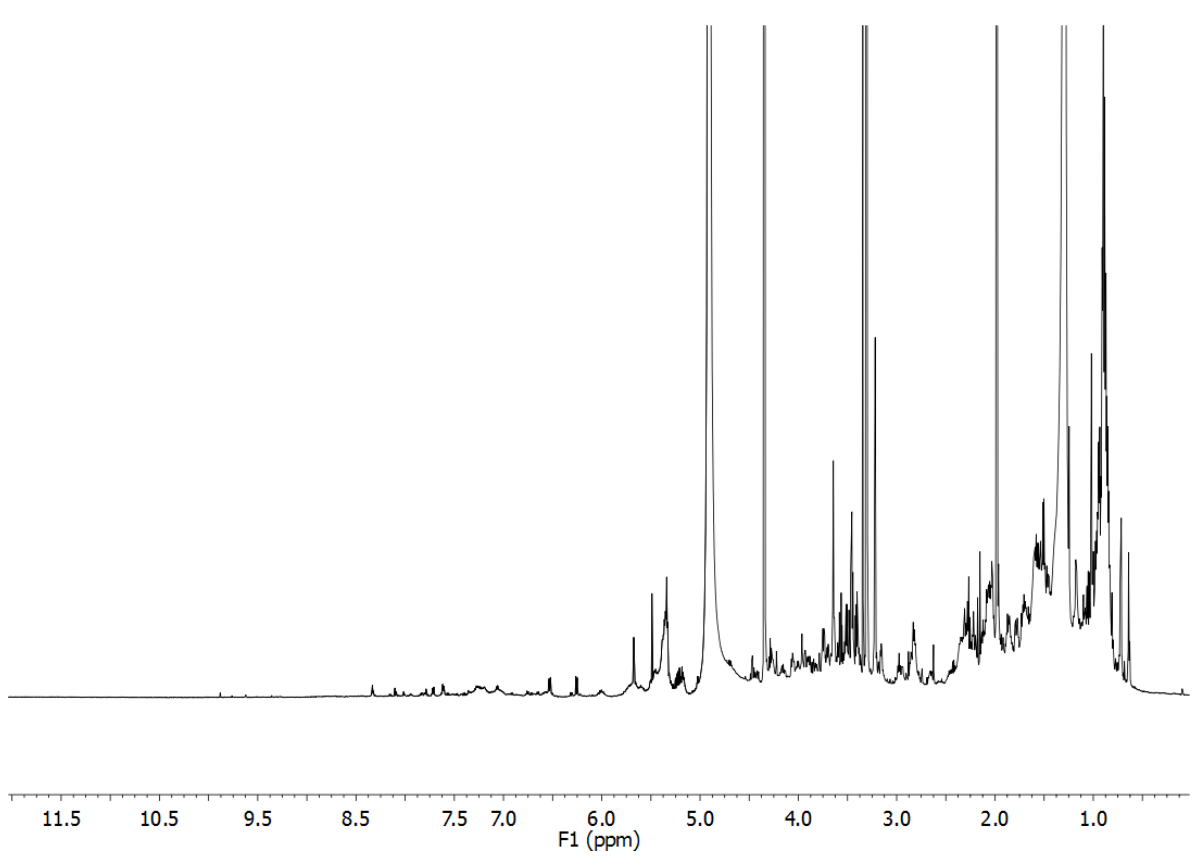

Figure 1.3 A generic ${ }^{1} \mathrm{H}$ NMR spectrum. 


\section{HMBC screening}

Analysis by an heteronuclear multiple bond correlation (HMBC) experiment at the first stage of purification is the natural progression in NMR screening. The HMBC spectrum (Figure 1.4) provides multiple-bond correlations from ${ }^{1} \mathrm{H}$ nuclei to both protonated and non-protonated ${ }^{13} \mathrm{C}$ centres. As such, the experiment should prove a powerful tool allowing the identification of entire families of compounds at an early stage of isolation. ${ }^{60}$ In combination with an HSQC screen, an interesting one-bond correlation can be extended upon by multiple-bond correlations in the HMBC spectrum. Although sub-structure screening can also be performed using the shorter COSY experiment (20 min vs $8 \frac{1}{4} \mathrm{~h}$ ), only protonated linkages are shown in the COSY spectrum and the experiment delivers less spectral dispersion as the ${ }^{1} \mathrm{H}$ spectrum forms both the real (F1) and synthetic (F2) dimensions.

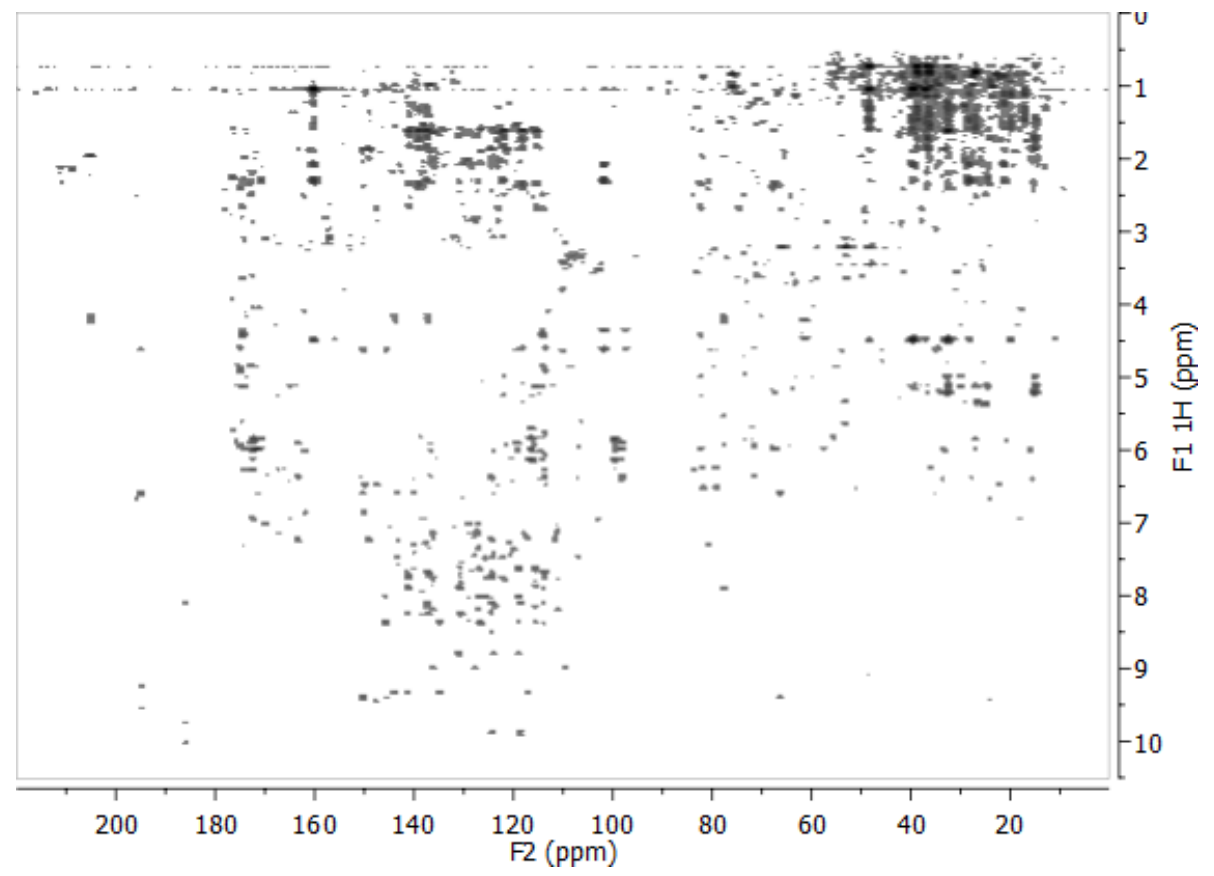

Figure 1.4 A generic HMBC spectrum.

Sub-structure analysis via the HMBC experiment was recently demonstrated by Bertelli et al. in the identification of sugar syrup contamination in various types of honey. ${ }^{67}$ The experiment allowed discrimination between spectroscopic signals of very similar chemical moieties in very similar molecules, namely the anomeric hydroxyls of sugars. Spectral correlations were statistically grouped according to their relative integrations across several fractions, allowing the identification of adulterated honey samples, and for the degree of adulteration to be quantified. ${ }^{67}$ The same approach has been used to determine the geographical origin of foodstuffs ${ }^{68}$ and to assess age-related tainting. ${ }^{69}$ Natural product studies have employed the techniques for chemotaxonomy, identifying the general classes of metabolites present within organism extracts. One such study allowed distinctions to be made between 
the extrudes from the conifer families Araucariaceae, Cupressaceae, and Pinaceae and the resins produced by the angiosperm family Fabaceae. ${ }^{70}$

The premise behind NMR-based screening methodology is that a novel structure often corresponds to a novel biological activity, however, a novel structure still requires a biological application and as such, bioassay methods cannot be ignored. As the antithesis of bioassay-guided isolation, ignorance of potential biological application is the primary disadvantage of this structure-guided isolation method. For this reason, spectroscopy-guided isolation will in all likelihood remain subordinate to bioassay-guided studies, although the approach is a viable alternative for research groups without access to a wide variety of sophisticated biological assays.

\subsection{Assigning biological applications}

Assigning biological application to small molecules isolated in the absence of biological assessment requires a broad-spectrum assay approach. A viable option is to test pure compounds individually, or as part of a compound library against a series of selected cell lines or protein-based assays. Although library generation via either synthesis or isolation requires significant time and labour, the approach delivers highly specific and wide-ranging bioactivity information. Accurate determination of biological activities is invaluable to progression of drug leads through pre-clinical and clinical testing phases.

It is often assumed that structurally similar molecules will display similar modes of activity. This is not always the case, however. Despite structural similarities between members of the resorcyclic acid lactone family, the compounds display a wide variety of biological activities. ${ }^{71}$ Radicicol (21) potently and selectively competes for ATPbinding pocket of HSP90, a molecular chaperone responsible for the maturation and stability of several oncogenic genes. ${ }^{72}$ Conversely, zearalone (22) interacts with the estrogen pathway, and has been shown to adopt a conformation mimicing that of 17 -estradiol, ${ }^{73}$ and interact with the estrogen receptor in direct competition with the sterol. ${ }^{74}$<smiles>C[C@H]1C[C@H](O)/C=C\C=C/C(=O)Cc2c(Cl)c(O)cc(O)c2C1=O</smiles>

21

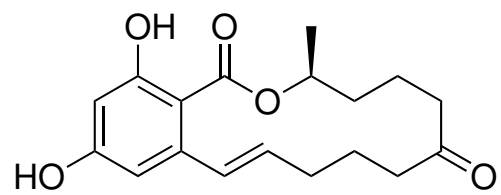

22

In light of these findings assay selection requires great consideration. The most 
logical approach would to test a pure compound or library against common screening assays, followed by more specific and sophisticated assays. This is, however, strictly limited by the type of assays available, and by the quantities of purified material. Ideally, one could assess multiple activities with a single assay, making judicious use of the compound. This can be delivered by microarray profiling assays, such as those carried out in the eukaryote model Saccharomyces cerevisiae (baking yeast). Although the assays may not identify the exact mechanism of a compound, the profiles will hint towards a given biological function indicating which protein-based assays would be most informative to deciphering a compound's biological function.

\subsubsection{Chemical genetic profiling}

Chemical genetic profiling via microarray analysis delivers broad-ranging information about biological interactions. During chemical genetic profiling assays, a pooled population of mutant yeast strains is assessed for growth under a certain growing condition, such as the presence of a xenobiotic. Each of the mutant strains carries the deletion for a single non-essential gene, which is replaced by a unique molecular barcode. These barcodes allow the growth characteristics of each strain to be quantified relative to a control population.

A fundamental assumption in chemical genetic screening is that small molecules bind specifically to a cellular component and alter its activity, functionally mimicking a mutation at the corresponding gene locus. ${ }^{75,76}$ Increased sensitivity of a specific deletion mutant strain to a small molecule indicates that the deleted gene is related to the molecule's cellular target in either function or pathway. These "guilt by association" interactions show that the deleted gene is required to buffer the effects of the small molecule, and can be used to elucidate its cellular target.

\subsubsection{Gene expression profiling}

Orthogonal to chemical genetic profiling, gene expression profiling analysis quantifies the up- and down-regulation of messenger RNA (mRNA) expression of genes in response to an exogenous substance. Fluctuations in levels of specific mRNA sequences suggest a changed requirement for the protein coded by the sequence; for example, cells or tissues will increase levels of mRNA encoding alcohol dehydrogenase in response to increased levels of ethanol in their environment. ${ }^{77}$

Both chemical genetic profiling and gene expression profiling techniques assess both the non-essential and essential gene cassettes simultaneously. However, under stress conditions, multiple gene products may be expressed but not be required for 
expedient adaptation to environmental changes, ${ }^{76}$ while genes essential for growth in particular environments may have an unchanged expression profile as their response is post-transcriptional. ${ }^{76}$ Chemical genetic profiling therefore has the power to identify the specific target of a small molecule inhibitor in the absence of any change in mRNA-expression. ${ }^{76}$

Chemical genetic profiling is an ideal form of biological assessment for small molecules isolated without knowledge of their biological activity. The profiles obtained from the assays will indicate which cellular functions are affected by a small molecule, aiding the identification of its precise cellular target and elucidation of its mechanism of action.

\subsection{Research objectives}

This thesis will encompass the isolation and structural elucidation of new secondary metabolites from Tongan marine sponges. To maximise the discovery of novel metabolites, the study of specimens with cyptic colouration and sparse distribution in geographically remote areas will be undertaken. This approach will hopefully uncover structural novelty from a well-examined region.

The principles of NMR-guided isolation will be applied throughout the chromatographic process, and the value of the HMBC experiment as a screening tool will be assessed through the identification of molecular substructures from within semipurified fractions.

Following isolation, all metabolites will be assessed for activity against the human promyelocytic leukemia cell line HL-60. Inhibition of Saccharomyces cerevisiae yeast will also be assessed with the aim of identifying compounds with antifungal activity that is sufficient to allow characterisation of their biological mechanisms with chemical genetic techniques.

\subsection{Summary}

With its multiple island groups spanning many latitudes, the Kingdom of Tonga offers a host of different marine environments, providing an ideal location to study marine natural products. In comparison to New Zealand, Tonga should offer a multitude of organisms whose investigation will hopefully culminate in the characterisation of new and interesting secondary metabolites. 
Bioassay-guided isolation has been used in the successful isolation of biologicallyactive secondary metabolites for decades. However, the partnership of spectroscopyguided isolation practices and chemical genetic screening offers an alternative. Chemical genetic screening techniques have the potential to identify the specific mode of activity of such biologically active metabolites. The approach is unbiased towards any particular mode of biological activity, and complements a structurebased isolation approach. Used in tandem, the two have the potential to uncover new metabolites and fully characterise their biological activities. 


\section{Chapter 2}

\section{Organism Screening and Selection}

Over the course of this study, 19 Tongan sponges were subjected to an NMR-based screening and selection process. $1 D$ and $2 D N M R$ spectra were acquired from the semi-purified screening fractions from each sponge, and the HMBC experiments in particular were assessed for apparent structural novelty. Nine specimens were selected for more thorough investigation on this basis. The metabolite profiles of each of these organisms is briefly detailed, and more comprehensive accounts of the structural classes from three organisms can be found in Chapters 3, 4 and 5.

\section{$2.1 \quad$ Collection areas}

Two excursions were made to the Kingdom of Tonga for sample collection yielding 260 sponge samples. In some cases multiple collections of the same organism were made, allowing study of changes in low-level metabolite profiles of specimens collected from geographically distinct locations. Collection sites visited during the first expedition in November 2008 included areas about the capital Nuku'alofa on the main island Tongatapu, the Houma blow-holes on the south side of the island, and the island of 'Eua to the east of Tongatapu. Several locations in the northern Vava'u island group were explored in November 2009.

\subsection{Organism selection}

Nineteen sponges that had been collected in small quantities from the Tongan islands were screened according to laboratory protocol (Appendix A). The crude methanol extracts of each sponge were individually partitioned over a column of HP20 resin beads, generating three $\mathrm{Me}_{2} \mathrm{CO}$ in $\mathrm{H}_{2} \mathrm{O}$ fractions of differing polarities, 30\%, $75 \%$ and $100 \% \mathrm{Me}_{2} \mathrm{CO}$ in $\mathrm{H}_{2} \mathrm{O}$. Full NMR data sets of the $100 \%$ and $75 \% \mathrm{Me}_{2} \mathrm{CO}$ in $\mathrm{H}_{2} \mathrm{O}$ fractions, including HMBC experiments, were acquired. Organism selection was 
largely based upon the structural motifs evident within the crude HMBC spectra, with an emphasis placed on sub-structures including non-protonated carbon centres. Using these guidelines, nine specimens were chosen for further investigation. The remaining ten samples were discontinued when the spectral interest offered within was superseded by others, and when insufficient quantities of the organism had been collected $(<10 \mathrm{~g})$. A compilation of the masses of screened sponge fractions extracted is presented in Table 2.1 .

\subsection{The Vava'u group}

The northern island group of Tonga, Vava'u, was visited in November 2009, and over 100 sponge samples were collected from eight dive sites within the island group. Comparison with sponge specimens also collected from 'Eua Island revealed subtle changes in metabolite profile between the two locations. In the case of the dictyoceratid sponge "luakuli" (PTN3_21E), new metabolites were isolated (see Chapter 3). Conversely, Vava'u collections of Cacospongia mycofijiensis investigated by Singh were deficient in the sought-after microtubule stabiliser zampanolide (20). ${ }^{60,62}$

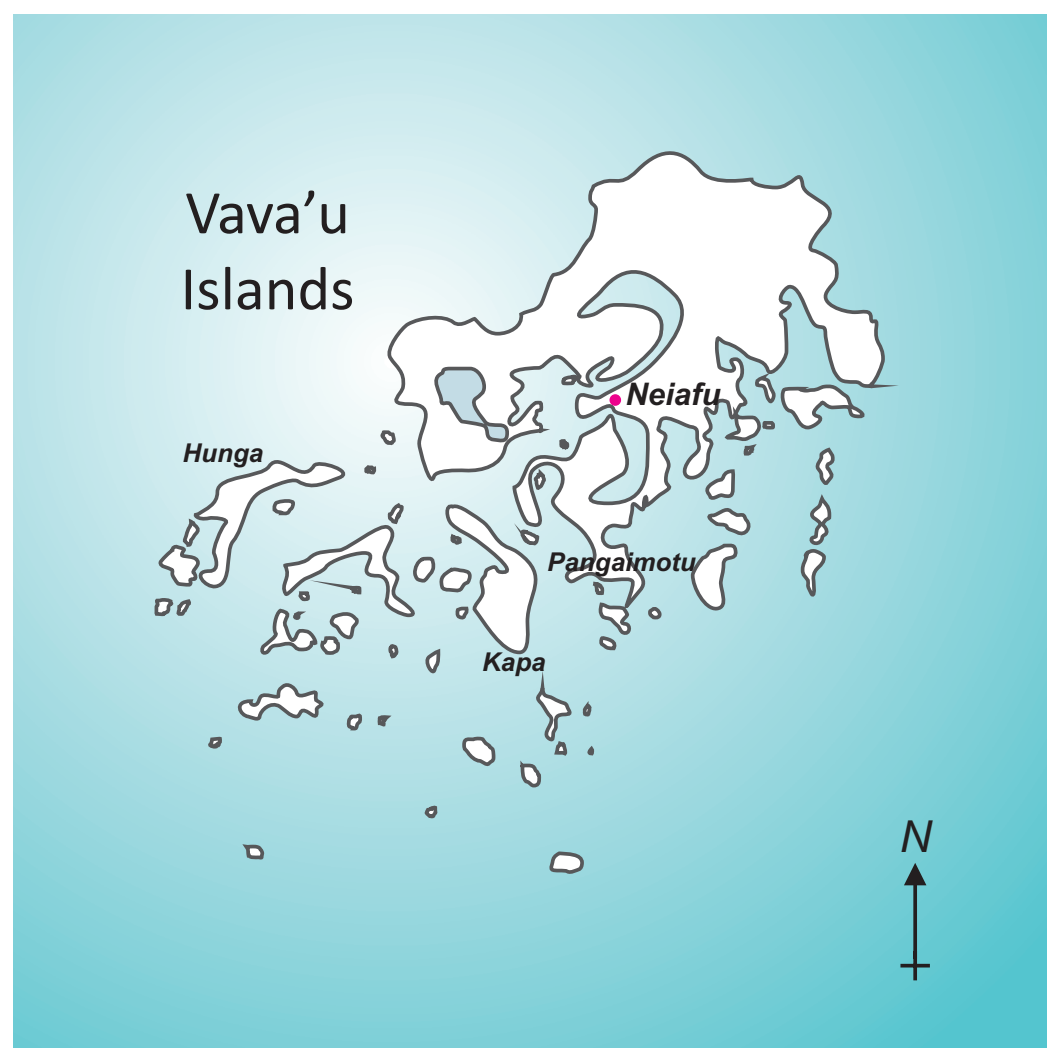

Figure 2.1 The Vava'u island group. 


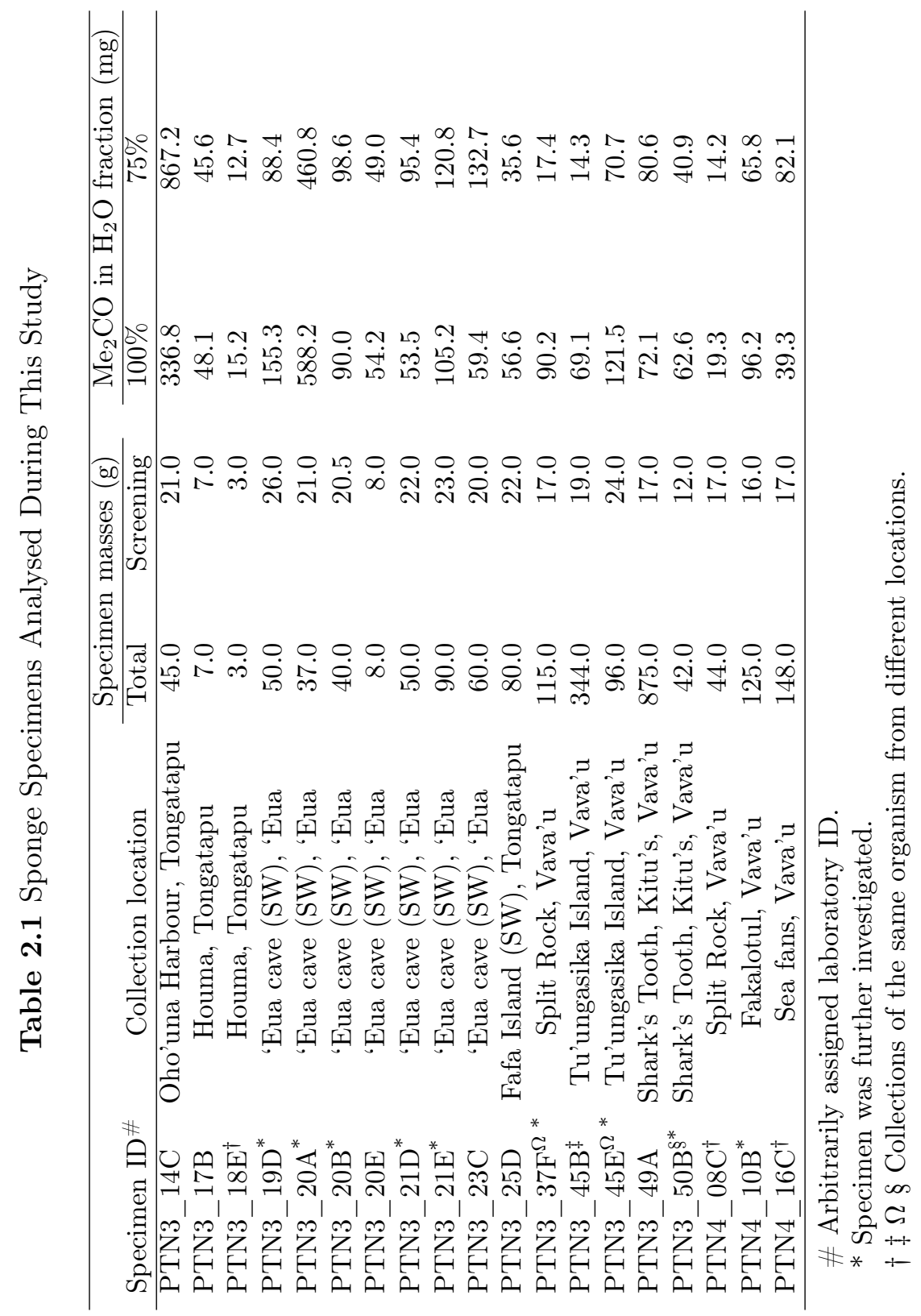




\subsection{1 $\quad$ PTN3 $37 \mathrm{~F}$ and PTN3 $45 \mathrm{E}$}

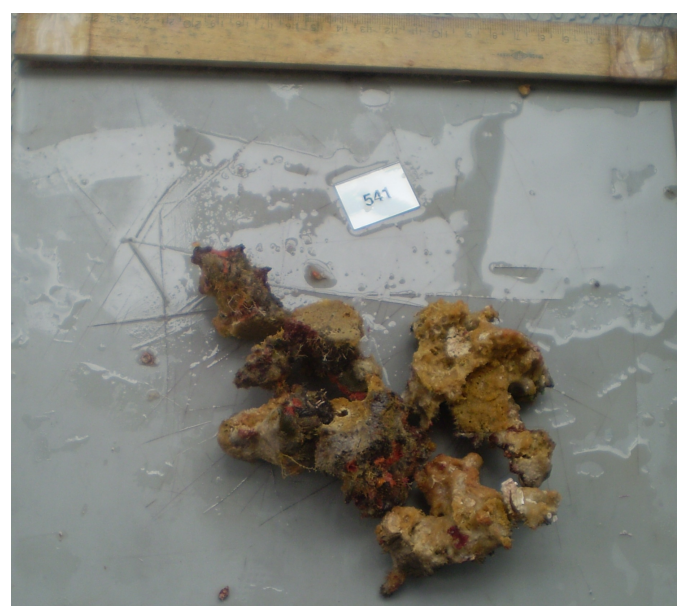

Figure 2.2 Surface photo of the sponge PTN3_45E.

Extraction of the demosponges PTN3_37F and PTN3_45E yielded two new diterpenes, iso-secothorectolide (23) and 1-hydroxyluffarin Q (24). The metabolites dominated the 1D and 2D NMR spectra of the $100 \%$ and $75 \% \mathrm{Me}_{2} \mathrm{CO}$ in $\mathrm{H}_{2} \mathrm{O}$ HP20 fractions (Figures 2.3 and 2.4). Concurrent isolation of the two unreported metabolites within this laboratory from a bright orange 'Euan sample of Fascaplysinopsis sp. (order Dictyoceratida, family Thorectidae) by Taufa ${ }^{78}$ aided structural elucidation of the compounds.<smiles>CC(C)=CCC/C(C)=C/CC/C(C)=C/CC/C(C=O)=C\CC(O)C1=CC(O)OC1=O</smiles>

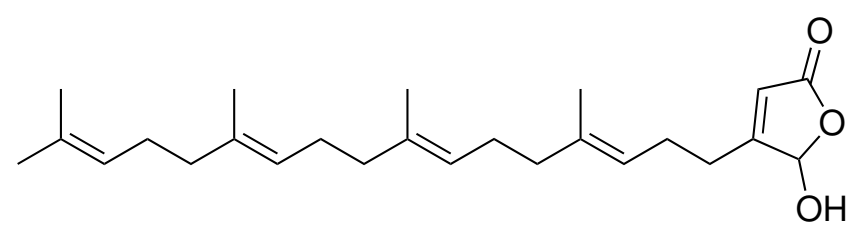




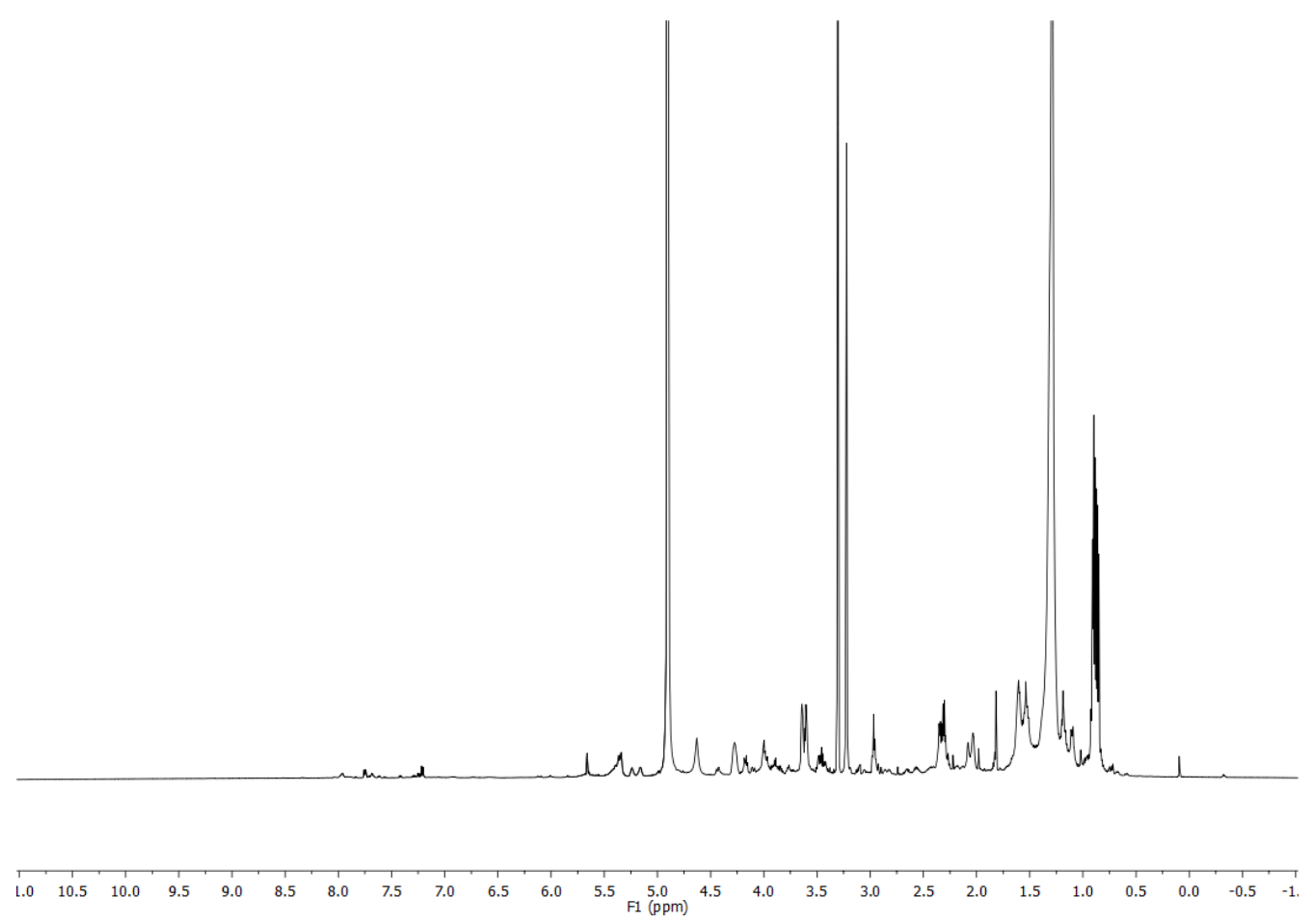

Figure $2.3{ }^{1} \mathrm{H}$ NMR spectrum $\left(\mathrm{CD}_{3} \mathrm{OD}\right)$ of the $75 \% \mathrm{Me}_{2} \mathrm{CO}$ in $\mathrm{H}_{2} \mathrm{O}$ HP20 fraction of PTN3 45E.

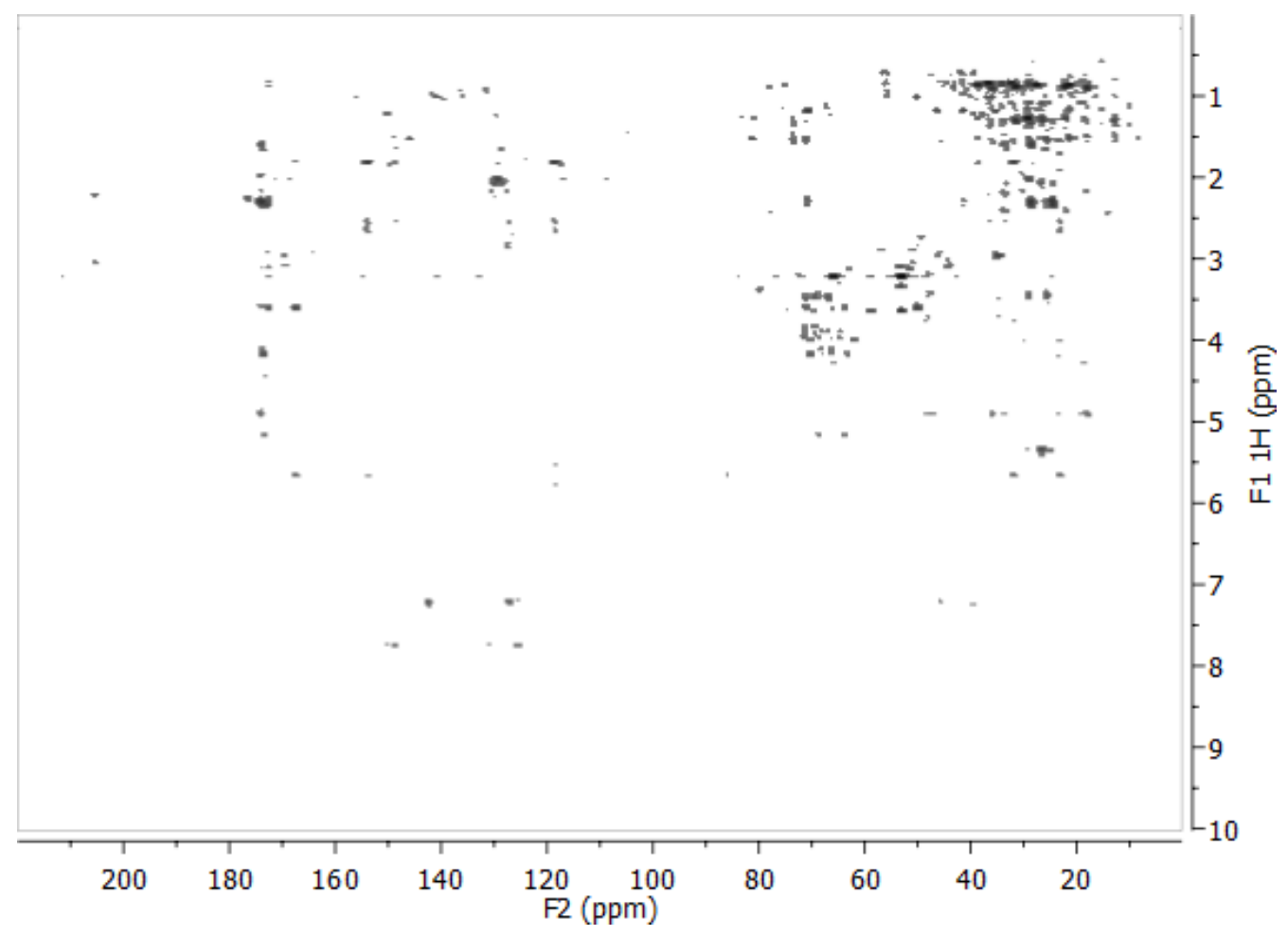

Figure 2.4 HMBC experiment $\left(\mathrm{CD}_{3} \mathrm{OD}\right)$ of the $75 \% \mathrm{Me}_{2} \mathrm{CO}$ in $\mathrm{H}_{2} \mathrm{O}$ HP20 fraction of PTN3_45E. 


\subsubsection{PTN4 10B}

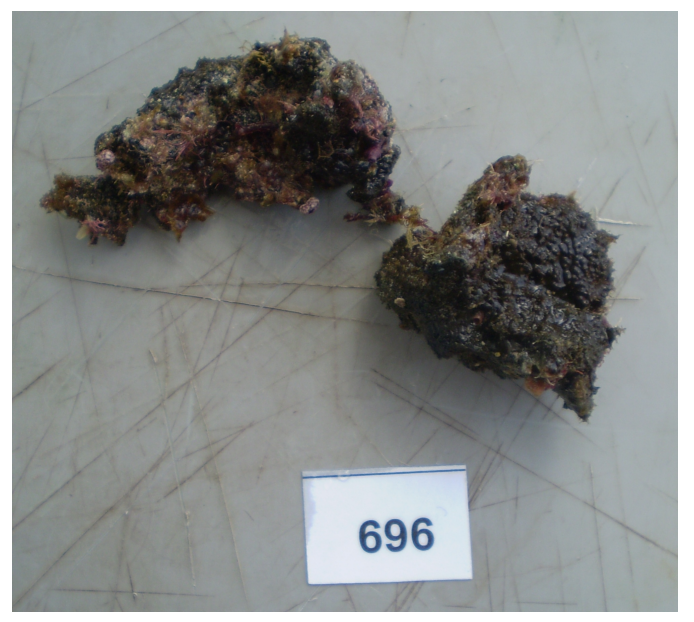

Figure 2.5 Surface photo of the sponge PTN4_10B

Several collections were made within the Vava'u island group of an unidentified demosponge, PTN4_10B. The lipophilic HP20 screening fraction (100\% $\mathrm{Me}_{2} \mathrm{CO}$ ) of PTN4_10B was dominated by two compounds: ambliol B (25) and luffarielolide (26), while the ${ }^{1} \mathrm{H}$ and $\mathrm{HMBC} \mathrm{NMR}$ spectra of the $75 \% \mathrm{Me}_{2} \mathrm{CO}$ in $\mathrm{H}_{2} \mathrm{O}$ fraction indicated the presence of other metabolites (Figures 2.6 and 2.7). Further analysis of this specimen was not undertaken in this study, although subsequent investigations by Woolner ${ }^{79}$ yielded several new members of the fascaplysin and homofascaplysin classes.

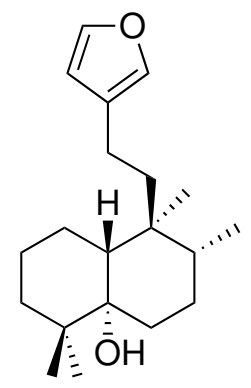<smiles>CC1=C(CC/C(C)=C/CC/C(C)=C/CCC2=CC(=O)O[C@@H]2O)C(C)(C)CCC1</smiles>

26

25 


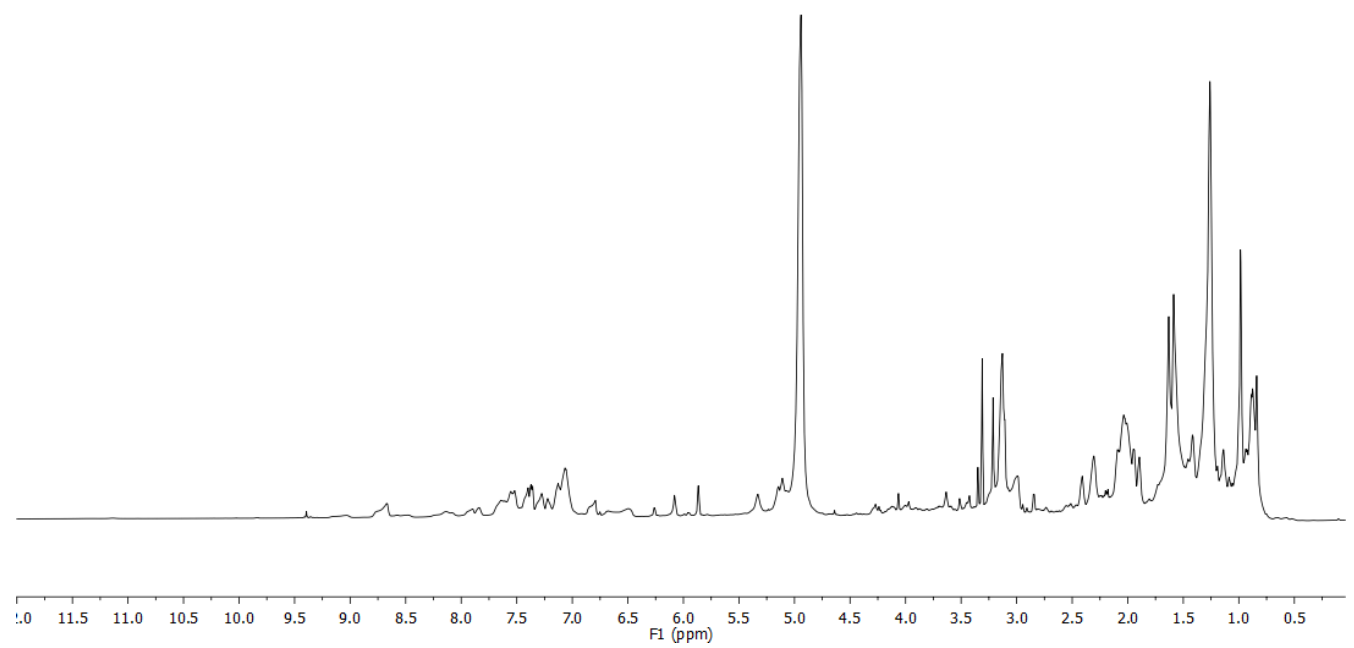

Figure 2.6 ${ }^{1} \mathrm{H}$ NMR spectrum $\left(\mathrm{CD}_{3} \mathrm{OD}\right)$ of the $75 \% \mathrm{Me}_{2} \mathrm{CO}$ in $\mathrm{H}_{2} \mathrm{O}$ HP20 fraction of PTN4_10B.

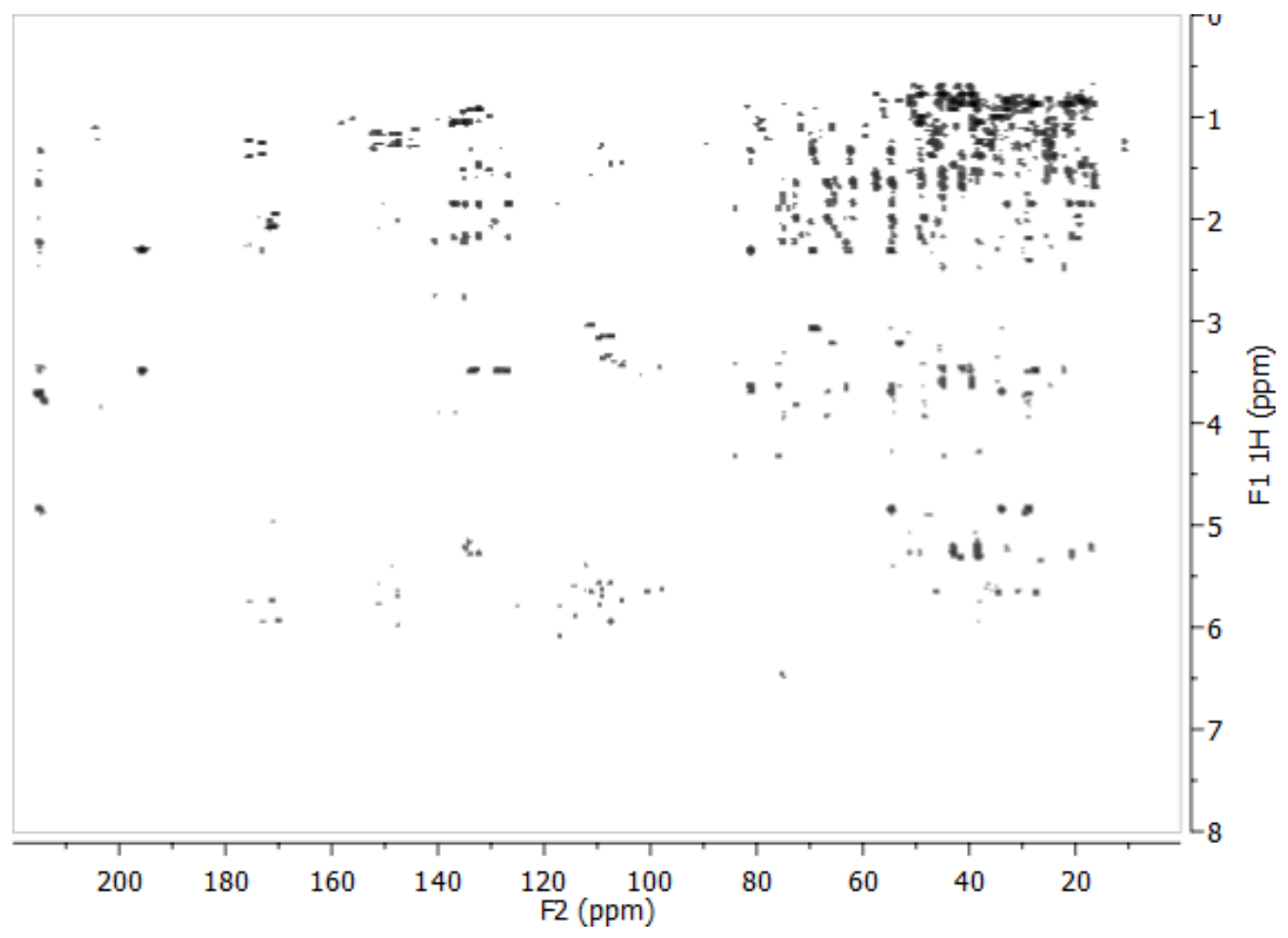

Figure 2.7 HMBC experiment $\left(\mathrm{CD}_{3} \mathrm{OD}\right)$ of the $75 \% \mathrm{Me}_{2} \mathrm{CO}$ in $\mathrm{H}_{2} \mathrm{O}$ HP20 fraction of PTN4_10B. 


\section{4 'Eua Island}

'Eua is the most ancient of Tongan islands, geologically distinct and geographically isolated from the other island groups. ${ }^{39}$ No report has been made of natural product investigations in the area, and it was hoped that the unique marine environment about 'Eua would produce novel chemistry.

A number of the specimens to receive further investigative attention during this work were collected at a depth of ca. $15 \mathrm{~m}$ in November 2008 from a marine cave on 'Eua island. A second collection venture to the area was intended, but had to be abandoned due to safety concerns. Recollection of particularly rich sponges, such as the Plakortis specimen PTN3_20A was attempted in the Vava'u group, although such attempts were for the most part unsuccessful, owing to organism rarity or difficulties in sample recognition.

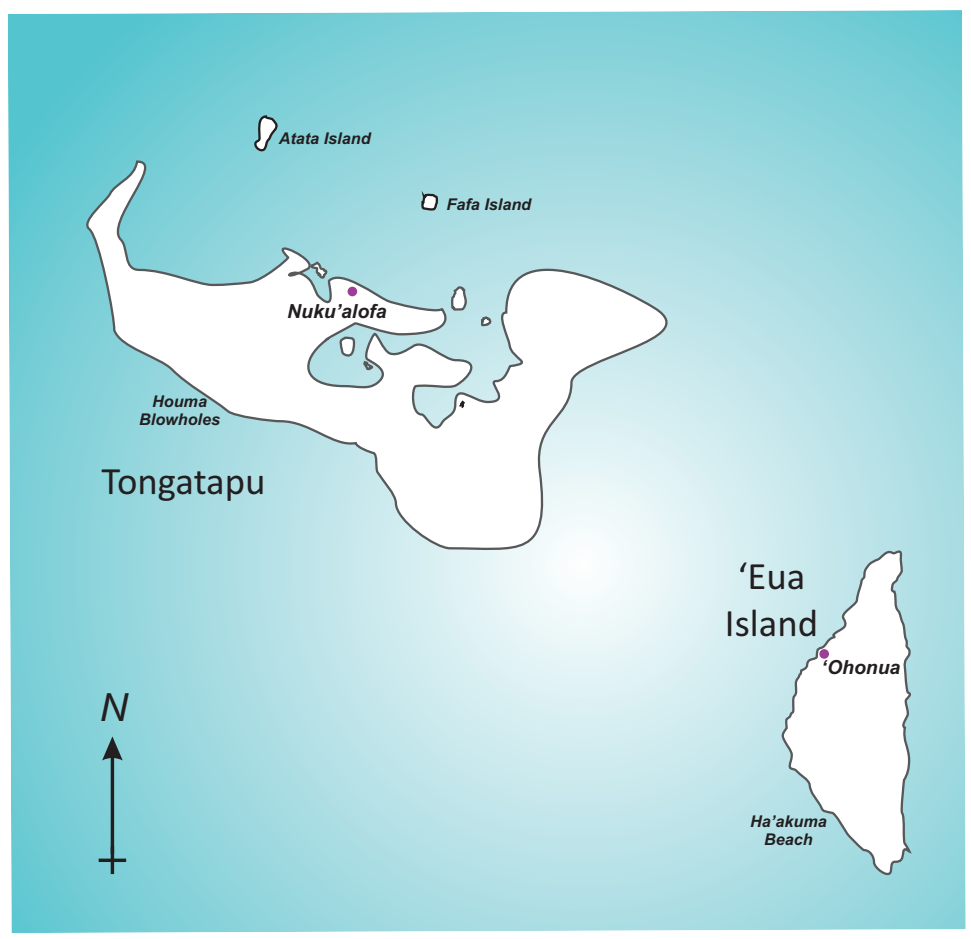

Figure 2.8 The Tongatapu island group. 


\subsubsection{PTN3 19D}

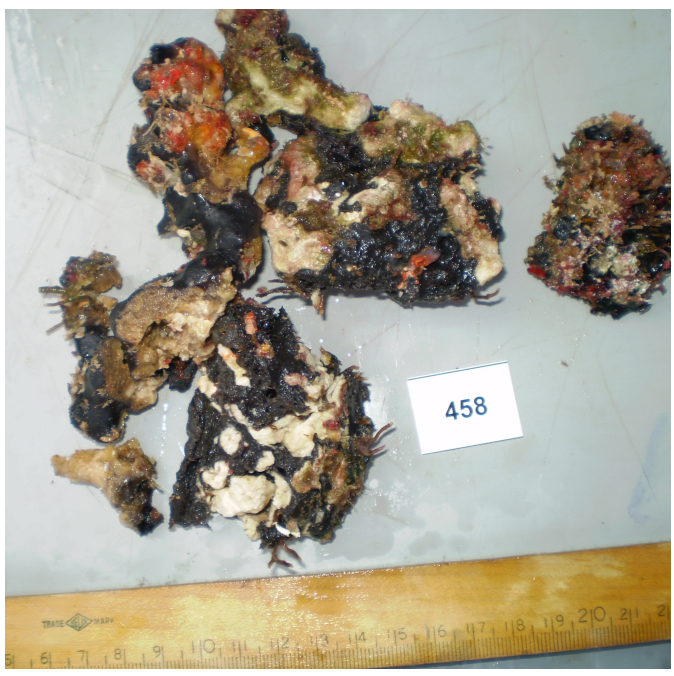

Figure 2.9 Surface photo of the plakinid specimen PTN3_19D.

Investigation of the thinly-encrusting black plakinid sponge PTN3_19D (50 g) yielded four new polyketide metabolites and two $5 \alpha, 8 \alpha$-epidioxysterols. Evident within the $\mathrm{HMBC}$ experiment of the $75 \% \mathrm{Me}_{2} \mathrm{CO}$ in $\mathrm{H}_{2} \mathrm{O}$ fraction were correlations from the protons of deshielded diastereotopic methylenes to oxycarbonyls and oxyquarternary centres (Figures 2.10 and 2.11). These interesting oxygenated structural fragments encouraged investigation of the specimen.

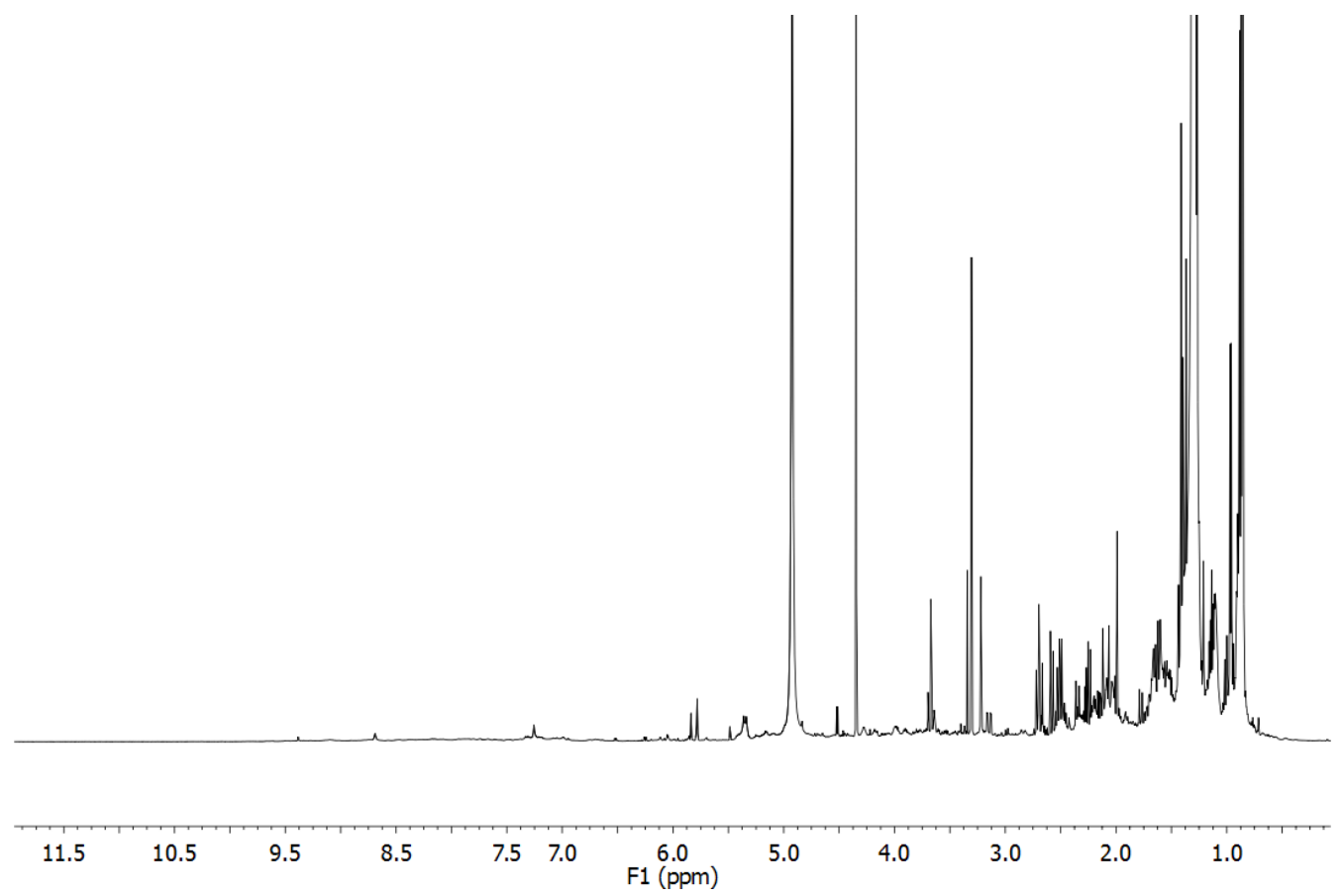

Figure 2.10 ${ }^{1} \mathrm{H}$ NMR spectrum $\left(\mathrm{CD}_{3} \mathrm{OD}\right)$ of the $75 \% \mathrm{Me}_{2} \mathrm{CO}$ in $\mathrm{H}_{2} \mathrm{O}$ HP20 fraction of PTN3 19D. 


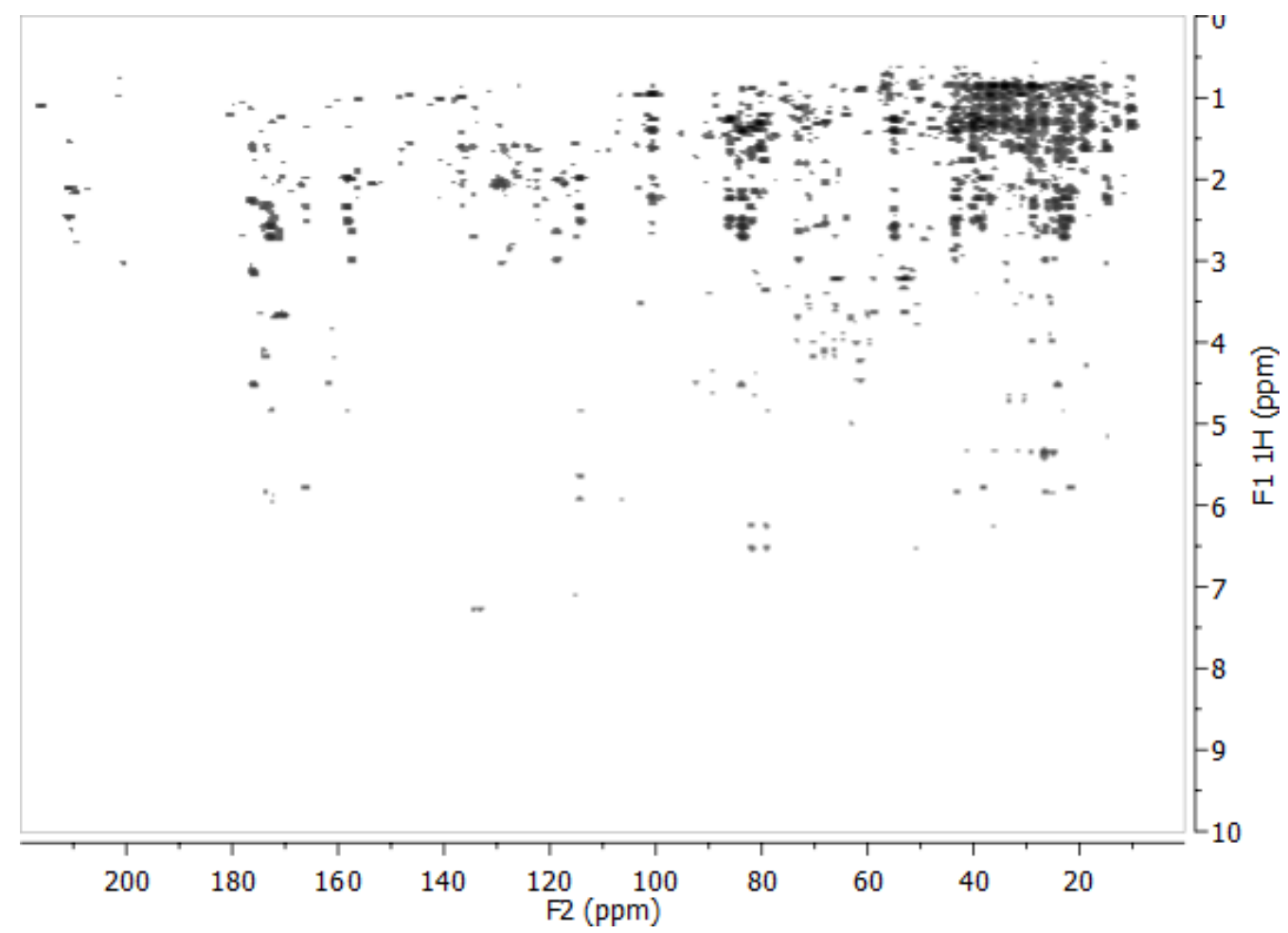

Figure 2.11 HMBC experiment $\left(\mathrm{CD}_{3} \mathrm{OD}\right)$ of the $75 \% \mathrm{Me}_{2} \mathrm{CO}$ in $\mathrm{H}_{2} \mathrm{O}$ HP20 fraction of PTN3_19D.

Two members of the $5 \alpha, 8 \alpha$-epidioxysterol class $\mathbf{2 7}$ and $\mathbf{2 8}$ were isolated from PTN3_19D. The metabolites were first reported in 1978 by Gunatilaka et al. and have subsequently been encountered in a number of different sponge species, ${ }^{80-82}$ the gorgonian Eunicell cavolini and the ascidian Trididemnum inarmatum. ${ }^{83}$ Stereochemical analysis of the sterols was not undertaken during this study.

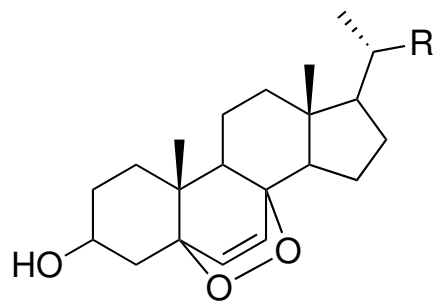

$27 \mathrm{R}=$

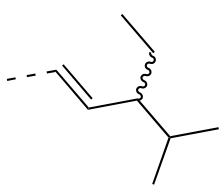

$28 \mathrm{R}=$

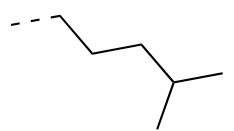

Investigation of PTN3_19D also afforded four new polyketide cyclic peroxides, the isolation and structural elucidation of which is detailed in Chapter 4. Biological assessment of the metabolites in Saccharomyces cerevisiae yeast follows in Chapter 5. 


\subsubsection{PTN3 20A}

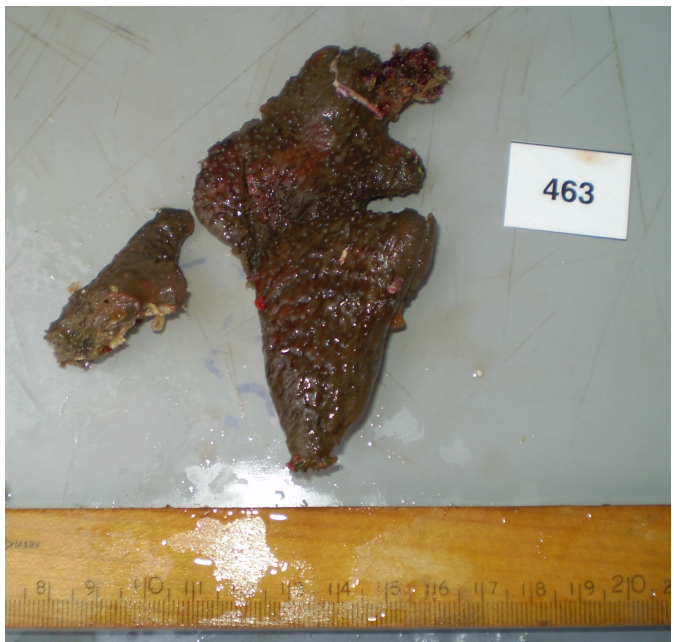

Figure 2.12 Surface photo of the Plakortis specimen PTN3_20A.

One of the first specimens to be analysed with our NMR-based screening protocol during this study was a dark purplish-brown Plakortis sponge, PTN3_20A. As detailed in Table 2.1, the $20 \mathrm{~g}$ screen sample yielded unusually high masses for the three $\mathrm{Me}_{2} \mathrm{CO}$ in $\mathrm{H}_{2} \mathrm{O}$ fractions. This was fortuitous as recollection of the sponge was never achieved.

Analysis of the ${ }^{1} \mathrm{H}$ NMR spectra of the $75 \%$ and $100 \% \mathrm{Me}_{2} \mathrm{CO}$ in $\mathrm{H}_{2} \mathrm{O}$ fractions drew attention to unusual combination of signals, including shielded methyl protons, oxymethyl protons, aromatic protons, and evidence of a long alkyl chain (Figure 2.13). However, the HMBC spectra indicated the most interesting structural relationships: correlations between deshielded, methyl groups, methylene centres and two isolated, highly polarised and substituted carbon double bonds (Figure 2.14). The seven new isolates from this organism are discussed in Chapter 4. 


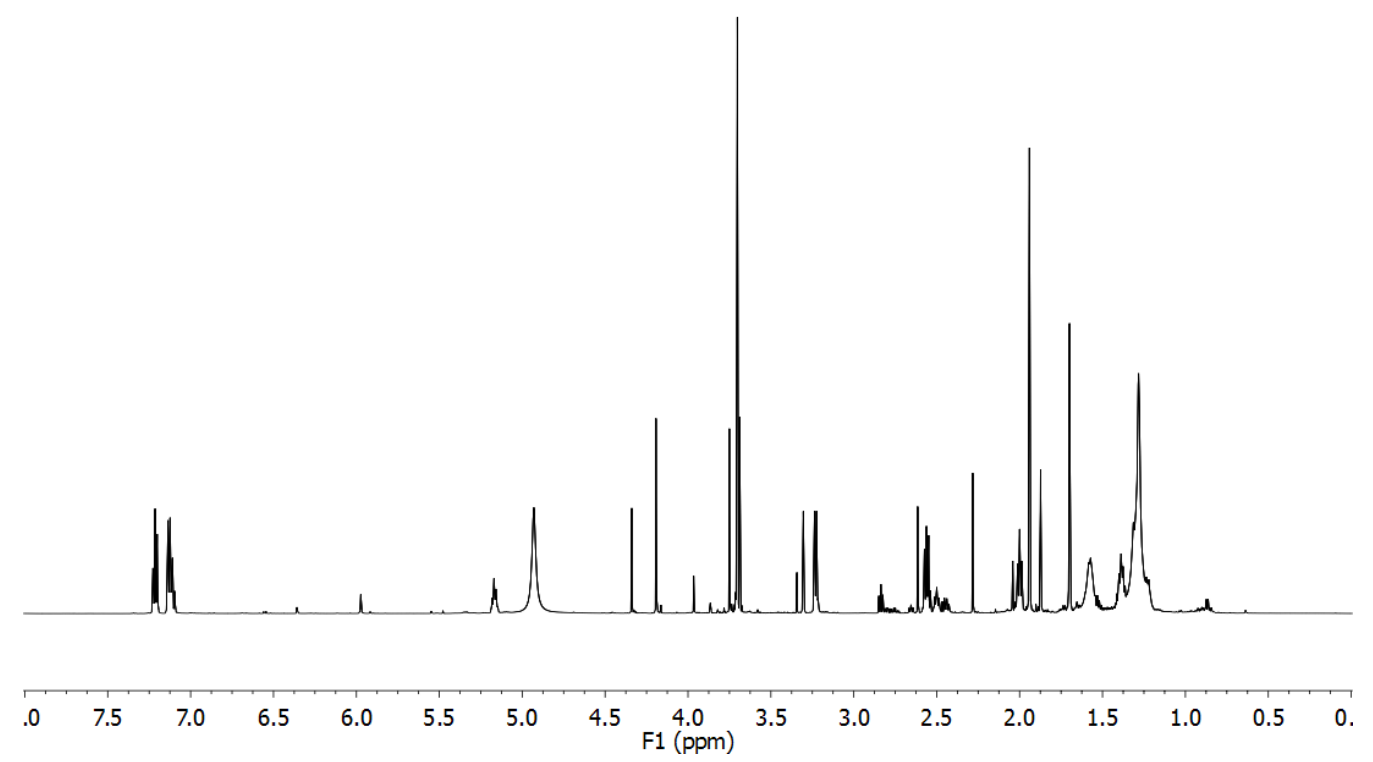

Figure $2.13{ }^{1} \mathrm{H}$ NMR spectrum $\left(\mathrm{CD}_{3} \mathrm{OD}\right)$ of the $75 \% \mathrm{Me}_{2} \mathrm{CO}$ in $\mathrm{H}_{2} \mathrm{O}$ HP20 fraction of PTN3_20A.

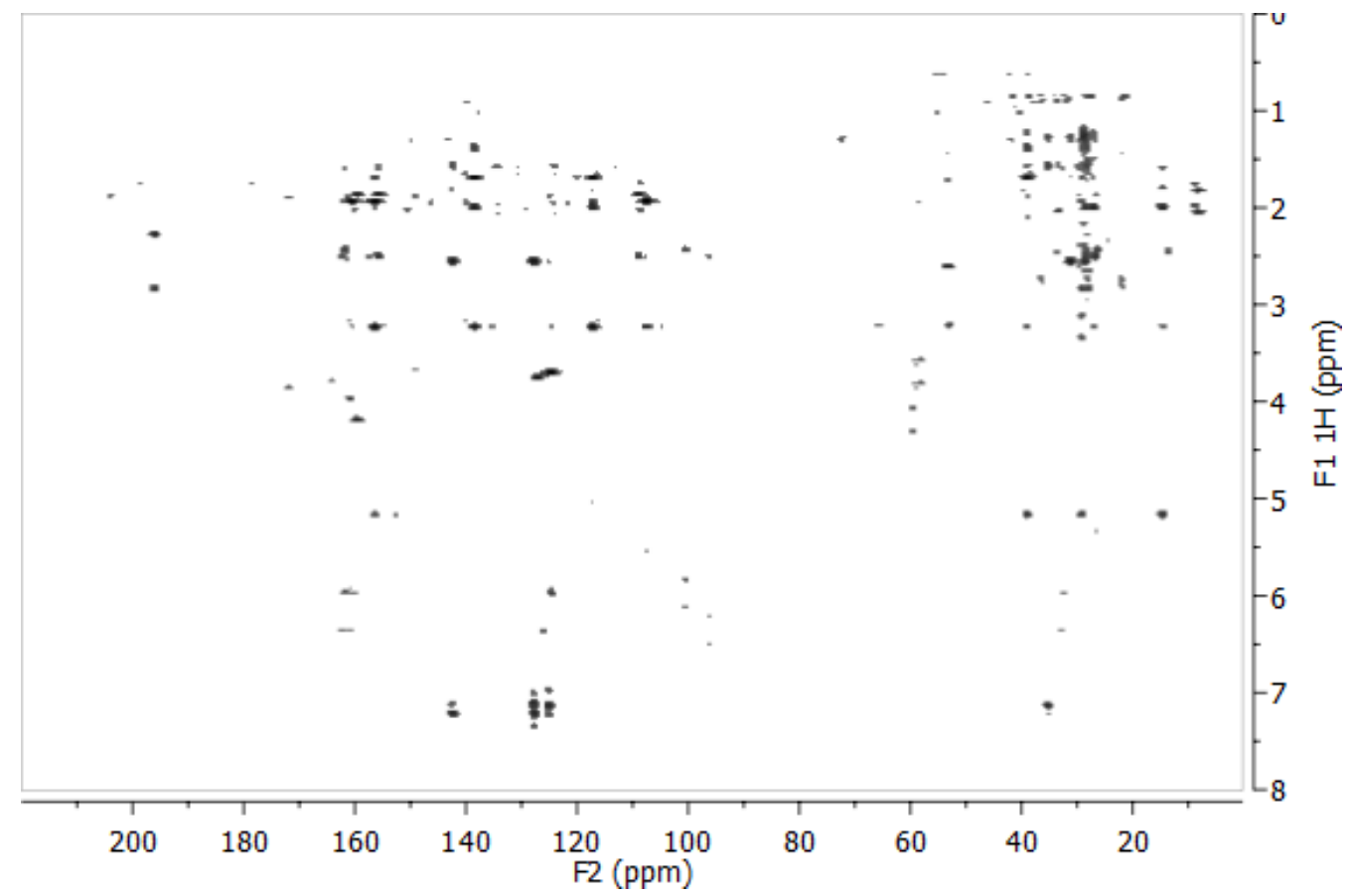

Figure 2.14 HMBC experiment $\left(\mathrm{CD}_{3} \mathrm{OD}\right)$ of the $75 \% \mathrm{Me}_{2} \mathrm{CO}$ in $\mathrm{H}_{2} \mathrm{O}$ HP20 fraction of PTN3 20A. 


\subsubsection{PTN3 20B}

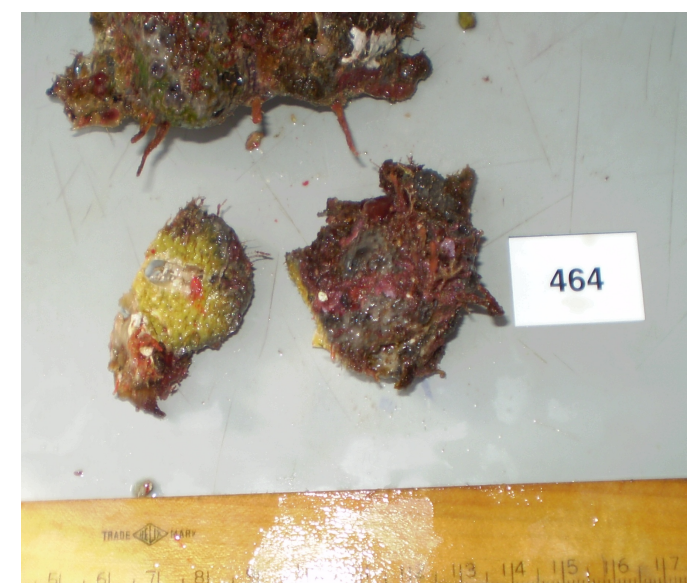

Figure 2.15 Surface photo of the slimy, mottled-green sponge PTN3_20B.

Analysis of the slimy, mottled-green demosponge PTN3_20B yielded the cytotoxic metabolite 14-bromohomofascaplysin (29). The alkaloid was present at low levels in the ${ }^{1} \mathrm{H}$ and HMBC NMR spectra of the $75 \% \mathrm{Me}_{2} \mathrm{CO}$ in $\mathrm{H}_{2} \mathrm{O}$ HP20 fraction (Figures 2.16 and 2.17). A new addition to the homofascaplysin class, 29 was later isolated in greater quantities from PTN4_10B by Woolner. ${ }^{79}$ The metabolite is a potent inhibitor of the HL-60 cell line, $\mathrm{IC}_{50} 33.8 \mathrm{nmol} \mathrm{L}{ }^{-1}$, and cell cycle analysis indicated that 29 induces S-phase delay. ${ }^{84}$ 14-bromohomofascaplysin $(\mathbf{2 9})$ was cytotoxic to wild type yeast with an $\mathrm{IC}_{50}$ value of $4.58 \mu \mathrm{mol} \mathrm{\textrm {L } ^ { - 1 }}$.

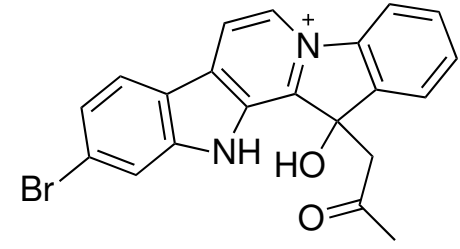

29

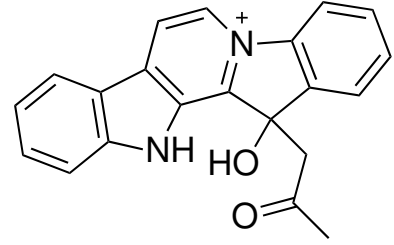

30

The des-bromo analogue $\mathbf{3 0}$ was isolated by Taufa from the orange 'Euan specimen PTN3_13A, ${ }^{78}$ allowing direct spectral and biological comparison between the compounds. Consistent with the trend observed by Crews et al, ${ }^{85}$ HL-60 cells exhibited increased sensitivity to the brominated form. The presence of this class of compounds in three apparently unrelated organisms (PTN3_13A, PTN3_20B and PTN4_10B), may indicate spongal origin of the compounds, or that the producing bacterial symbiont is not specific to sponges of a particular class. 


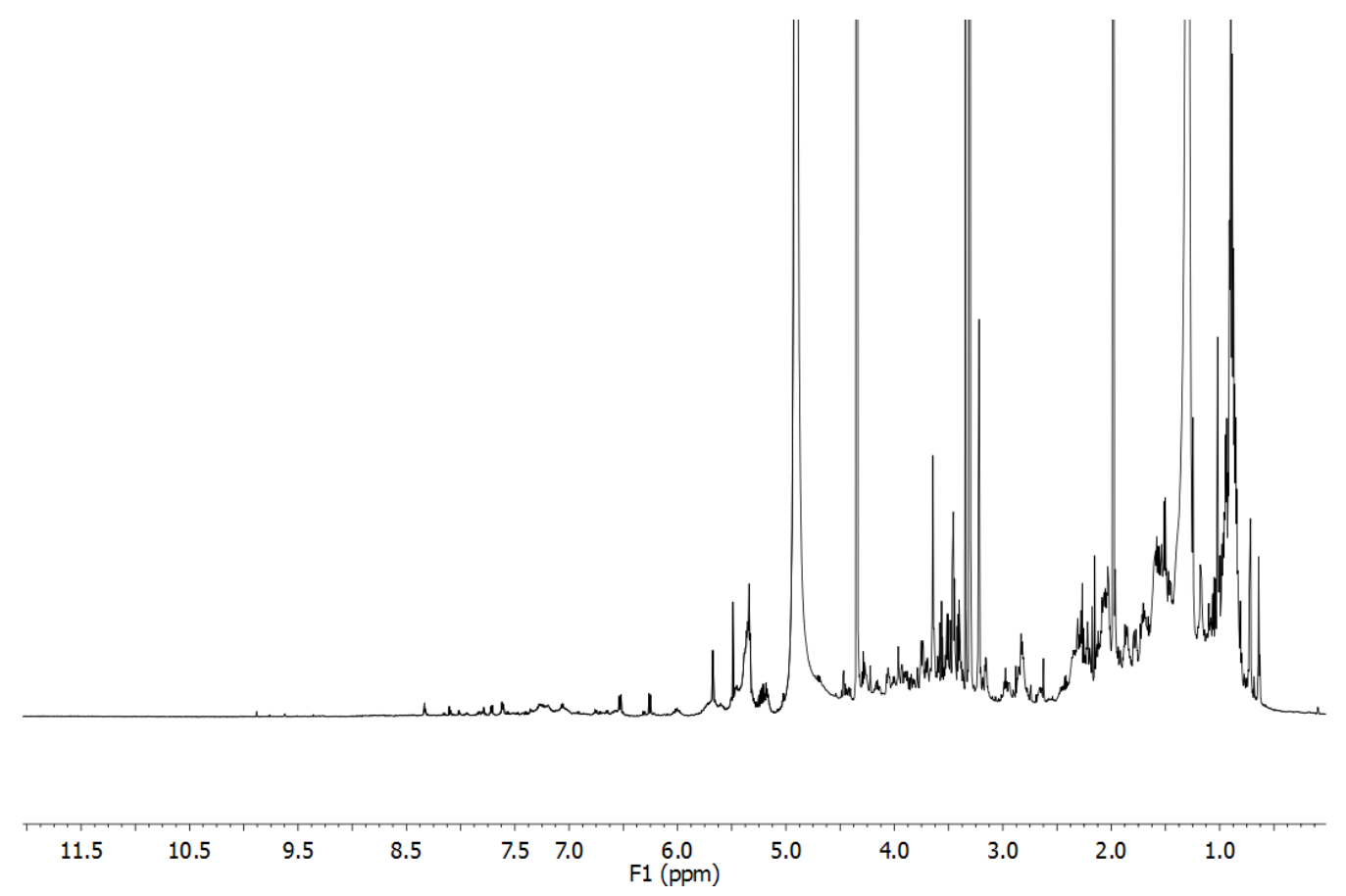

Figure 2.16 ${ }^{1} \mathrm{H}$ NMR spectrum $\left(\mathrm{CD}_{3} \mathrm{OD}\right)$ of the $75 \% \mathrm{Me}_{2} \mathrm{CO}$ in $\mathrm{H}_{2} \mathrm{O}$ HP20 fraction of PTN3_20B.

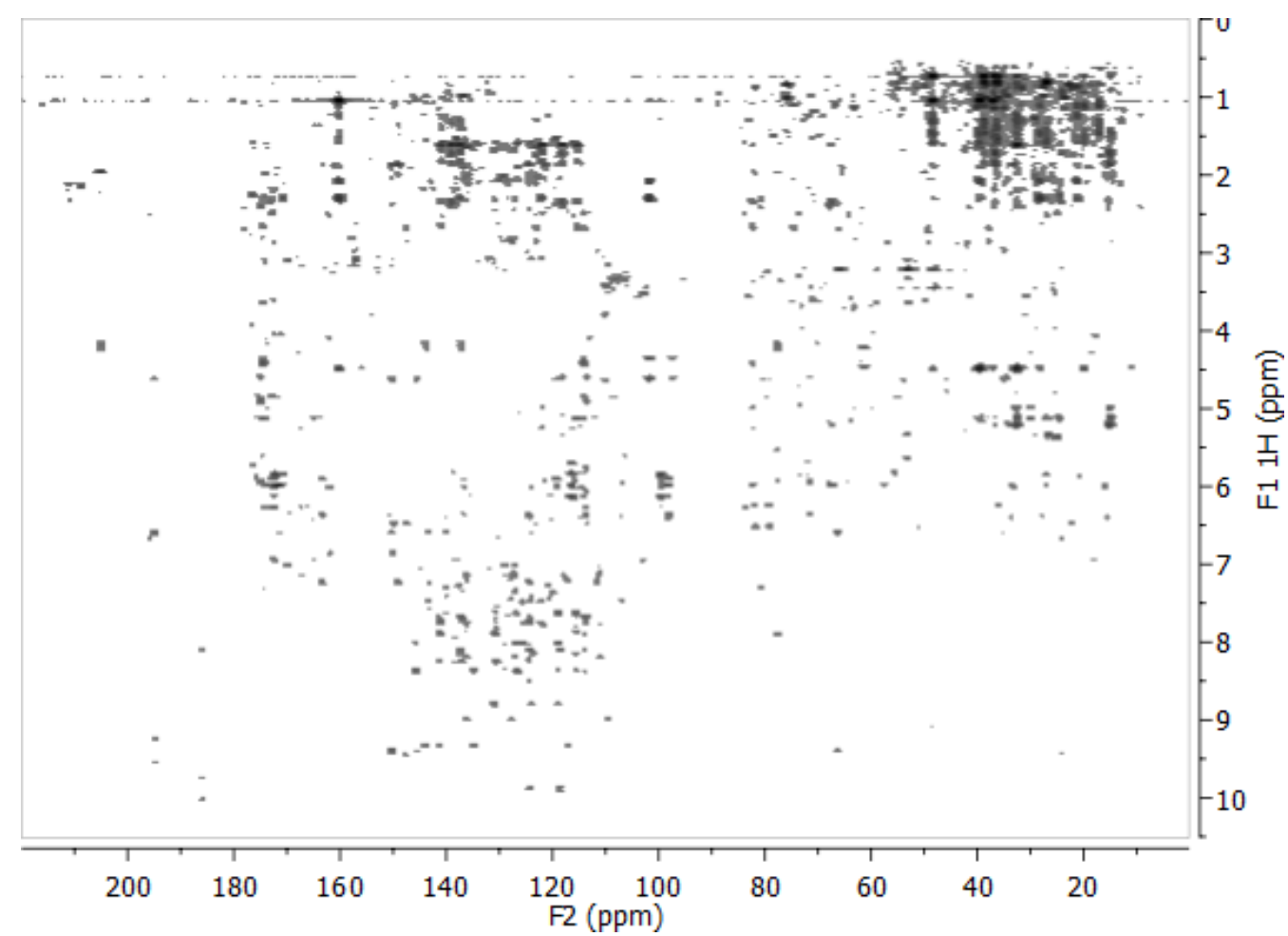

Figure 2.17 HMBC experiment $\left(\mathrm{CD}_{3} \mathrm{OD}\right)$ of the $75 \% \mathrm{Me}_{2} \mathrm{CO}$ in $\mathrm{H}_{2} \mathrm{O} \mathrm{HP} 20$ fraction of PTN3_20B. 


\subsubsection{PTN3 21D}
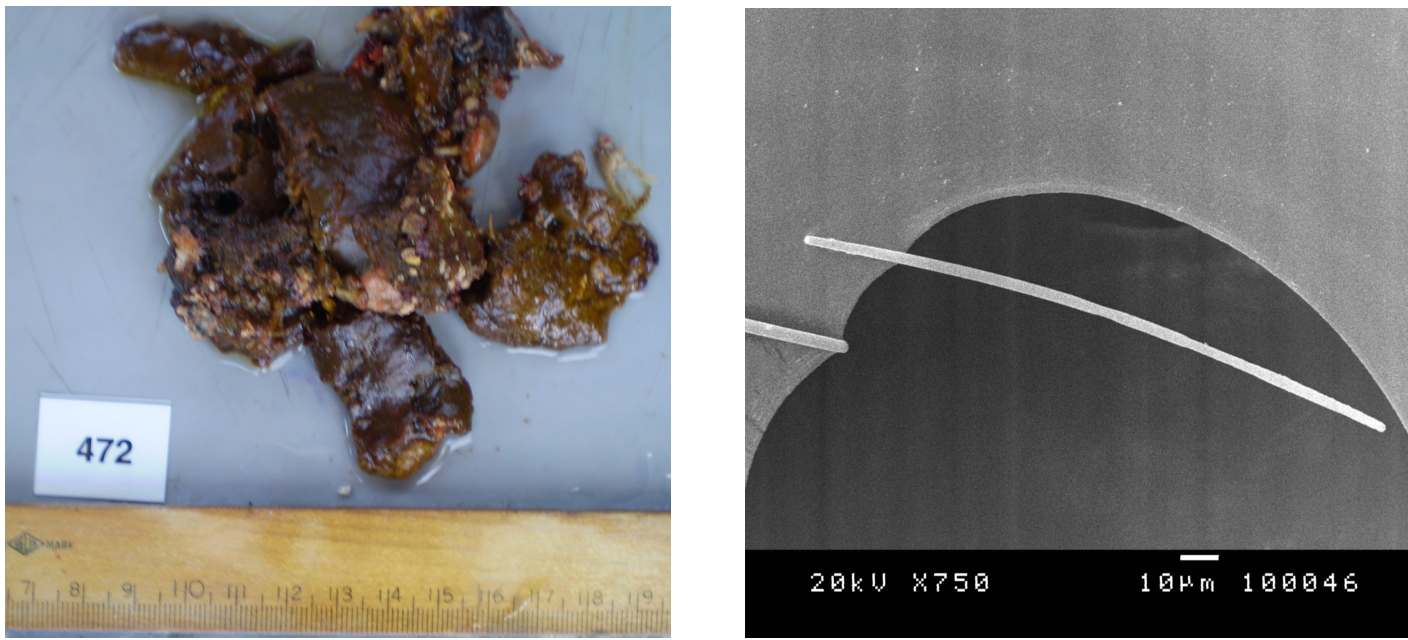

Figure 2.18 Surface photo of the sponge PTN3_21D and diod spicules recovered from the the organism.

Extraction of the blood red sponge specimen PTN3_21D (ca. 50 g) led to the isolation of the polyketide halenaquinone (31). ${ }^{86}$ The compound dominated the ${ }^{1} \mathrm{H}$ and HMBC NMR spectra of the $100 \%$ and $75 \% \mathrm{Me}_{2} \mathrm{CO}$ in $\mathrm{H}_{2} \mathrm{O}$ fractions from the initial HP20 screening column (Figures 2.19 and 2.20).

Identification of $\mathbf{3 1}$ was difficult; low level protonation about the fused penta cycle hindered structural elucidation. Connections between the tri-substituted furan and the adjoining $\alpha, \beta$-unsaturated ketone were particularly difficult to establish. Further inspection of the $1 \mathrm{D}$ and $2 \mathrm{D}$ NMR spectra indicated the presence of the known dihydroxy reduction derivative $32,{ }^{87}$ and other related compounds. Analysis of the sponge was problematic however; only MeCN appeared to dissolve the metabolites adequately, but also caused degradation of the metabolites. In light of solubility and stability issues and the presence of known metabolites within the organism, investigation of this specimen was discontinued. Diod spicules were recovered from the organism following $\mathrm{HNO}_{3}$ tissue digest (Figure 2.18).<smiles>CC12CCC(=O)c3coc(c31)C(=O)c1cc3c(cc12)C(=O)C=CC3=O</smiles>

31<smiles>CC12CCC(=O)c3coc(c31)C(=O)c1cc3c(O)ccc(O)c3cc12</smiles>

32 


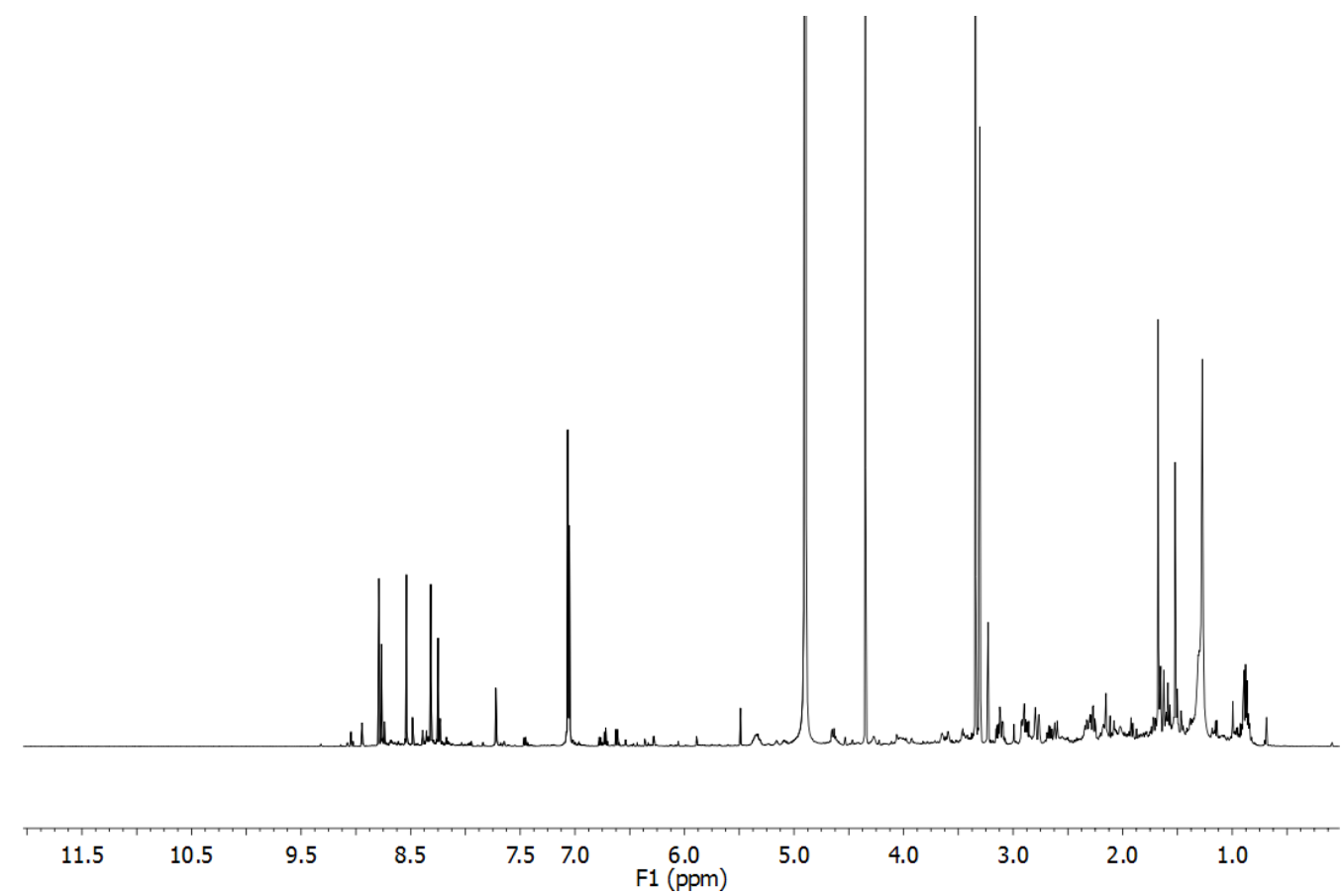

Figure 2.19 ${ }^{1} \mathrm{H}$ NMR spectrum $\left(\mathrm{CD}_{3} \mathrm{OD}\right)$ of the $75 \% \mathrm{Me}_{2} \mathrm{CO}$ in $\mathrm{H}_{2} \mathrm{O}$ HP20 fraction of PTN3_21D.

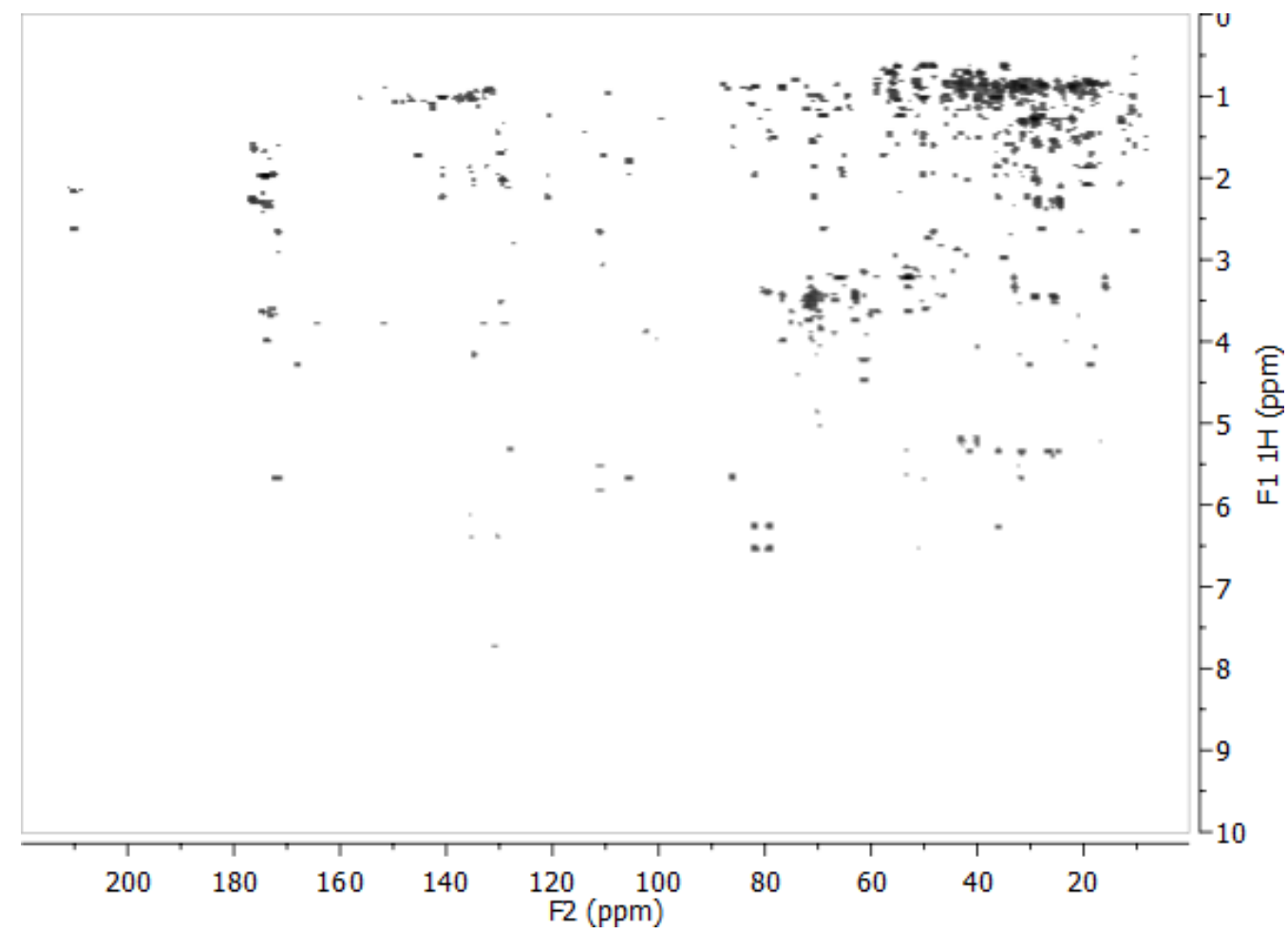

Figure 2.20 HMBC experiment $\left(\mathrm{CD}_{3} \mathrm{OD}\right)$ of the $75 \% \mathrm{Me}_{2} \mathrm{CO}$ in $\mathrm{H}_{2} \mathrm{O} \mathrm{HP} 20$ fraction of PTN3_21D. 


\subsubsection{PTN3 21E and PTN3 50B}

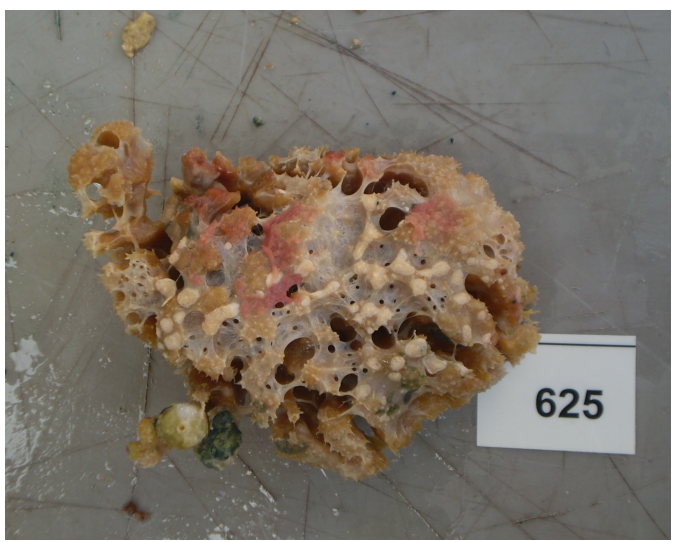

Figure 2.21 Surface photo of the Vava'u collection of the dictyoceratid sponge PTN3 50B.

A small quantity of an unidentified dictyoceratid sponge (see Figure 2.21) was collected from 'Eua island (PTN3_21E; 90 g, November 2008), and dubbed "luakuli"* owing to its unappetising appearance. Two closely related metabolites dominated the $75 \%$ and $100 \% \mathrm{Me}_{2} \mathrm{CO}$ in $\mathrm{H}_{2} \mathrm{O}$ fractions; correlations characteristic of a gem-dimethyl pair were immediately apparent in the HMBC spectum, while a 3 -substituted furan ring was evident in the ${ }^{1} \mathrm{H}$ and HMBC spectra (Figures 2.22 and $2.23)$.

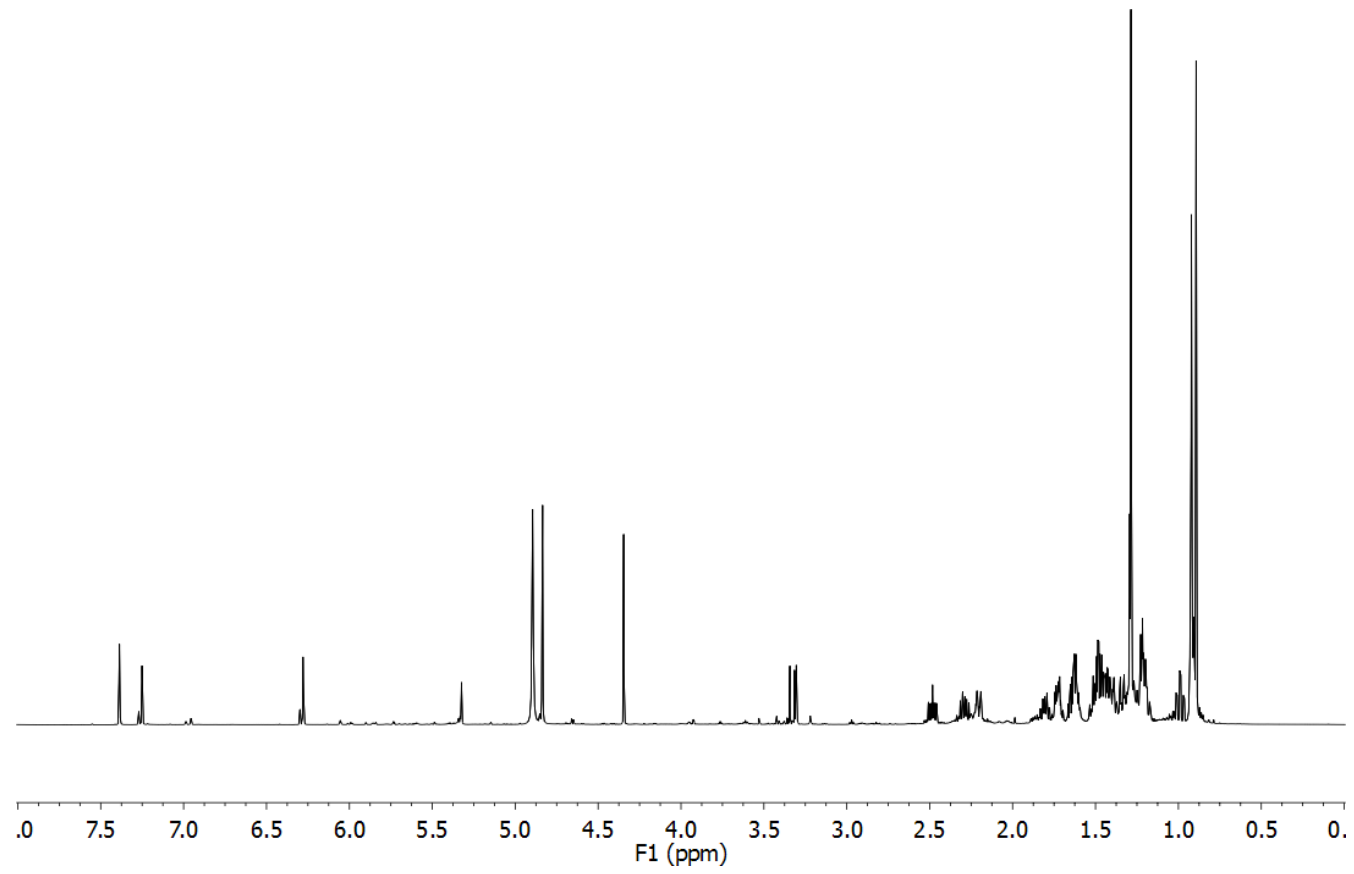

Figure $2.22{ }^{1} \mathrm{H}$ NMR spectrum $\left(\mathrm{CD}_{3} \mathrm{OD}\right)$ of the $75 \% \mathrm{Me}_{2} \mathrm{CO}$ in $\mathrm{H}_{2} \mathrm{O} \mathrm{HP} 20$ fraction of PTN3 21E.

*Luakuli, Tongan term for the product(s) of canine stomach upheaval. 


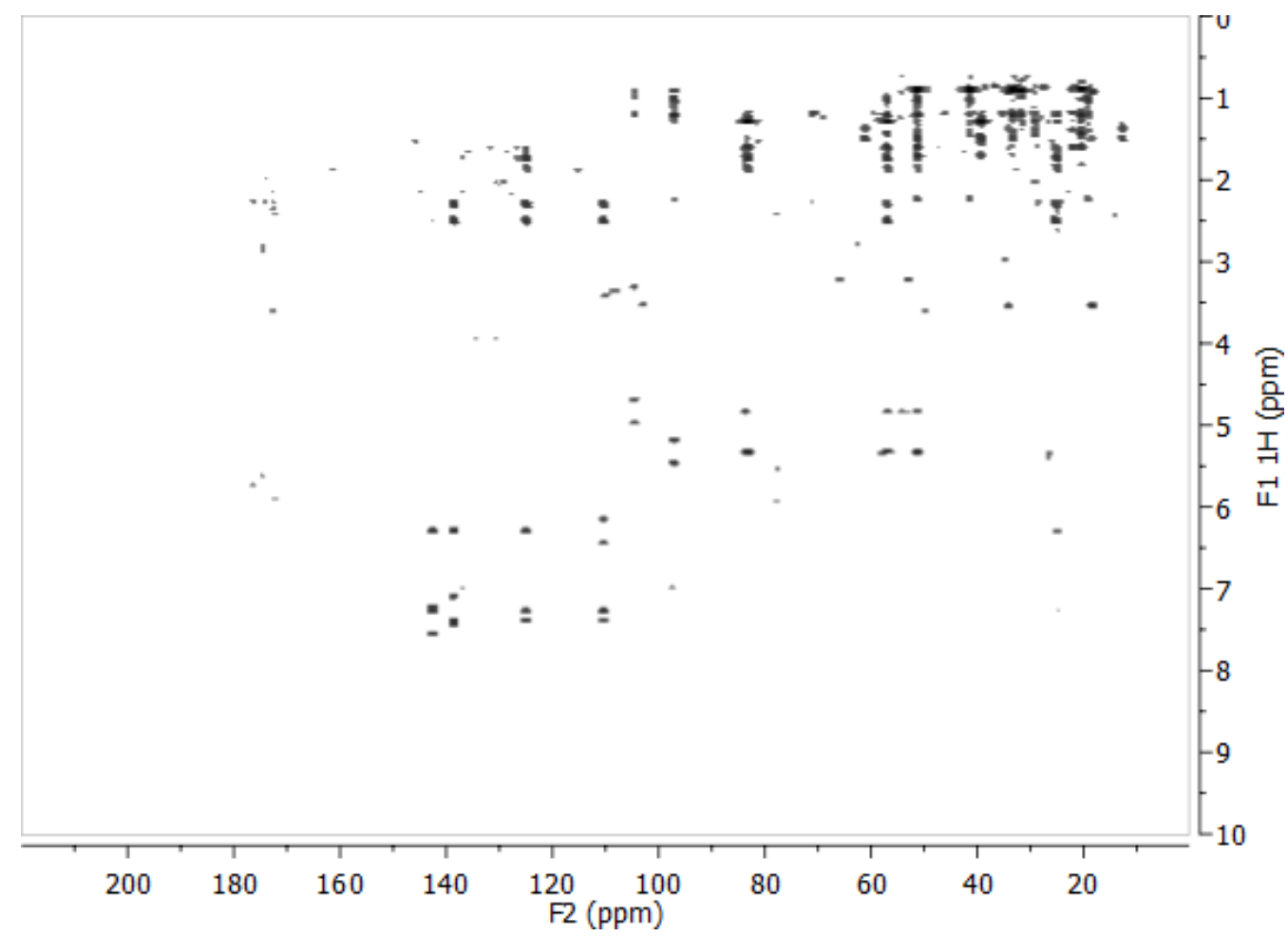

Figure 2.23 HMBC experiment $\left(\mathrm{CD}_{3} \mathrm{OD}\right)$ of the $75 \% \mathrm{Me}_{2} \mathrm{CO}$ in $\mathrm{H}_{2} \mathrm{O}$ HP20 fraction of PTN3_21E.

Subsequent study generated large quantities of a new labdane diterpene and its methyl acetal derivative. Targeted recollection of the "luakuli" sponge was achieved in the Vava'u group, and two minor metabolites were identified. An account of their isolation can be found in Chapter 3 .

\subsection{Concluding remarks}

Investigation of nine Tongan sponges culminated in the isolation of a variety of terpene, alkaloid and polyketide metabolites. As postulated, the metabolite profiles of sponges, such as the dictyoceratid specimens PTN3_21E and PTN3_50B, were seen to vary subtly depending on the geographical origin of the specimen.

Organisms collected from the most ancient of the Tongan islands, ${ }^{88}$ 'Eua, appear to be chemically distinct from those collected in the Tongatapu and Vava'u island groups, yielding a greater proportion of new structures. Although the Vava'u island group boasts a wealth of marine life, organisms collected within the Vava'u group have displayed chemistry more similar to that observed in organisms from the neighbouring Fijian islands. This indicates that further investigation of organisms from 'Eua island in particular should be undertaken. 


\section{Chapter 3}

\section{Luakuliides A-C}

Analysis of an unidentified dictyoceratid sponge yielded three new labdane diterpenes; luakuliides $A-C$, which exhibit interesting immunomodulatory activity. Investigation of the specimen was undertaken following identification of the gem-dimethyl motif, characteristic of the cyclised labdane skeleton, in the HMBC screening spectrum.

\subsection{The order Dictyoceratida}

Dictyoceratida is an order in the class Demospongiae (sub-class Ceractinomorpha), and further divided into four families: Dysideidae, Irciniidae, Spongiidae and Thorectidae (Figure 3.1). Dicytoceratid sponges are characteristically devoid of an easily classified skeletal component, such as spicules, making their taxonomic identification particularly difficult. In place of spicules, they possess complex and ordered network of spongin fibres. This produces a tough, flexible tissue with a soft texture, while the textured surface is typically reticulated or conulose (marked with cone-shaped elevations). Modulation of surface pigmentation is frequently observed in demosponges - changes in light exposure can affect differences within the same organism. Hence, organisms growing in light exhibit a dark exterior with interiors ranging from white through to yellow and pale brown. ${ }^{38}$

Order Dictyoceratida

Family Dysideidae

Family Irciniidae

Family Spongiidae

Family Thorectidae

Subfamily Phyllospongiinae

Subfamily Thorectinae

Figure 3.1 Taxonomic classification of Dictyoceratida, after Hooper and van Soest. ${ }^{38}$

Owing to frequent phylogenic restructuring and the ensuing taxonomic inconsistencies within the literature, a detailed account of dictyoceratid sponge metabolites is 


\subsection{Labdane diterpenoids from the dictyoceratid sponge "luakuli"}

The sponge PTN3_21E was initially collected from a dimly illuminated marine cave on the island of 'Eua, at a depth of ca. $15 \mathrm{~m}$ in November 2008. Tentatively assigned as belonging to the order Dictyoceratida, the sponge is porous and firm, with a reticulated surface and oscules of two size classes (see Figure 2.21). The organism contains no silicaceous spicules and there is little difference in the pigmentation of its pinky-beige exterior and interior. Its nondescript appearance and sparce distribution made recollection in the Vava'u group difficult (November 2009), and only one small specimen (PTN3_50B) was recovered from the vertical interior of a cave.

\subsubsection{Isolation and identification of luakuliides $\mathrm{A}-\mathrm{C}$}

The methanol extracts of a ca. $20 \mathrm{~g}$ screening sub-sample were chromatographically separated over HP20 resin (see Appendix A). Both the $100 \%$ and $75 \%$ and $\mathrm{Me}_{2} \mathrm{CO}$ fractions displayed interesting NMR spectral signals, which appeared to belong to two closely related metabolites. The ${ }^{1} \mathrm{H}$ NMR spectrum contained the broad, deshielded signals of three heterocyclic olefinic methines, a hemi-acetal methine, deshielded diastereotopic methylene protons and shielded methyl singlets. Similarly, the ${ }^{1} \mathrm{H}$ and $\mathrm{HMBC}$ spectra of these fractions contained patterns characteristic of a gem-dimethyl pair and a 3-substituted furan system (Figure 3.2).

Portions of both HP20 fractions were partitioned further over HP20SS. The labdane diterpene luakuliide $\mathrm{A}$ (33) eluted in $60 \%$ and $70 \% \mathrm{Me}_{2} \mathrm{CO}$ in $\mathrm{H}_{2} \mathrm{O}$ fractions, while further normal-phase purification of the $90 \%$ and $100 \% \mathrm{Me}_{2} \mathrm{CO}$ in $\mathrm{H}_{2} \mathrm{O}$ fractions over DIOL yielded the presumed methanol artifact 34, in the $100 \% \mathrm{CH}_{2} \mathrm{Cl}_{2}$ fraction. Flash chromatography on DIOL of the $80 \% \mathrm{Me}_{2} \mathrm{CO}$ in $\mathrm{H}_{2} \mathrm{O}$ HP20SS fraction generated further quantities of 33, while the methoxy congener 34 eluted in the $10 \% \mathrm{CH}_{2} \mathrm{Cl}_{2}$ in hexanes fraction.

The Vava'u specimen was extracted and partitioned over HP20 and HP20SS in a similar manner to the 'Eua specimen. Quantities of $\mathbf{3 3}$ and $\mathbf{3 4}$ were isolated following DIOL flash chromatography of the $100 \% \mathrm{Me}_{2} \mathrm{CO}$ in $\mathrm{H}_{2} \mathrm{O}$ HP20SS fraction, and further purification of the $33 \%$ EtOAc in $\mathrm{CH}_{2} \mathrm{Cl}_{2}$ DIOL fraction by reversedphase HPLC $\left(\mathrm{C}_{18}, 70 \% \mathrm{MeCN}\right.$ in $\left.\mathrm{H}_{2} \mathrm{O}\right)$ yielded luakuliides $\mathrm{B}$ and $\mathrm{C}$ (35 and 36). An overview of the isolation of the individual luakuliides is depicted in Figure 3.3. 
A

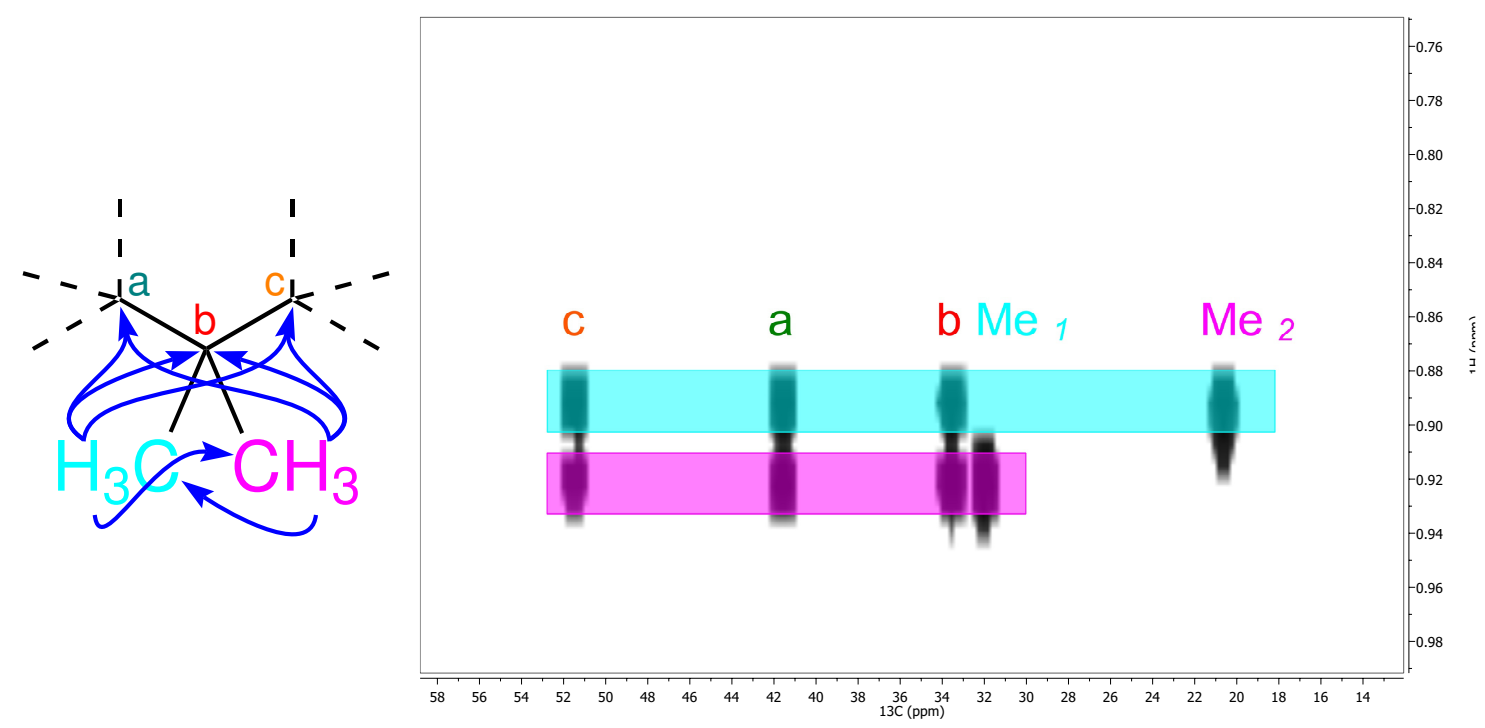

B

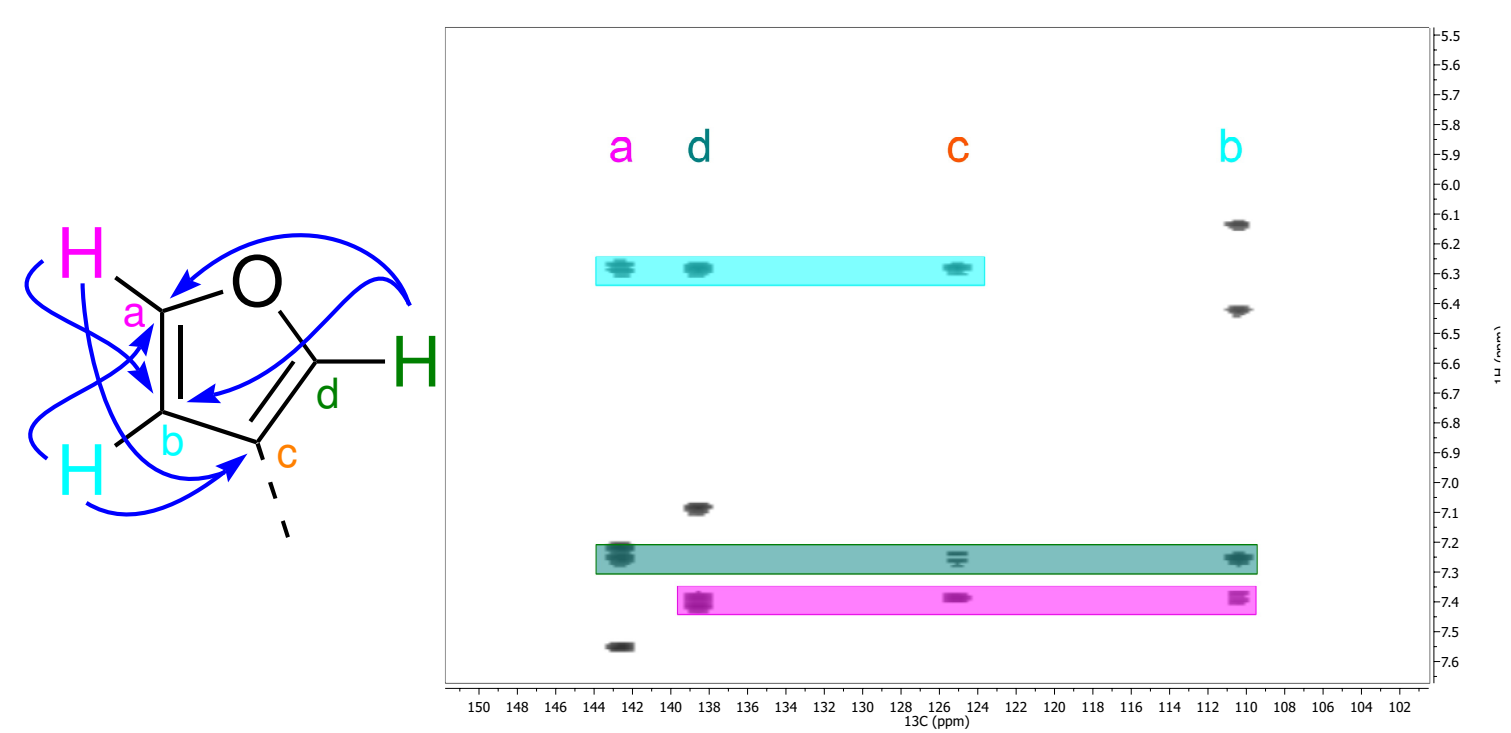

Figure 3.2 Proton to carbon correlations evidencing substructures in the HMBC spectrum of the $75 \% \mathrm{Me}_{2} \mathrm{CO}$ in $\mathrm{H}_{2} \mathrm{O}$ HP20 screen fraction of PTN3_21E. A: the gem-dimethyl pair and $\mathrm{B}$ : the 3-substituted furan ring. 


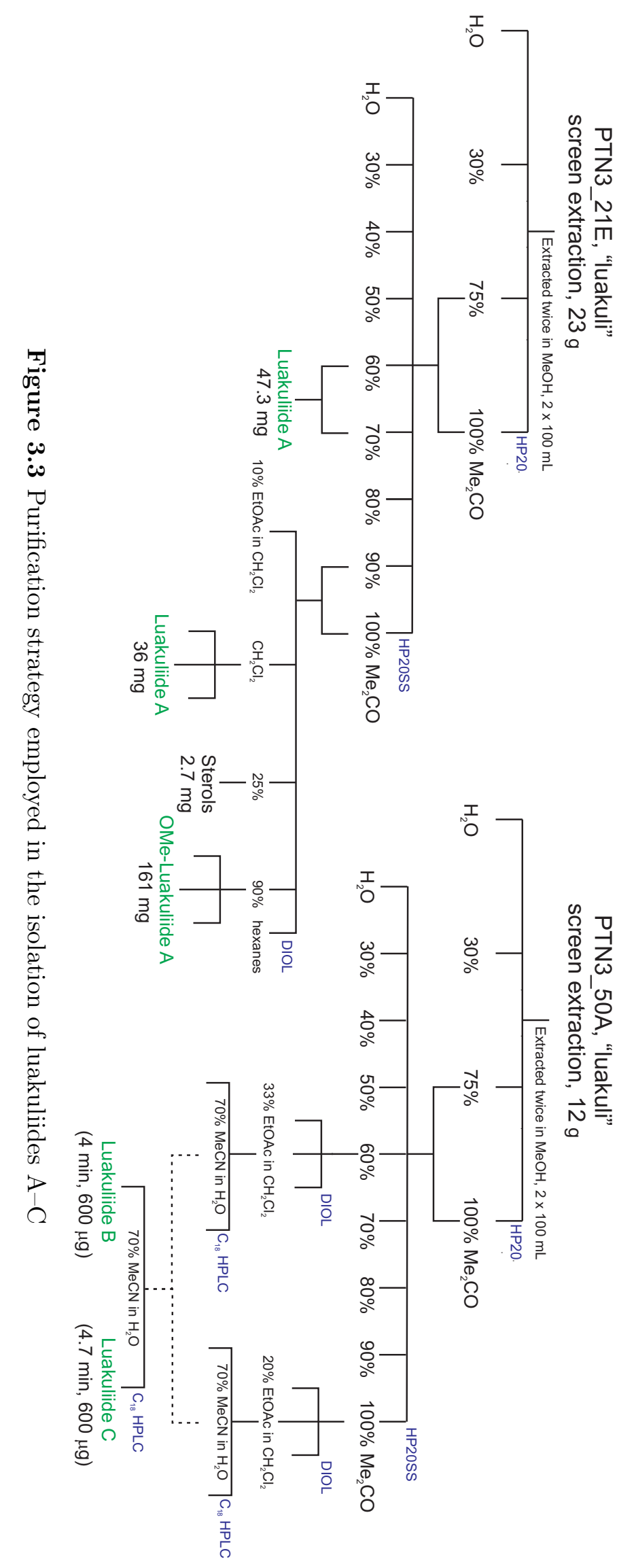




\section{Luakuliide A (33)}

Luakuliide $\mathrm{A}(\mathbf{3 3}),[\alpha]_{D}^{25.4}-10.8^{\circ}\left(c 18.5, \mathrm{CHCl}_{3}\right)$ was isolated as a colourless oil $(92.7$ $\mathrm{mg}$ in total). Positive-mode HRESIMS analysis of $\mathbf{3 3}$ revealed a $[\mathrm{M}+\mathrm{Na}]^{+}$pseudomolecular ion peak at $m / z 341.2093$, indicative of the molecular formula $\mathrm{C}_{20} \mathrm{H}_{30} \mathrm{O}_{3}$, requiring six degrees of unsaturation. The ${ }^{13} \mathrm{C}$ NMR spectrum contained 20 distinct resonances, including four $s p^{2}$ centres, while the fully-coupled HSQC spectrum accounted for 29 of the 30 protons, indicating the presence of an exchangeable proton.

Analysis of the ${ }^{1} \mathrm{H}$ and HSQC NMR spectra $\left(\mathrm{CDCl}_{3}\right)$ suggested the presence of a 3 -substituted furan system $\left[\left(\delta_{\mathrm{C}} 111.0, \delta_{\mathrm{H}} 6.26\right),\left(\delta_{\mathrm{C}} 138.9, \delta_{\mathrm{H}} 7.21\right)\right.$ and $\left(\delta_{\mathrm{C}} 142.9\right.$, $\left.\left.\delta_{\mathrm{H}} 7.34\right)\right]$, a hemi-acetal methine $\left(\delta_{\mathrm{C}} 98.0, \delta_{\mathrm{H}} 5.33\right)$, two deshielded diastereotopic methylene pairs $\left[\left(\delta_{\mathrm{C}} 25.8, \delta_{\mathrm{H}}\right.\right.$ a $\left.2.52, \mathrm{~b} 2.29\right)$ and $\left(\delta_{\mathrm{C}} 26.0, \delta_{\mathrm{H}}\right.$ a $\left.\left.1.88, \mathrm{~b} 1.72\right)\right]$, a deshielded methyl singlet $\left(\delta_{\mathrm{C}} 23.0, \delta_{\mathrm{H}} 1.31\right)$, two methines $\left[\left(\delta_{\mathrm{C}} 51.7, \delta_{\mathrm{H}} 1.18\right)\right.$ and $\left.\left(\delta_{\mathrm{C}} 57.4, \delta_{\mathrm{H}} 1.15\right)\right]$, a gem-dimethyl pair $\left[\left(\delta_{\mathrm{C}} 21.7, \delta_{\mathrm{H}} 0.89\right)\right.$ and $\left.\left(\delta_{\mathrm{C}} 33.0, \delta_{\mathrm{H}} 0.88\right)\right]$ and a region of diastereotopic methylene protons integrating for 10 resonances. Four spin systems were identified in the COSY spectrum: the $s p^{2}$ hybridised furan system, two isolated segments of contiguous deshielded diastereotopic methylenes and a methine, and a further isolated segment of three contiguous diastereotopic methylenes. Immediately apparent from the HMBC were the characteristic patterns of a gem-dimethyl pair and the 3-substituted furan system.

Olefinic methines CH-16 $\left(\delta_{\mathrm{C}} 138.9, \delta_{\mathrm{H}} 7.21\right)$ and $\mathrm{CH}-14\left(\delta_{\mathrm{C}} 111.0, \delta_{\mathrm{H}} 6.26\right)$ share reciprocal allylic COSY couplings and strong three-bond HMBC correlations. Vicinal COSY and HMBC correlations were observed between CH-14 and CH-15 $\left(\delta_{\mathrm{C}} 142.9, \delta_{\mathrm{H}} 7.34\right)$ and further HMBC correlations were observed from the three methines to the non-protonated olefinic carbon centre $\mathrm{C}-13\left(\delta_{\mathrm{C}} 125.1\right)$, as depicted in Figure 3.4. Methines CH-16 and CH-15 both display large ${ }^{1} J_{\mathrm{CH}}$ couplings of $203 \mathrm{~Hz}$ and $201 \mathrm{~Hz}$ respectively, consistent with oxygen-substituted $s p^{2}$ centres, whereas $\mathrm{CH}-14$ displays a smaller coupling consistent with carbon substitution. The ${ }^{13} \mathrm{C}$ chemical shifts of the four centres were consistent with oxygen substitution of $\mathrm{CH}$ 15 and $\mathrm{CH}-16$. Placement of the oxygen between $\mathrm{CH}-15$ and $\mathrm{CH}-16$ completed the 3 -substituted furan system, accounting for three degrees of molecular unsaturation.

As depicted in Figure 3.5, connection between $\mathrm{CH}_{2}-12\left(\delta_{\mathrm{C}} 25.8, \delta_{\mathrm{H}}\right.$ a 2.52 , b 2.29$)$ and $\mathrm{CH}_{2}-11\left(\delta_{\mathrm{C}} 26.0, \delta_{\mathrm{H}}\right.$ a $\left.1.88, \mathrm{~b} 1.72\right)$ was evidenced by strong correlations in the COSY spectrum, corroborated by reciprocal HMBC correlations. Protons of $\mathrm{CH}_{2}-11$ shared further COSY correlations with methine $\mathrm{CH}-9\left(\delta_{\mathrm{C}} 57.4, \delta_{\mathrm{H}} 1.15\right)$, and reciprocal HMBC correlations were observed between the centres. 

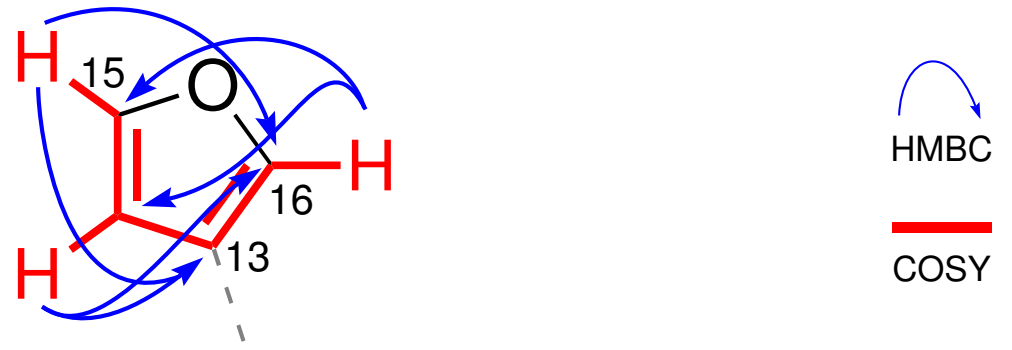

Figure 3.4 COSY and selected HMBC $(\mathrm{H} \rightarrow \mathrm{C})$ correlations observed within the furan system of 33.

The third COSY spin system extends from methine $\mathrm{CH}-5\left(\delta_{\mathrm{C}} 51.7, \delta_{\mathrm{H}} 1.18\right)$ to $\mathrm{CH}_{2^{-}}$ $7\left(\delta_{\mathrm{C}} 39.8, \delta_{\mathrm{H}}\right.$ a $\left.1.62, \mathrm{~b} 1.42\right)$. Strong COSY coupling was observed between CH-5 and methylene $\mathrm{CH}_{2}-6\left(\delta_{\mathrm{C}} 21.0, \delta_{\mathrm{H}}\right.$ a 1.69 , b 1.48), with the large coupling (17.4 $\mathrm{Hz}$ ) between $\mathrm{H}-5$ and $\mathrm{H}_{2}-6$ a indicating an anti-periplanar relationship. Protons of $\mathrm{CH}_{2}-6$ further correlated with those of $\mathrm{CH}_{2}-7$, with the connectivities corroborated by HMBC correlations as shown in Figure 3.5.

Identification of the final spin system by the COSY experiment began with the diastereotopic methylene $\mathrm{CH}_{2}-1\left(\delta_{\mathrm{C}} 29.6, \delta_{\mathrm{H}}\right.$ a 2.21 , b 1.03), the protons of which shared reciprocal COSY correlations with $\mathrm{CH}_{2}-2\left(\delta_{\mathrm{C}} 19.9, \delta_{\mathrm{H}}\right.$ a $\left.1.47, \mathrm{~b} 1.38\right) . \mathrm{CH}_{2}-2$ displayed further COSY correlations to a third methylene $\mathrm{CH}_{2}-3\left(\delta_{\mathrm{C}} 41.9, \delta_{\mathrm{H}}\right.$ a 1.39 , b 1.16). Selective irradiation of the $\mathrm{H}-1 \mathrm{a}$ methylene proton with a 1DTOCSY experiment provided further evidence of the C-1 to C-3 segment. Over spin-lock mixing times of 20-60 ms, geminal partner $\mathrm{H}-1 \mathrm{~b}$, both $\mathrm{H}_{2}-2$ protons and the resonances of $\mathrm{H}_{2}-3$ were sequentially revealed, proving connection of $\mathrm{CH}_{2}-1$ to $\mathrm{CH}_{2}-3$ in this order (Figure 3.5).
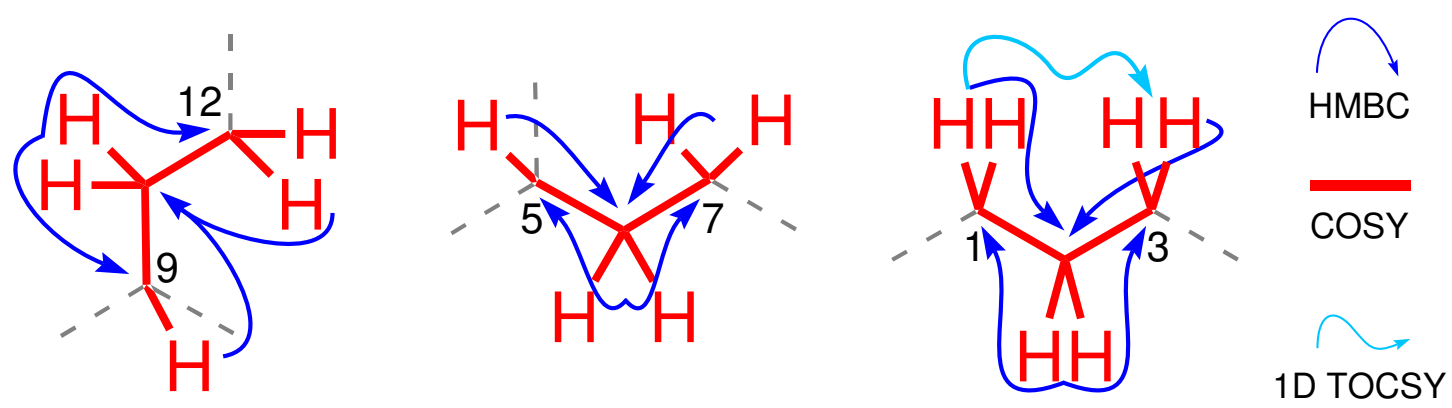

Figure 3.5 COSY, selected HMBC and 1D TOCSY correlations establishing the connectivities C-9 to C-12, C-5 to C-7 and C-1 to C-3 in 33.

A gem-dimethyl pair, $\mathrm{CH}_{3}-18\left(\delta_{\mathrm{C}} 21.7, \delta_{\mathrm{H}} 0.89\right)$ and $\mathrm{CH}_{3}-19\left(\delta_{\mathrm{C}} 33.0, \delta_{\mathrm{H}} 0.88\right)$ was evidenced by reciprocal HMBC correlations between the methyls, and shared correlations to a quaternary centre C-4 $\left(\delta_{\mathrm{C}} 34.1\right)$, methine $\mathrm{CH}-5$, and methylene $\mathrm{CH}_{2}-3$. Further HMBC correlations from the protons of $\mathrm{CH}_{2}-3$ to $\mathrm{C}-4$ and $\mathrm{CH}-5$, and from $\mathrm{CH}-5$ to $\mathrm{C}-4$, established the connection between the C-1 to C-3 methylene series and the gem-dimethyl pair (Figure 3.6). 

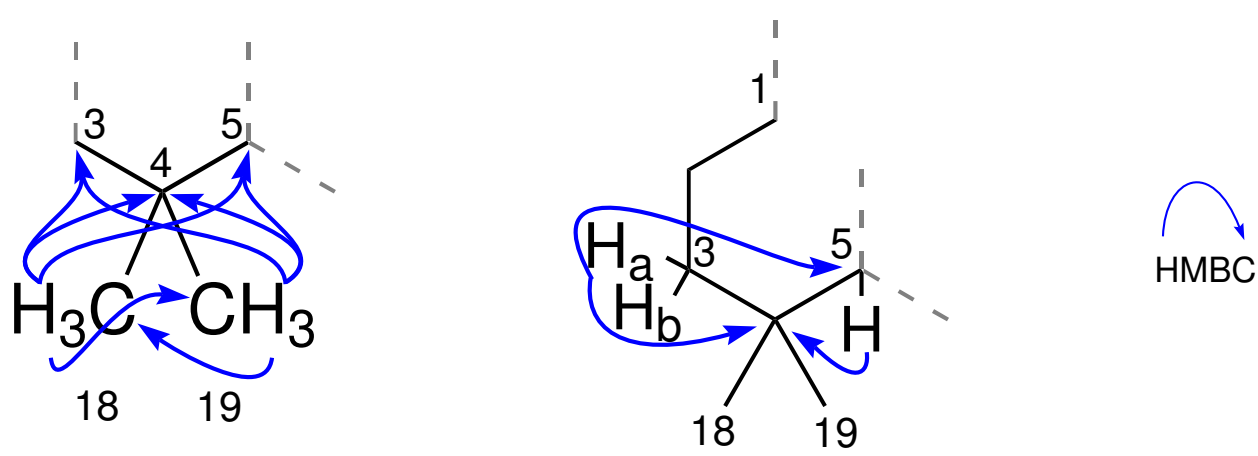

HMBC

Figure 3.6 HMBC correlations establishing the gem-dimethyl substructure in $\mathbf{3 3}$, and its connection to the $\mathrm{C}-1$ to $\mathrm{C}-3$ methylene segment.

The protons of methyl singlet $\mathrm{CH}_{3}-17\left(\delta_{\mathrm{C}} 23.0, \delta_{\mathrm{H}} 1.31\right)$ shared weak COSY correlations with $\mathrm{H}-9$ and $\mathrm{H}_{2}-7$, and correlated strongly in the HMBC experiment with the two protonated centres and C-8. These correlations completed the linear sequence extending from $\mathrm{CH}_{2}-1$ to $\mathrm{CH}-9$ as shown in Figure 3.7.

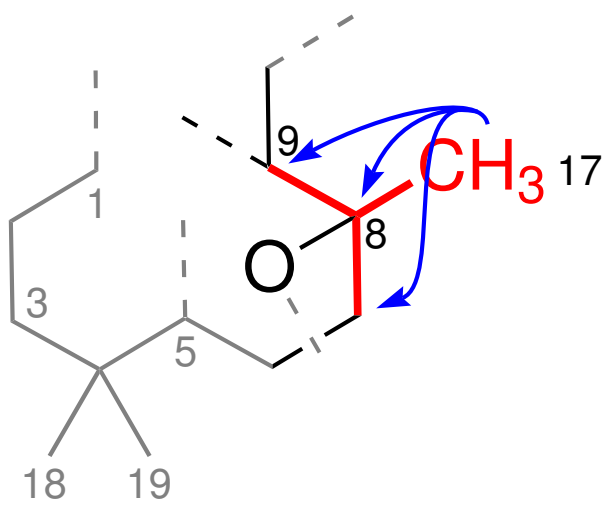

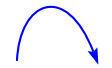

HMBC

cosy

Figure 3.7 COSY and selected HMBC correlations establishing the linear connections from C-7 to C-9 and methyl substitution of C-8 in $\mathbf{3 3}$.

Observation of $\mathrm{HMBC}$ correlations from the protons of $\mathrm{CH}_{2}-2$ to the quaternary centre C-10 established the bond between C-1 and C-10, extending the linear sequence by one carbon atom. Similarly, an HMBC correlation from H-1b to C-9 established the C-10-CH-9 bond. Corroborating correlations were observed from H-9 to the oxygen-substituted C-8 $\left(\delta_{\mathrm{C}} 84.0\right)$ and fully carbon-substituted C-10 $\left(\delta_{\mathrm{C}} 51.9\right)$ quaternary centres. These correlations completed the cyclodecane system C-1 to C10, accounting for a further degree of molecular unsaturation. Connection between the furan system (C-13 to C-16) and the substituted cyclodecane segment C-1 to C-12 was established by HMBC correlations from the protons of the diastereotopic methylene $\mathrm{CH}_{2}-12$ to the $s p^{2}$ centres $\mathrm{C}-13, \mathrm{CH}-14$ and $\mathrm{CH}-16$. This completed the carbon skeleton from C-1 to C-16 as shown in Figure 3.8. 

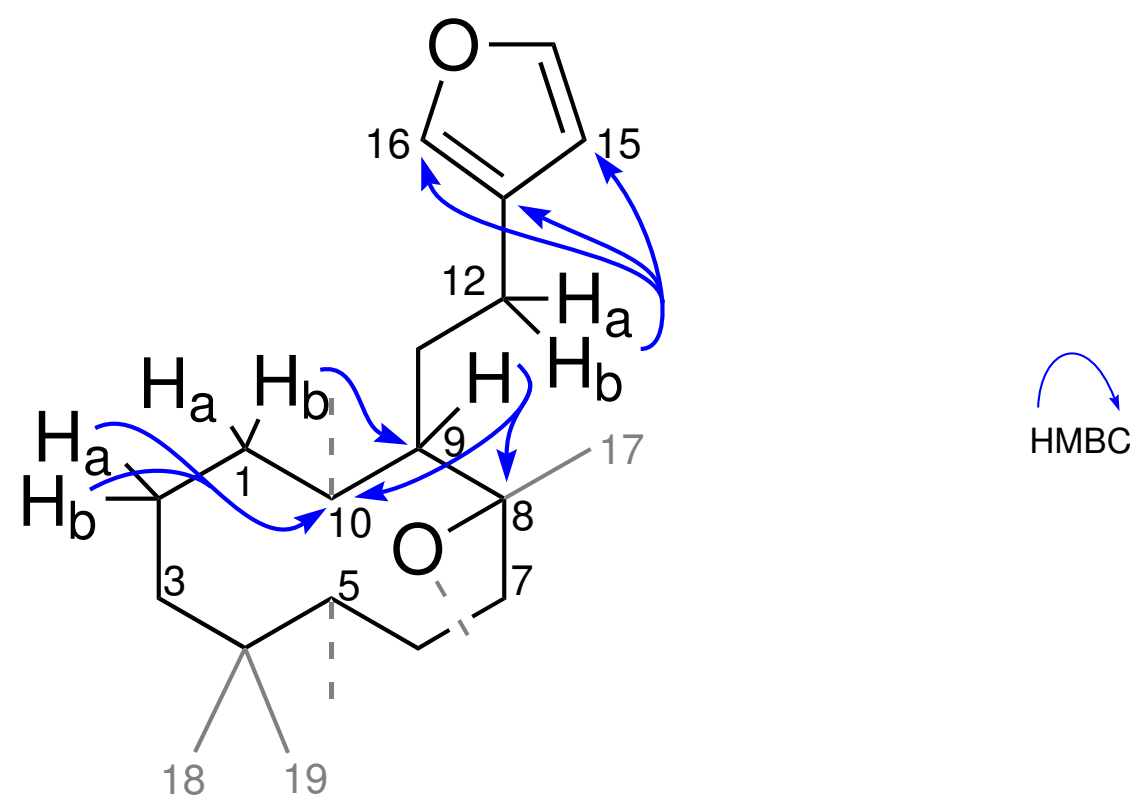

$\mathrm{HMBC}$

Figure 3.8 Selected HMBC correlations establishing the cyclodecane system of $\mathbf{3 3}$, and attachment of the furan moiety.

All but the carbon resonance, CH-20 $\left(\delta_{\mathrm{C}} 98.0, \delta_{\mathrm{H}} 5.33,{ }^{1} J_{\mathrm{CH}} 170 \mathrm{~Hz}\right)$, was accounted for the by furan-subsituted cyclodecane system, C-1 to C-19. The deshielded chemical shift and large ${ }^{1} J_{\mathrm{CH}}$ value of $\mathrm{CH}-20$ are consistent with a hemi-acetal functionality. The exchangeable hydroxyl was evidenced by the IR and ${ }^{1} \mathrm{H}$ NMR spectra $\left(\nu_{\max } 3394 \mathrm{~cm}^{-1}, \delta_{\mathrm{H}} 4.08\right)$, and a COSY correlation observed with $\mathrm{H}-20$. The hemi-acetal methine H-20 displayed HMBC correlations to C-10, and the methines CH-5 and CH-9, and a further HMBC correlation was observed from H-5 to C10. These correlations established the bond between $\mathrm{CH}-5$ and $\mathrm{C}-10$, forming the two fused six-membered rings of the [4.4.0]-bicyclodecane system in luakuliide A (33), accounting for another double-bond equivalent in the fused bicyclic system. A weak HMBC correlation was observed from H-20 to the oxygen substituted C-8 $\left(\delta_{\mathrm{C}} 84.0\right)$ establishing a hemi-acetal bridge. Somewhat surprisingly, reciprocal fivebond correlations were observed in the COSY experiment between $\mathrm{H}-20$ and $\mathrm{H}_{3}-17$, providing further evidence of their connection. The hemi-acetal bridge between $\mathrm{C}$ 10 and the oxygen-substituted C-8 completed the structure of $\mathbf{3 3}$ and accounted for the final degree of unsaturation in the tricyclic diterpene (Figure 3.9).

Requisite of the fused tricyclic system is the 1,3-diaxial positioning of the hemiacetal, CH-20, and oxygen substituent of $\mathrm{C}-8$, placing $\mathrm{CH}_{3}-17$ in an equatorial position on ring B. A weak COSY coupling between $\mathrm{H}-5$ and $\mathrm{CH}_{3}-19$ established their 1,2-diaxial relationship on ring $\mathrm{A}$, and the large coupling $(12.8 \mathrm{~Hz})$ between H-5 and H-6a indicated their 1,2-diaxial relationship on ring B (Figure 3.10). 

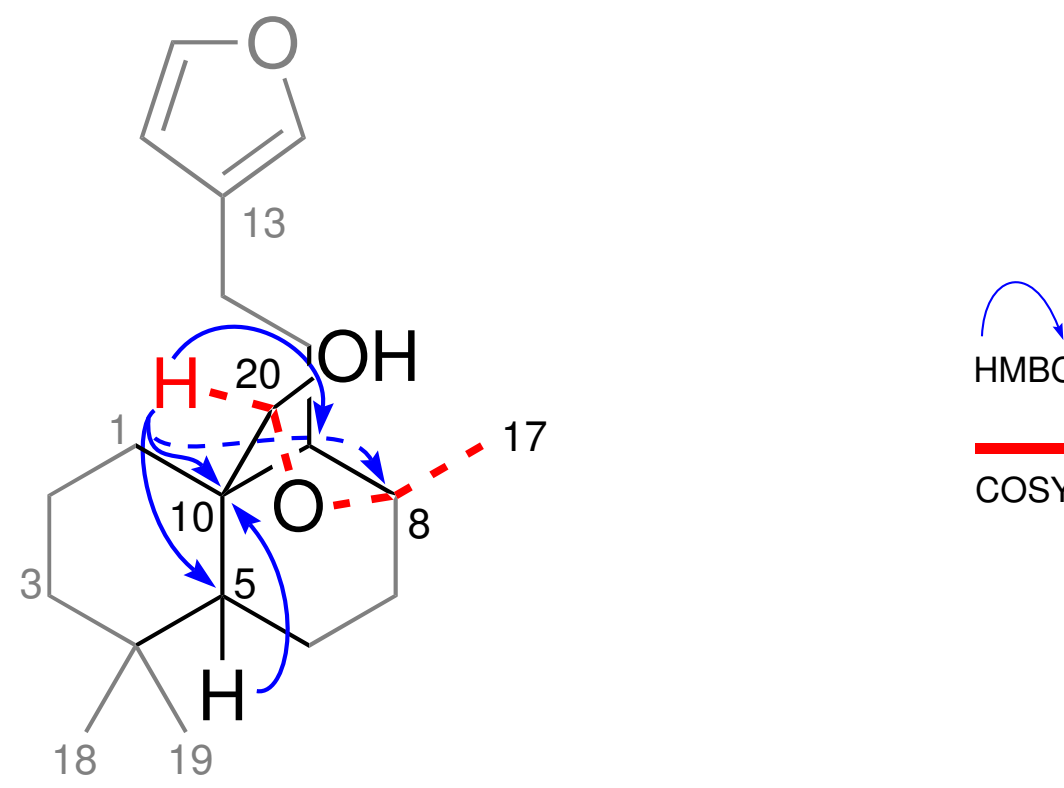

HMBC

COSY

Figure 3.9 COSY and HMBC correlations establishing the hemi-acetal bridge between C-10 and C-8 in 33. Dashed lines and arrows indicate a weak correlations.

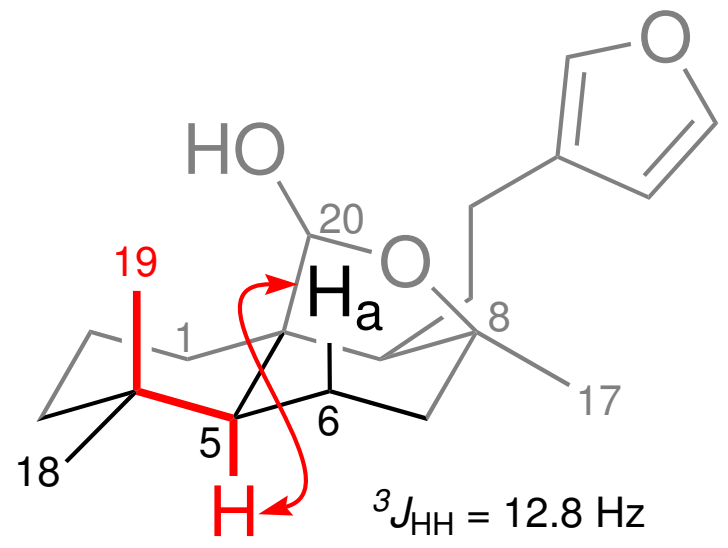

$\cos Y$

Figure 3.10 The trans-fused [4.4.0]-bicyclodecane system of $\mathbf{3 3}$ as evidenced by ${ }^{1} \mathrm{H}-{ }^{1} \mathrm{H}$ coupling between $\mathrm{H}-5$ and $\mathrm{H}-6 \mathrm{a}\left({ }^{3} J_{\mathrm{HH}}=12.8 \mathrm{~Hz}\right)$ and COSY correlations between $\mathrm{H}-5$ and $\mathrm{H}_{3}-19$.

Following selective irradiation of the hemi-acetal methine H-20 in a $1 \mathrm{D}$ NOE experiment, positive enhancements of $\mathrm{H}_{3}-19$ and axial $\mathrm{H}-6$ a were observed, placing these protons on the same face of the [4.4.0]-bicyclodecane system. Irradiation of $\mathrm{H}$ 11a with a $1 \mathrm{D}$ TOCSY experiment revealed H-9 $\left(\delta_{\mathrm{H}} 1.15\right)$ after a $20 \mathrm{~ms}$ mixing time. The excited H-9 resonance provided a resolved target for a selective 1D NOE experiment. The experiment produced positive enhancement of the equatorial methyl $\mathrm{H}_{3}-17$, and the protons $\mathrm{H}-5\left(\delta_{\mathrm{H}} 1.18\right), \mathrm{H}-1 \mathrm{~b}\left(\delta_{\mathrm{H}} 1.03\right)$ and $\mathrm{H}-7 \mathrm{~b}\left(\delta_{\mathrm{H}} 1.42\right)$, placing them on the same face of the bicycle (Figure 3.11). These correlations firmly established the trans-fusion of the [4.4.0]-bicyclodecane system, and equatorial attachment of the furan-bearing segment to ring B. 


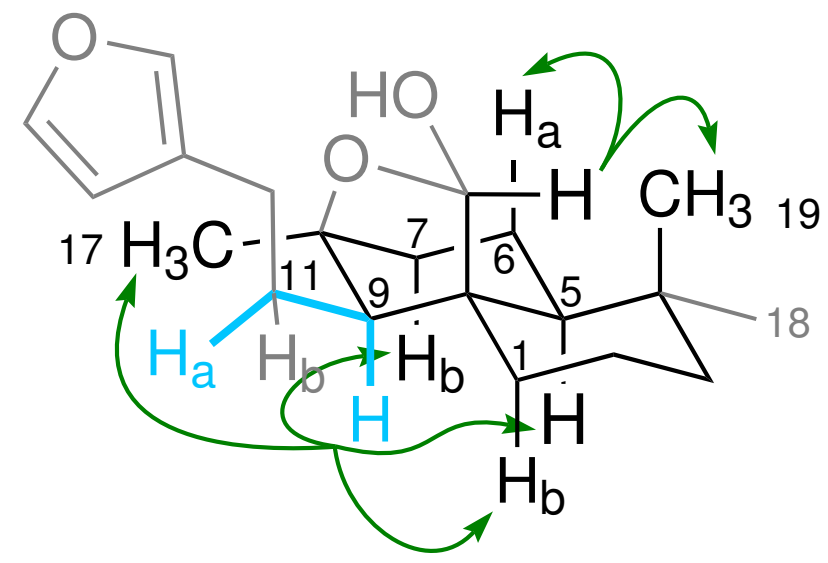

1D TOCSY<smiles>C1CCC1</smiles>

1D NOE

Figure 3.11 1D TOCSY-NOESY and 1D NOE correlations used to determination the relative stereochemistry about the trans-[4.4.0]-bicyclodecane system in $\mathbf{3 3}$.

Isolation of the methyl-acetal analogue 34 and facile conversion of 33 into 34 confirmed the hemi-acetal functionality of luakuliide A (33). Positive ion-mode HRESIMS of the derivative $\mathbf{3 4}$ generated a pseudo-molecular ion corresponding to the molecular formula $\mathrm{C}_{21} \mathrm{H}_{32} \mathrm{O}_{3}\left(\mathrm{~m} / z 355.2249[\mathrm{M}+\mathrm{Na}]^{+}, \Delta 2.3 \mathrm{ppm}\right)$, and the two displayed similar optical rotations; $33,[\alpha]_{D}^{25.4}-10.8^{\circ}\left(c 18.5, \mathrm{CHCl}_{3}\right)$ and $\mathbf{3 4},[\alpha]_{D}^{25.4}$ $-75.0^{\circ}\left(c 0.14, \mathrm{CHCl}_{3}\right)$. With the exception of the strong methoxy singlet signal $\left(\delta_{\mathrm{C}} 55.4, \delta_{\mathrm{H}} 3.34\right)$ and the up-field shift of the acetal position (from $\delta_{\mathrm{C}} 98.0, \delta_{\mathrm{H}} 5.33$ to $\left.\delta_{\mathrm{C}} 104.9, \delta_{\mathrm{H}} 4.83\right)$, the two compounds proved to be very spectroscopically similar as shown in Table 3.1.

Table 3.1 NMR Spectral Data for the Acetal Moieties of $\mathbf{3 3}$ and $\mathbf{3 4},\left(600 \mathrm{MHz}, \mathrm{CDCl}_{3}\right)$

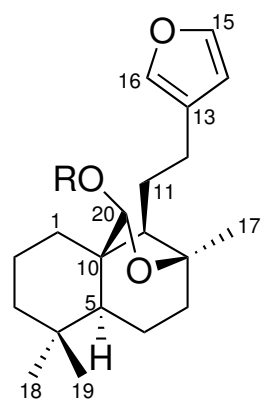

$$
\begin{array}{ll}
33 & \mathrm{R}=\mathrm{H} \\
34 & \mathrm{R}=\mathrm{Me}
\end{array}
$$

\begin{tabular}{|c|c|c|c|c|c|c|c|}
\hline \multirow[b]{2}{*}{ Position } & \multirow[b]{2}{*}{ mult } & \multicolumn{2}{|c|}{33} & \multicolumn{2}{|c|}{34} & \multicolumn{2}{|c|}{ Difference } \\
\hline & & $\overline{\delta_{\mathrm{C}}}(\mathrm{ppm})$ & $\delta_{\mathrm{H}}(\mathrm{ppm})$ & $\delta_{\mathrm{C}}(\mathrm{ppm})$ & $\delta_{\mathrm{H}}(\mathrm{ppm})$ & $\Delta \delta_{\mathrm{C}}(\mathrm{ppm})$ & $\Delta \delta_{\mathrm{H}}(\mathrm{ppm})$ \\
\hline 8 & $\mathrm{C}$ & 84.0 & & 83.7 & & -0.3 & \\
\hline 9 & $\mathrm{CH}$ & 57.4 & 1.15 & 57.5 & 1.15 & 0.1 & 0.0 \\
\hline 10 & $\mathrm{C}$ & 51.9 & & 52.0 & & 0.1 & \\
\hline 20 & $\mathrm{CH}$ & 98.0 & 5.44 & 104.9 & 4.83 & 5.9 & -0.50 \\
\hline $\mathrm{OCH}_{3}$ & $\mathrm{CH}_{3}$ & & & 55.4 & 3.34 & & \\
\hline
\end{tabular}

$* \Delta \delta=\delta_{\mathbf{3 4}}-\delta_{\mathbf{3 3}}$

Absolute configuration is arbitrary. 


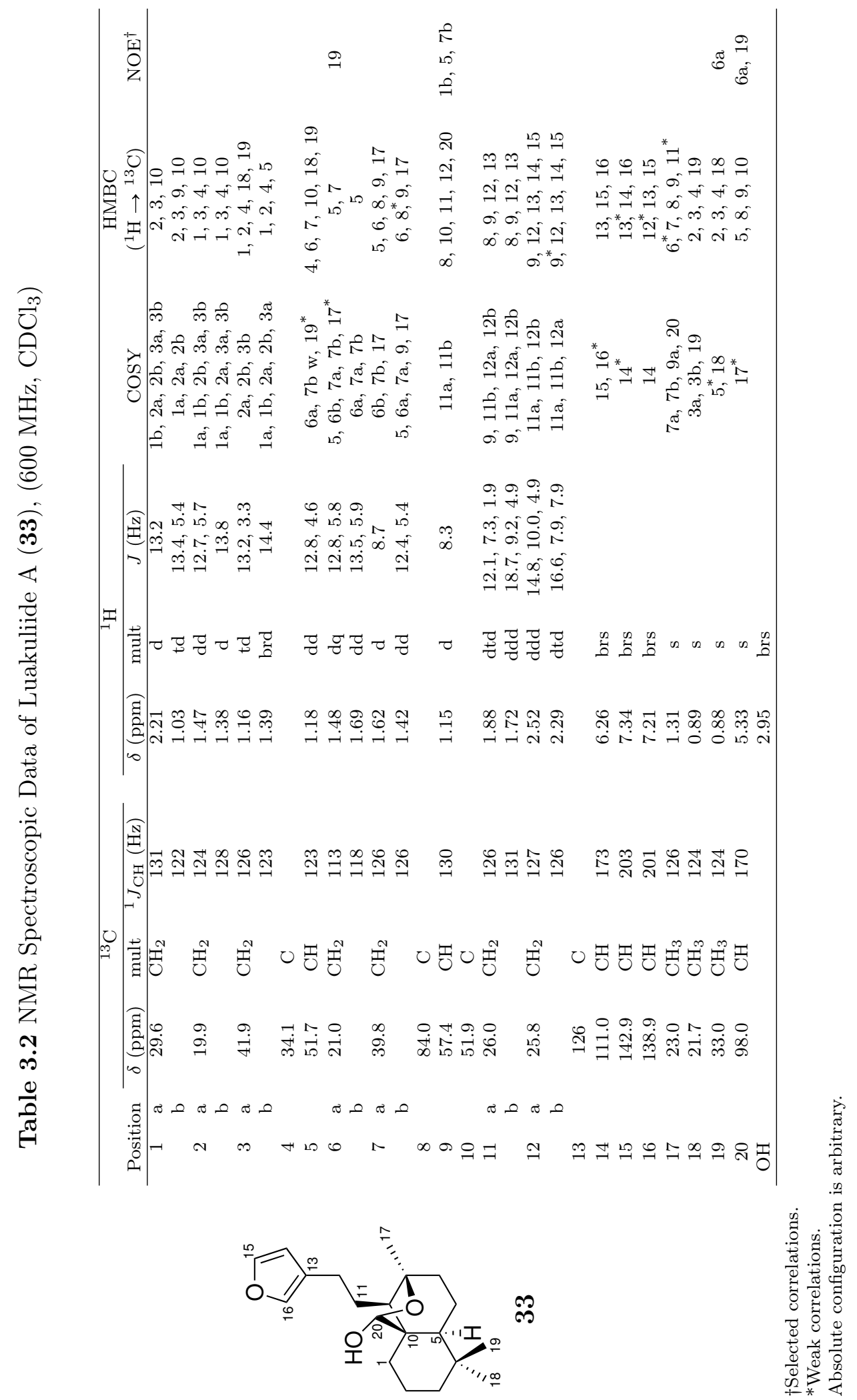



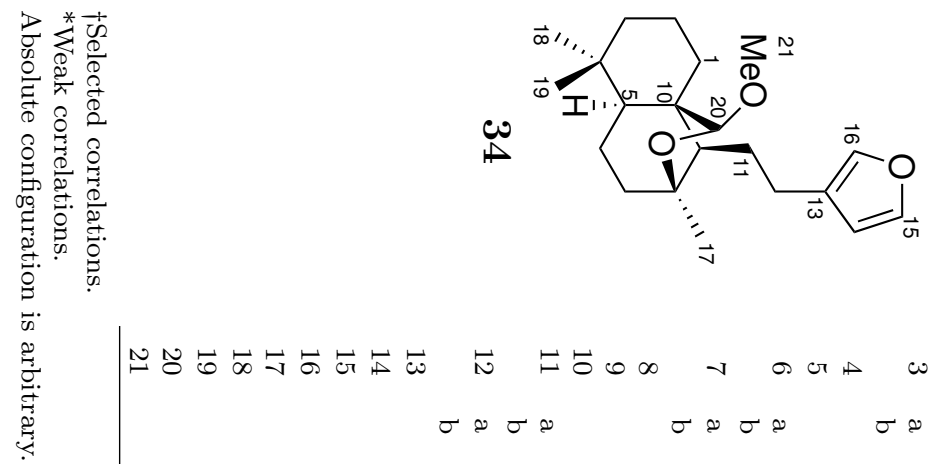

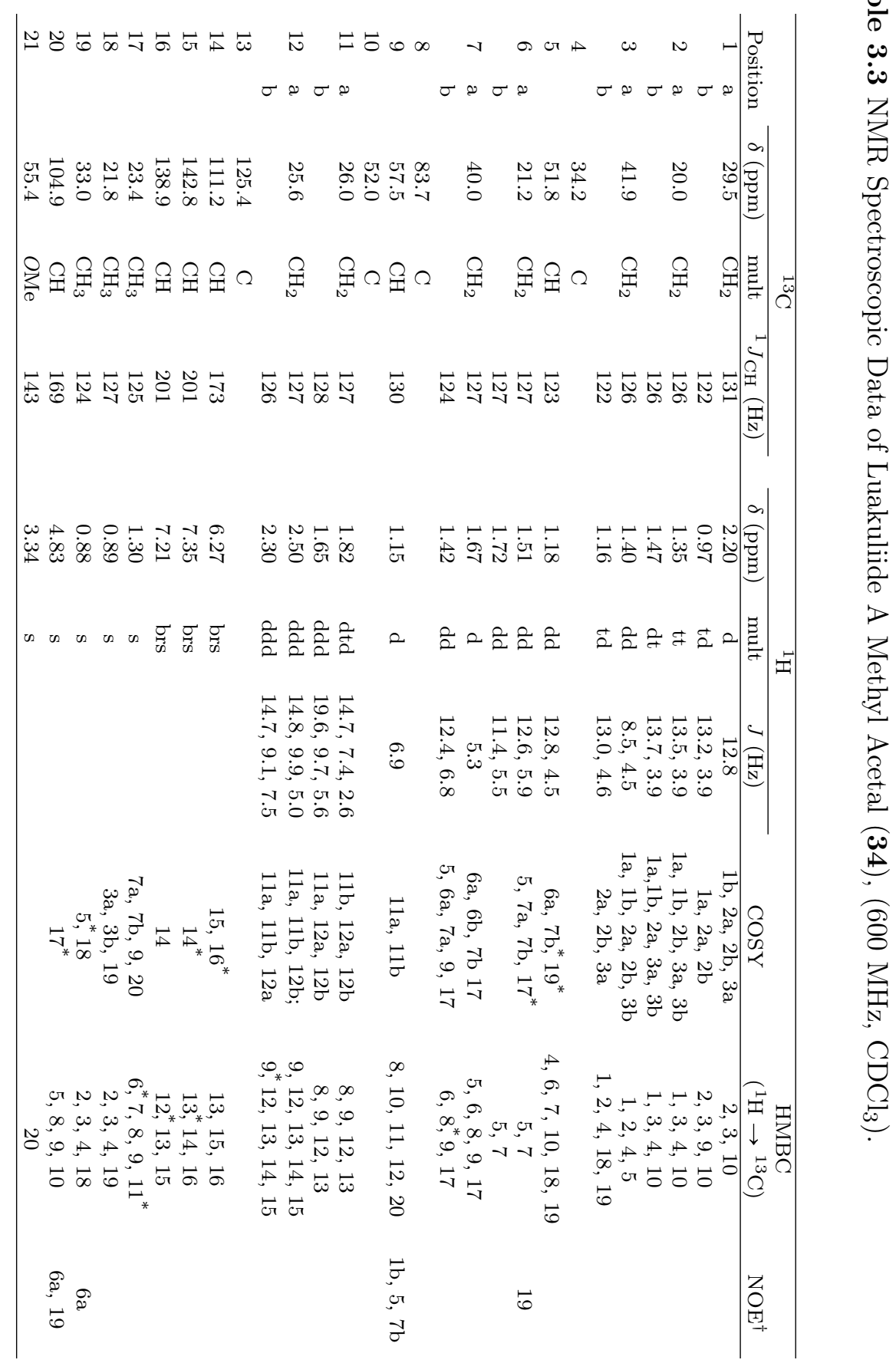




\section{Luakuliide B (35)}

Analysis of luakuliide B $(\mathbf{3 5})$ by positive ion-mode HRESIMS revealed a $[\mathrm{M}+\mathrm{Na}]^{+}$ pseudo-molecular ion peak at $m / z 373.1996$, corresponding to the molecular formula $\mathrm{C}_{20} \mathrm{H}_{30} \mathrm{O}_{5}$. The ${ }^{13} \mathrm{C}$ NMR spectrum displayed all 20 expected resonances, and analysis of the fully-coupled HSQC spectrum in conjunction with the ${ }^{1} \mathrm{H}$ NMR spectrum accounted for all but two hydrogens, indicating the presence of an additional exchangeable proton relative to compound 33 .

Inspection of the ${ }^{1} \mathrm{H},{ }^{13} \mathrm{C}$ NMR spectra and HSQC experiment of luakuliide B (35) revealed much structural similarity with that of $\mathbf{3 3}$, particularly in the aliphatic region. As such, the structure was elucidated in the same fashion as luakuliide A (33). The characteristic furan signals were, however, replaced by an ester carbonyl $\left(\mathrm{C}-16: \delta_{\mathrm{C}} 171.5\right)$, an olefinic methine $\left(\delta_{\mathrm{C}} 143.0, \delta_{\mathrm{H}} 6.87\right)$, a non-protonated olefinic centre $\left(\delta_{\mathrm{C}} 131.0\right)$ and a hemi-acetal methine $\left(\delta_{\mathrm{C}} 96.7, \delta_{\mathrm{H}} 6.10,{ }^{1} J_{\mathrm{CH}} 176 \mathrm{~Hz}\right)$.

As illustrated in Figure 3.12, the methylene centres $\mathrm{CH}_{2}-11$ and $\mathrm{CH}_{2}-12$ appear at a slightly higher chemical shift, relative to those in $33\left[\left(\mathrm{CH}_{2}-11: \delta_{\mathrm{C}} 23.6\right.\right.$, $\delta_{\mathrm{H}}$ a $\left.1.91, \mathrm{~b} 1.73\right)$ and $\left(\mathrm{CH}_{2}-12: \delta_{\mathrm{C}} 26.0, \delta_{\mathrm{H}}\right.$ a $\left.\left.2.41, \mathrm{~b} 2.21\right)\right]$, and share reciprocal COSY and HMBC correlations with each other and the methine CH-9 $\left(\delta_{\mathrm{C}} 58.0\right.$, $\left.\delta_{\mathrm{H}} 1.22\right)$. Connection from $\mathrm{CH}-5$ to $\mathrm{CH}_{2}-7$ [ $\left(\mathrm{CH}-5: \delta_{\mathrm{C}} 51.8, \delta_{\mathrm{H}} 1.22\right),\left(\mathrm{CH}_{2}-6 \delta_{\mathrm{C}} 21.0\right.$, $\delta_{\mathrm{H}}$ a $\left.1.50, \mathrm{~b} 1.72\right)$ and $\left(\mathrm{CH}_{2}-7 \delta_{\mathrm{C}} 41.9, \delta_{\mathrm{H}}\right.$ a 1.68 , b 1.42$\left.)\right]$ was evidenced by COSY correlations between the centres, and corroborating HMBC correlations. Further COSY and HMBC correlations established the C-1 to $\mathrm{C}-3$ spin system $\left[\left(\mathrm{CH}_{2}-1\right.\right.$ : $\delta_{\mathrm{C}} 29.6, \delta_{\mathrm{H}}$ a $\left.2.21, \mathrm{~b} 1.08\right),\left(\mathrm{CH}_{2}-2: \delta_{\mathrm{C}} 19.9, \delta_{\mathrm{H}}\right.$ a $\left.1.51, \mathrm{~b} 1.39\right)$ and $\left(\mathrm{CH}_{2}-3: \delta_{\mathrm{C}} 39.8\right.$, $\delta_{\mathrm{H}}$ a 1.43 , b 1.19)], which was confirmed by a $1 \mathrm{D}$ TOCSY experiment as seen in 33.
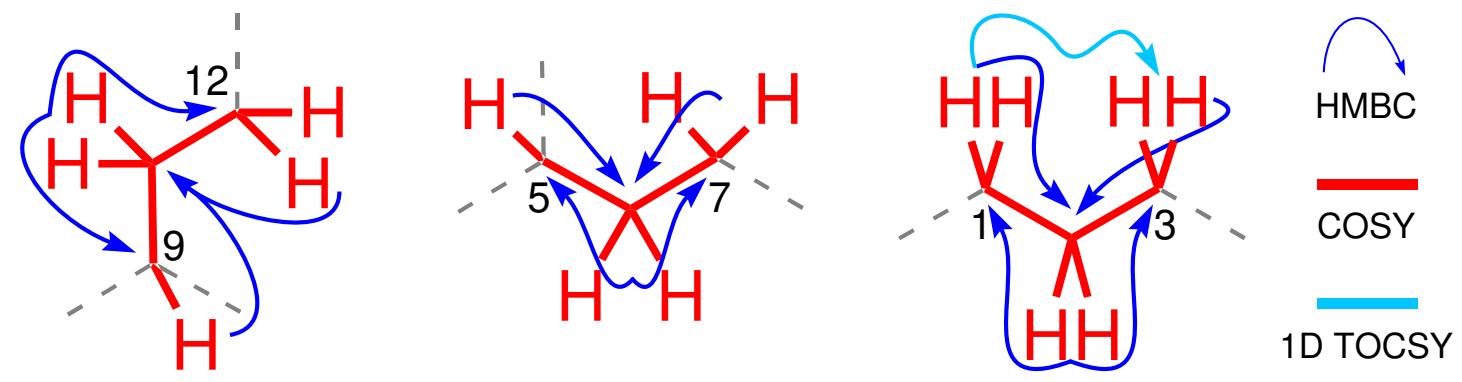

Figure 3.12 COSY, HMBC and 1D TOCSY correlations establishing the connections from C-9 to C-12, C-5 to C-7 and from C-1 to C-3 in $\mathbf{3 5 .}$

The gem-dimethyl pair $\left[\left(\mathrm{CH}_{3}-18: \delta_{\mathrm{C}} 21.8, \delta_{\mathrm{H}} 0.90\right)\right.$ and $\left.\left(\mathrm{CH}_{3}-19: \delta_{\mathrm{C}} 33.0, \delta_{\mathrm{H}} 0.90\right)\right]$ was again evidenced by the display of reciprocal HMBC correlations between the methyls and shared correlations to $\mathrm{CH}-5\left(\delta_{\mathrm{C}} 51.8, \delta_{\mathrm{H}} 1.22\right)$, C-4 $\left(\delta_{\mathrm{C}} 34.2\right)$ and $\mathrm{CH}_{2}-3$. As with 33, HMBC correlations were observed from $\mathrm{H}_{2}-3$ and $\mathrm{H}-5$ to the quaternary carbon $\mathrm{C}-4$, and from $\mathrm{H}_{2}-3$ to $\mathrm{CH}-5$, establishing the linear connections from C-1 to C-5 (Figure 3.13). 

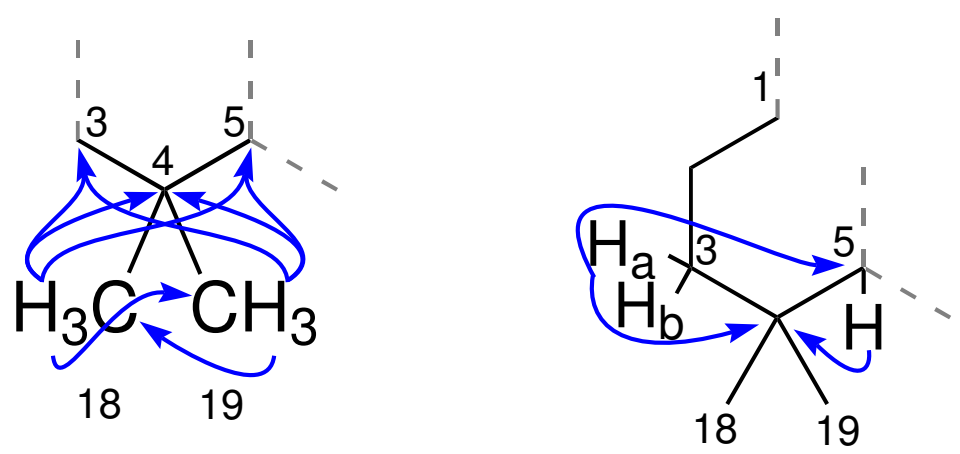

$\bigcap_{\text {HMBC }}$

Figure 3.13 HMBC correlations establishing the gem-dimethyl substructure in $\mathbf{3 5}$ and the connections from $\mathrm{C}-1$ to $\mathrm{C}-5$.

As observed in compound 33, the methyl singlet of $\mathbf{3 5}, \mathrm{CH}_{3}-17\left(\delta_{\mathrm{C}} 23.3, \delta_{\mathrm{H}} 1.31\right)$ shared weak COSY correlations with $\mathrm{H}-9$ and $\mathrm{CH}_{2}-7\left(\delta_{\mathrm{C}} 41.9, \delta_{\mathrm{H}}\right.$ a $\left.1.68, \mathrm{~b} 1.42\right)$, and displayed HMBC correlations to the oxygen-substituted C-8 $\left(\delta_{\mathrm{C}} 84.0\right), \mathrm{CH}-9$ and $\mathrm{CH}_{2}-7$. Protons of the methylenes $\mathrm{CH}_{2}-2$ and $\mathrm{CH}_{2}-1$ correlated to $\mathrm{C}-10$ and C-9, respectively, completing the linear connectivity from C-1 to C-10, corroborated by HMBC correlations from H-9 to quaternary centres C-8 and C-10 (Figure 3.14).
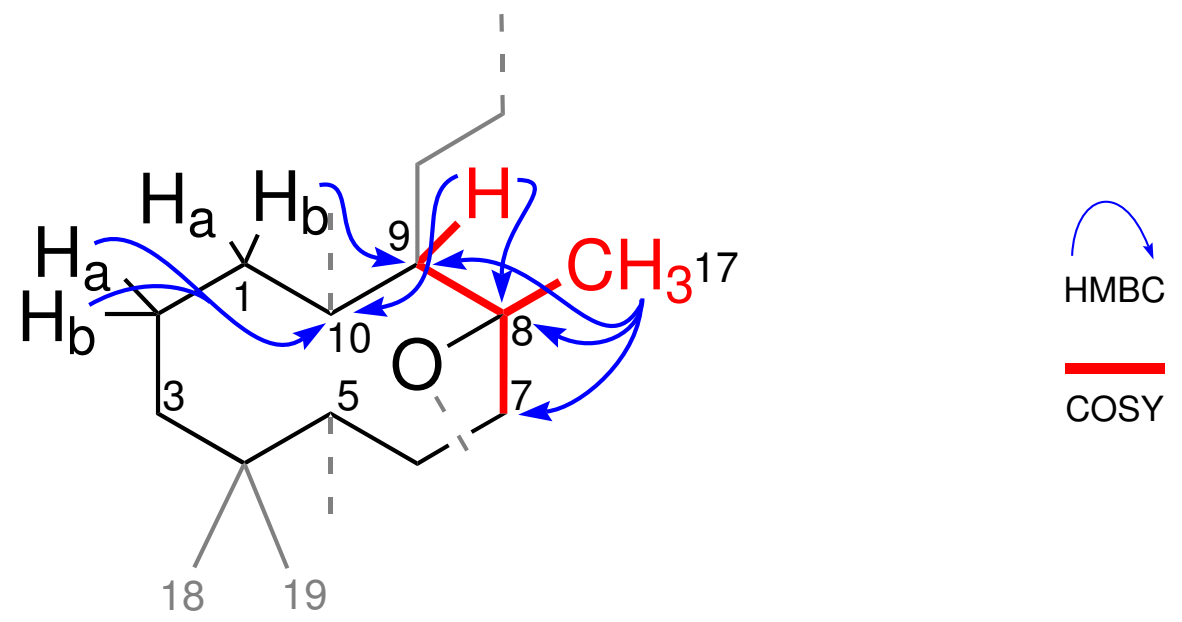

Figure 3.14 COSY and selected HMBC correlations completing the cyclodecane system of 35 .

The hemi-acetal methine CH-20 ( $\delta_{\mathrm{C}}$ 98.0, $\delta_{\mathrm{H}}$ 5.35), displayed strong HMBC correlations to C-10 and weaker correlations to C-5 and C-9. These correlations reestablished the [4.4.0]-bicyclodecane system. Again, a weak HMBC correlation was observed from H-20 to C-8, establishing the hemi-acetal link in $\mathbf{3 5}$, as shown in Figure 3.15.

As required by constraints of the fused tricyclic system, $\mathrm{CH}-20$ and the oxygensubstituent of $\mathrm{C}-8$ occupy 1,3-diaxial positions on ring $\mathrm{A}$, with $\mathrm{CH}_{3}-17$ equatorial at C-8. As in 33, the large ${ }^{3} J_{\mathrm{HH}}$ coupling between H-5 and H-6a $(13.6 \mathrm{~Hz})$ was indicative of their 1,2-diaxial relationship on ring B. Similarly, the observation of 
a weak COSY correlation between $\mathrm{H}-5$ and $\mathrm{H}_{3}-19$ placed these resonances as 1,2diaxial with respect to ring A, confirming trans-fusion of the [4.4.0]-bicyclodecane system. The 1D TOCSY-NOESY experiment utilised in 33 to establishing configuration at CH-9 could not be employed here owing to small sample size. However, similarities in chemical shift and ${ }^{3} J_{\mathrm{HH}}$ couplings of CH-9 between 35 and 33 suggests retention of relative stereochemistry about C-9, placing the $\mathrm{CH}_{2}$ 11 to $\mathrm{C}-16$ substructure in an equatorial position.
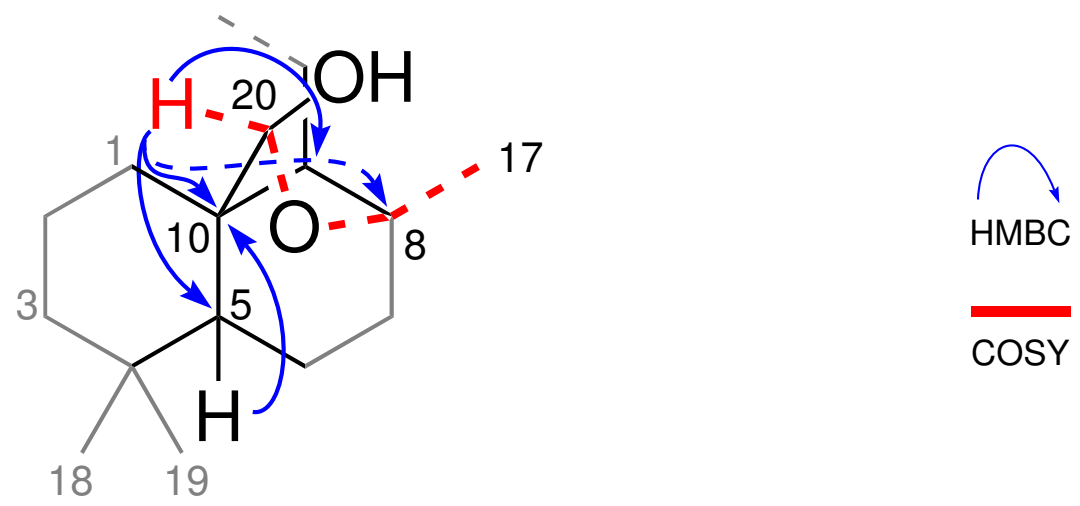

Figure 3.15 COSY and selected HMBC correlations used in completion of the [4.4.0]bicyclodecane system and assignment of the bridging hemi-acetal moiety in 35. Dashed lines and arrows indicate weak correlations.

Remaining unaccounted for by the tricyclic system were three $s p^{2}$ carbon resonances [(C-13: $\left.\delta_{\mathrm{C}} 131.0\right),\left(\mathrm{CH}-14: \delta_{\mathrm{C}} 143.0, \delta_{\mathrm{H}} 6.87^{1} J_{\mathrm{CH}} 177 \mathrm{~Hz}\right)$ and $\left.\left(\mathrm{C}-16: \delta_{\mathrm{C}} 171.5\right)\right]$, and an oxygen-substituted methine (CH-15: $\left.\delta_{\mathrm{C}} 96.7, \delta_{\mathrm{H}} 6.10,{ }^{1} J_{\mathrm{CH}} 176 \mathrm{~Hz}\right)$. On the basis of chemical shift and ${ }^{1} J_{\mathrm{CH}}$ couplings the resonances were assigned as a tri-substituted alkene, an ester carbonyl and a second hemi-acetal methine. The vicinal positioning of CH-14 and CH-15 was evidenced by reciprocal COSY and HMBC correlations. The C-12 to C-13 bond was established by HMBC correlations $\mathrm{H}-14$ to quaternary $\mathrm{C}-13$ and $\mathrm{CH}_{2}-12$. Further HMBC correlations were observed from the $\mathrm{CH}_{2}-12$ methylene protons to quaternary $\mathrm{C}-13, \mathrm{CH}-14$ and carbonyl C-16 confirming bonds from C-13 to C-14 and C-16. This established the $\alpha, \beta$-unsaturated ester function, C-16-C-13-CH-14 (Figure 3.16).

The substituted [4.4.0]-bicyclodecane tricyclic system (C-1 to C-20) and the C14 to C-16 enoate accounted for all but one degree of unsaturation associated with the molecular formula. The combination of chemical shift and high ${ }^{1} J_{\mathrm{CH}}$ for $\mathrm{CH}-15$ $\left(\delta_{\mathrm{C}} 96.7, \delta_{\mathrm{H}} 6.10,{ }^{1} J_{\mathrm{CH}} 176 \mathrm{~Hz}\right)$ is consistent with dioxy-substitution of the centre. As such, an $\alpha$-substituted $\gamma$-hydroxybutenolide moiety linking the hemi-acetal CH15 and carbonyl C-16 is proposed (Figure 3.17). This final substructure satisfies the oxygen count and unsaturation requirements of the molecular formula. 


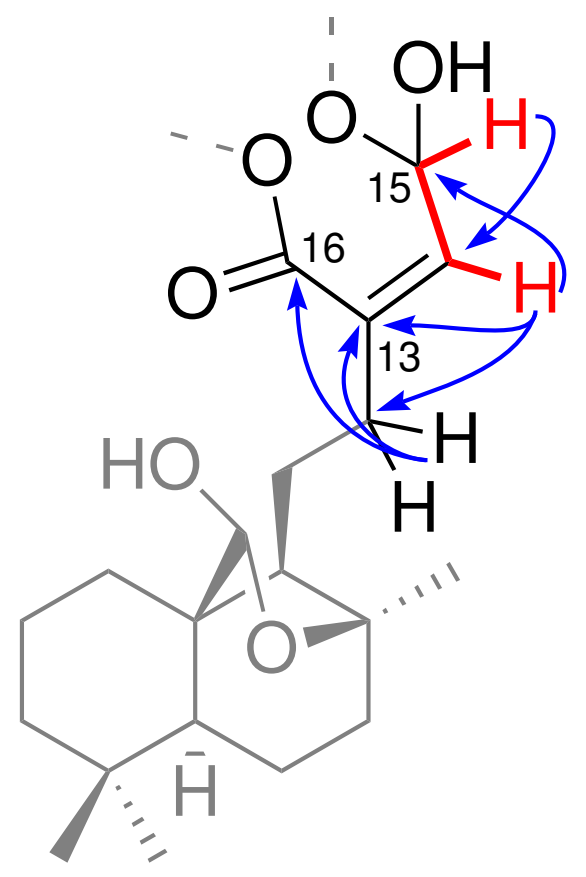

\section{๑ \\ HMBC \\ $\overline{\cos Y}$}

Figure 3.16 COSY and HMBC correlations evidencing the substituted $\alpha, \beta$-unsaturated ester (C16-C-13-C-14) and $\gamma$-hemi-acetal methine (C-15) in $\mathbf{3 5}$.

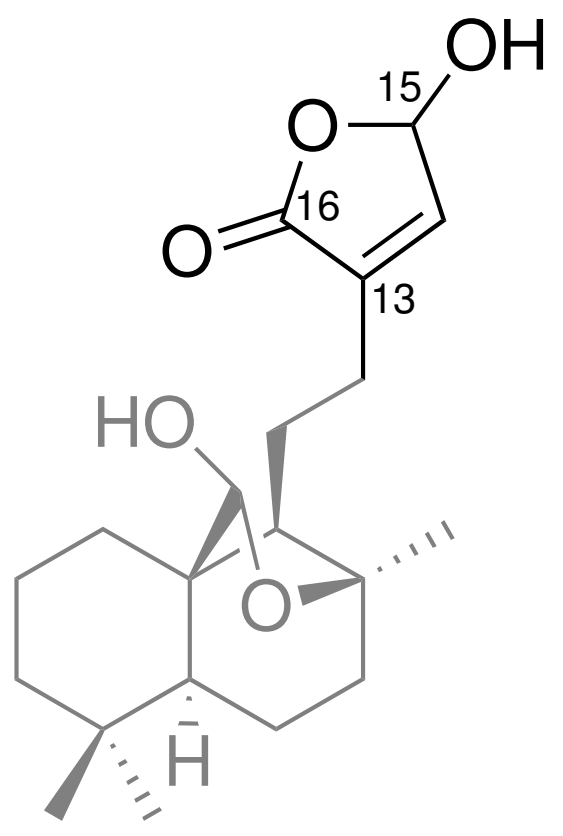

Figure 3.17 The $\alpha$-substituted $\gamma$-hydroxybutenolide moiety of $\mathbf{3 5}$. 


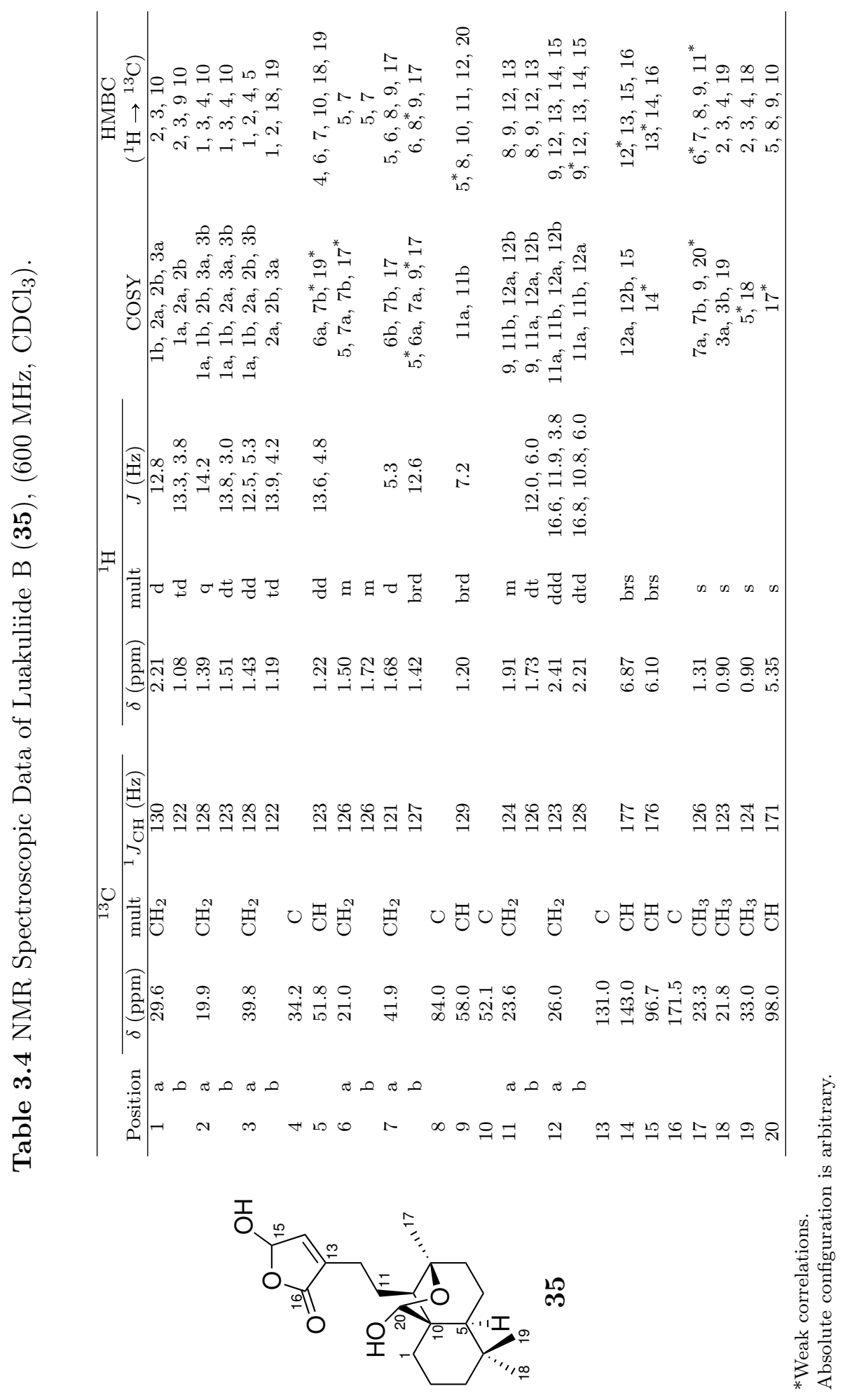


The observation of a $[\mathrm{M}+\mathrm{Na}]^{+}$pseudomolecular ion peak $(m / z$ 373.1990) by positive ion-mode HRESIMS analysis of luakuliide $\mathrm{C}(\mathbf{3 6})$, indicated that $\mathbf{3 6}$ has a molecular formula of $\mathrm{C}_{20} \mathrm{H}_{30} \mathrm{O}_{5}$, like that of 35 . The differences in HPLC retention times between 35 and 36 implied an isomeric relationship between the metabolites. Inspection of the $1 \mathrm{D}$ and $2 \mathrm{D}$ NMR spectra of $\mathbf{3 6}$ indicated that the [4.4.0]-bicyclodecane system (C-1 to C-12) observed in 33-35 is conserved. However, subtle changes in chemical shift of the ${ }^{1} \mathrm{H}$ and ${ }^{13} \mathrm{C}$ resonances associated with the $\gamma$-hydroxybutenolide moiety suggested some change in functionality.

Consistent with the isomeric relationship between 35 and 36, the resonances C-13 to C-16 were assigned once more as an ester carbonyl $\left(\delta_{\mathrm{C}} 171.0\right)$, a trisubstituted alkene $\left(\delta_{\mathrm{C}} 168.9 ; \delta_{\mathrm{C}} 117.9, \delta_{\mathrm{H}} 5.90,{ }^{1} J_{\mathrm{CH}} 181 \mathrm{~Hz}\right)$, and a hemi-acetal methine $\left(\delta_{\mathrm{C}} 98.6, \delta_{\mathrm{H}} 5.99,{ }^{1} J_{\mathrm{CH}} 177 \mathrm{~Hz}\right)$. Interpretation of COSY and HMBC correlations between the resonances, centres were assigned as a $\beta$-substituted- $\gamma$ hydroxybutenolide (Figure 3.18). The change in polarisation of the C-13 to CH-14 double bond in 36, $\delta_{\mathrm{C}} 168.9$ and 117.9, compared to that of $\mathbf{3 5}, \delta_{\mathrm{C}} 131.0$ and 143.0, is consistent with the change in alkyl substitution relative to the carbonyl centre.

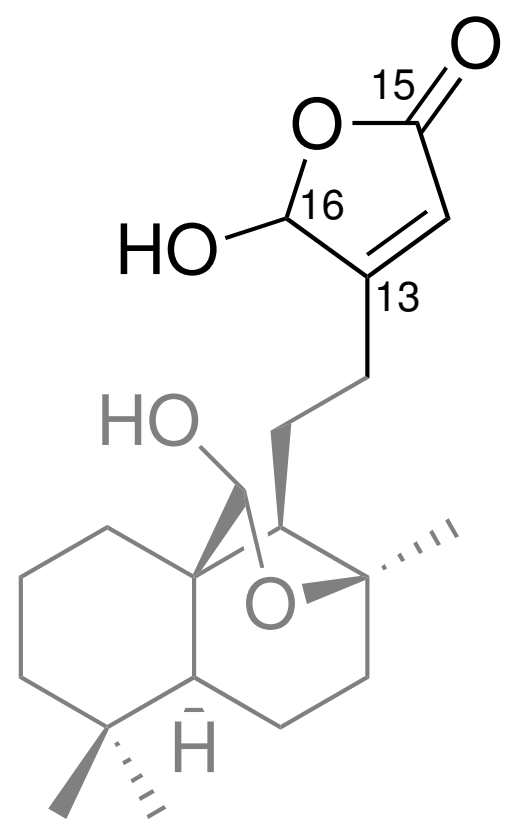

Figure 3.18 The $\beta$-substituted- $\gamma$-hydroxybutenolide moiety of $\mathbf{3 6}$.

The same trends in chemical shift are also observed in the $\gamma$-hydroxybutenolide moieties of iso-secothorectolide (23), luffarin Q (24) and luffarielolide (26) isolated during this study (Tables C.1, C.2 and C.4). Similar trends in bond polarisation are also observed in the $\gamma$-hydroxybutenolides teuponin $(\mathbf{3 7})^{89}$ and acuminolide $(\mathbf{3 8})^{90}$, which were isolated from the terrestrial organisms Teucrium japonicum and Neouvaria acuminatissima, respectively. ${ }^{89,90}$ Single crystal X-ray analysis of 
teuponin (37) and acuminolide (38) confirmed the substitution patterns of the two $\gamma$-hydroxybutenolide groups. Differences in hybridisation of the carbonyl and hemiacetal centres, and lengths of the $\mathrm{C}-\mathrm{O}$ and $\mathrm{C}=\mathrm{O}$ bonds are clearly illustrated in the crystal structures. Comparisons of the ${ }^{1} \mathrm{H}$ and ${ }^{13} \mathrm{C}$ NMR spectra of $\mathbf{3 5}$ and $\mathbf{3 7}$, and 36 and 38, are shown below in Table 3.5, providing support for the structural assignment of 35 and 36 .

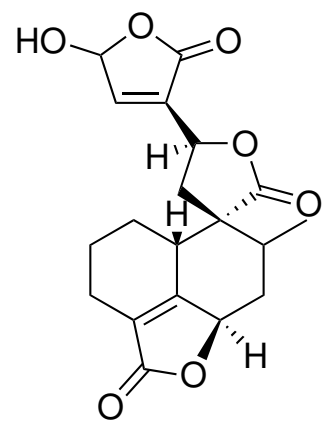

37

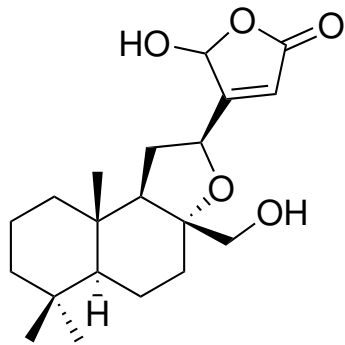

38

Table 3.5 NMR Spectral Data for the $\gamma$-Hydroxybutenolide Moieties in 35-38.

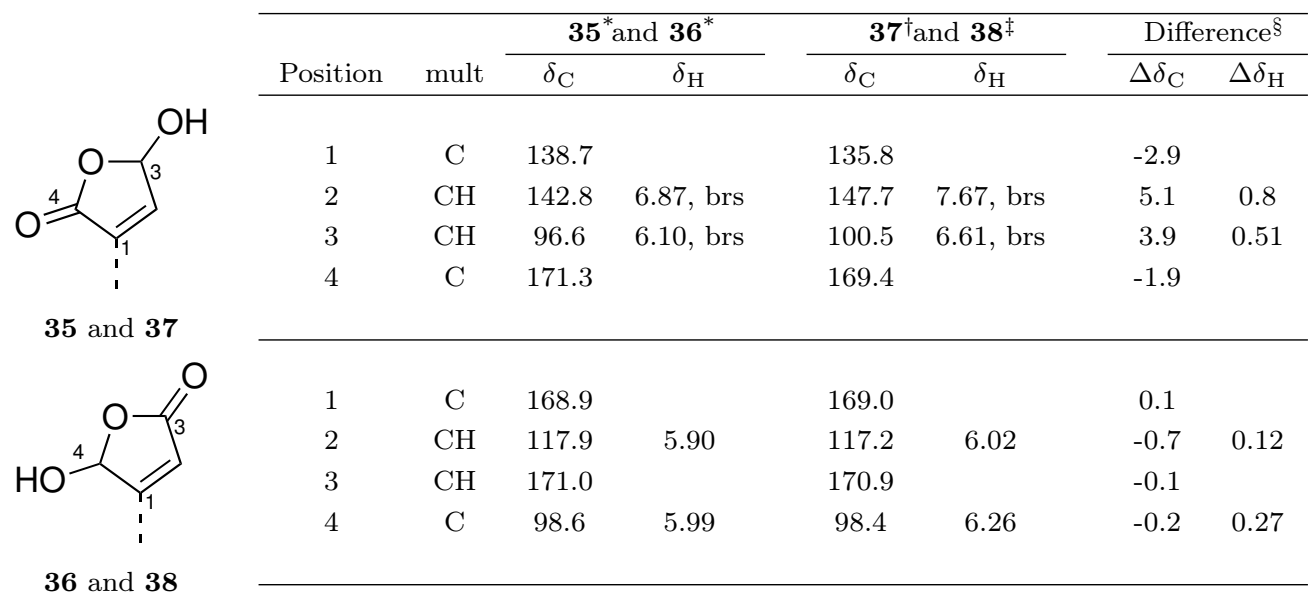

${ }^{*} \mathrm{CDCl}_{3}, 600 \mathrm{MHz}$

$\dagger d_{5}$-pyridine, $400 \mathrm{MHz}$

$\ddagger \mathrm{CDCl}_{3}, 300 \mathrm{MHz}$

$\S \Delta \delta=\delta_{37-38}-\delta_{35-36}$

The high ${ }^{1} J_{\mathrm{CH}}$ values of the methines CH-15 and CH-16 in the $\gamma$-hydroxybutenolide moieties of 35 and 36 are noteworthy at $177 \mathrm{~Hz}$ and $181 \mathrm{~Hz}$, respectively. An equivalent carbon-substituted $s p^{2}$-hybridised centre could be expected to have a ${ }^{1} J_{\mathrm{CH}}$ value of ca. $160 \mathrm{~Hz}$, and the coupling constants appear to have increased in magnitude due to the inductive electron-withdrawing qualities of the nearby oxygen atoms and strain imposed by the five-membered ring. Easily extracted from ${ }^{13} \mathrm{C}$-coupled HSQC spectra, ${ }^{1} J_{\mathrm{CH}}$ values are frequently invoked in functional group determination, there is value in comprehensively compiling $J$-coupling values associated with these commonly isolated heterocycles, in addition to those found in more unusual synthetic substrates. Such a resource would be invaluable to spectroscopic structural elucidation. 


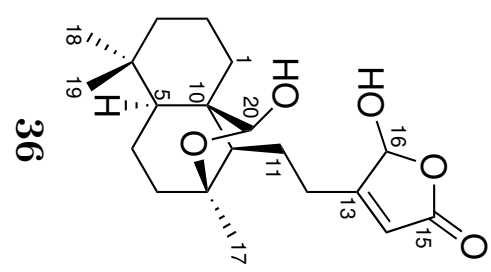

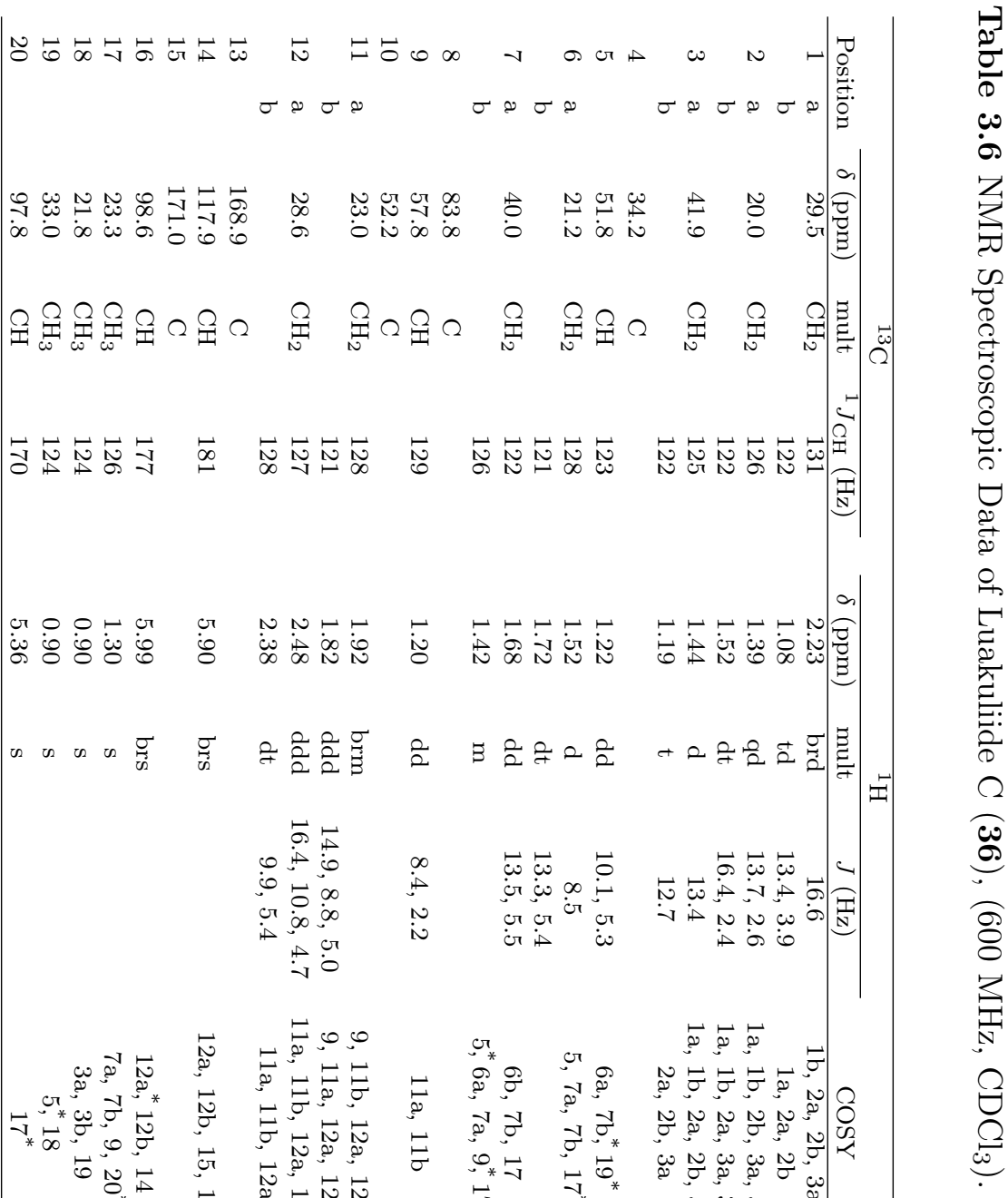

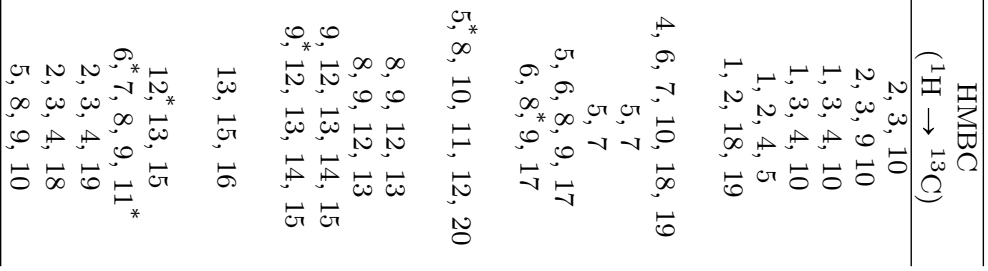




\subsubsection{Biological activity of the luakuliides}

Labdane diterpenes have been associated with antimicrobial, antiinflammatory, immuno- and enzyme-modulatory biological activities. ${ }^{91}$ As such, 33 and $\mathbf{3 4}$ were assessed for cytoxicity, antifungal and immunomodulatory activity. ${ }^{92}$ In a standard 48 h proliferation MTT assay 33 and $\mathbf{3 4}$ displayed weak inhibition HL-60 cell line with $\mathrm{IC}_{50}$ values of 21.7 and $43.5 \mu \mathrm{mol} \mathrm{L}{ }^{-1}$, respectively, and negligible inhibition of a drug-sensitive $S$. cerevisiae strain. ${ }^{93}$ Their immunomodulatory activities were far more significant. Luakuliide A (33) and the methyl acetal 34 inhibited production of nitric oxide (NO) in stimulated splenocytes, while $\mathbf{3 4}$ also affected an increase in interferon- $\gamma(\mathrm{INF}-\gamma) .{ }^{94}$ Increased production of the proinflammatory cytokine, tumor necrosis factor- $\alpha$ (TNF- $\alpha)$ in stimulated macrophages was also observed and lesser response was affected in non-stimulated cells. ${ }^{92}$

Inflammation is fundamental to many pathological conditions, from triggering and perpetuating autoimmune diseases through to immune responses to foreign bodies. ${ }^{95}$ The macrophage white blood cells line are key to both the initiation and direction of inflammatory processes via TNF- $\alpha$ and NO. ${ }^{96}$ TNF- $\alpha$ acts in transduction of signals pertaining to cell activation, proliferation and apoptosis, enhancing the overall inflammatory response. ${ }^{97}$ Nitrous oxide has roles in the innate and adaptive immune systems including macrophage phagocytosis, neutrophil migration, and natural killer T-cell activity. ${ }^{98}$ Hence, small molecules which affect the regulation of T-cell activation and influence NO and TNF- $\alpha$ are of interest with respect to the treatment of autoimmune and inflammatory disorders. ${ }^{92,99}$

\subsubsection{Labdane diterpenes}

As products of the ubiquitous isoprenoid pathway, cyclised diterpene metabolites have been extensively isolated from terrestrial sources, especially the Angiosperm families Asteraceae and Labiatae. 100 The skeleton name is derived from "labdanum", a resin extracted from the rockroses Cistus (family Cistaceae), from which the first members of the class were initially obtained. ${ }^{101}$

The labdane skeleton is infrequently encountered within the marine environment, examples including cacofurans A (39) and B (40), isolated from an Okinawan sponge specimen of Cacospongia sp. (order Dictyoceratida, family Thorectidae). ${ }^{102}$ As seen in the luakuliide skeleton, oxidation of C-8 and subsequent nucleophilic attack on the $\mathrm{CH}_{3}-20$ methyl (aldehyde) generates the $\mathrm{C}-8$ to $\mathrm{C}-20$ ether or hemi-acetal bridge. The source of differentiation between the luakuliides and cacofurans is oxidation at C-11 compared to further oxidation at C-20. ${ }^{102}$ Investigation of another Cacospongia specimen from the Philipines yielded several furano and meroquinone diterpenes 
metabolites, including 41, where the C-8 methyl substituent is replaced by an exocyclic methylidene. Sesterterpene luffariellolide (26, isolated from PTN4_10B) was encountered during the same investigation, illustrating the promiscuity of the compound. ${ }^{103}$

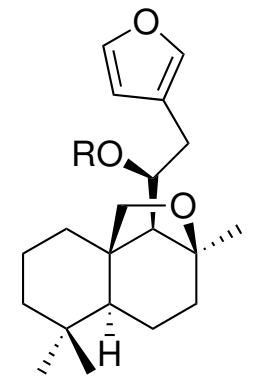

$39 \mathrm{R}=\mathrm{H}$

$40 \mathrm{R}=\mathrm{Ac}$

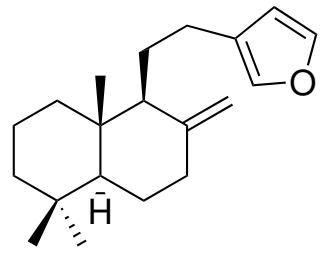

41

Furan moeities, such as that of luakuliide A (33), frequently undergo further oxidation forming the entities such as butenolide or 2-furanone functions, and the $\gamma$-hydroxybutenolide moieties featured in 35 and 36. Many metabolites featuring both these functions have been reported, although this oxidation could conceivably occur naturally within the organism or during isolation. ${ }^{104}$

Intertidal limpets of the Trimusculus genus, are also rich sources of such cyclic diterpenes. Collections from central Chile of $T$. peruvianus yielded four new diterpenes metabolites with the characteristic labdane bicycle and an acyclic terminal isoprene unit. ${ }^{105}$ Further investigation of the same species led to the isolation of several new additions to this class including 42-47, which differ in oxidative substitution of the labdane bicycle. ${ }^{106,107}$ South African collections of $T$. costatus yielded similar labdanes, ${ }^{108}$ in addition to $5 \alpha, 8 \alpha$-epidioxy sterols similar to $\mathbf{2 7}$ and $\mathbf{2 8}$ isolated from the plakinid sponge PTN3_19D. ${ }^{109}$

\subsubsection{Biogenesis of luakuliides $\mathrm{A}-\mathrm{C}$}

Isoprene biosynthesis and formation of the different terpene classes is well documented. Formation of many carbon skeletons, including that of the labdanes, has also been well explored and confirmed via both the mevalonate and non-mevalonate pathways. ${ }^{110}$

Cyclisation of geranylgeranyl pyrophosphate (GGPP) by either of two ways forms the four diastereomers of the labdane skeleton, differing in configuration at $\mathrm{C}$ 5 and C-9. ${ }^{104}$ The labdane skeletion is a precursor to many other diterpenes skeletons; diastereomers of the labdadienyl cation can undergo a series of 1,2-hydride and methyl shifts. Concerted migrations generate a trans-decalin product, and 
cis- relationship between C-8 and C-10 methyls. However, the series of stepwise migrations pauses at the $\mathrm{C}-5$ cation intermediate, giving rise to either cis- or transfused clerodane products depending on which of the two C-4 methyl substituents migrates. This process is depicted in Scheme 3.1 for one labdadienyl diastereomer. Alternatively, capture of the C-5 cation of the halimane skeleton by an exogenous nucleophile generates species including ambliol B (25), ${ }^{111}$ isolated in this study from PTN4_10B. This theory of dual mechanisms is supported by the high proportion of trans- over cis-decalin products, and the isolation of partially rearranged labdanes such as the halimane $\mathbf{2 5}$.<smiles>CC(=CCO)CCC1=C(C)C[C@@H](O)C2C(C)(C)CCC[C@]12C</smiles>

42<smiles>CC1=C(CC/C(C)=C/C(=O)O)[C@@]2(C)CC[C@@H](O)[C@](C)(CO)C2[C@H](O)C1</smiles>

45<smiles>C=C1C[C@H](O)[C@@H]2[C@](C)(CC[C@@H](OC(=O)C(C)CC)[C@]2(C)COC(=O)C(C)CC)[C@H]1OCC/C(C)=C/C(=O)O</smiles>

43<smiles>CCC(C)C(=O)O[C@H]1CC[C@]2(C)C(CC/C(C)=C/C(=O)O)=C(C)C[C@H](O)C2[C@]1(C)CO</smiles>

46<smiles>CCC(C)C(=O)OC[C@]1(C)CCC[C@]2(C)C(CC/C(C)=C/C(=O)O)=C(C)C[C@H](O)[C@@H]21</smiles>

44

47

Historical confusion within the literature has arisen over the absolute stereochemistry of the labdane, halimane and clerodane skeletons and the governing nomenclature. Stereochemical classification is now made in reference to the original isolate clerodin (48); those with the same absolute configuration are neo- while the enantiomeric forms are ent-neo-labdanes and -clerodanes. As indicated in Scheme 3.1, further division is made in reference to the cis- or trans-decalin ring junction. Relative orientation of C- 8 and C-9 methyls is the final source of differentiation within the skeletons. 

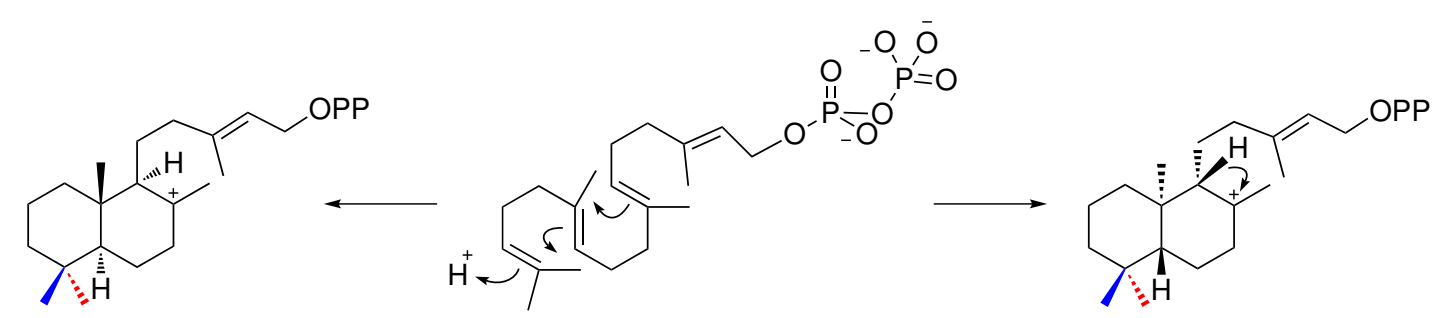

Labdane

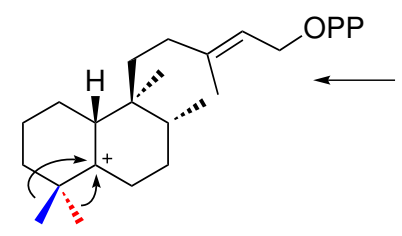
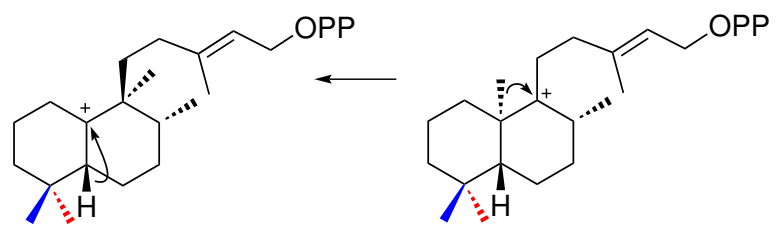

Halimane

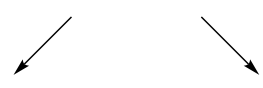<smiles>CC1CCC[C@@H]2[C@@H](C)C/C(=C\COP)CC[C@@H]12</smiles>

trans-Clerodane

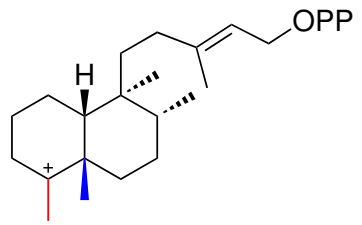

cis-Clerodane

Scheme 3.1 Formation of the labdane, halimane and clerodane skeletons via cyclisation of geranylgeranyl pyrophosphate.

In the case of luakuliides $\mathrm{A}-\mathrm{C}$, the electrophilic $\mathrm{C}-8$ of the labdadienyl cation most probably undergoes enzyme-mediated hydration, forming an internal hydroxyl nucleophile. As shown in Scheme 3.2, oxidation of the C-20 methyl centre to a aldehyde function, and subsequent nucleophilic attack of the carbonyl by the C-8 hydroxyl generates the bridging hemi-acetal of 33-36 in its entirety. Surprisingly, few C-8 hydration products have been reported, ${ }^{104}$ exceptions being the Cacospongia isolates cacofurans A and B (39 and 40), the luakuliides and 49, a recent isolate from the essential oil of Acalypha plicata leaves. ${ }^{112}$ The furan function is thought to be installed via oxidative cyclisation, ejecting the OPP moiety. Further oxidation of the diene, potentially via addition of molecular oxygen across the diene generates the $\gamma$-hydroxybutenolide functions of $\mathbf{3 5}$ and 36. Lastly, although the relative configuration of the luakuliides has been determined, it is not yet known whether the molecules can be categorised as either neo- or ent-neo-labdanes.

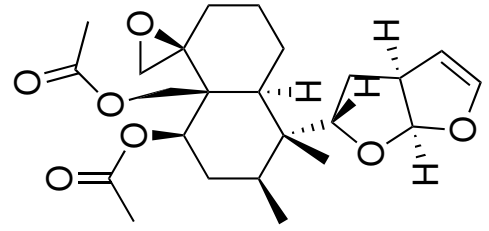

48<smiles>CC1(C)CCC[C@@]2(C)C1CC[C@](C)(O)[C@H]2CCc1ccoc1</smiles>

49 

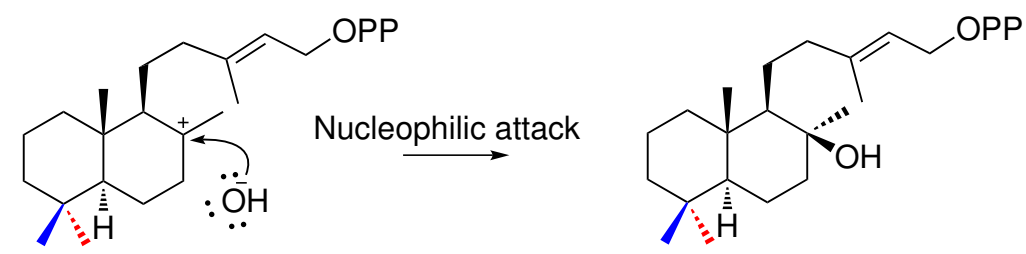

$\downarrow$ Oxidation

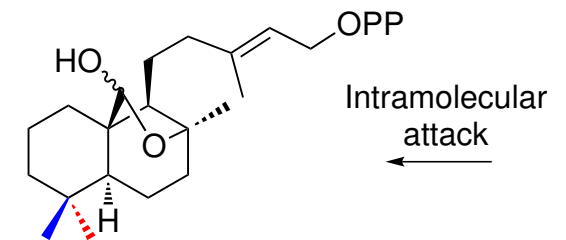

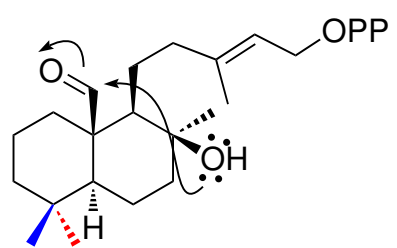

Cyclisation

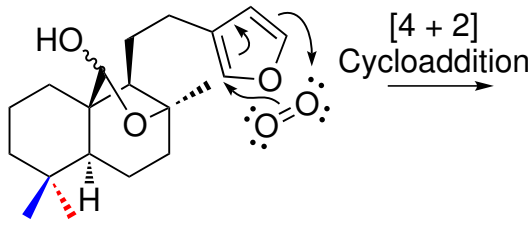

Luakuliide A

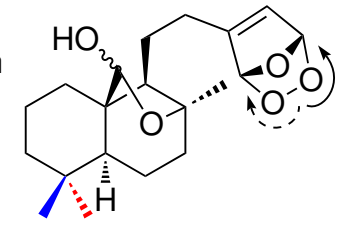

Ring opening $+\mathrm{H}^{+}$

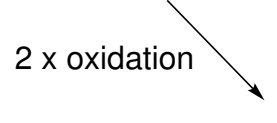

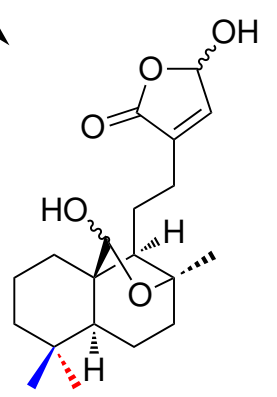

Luakuliide B

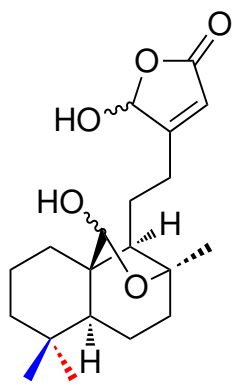

Luakuliide C

Scheme 3.2 Proposed pathway for generation of the hemi-acetal functions, furan and $\gamma$-hydroxybutenolide moieties of luakuliides A-C (33-36). 


\subsubsection{Concluding remarks}

Labdane metabolites are infrequently encountered in marine organisms. In this case their characteristic gem-dimethyl feature was identified early during analysis of the HMBC spectra from crude fractions, prompting further purification and eventual isolation of the luakuliides. Differentiating these structures from other labdanes is the hemi-acetal bridge from $\mathrm{C}-20$ to $\mathrm{C}-8$, which also provides evidence for capture of the C-8 labdadienyl cation. The 1D TOCSY-NOESY NMR experiment was integral to assigning the trans-fusion of the [4.4.0]-bicyclodecane system and relative stereochemistry of the luakuliides.

Luakuliide A (33) and it methyl acetal derivative (34) display interesting biological activity. The metabolites have significant macrophage stimulatory activity, promoting a proinflammatory response, which is complemented by increased activity in Tcells. Although in contrast to antiinflammatory properties frequently demonstrated by labdane diterpenes, these findings illustrate the potential of the luakuliides as new immunomodulatory agents. 


\section{Chapter 4}

\section{Chemical Analyses of Plakinid Sponges}

Investigation of two plakinid sponge specimens yielded several new polyketide metabolites: the $\alpha$-pyrones lehualides $E-K$ and four cyclic peroxides. Analysis of the $H M B C$ experiments of semi-purified fractions from each of the organisms was integral to their selection for further study. During the selection process the $\alpha$-pyrone of the lehualides, and the oxygenated backbones of the cyclic peroxides were partially elucidated, prompting further investigation of the organisms.

\subsection{The family Plakinidae}

Plakinidae, the sole family of the order Homosclerophorida ${ }^{113}$ encompasses several valid genera: Plakina, Plakortis, Plakinastrella, Placinolopha, Corticium, Pseudocorticium, and Oscarella (Figure 4.1). Except for Oscarella, these genera possess a siliceous skeleton, formed by either a uniform arrangement of calthropes, diod and triod spicules, or a combination of the two. Found mainly in warm waters, the encrusting sponges are generally dark purple and grey in appearance, with a lighter tan and beige interior. With cork-like, dense and rubbery textures, their velveteen surfaces are frequently covered with wide, shallow nodules. ${ }^{38,113}$

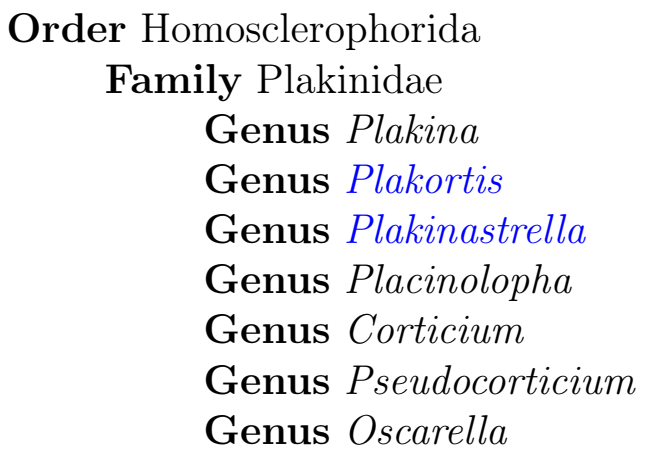

Figure 4.1 Taxonomic classification of the order Homosclerophorida, after Hooper and van Soest. ${ }^{38}$ Genera encountered during this study are shown in blue. 


\subsubsection{The Plakortis and Plakinastrella genera}

The phylum Porifera is notorious for its taxonomic uncertainty. In the case of the closely related genera Plakortis and Plakinastrella, their simple spiculation makes generic distinction and further speciation particularly difficult to establish. The spicule profile of Plakortis specimens is mainly composed of irregular diods in a single size-class and the occasional triod, while Plakinastrella sponges are thought to exhibit up to three size-classes of diods, triod spicules and calthropes.

A range of biologically active compounds have been reported from species within these two genera. A series of $\beta$-carbolines, the plakortamines (50-52), were isolated in conjunction with epiplakinic acids. ${ }^{114}$ These simple brominated alkaloids are often associated with ascidians (phylum Chordata), such as the antiviral eudistomins from the Caribbean ascidian Eudistoma olivaceum. ${ }^{115}$ Although uncommon, this class of metabolites is precedented within sponges, sharing structural features with the polycyclic fascaplysin alkaloids, such as 14-bromohomofascaplysin (29).<smiles>CN(C)CCc1nccc2c1[nH]c1cc(Br)ccc12</smiles>

50

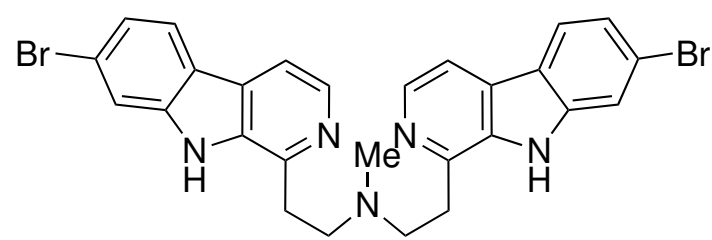

52<smiles>C=Cc1nccc2c1[nH]c1cc(Br)ccc12</smiles>

51<smiles>C[N+](C)(C)C(Cc1cc2cc(I)c(I)c(I)c2[nH]1)C(=O)O</smiles>

53

Chemical investigations by Costantino and co-workers of Caribbean collections of Plakortis simplex have also yielded a series of iodoindole metabolites. ${ }^{116,117}$ Plakohypaphorine E (53) and others of the class represent the first naturally occurring iodoindole metabolites. Subsequently, the same research group reported the pyridinium derivatives simplakidine $(\mathbf{5 4})^{118}$ and simplexidine ${ }^{119}$ from the same organism. Simplakadine combines the pyridinium nucleus shared by simplexidine with the polyketide framework observed in plakortether B (55), an archetypal isolate of sponges from this family.

A rich complement of type I polyketide metabolites have been isolated from Plakortis sponges. The type I polyketide synthesis pathway (PKS1) is also active in macrolactone biosynthesis, although the Plakortis metabolites generally feature an acid-substituted five- or six-membered heterocycle within a long, largely saturated carbon skeleton. Such carbon backbones, in combination with a terminal carboxylic 
<smiles>CC[C@H](C[C@]1(C)C[C@@H](CC)[C@H]([C@H](CO)c2cc[n+](C)cc2C(=O)[O-])O1)[C@@H](O)CC(=O)O</smiles>

54<smiles>CCC[C@H]1O[C@](C)(C[C@H](CC)[C@H](O)CC(=O)OC)C[C@@H]1CC</smiles>

55

acid, contributed to their assumed origins in fatty acid synthesis. ${ }^{120}$ The mechanisms of PKS1 and fatty acid synthesis both involve chain growth by $\mathrm{C}_{2}$, however, PKS1 utilises a great variety of starter units, and the different propagation units specify acetate, propionate, butyrate and methyl-propionate groups. ${ }^{121}$ These generate a carbon skeleton featuring methyl, ethyl, propyl and gem-dimethyl side-branches, as observed in plakortether B (55, Figure 4.2). Products of PKS1 frequently exhibit great variation in oxidation, and the various reduction units used in PKS1 are also enantiospecific.

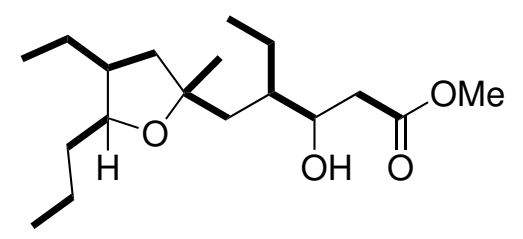

Figure 4.2 Depiction of the PKS1 propagation units used in the biosynthesis of plakortether B (55). The metabolite comprises three acetate, one propionate and two butyrate units.

Cyclic peroxides are a distinctive class of polyketide metabolite frequently isolated from both the Plakortis and Plakinastrella genera. The first example, plakortin (56), a potent antibiotic, was isolated from a Plakortis halichondrioides specimen in 1978. ${ }^{122}$ Rich augmentation of both the acid-substitued 1,2-dioxolane nucleus and substituents of $\mathbf{5 6}$ has given rise to a contingent of structurally related compounds categorised into several arbitrary classes. Plakortide F (57), retains the acidsubstituted peroxide nucleus of $\mathbf{5 6}$ although the compounds differ in absolute configuration. Variation in substitution about the cyclic peroxide core at C-4 and C-6 reflects a change in propagation units during biosynthesis, while the inclusion of an additional acetate monomer during formation of $\mathbf{5 7}$ (C-7-C-8) accounts for the difference in chain length.

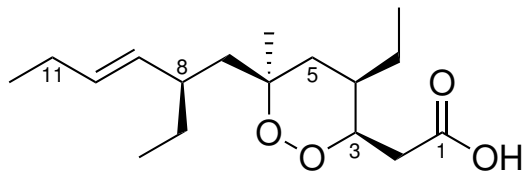

56<smiles>CC/C=C/C(CC)CCC[C@]1(CC)C[C@H](CC)[C@@H](CC(=O)OC)OO1</smiles>

57

Cyclisation of the acid forms the peroxy-lactone bicyclic system, characteristic of 
the plakortolide class. The first example was described in 1980, although not fully characterised until 1991 by Davidson et al. ${ }^{123}$ The absence of the free acid in the plakortolide skeleton frequently corresponds to decreased biological activity compared to the carboxylic acid analogue. Although the ring fusion is exclusively cis, varied configuration at C-6 forms two diastereomeric carbon skeletons. The two are easily distinguishable by NOE correlations between the C- 4 and C- 6 methyl substituents and changes in the ${ }^{1} \mathrm{H}$ chemical shift of $\mathrm{H}-5 \mathrm{a}$, ca. $0.1 \mathrm{ppm}$.

Ring-opening of the plakortolide cyclic peroxide generates the seco-plakortolide diols. Loss of ring-strain lessens diastereotopic differentiation of the $\mathrm{CH}_{2}-5$ methylene protons allowing spectroscopic distinction between the two forms. Upon isolation, seco-plakortolides are known to undergo facile intramolecular condensation and Michael-type addition of the C-6 hydroxyl to C-3 of a butenolide intermediate forming hydrofuran derivatives, plakortones (Scheme 4.1). ${ }^{124}$ Co-isolation of all three forms of the same carbon skeleton could cast doubt over the natural origins of secoplakortolide and plakortone structures. ${ }^{125}$ However, plakortone skeletons bearing ethyl substituents at C-4 and C-6 have been isolated in the absence of plakortolide analogues from Caribbean Plakortis halicondrioides and P. simplex specimens. ${ }^{126,127}$

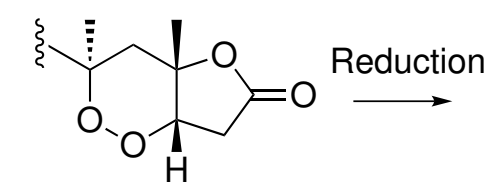

Plakortolide

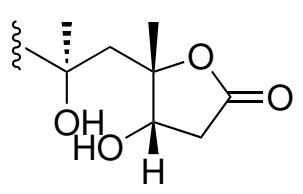

seco-Plakortolide

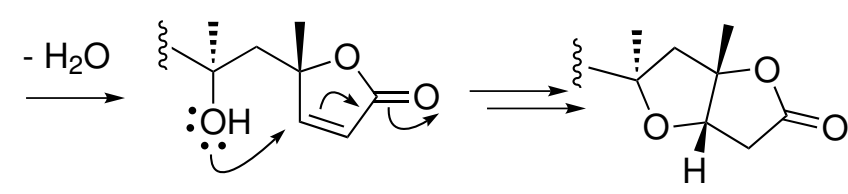

Plakortone

Scheme 4.1 Conversion of plakortolides to plakortones via seco-plakortolides by reduction, intramolecular condensation and Michael-type addition, after Yong et al.. ${ }^{124}$

This parade of polyketide metabolites displays further structural variation. Isolated from a Dominican Plakortis halichondrioides specimen, glánvillic acids A and B $(\mathrm{A}, 58),{ }^{128}$ for which stereochemistry has not been defined, bear an aromatic furan ring (C-3-C-6). A host of other Plakortis metabolites bear an alkyl group varying in saturation, branching and chain length. Further additions to the contingent of cyclic peroxides are the plakinic and epiplakinic acids, which were first encountered from Palauan specimens of $P$. nigra. As demonstrated by 59 and 60, the two classes possess lengthy methyl-branched alkyl backbones terminating in a phenyl ring, derived from a benzoate starting unit. ${ }^{114}$ These structural characteristics are shared with members of the plakortolide class. ${ }^{124}$ The two acid classes are differentiated by 
the orientation of the C-3 and C-5 methyl substituents about the 1,2-dioxane ring, cis- in the plakinic acids (59), and trans- in the epiplakinic acids (60). One example of epiplakinic acid decarboxylation has been reported. ${ }^{129}$<smiles>CC/C=C/C(CC)CC(C)Cc1cc(CC)c(CC(=O)O)o1</smiles>

58<smiles>CC(CCCCCCCc1ccccc1)CC(C)CC1(C)CC(C)(CC(=O)O)OO1</smiles>

59<smiles>CC(CCCCCCCc1ccccc1)CC(C)C[C@]1(C)C[C@](C)(CC(=O)O)OO1</smiles>

60

Spiculoic acids A and B (61 and 62) were isolated from a P. angulospiculatus specimen, ${ }^{130}$ and possess an unprecedented carbon skeleton. The biosynthetic origins of the polyketide-derived structures are not clear, but the skeleton is thought to incorporate a phenyl acetate starter unit and four butyrate units. A post-assembly enzyme-catalysed intramolecular [4+2] cycloaddition is proposed for formation of the fused bicycle, which contains the expected cyclohexene ring. ${ }^{130}$ Recent investigation of a $P$. zyggompha specimen by Berrue et al. uncovered three further spiculoic acids, and two zyggomphic acids (63 and 64). ${ }^{131}$ Common to both classes is the fused bicyclic nucleus, with structural variation arising from the inclusion of different PKS1 monomers.

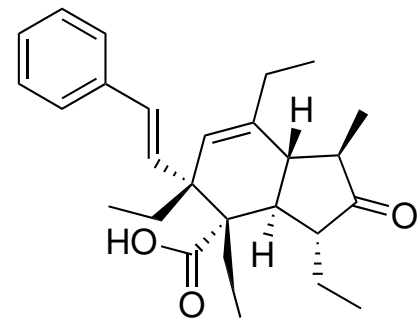

61

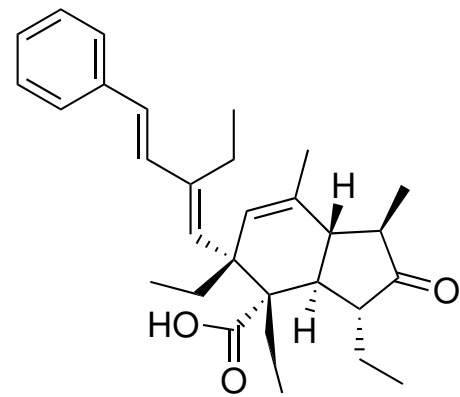

63

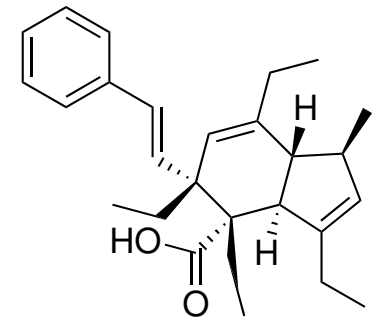

62

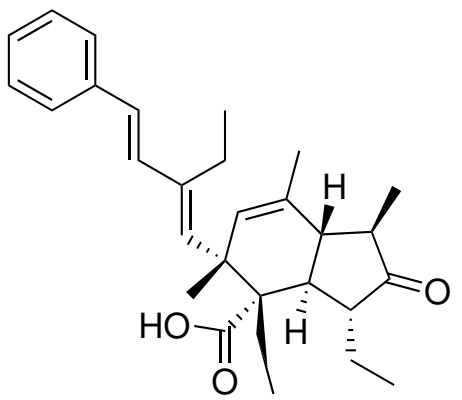

64 
While this series of polyketide metabolites are most probably of bacterial origin, they appear to be exclusive to sponges of the Plakortis and Plakinastrella genera, indicating a taxonomically-specific symbiosis. Another class of polyketide metabolites unique to plakinid sponges is the lehualides. The class differs from more common plakinid isolates, bearing $\alpha$ - or $\gamma$-pyrone moieties in place of the cyclic peroxides. The first members of the class have a pseudo-terpenoid appearance owing to the number of methyl-substituted alkene functions present in the extended alkyl chain. Terminal substituents encountered in the lehualides include phenyl rings, and a variety of sulfur functionalities of which the biosynthetic origins are unclear. The first members of this series, lehualides A-D (65-68), were reported in 2005 from a Hawai'ian Plakortis specimen, ${ }^{132}$ and the succinct synthesis of lehualide B (66) was reported in 2010 by Jeso et al. ${ }^{133}$ Seven additions to this class, lehualides E-K (6975), were isolated during this study from a Tongan Plakortis sp. and are detailed in the following sections. ${ }^{134}$<smiles>C/C=C\C=C/C/C=C(\C)CC/C=C(\C)C/C(C)=C/Cc1ccccc1</smiles>

65<smiles>COc1oc(C/C=C(\C)CC/C=C(/C)C/C(C)=C/Cc2ccccc2)c(C)c(=O)c1OC</smiles>

66<smiles>[R]SCCCCCCCCC/C(C)=C/Cc1oc(OC)c(OC)c(=O)c1C</smiles>

$67 \mathrm{R}=\mathrm{Ac}$

$68 \mathrm{R}=\mathrm{H}$

\section{2 $\alpha$-Pyrone metabolites from Plakortis sp.}

The sponge specimen PTN3_20A (Figure 2.12) was collected at a depth of 12-15 $\mathrm{m}$ from the ceiling of a large cave on 'Eua Island in November 2008. Identified as a species of Plakortis, similar to P. ceylonica (Dendy, 1905), the sponge morphologically formed small oval pendant encrustations about $3 \mathrm{~cm}$ thick, with a cork-like, rubbery and dense texture. Smooth to the touch, the surface was dark chocolate-purplish brown in colour and covered in wide shallow nodules ca. $3 \mathrm{~mm}$ high. Diod spicules (180-250 $\mu \mathrm{m}$ long, Figure 4.3) were isolated via centrifuge following $\mathrm{HNO}_{3}$ treatment of the tissue, aiding classification of the organism as a Plakortis species. Attempts were made to recollect the organism in the Vava'u group but were unsuccessful. The nondescript appearance and growth of the sponge 
in dimly-lit caves may have both been contributing factors leading to specimens going unnoticed.

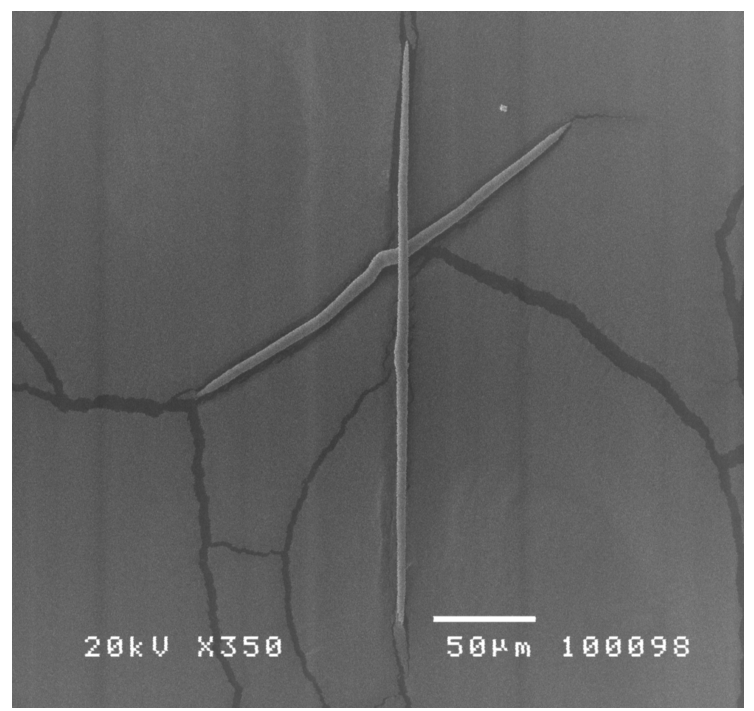

Figure 4.3 Diod spicules recovered from the Plakortis sponge PTN3_20A.

\subsubsection{Isolation and identification of lehualides $\mathrm{E}-\mathrm{K}$}

Methanolic extracts of the Plakortis specimen PTN3_20A were partitioned over reversed-phase HP20 resin, generating three fractions with differing polarity; 30\%, $75 \%$ and $100 \% \mathrm{Me}_{2} \mathrm{CO}$ in $\mathrm{H}_{2} \mathrm{O}$. NMR analysis of the less polar $75 \%$ and $100 \%$ $\mathrm{Me}_{2} \mathrm{CO}$ in $\mathrm{H}_{2} \mathrm{O}$ fractions showed the presence of one major component and several closely related compounds. Immediately apparent in the ${ }^{1} \mathrm{H}$ NMR spectra of the two fractions were the resonances of an aromatic system, olefinic methines $\left(\delta_{\mathrm{H}} 5.20 \mathrm{ppm}\right)$, methoxy groups $\left(\delta_{\mathrm{H}} 3.80-4.20 \mathrm{ppm}\right)$, deshielded methylenes and methyls $\left(\delta_{\mathrm{H}} 2.60-\right.$ 3.20 and $1.60-1.90 \mathrm{ppm})$, and a large aliphatic methylene envelope $\left(\delta_{\mathrm{H}} 1.15-1.30\right.$ $\mathrm{ppm})$.

Analysis of correlations within the HMBC spectra of the $75 \%$ and $100 \% \mathrm{Me}_{2} \mathrm{CO}$ in $\mathrm{H}_{2} \mathrm{O}$ fractions confirmed the value of investigating the organism further. Protons of deshielded methylene doublets displayed correlations to two separate methylsubstituted carbon double bonds (Figure 4.4). One of the double bonds appeared to be oxy-substituted, and another deshielded methylene correlated to a monosubstituted phenyl ring. Interpretation of these structural fragments indicated that the structure in question could be closely related to the Plakortis isolates lehualides A-D (65-68). The lehualides were originally isolated from a Plakortis sponge collected from the vertical interior of large caves on Niihua Island, Hawai'i, which appeared morpholigicaly similar to the specimen from 'Eua. ${ }^{132}$ The moderate toxicity associated with lehualides $\mathrm{A}-\mathrm{D}$, coupled with subtle variations in the NMR 
spectra of the 'Eua sample pointed towards a targeted and spectroscopically guided chemical evaluation of the Plakortis sp.

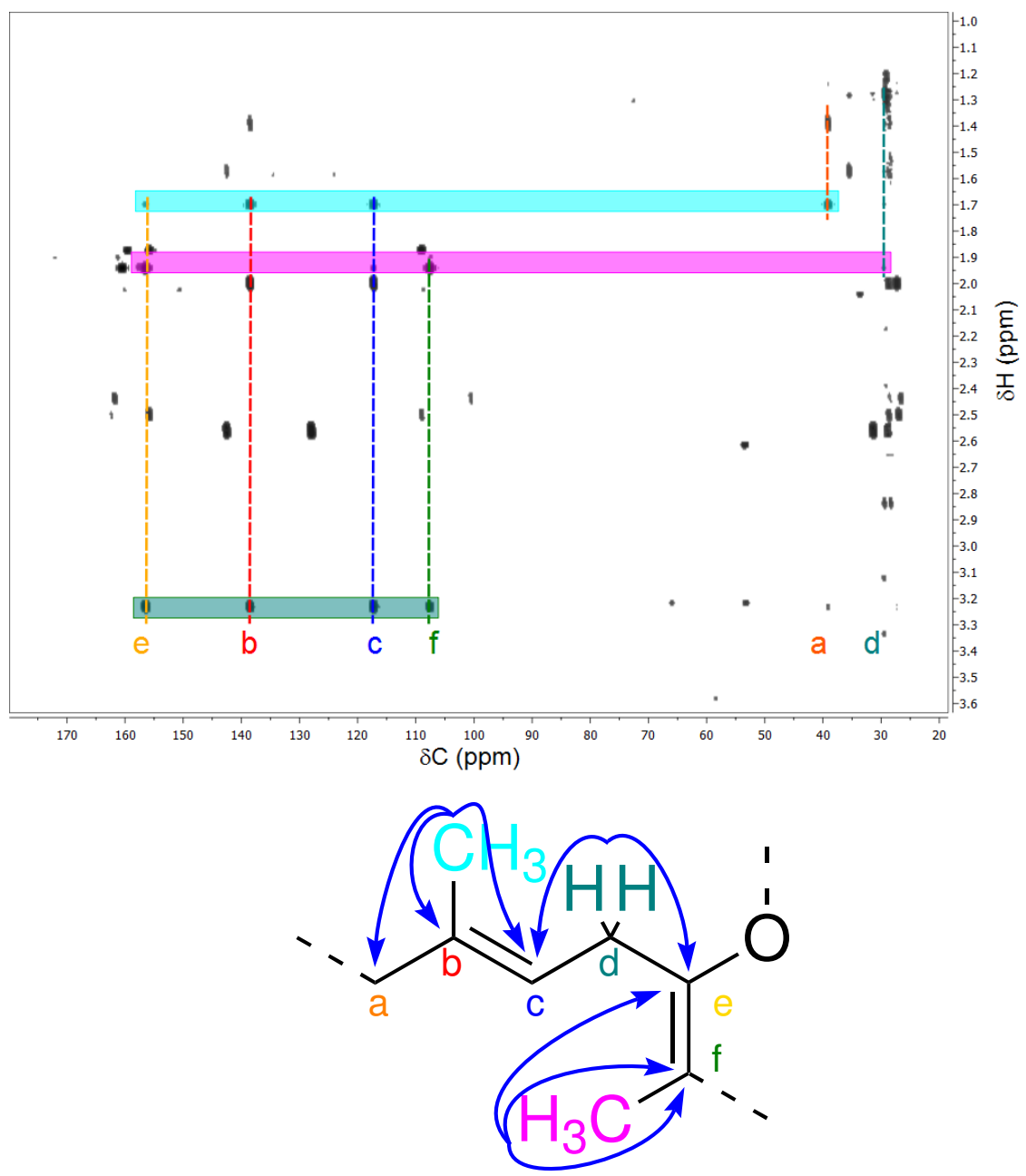

Figure 4.4 Proton to carbon correlations evidencing the methyl-substituted carbon double bonds present in the HMBC spectrum of the $75 \% \mathrm{Me}_{2} \mathrm{CO}$ in $\mathrm{H}_{2} \mathrm{O}$ screen fraction of PTN3_20A.

Following the initial partitioning over HP20, the non-polar fractions derived from PTN3_20A were subjected to further reversed-phase chromatography (HP20SS, $\mathrm{Me}_{2} \mathrm{CO}$ in $\left.\mathrm{H}_{2} \mathrm{O}\right)$. All fractions retained signals associated with the oxy-substituted double bond and tri-substituted double bond, indicating the separation was dependent upon a secondary structural feature. Relatively good separation was apparent on silica gel thin layer chromotography (TLC) plates, however, the $\alpha$ - and $\gamma$-pyrone groups present in $\mathbf{6 5 - 6 8}$ were suspected to be acid-sensitive. In light of this, and given the small quantities of the specimen available for analysis, DIOL was chosen as a normal-phase material for isolation. The closely related compounds were found to elute from DIOL in $\mathrm{CH}_{2} \mathrm{Cl}_{2}$, and final purification of the DIOL fractions was completed by $\mathrm{C}_{18}$ HPLC. The overall isolation strategy of lehualides $\mathrm{E}-\mathrm{K}$ (69-75) is summarised in Figure 4.5. 


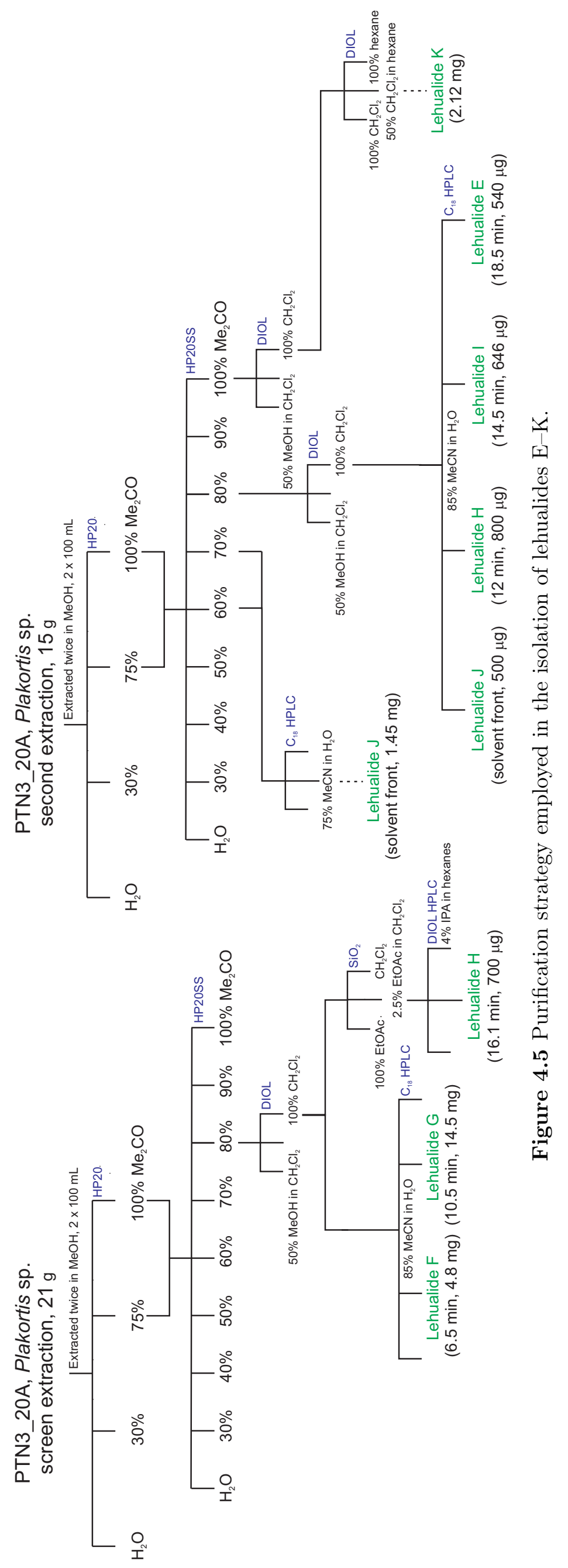




\section{Lehualide E (69)}

The positive-ion mode HRESIMS data of 69 showed a $[\mathrm{M}+\mathrm{Na}]^{+}$pseudo-molecular ion peak at $m / z 385.2399$, consistent with the molecular formula of $\mathrm{C}_{24} \mathrm{H}_{32} \mathrm{O}_{4}$, requiring nine degrees of unsaturation. The ${ }^{13} \mathrm{C}$ NMR spectrum contained 22 distinct resonances, including $11 s p^{2}$ centres, indicating some form of molecular symmetry, while interpretation of the ${ }^{1} \mathrm{H}$ and the fully-coupled HSQC NMR spectra accounted for all of the 32 protons.

Immediately apparent in the $\mathrm{CDCl}_{3}{ }^{1} \mathrm{H}$ NMR spectrum were the resonances of a mono alkyl-substituted benzene ring $\left(\delta_{\mathrm{H}} 7.27-7.16\right)$ an olefinic methine $\left(\delta_{\mathrm{H}} 5.18\right)$, two methoxy singlets $\left(\delta_{\mathrm{H}} 4.19,3.81\right)$, a deshielded methylene doublet and triplet each integrating for two protons $\left(\delta_{\mathrm{H}} 3.17,2.57\right)$, two olefinic methyl singlets $\left(\delta_{\mathrm{H}} 1.85\right.$, 1.66) and an aliphatic methylene envelope integrating for 12 protons.

Three spin systems were identified from the COSY spectrum: a mono alkylsubstituted phenyl ring, an isolated methyl-substituted alkene and an extended aliphatic chain. As illustrated in Figure 4.6, COSY and HMBC correlations allowed the facile assembly of the mono alkyl-substituted benzene ring C-16 to C-21 [(C-16: $\left.\delta_{\mathrm{C}} 143.1\right),\left(\mathrm{CH}-17\right.$ and $\left.\mathrm{CH}-21: \delta_{\mathrm{C}} 128.3, \delta_{\mathrm{H}} 7.27,2 \mathrm{H}\right),\left(\mathrm{CH}-18\right.$ and $\mathrm{CH}-20: \delta_{\mathrm{C}} 128.5$, $\left.\delta_{\mathrm{C}} 7.17,2 \mathrm{H}\right)$ and $\left.\left(\mathrm{CH}-19: \delta_{\mathrm{C}} 125.7, \delta_{\mathrm{H}} 7.16,1 \mathrm{H}\right)\right]$. The ring accounted for four of the 11 observed $s p^{2}$ resonances, four of the degrees of unsaturation required by the molecular formula and the element of molecular symmetry indicated by the ${ }^{13} \mathrm{C}$ spectrum. HMBC correlations were observed between the protons of a methylene triplet $\mathrm{CH}_{2}-15\left(\delta_{\mathrm{C}} 36.1, \delta_{\mathrm{H}} 2.57\right)$ and $\mathrm{C}-16$, while reciprocal correlations between the methines $\mathrm{CH}-17 /-21$ and $\mathrm{CH}_{2}-15$ established the attachment point of the aromatic ring.
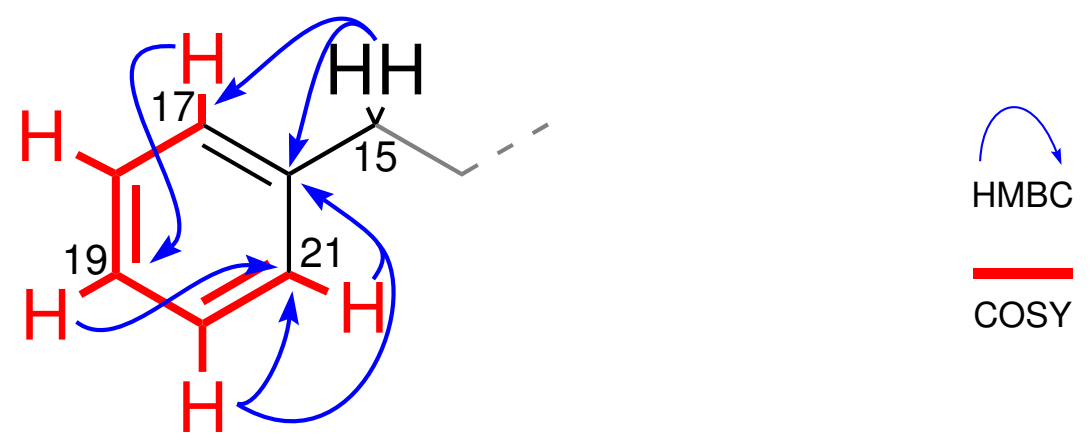

Figure 4.6 The mono alkyl-substituted benzene ring (C-16 to C-21) of $\mathbf{6 9}$ as evidenced by COSY and HMBC correlations.

The protons of the upfield olefinic methyl singlet $\mathrm{CH}_{3}-23\left(\delta_{\mathrm{C}} 16.4, \delta_{\mathrm{H}} 1.66\right)$ showed HMBC correlations to two $s p^{2}$ carbons $\mathrm{CH}-8\left(\delta_{\mathrm{C}} 118.2, \delta_{\mathrm{H}} 5.18\right)$, and C-9 $\left(\delta_{\mathrm{C}}\right.$ 138.7), and shared an allylic COSY correlation with H-8. A further HMBC 
correlation from $\mathrm{H}_{3}-23$ to a methylene carbon $\mathrm{CH}_{2}-10\left(\delta_{\mathrm{C}} 39.7, \delta_{\mathrm{H}} 1.96\right)$, and a COSY correlation from $\mathrm{H}-8$ to the protons of another methylene $\mathrm{CH}_{2}-7\left(\delta_{\mathrm{C}} 30.2\right.$, $\left.\delta_{\mathrm{H}} 3.17\right)$ established an isolated, tri-substituted double bond. The $E$ geometry of the alkene was established from selective irradiation of $\mathrm{H}_{3}-23$ in a 1D NOE experiment and subsequent enhancement of $\mathrm{H}_{2}-7$ and $\mathrm{H}-11$. Methylenes $\mathrm{H}_{2}-7$ and $\mathrm{H}_{2}-10$ were similarly enhanced after irradiation of H-8, confirming the $E$ geometry of the trisubstituted double bond (Figure 4.7).
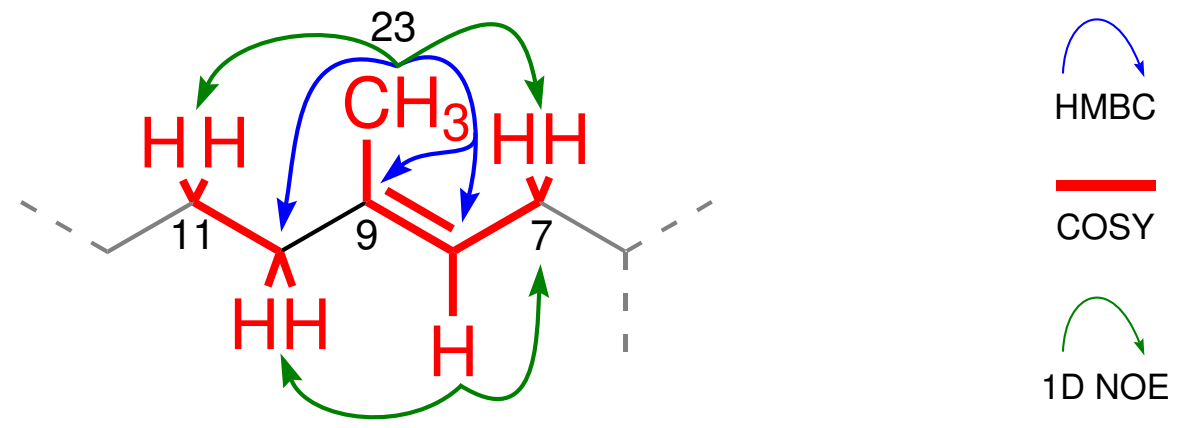

Figure 4.7 COSY, HMBC and 1D NOE correlations confirming the geometry and connectivity of the C-7 to C-11 segment in $\mathbf{6 9}$.

The remaining five non-protonated $s p^{2}$ carbons C-2 $\left(\delta_{\mathrm{C}} 162.7\right), \mathrm{C}-3\left(\delta_{\mathrm{C}} 128.3\right), \mathrm{C}-4$ $\left(\delta_{\mathrm{C}} 159.0\right), \mathrm{C}-5\left(\delta_{\mathrm{C}} 108.3\right)$ and $\mathrm{C}-6\left(\delta_{\mathrm{C}} 154.8\right)$ could only be accounted for by two fully substituted carbon-carbon double bonds and a carbonyl. HMBC correlations were observed from $\mathrm{H}_{2}-7$ to $\mathrm{C}-5$ and $\mathrm{C}-6$, indicating the double-allylic character of $\mathrm{H}_{2}-7$, consistent with its chemical shift $\left(\delta_{\mathrm{C}} 30.2, \delta_{\mathrm{H}} 3.17\right)$. An HMBC correlation from H-8 to C-6, established the C-6-C-7 bond. Further HMBC correlations were observed from the protons of the final olefinic methyl $\mathrm{CH}_{3}-22\left(\delta_{\mathrm{C}} 10.1, \delta_{\mathrm{H}} 1.85\right)$ to C-4, C-5 and C-6, which combined with a homoallylic COSY correlation between $\mathrm{H}_{2}-7$ and $\mathrm{H}_{3}-22$ established attachment of the methyl at C-5 and the connections from C-4 to C-5 and from C-5 to C-6 (Figure 4.8).
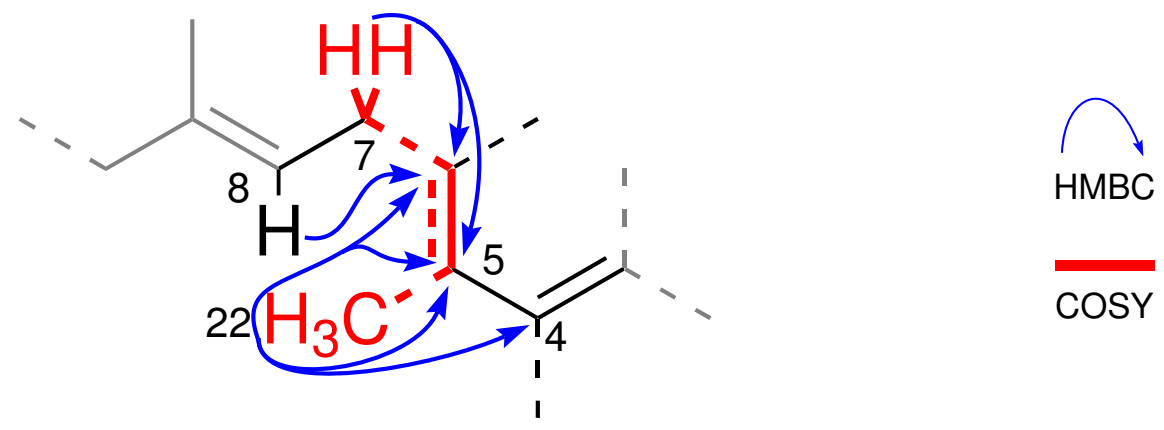

Figure 4.8 COSY and HMBC correlations firmly establishing the C-5 to C-8 connections in 69. Dashed lines indicate weak correlations.

The substructure was extended by an HMBC correlation from the protons of a methoxy $\mathrm{OCH}_{3}-4\left(\delta_{\mathrm{C}} 60.5, \delta_{\mathrm{H}} 4.19\right)$ to $\mathrm{C}-4$, while a second methoxy $\mathrm{OCH}_{3}-3$ 
$\left(\delta_{\mathrm{C}} 60.4, \delta_{\mathrm{H}} 3.81\right)$ correlated with $\mathrm{C}-3$, revealing the final carbon-carbon double bond. Reciprocal NOE correlations between $\mathrm{OCH}_{3}-3$ and $\mathrm{OCH}_{3}-4$ established the C-3 to C-4 connectivity and the cis relationship of the methoxy substituents. NOE correlations between $\mathrm{OCH}_{3}-4$ and $\mathrm{H}_{3}-22$, and $\mathrm{H}_{2}-7$ to $\mathrm{H}_{3}-22$ confirmed the tetrasubstituted diene $\mathrm{C}-3$ to $\mathrm{C}-6$, with $\mathrm{CH}_{2}-7$ and $\mathrm{CH}_{3}-22$ on the same side of the $\mathrm{C}$ 5 to C-6 double bond. With one carbon and two oxygen atoms remaining from the molecular formula and two double-bond equivalents, the remaining $s p^{2}$ centre C-2 $\left(\delta_{\mathrm{C}} 162.7\right)$, which must be a carbonyl was therefore assigned as an $\alpha, \beta$-unsaturated ester on the basis of its shielded chemical shift (Figure 4.9). The shielded chemical shift of the oxygenated $\mathrm{C}-3\left(\delta_{\mathrm{C}} 126.2\right)$ is also consistent with the attachment of the carbonyl. ${ }^{132,135}$

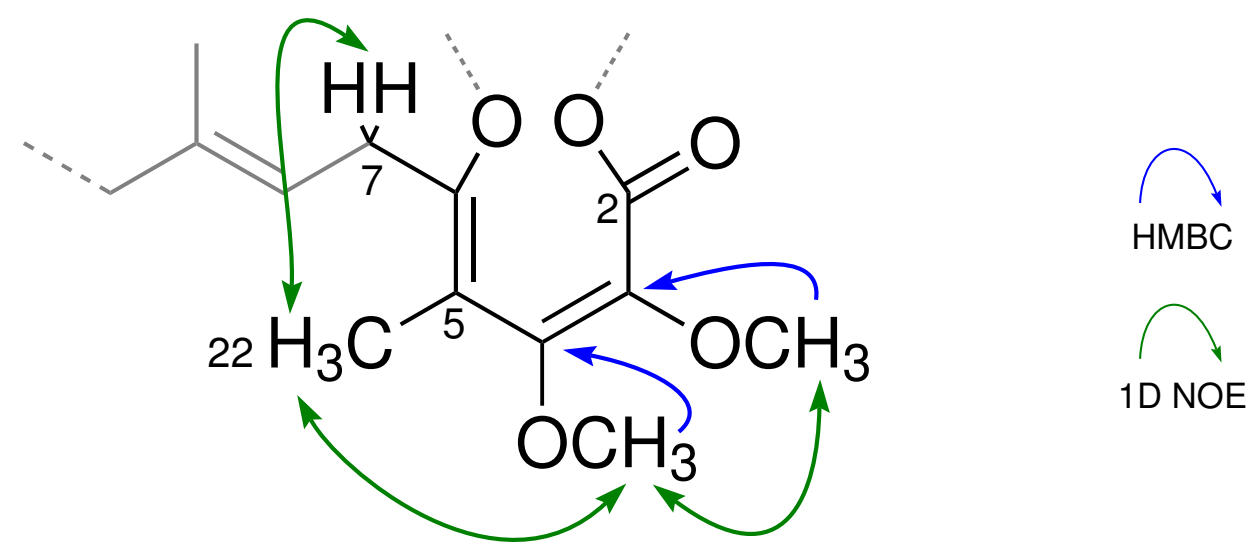

Figure 4.9 HMBC and 1D NOE correlations used to establish the C-3 to C-6 tetrasubstituted diene in $\mathbf{6 9}$, and the geometry of the substituents. Double headed arrows indicate reciprocal correlations.

All but one degree of unsaturation associated with the molecular formula were accounted for by the phenyl ring, the isolated double bond C-8-C-9, and the C2 to C-6 dienoate. Furthermore, the four oxygen atoms required by the molecular formula are accounted for by the two oxymethyls and the ester functionality. The deshielded chemical shift of C-6 $\left(\delta_{\mathrm{C}} 154.8\right)$ is consistent with oxygen substitution, and therefore an $\alpha$-pyrone moiety, C-2 to C-6, similar to that of lehualide A (65), is established (Figure 4.10). UV absorption maxima $\left(\lambda_{\max } 291 \mathrm{~nm}\right)$, and IR stretching frequencies $\left(\nu_{\max } 1686,1649 \mathrm{~cm}^{-1}\right)$ support this assignment, ${ }^{136}$ while the chemical shifts of the $s p^{2}$ carbon resonances are consistent with those reported for similar systems. ${ }^{132}$

A series of sequential COSY and HMBC correlations, beginning at the $\mathrm{H}_{2}-15$ methylene triplet, and terminating with $\mathrm{H}_{2}-10$ established a $\mathrm{C}_{6}$ methylene chain linking the mono alkyl-substituted benzene ring and the $\mathrm{C}-2$ to $\mathrm{CH}_{2}-10$ segment. Selective excitation of the methylenes $\mathrm{CH}_{2}-10\left(\delta_{\mathrm{C}} 39.7 \delta_{\mathrm{H}} 1.96\right)$ and $\mathrm{CH}_{2}-15\left(\delta_{\mathrm{C}} 36.1\right.$ $\left.\delta_{\mathrm{H}} 2.57\right)$ with mixing times of $20-120 \mathrm{~ms}$ revealed the resonances within the methylene envelope $\left(\mathrm{CH}_{2}-12-\mathrm{CH}_{2}-14: \delta_{\mathrm{C}} 27.8,29.9,29.3,31.6 \delta_{\mathrm{H}} 1.24-1.38\right)$ and 
gave reciprocal revelation of $\mathrm{CH}_{2}-10$ and $\mathrm{CH}_{2}-15$ (Figure 4.10). This completed the structure of lehualide E (69). NMR data for $\mathbf{6 9}$ is presented in Table 4.1.

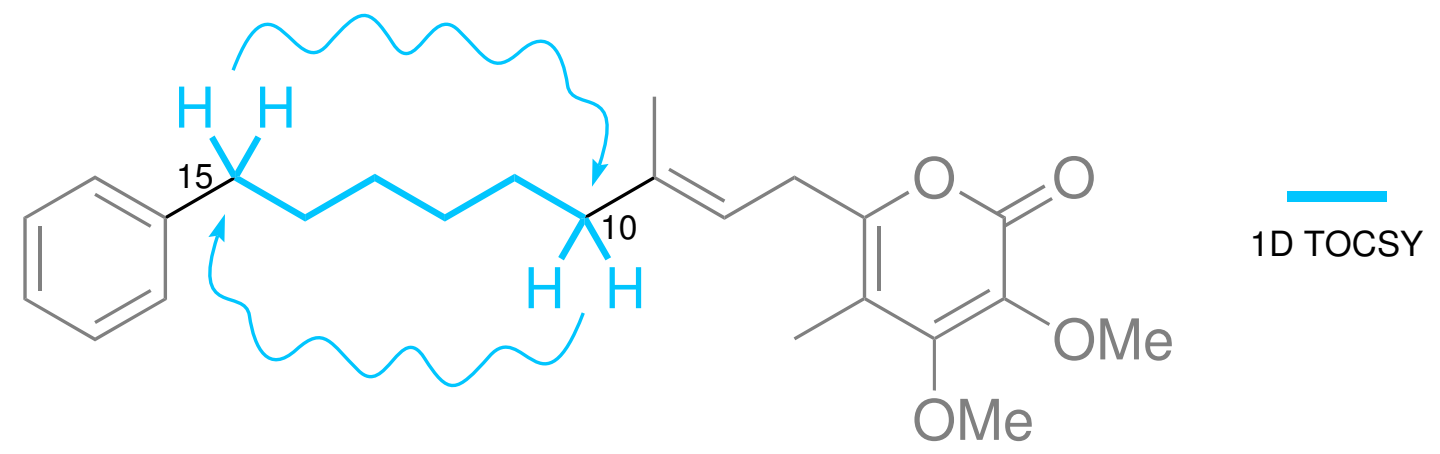

Figure 4.10 1D TOCSY correlations confirming connection between the extended $\alpha$ pyrone segment (C-2 to C-11) and the mono alkyl-substituted benzene ring (C-15 to C-21) in 69. 

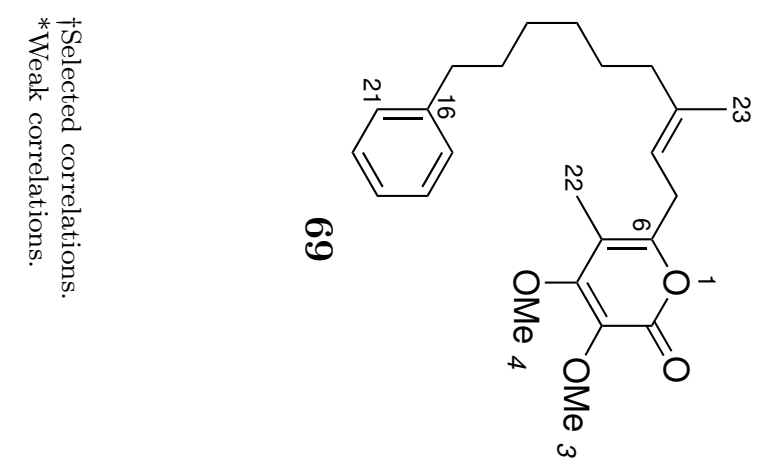

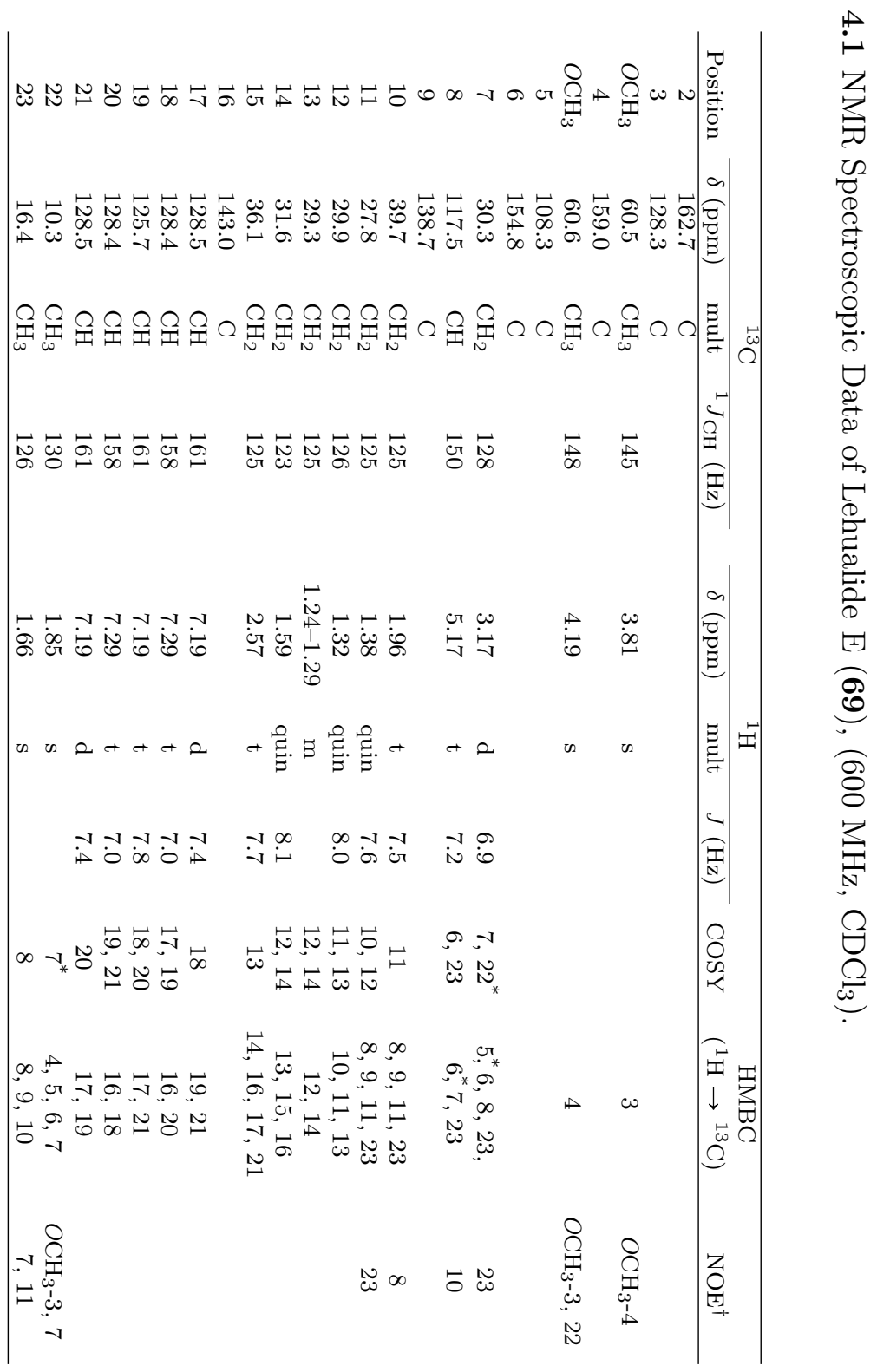




\section{Lehualide F (70)}

Lehualide F (70) was isolated as a white solid. Positive-ion mode HRESIMS analysis of 70 revealed a $[\mathrm{M}+\mathrm{Na}]^{+}$pseudo-molecular ion peak at $m / z$ 393.2042. This indicated the molecular formula $\mathrm{C}_{23} \mathrm{H}_{30} \mathrm{O}_{4}$, differing from that of 69 by an additional $\mathrm{CH}_{2}$ equivalent. The multiplicity-edited HSQC spectrum accounted for 32 of the 33 protons, indicating the presence of one exchangeable proton, while the ${ }^{1} \mathrm{H}$ spectrum of $\mathbf{7 0}$ displayed resonances for a mono alkyl-substituted benzene ring $\left(\delta_{\mathrm{H}} 7.22-7.16\right)$ an olefinic methine $\left(\delta_{\mathrm{H}} 5.17\right)$, a deshielded methylene doublet and triplet $\left(\delta_{\mathrm{H}} 3.20\right.$, $2.57)$, two olefinic methyl singlets $\left(\delta_{\mathrm{H}} 1.85,1.66\right)$ and an aliphatic methylene envelope integrating for 12 protons. As demonstrated in Figure 4.11, the ${ }^{1} \mathrm{H}$ NMR spectrum of 70 appeared very similar to that of lehualide $\mathrm{E}(69)$, except for the broad resonance of an exchangeable proton $\left(\delta_{\mathrm{H}} 6.50\right)$, the presence of only one methoxy $\left(\delta_{\mathrm{H}} 3.90\right)$ and slight downfield change in the chemical shift of the $\mathrm{CH}_{3}-22$ methyl signal $\left(\delta_{\mathrm{H}} 1.96\right)$.

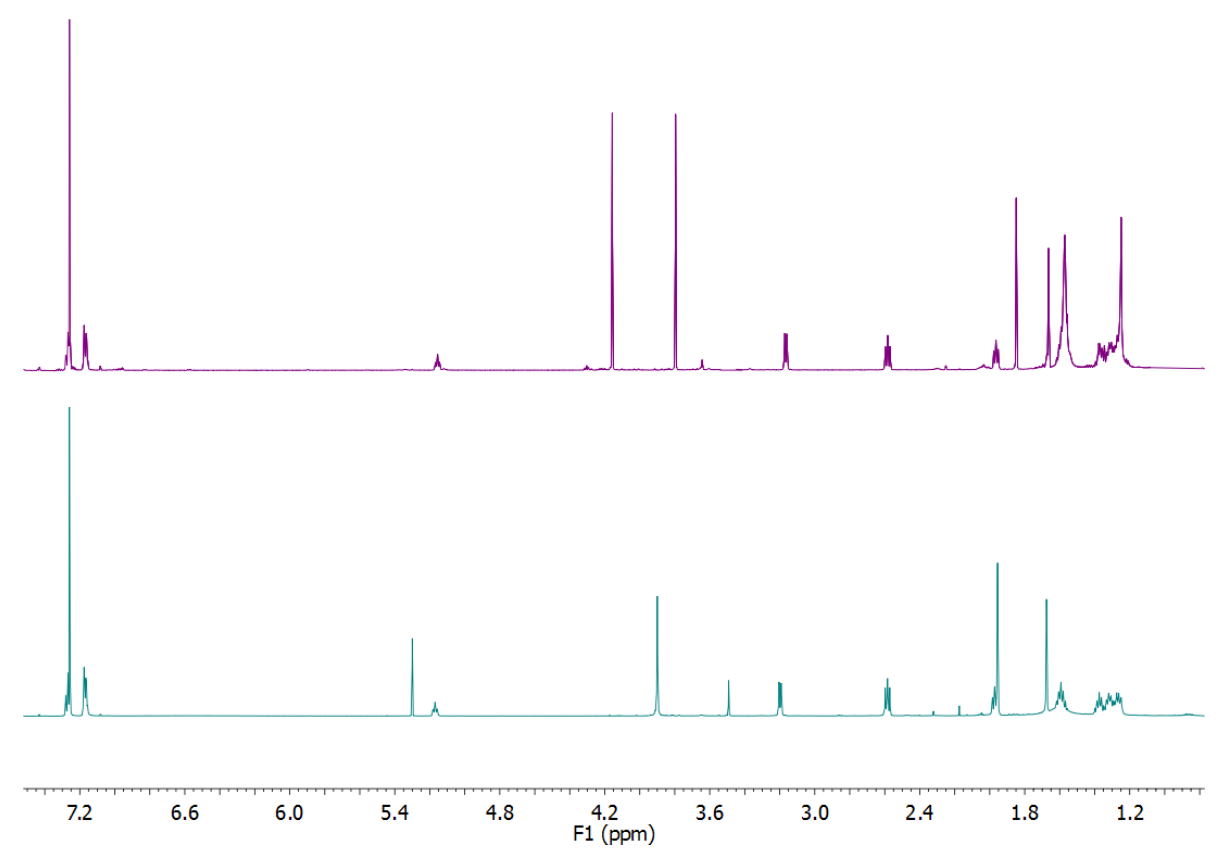

Figure $4.11{ }^{1} \mathrm{H}$ NMR spectra of lehualides E (69) and F (70); differing in the number of methoxy signals and chemical shift of $\mathrm{CH}_{3}-22\left(\mathbf{6 9} \delta_{\mathrm{H}} 1.85 ; \mathbf{7 0} \delta_{\mathrm{H}} 1.96\right)$.

As with 69, COSY and HMBC correlations allowed the facile assembly of the mono alkyl-substituted benzene ring $\mathrm{C}-16$ to $\mathrm{C}-21$ [(C-16: $\left.\delta_{\mathrm{C}} 143.0\right),(\mathrm{CH}-17$ and $\mathrm{CH}-$ 21: $\left.\delta_{\mathrm{C}} 128.5, \delta_{\mathrm{H}} 7.17,2 \mathrm{H}\right),\left(\mathrm{CH}-18\right.$ and $\left.\mathrm{CH}-20: \delta_{\mathrm{C}} 128.4, \delta_{\mathrm{H}} 7.22,2 \mathrm{H}\right)$ and $(\mathrm{CH}-19$ : $\left.\delta_{\mathrm{C}} 125.6, \delta_{\mathrm{H}} 7.16,1 \mathrm{H}\right)$ ]. Substitution of the aromatic ring was established via HMBC correlation from protons of a methylene triplet $\mathrm{CH}_{2}-15\left(\delta_{\mathrm{C}} 36.1, \delta_{\mathrm{H}} 2.57\right)$ to $\mathrm{C}-16$, and reciprocal correlations between the methines $\mathrm{CH}-17 /-21$ and $\mathrm{CH}_{2}-15$ (Figure 4.12). 


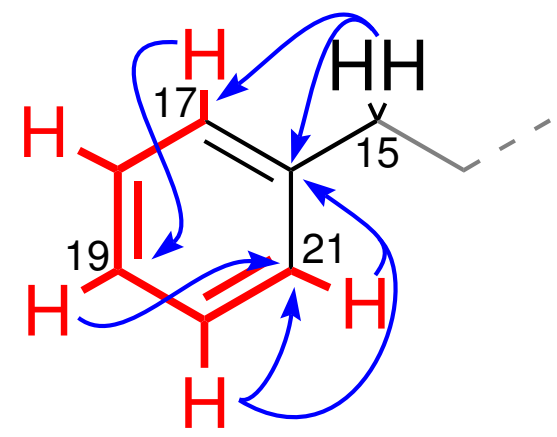

\section{冈 \\ HMBC \\ $\cos Y$}

Figure 4.12 COSY and selected HMBC correlations establishing the C-17 to C-23 segment of 70 .

The isolated double bond C-8-C-9 was again assembled on the basis of HMBC correlations from protons of the olefinic methyl singlet $\mathrm{CH}_{3}-23\left(\delta_{\mathrm{C}} 16.3, \delta_{\mathrm{H}} 1.68\right)$ to the $s p^{2}$ carbons $\mathrm{CH}-8\left(\delta_{\mathrm{C}} 117.2, \delta_{\mathrm{H}} 5.17\right)$, and C-9 $\left(\delta_{\mathrm{C}} 139.0\right)$. A further HMBC correlation from $\mathrm{H}_{3}-23$ to a methylene carbon $\mathrm{CH}_{2}-10\left(\delta_{\mathrm{C}} 39.7, \delta_{\mathrm{H}} 1.96\right)$, and a COSY correlation from $\mathrm{H}-8$ to the protons of another methylene $\mathrm{CH}_{2}-7\left(\delta_{\mathrm{C}} 30.2\right.$, $\delta_{\mathrm{H}} 3.17$ ) extended the $s p^{2}$ substructure. Selective irradiation of $\mathrm{H}_{3}-23$ in a $1 \mathrm{D} \mathrm{NOE}$ experiment induced enhancement of $\mathrm{H}_{2}-7$ and $\mathrm{H}_{2}-11$, while $\mathrm{H}_{2}-7$ and $\mathrm{H}_{2}-10$ were similarly enhanced after irradiation of H-8. This established the $E$ geometry of the C-8 to C-9 alkene.

An $\alpha$-pyrone $\left(\lambda_{\max } 298 \mathrm{~nm}\right)$ similar to that of $\mathbf{6 9}$ was quickly identified. The oxysubstituted double bond between C-5 and C-6 was established on the basis of the strong HMBC correlations from the protons of the $s p^{2}$ methyl $\mathrm{H}_{3}-21$ and methylene $\mathrm{H}_{2}-7$ to both C-5 and C-6. Evidence of weak homoallylic COSY coupling between $\mathrm{H}_{3}-21$ and $\mathrm{H}_{2}-7$ confirmed the $\mathrm{CH}_{2}-7-\mathrm{C}-6-\mathrm{C}-5-\mathrm{CH}_{3}-21$ bonds (Figure 4.13).
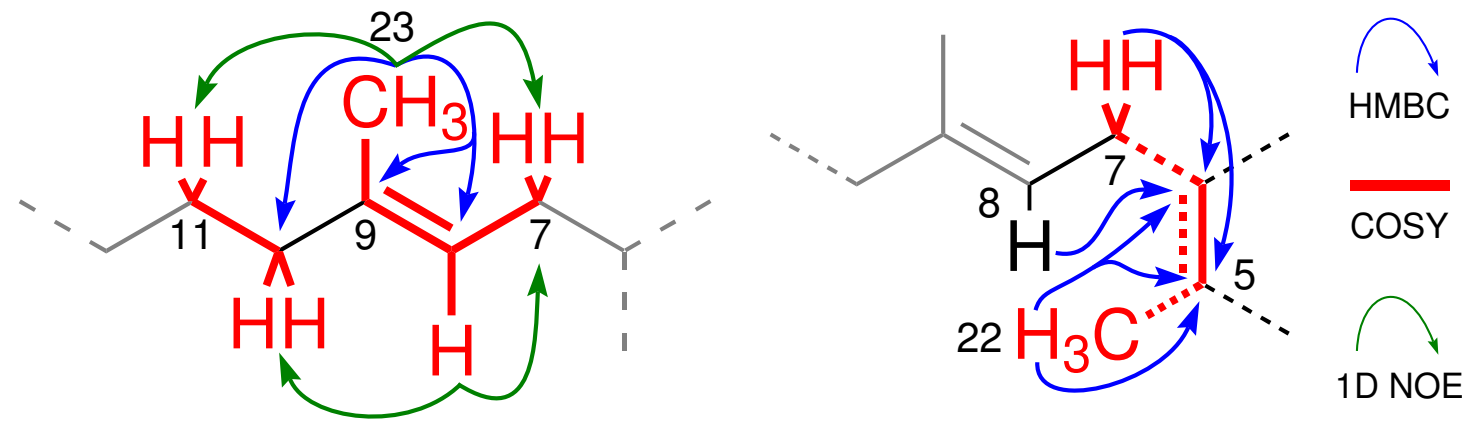

Figure 4.13 COSY, HMBC and 1D NOE correlations confirming the tri-substitution of the $E$ alkene C-8-C-9, and the tetra-substituted alkene C-5-C-6 in 70. Dashed lines indicate weak correlations.

A further HMBC correlation from $\mathrm{H}_{3}-22$ to $\mathrm{C}-4\left(\delta_{\mathrm{C}} 162.1\right)$ was observed, the deshielded chemical shift again indicating oxygen-substitution of C-4. However, the methoxy observed in $\mathbf{6 9}$ was absent suggesting a hydroxyl functionality. This accounted for the exchangeable proton (IR: $\nu_{\max } 3250 \mathrm{~cm}^{-1}, \delta_{\mathrm{H}} 6.50 \mathrm{ppm}$ ). HMBC 
correlations from protons of the lone methoxy ${ } \mathrm{CH}_{3}-3\left(\delta_{\mathrm{C}} 60.0, \delta_{\mathrm{H}} 3.86\right)$ to $\mathrm{C}-3$ $\left(\delta_{\mathrm{C}} 125.0\right)$ were observed as in $\mathbf{6 9}$, as was an NOE correlation between the protons of $\mathrm{CH}_{2}-7$ and $\mathrm{CH}_{3}-21$ (Figure 4.14).
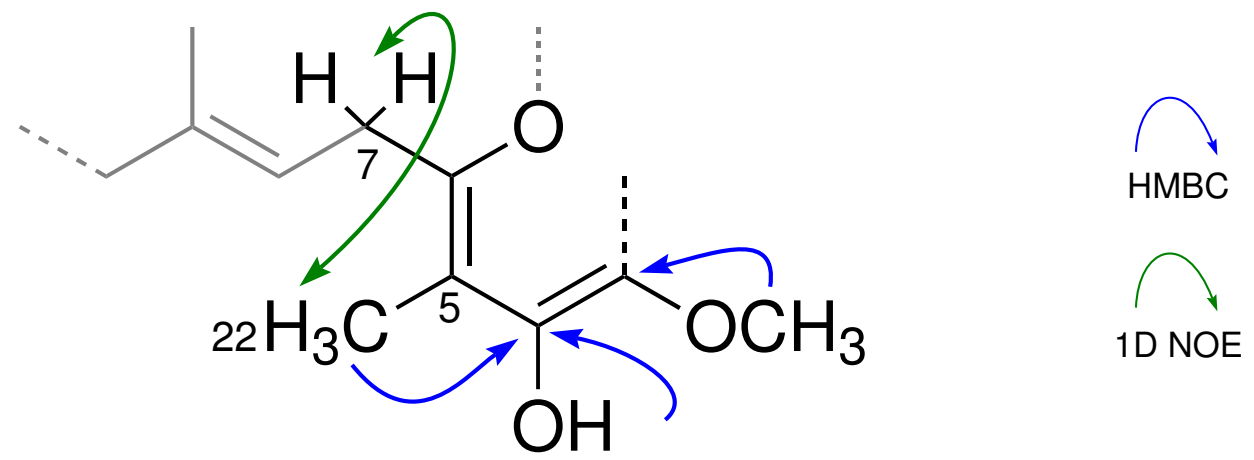

Figure 4.14 HMBC and 1D NOE correlations establishing the C-4-C-5 bond and methoxy substitution of C-3 in 70. Double headed arrows indicate reciprocal correlations.

The $\alpha$-pyrone system was completed by the $\alpha, \beta$-unsaturated ester carbonyl C-2 $\left(\delta_{\mathrm{C}} 160.4\right)$, Figure 4.15. All other chemical shifts and correlations observed in the NMR spectra were essentially identical to that of $\mathbf{6 9}$, thereby establishing $\mathbf{7 0}$ as the C-4 des-methoxy congener of $\mathbf{6 9}$. NMR data for $\mathbf{7 0}$ is presented in Table 4.2.

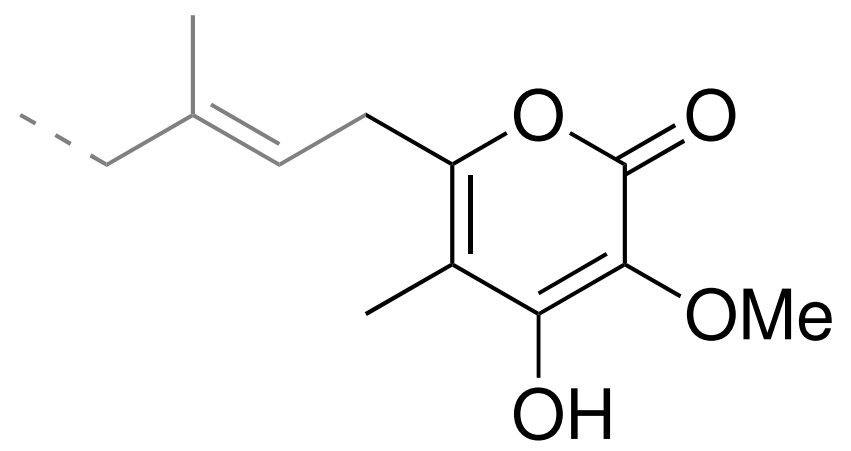

Figure 4.15 The $\alpha$-pyrone system present in $\mathbf{7 0}$, the des-methoxy congener of $\mathbf{6 9}$. 

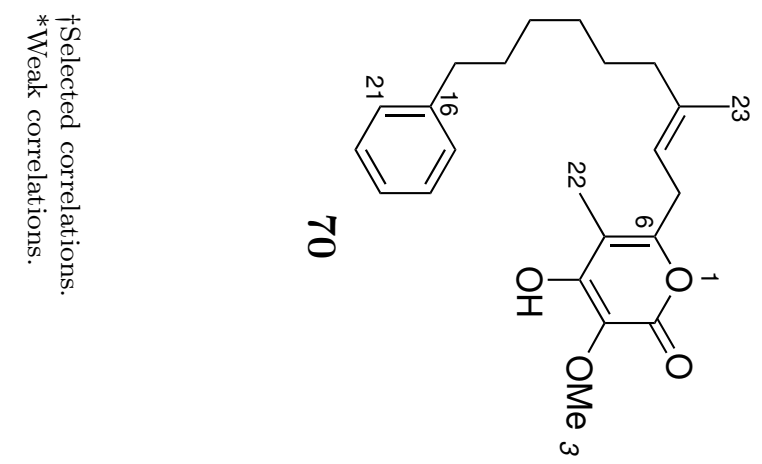

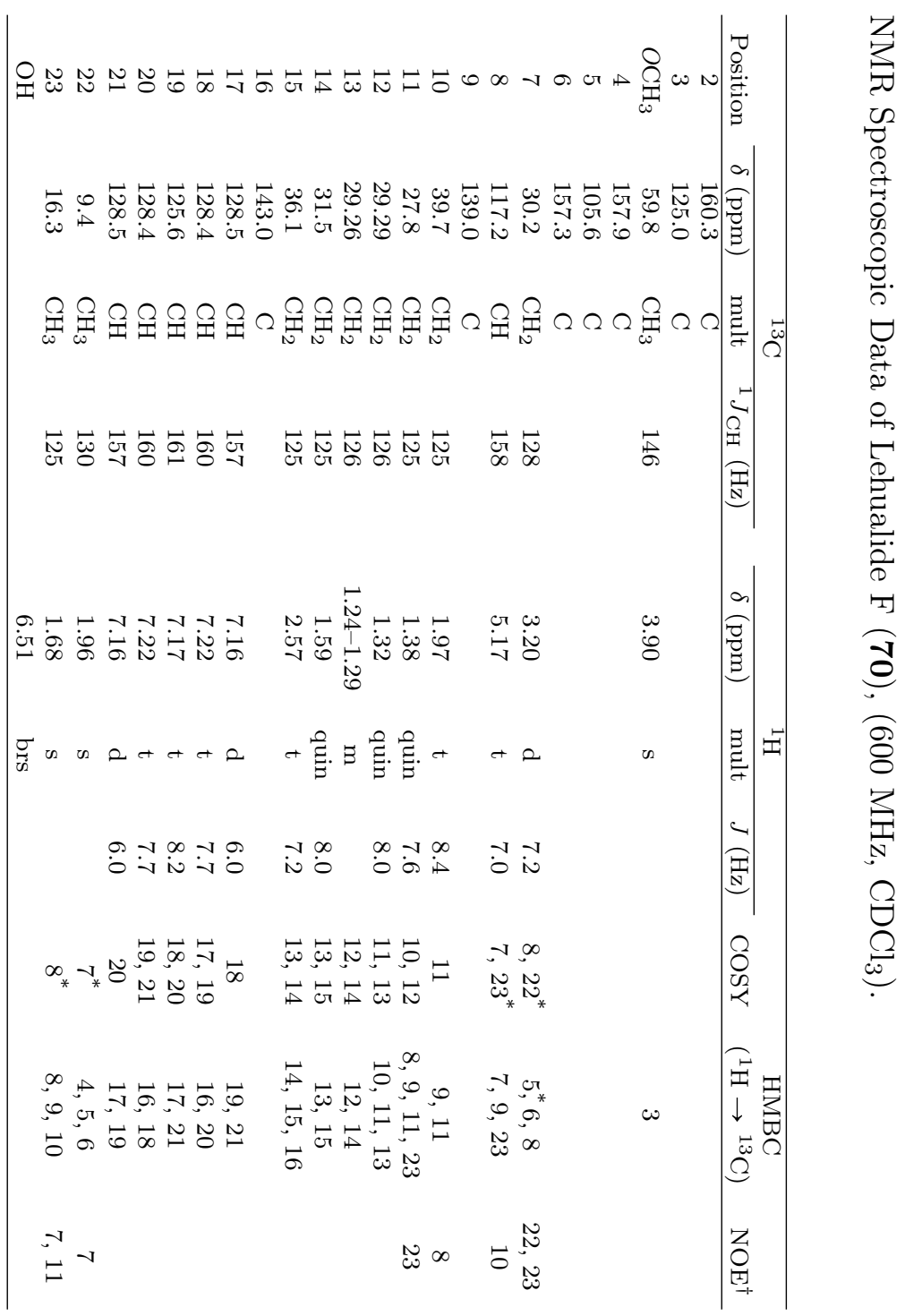




\section{Lehualide G (71)}

$\mathrm{A}[\mathrm{M}+\mathrm{Na}]^{+}$pseudo-molecular ion peak observed in the positive-ion mode HRESIMS spectrum for lehualide $\mathrm{G}(\mathbf{7 1})$ at $m / z$ 421.2355, indicated a molecular formula of $\mathrm{C}_{25} \mathrm{H}_{34} \mathrm{O}_{4}$, differing from $\mathbf{7 0}$ by an additional $\mathrm{C}_{2} \mathrm{H}_{2}$ equivalent. Detailed analysis of the 1D and 2D NMR spectra of $\mathbf{7 0}$ and $\mathbf{7 1}$ determined that the two were chainlength congeners. The only perceivable differences between the spectra of the two compounds were the methylene regions of the ${ }^{1} \mathrm{H}$ and ${ }^{13} \mathrm{C}$ spectra (Figure 4.16).

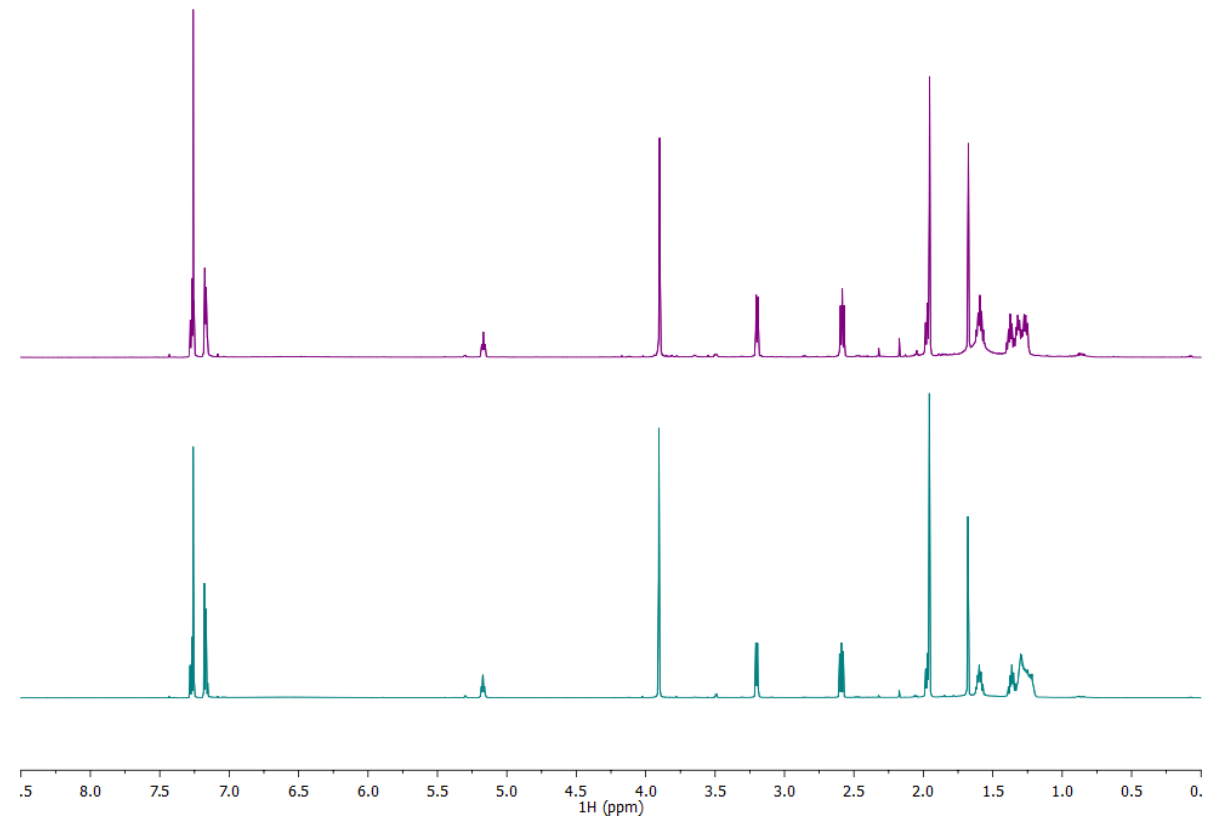

Figure 4.16 ${ }^{1} \mathrm{H}$ NMR spectra of lehualides F (70) and G (71). The compounds differ only integration of the methylene region $\left(\delta_{\mathrm{H}} 1.28-1.45 \mathrm{ppm}, 696 \mathrm{H}, 708 \mathrm{H}\right)$.

Much of the 1D and 2D NMR data of 71 were consistent with the $\alpha$-pyrone moiety present in 70, and UV and IR spectroscopic data ( $\lambda_{\max } 235$ and $291 \mathrm{~nm}, \nu_{\max } 3250$ $\mathrm{cm}^{-1}$ ) supported this assignment. The extended methylene chain of $\mathbf{7 1}, \mathrm{C}-10$ to $\mathrm{C}-$ 17 , was established by selective 1D TOCSY irradiation of $\mathrm{CH}_{2}-17\left(\delta_{\mathrm{C}} 36.1, \delta_{\mathrm{H}} 2.61\right)$. As the mixing time was increased from 0 to $120 \mathrm{~ms}$, the methylene resonances $\mathrm{H}_{2}$ 16 to $\mathrm{H}_{2}-10\left(\mathrm{C}-10: \delta_{\mathrm{C}} 40.5, \delta_{\mathrm{H}} 2.01\right)$ were sequentially revealed. Analogous 1D TOCSY irradiation of $\mathrm{H}_{2}-10$ coupled with COSY and HMBC correlations within the chain provided confirmation of this assignment and the connection between the methylenes (Figure 4.17).

HSQC-TOCSY analysis of 71 in $\mathrm{CD}_{3} \mathrm{OD}$ over a selected band-width, $\delta_{\mathrm{C}} 10-50 \mathrm{ppm}$, allowed elucidation of the aliphatic chain: [(C-12 to C-15: $\delta_{\mathrm{C}} 32.8,30.6,30.5,30.3$, $\left.\delta_{\mathrm{H}} 1.25-1.29\right)$ and $\left.\left(\mathrm{C}-16: \delta_{\mathrm{C}} 30.1, \delta_{\mathrm{C}} 1.22\right)\right]$. Using this method the overlapping methylene resonances from C-12 to C-16 were clearly resolved, while the TOCSY correlations aided concise ordering of the resonances. NMR data for $\mathbf{7 1}$ is presented in Table 4.3. 


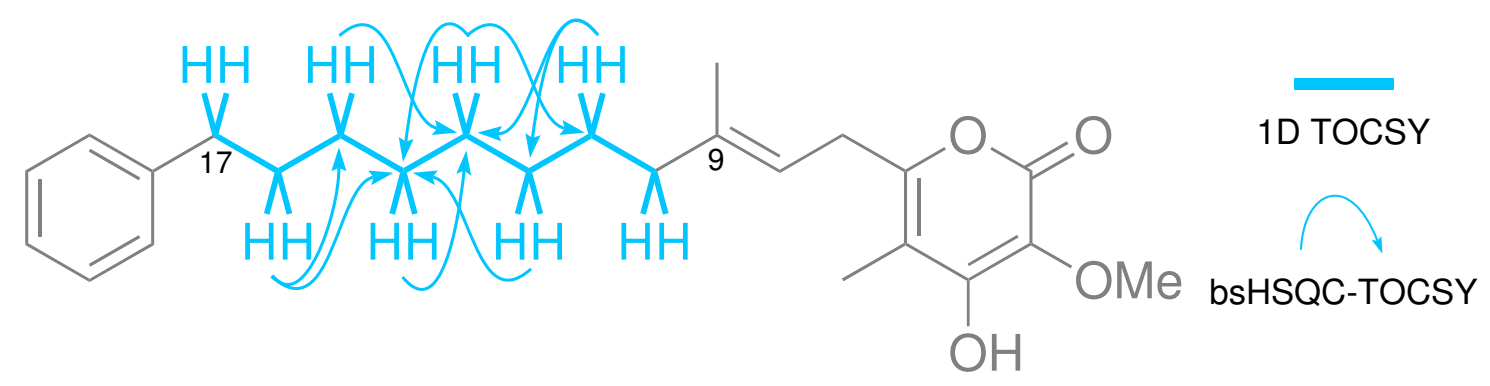

Figure 4.17 1D TOCSY and bsHSQC-TOCSY correlations established the connection between methylenes $\mathrm{CH}_{2}-10$ and $\mathrm{CH}_{2}-17$ in the extended aliphatic chain of $\mathbf{7 1}$.

The structural similarities between lehualides $F$ and $G$ made resolution of the chain-length congeners impossible under normal-phase chromatographic conditions. With no discernable difference in polarity, the two compounds would co-elute in an approximate 1:20 ratio. Exploitation of the differences in lipophilicity granted separation of the two congeners under reversed-phase conditions $\left(\mathrm{C}_{18}\right.$ HPLC, $85 \%$ $\mathrm{MeCN}$ in $\mathrm{H}_{2} \mathrm{O}$ ). Conversely, resolution of mixed fractions of lehualides $\mathrm{E}$ and $\mathrm{F}$ could be nearly achieved under normal-phased conditions. Although the lipophilic chains induced streaking across the stationary phase, polarity differences between the methoxy and hydroxyl forms were sufficient to afford some separation. 


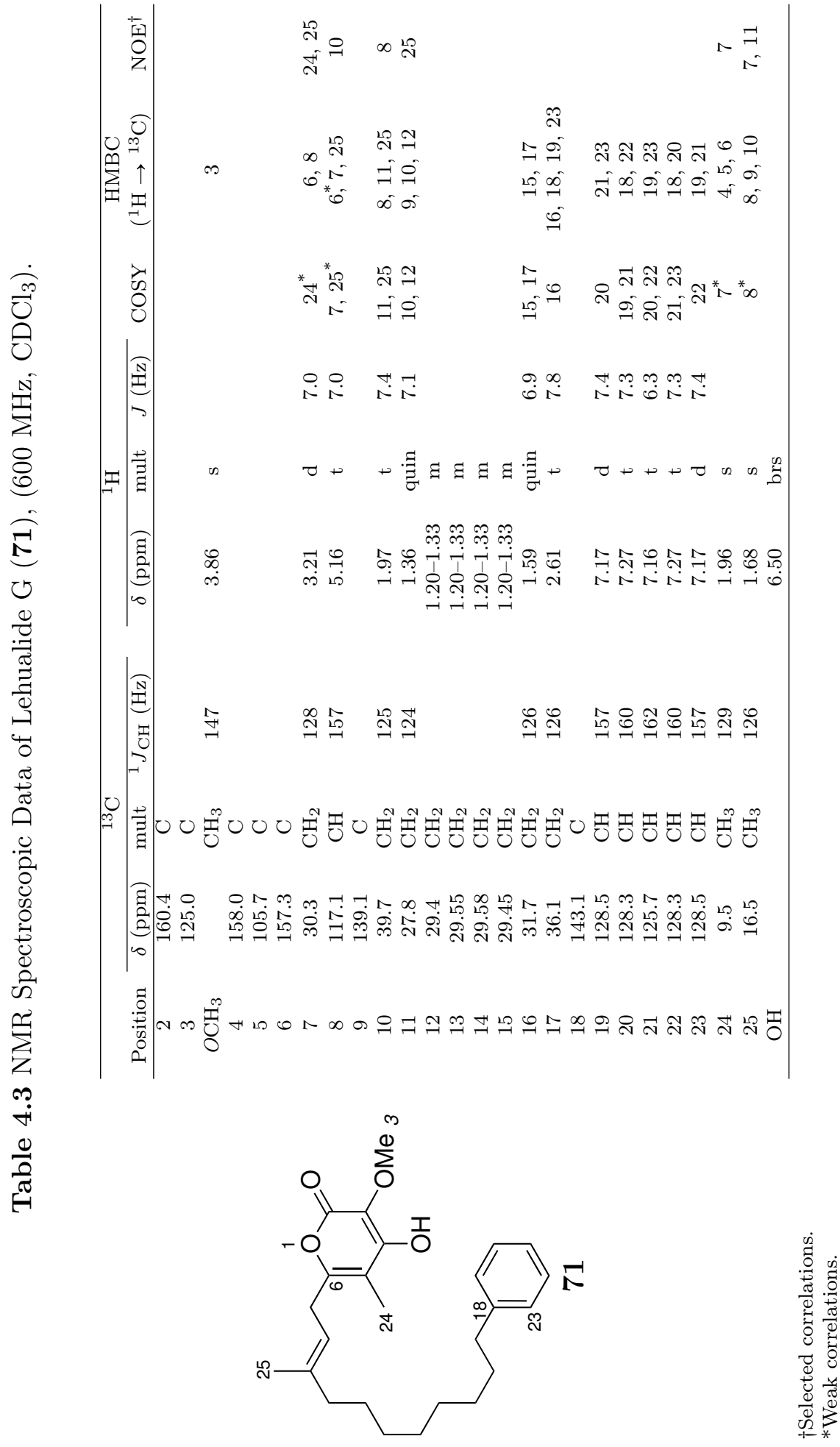




\section{Lehualide H (72)}

Positive-ion mode HRESIMS analysis of lehualide $\mathrm{H}(\mathbf{7 2})$ generated a $[\mathrm{M}+\mathrm{Na}]^{+}$ pseudo-molecular ion peak at $m / z$ 407.1864, suitable for the formula $\mathrm{C}_{20} \mathrm{H}_{32} \mathrm{O}_{5} \mathrm{~S}$, requiring five degrees of unsaturation, and incorporation of a sulfur atom. Analysis of the ${ }^{1} \mathrm{H}$ NMR spectrum of $\mathbf{7 2}$ revealed, in contrast with the lehualides $\mathrm{E}-\mathrm{G}$, the absence of the phenyl moiety, olefinic proton, and doubly allylic methylene signals.

Similar 2D NMR, IR and UV spectroscopic data to that of $\mathbf{6 9}$, and the presence of two methoxy signals indicated retention of the dimethoxy $\alpha$-pyrone. This accounted for four of the five oxygen atoms, and four degrees of molecular unsaturation. As with 69, the non-protonated $\alpha$-pyrone ring was assigned on the basis of HMBC and NOE correlations between $\mathrm{H}_{2}-7, \mathrm{H}_{3}-21$ and the methoxy groups $O_{\mathrm{CH}_{3}}-4$ and $O_{\mathrm{CH}_{3}-}$ 3. The doubly allylic methylene doublet of $\mathbf{6 9}, \mathrm{CH}_{2}-7\left(\delta_{\mathrm{C}} 30.2, \delta_{\mathrm{H}} 3.17\right)$, is replaced in 72 by a singly allylic methylene triplet, $\mathrm{CH}_{2}-7\left(\delta_{\mathrm{C}} 30.9, \delta_{\mathrm{H}} 2.44\right)$, as evidenced by homoallylic COSY correlation between $\mathrm{CH}_{2}-7$ and $\mathrm{H}_{3}-21$, and HMBC correlations to C-5 and C-6 (Figure 4.18). These data confirmed the absence of the isolated double bond and associated olefinic methyl of the previously described structures.
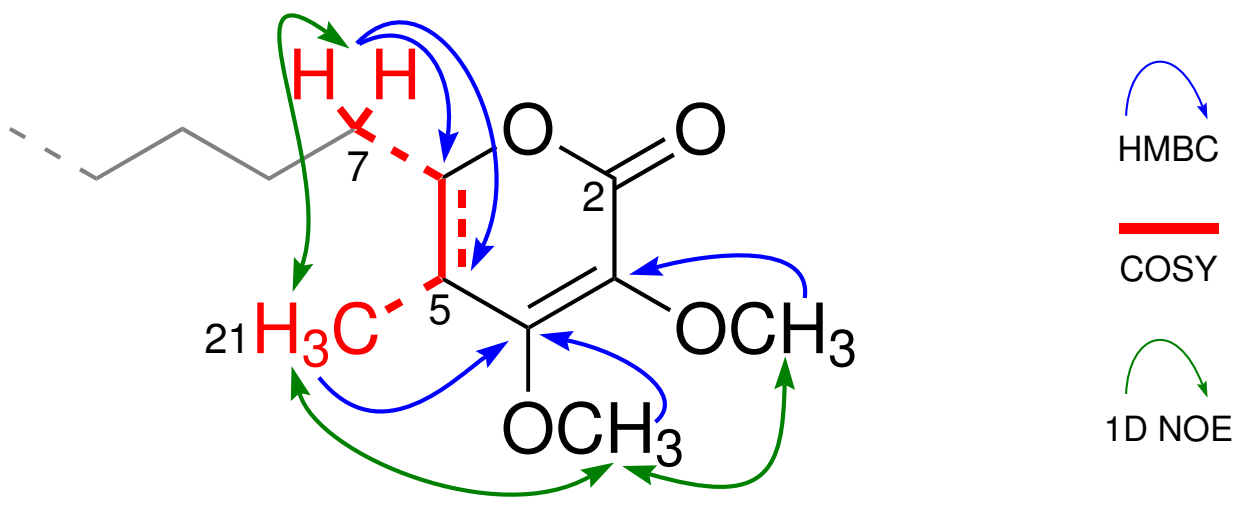

Figure 4.18 The dimethoxy-substituted $\alpha$-pyrone and singly-allylic methylene substituent of $\mathbf{7 2}$ as evidenced by COSY, HMBC and 1D NOE correlations. Double headed arrows indicate reciprocal correlations, and dashed lines indicate weak correlations.

The long alkyl chain characteristic of the lehualides extended between $\mathrm{CH}_{2}-7$ and $\mathrm{CH}_{2}-16\left(\delta_{\mathrm{C}} 29.2, \delta_{\mathrm{H}} 2.86\right)$, selective 1D TOCSY irradiations of methylenes $\mathrm{H}_{2}-7$ and $\mathrm{H}_{2}$-16, with increasing mixing times (0-200 ms) established the connection between the two centres.

The final substructure began at the deshielded methyl singlet terminus $\left(\delta_{\mathrm{C}} 30.8\right.$, $\left.\delta_{\mathrm{H}} 2.35\right)$. Strong HMBC correlations from the protons of both the methyl and $\mathrm{H}_{2^{-}}$ 16 to an ester carbonyl $\left(\delta_{\mathrm{C}} 196.3\right)$ were observed. The large ${ }^{1} J_{\mathrm{CH}}$ coupling constant and low ${ }^{1} \mathrm{H}$ and ${ }^{13} \mathrm{C}$ chemical shifts of the $\mathrm{C}-16$ methylene $\left(\delta_{\mathrm{C}} 29.2, \delta_{\mathrm{H}} 2.86,{ }^{1} J_{\mathrm{CH}} 141\right.$ $\mathrm{Hz}$ ) indicated sulfur attachment. ${ }^{137,138}$ The chemical shift of the acetate carbonyl $\left(\delta_{\mathrm{C}} 196.3\right)$ is consistent with the thioacetate moiety previously observed in lehualide 
C (67). As illustrated in Figure 4.19, reciprocal weak HMBC correlations observed from the protons of methylene $\mathrm{H}_{2}-16$ and methyl terminus to the respective carbons confirmed the thioacetate linkage. Elucidation of this substructure accounted for the sulfur atom, the fifth oxygen atom, and the final degree of unsaturation required by the molecular formula. This completed the structure of lehualide $\mathrm{H}(\mathbf{7 2})$, the NMR data of which is presented in Table 4.4 .
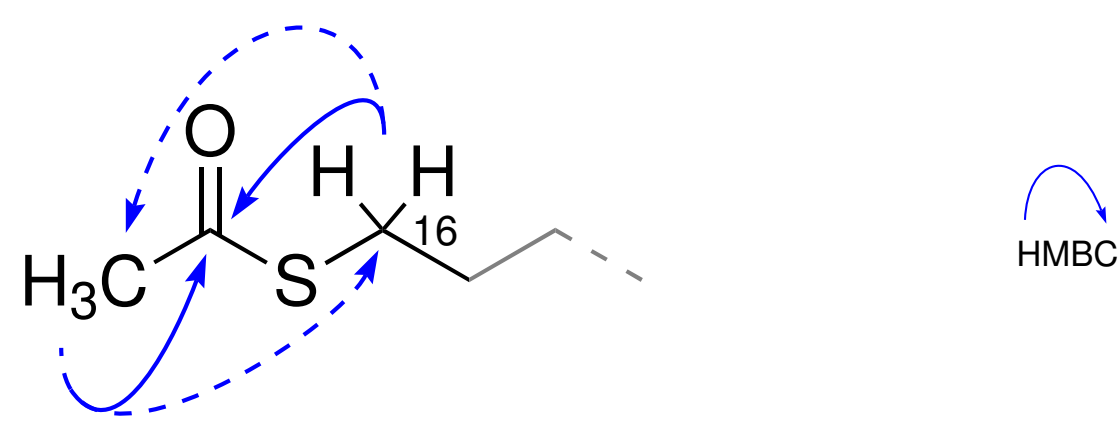

Figure 4.19 The thioacetate moiety of $\mathbf{7 2}$ as evidenced by HMBC correlations. Dashed arrows indicate weak correlations. 

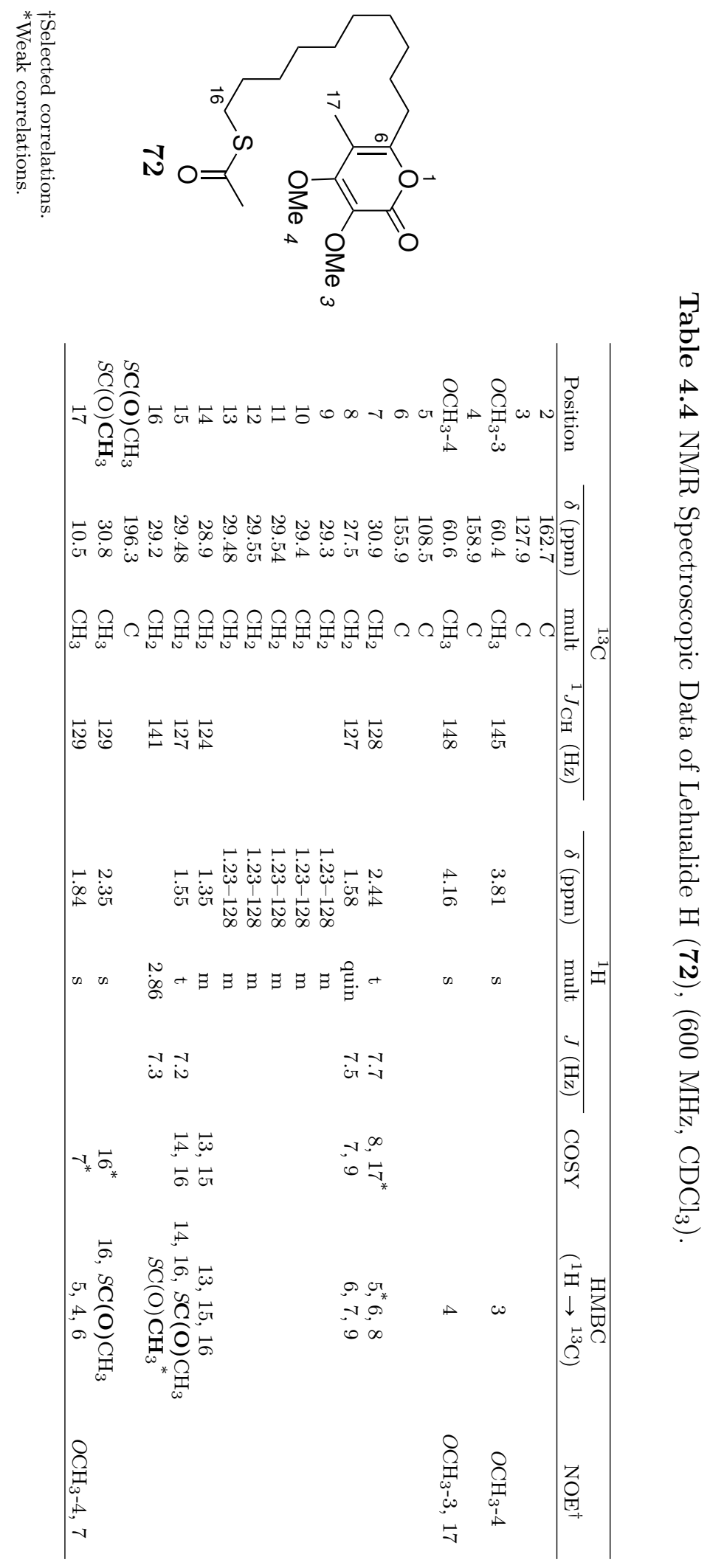


\section{Lehualides I and J (73 and 74)}

The sulfur functionalities in lehualides I and J presented challenges during structural elucidation of the compounds, beginning with establishing their respective molecular formulae. Interpretation of the positive-ion mode HRESIMS spectrum of lehualide $\mathrm{I}$ (73) led to a proposed molecular formula of $\mathrm{C}_{19} \mathrm{H}_{32} \mathrm{O}_{5} \mathrm{~S}\left(\mathrm{~m} / z 395.1867[\mathrm{M}+\mathrm{Na}]^{+}\right.$, $\left.m / z 379.1920[\mathrm{M}-16+\mathrm{Na}]^{+}\right)$. A pseudo-molecular ion peak correlating to the same formula was also observed in the HRESIMS spectrum of 74: $\mathrm{C}_{19} \mathrm{H}_{32} \mathrm{O}_{5} \mathrm{~S}$ $\left(m / z 395.1868[\mathrm{M}+\mathrm{Na}]^{+}\right)$(Figure 4.20). Relative peak heights of the $[\mathrm{M}+\mathrm{Na}]^{+}$ and $[\mathrm{M}-16+\mathrm{Na}]^{+}$signals in the spectrum of 73 varied slightly between injections, suggesting they may not be products of fragmentation. Following further interpretation of the HRESIMS and NMR spectra of both compounds, the formula of 73 was revised to $\mathrm{C}_{19} \mathrm{H}_{32} \mathrm{O}_{4} \mathrm{~S}\left(\mathrm{~m} / z 379.1920[\mathrm{M}+\mathrm{Na}]^{+}, 395.1867[\mathrm{M}+16+\mathrm{Na}]^{+}\right)$. This new formula contains one less oxygen atom than that of $\mathbf{7 4}$, and the strong $[\mathrm{M}+16+\mathrm{Na}]^{+}$peak was thought to represent an oxygen adduct formed in the positive-ion mode conditions.

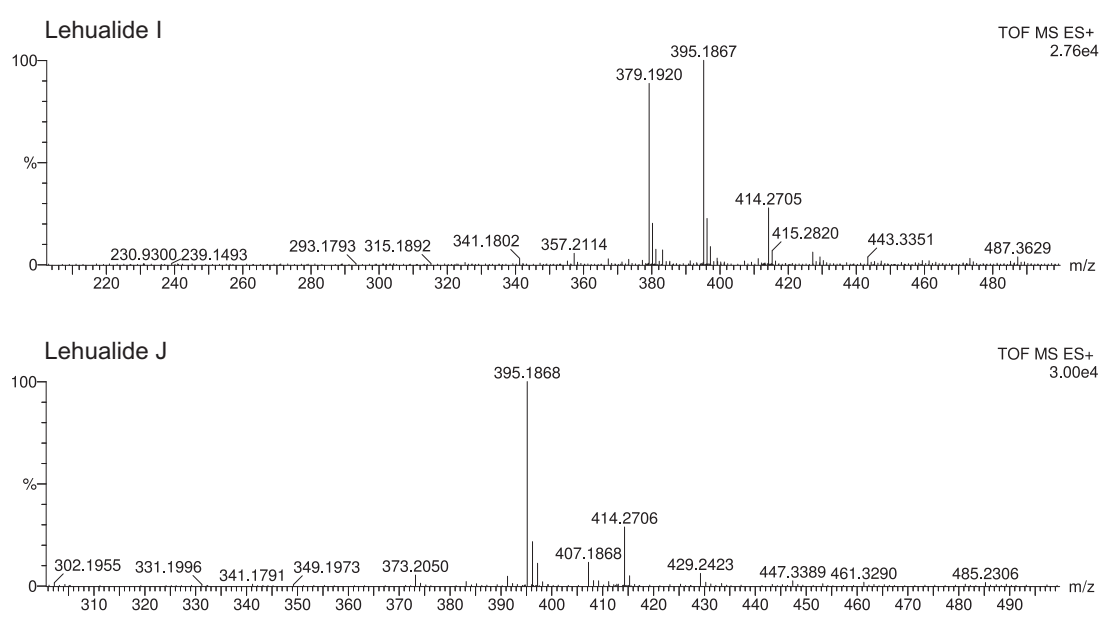

Figure 4.20 Positive-ion mode HRESIMS spectra of lehualides I and J. Both compounds appear to have the same molecular weight and corresponding chemical formula.

Analysis of the 1D and 2D NMR spectroscopic data of lehualide I (73), in conjunction with IR and UV spectra, confirmed the same C-6 alkyl-substituted $\alpha$-pyrone system present in $\mathbf{7 2}$. This accounted for the four degrees of unsaturation, and the four oxygen atoms required by the molecular formula. The alkyl chain was again elucidated by irradiation of methylenes $\mathrm{H}_{2}-16$ and $\mathrm{H}_{2}-7$ using a 1D TOCSY pulse with sequentially increasing mixing times. When acquired in $\mathrm{CDCl}_{3}$, the ${ }^{13} \mathrm{C}$ NMR spectrum was devoid of one methylene resonance, although no discernable differences in peak height or line width were observed between resonances in the region. Analysis of 73 in both $\mathrm{C}_{6} \mathrm{D}_{6}$ and $\mathrm{CD}_{3} \mathrm{OD}$ allowed resolution and observation of the missing methylene centre.

Similar to lehualide $\mathrm{H}(\mathbf{7 2})$, a methyl singlet $\left(\mathrm{CH}_{3}-17: \delta_{\mathrm{C}} 15.6, \delta_{\mathrm{H}} 2.09\right)$ was observed 
in the ${ }^{1} \mathrm{H}$ spectrum of lehualide $\mathrm{I}(\mathbf{7 3})$. Inspection of 2D NMR spectroscopic data revealed weak HMBC and COSY correlations between $\mathrm{CH}_{3}-17$ and the $\mathrm{CH}_{2}-16$ methylene $\left(\delta_{\mathrm{C}} 34.3, \delta_{\mathrm{H}} 2.49\right)$, indicating the presence of a heteroatom between them (Figure 4.21). The high ${ }^{1} J_{\mathrm{CH}}$ values and shielded chemical shifts of the aliphatic centres $\mathrm{CH}_{2}-16\left({ }^{1} J_{\mathrm{CH}} 137 \mathrm{~Hz}\right)$ and $\mathrm{CH}_{3}-17\left({ }^{1} J_{\mathrm{CH}} 137 \mathrm{~Hz}\right)$ indicated the linkage was a sulfur atom. ${ }^{137}$ This was corroborated by a weak C-S stretch present in the IR spectrum at $698 \mathrm{~cm}^{-1}$ indicative of a sulfide function.

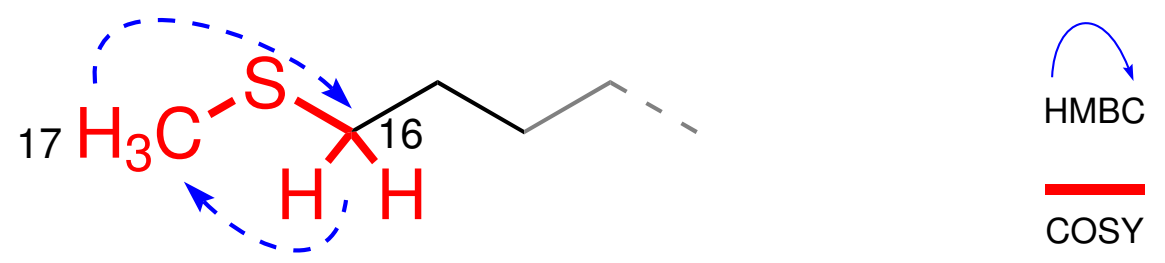

Figure 4.21 The methyl sulfide function of $\mathbf{7 3}$ as evidenced by COSY and HMBC correlations. Dashed arrows indicate weak correlations.

As shown in Figure 4.22, acquisition of the ${ }^{1} \mathrm{H}$ NMR spectrum of lehualide I (73) in $\mathrm{CDCl}_{3}$ resulted in broadening of the signals for the centres adjacent to the sulfur $\left(\mathrm{CH}_{3}-17\right.$ and $\left.\mathrm{CH}_{2}-16\right)$ after an hour in solution. The effect was initially attributed to acid-catalysed H-D exchange. Acidic $\alpha$-protons of dicarbonyl sulfone centres have been observed to undergo H-D exchange, ${ }^{139}$ although presence of the function was negated by the HRESIMS evidence. Dissolution of the compound in a polar solvents such as $\mathrm{CD}_{3} \mathrm{OD}$ alleviated the broadness, presumably due to hydrogenbonding between the solvent and the sulfur centre. The broadness observed in $\mathrm{CDCl}_{3}$ is therefore attributed to fluctuating intramolecular interactions between the sulfur and the pyrone core in the absence of intermolecular hydrogen-bonding.

The ${ }^{1} \mathrm{H}$ NMR spectrum of lehualide $\mathrm{J}(\mathbf{7 4})$ was very similar to that of lehualides $\mathrm{H}$ and $\mathrm{I}$ (72 and 73), the primary difference being the presence of a deshielded diastereotopic methylene $\left(\delta_{\mathrm{C}} 54.9, \delta_{\mathrm{H}}\right.$ a 2.73 , b 2.66), observed in the HSQC experiment. As with compounds $\mathbf{7 2}$ and 73, the C-6 alkyl-substituted $\alpha$-pyrone system was identified through detailed analysis of both 1D and 2D NMR data, corroborated by UV and IR spectroscopic data. In this case, the aliphatic chain extending from the pyrone terminates in the methylene centre $\mathrm{CH}_{2}-16\left(\delta_{\mathrm{C}} 54.9, \delta_{\mathrm{H}}\right.$ a 2.73 , b 2.66), the diastereotopic nature of which indicated close proximity to a chiral centre. A deshielded methyl singlet $\mathrm{CH}_{3}-17\left(\delta_{\mathrm{C}} 38.7, \delta_{\mathrm{H}} 2.56\right)$ was also observed at significantly higher chemical shift to that of 73. As depicted in Figure 4.23, the centres displayed weak reciprocal COSY and HMBC correlations, indicating the presence of a heteroatom between them, while the large ${ }^{1} J_{\mathrm{CH}}$ couplings associated with the centres $\left(\mathrm{CH}_{2}-16:{ }^{1} J_{\mathrm{CH}} \mathrm{Ha} 137 \mathrm{~Hz}, \mathrm{Hb} 135 \mathrm{~Hz} ; \mathrm{CH}_{3}-17:{ }^{1} J_{\mathrm{CH}} 137 \mathrm{~Hz}\right)$ in conjunction with their chemical shifts was again indicative of a bridging sulfur functionality. ${ }^{137}$ Incorporation of an oxygen-bearing stereogenic sulfoxide centre 
A
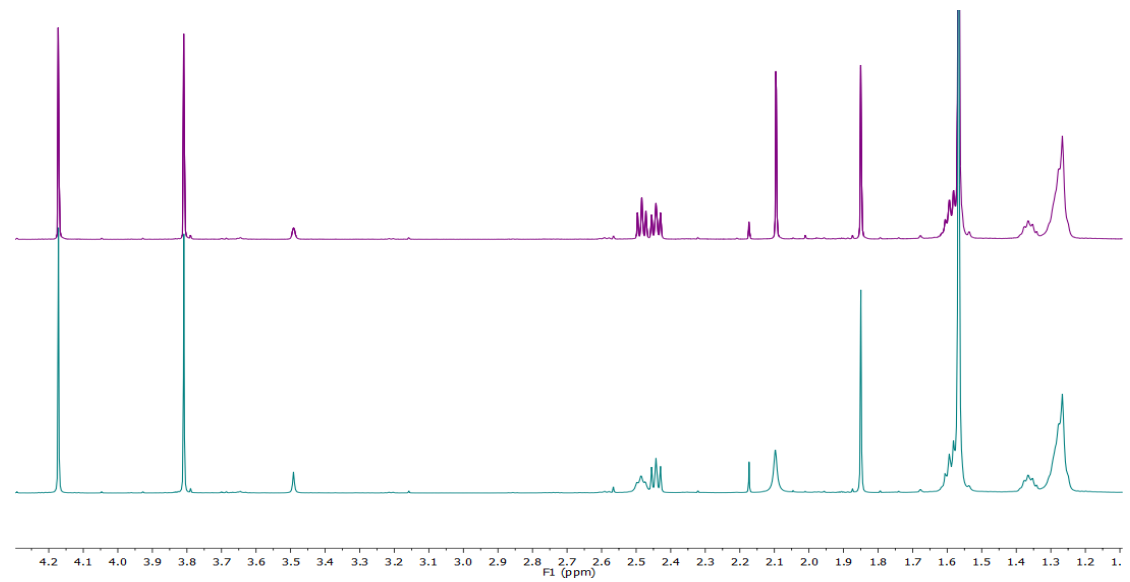

B

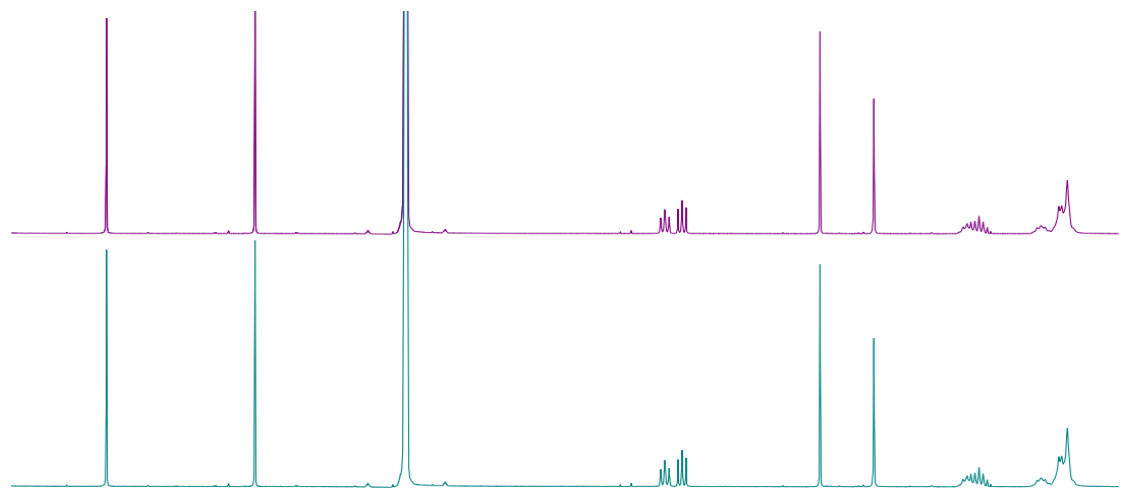

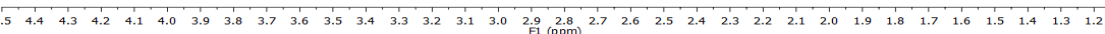

Figure 4.22 ${ }^{1} \mathrm{H}$ NMR spectrum of lehualide I (73) acquired in $\mathrm{A}: \mathrm{CDCl}_{3}$ and $\mathrm{B}: \mathrm{CD}_{3} \mathrm{OD}$. Broadening of the methylene and methyl signals $\left(\delta_{\mathrm{H}} 2.48,2.08 \mathrm{ppm}\right)$ adjacent to the sulfur centre is apparent after $60 \mathrm{~min}$ in $\mathrm{CDCl}_{3}$, but not observed in $\mathrm{CD}_{3} \mathrm{OD}$.

accounted for both the diastereotopic nature of $\mathrm{CH}_{2}-16$ and deshielded chemical shifts of the methylene and $\mathrm{CH}_{3}-17$, while incorporating the remaining oxygen indicated by the molecular formula. The IR stretch of the functionality was observed at $1027 \mathrm{~cm}^{-1}{ }^{140}$ It is unclear, however, whether the apparent lack of optical activity $\left([\alpha]_{D}^{19.1} 0.0^{\circ}\left(c 3.69 \times 10^{-3}, \mathrm{CHCl}_{3}\right)\right)$ is due to natural occurrence of lehualide $\mathrm{J}(\mathbf{7 4})$ as a racemic mixture or small sample size. NMR data for the structures of lehualides I and lehualide $\mathrm{J}$ (73 and $\mathbf{7 4}$ ) is presented in Tables 4.5 and 4.6.

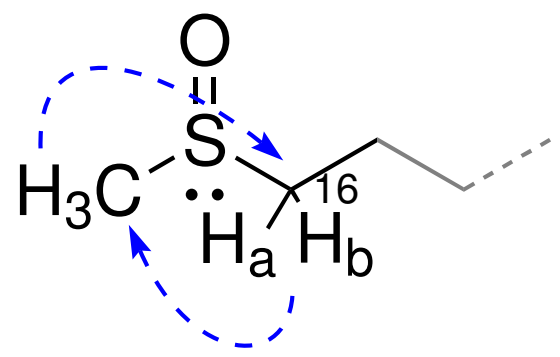

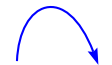

$\mathrm{HMBC}$

Figure 4.23 The stereogenic sulfoxide centre of $\mathbf{7 4}$ as evidenced by HMBC correlations. Dashed arrows indicate weak correlations. 


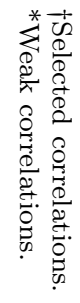

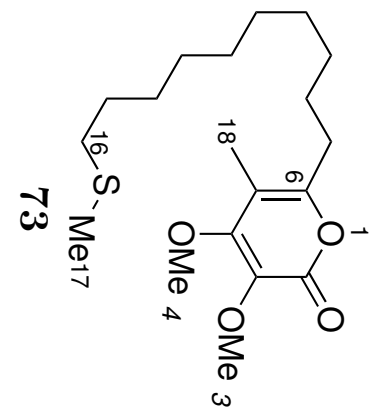

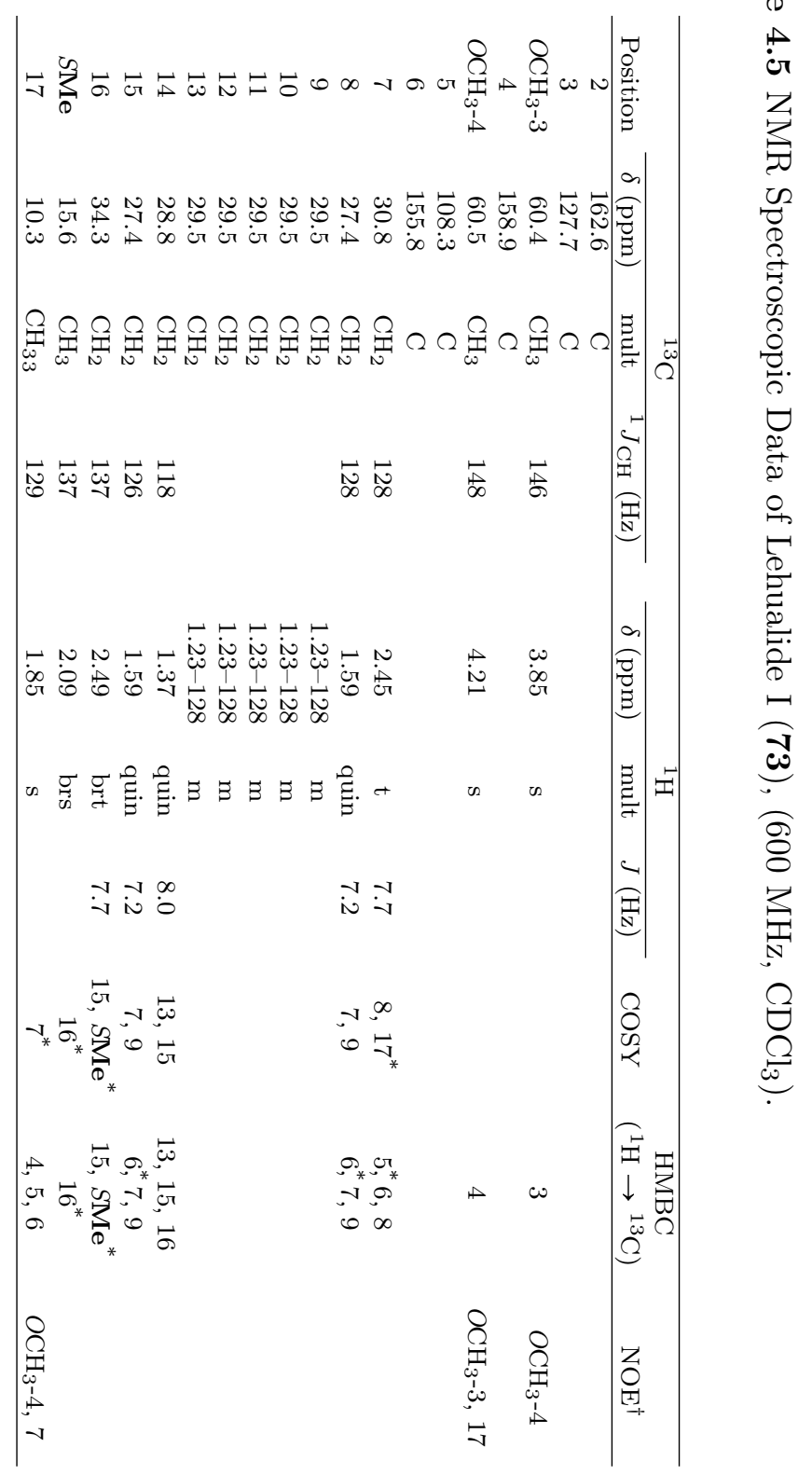



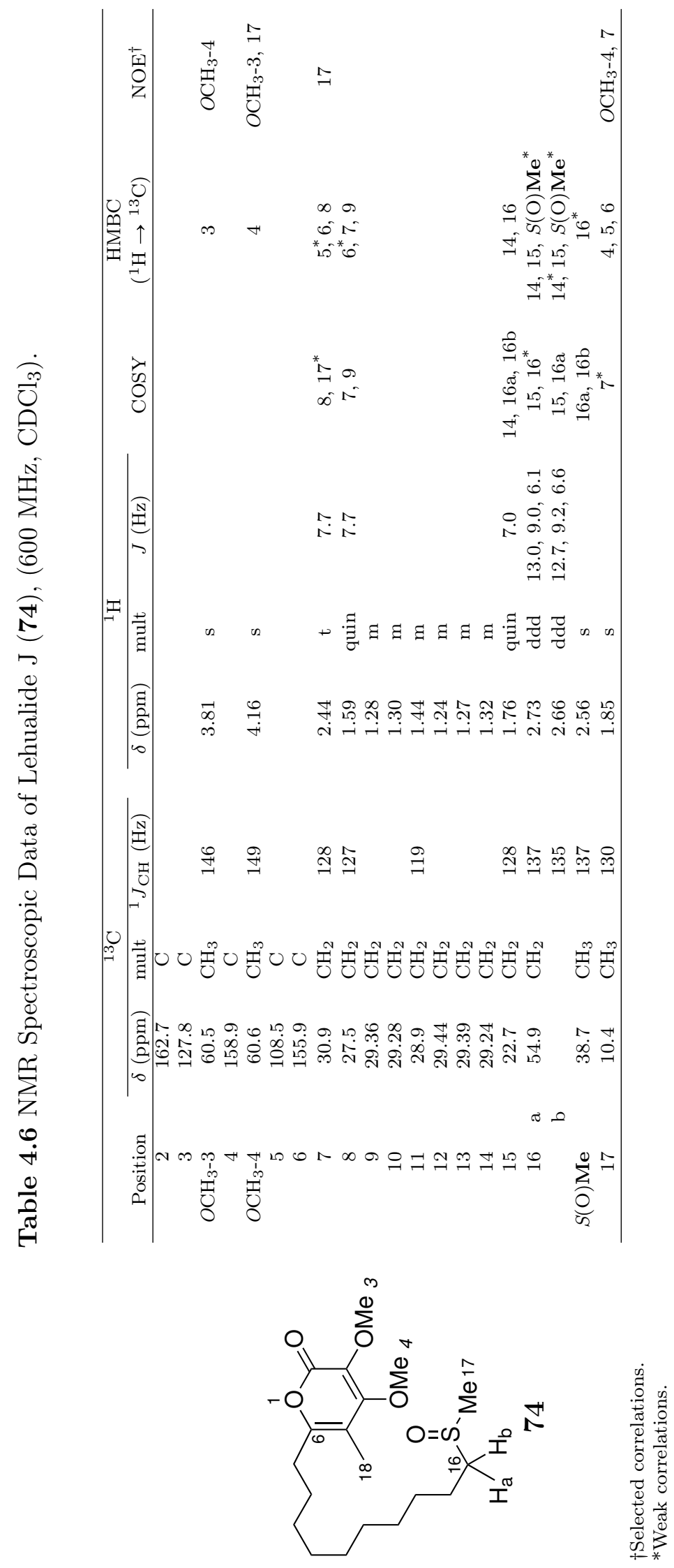
The final addition to the lehualide suite, K (75) was not isolated in any purity exceeding approximately 85\%. Persistent impurities of $\mathbf{7 0}$ and $\mathbf{7 2}$ remained within the sample despite multiple normal phase column chromatographic purifications, and attempted isolation by $\mathrm{C}_{18}$ and DIOL HPLC under various solvent conditions. The ${ }^{1} \mathrm{H}$ and ${ }^{13} \mathrm{C}$ NMR spectra of $\mathbf{7 5}$ are remarkably similar to those of $\mathbf{7 2}$, except the absence of the acetyl group. As is typical of the latter lehualides, compounds 72-74, the terminating methylene of the extended aliphatic chain, $\mathrm{CH}_{2}-16$ has a large ${ }^{1} J_{\mathrm{CH}}$ value $\left(\delta_{\mathrm{C}} 39.3, \delta_{\mathrm{H}} 2.67,{ }^{1} J_{\mathrm{CH}} 139 \mathrm{~Hz}\right)$ and shielded chemical shift, consistent with a terminal sulfur-containing functional group. Further analysis of both the 1D and 2D NMR spectra suggested 75 to be the thiol analogue of $\mathbf{7 2}$. The signal attributed to the $\mathrm{S}-\mathrm{H}$ proton diminished during the purification process, however, while the positive-ion mode HRESIMS spectrum showed a $[\mathrm{M}+\mathrm{Na}]^{+}$ pseudo-molecular ion peak at $m / z$ 705.3477. This mass was consistent with the molecular formula $\mathrm{C}_{36} \mathrm{H}_{58} \mathrm{O}_{8} \mathrm{~S}_{2}$, indicating formation of a disulfide dimer. In light of the isolation of the thiol lehualide D (68), dimerisation of 75 during the isolation process is suspected. NMR data for the disulfide $\mathbf{7 5}$ is presented in Table 4.8.

\subsubsection{Bioactivity of the lehualides}

Lehualides F-I (70-73) displayed mild growth inhibition (60-70\%) of a drugsensitive Saccharomyces cerevisae strain at $100 \mu \mathrm{mol} \mathrm{L} \mathrm{L}^{-1}$, and mild cytotoxicity against the human promyelocytic leukemia (HL-60) cell line (Table 4.7). Unfortunately, the limited quantities of material available for assessment combined with compound instability prevented further biological investigation of the lehualides.

Table 4.7 Inhibition of HL-60 Cells by Lehualides F-I (70-73).

\begin{tabular}{cc}
\hline Compound & $\mathrm{IC}_{50}\left(\mu \mathrm{mol} \mathrm{L}^{-1}\right)$ \\
\hline \hline $\mathbf{7 0}$ & 6.2 \\
$\mathbf{7 1}$ & 5.4 \\
$\mathbf{7 2}$ & 14.6 \\
$\mathbf{7 3}$ & 10.8 \\
\hline
\end{tabular}




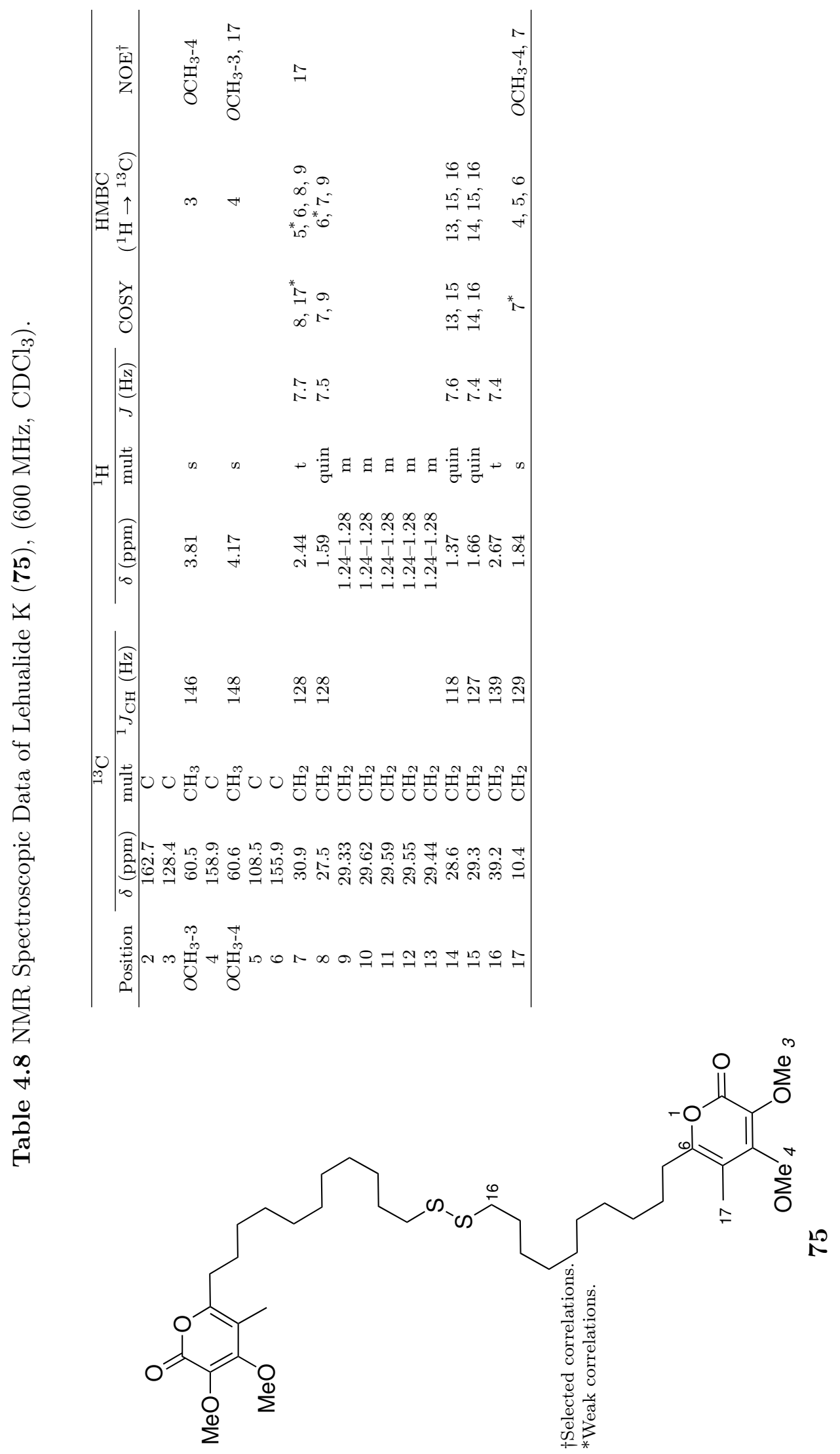




\subsubsection{Determination of sulfur functionalities}

The NMR-silent nature of the ${ }^{32} \mathrm{~S}$ nuclei present in lehualides $\mathrm{H}-\mathrm{K}(\mathbf{7 2}-\mathbf{7 5})$ presented particular difficulties during characterisation of the compounds via NMR. A suite of sulfur functional groups were prepared in order to confirm the structures of $\mathbf{7 2 - 7 4}$ through comparison of spectroscopic data (Scheme 4.2).

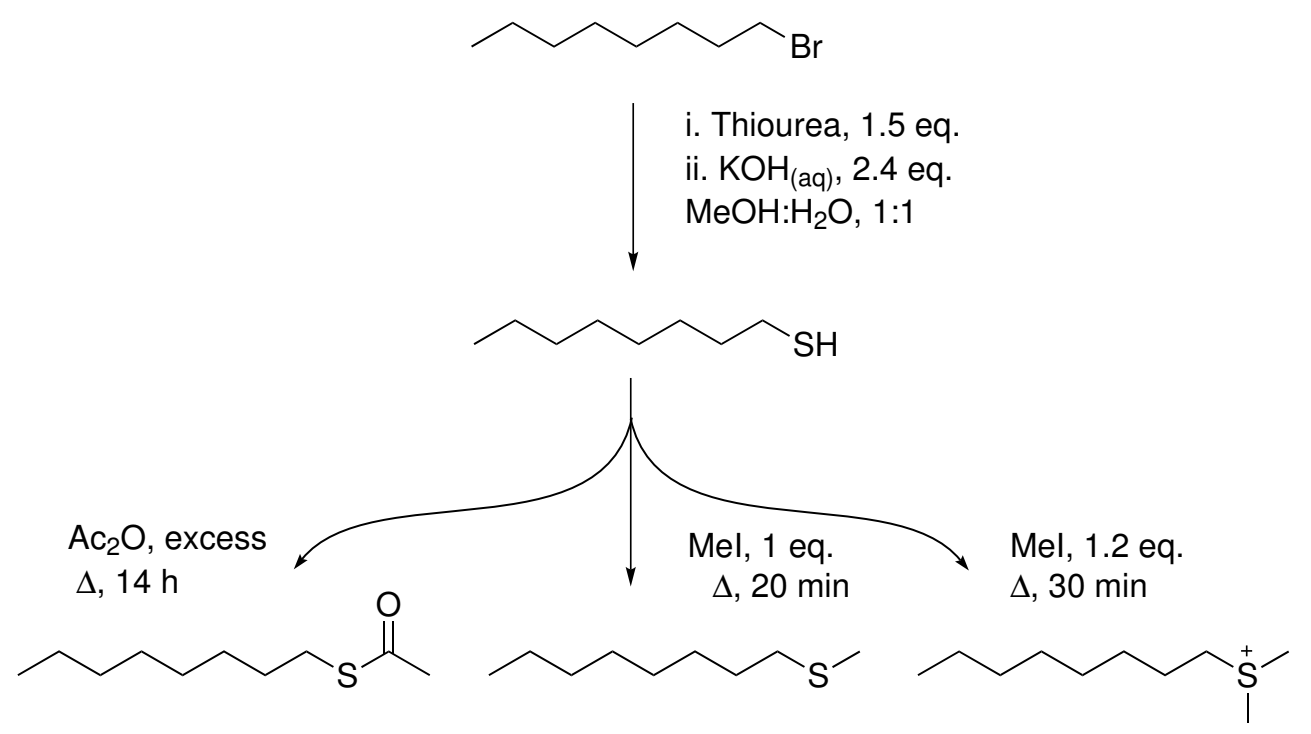

Octanethioacetate, $80 \% \quad$ Methyloctylsulfide, $40 \% \quad$ di-methyloctylsulfide, $90 \%$

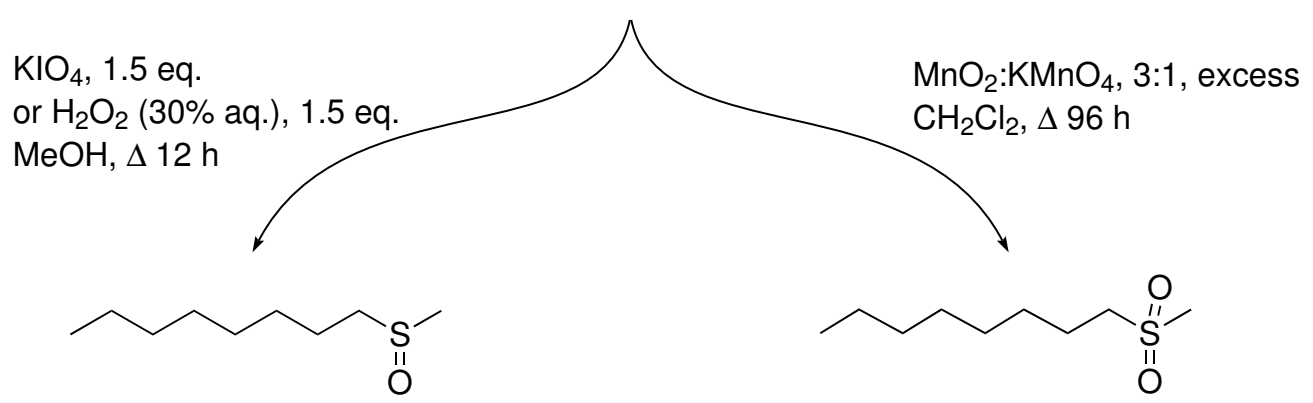

Methyloctylsulfoxide, $100 \%$

Methyloctylsulfone, $100 \%$

Scheme 4.2 Synthesis of the spectroscopic model compounds of lehualides H-J: octanethioacetate, methyloctylsulfide and methyloctylsulfoxide.

Octanethiol was prepared and derivatised to yield octanethioacetate, ${ }^{141}$ the monoand di-methylsulfide products and the corresponding sulfoxide and sulfone species. The thioacetate proved a spectroscopic match for the aliphatic portion of lehualide $\mathrm{H}(\mathbf{7 2})$, supporting the proposal of $\mathbf{7 2}$ as its final structure. Methyloctylsulfide was found to have identical spectroscopic characteristics to the aliphatic portion of the natural product 73 , confirming the methylsulfide substructure of lehualide I and its final structure as 73. Stoichiometric oxidation of methyloctyl sulfide with both $\mathrm{H}_{2} \mathrm{O}_{2}$ and $\mathrm{KIO}_{4}$ provided the sulfoxide product; ${ }^{142,143}$ a spectroscopic match for the aliphatic portion of the metabolite $\mathbf{7 4}$ in chemical shift and ${ }^{1} J_{\mathrm{CH}}$ coupling constants, confirming the structure of lehualide $\mathrm{J}$ as $\mathbf{7 4}$ (Figures 4.24-4.26). 


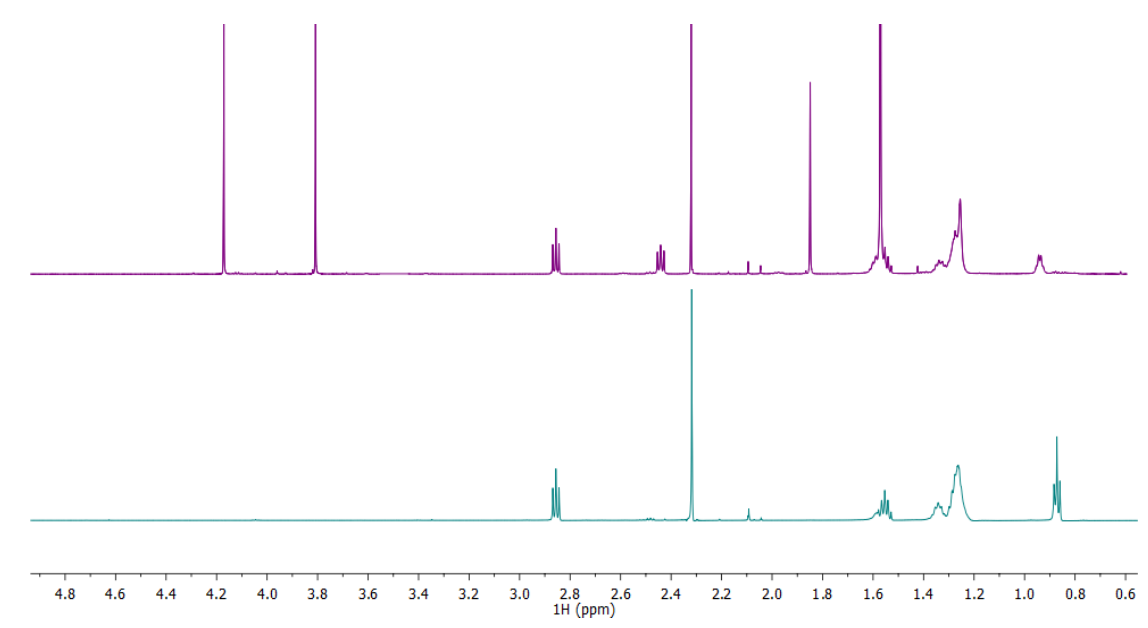

Figure $4.24{ }^{1} \mathrm{H}$ NMR spectral comparison of lehualide $\mathrm{H}(\mathbf{7 2})$ and octanethioacetate. The methylene $\left(\delta_{\mathrm{H}} 2.86 \mathrm{ppm}\right)$ and methyl $\left(\delta_{\mathrm{H}} 1.84 \mathrm{ppm}\right)$ centres adjacent to the sulfur moiety share chemical shifts.

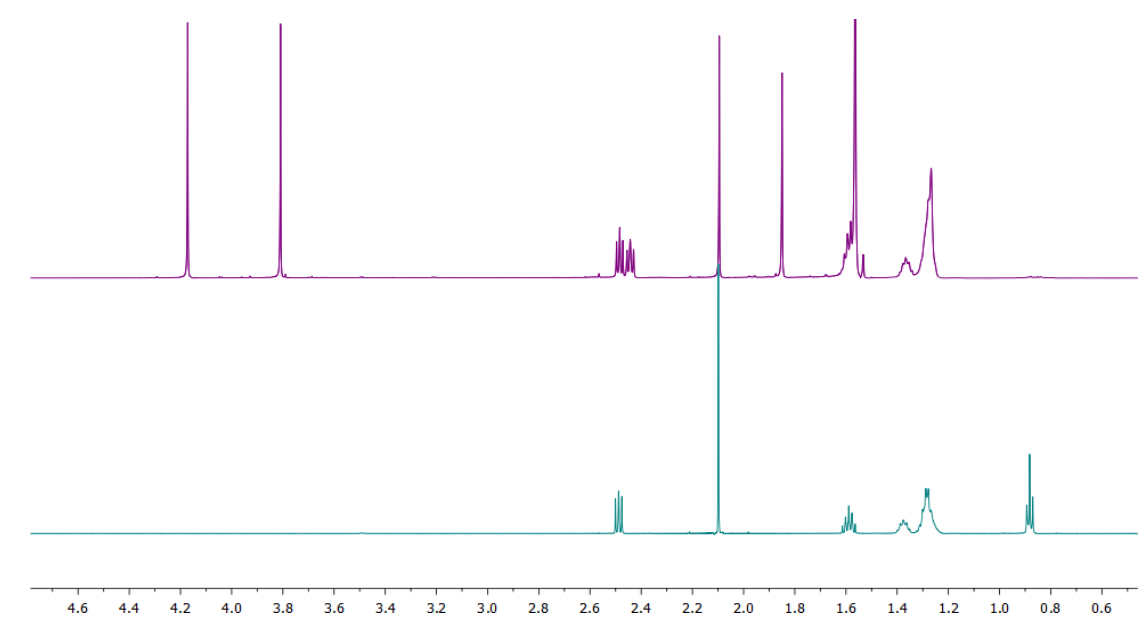

Figure 4.25 ${ }^{1} \mathrm{H}$ NMR spectral comparison of lehualide I (73) and methyloctylsulfide. The methylene $\left(\delta_{\mathrm{H}} 2.49 \mathrm{ppm}\right)$ and methyl $\left(\delta_{\mathrm{H}} 2.09 \mathrm{ppm}\right)$ centres adjacent to the sulfur nucleus share chemical shifts.

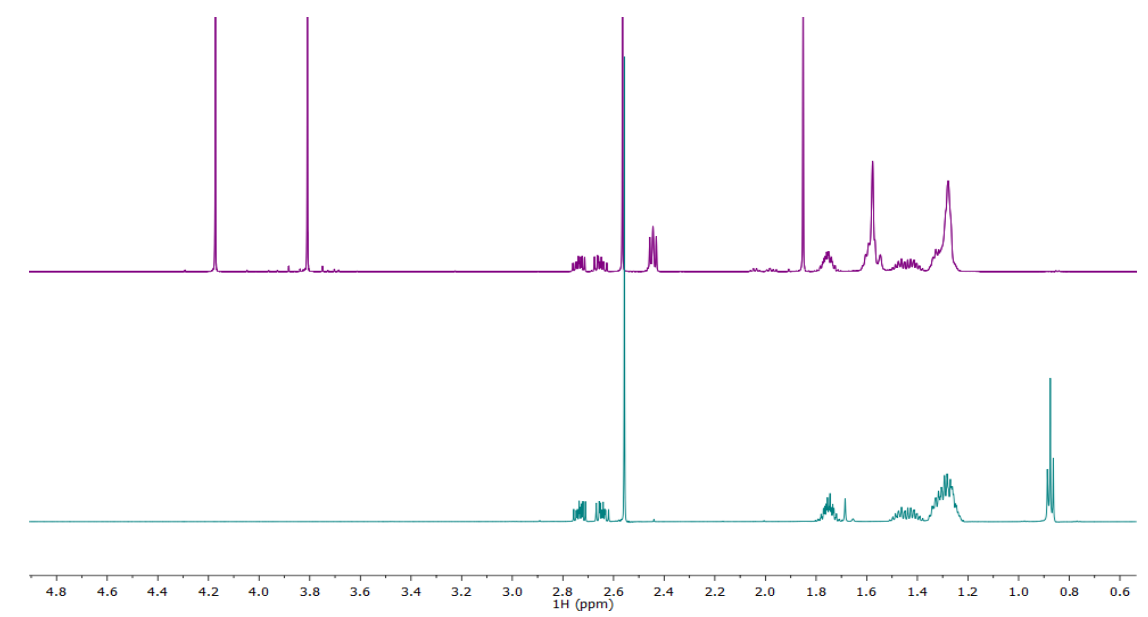

Figure 4.26 ${ }^{1} \mathrm{H}$ NMR spectral comparison of lehualide $J(\mathbf{7 4})$ and methyloctylsulfoxide. The methylene $\left(\delta_{\mathrm{H}} 2.73,2.66 \mathrm{ppm}\right)$ and methyl $\left(\delta_{\mathrm{H}} 2.56 \mathrm{ppm}\right)$ centres adjacent to the sulfoxide centre share chemical shifts.

For further spectroscopic comparison with the natural products dimethyloctylsulfide and methyloctylsulfone were prepared via established methods, ${ }^{144}$ and were found 
to display significant spectroscopic differences (see Table 4.9). Analysis by TLC of the three model compounds and the natural products gave corroborating evidence for the nature of the sulfur moieties.

\section{NMR detection of sulfur functionalities}

Direct bonding to sulfur has little effect on the chemical shifts of spin-active carbon and hydrogen nuclei, as the three elements have similar relative electronegativities. ${ }^{145}$ In the absence of a deshielding effect upon chemical shifts, analysis of the ${ }^{1} J_{\mathrm{CH}}$ coupling of adjacent $\mathrm{C}-\mathrm{H}$ bonds is the best way to analyse sulfur in an NMR spectrum. Orientation of the sulfur lone pairs increases the ${ }^{1} J_{\mathrm{CH}}$ values of adjacent $\mathrm{C}-\mathrm{H}$ bonds, as interaction with the filled $p$-orbitals decreases the $s$ character of the $\mathrm{C}-\mathrm{H}$ bonds. Interaction with $\pi$-systems has a similar effect. ${ }^{137,145}$ ${ }^{1} J_{\mathrm{CH}}$ values can be obtained from fully-coupled HSQC spectra (and strong HMBC data), but as it is common practice to conduct decoupled HSQC experiments, there are few literature references. Bonding to oxygen similarly affects ${ }^{1} J_{\mathrm{CH}}$ values, but the electronegativity of the nucleus also significantly deshields adjacent nuclei, facilitating indirect observation of the nucleus through the changes in chemical shift. Only when the sulfur itself is bonded to a more electronegative element are chemical shifts significantly affected. As observed in 73 and its spectroscopic model, the sulfur lone-pair can act as a fourth substituent, thereby forming a chiral centre and inducing diastereotopic splitting of adjacent methylenes. The deshielding oxygen substituent induces downfield shifts of ca. $0.5 \mathrm{ppm}$ in the ${ }^{1} \mathrm{H}$ spectrum, while dioxysubstituted sulfones can deshield chemical shifts by ca. $1 \mathrm{ppm}$, relative to aliphatic chemical shifts. ${ }^{146}$ Formation of the oxygen-adduct of $\mathbf{7 3}$ during HRESIMS analysis illustrates the potential danger of relying solely upon MS data for confirmation of molecular formula(e). In the absence of isolation of $\mathbf{7 4}$, the sulfide analogue $\mathbf{7 3}$ may have been mischaracterised and falsely reported.

\section{Sulfur functionalities in the marine environment}

While the sulfur functionalities of lehualides $\mathrm{H}$ and $\mathrm{K}$ (72 and 75) have been previously encountered in lehualides $\mathrm{C}$ and D (67 and 68), the methyl sulfide and sulfoxide functionalities present in lehualides I and J (73 and 74) appear to be unprecedented in metabolites from sponges of this genus. Sulfoxides and sulfides are reported rarely from marine organisms, although there have been numerous examples reported from terrestrial plants including methionine and cysteine sulfoxides from brassicaceous and alliaceous vegetables, ${ }^{140,147}$ and trisulfides from the roots of some angiosperms. ${ }^{148}$ Examples from sponge literature include 


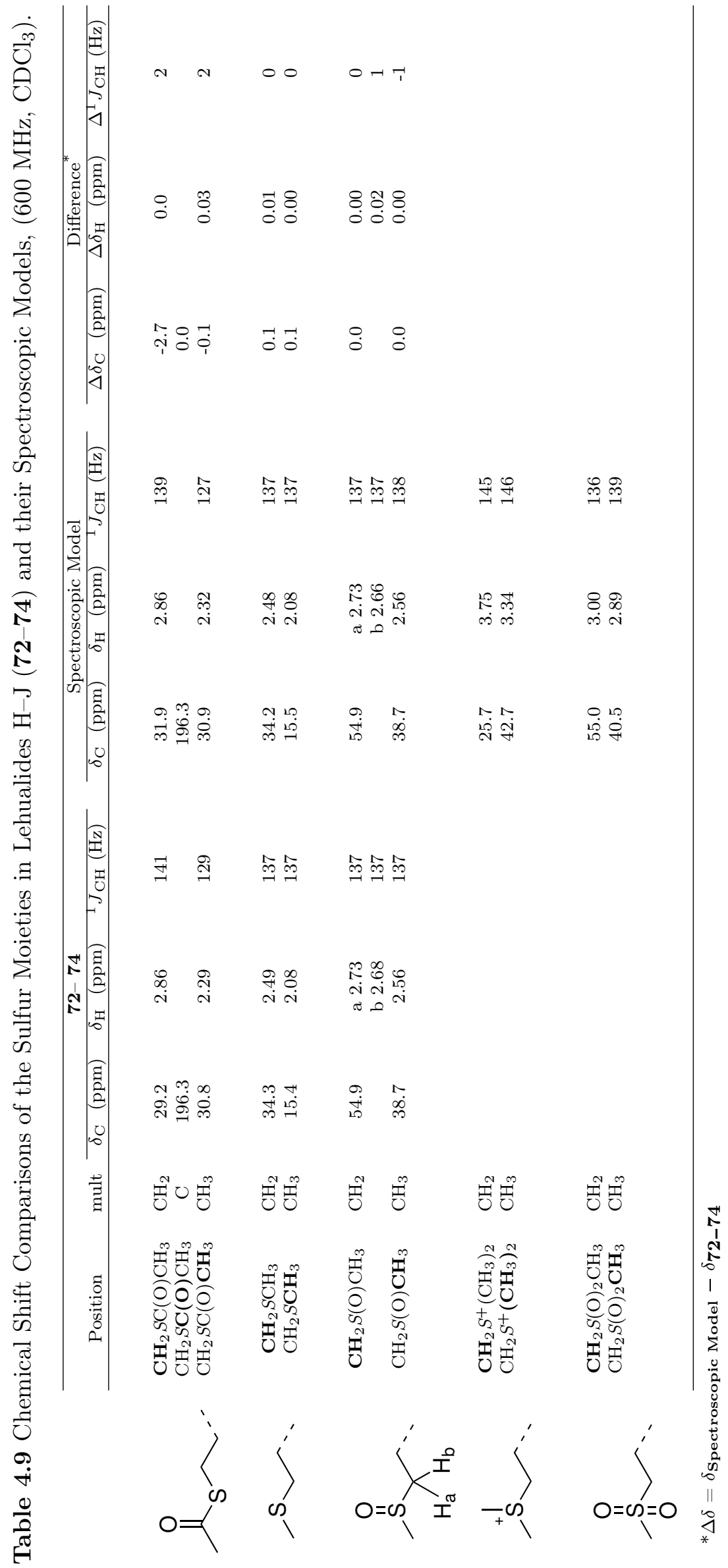


psammaplin N (76) ${ }^{149}$ eudistomin $\mathrm{K}(\mathbf{7 7}),{ }^{150}$ didemnolines A-D (78-81), ${ }^{138}$ and the sulfide benzylthiocrellidone (82) from Crella spinulata. ${ }^{151}$<smiles>CS(=O)CCNC(=O)/C(Cc1ccc(O)c(Br)c1)=N/O</smiles><smiles>NC(CS(=O)CO)C1NCCc2c1[nH]c1cc(Br)ccc21</smiles>

77

76

Formation of disulfide species, as is suspected in the case of $\mathbf{7 5}$, has also been encountered in the marine environment. Psammaplin A (83), isolated from a Tongan specimen of Psammaplysilla sp. ${ }^{152}$ was the first example of a disulfide species originating from a marine sponge. The isolation of lehualides $\mathrm{E}-\mathrm{K}$ (6975), particularly in light of the sulfur functionalities present in the latter members of the class, adds to the diversity of chemical functionality obtained from Plakortis sponges.<smiles>[X]c1ccc2c3ccncc3n(Cc3nc[nH]c3SC)c2c1</smiles>

$$
\mathrm{X}=\mathrm{Br} \quad 78
$$$$
\mathrm{X}=\mathrm{H} \quad 79
$$<smiles>[X]c1ccc2c3ccncc3n(Cc3nc[nH]c3S(C)=O)c2c1</smiles>

$\mathrm{X}=\mathrm{Br} \quad 80$

$\mathrm{X}=\mathrm{H} \quad 81$<smiles>CC1(C)CC(=O)C(=C(SCc2ccccc2)C2=C(O)CC(C)(C)CC2=O)C(=O)C1</smiles>

82<smiles>O=C(NCCSSCCNC(=O)/C(Cc1ccc(O)c(Br)c1)=N/O)/C(Cc1ccc(O)c(Br)c1)=N\O</smiles>

83

\subsection{4 $\alpha$ - and $\gamma$-Pyrone metabolites from marine invertebrates}

Lehualides $\mathrm{A}-\mathrm{K}$ are the stand-alone pyrone metabolites from Plakinidae sponges, although the heterocycle function has been encountered within a variety of sponge metabolites and cultures of microbial sponge symbiotes. Examples include nocapyrone A (84), discovered within cultures of the Nocardiopsis strain HB383, 
isolated from a Halichondria panacea specimen. Successful ex-host culturing also allowed biosynthetic characterisation of the $\gamma$-pyrones via feeding experiments with ${ }^{13} \mathrm{C}$-labeled acetate, propionate, and methionine. Feeding of $1{ }^{13} \mathrm{C}$-propionate led to an enhancement of the ${ }^{13} \mathrm{C}$ NMR signals of $\mathrm{C}-1$ and $\mathrm{C}-3$, while $1-{ }^{13} \mathrm{C}$-acetate enhanced the carbon resonances of C-5 and C-7. The signal of C-15 was enhanced after feeding with $S \mathrm{Me}^{13} \mathrm{C}$-methionine, indicating that the $\mathrm{C}-1$ methoxy substituent is derived from $S$-adenosylmethionine (SAM). ${ }^{153}$ These observations led Schneeman and co-workers to propose the initial formation of an $\alpha$-pyrone, which tautomerises into the $\gamma$-pyrone in the course of methylation by an $O$-methyltransferase. ${ }^{153}$

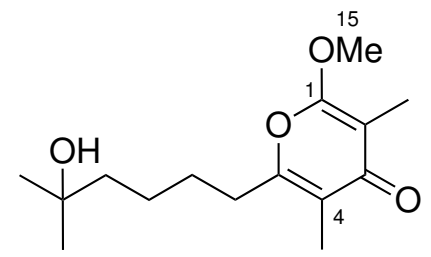

84

Marine fungi have produced many pyrone metabolites, such as the phenethyl$\alpha$-pyrone derivatives from a marine Aspergillus niger strain (EN-13). ${ }^{154}$ The endophytic fungus was isolated from the inner tissue of the brown algae Colpomenia sinuosa, cultured ex vivo to yield four pyrone metabolites, and three cyclodipeptides. ${ }^{154}$ Similar ex vivo culturing of another endophytic A. niger strain (MA132), isolated from the marine mangrove plant Avicennia marina, afforded the $\alpha$-pyrone metabolites nigerapyrones A-H. ${ }^{155}$ Similar to lehualides A and B, the nigerapyrones have tails with varied regiochemistry about the methyl-substituted double bonds. The structures of nigerapyrones $\mathrm{F}-\mathrm{H}(\mathbf{8 5}-\mathbf{8 7})$ terminate with a phenyl function. ${ }^{155}$

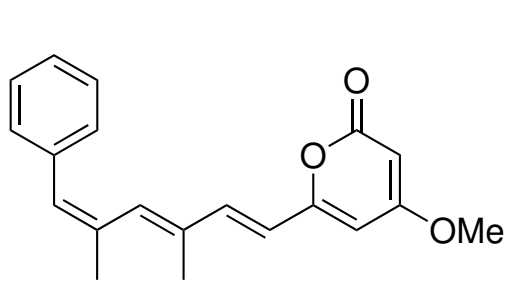

85

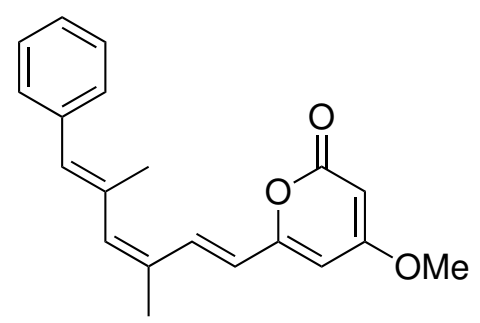

86<smiles>COc1cc(/C=C\C(C)=C/C(C)=C/c2ccccc2)oc(=O)c1</smiles>

87

The new $\alpha$-pyrone nigrosporapyrone (88) was isolated from cultured Nigrospora sp. fungus (PSU-F5) harvested from an Annella sp. sea fan. ${ }^{156}$ The fungus Microsphaeropsis sp. was isolated from the Mediterranean sponge Aplysina aerophoba and subsequent investigation yielded the $\gamma$-pyrones microsphaerones $\mathrm{A}$ (89) and B. ${ }^{157}$ Investigation of other marine invertebrates has also yielded examples of this heterocycle. Isolated from the Mediterranean mollusc Placida dendritica 
(order Sacoglossa), placidenes C-F (90-93) exhibit $\alpha$-pyrone groups, differing from plakinid polyketides in that they comprise entirely of propionate groups. ${ }^{158}$

More akin to the lehualides, recent isolates from another mollusc, Aplysiopsis formosa, the aplysiopsenes have a mixed acetate/propionate pathway. ${ }^{159}$ Polypropionate skeletons are frequently uncovered within marine gastropods: Chilean specimens of Siphonaria lessoni yielded the $\alpha$-pyrone norpectinatone in addition to acyclic congeners, ${ }^{160}$ while collections of Siphonaria diemenensis from Sydney Harbour, Australia yielded the $\alpha$-pyrone diemenensin A (94). ${ }^{161}$ Like lehualides A and E-K, 94 bears a C-4 hydroxyl and an olefinic methyl at C-5, yet differs in methylation in place of oxygen substitution at C-3.<smiles>COc1cc(=O)oc(C(C)O)c1C</smiles>

88<smiles>C[C@H](CC(=O)NC(=O)c1coc(/C=C/C=C/CCCCCCC(=O)O)cc1=O)C(=O)O</smiles>

89<smiles>[R]CC(C)/C=C/c1oc(=O)cc(OC)c1C</smiles>

$90 \mathrm{R}=\mathrm{CH}_{3}$

$91 \mathrm{R}=\mathrm{CH}_{2} \mathrm{CH}_{3}$<smiles>C/C=C(/C)C=C(C)c1oc(=O)cc(OC)c1C</smiles>

$92 \mathrm{R}=\mathrm{H}$

$93 \mathrm{R}=\mathrm{Me} \Delta_{7,8} \Delta_{9,10}$<smiles>CCCC(C)CC(C)/C=C(C)/C=C(\C)c1oc(=O)c(C)c(O)c1C</smiles>

94

Oxidative degradation of $\alpha$-pyrone tautomers is frequently encountered, particularly in C-3 carbon-substituted systems. Kong et al. observed transformation of 3,6alkyl-substituted 4-hydroxy- $\alpha$-pyrones into 3-furanone substrates following storage of the metabolites in $\mathrm{CDCl}_{3}$ at ambient temperatures for 11 days. ${ }^{162,163}$ Scheme 4.3 depicts their proposed mechanism, which entails $\mathrm{C}-3$ oxidation by $\mathrm{O}_{2}$, followed by hydrolysis of the lactone, decarboxylation of the C-2 carboxylate and intramolecular cyclisation. Popplewell et al. proposed a similar degradation pathway for similarly substituted oxylipin pyrone substrates from a New Zealand red macroalgae Phacelocarpus labillardieri. ${ }^{66}$ To circumvent such degradation lehualides E-K were 
stored frozen in benzene/ethanol solutions, a more practical storage method than some methods previously employed. ${ }^{66}$

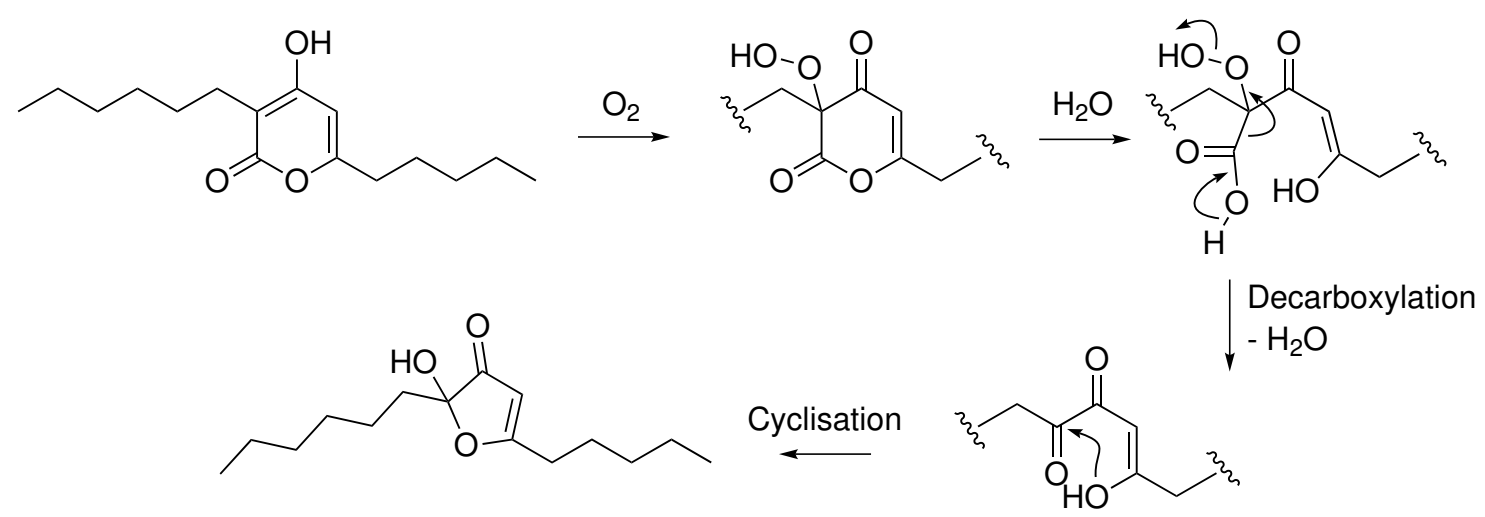

Scheme 4.3 Mechanism for degradation of pseudopyrone B into furanone 3 as proposed by Kong et al. ${ }^{162}$

\subsubsection{Biogenesis of the lehualides}

The polyketide derivation of the lehualides is apparent; the skeletons primarily comprise fully reduced acetate monomers, with little post-translational modification required to introduce chemical functionalities such as the C-2 methoxy groups. The terminal phenyl group of 65, 66 and 69-71 involves a phenylacetate chain starter unit. Incorporation of propionate monomers into the predominantly acetate-based chain introduces the C-5 methyl substituent of the $\alpha$-pyrone ring, and the trisubstituted $\Delta_{8,9}$ alkene of lehualides $\mathrm{E}-\mathrm{G}$ (69-71). Installation of the $\alpha$-pyrone occurs during cleavage of the final module from the ACP domain.

Occurrence of the four different sulfur functionalities in lehualides $\mathrm{H}-\mathrm{J}$ pose interesting biosynthetic questions. While synthetic introduction of C-S bonds often involves (facile) attack of an electrophilic carbon by a sulfur nucleophile, biological formation of $\mathrm{C}-\mathrm{S}$ bonds is more complex. Multiple sulfur cofactors have been identified in biological systems, these include coenzymes A and M, SAM, thiamine pyrophosphate, biotin, molybdopterin, lipoic acid and $\mathrm{N}$-(7mercaptoheptanoyl)threonine phosphate. ${ }^{164}$ Introduction of thiol functionalities to the lehualide polyketide skeleton is most probably analogous to the conversion of L-cysteine and sulfoactaldehyde into coenzyme M. As depicted in Scheme 4.4, sulfoactaldehyde is attacked by the cysteine $\mathrm{SH}$ nucleophile forming a thiazolidine adduct. The new C-N bond undergoes reductive ring-opening generating S(sulfoethyl)-L-cysteine. Pyridoxyl-P assists $\beta$-elimination in the 2-aminoacrylic acid portion generating coenzyme $\mathrm{M}$ and ammoniumpyruvate. ${ }^{165}$

The uneven number of carbons in the backbones of lehualides $\mathrm{H}-\mathrm{J}$ indicates chain-truncation within the structures, similar to that observed by Yong et al.. ${ }^{166}$ 
<smiles>NC(CS)C(=O)O</smiles><smiles>COSCC1NC(C(=O)OC)CS1</smiles>

Cysteine Sulfoacetaldehyde<smiles>CC(=O)C(=O)[O-]</smiles>

Coenzyme M Ammoniumpyruvate

Scheme 4.4 Thiolation of sulfoactaldehyde by L-cysteine to form coenzyme M.

The lehualides may share a common phenylacetate starter unit, with incomplete reduction of an adjacent acetate group allowing for oxidative cleavage of the alkene producing an aldehyde function. A similar reaction to that above between L-cysteine and the new aldehyde functionality in the polyketide can be envisaged generating a monomeric form of lehualide K (75). As would be the case in organic synthesis, the thioacetate of lehualide $\mathrm{H}$ (72) could easily form via nucleophilic attack of an electrophilic centre such as ethanoic acid by the thiol function of the polyketide product. Similarly, enzyme-mediated methyl transfer to the thiol group would give rise to the sulfide lehualide I (73). Oxidation of the methylsulfide group forming the sulfoxide functionality present in lehualide $\mathrm{J}(\mathbf{7 4})$, and hypothetically a sulfone moiety, could proceed by a host of enzymatic processes. Synthetic experimentation with the heme enzyme chloroperoxidase of Caldariomyces fumago and the vanadiumdependant bromoperoxidase of Ascophyllum nodosum has proven the applicability of the enzymes to a range of synthetic substrates and such enzymes could play a role in this biosynthesis. ${ }^{167}$ 

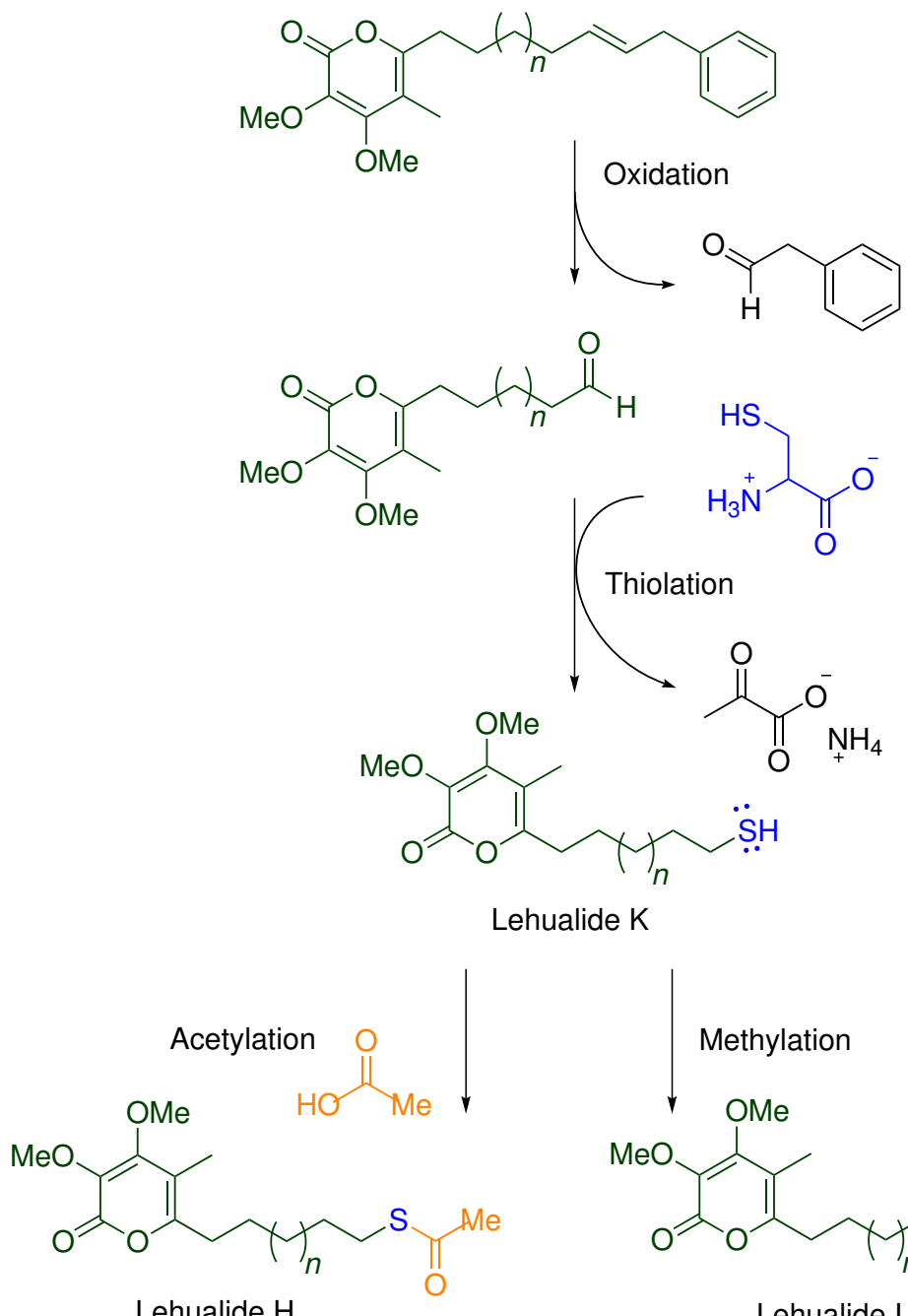

Lehualide $\mathrm{H}$

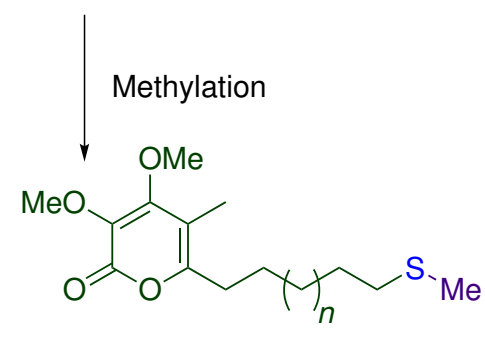

Lehualide I
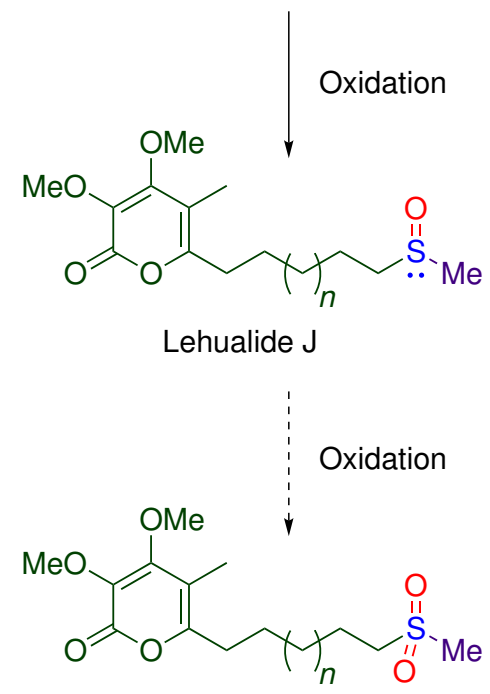

Scheme 4.5 Proposed biogenesis for lehualides $\mathrm{H}-\mathrm{K}(\mathbf{7 2}-\mathbf{7 5}), n=6$. 


\subsubsection{Concluding remarks}

Analysis of the HMBC spectra of screening fractions from the Plakortis specimen PTN3_20A was of great import identifying the potential for structural novelty within the extracts and selection of this sponge for further evaluation. Further use of the HMBC NMR spectrum in the early stages of purification led to determination of the non-protonated $\alpha$-pyrone substructure, further demonstration of the value in analysing HMBC spectra early on in the isolation process.

The isolation of lehualides $\mathrm{E}-\mathrm{K}$ has added to chemical knowledge pertaining to spectral characteristics of substituted pyrone systems. Structural elucidation of the fully-substituted $\alpha$-pyrone system of lehualides $\mathrm{E}-\mathrm{K}$ (69-75) presented a challenge. Similarly unprotonated systems have previously been proposed on the basis of chemical shifts alone, however, the substitution pattern here allowed for the indisputable establishment of the $\alpha$-pyrone substructure.

The suite of sulfur functional groups isolated during this study are of interest biosynthetically and from a characterisation perspective. Unequivocal identification of the moieties containing the NMR-silent ${ }^{32} \mathrm{~S}$ nucleus was provided by generating model compounds for comparison of their ${ }^{1} \mathrm{H}$ and ${ }^{13} \mathrm{C}$ NMR spectra. Although in agreement with the proposed structures, this experience highlights the necessity of structural determination via some means other than NMR spectroscopic analysis.

Finally, as encountered throughout this work, investigation of metabolites from infrequently identified organisms is double-edged. The benefits of expedient isolation of new metabolites are countered by the realities of isolation, especially when the metabolites in question are potentially structurally unstable. This also impacted on the quantities of isolated material available for biological assessment. 


\subsection{Cyclic peroxides from a plakinid sponge}

Identified as a species of Plakinastrella or Plakortis, the sponge specimen PTN3_19D (Figure 2.9), was collected at a depth of 12-15 $\mathrm{m}$ from the horizontal ceiling of a large cave on 'Eua Island in November 2008. The specimen formed thick, dense encrustations $0.5-1.5 \mathrm{~cm}$ thick, appearing smooth but with a rough reticulated surface covered in wide shallow nodules ca. $3 \mathrm{~mm}$ high. The black-grey exterior contrasted against the tan-pink interior, which turned grey during methanol extraction, and the texture was dense and cork-like. The abundant diod spicules (40-60 $\mu \mathrm{m} \times 1-1.5 \mu \mathrm{m}$, Figure 4.27) were isolated via centrifuge following $\mathrm{HNO}_{3}$ treatment of the tissue, aiding classification of the specimen as a plakinid sponge. As with the Plakortis specimen which yielded the lehualides, unsuccessful recollection attempts were made in the Vava'u group. The nondescript appearance and growth of the specimen in dimly-lit caves may have again been contributing factors leading to specimens going unnoticed.

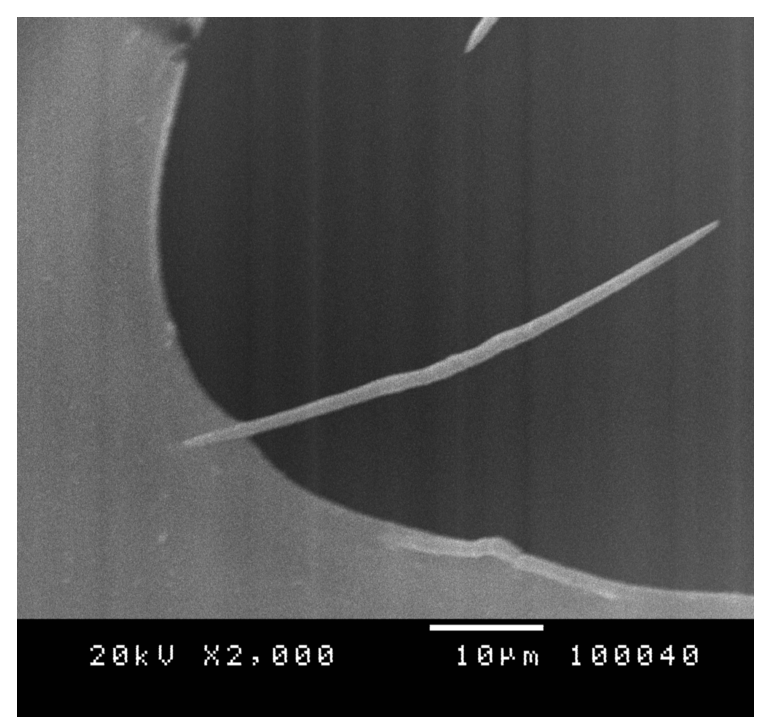

Figure 4.27 A diod spicule recovered from the plakinid sponge PTN3_19D.

\subsubsection{Isolation and identification of cyclic peroxides}

The $\mathrm{MeOH}$ extracts of the frozen screen sample of PTN3_19D were partitioned over $\mathrm{HP} 20$ resin beads, generating fractions of $30 \%, 75 \%$ and $100 \% \mathrm{Me}_{2} \mathrm{CO}$ in $\mathrm{H}_{2} \mathrm{O}$. HMBC NMR analysis of the less polar $75 \%$ and $100 \% \mathrm{Me}_{2} \mathrm{CO}$ in $\mathrm{H}_{2} \mathrm{O}$ fractions showed the presence of several closely related compounds containing multiple deshielded diastereotopic methylenes correlating to oxycarbonyls $\left(\delta_{\mathrm{C}} 170-174 \mathrm{ppm}\right)$ and oxyquaternary centres $\left(\delta_{\mathrm{C}} 80-84 \mathrm{ppm}\right)$. Similarly deshielded methyl doublet and singlet signals shared HMBC correlations with the same oxyquaternaries (Figure 4.28). The ${ }^{1} \mathrm{H}$ and ${ }^{13} \mathrm{C}$ NMR spectra also showed the presence of saturated alkyl 
systems, evidenced by COSY and HMBC correlations. This interesting combination of NMR correlations suggested that this plakinid specimen would be ideal for an NMR-guided isolation. Furthermore, reports of plakinid isolates displaying antifungal activity ${ }^{168-171}$ indicated that any isolates could be suitable for analysis by chemical genetic methods in Saccharomyces cerevisiae yeast.

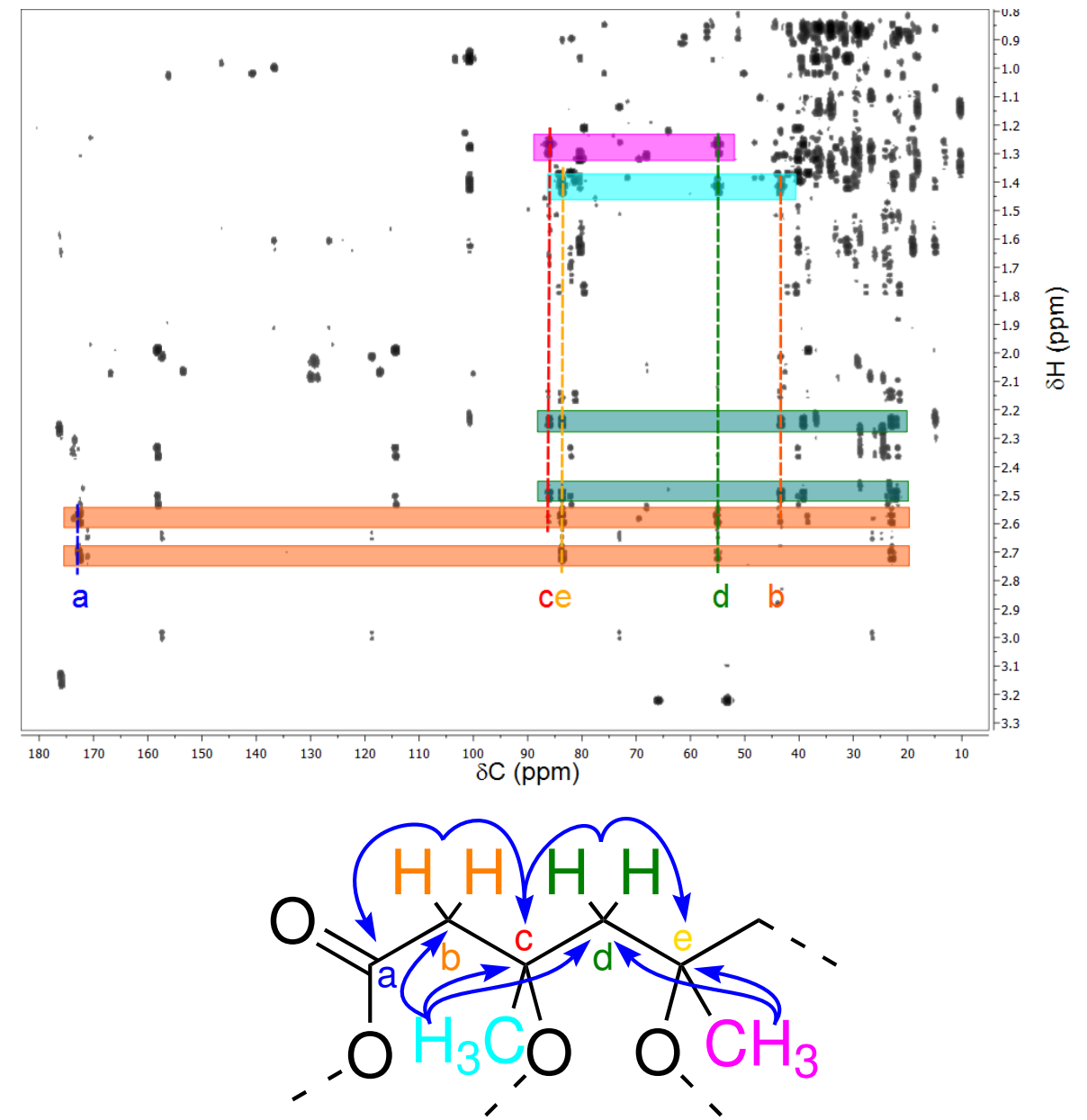

Figure 4.28 Correlations evidencing the methyl-substituted oxyquaternary centres and nearby diastereotopic methylenes and oxycarbonyls present in the HMBC spectrum of the $\mathrm{Me}_{2} \mathrm{CO}$ in $\mathrm{H}_{2} \mathrm{O}$ screen fraction of PTN3 $19 \mathrm{D}$.

Following initial partitioning of the crude methanol extracts over HP20, the nonpolar fractions were subjected to further reversed-phase chromatography (HP20SS, $\mathrm{Me}_{2} \mathrm{O}$ in $\mathrm{H}_{2} \mathrm{O}$ ). Analysis by ${ }^{1} \mathrm{H}$ NMR of the non-polar $80-100 \% \mathrm{Me}_{2} \mathrm{CO}$ in $\mathrm{H}_{2} \mathrm{O}$ fractions showed retention of the diastereotopic methylene signals associated with the oxygenated quaternary centres, indicating relatively non-polar compounds despite the oxygenation.

Relatively good separation was apparent on silica gel TLC plates, however the oxygenated functionalities were anticipated to be acid-sensitive, as such DIOL was chosen as a normal-phase material for further purification. Batch elution on DIOL resulted in elution of the closely related compounds in the $\mathrm{CH}_{2} \mathrm{Cl}_{2}$ fraction, indicative of significant lipophilicity within the structures. As the structures were deficient in a chromophore, the choice of chromatographic methods for the isolation of the 
metabolites was limited. Cyclic peroxides epiplakinic acid I (95), its methyl ester derivative (96), plakortolide X (97) and an unusual 1-hydroxy-1,2-dioxane (98) were all isolated via LH20 size-exclusion chromatography followed by cycles of bench-top DIOL chromatography with mixtures of hexanes, $\mathrm{CH}_{2} \mathrm{Cl}_{2}$ and $\mathrm{MeOH}$. Although DAD-HPLC was used successfully in the isolation of the lehualides, the technique could not be applied here, excepting final purification of the $5 \alpha, 8 \alpha$-epidioxysterols (27) and (28) $\left(\mathrm{C}_{18}\right.$ HPLC, MeCN in $\left.\mathrm{H}_{2} \mathrm{O}\right)$. A summary of the overall isolation strategy for the cyclic peroxides is depicted in Figure 4.29. 


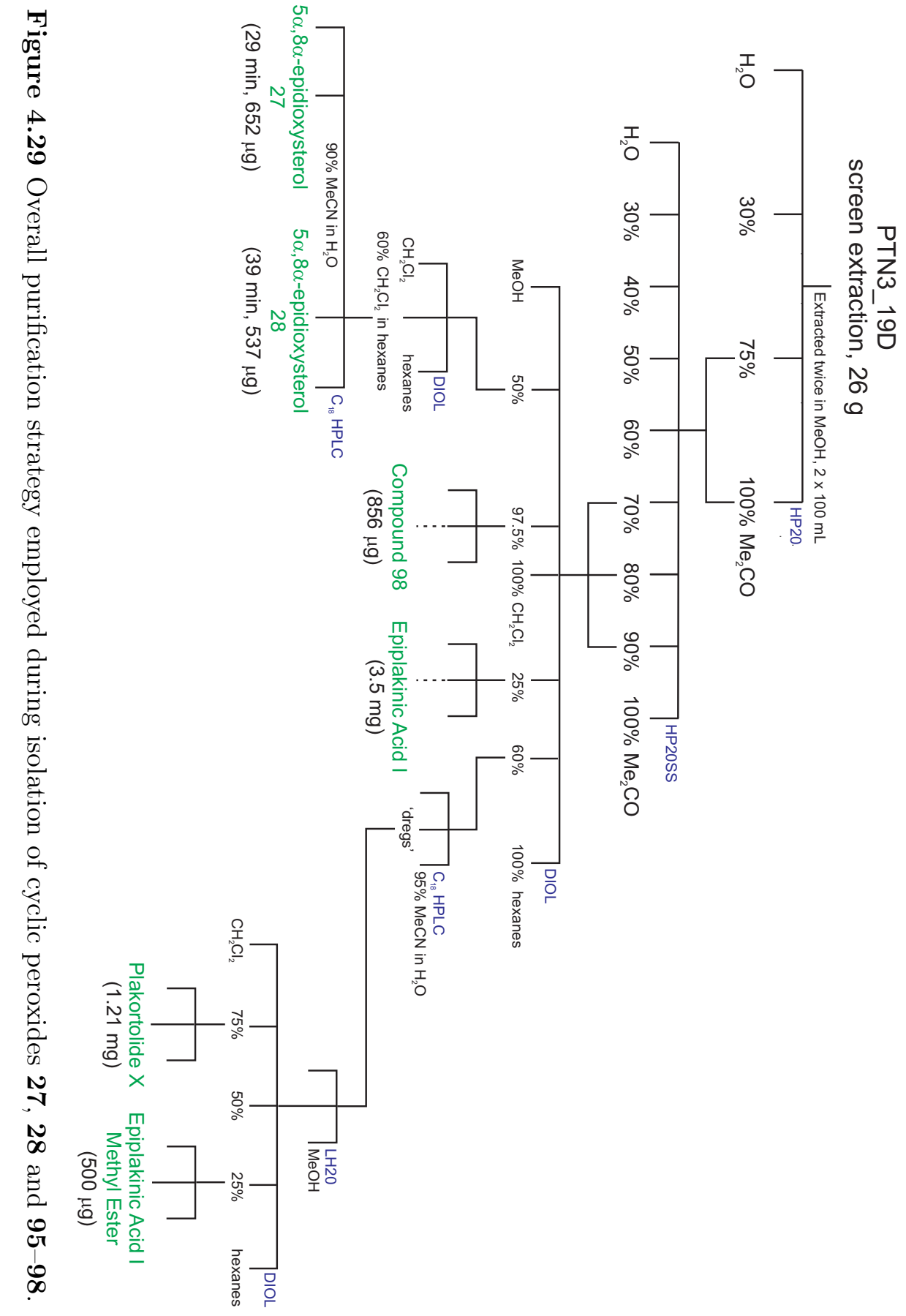




\section{Epiplakinic acid I (95)}

Epiplakinic acid I (95), $[\alpha]_{D}^{25.2}+1.9^{\circ}\left(c 0.068, \mathrm{CHCl}_{3}\right)$, was isolated as a colourless oil. Positive-ion mode HRESIMS analysis of $\mathbf{9 5}$ generated a $[\mathrm{M}+\mathrm{Na}]^{+}$pseudomolecular ion peak at $m / z 365.2668$, suitable for the formula $\mathrm{C}_{20} \mathrm{H}_{38} \mathrm{O}_{4}$, which required two degrees of unsaturation. This was supported by negative-ion mode HRESIMS analysis of the compound. Analysis of the ${ }^{1} \mathrm{H}$ and ${ }^{13} \mathrm{C}$ data showed a carboxylic acid carbonyl $\left(\delta_{\mathrm{C}} 174.3\right)$, two oxyquaternary centres $\left(\delta_{\mathrm{C}} 86.8,84.0\right)$, two isolated deshielded diastereotopic methylenes $\left[\left(\delta_{\mathrm{C}} 55.7, \delta_{\mathrm{H}}\right.\right.$ a 2.45 , b 2.25$)$ and $\left(\delta_{\mathrm{C}} 39.9, \delta_{\mathrm{H}}\right.$ a $\left.\left.1.70, \mathrm{~b} 1.53\right)\right]$, a third diastereotopic aliphatic methylene $\left(\delta_{\mathrm{C}} 36.8\right.$, $\delta_{\mathrm{H}}$ a 1.26 , b 1.06), resonances corresponding to an extended aliphatic chain, two methyl singlets $\left[\left(\delta_{\mathrm{C}} 23.9, \delta_{\mathrm{H}} 1.45\right)\right.$ and $\left.\left(\delta_{\mathrm{C}} 23.3, \delta_{\mathrm{H}} 1.29\right)\right]$, an aliphatic methine $\left(\delta_{\mathrm{C}} 34.5, \delta_{\mathrm{H}} 1.28\right)$, an aliphatic methyl doublet $\left(\delta_{\mathrm{C}} 19.7, \delta_{\mathrm{H}} 0.83\right)$ and a terminal aliphatic methyl triplet $\left(\delta_{\mathrm{C}} 11.6, \delta_{\mathrm{H}}\right.$ 0.84). All but one hydrogen atom was accounted for by the fully-coupled HSQC spectrum; with corroborating evidence for an acid functionality indicated by ${ }^{13} \mathrm{C}$ NMR and IR spectra $\left(\nu_{\max } 3428(\mathrm{OH})\right.$, $\left.1717(\mathrm{C}=\mathrm{O}) \mathrm{cm}^{-1}\right)$.

As illustrated by Figure 4.30, HMBC NMR correlations were observed from the protons of a methyl singlet $\mathrm{CH}_{3}-18\left(\delta_{\mathrm{C}} 23.9, \delta_{\mathrm{C}} 1.45\right)$ to methylenes $\mathrm{CH}_{2}-2$ and $\mathrm{CH}_{2}-4\left[\left(\mathrm{CH}_{2}-2: \delta_{\mathrm{C}} 44.1, \delta_{\mathrm{H}}\right.\right.$ a 2.80, b 2.72$)$ and $\left.\left(\mathrm{CH}_{2}-4: \delta_{\mathrm{C}} 55.7, \delta_{\mathrm{H}} 2.45,2.25\right)\right]$ and oxyquaternary C-3 $\left(\delta_{\mathrm{C}} 84.0\right)$. This established connection between the methyl singlet $\mathrm{CH}_{3}-18$ and the non-protonated oxyquaternary centre, and the adjacent positioning of methylenes $\mathrm{CH}_{2}-2$ and $\mathrm{CH}_{2}-4$. A weak COSY correlation between $\mathrm{H}_{3}-18$ and $\mathrm{H}_{2}-2$ confirmed the $\mathrm{CH}_{2}-2-\mathrm{C}-3$ bond, corroborated by an HMBC correlation from $\mathrm{H}_{2}-2$ to C-3. Further HMBC correlations from the protons of $\mathrm{H}_{2}-2$ to a carbonyl C-1 $\left(\delta_{\mathrm{C}} 174.3\right)$ extended the linear segment $\mathrm{C}-1$ to $\mathrm{C}-4$. The absence of further spectral correlations to the carbonyl $\mathrm{C}-1$ indicated an acid function.
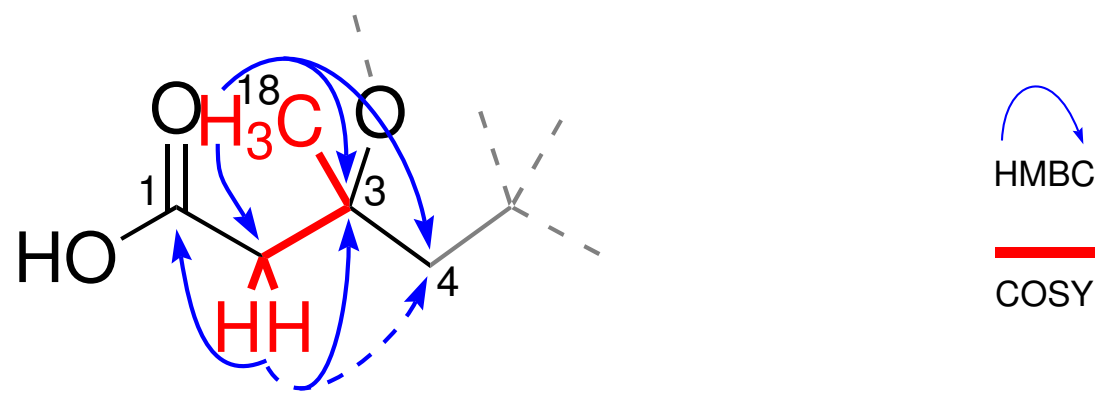

Figure 4.30 Salient COSY and HMBC NMR correlations establishing the C-1 to C-4 linkages in 95, and methyl substitution of C-4. Dashed arrows indicate weak correlations.

The second methyl singlet $\mathrm{CH}_{3}-19\left(\delta_{\mathrm{C}} 23.3, \delta_{\mathrm{H}} 1.29\right)$ displayed HMBC correlations to a second oxyquaternary $\mathrm{C}-5\left(\delta_{\mathrm{C}} 86.8\right)$, and methylenes $\mathrm{CH}_{2}-4$ and $\mathrm{CH}_{2}-6\left(\delta_{\mathrm{C}} 39.9\right.$, $\delta_{\mathrm{H}}$ a $\left.1.70, \mathrm{~b} 1.53\right)$. This established substitution of the non-protonated C-5 with the 
methyl singlet $\mathrm{CH}_{3}-19$, and adjacent positioning of the methylenes $\mathrm{CH}_{2}-4$ and $\mathrm{CH}_{2}-6$ (Figure 4.31). Corroborating HMBC correlations were observed from $\mathrm{H}_{2}-4$ to both quaternary centres, C-3 and C-5, confirming the linear sequence from C-1 to $\mathrm{CH}_{2}-6$.

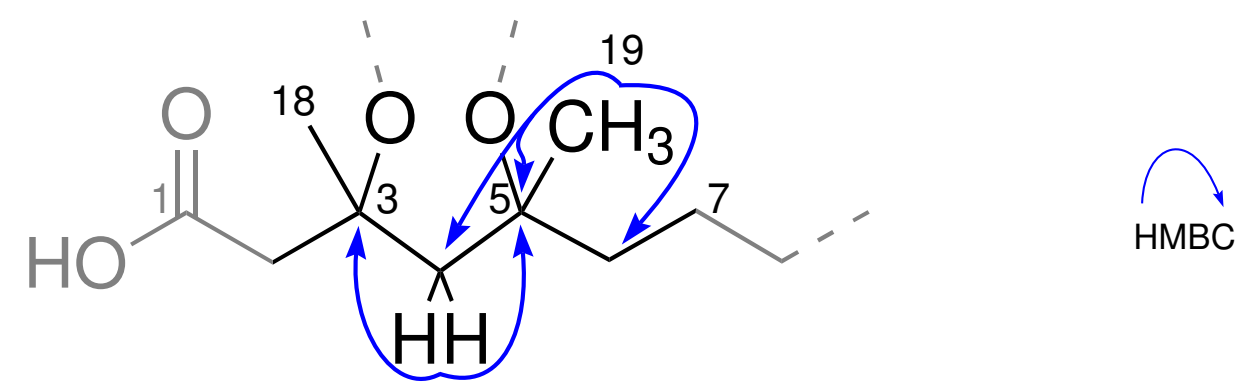

Figure 4.31 Salient HMBC NMR correlations establishing the C-3 to C-6 linkages of $\mathbf{9 5}$, and methyl substitution of C-5.

COSY correlations clearly established the connectivity between the terminal methyl triplet $\mathrm{CH}_{3}-17\left(\delta_{\mathrm{C}} 11.6, \delta_{\mathrm{H}} 0.84\right)$, aliphatic methylene $\mathrm{CH}_{2}-16\left(\delta_{\mathrm{C}} 29.73, \delta_{\mathrm{H}} 1.11\right)$, methine $\mathrm{CH}-15\left(\delta_{\mathrm{C}} 34.5, \delta_{\mathrm{H}} 1.28\right)$, its methyl doublet substituent $\mathrm{CH}_{3}-20\left(\delta_{\mathrm{C}} 19.7\right.$, $\left.\delta_{\mathrm{H}} 0.83\right)$ and a second aliphatic methylene $\mathrm{CH}_{2}-14\left(\delta_{\mathrm{C}} 36.8, \delta_{\mathrm{H}}\right.$ a $\left.1.26, \mathrm{~b} 1.06\right)$. This series was corroborated by HMBC correlations from methyl doublet $\mathrm{CH}_{3}-20$ to the methine $\mathrm{CH}-15$ and the adjacent methylenes $\mathrm{CH}_{2}-14$ and $\mathrm{CH}_{2}-16$. Selective excitation of $\mathrm{CH}_{3}-17$ with a 1D TOCSY experiment over mixing times of 20-80 ms, confirmed connection between the protonated centres (Figure 4.32).
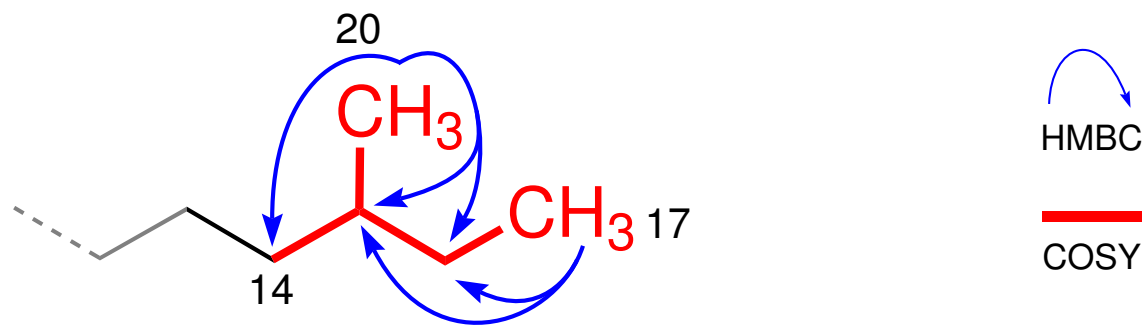

Figure 4.32 COSY and HMBC NMR correlations establishing $\gamma$-methyl-substituted motif C-14 to C-17 of $\mathbf{9 5 .}$

A $\mathrm{C}_{9}$ methylene chain beginning with $\mathrm{CH}_{2}-6$, terminating at $\mathrm{CH}_{2}-14$, was established by a series of sequential COSY and HMBC correlations. Selective irradiation of both methylene centres $\left(\mathrm{CH}_{2}-6\right.$ and $\left.\mathrm{CH}_{2}-14\right)$ and subsequent revelation of the other in a series of 1D TOCSY experiments with mixing times of 20-120 ms confirmed the connection (Figure 4.33).

As all of the protons of the molecular formula were accounted for, a cyclic structure is required to account for the final degree of unsaturation associated with the molecular formula. Two of the oxygen atoms were incorporated into the acid functionality, 


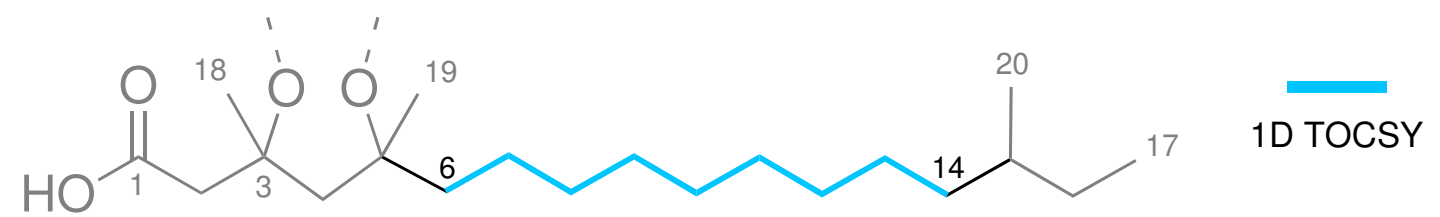

Figure 4.33 1D TOCSY correlations establishing the $\mathrm{C}_{9}$ methylene chain of $\mathbf{9 5}$.

implying substitution of the oxyquaternaries C-3 and C-5 with different oxygen atoms. A peroxide linkage between the oxyquaternary centres is therefore proposed, completing the structure of $\mathbf{9 5}$.

The 1,2-dioxolane core of $\mathbf{9 5}$ is consistent with common Plakortis and Plakinastrella metabolites, the plakinic and epiplakinic acids. The classes are diastereomeric in their orientation of alkyl substituents about the 1,2-dioxolane nucleus, cis in plakinic acids, and trans in the epiplakinic acids. Comparison of the ${ }^{1} \mathrm{H}$ and ${ }^{13} \mathrm{C} \mathrm{NMR}$ chemical shifts of the methyl groups in known plakinic and epiplakinic acids was consistent with trans configuration of the methyl groups in $\mathbf{9 5} .{ }^{171}$ The cis orientation of methyls within plakinic acids increases diastereotopic differentiation of the $\mathrm{CH}_{2}$ 4 protons in the ${ }^{1} \mathrm{H}$ NMR spectra, and the C-5 methyl substituent has a higher chemical shift relative to the analogous positions in epiplakinic acids. ${ }^{114,123}$

Trans orientation of $\mathrm{CH}_{3}-18$ and $\mathrm{CH}_{3}-19$ about the 1,2-dioxolane nucleus of 95 was confirmed by a series of 1D NOE experiments. Selective irradiation of H-4a and H$4 \mathrm{~b}$ gave exclusive enhancement of $\mathrm{H}_{3}-19$ and $\mathrm{H}_{3}-18$ respectively, while irradiation of the $\mathrm{H}_{3}-18$ and $\mathrm{H}_{3}-19$ methyls resulted in reciprocal enhancements of the methylene protons, and only weak enhancement of the other methyl (Figure 4.34).
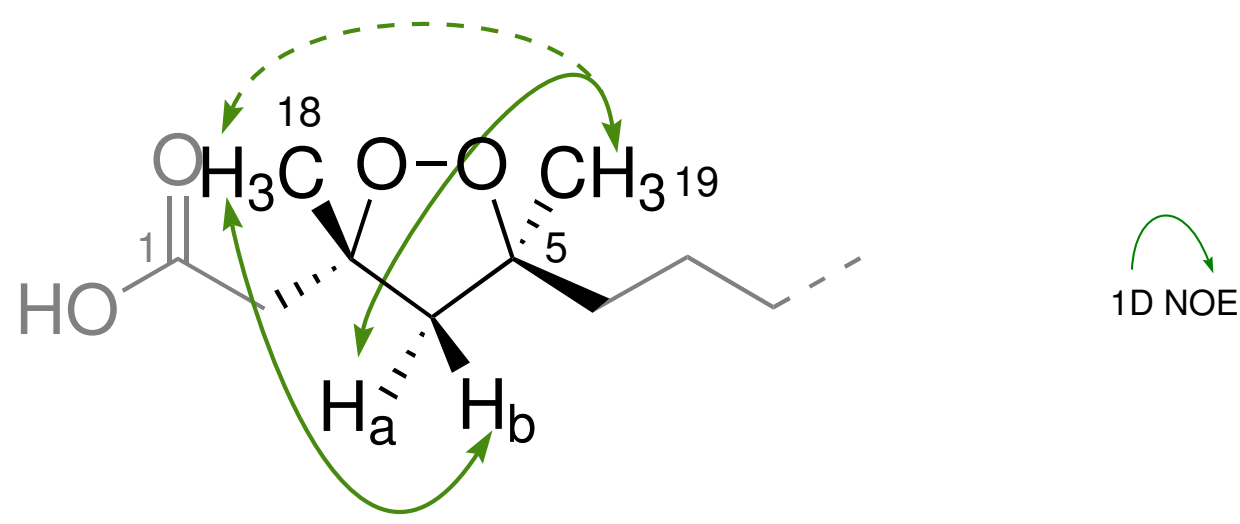

Figure 4.34 1D NOE correlations used to establish relative stereochemistry in the 1,2dioxolane nucleus of $\mathbf{9 5}$. Double headed arrows indicate reciprocal correlations, and dashed arrows indicate weak correlations. 

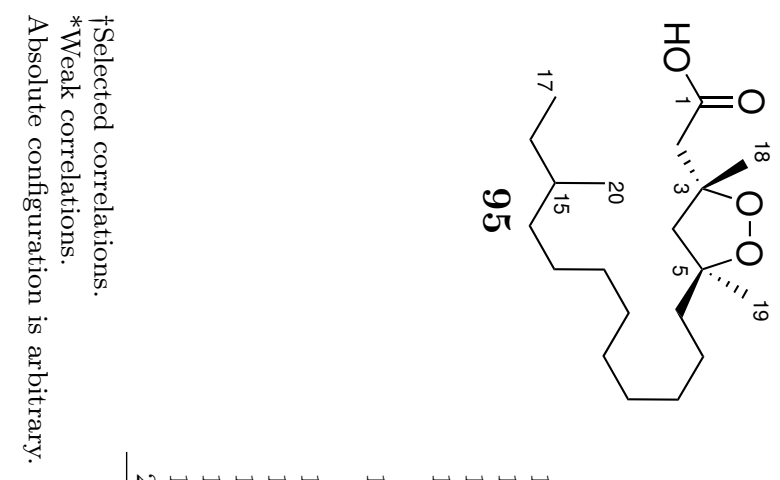

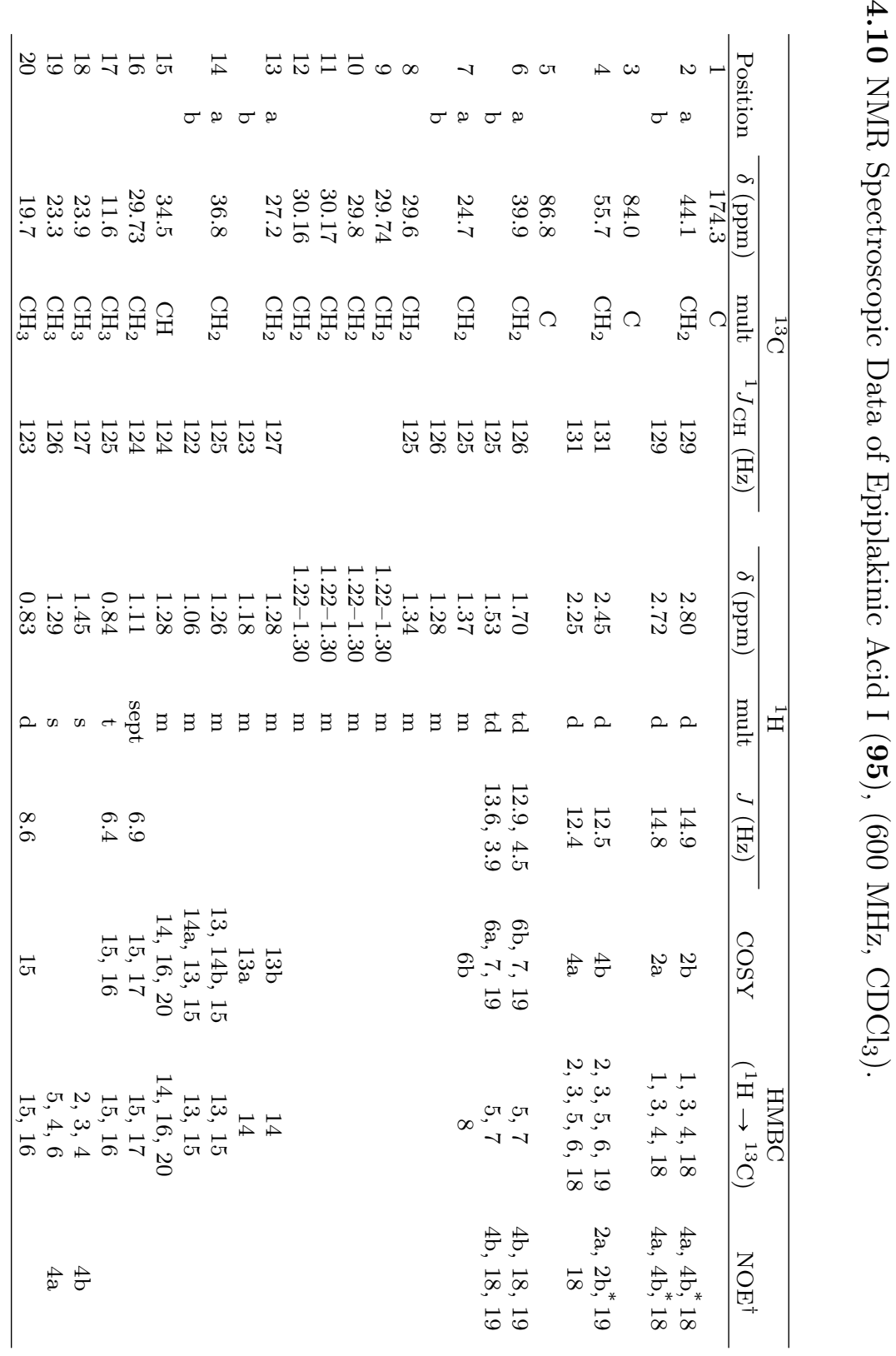




\section{Epiplakinic acid I methyl ester (96)}

The methyl ester 96 eluted earlier from DIOL than its acid analogue 95. Analysis by positive-ion mode HRESIMS revealed a $[\mathrm{M}+\mathrm{Na}]^{+}$pseudo-molecular ion peak at $m / z 371.2790$, which correlated to the formula $\mathrm{C}_{21} \mathrm{H}_{39} \mathrm{O}_{5}$. This established the presence of an additional $\mathrm{CH}_{2}$ equivalent in the structure, relative to 95. Comparison of $1 \mathrm{D}$ and 2D NMR data showed compounds 95 and 96 to be identical in structure and relative stereochemistry, except for the presence of a methoxy singlet $\left(\mathrm{CH}_{3}-21\right.$ : $\left.\delta_{\mathrm{C}} 52.0, \delta_{\mathrm{H}} 3.69\right)$ in 96. The observation of an $\mathrm{HMBC}$ correlation from it $\left(\mathrm{CH}_{3}-21\right)$ to an ester carbonyl $\left(\mathrm{C}-1: \delta_{\mathrm{C}} 171.4\right)$ confirmed the methyl ester function (Figure $4.35)$.
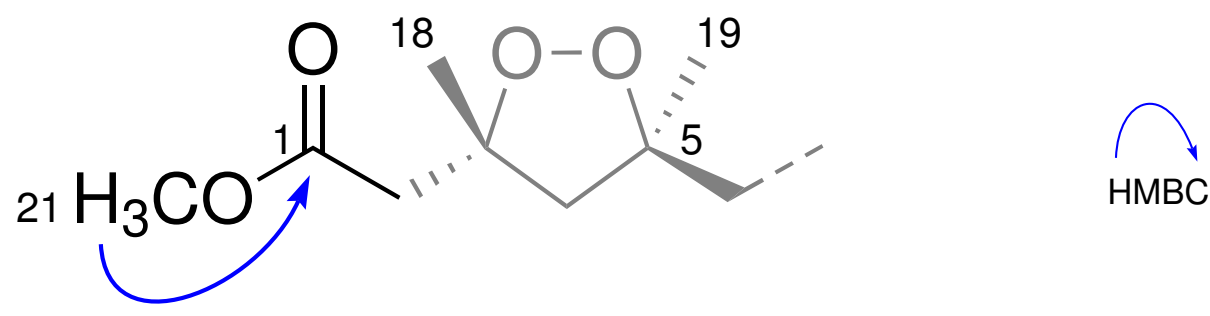

Figure 4.35 HMBC NMR correlation establishing the C-21 methyl ester moiety of 96. 

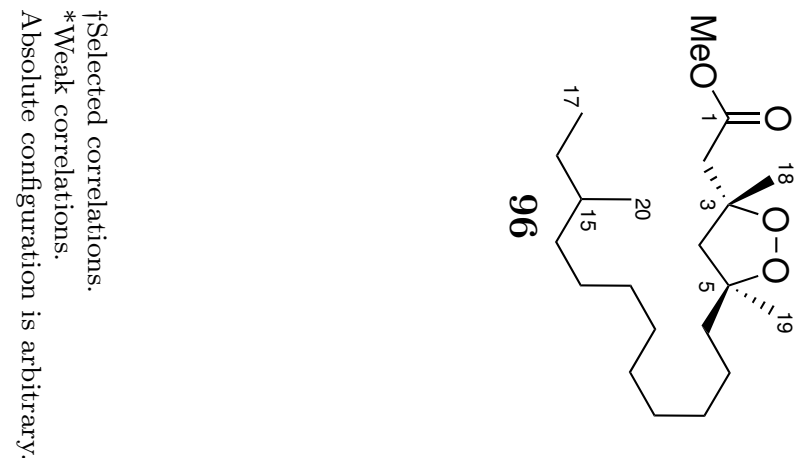

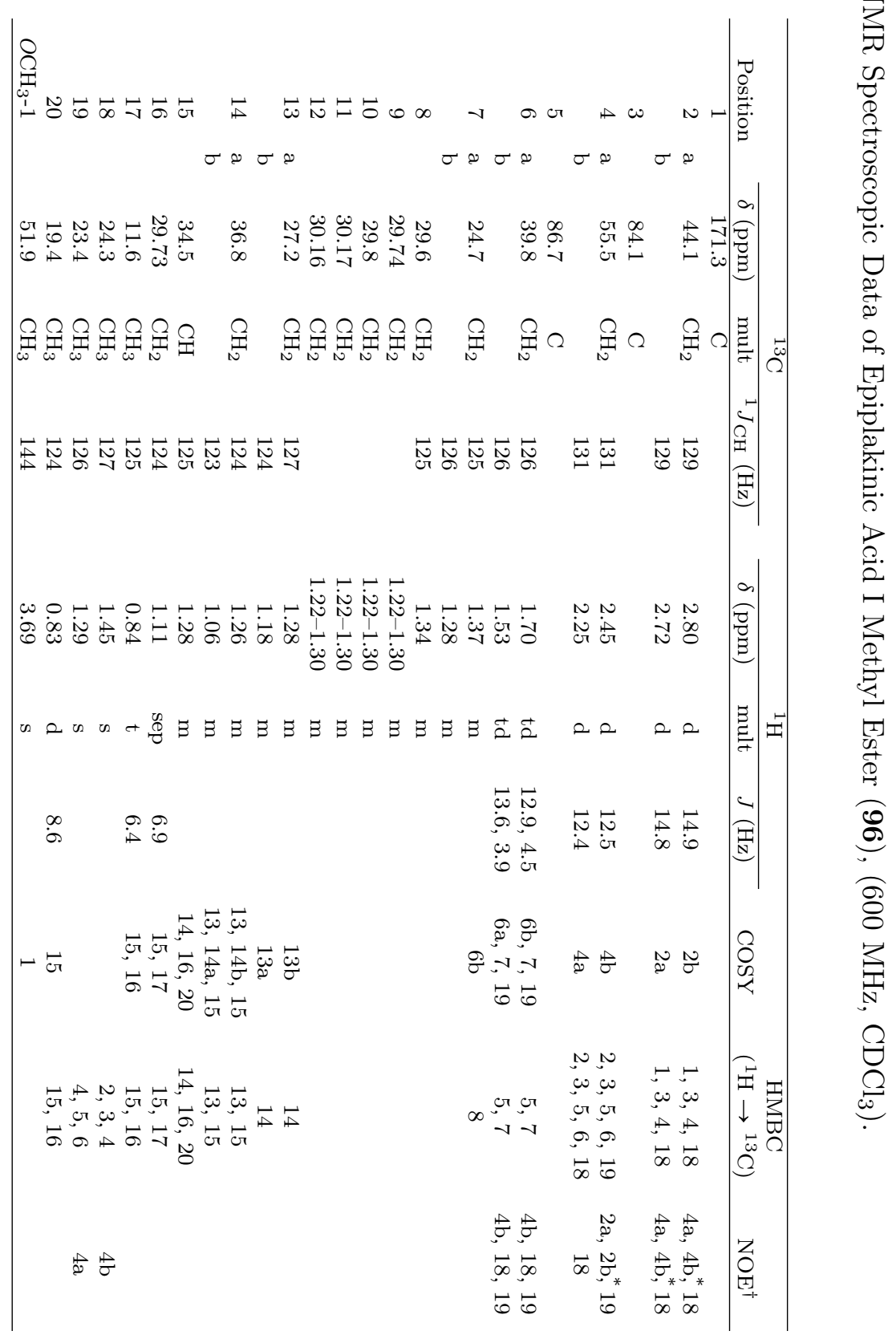




\section{Plakortolide X (97)}

Plakortolide $\mathrm{X}(\mathbf{9 7}),[\alpha]_{D}^{24.6}+0.0^{\circ}\left(c 0.08, \mathrm{CHCl}_{3}\right)$, was isolated as a colourless oil. The apparent lack of rotation may indicate the isolation of a racemic mixture. Positive-ion mode HRESIMS analysis of $\mathbf{9 7}$ revealed a $[\mathrm{M}+\mathrm{H}]^{+}$pseudo-molecular ion peak $\left(\mathrm{m} / z\right.$ 349.2355) correlating to the molecular formula $\mathrm{C}_{20} \mathrm{H}_{38} \mathrm{O}_{4}$, which required three degrees of unsaturation. A $[\mathrm{M}-\mathrm{H}]^{-}$ion peak was observed at $\mathrm{m} / z$ 325.2379 by negative-ion mode analysis, confirming this formula. Evident in the 1D and 2D NMR data was conservation of the $\gamma$-methyl branched alkyl chain seen in 95 and 96. The oxygenated end of the molecule displayed NMR resonances significantly different from those of $\mathbf{9 5}$ and 96, indicating a different arrangement of functionality. The absence of an $\mathrm{OH}$ stretch in the IR spectrum and the continued presence of an oxy-substituted carbonyl $\left(\delta_{\mathrm{C}} 174.3\right)$ and an additional oxy-substituted methine $\left(\delta_{\mathrm{C}} 81.3, \delta_{\mathrm{H}} 4.46\right)$ suggested the presence of a lactone.

Strong HMBC correlations were observed from the protons of a methyl singlet, $\mathrm{CH}_{3}$ $18\left(\delta_{\mathrm{C}} 25.9, \delta_{\mathrm{H}} 1.39\right)$ to three carbon centres; oxymethine $\mathrm{CH}-3\left(\delta_{\mathrm{C}} 81.3, \delta_{\mathrm{H}} 4.46\right)$, oxyquaternary C-4 $\left(\delta_{\mathrm{C}} 83.0\right)$ and an isolated methylene $\mathrm{CH}_{2}-5\left(\delta_{\mathrm{C}} 40.5, \delta_{\mathrm{H}}\right.$ a 2.17 , $\mathrm{b}$ 1.71). These correlations established attachment of the methyl singlet to the non-protonated C-4, and the bonds from CH-3 to C-4 and between C-4 and $\mathrm{CH}_{2}-5$. COSY correlations between the methine $\mathrm{CH}-3$ and a second diastereotopic methylene $\mathrm{CH}_{2}-2\left(\delta_{\mathrm{C}} 34.3, \delta_{\mathrm{H}}\right.$ a 2.92 , b 2.62) extended the substructure. HMBC correlations from the protons of $\mathrm{CH}_{2}-2$ to the oxycarbonyl C-1 $\left(\delta_{\mathrm{C}} 174.3\right)$ indicated linkage from C-1 to C-2, firmly establishing the $\mathrm{C}-1$ to $\mathrm{C}-5$ terminus of the molecule (Figure $4.36)$.
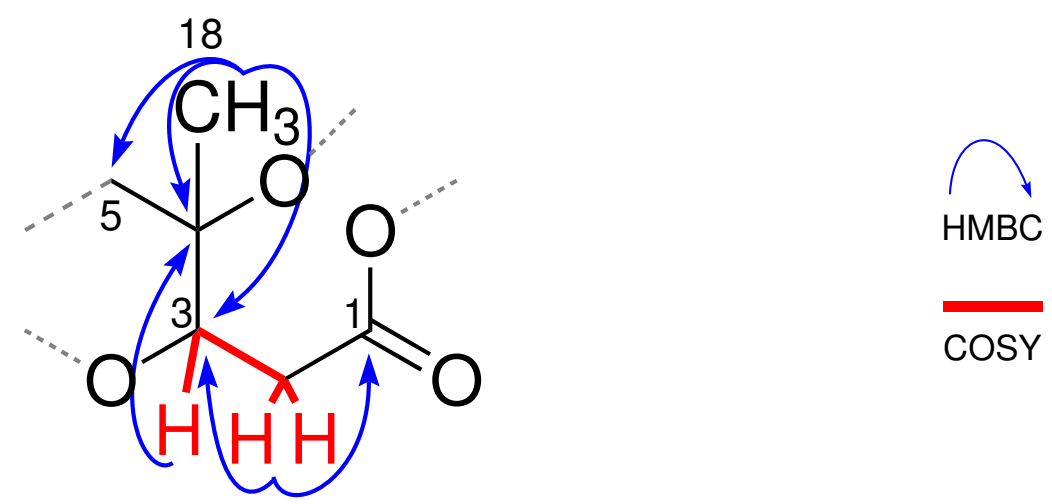

Figure 4.36 COSY and HMBC correlations establishing the C-1 to C-5 linkages in $\mathbf{9 7 .}$

Further evidence of the bond between $\mathrm{C}-4$ and the isolated methylene $\mathrm{CH}_{2}-5$ was given by $\mathrm{HMBC}$ correlations from the protons of $\mathrm{CH}_{2}-5$ to the oxyquaternary $\mathrm{C}$ 4 and oxymethine $\mathrm{CH}-3$, further extending the $\delta$-methyl branched carbon chain (Figure 4.37). The remaining methyl singlet $\mathrm{CH}_{3}-19\left(\delta_{\mathrm{C}} 22.4, \delta_{\mathrm{H}} 1.29\right)$, also showed strong $\mathrm{HMBC}$ correlations to $\mathrm{CH}_{2}-5$, its non-protonated attachment point 
C-6 $\left(\delta_{\mathrm{C}} 80.3\right)$ and a further methylene $\mathrm{CH}_{2}-7\left(\delta_{\mathrm{C}} 41.0, \delta_{\mathrm{H}} 1.48\right)$. Corroborating HMBC correlations were observed from $\mathrm{H}_{2}-5$ to $\mathrm{C}-6$ and $\mathrm{CH}_{2}-7$. These correlations fully established the 4,6-dimethyl-substituted carbon chain with oxy-substitution at carbonyl C-1 and at CH-3, C-4 and C-6 (Figure 4.38).

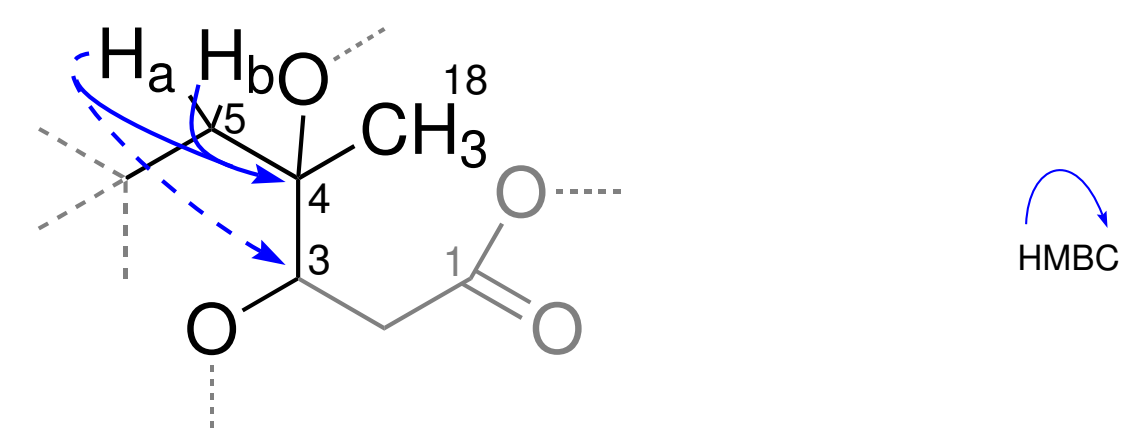

Figure 4.37 COSY and selected HMBC correlations confirming the C-3 to C-5 connections in 97. Dashed arrows indicate weak correlations.

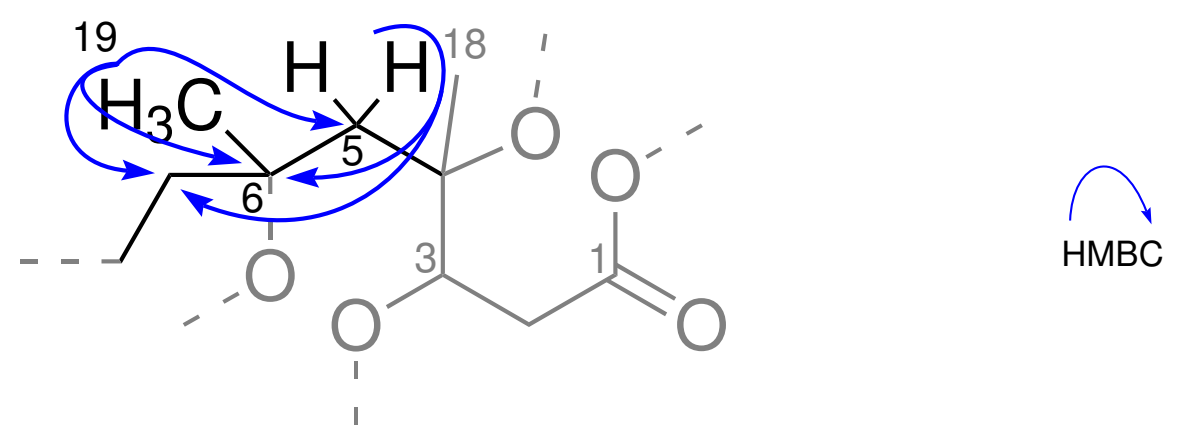

Figure 4.38 HMBC correlations establishing the linkages from C-5 to C-7 in $\mathbf{9 7 .}$

The remaining carbon resonances of the molecule included one methine, eight methylenes and two methyl groups. These centres were readily assembled into the $\gamma$-methyl substituted alkyl chain observed in $\mathbf{9 5}$ and $\mathbf{9 6}$ through interpretation of COSY and HMBC correlations. The attachment of this chain to $\mathrm{CH}_{2}-7$ was confirmed by selective irradiation of the methylenes $\mathrm{H}_{2}-7$ and $\mathrm{H}_{2}-14$ with a $1 \mathrm{D}$ TOCSY experiment. Over mixing times of 20-120 ms the other resonance was revealed, confirming the connection between the $\mathrm{C}-1$ to $\mathrm{C}-7$ and $\mathrm{C}-14$ to $\mathrm{C}-17$ segments.

The $\gamma$-methyl substituted alkyl chain, C-8 to C-20, accounted for all other protons associated with the molecular formula, however, two degrees of unsaturation remained and four oxygenated linkages required incorporation via three oxygen atoms. These criteria are satisfied by formation of a bicyclic structure, with a lactone linkage between C-1 and C-4, and a peroxy bridge between C-3 and C-6 as seen in the plakortolide skeleton (Figure 4.39). 


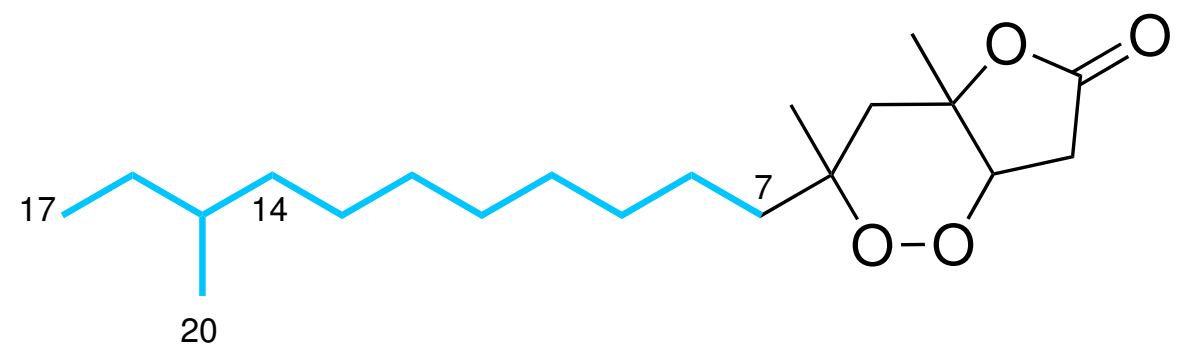

1D TOCSY

Figure 4.39 1D TOCSY correlations in 97, confirming connection between the segments C-1 to C-7 and C-14 to C-17. Incorporation of the two final degrees of molecular unsaturation completes the structure of $\mathbf{9 7}$.

Selective irradiation of $\mathrm{H}_{3}-18$ and $\mathrm{H}-3$ during 1D NOE experiments gave reciprocal enhancement of these resonances, confirming the configurations at C-3 and C-4, and the cis fusion of peroxy lactone bicycle. Reciprocal 1,3-diaxial correlations observed between H-3 and H-5a, and weaker correlations between $\mathrm{H}_{3}-18$ and $\mathrm{H}-5 \mathrm{a}$, placed the resonances on the same side of the six-membered 1,2-dioxane ring, while $\mathrm{H}_{3}-19$ shared a strong NOE correlation with $\mathrm{H}-5 \mathrm{~b}$ and a weaker correlation with $\mathrm{H}-5 \mathrm{a}$. Following 1D NOE irradiation of $\mathrm{H}_{3}-19$ and $\mathrm{H}_{3}-18$, enhancement of neither methyl singlet was observed, indicating that the two methyls occupy opposing faces of the 1,2-dioxane ring (Figure 4.40).

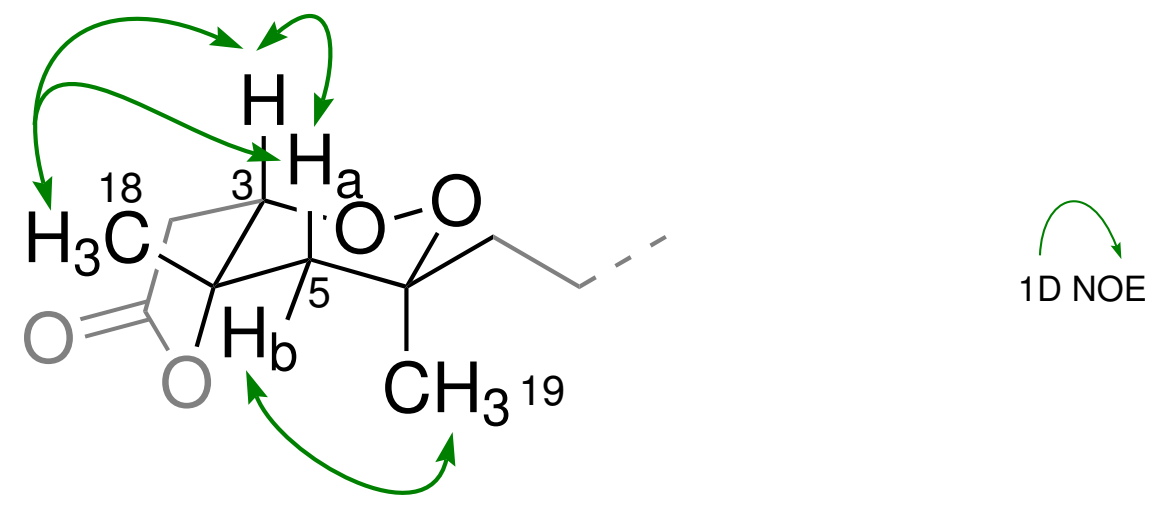

Figure 4.40 Stereochemistry about the bicyclic nucleus of $\mathbf{9 7}$ as evidenced by selected 1D NOE correlations. Double headed arrows indicate reciprocal correlations.

The plakortolide class has primarily been isolated from sponges identified as Plakinastrella sp. although some examples have been reported from specimens identified as Plakortis sponges. Comparison of the NMR spectral data of $\mathbf{9 7}$ with that of plakortolide $\mathrm{T},{ }^{166}$ as shown in Table 4.12, supported the structural assignment of $\mathbf{9 7}$. A complete tabulation of the NMR spectroscopic data for $\mathbf{9 7}$ can be found in Table 4.13 .

Fusion of the plakortolide bicycle at C-3 and C-4 is exclusively cis, although varied configuration at C-6 forms two diastereomeric carbon skeletons, which display significant differences in ${ }^{1} \mathrm{H}$ chemical shift of $\mathrm{CH}_{2}-5$ and the $\mathrm{C}-4$ methyl 
substituent. ${ }^{172}$ Orientation of the C-4 and C-6 methyls on opposing faces decreases diastereotopic differentiation of the $\mathrm{H}_{2}-5$ protons, and ${ }^{1} \mathrm{H}$ NMR chemical shift of the C-4 methyl increases ca. 0.1 ppm. ${ }^{124}$ Hydrolysis of the peroxide bridge produces another structural variant, the seco-plakortolide diols, which have been reported as both true isolates and as synthetic degradation products of plakortolides. ${ }^{124}$ One can easily distinguish the peroxide and diol forms through analysis of the $\mathrm{H}_{2}-5{ }^{1} \mathrm{H} \mathrm{NMR}$ shifts. Ring strain in the peroxide form increases the diastereotopic differentiation of $\mathrm{H}-5 \mathrm{a}$ and $\mathrm{H}-5 \mathrm{~b}$ relative to the diol form. Furthermore, the ${ }^{1} \mathrm{H}$ NMR shifts of the C-4 and C-6 methyl groups are deshielded by ca. 0.1 ppm in plakortolides, relative to seco-plakortolides. ${ }^{124}$

Table 4.12 Comparison of Salient NMR Chemical Shifts in Plakortolide $\mathrm{T}^{166}$ relative to those of Plakortolide X (97).

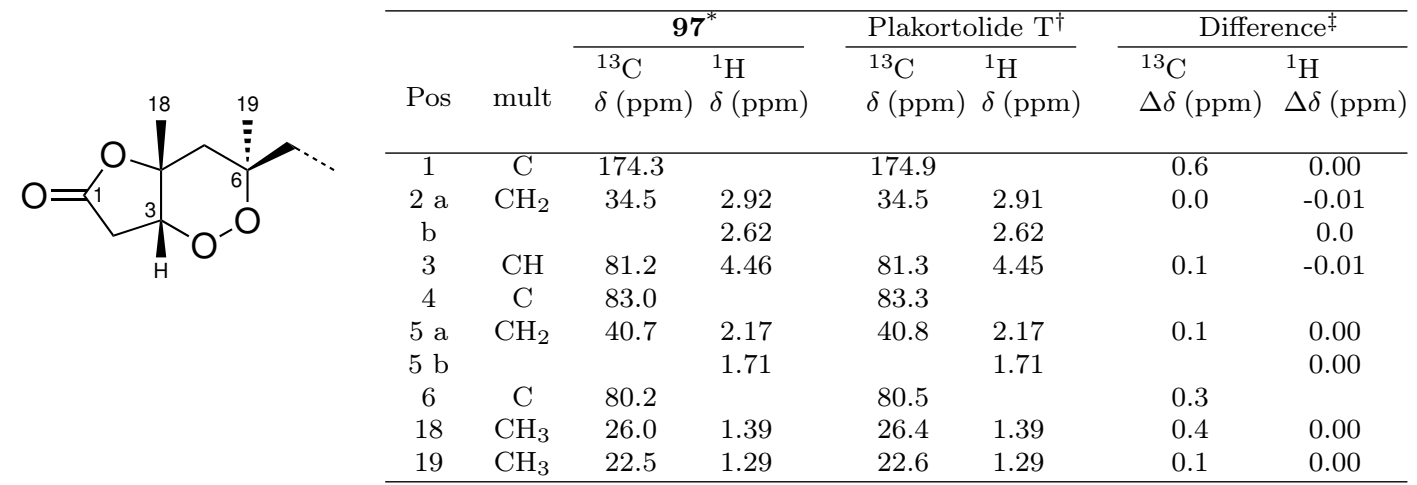

${ }^{*} \mathrm{CDCl}_{3},{ }^{1} \mathrm{H} 600 \mathrm{MHz} ;{ }^{13} \mathrm{C} 150 \mathrm{MHz}$.

$\mathrm{fCDCl}_{3},{ }^{1} \mathrm{H} 500 \mathrm{MHz} ;{ }^{13} \mathrm{C} 125 \mathrm{MHz}$.

$\ddagger \Delta \delta=\delta$ Plakortolide $\mathbf{T}-\delta \mathbf{9 7}$ 

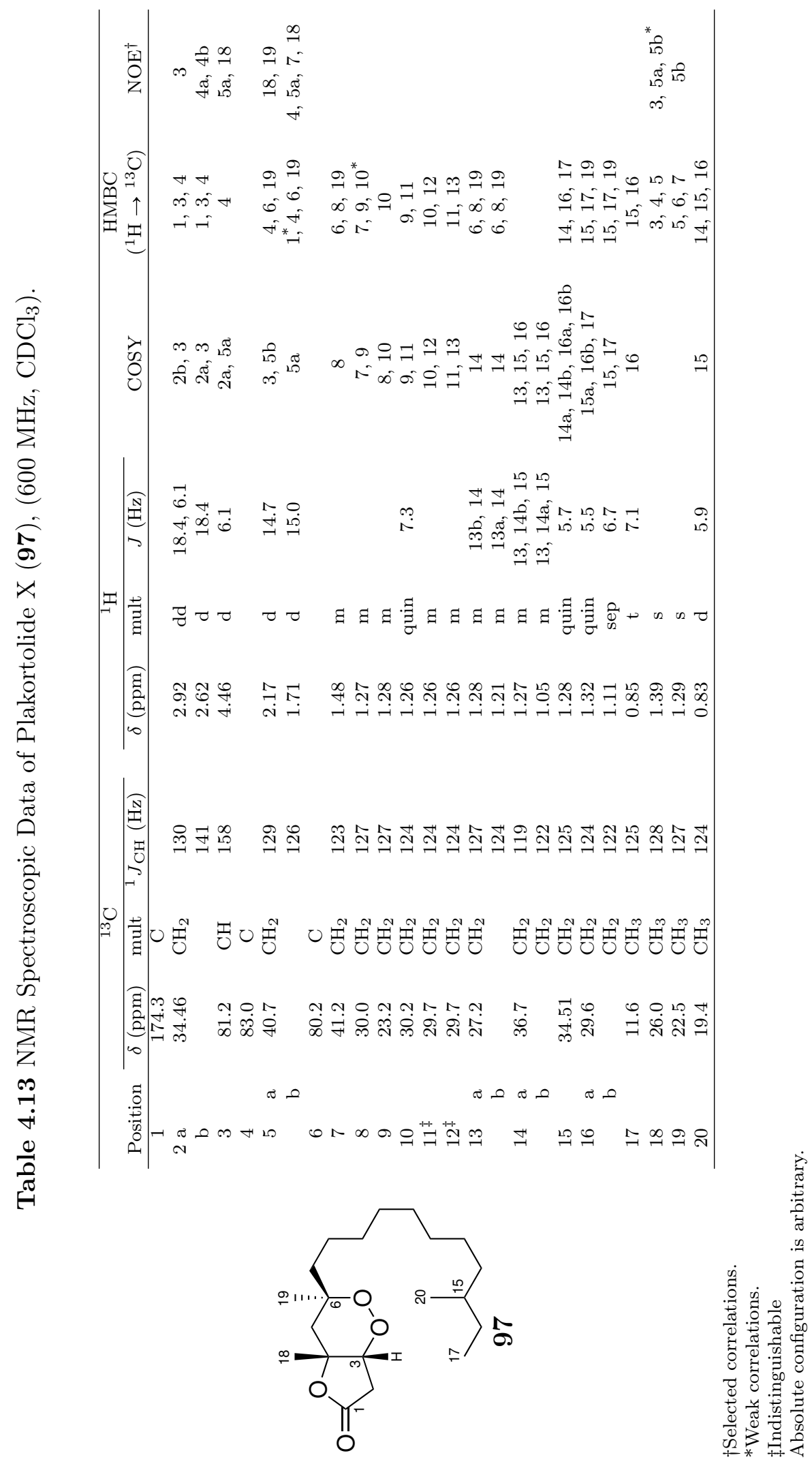


\section{Cyclic peroxide 98}

Isolated as colourless oil, positive- and negative-ion mode HRESIMS analysis of 98, $[\alpha]_{D}^{24.6}+4.1^{\circ}\left(c 0.03, \mathrm{CHCl}_{3}\right)$, indicated a molecular formula of $\mathrm{C}_{21} \mathrm{H}_{40} \mathrm{O}_{5}$; $\left[\left(m / z 395.2771[\mathrm{M}+\mathrm{Na}]^{+}, \Delta-0.8 \mathrm{ppm}\right)\right.$ and $\left(\mathrm{m} / z 371.2790[\mathrm{M}-\mathrm{H}]^{-}, \Delta-1.9\right.$ $\mathrm{ppm})$. This formula required the incorporation of an additional oxygen atom, relative to compounds 95-97. Evident in the 1D and 2D NMR data was conservation of the $\gamma$-methyl branched alkyl chain. However, the oxygenated terminus of the molecule displayed NMR resonances differing again from those observed in the spectra of compounds 95-97, indicating a further variation in functional arrangement. In particular, the chemical shift of one of the oxyquaternary centres had increased significantly to $\delta_{\mathrm{C}} 100.9 \mathrm{ppm}$, from the $\delta_{\mathrm{C}} 80-84 \mathrm{ppm}$ observed in the previous three compounds. An $\mathrm{OH}$ stretch was observed in the IR spectrum $\left(\nu_{\max }\right.$ $\left.3374 \mathrm{~cm}^{-1}\right)$, which in combination with an oxy-substituted carbonyl $\left(\delta_{\mathrm{C}} 174.7 ; \nu_{\max }\right.$ $1717 \mathrm{~cm}^{-1}$ ) was again suggestive of a carboxylic acid functionality.

As illustrated in Figure 4.41, correlations were observed in the COSY experiment between the aliphatic methyl doublet $\mathrm{CH}_{3}-19\left(\delta_{\mathrm{C}} 16.2, \delta_{\mathrm{H}} 0.99\right)$, methine $\mathrm{CH}-4$ $\left(\delta_{\mathrm{C}} 32.7, \delta_{\mathrm{H}} 1.99\right)$ and the diastereotopic methylene $\mathrm{CH}_{2}-5\left(\delta_{\mathrm{C}} 37.3, \delta_{\mathrm{H}}\right.$ a 1.66 , b 1.41). The C-19-C-4-C-5 bonds were confirmed by HMBC correlations from the methyl doublet $\mathrm{CH}_{3}-19$ to the methine $\mathrm{CH}-4$ and the adjacent methylene $\mathrm{CH}_{2}-5$. A third HMBC correlation was observed from $\mathrm{CH}_{3}-19$ to the highly deshielded oxyquaternary $\mathrm{C}-3\left(\delta_{\mathrm{C}} 100.9\right)$, whose chemical shift is consistent with dioxy-substitution. These correlations established the connections from C-3 to C-5.
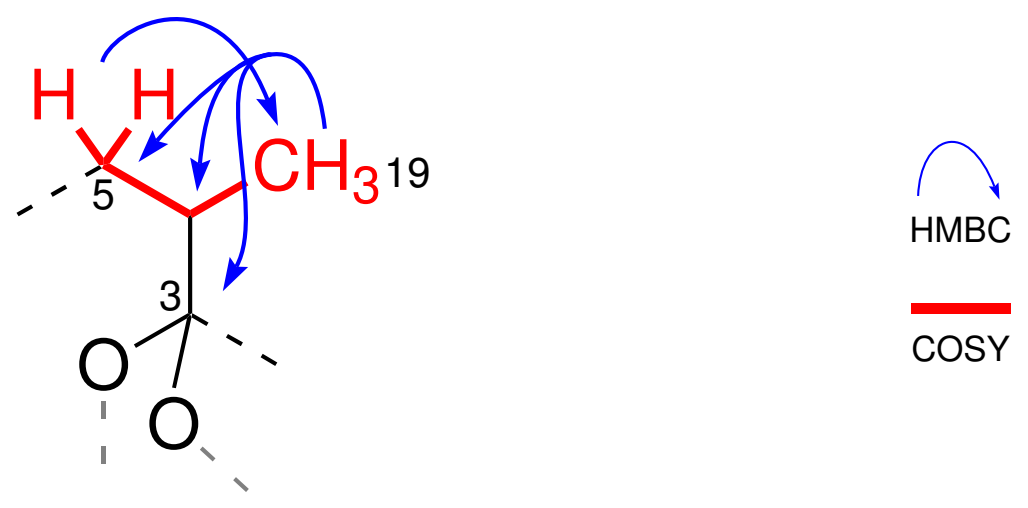

Figure 4.41 COSY and selected HMBC correlations evidencing the C-3 to C-5 connections and methyl substitution of C-4 in $\mathbf{9 8}$.

An isolated diasterotopic methylene $\mathrm{CH}_{2}-2\left(\delta_{\mathrm{C}} 39.4, \delta_{\mathrm{H}}\right.$ a 2.89 , b 2.55) displayed HMBC correlations to C-4 and C-3, confirming the C-3-C-4 bond and connection between $\mathrm{CH}_{2}-2$ and $\mathrm{C}-3$. HMBC correlations from both protons of the methylene $\mathrm{CH}_{2}-2$ were observed to the oxy-substituted carbonyl C-1 completing the $\gamma$-methyl 
substituted carbon chain C-1 to C-5. Strong HMBC correlations were observed from the methyl singlet $\mathrm{CH}_{3}-20\left(\delta_{\mathrm{C}} 20.1, \delta_{\mathrm{H}} 1.34\right)$ to the methylene $\mathrm{CH}_{2}-5$, its attachment point the oxyquaternary centre $\mathrm{C}-6\left(\delta_{\mathrm{C}} 81.1\right)$ and another methylene $\mathrm{CH}_{2}-7\left(\delta_{\mathrm{C}} 40.6\right.$, $\delta_{\mathrm{H}}$ 1.42). These were corroborated by HMBC correlations observed from $\mathrm{H}_{2}-5$ to both C-4 and C-6. As shown in Figure 4.42, these correlations established the linear connectivity from $\mathrm{C}-1$ to $\mathrm{C}-7$ with oxycarbonyl substitution at $\mathrm{C}-1$, dioxysubstitution at C-3 and monooxygenation at C-6.
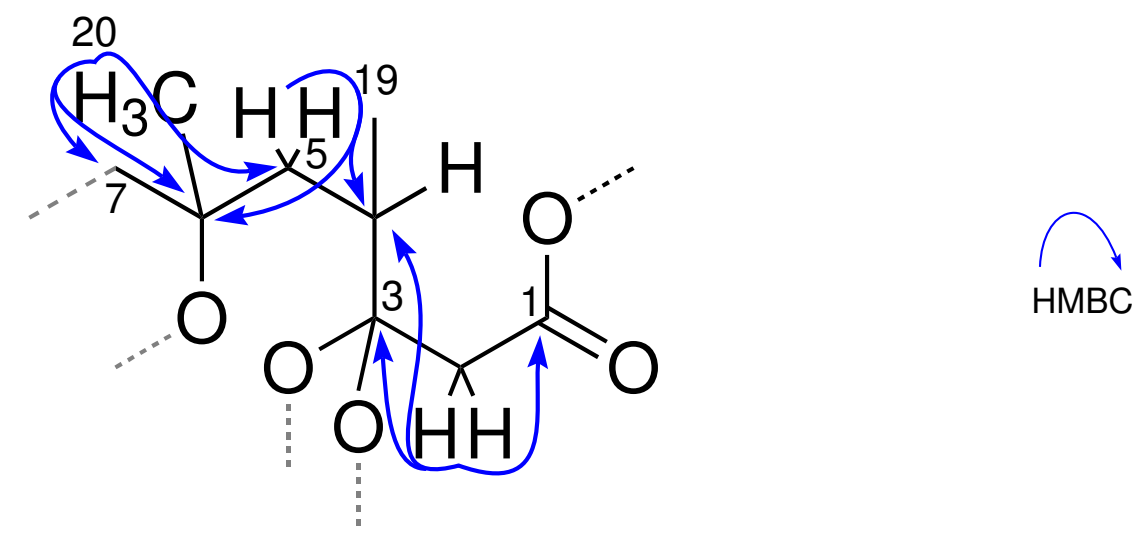

Figure 4.42 HMBC correlations proving the connections C- 1 to C-4 and C-5 to C-7 and methyl substitution of C-6 in $\mathbf{9 8}$.

A series of sequential COSY and HMBC correlations established the aliphatic chain extending from $\mathrm{CH}_{2}-7$ to $\mathrm{CH}_{3}-18$, with methyl substitution at $\mathrm{CH}-16$. Connection between the protonated centres $\mathrm{H}_{2}-7$ to $\mathrm{H}_{3}-18$ was proven via irradiation of $\mathrm{H}_{2}-7$, $\mathrm{H}_{2}-14$ and $\mathrm{H}_{3}-18$ with $1 \mathrm{D}$ TOCSY experiments over mixing times of $20-200 \mathrm{~ms}$, as shown in Figure 4.43.
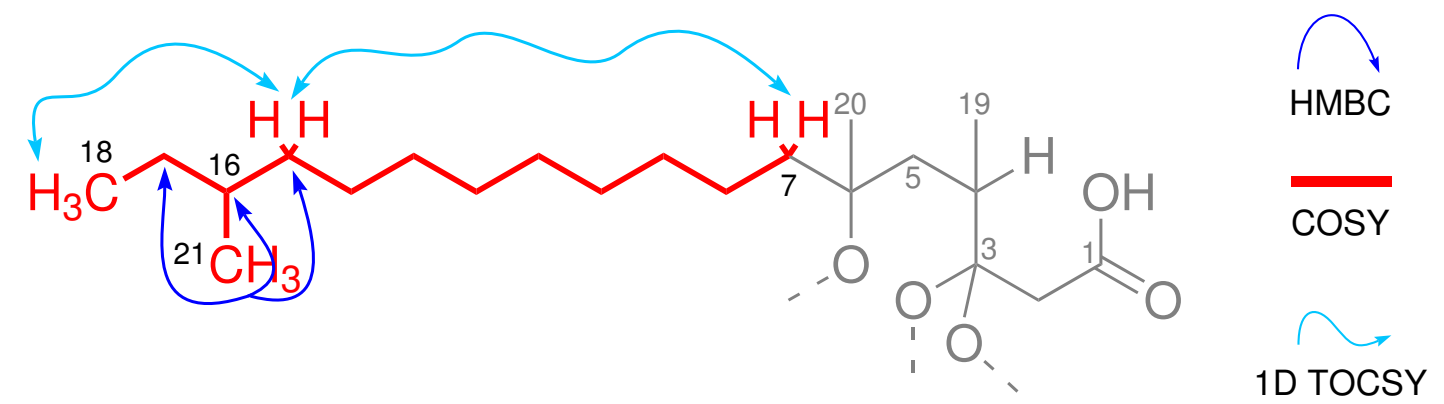

Figure 4.43 The completed linear structure of $\mathbf{9 8}$ as evidenced by COSY, 1D TOCSY and selected HMBC correlations. Double headed arrows indicate reciprocal correlations.

The presence of five oxygen atoms in the molecular formula, implied that C-1, C-3 and C-6 are substituted by different oxygens. As only two hydrogen atoms remained from the molecular formula, another degree of unsaturation required incorporation. Formation of a six-membered cyclic peroxide function bridging from the hemi-acetal centre C-3 to C-6, and an acid function at C-1 satisfied these requirements. The strong, reciprocal NOE correlations observed between methine $\mathrm{CH}-4$ and the C-6 
substituent, $\mathrm{CH}_{3}-20$, are consistent with their 1,3-diaxial positioning on the sixmembered peroxide ring. The methyl $\mathrm{H}_{3}-20$ and $\mathrm{CH}-4$ shared further strong NOE correlations with $\mathrm{H}-5 \mathrm{~b}$, while the $\mathrm{C}-4$ substituent $\mathrm{H}_{3}-19$ correlated strongly with $\mathrm{H}-$ $5 \mathrm{a}$ and weakly with $\mathrm{H}-5 \mathrm{~b}$. NOE enhancement was not observed between $\mathrm{H}_{3}-19$ and $\mathrm{H}_{3}$-20, indicating that they occupied opposing faces on the 1,2-dioxane ring (Figure $4.44)$.
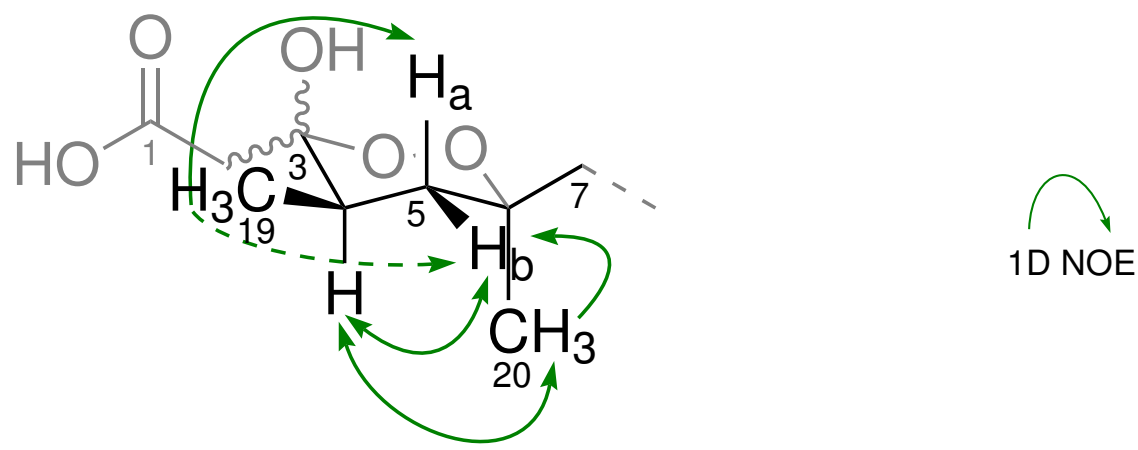

Figure 4.44 1D NOE correlations evidencing the 1,3-diaxial positioning of $\mathrm{CH}-4$ and $\mathrm{CH}_{3}-20$ and relative configuration at C-4 and C-6 in 98. Double headed arrows indicate reciprocal correlations.

The only other report of the 1-hydroxy-1,2-dioxane moiety of $\mathbf{9 8}$ is in compounds 99 and 100, metabolites isolated from a Plakortis (or Plakinastrella) specimen collected in Guam. ${ }^{173}$ Manzo et al. reported that 99 and 100 underwent degradative decarboxylation during the isolation process (reversed-phase HPLC in MeOH). ${ }^{173}$ Such degradation was not encountered during this study. Compound $\mathbf{9 8}$ also bears structural similarity to plakorin (101), ${ }^{120}$ although differing in the position of the hemi-acetal oxygen.
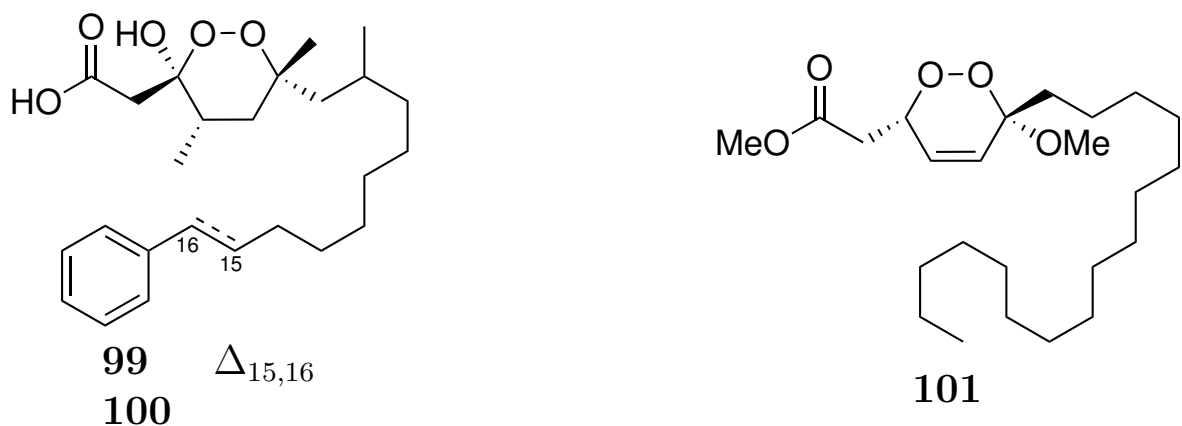

101

While the configuration at C-4 and C-6 in 98 was readily assigned by NOE correlations, configuration of C-3 was more difficult to determine, and was assigned through spectroscopic comparison with methyl capucinoate (102). Isolated from a Caribbean Plakinastrella onkodes specimen, ${ }^{128} 102$ shares the methylation pattern of 98, 99 and 100 but bears an axial hydrogen at C-3 in place of the hydroxyl group. 


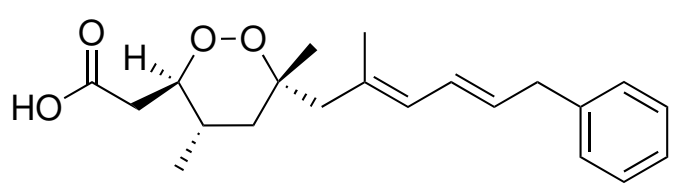

102

As with 98, the 1,3-diaxial orientation of H-4 and the C-6 methyl in compounds 99 and 100 was assigned on the basis of 1D NOE experiments. ${ }^{173}$ Chemical shift comparison of 99 and 100 with 102 led Manzo et al. to position the C-3 hydroxyl in 99 and 100 axially. The configuration at C-3 in 99 and 100 was inferred from the downfield chemical shift of the $\mathrm{H}-5_{a x}$ proton in 99 relative to $102 .{ }^{173}$ The same argument can be applied to 98. Consistent with 1,3-diaxial interactions with a hydroxyl function, the $\mathrm{H}-5_{a x}$ proton resonance appears $0.31 \mathrm{ppm}$ further downfield in 98 and 99 compared with the corresponding proton in $102\left[\left(\mathbf{9 8}: \delta_{\mathrm{H}} 1.66\right.\right.$, t, $J=12.8 \mathrm{~Hz}),\left(\mathbf{9 9}: \delta_{\mathrm{H}} 1.70, \mathrm{dd}, J=13.1,13.1 \mathrm{~Hz}\right)$ and $\left(\mathbf{1 0 2}: \delta_{\mathrm{H}} 1.39, \mathrm{t}\right.$, $J=12.8)$ ]. ${ }^{173}$ Other spectral data about the peroxide cores of compounds $\mathbf{9 8}-\mathbf{1 0 0}$ are virtually identical, implying conservation of relative configuration between the three compounds. Comparison of the chemical shift differences about the cyclic peroxide nuclei of $\mathbf{9 8}$ and $\mathbf{9 9}$ can be found in Table 4.14. NMR spectroscopic data for $\mathbf{9 8}$ is detailed in Table 4.15 .

Table 4.14 Comparison of Salient NMR Chemical Shifts in $\mathbf{9 8}$ and $\mathbf{9 9 .}$

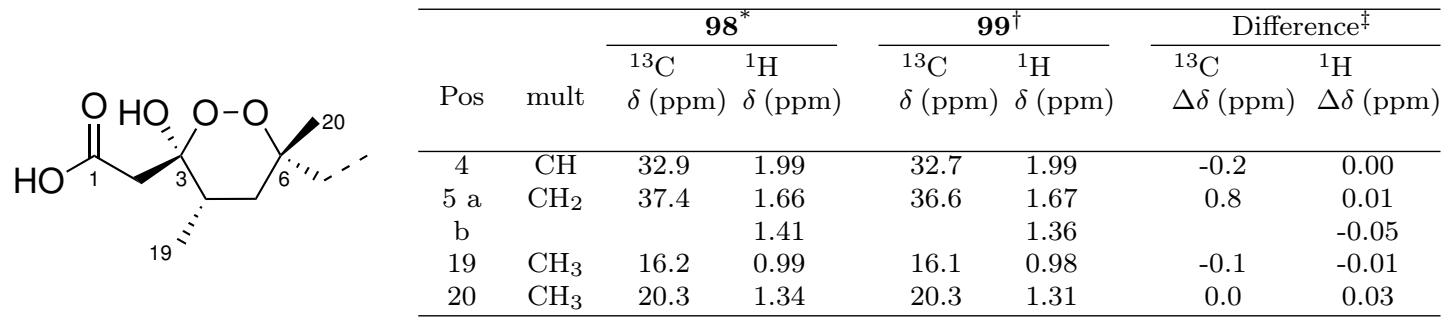

${ }^{*} \mathrm{CDCl}_{3},{ }^{1} \mathrm{H} 600 \mathrm{MHz} ;{ }^{13} \mathrm{C} 150 \mathrm{MHz}$.

$\dagger \mathrm{CDCl}_{3},{ }^{1} \mathrm{H} 400 \mathrm{MHz} ;{ }^{13} \mathrm{C} 75 \mathrm{MHz}$.

$\ddagger \Delta \delta=\delta_{\mathbf{9 9}}-\delta_{\mathbf{9 8}}$ 

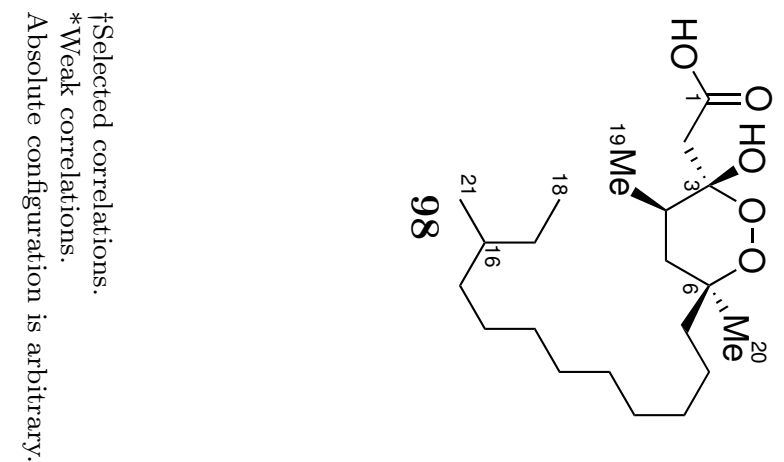

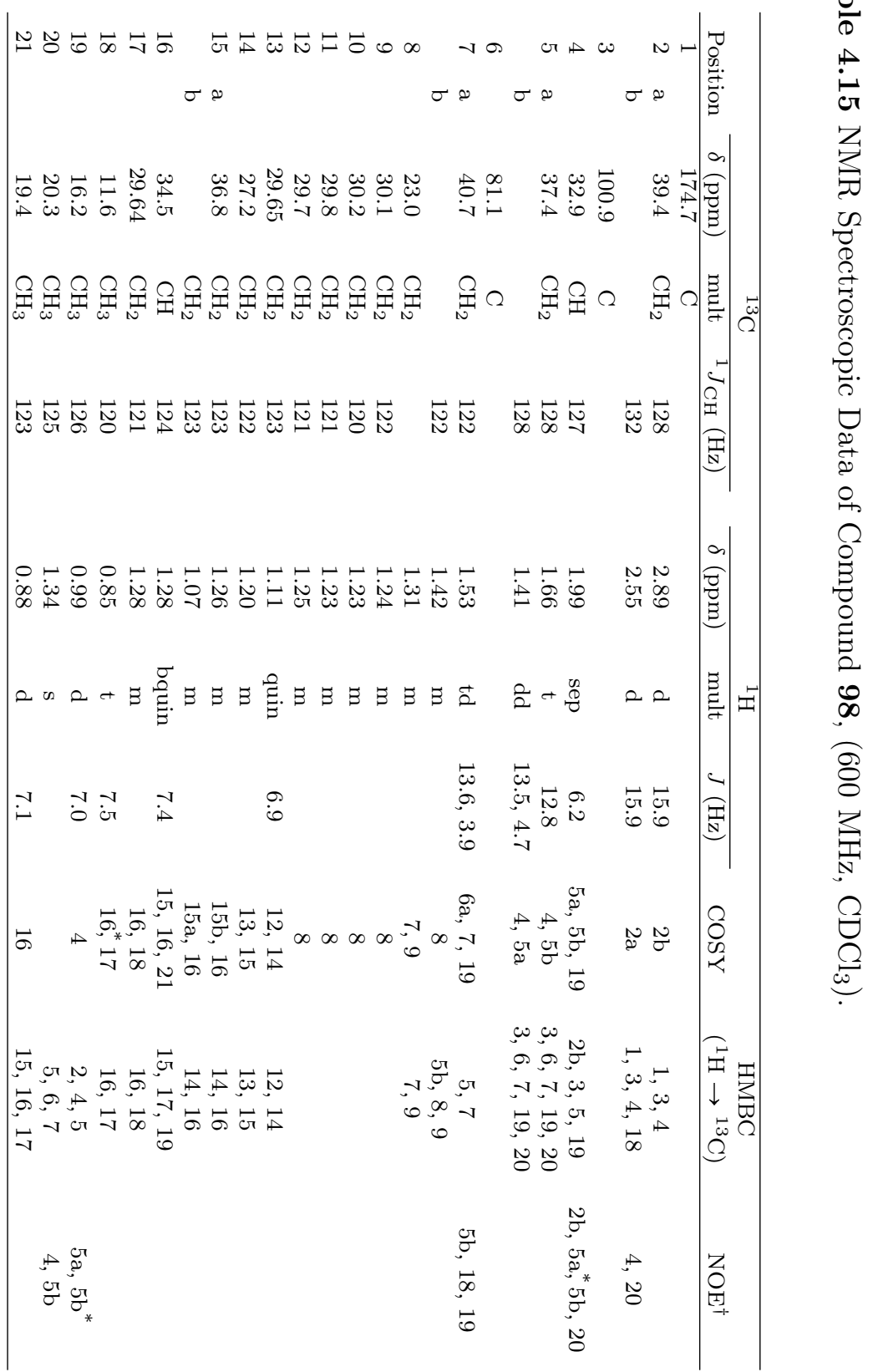




\subsubsection{Growth inhibition of eukaryotes}

Cyclic peroxides 95, 97 and 98 displayed cytotoxicity against the HL-60 cell line (Figure 4.45). ${ }^{93}$ Using a standard $48 \mathrm{~h}$ cell proliferation MTT assay, 95, 97 and 98 exhibited $\mathrm{IC}_{50}$ values for growth inhibition of $13.43,285.09$ and $3.59 \mathrm{nmol} \mathrm{L}-1$ respectively, while $5 \alpha, 8 \alpha$-epidioxysterols $\mathbf{2 7}$ and $\mathbf{2 8}$ showed far weaker inhibition of the same cell line with respective $\mathrm{IC}_{50}$ values of 6.48 and $7.90 \mu \mathrm{mol} \mathrm{L}{ }^{-1}$ (not shown). ${ }^{93}$

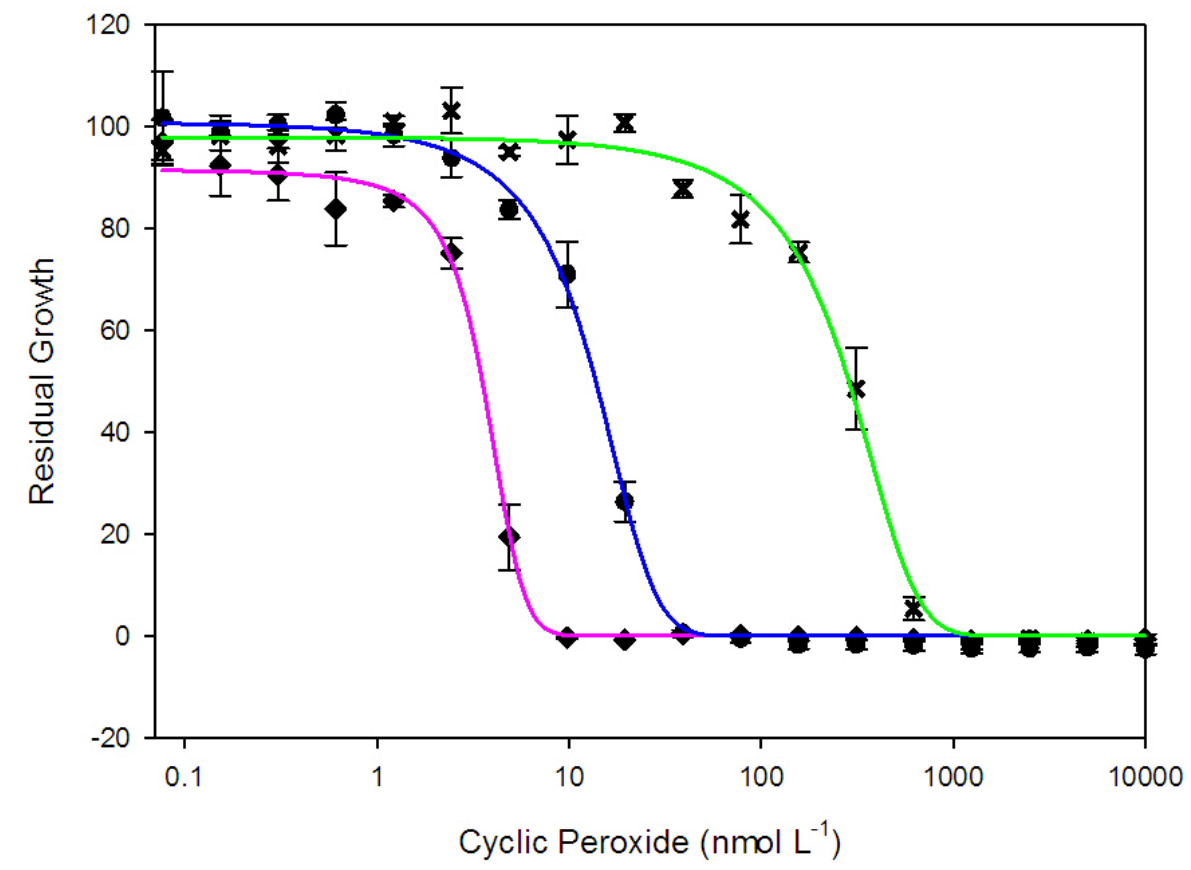

Figure 4.45 MTT-assay showing inhibition of the HL-60 cell line by $95(-\bullet-), \mathrm{IC}_{50}$ $13.43 \mathrm{nmol} \mathrm{L}-1 ; 97(-\times-), \mathrm{IC}_{50} 285.09 \mathrm{nmol} \mathrm{L}{ }^{-1}$; and $98(-\diamond), \mathrm{IC}_{50} 3.59 \mathrm{nmol} \mathrm{L}^{-1}$.

The biological activities of epiplakinic acid I (95) and plakortolide X (97) were further investigated using two strains of Saccharomyces cerevisiae yeast, a wild-type (WT) and a drug-sensitive mutant strain $(\Delta p d r)$. The $\Delta p d r$ strain carries deletions for the transcription factor genes, PDR3 and PDR3, which regulate expression of the pleiotropic drug resistance pump network. ${ }^{174}$ Unable to upregulate the PDR response, the $\Delta p d r$ strain is up to $3-100$-fold more sensitive to some xenobiotics than WT yeast. ${ }^{175}$ Interestingly, 95 displayed similar inhibition of WT and the $\Delta p d r$ mutant strain, $\mathrm{IC}_{50} 3.36$ and $3.50 \mu \mathrm{mol} \mathrm{L}{ }^{-1}$ respectively, indicating 95 is not a substrate for the PDR pump network. Conversely, 97 was ineffective against WT, and inhibited the $\Delta p d r$ strain $\left(\mathrm{IC}_{50} 4.45 \mu \mathrm{mol} \mathrm{L}{ }^{-1}\right)$. This suggests that 97 is a substrate for one of the PDR pumps; a change in biological activity that may be due to the change in carbonyl functionality. Chemical genetic screening of $\mathbf{9 5}$ and 97 is discussed in Chapter 5. 


\subsubsection{Organism classification}

Chemotaxonomic analysis of the sponge PTN3_19D, in combination with physical examination, points irrevocably to the plakinid genera Plakinastrella and Plakortis, although further distinction is difficult. A larger proportion of cyclic peroxides have been isolated from the Plakinastrella genus in comparison to Plakortis. Epiplakinic acids and plakortolide compounds have been found almost exclusively in Plakinastrella specimens, although the single report of the 1-hydroxy-1,2-dioxane nucleus of compound $\mathbf{9 8}$ is from a specimen of Plakortis sp. ${ }^{173}$ In all these cases the initial organism identification can be debated given the close relationship between the genera. In the case of 99 and 100, the specimen was originally identified as Plakinastrealla clathrata (Kilpatrick, 1911), but only one size-class of diod spicules and few irregularly-shaped triods were observed and the organisms was eventually reclassified as Plakortis. ${ }^{173}$

The exclusive occurrence of cyclic peroxides such as 95-98 in sponges of the Plakortis and Plakinastrella genera may indicate singular symbiosis of specific bacteria with sponges from the Plakinidae family, similar to that observed in Myxilla and Haliclona sponges. ${ }^{52}$ Microbial origins for the plakinid polyketides further explains subtle changes in metabolite profiles between specimens collected from different environments and locations. ${ }^{53}$ In stark contrast, the $5 \alpha, 8 \alpha$-epidioxysterols $\mathbf{2 7}$ and 28 have been isolated from sponges of many orders from different locations. This may indicate that $\mathbf{2 7}$ and $\mathbf{2 8}$ are products of sponge metabolism, which is less affected by subtle environmental variation, or simply products of bacteria common to both locations and sponge orders.

\subsubsection{Cyclic peroxide natural products}

A great number of cyclic peroxide sponge metabolites of both polyketide and terpenoid derivation have been isolated in addition to those from plakinidae sponges, a selection of which are detailed below.

Plakorin (101) was first isolated from an Okinawan Plakortis specimen, ${ }^{120}$ while its C-3 epimer chondrillin (103) has been encountered in Plakortis ${ }^{176,177}$ and Chondrilla sponges. ${ }^{178}$ Six plakorin-like metabolites were reported in 1993 from another collection of Okinawan Plakortis sponge, the crude extracts of which displayed potent antifungal activity. ${ }^{168}$ However, the metabolites were found to be too reactive to allow isolation by either (preparative) TLC or HPLC (the stationary phase was not specified), and their structures were elucidated from a semi-pure mixture. ${ }^{168}$ A specimen of Callyspongia sp. collected in New Guinea was found to contain two 
modestly cytotoxic alkyl-branched 1,2-dioxane carboxylates 104 and 105, ${ }^{179}$ sharing great structural similarity with Plakortis isolates the plakortides. ${ }^{127,180}$

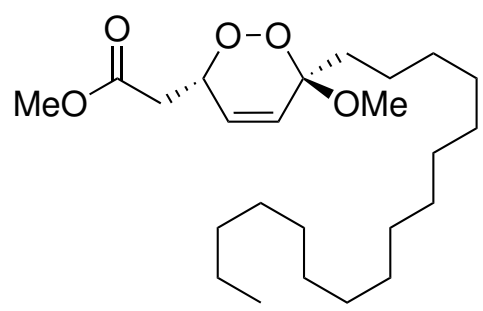

103<smiles>[R]C1C[C@](CC)(CCCC)OOC1C(=O)O</smiles>

$104 \mathrm{R}=\mathrm{CH}\left(\mathrm{CH}_{3}\right)_{2}$

$105 \mathrm{R}=\mathrm{CH}_{2} \mathrm{CH}_{3}$

A Thai collection of Mycale sp. yielded two norsesterterpene 1,2-dioxanes 106 and 107. ${ }^{181}$ differing by the hydroxyl and methyl substitution of C-9 and C10. The two compounds show diverse biological activity: inhibition of several tumor cell lines, antiviral activity against the vesicular stomatitis virus and HSV1, and growth inhibition of some gram positive bacteria. An Australian collection of the same genus afforded several similar norsesterterpene 1,2-dioxanes including 108, while an Australian Latrunculia conulosa specimen yielded several rearranged norsesterterpenes including 109, and the aptly named pentacycle contrunculin B (110). ${ }^{182}$<smiles>CCC(C)(C[C@@H]1CCC2C(C)(C)CCCC2(O)C1C)OOC(C)C(C)C(=O)OC</smiles>

106

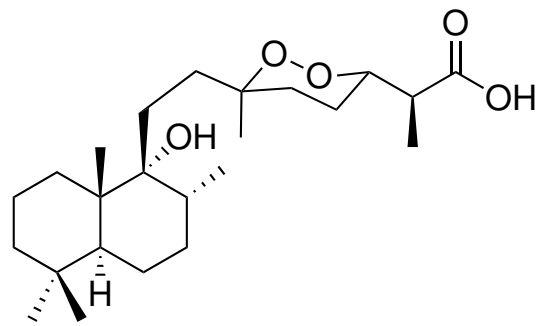

107

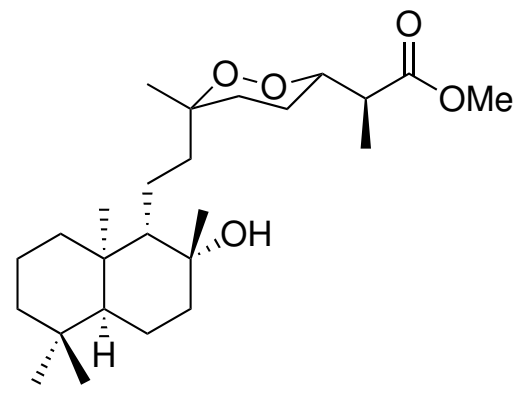

108<smiles>Cc1ccc2c(c1)C[C@H](C)[C@@H]1CC(C3(C)CCC([C@H](C)C(=O)O)OO3)O[C@H]21</smiles>

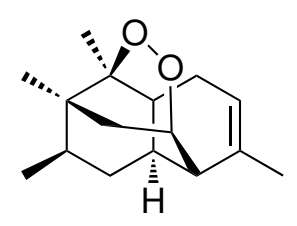

110 
Cyclic peroxides have widely been encountered in sponges, other marine invertebrates and their predators. The ascidian Stolonica socialis has produced metabolites most similar to those of plakinid origins. ${ }^{183}$ The stolonoxides feature a 2,5-trans-substitued tetrahydrofuran ring adjoined by a 1,2-dioxolane carboxylate, and a partially unsaturated long alkyl chain extending from the 5-position of the tetrahydrofuran ring. Recent additions to the class, stolonoxide E (111) and its diastereomer stolonoxide F, were isolated from a Spanish specimen of the ascidian. Both terminate in an unusual cyclooctatriene function, thought to form from an unstable terminal tetraene function. As stolonoxides A and C (112 and 113) both posses a terminal alkene separated from an internal diene by two methylene groups, the formation of a tetraene is not implausible. Whether formation of the 2,4,6cyclooctatriene moiety occurs in vivo or during isolation is uncertain, however. ${ }^{184}$ The stolonoxides all display low micromolar cytotoxicity across several mammalian cancer cell lines including the breast cancer (MDA-MB-231), colon adenocarcinoma (HT-29) and lung epithelial cell adenocarcinoma (A549). ${ }^{183}$

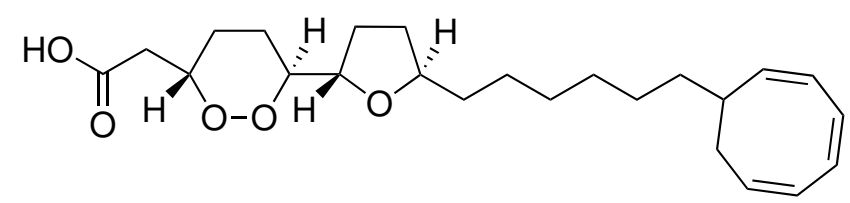

111

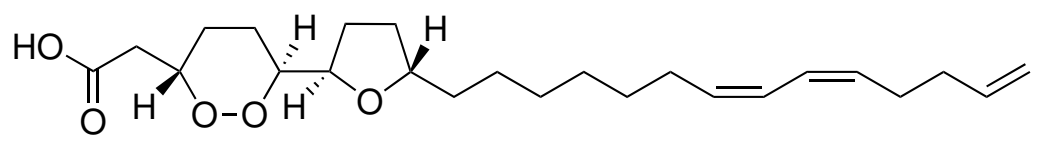

112

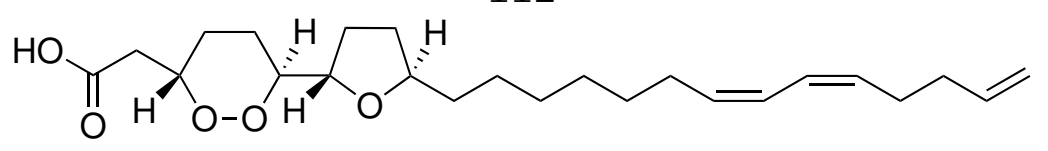

113

Soft corals, such as Labophytum denticulatum and other species within the genus have also been observed to incorporate peroxide linkages within their characteristic cembranolide diterpene carbon skeletons giving rise to structures such as 7epidenticulatolide (114). ${ }^{185}$ Hydroperoxides are also encountered within the marine environment, such as cembranoid $\mathbf{1 1 5}$ from the mollusc Planaxis sulcatus. ${ }^{186}$
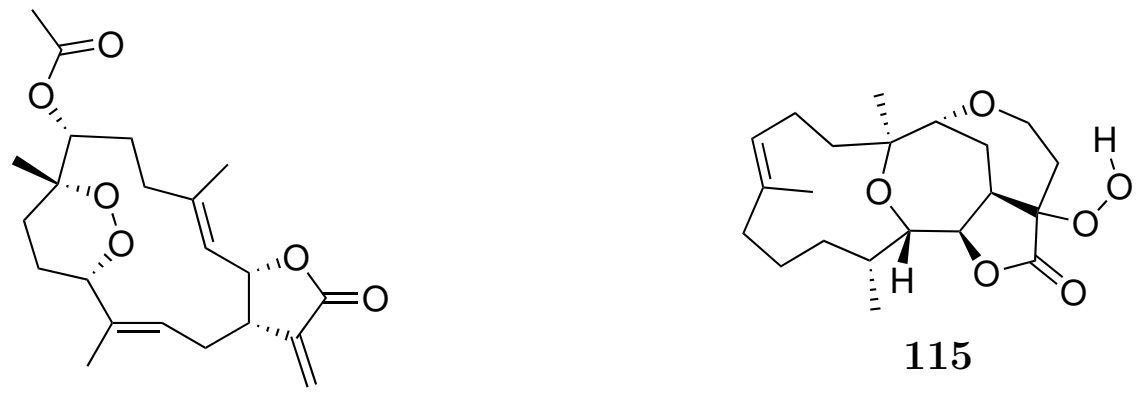

115

114 
Derived from the sweet wormwood Artemisia annua, the sesquiterpene artemisinins are the most noted terrestrial cyclic peroxide metabolites. ${ }^{187}$ Derivatives of the parent artemisinin skeleton (116), first reported in 1971, are now the standard treatment worldwide for Plasmodium falciparum malaria, delivering the most rapid action of all pharmaceuticals currently available to combat the disease. ${ }^{188}$

A wide-range of monoterpene and diterpene cyclic peroxide metabolites have also been reported from terrestrial sources, as have steroidal peroxides such as the ubiquitous $5 \alpha, 8 \alpha$-epidioxysterols. These classes are encountered widely in sponges, ${ }^{80}$ gorgonians and ascidians, ${ }^{83}$ with the peroxide function thought to form by addition of molecular oxygen across the $\Delta_{5,7}$ diene. Both the $5 \alpha, 8 \alpha$ - and $5 \beta, 8 \beta$ epidioxysterols have been the focus of many biological studies attributing antiviral, ${ }^{80}$ cytotoxic, ${ }^{80,83}$ immunosuppressive, ${ }^{189}$ and modest antibacterial ${ }^{190}$ activities to the carbon skeletons.

Fatty acid metabolites of both marine and terrestrial origins are final additions to this complement of cyclic peroxides. Lipoxygenase enzymes, present within a variety of organisms, act upon arachidonic acid and derivatives of linoleic acid producing a number of hydroperoxy-eicosatetraenoic acids such as $\mathbf{1 1 7}$, which affect a wide range of biological functions. ${ }^{191}$

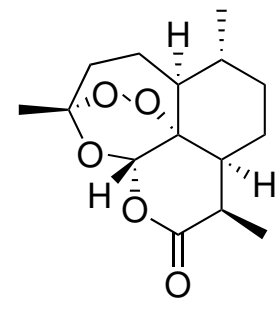

116<smiles>CCCCC/C=C/C=C/[C@H](CCCC(=O)O)OO</smiles>

117 


\subsubsection{Biogenesis of cyclic peroxides from plakinid sponges}

Cyclic peroxides 95-98 are of polyketide derivation, comprised predominantly of acetate monomers with three propionate units forming the methyl side-branches. There are two primary mechanisms proposed for formation of the cyclic peroxide moiety. The first involves Diels-Alder cycloaddition of singlet oxygen across a diene. ${ }^{192,193}$ Such an approach was employed in the synthesis of 6-epi-plakortolide $\mathrm{E},{ }^{194}$ however, as shown in Scheme 4.6, the regiospecific mechanism cannot account for the stereochemistry evident within some natural product classes. ${ }^{124}$ Attack of molecular oxygen at C-6 from the bottom face installs an arbitrary $6 S$ configuration, and a $(3 E, 5 Z)$-diene acid provides trans configuration of methyl groups as seen in 97. Yong et al. ${ }^{124}$ have noted that generation of cis plakortolides is unlikely by this mechanism, requiring either a change in diene configuration to $3 Z, 5 Z$ introducing unfavourable steric congestion, or attack of oxygen from the top face of a $3 E, 5 Z$ precursor disallowing $6 S$ configuration.

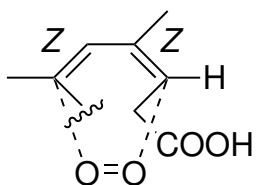
Diels-Alder
disfavoured<smiles>CC1(C)C=CCCCC1CC(=O)O</smiles><smiles>[3H][PH2+]</smiles><smiles>CC1(C)C[C@H]2CC[C@](C)(C1)OO2</smiles>

cis<smiles></smiles>

Diels-Alder favoured<smiles>CCC1(C)CC(C)CCC1(C)CC(=O)O</smiles><smiles>[PH2+][I-]</smiles><smiles>CC1(C)C[C@]2(C)OC(=O)C[C@H]2OO1</smiles>

trans

Scheme 4.6 Biosynthesis of cyclic peroxides via Diels-Alder cycloaddition of molecular oxygen as proposed by Yong et al. ${ }^{124}$

An alternate model from Ovenden and Capon rationalises biosynthesis of the norterpene sigmosceptrellins, such as 118, in a manner comparable to oxylipin 
biosynthesis, ${ }^{191}$ and can be applied to plakinid polyketide metabolites. ${ }^{195}$ As shown in Scheme 4.7, stereochemistry is defined in the first step where hydroperoxidation occurs on the re or si face of a skipped diene. The adjacent carbonyl activates the alkene to oxa-Michael addition by the hydroperoxide nucleophile, defining stereochemistry about the C-3 position. Conceivably, the overall mechanism could occur pre- or post-cleavage from the polyketide ACP domain. Support for the theory of carbonyl activation can be found in the structure of 118, which bears a $\gamma$-alkene and hydroperoxide in addition to an acid-substituted cyclic peroxide moiety. The absence of an activating carbonyl group appears to have impeded cyclisation of the hydroperoxide and $\gamma$-alkene functions. ${ }^{196}$

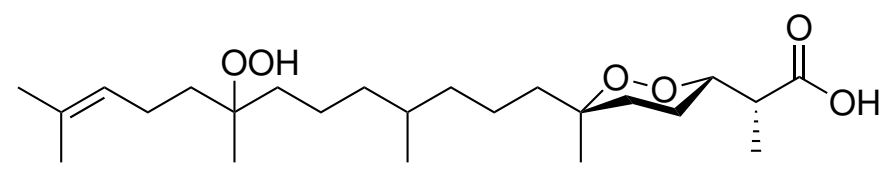

118

Application of the Ovenden-Capon mechanism to conjugated diene substrates generates diastereomeric plakinic and epiplakinic acids (see Scheme 4.8). As noted by Yong et al., migration of the $\Delta_{5}$ alkene to $\Delta_{4}$ via a 1,3-hydride shift prior to the initial hydroperoxidation introduces unsaturation observed in the plakortide skeleton (see Scheme 4.9). This provides a suitable electrophile for re or si attack by the terminal carboxylic acid generating both diastereomers of the plakortolide bicycle. ${ }^{124}$ Finally, as depicted in Scheme 4.10, the same mechanism can be invoked in the proposed biosynthesis of the 1-hydroxy-1,2-dioxane core of $\mathbf{9 8}$. The presence of a ketone at C-3 allows formation of both epimers of the hemi-acetal function.

However, the bis-1,2-dioxene polyketides, represented by 119, isolated from South African Plakortis aff. simplex specimens question the theory of carbonyl-activated cyclisation, or raises the possibility of cyclisation occurring by more than one mechanism within the same substrate. The second cyclic peroxide group could be a product of a $[4+2]$ cycloaddition of singlet oxygen across a diene; the stereochemical constraints discrediting this mechanism in some substrates are not present here. ${ }^{129}$

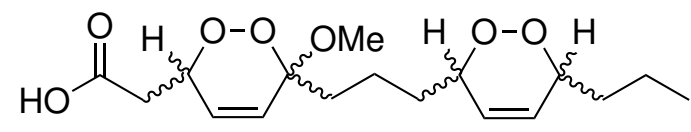

119 


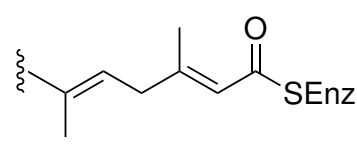<smiles>CCCCC(=O)C=C(C)CCC(C)(C)O</smiles>

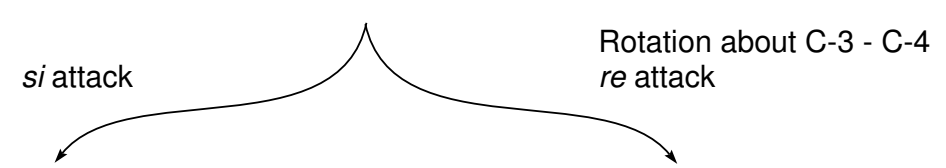

Followed by thioesterase-catalysed cleavage
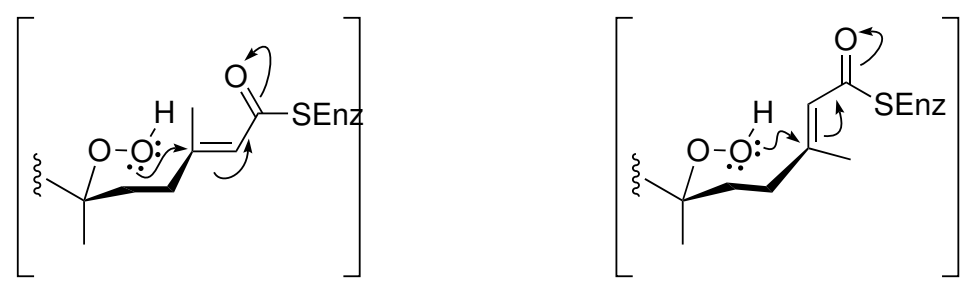

$\downarrow-\mathrm{H}^{+}$

$\downarrow-\mathrm{H}^{+}$
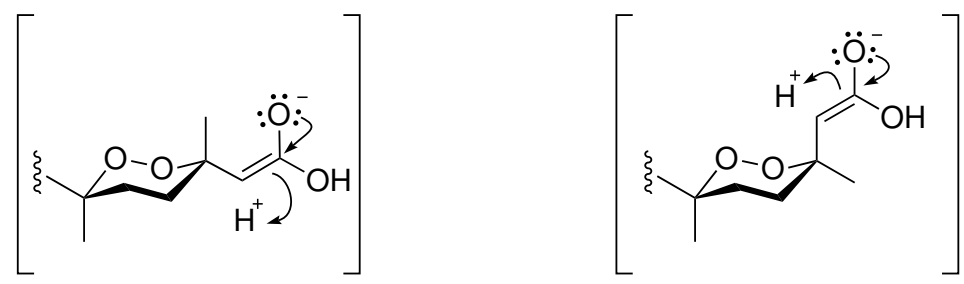

$\downarrow+\mathrm{H}^{+}$

$\downarrow+\mathrm{H}^{+}$<smiles>CC1(C)CC(C)(CC(=O)O)OO1</smiles><smiles>CC(C)(C)OOC(C)(CO)CC(=O)O</smiles>

trans

cis

Scheme 4.7 Proposed biosynthesis of terpenoid and polyketide cyclic peroxides via hydroperoxidation and subsequent oxa-Michael addition across an $\alpha, \beta$-unsaturated acid or ester, after Ovenden et al. ${ }^{195}$ 

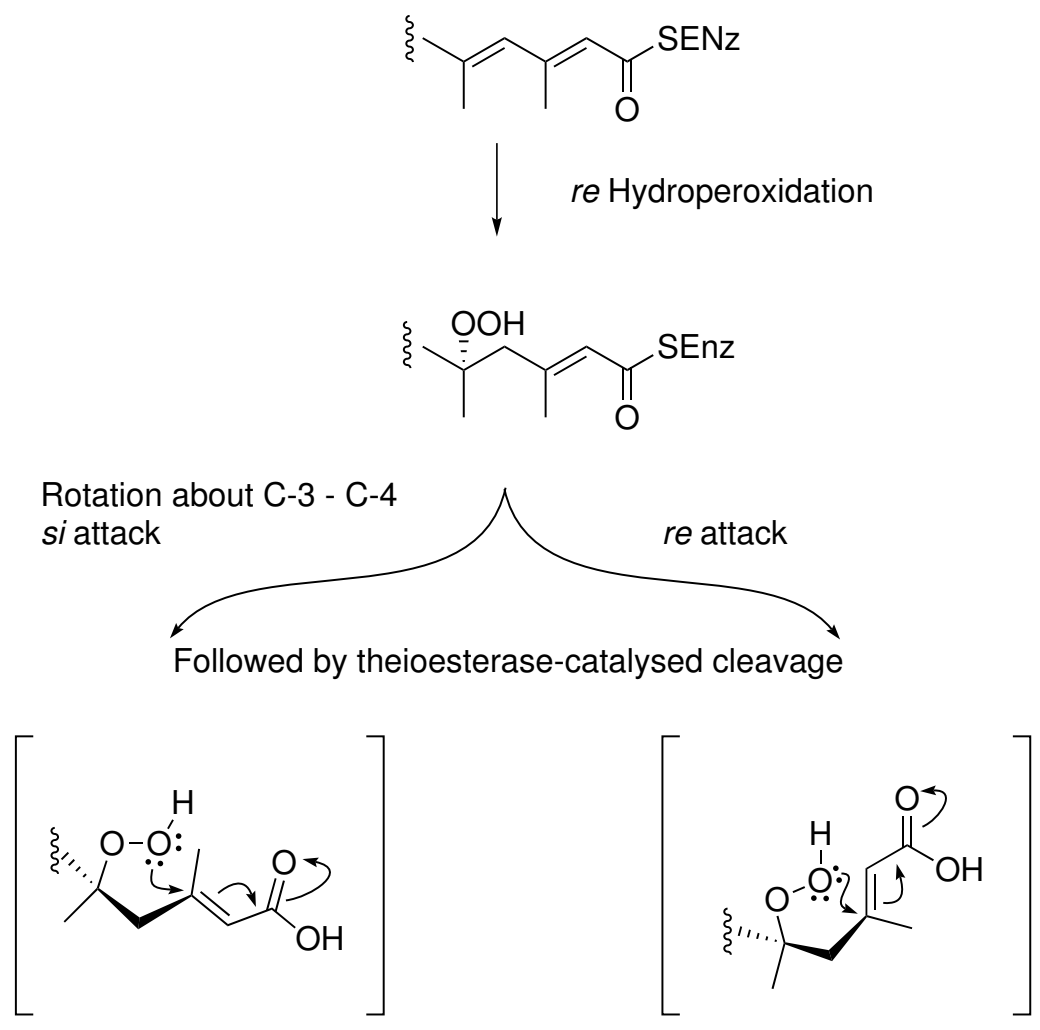

$\downarrow-\mathrm{H}^{+}$
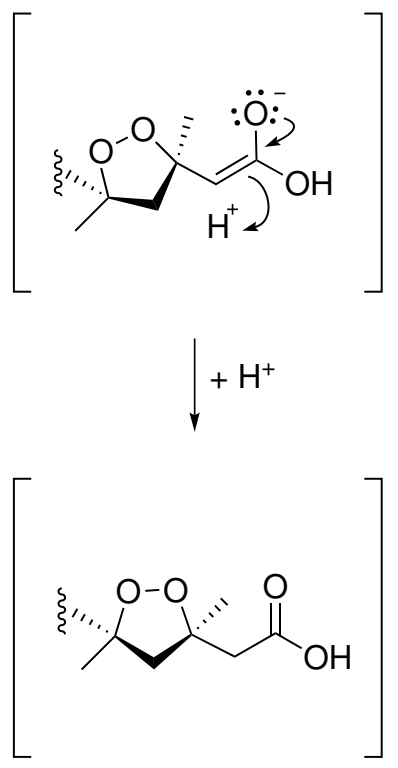

Epiplakinic acids

$\downarrow-\mathrm{H}^{+}$

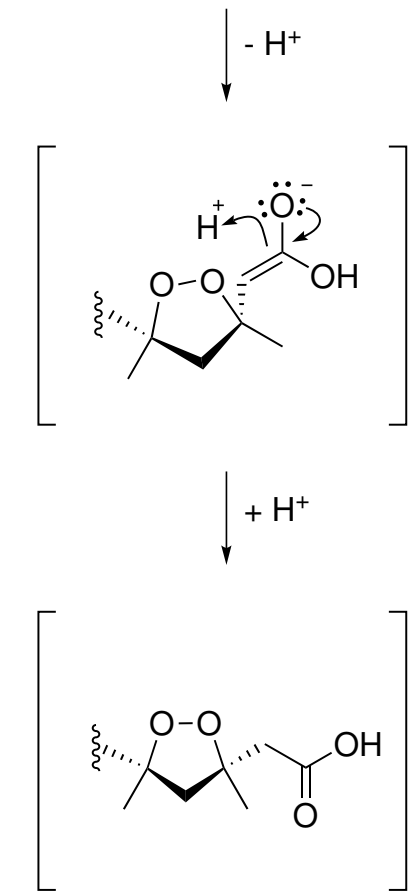

Plakinic acids

Scheme 4.8 Proposed biosynthesis of the 1,2-dioxane nuclei of plakinic and epiplakinic acids via hydroperoxidation and oxa-Michael addition. 

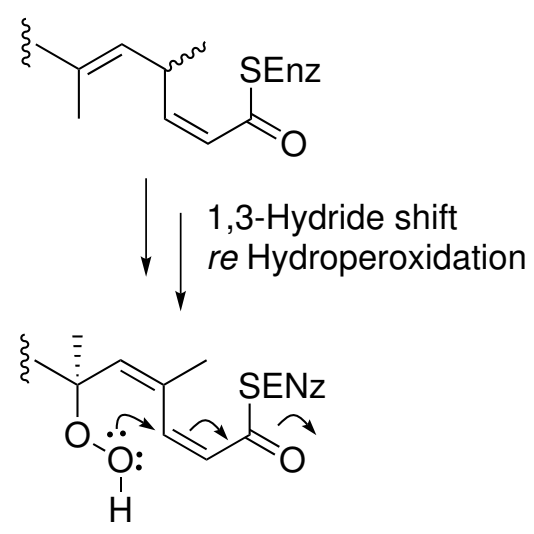

Followed by thioesterase-catalysed cleavage
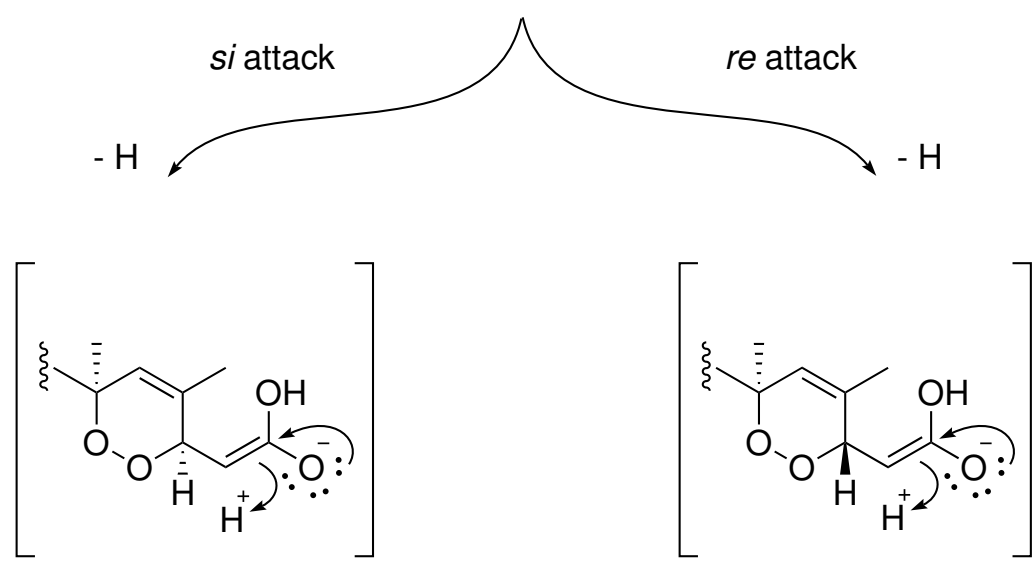

$\downarrow+\mathrm{H}^{+}$<smiles>[3H][PH3+]</smiles>

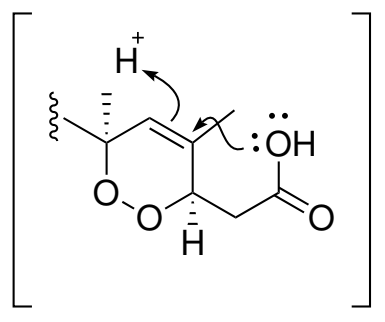

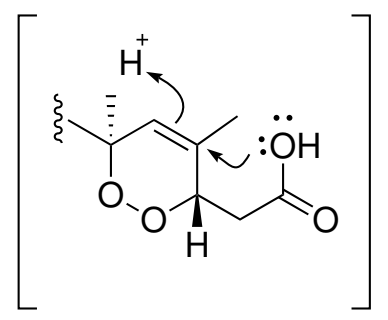<smiles>[3H][PH3+]</smiles><smiles>[3H][PH3+]</smiles><smiles>CC1C[C@H]2OC(=O)CC2OO1</smiles>

cis

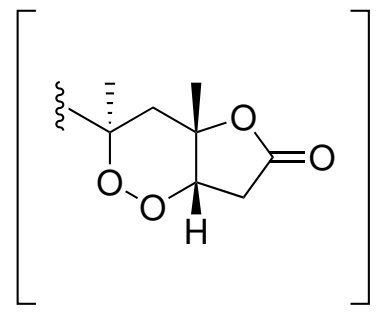

trans

Scheme 4.9 Proposed biosynthesis of the plakortolide bicycle by intramolecular cyclisation, after Yong et al. ${ }^{124}$ 
<smiles>CCCCCCCCCC(=O)CC(C)C=C(C)C</smiles>

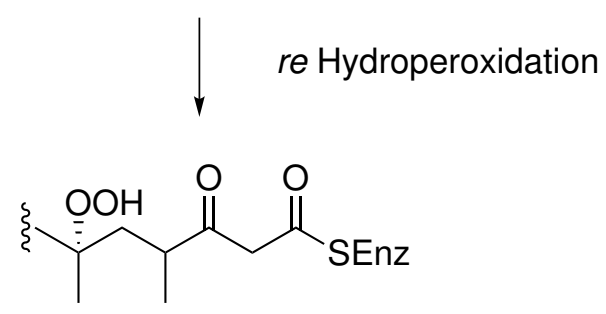

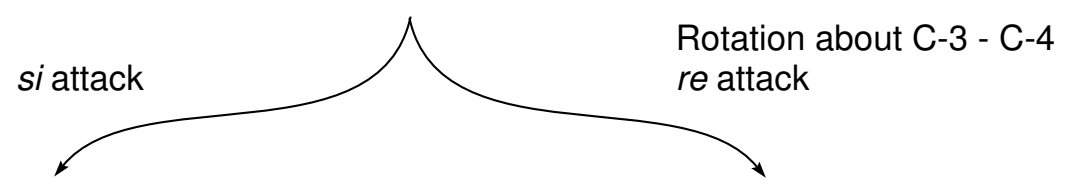

Followed by thioesterase-catalysed cleavage
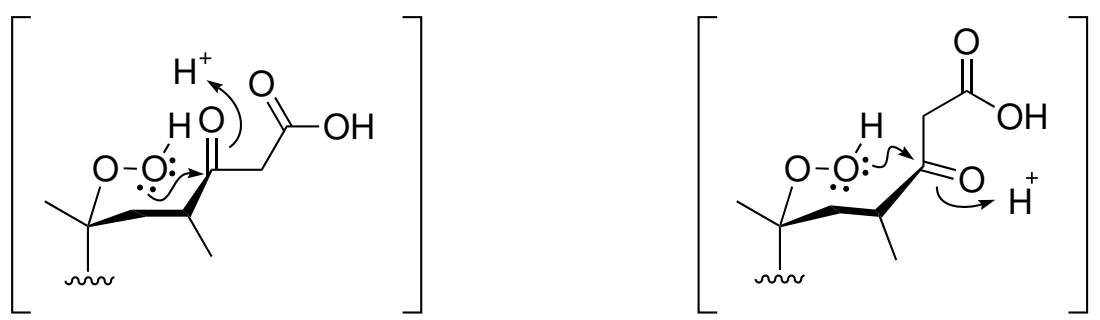

$\downarrow+\mathrm{H}^{+}$

$\downarrow+\mathrm{H}^{+}$

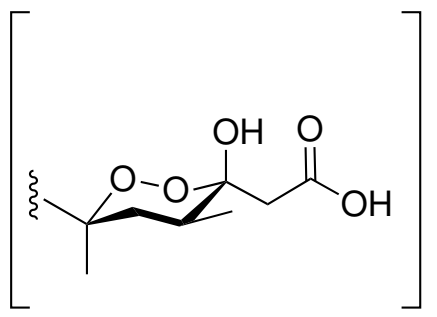

Axial

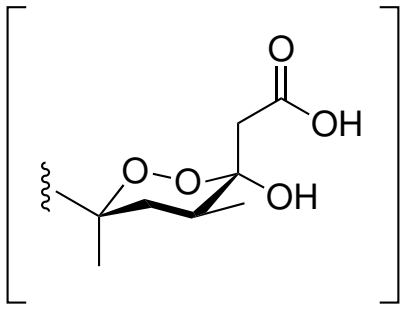

Equatorial

Scheme 4.10 Proposed biosynthesis of both epimeric forms of the 1-hydroxy-1,2-dioxane nucleus of $\mathbf{9 8}$. 


\subsubsection{Concluding remarks}

Overall, this study has furthered the chemical knowledge pertaining to the cyclic peroxide complement of plakinid sponges. Identification of the substituted peroxide functions was facilitated greatly by analysis of HMBC spectra during organism screening and selection. Correlations were observed from diastereotopic methylenes to the oxyquaternary centres within the cyclic peroxide moieties and to the adjacent oxycarbonyl centres. Identification of these oxygenated structural fragments at the screening stage was integral to the selection of this organism for further analysis.

Examples from three different cyclic peroxide classes were encountered: compounds 95 and 96 display the 1,2-dioxane moiety of the epiplakinic acids, the peroxide lactone bicycle of the plakortolide class is demonstrated by $\mathbf{9 7}$, while an uncommon 1-hydroxy-1,2-dioxane moiety was encountered in 98. These cyclic peroxides all exhibited surprising stability to oxidative degradation, despite precedence for degradation among similar compounds.

Metabolites 95, 97 and 98 were potently active against the HL-60 cell line, and their assessment against other cell lines will be undertaken in the future. The decreased sensitivity of HL-60 cells to $\mathbf{9 7}$ compared with $\mathbf{9 5}$ and $\mathbf{9 8}$ may reflect the absence of the free carboxylic acid moiety, an observation that may be useful to future development of the structures as structural leads. 


\section{Chapter 5}

\section{Chemical Genetic Profiling of Marine Cyclic Peroxides}

The spectroscopy-guided isolation approach applied during this study requires an unbiased assay technique for the biological characterisation of small molecules following their isolation. The suitability of genome-wide screening techniques as such an approach was explored. Chemical genetic profiling assays were carried out in Saccharomyces cerevisiae yeast in an effort to elucidate the precise biological mechanism of the cyclic peroxides presented in Chapter 4.

\subsection{The biological effects of marine cyclic peroxides}

Since the late 1980s, cyclic peroxide polyketides have been implicated in $\mathrm{Ca}^{2+}$ modulation. ${ }^{120,169,173,180}$ Plakorin (101) was shown to increase $\mathrm{Ca}^{2+}$ uptake by the cardiac sarcoplasmic reticulum ATPase in rabbit white skeletal muscle in 1989, ${ }^{120}$ and the same activity was assigned to the plakortone class in $1996 .{ }^{197}$ Excessive release of $\mathrm{Ca}^{2+}$ inside cardiac muscle can cause sudden cardiac death in heart failure patients. ${ }^{198}$ As such, the therapeutic potential of the plakortones in treating some forms of heart failure prompted efforts towards the synthesis of the 2,6dioxabicyclo[3.3.0]octane-3-one core of the class. ${ }^{199}$ Molluscan isolates, stolonoxides $\mathrm{A}$ and $\mathrm{C}$ (112 and 113), have been identified as inhibitors of the mitochondrial respiratory chain, ${ }^{184}$ specifically affecting the function of two protein complexes that are reliant upon $\mathrm{Ca}^{2+}$-signaling: complex II (succinate: ubiquinone oxioreductase) and complex III (ubiquinol: cytochrome C oxioreductase). ${ }^{200}$ Similarly, the plakortides have also been found to promote activation of the ATP-dependent sarcoplasmic reticulum (SR) $\mathrm{Ca}^{2+}$ pump. ${ }^{180}$ The antimalarial drug artemisinin (116) has also been shown to inhibit the SERCA-type $\mathrm{Ca}^{2+}$ transporter of the malaria parasite Plasmodium falciparum. ${ }^{201}$ Although structurally distinct from plakinid isolates, the $\mathrm{Ca}^{2+}$-modulatory activity of $\mathbf{1 1 6}$ is attributed to the peroxide moiety, and is 
thought to contribute to its antimalarial activity. ${ }^{201}$ The terpenoid metabolites $\mathbf{1 2 0}$ and the labdane $\mathbf{1 2 1}$ also inhibit $P$. falciparum in a manner similar to $\mathbf{1 1 6},{ }^{195,196,202}$ and again the cyclic peroxide group is thought to cause these effects.<smiles>CC1=C2CC/C(C)=C/CCC(C)(CC3(C)CC(C)(C(C)C(=O)OCc4ccccc4)O3)C1CCCC2(C)C</smiles>

120<smiles>CC1=C(CCC2(C)CCC([C@@H](C)C(=O)OCc3ccccc3)OO2)[C@H]2CCCC(C)(C)[C@@H]2CC1</smiles>

121

Recent investigations of plakortide $\mathrm{F}$ acid (122) by gene expression analysis in Saccharomyces cerevisiae, revealed significant changes in the levels of mRNA encoding proteins involved in $\mathrm{Ca}^{2+}$ homeostasis. ${ }^{169}$ The observed upregulation of these genes in response to $\mathbf{1 2 2}$ provided the first evidence for the widely acknowledged activity of this metabolite class in yeast.<smiles>CC/C=C/C(CC)CCC[C@]1(CC)C[C@H](CC)[C@@H](CC(=O)O)OO1</smiles>

122

Using these studies as guides an investigation of the cyclic peroxides detailed in Chapter 4 was embarked upon. Our aim was to characterise the biological mechanism of the plakinid cyclic peroxides presented in Chapter 4 by chemical genetic methods. Given the recognised lack of overlap between genes whose deletion causes sensitivity to a condition and mRNA's that are differentially regulated in the presence of the condition, ${ }^{76}$ the chemical genetic profiling methods utilised here should provide information complementary to that gained from the gene expression profiling by $\mathrm{Xu}$ et al.. ${ }^{169}$

\subsection{Biological assessment in S. cerevisiae}

The budding bakers yeast $S$. cerevisiae is an ideal model organism for higher eukaryote systems. The single-celled eukaryote is inexpensive, readily available, 
easily cultured in defined media, and exhibits obvious phenotypes such as growth inhibition and morphological changes. Furthermore, approximately $31 \%$ of the human genes implicated in disease have an orthologue in $S$ cerevisiae. ${ }^{203}$ The cell cycle of $S$ cerevisiae has been well studied and its extremely short replication time (ca. $90 \mathrm{~min}$ ) aids culturing in a laboratory environment, significantly decreasing growth-based assay times. The robust organism can be stably maintained in both haploid and diploid states, facilitating the generation of deletion strain libraries.

Yeast cells of all species are remarkably robust and resistant to many xenobiotics. Much of this innate resistance is due to their extensive network of membrane-bound pumps, termed the pleiotropic drug resistance (PDR) network. The PDR pumps presumably defend the cell, acting to eject xenobiotics within the cell before they can reach levels that affect growth. ${ }^{204}$ The transcription factor genes PDR1 and PDR3 encode the master regulators of the PDR pump network, and their simultaneous deletion in a haploid strain $(\Delta p d r)$ confers 3-100-fold greater sensitivity to inhibitory compounds compared to a wild type (WT) strain. ${ }^{175}$ Hence, molecules which display little or no activity in the WT can often be assessed in the drug-sensitive $\Delta p d r$ background, when the lack of activity is due to drug efflux.

\subsection{Chemical genetic profiling in $S$. cerevisiae}

The complete deletion of anyone of around $80 \%$ of $S$. cerevisiae genes has no obvious effect on cell viability, ${ }^{205}$ a phenomenon attributed to the functional overlap exhibited by many genes, termed genetic buffering. This buffering mechanism means the deletion of a single gene can be compensated for by other genes that can functionally assume its role. Chemical genetic interactions are observed when a deletion strain is exposed to a small molecule whose cellular target is encoded by another gene that is genetically buffering the initial deletion. These interactions are manifested as inhibition or death of the deletion strain, termed synthetic sickness or synthetic lethality, indicating processes or pathways that are affected by the small molecule and require compensation in its presence. In this way chemical genetic interactions identify genetic networks that buffer the phenotype induced by the small molecule.

Since completion of the $S$. cerevisiae genome sequence and functional annotation of much of the genome, ${ }^{206,207}$ a comprehensive and ordered array of haploid and diploid Yeast Knock-Out (YKO) strains covering the entire yeast genome have been described. ${ }^{75}$ Each carrying deletions for a single gene, the YKO strains have been utilised in the study of biologically active small molecules, aiding elucidation of biological mechanisms and identification of cellular targets. ${ }^{208}$ 
The presence of a unique molecular barcode within each deletion strain is integral to chemical genetic assays used in this study. In each strain the open reading frame is replaced by a growth-selection marker for resistance to the antifungal agent kanamycin. The marker is flanked by two 20-nucleotide barcodes (Up and Dn tags) unique to each yeast strain, which are adjoined on either side by a universal primer sequence common to the strains (Figure 5.1). These features allow identification of all deletion strains, and quantification of their relative representation from within a mixed population by PCR amplification. Incorporation of the universal primer sites allows tandem amplification of all barcodes, while the use of two tags makes for more robust data. ${ }^{75}$

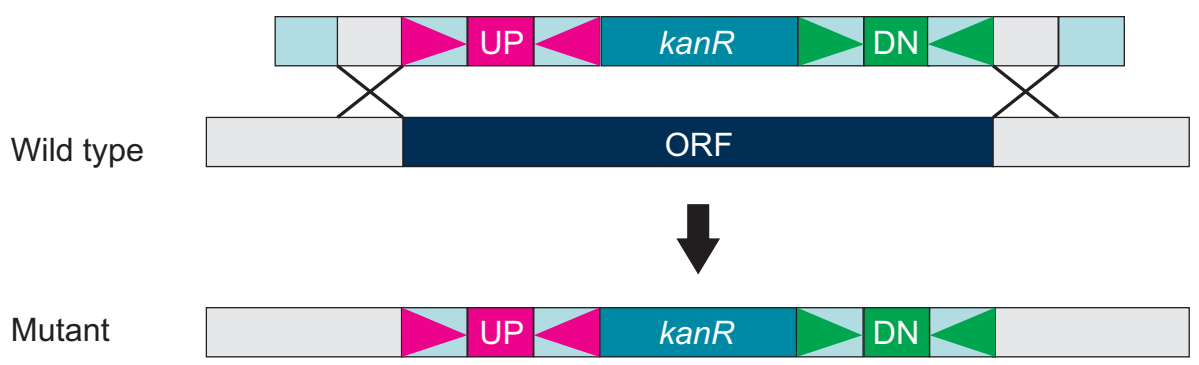

Figure 5.1 Deletion of the open reading frame deletion within the S. cerevisiae genome and insertion of the kanamycin resistance marker, after Boone et al. ${ }^{75} \mathrm{In}$ each YKO strain, the kanamycin resistance marker is flanked by two unique molecular barcode tags, each adjoined by universal primers. ${ }^{75}$

As depicted in Figure 5.2, under assay conditions a mixed culture containing every deletion mutant is grown in the presence of a potential drug at a concentration that inhibits $30 \%$ of the population - $\mathrm{IC}_{30}$. The genomic DNA is then extracted and the tags of each strain amplified in two tandem PCR reactions for the Up and Dn tags. The barcode amplicons of each YKO are hybridised to an oligonucleotide microarray, which carries the the complementary barcode sequences for the tags of each YKO. From the array, the relative abundance of each deletion strain can be quantified via the fluorescence signal intensity of their respective barcodes. High or low intensity of a barcode signal corresponds to high or low growth of that particular deletion strain within the population pool. ${ }^{75,203,209}$

Mutants carrying deletions for genes that are important for growth under a specific condition compete less effectively within the assay pool and diminish in population size decreasing the signal intensity of their molecular barcode. Hence, one experiment can identify the genes required for growth under a given condition, and rank them in order of their importance to fitness, i.e. their sensitivity to the experimental conditions. Homozygous profiling (HOP) analyses ca. 4,000 nonessential gene deletions using the homozygous diploid deletion set in which each strain is deleted for both alleles of a single non-essential gene. ${ }^{210}$ Interactions with essential genes are probed via haploinsufficiency profiling (HIP), which is conducted 
using the genome-wide heterozygous diploid deletion set in which only one allele of a given gene (essential and non-essential) is removed from a strain. ${ }^{76,209,211}$

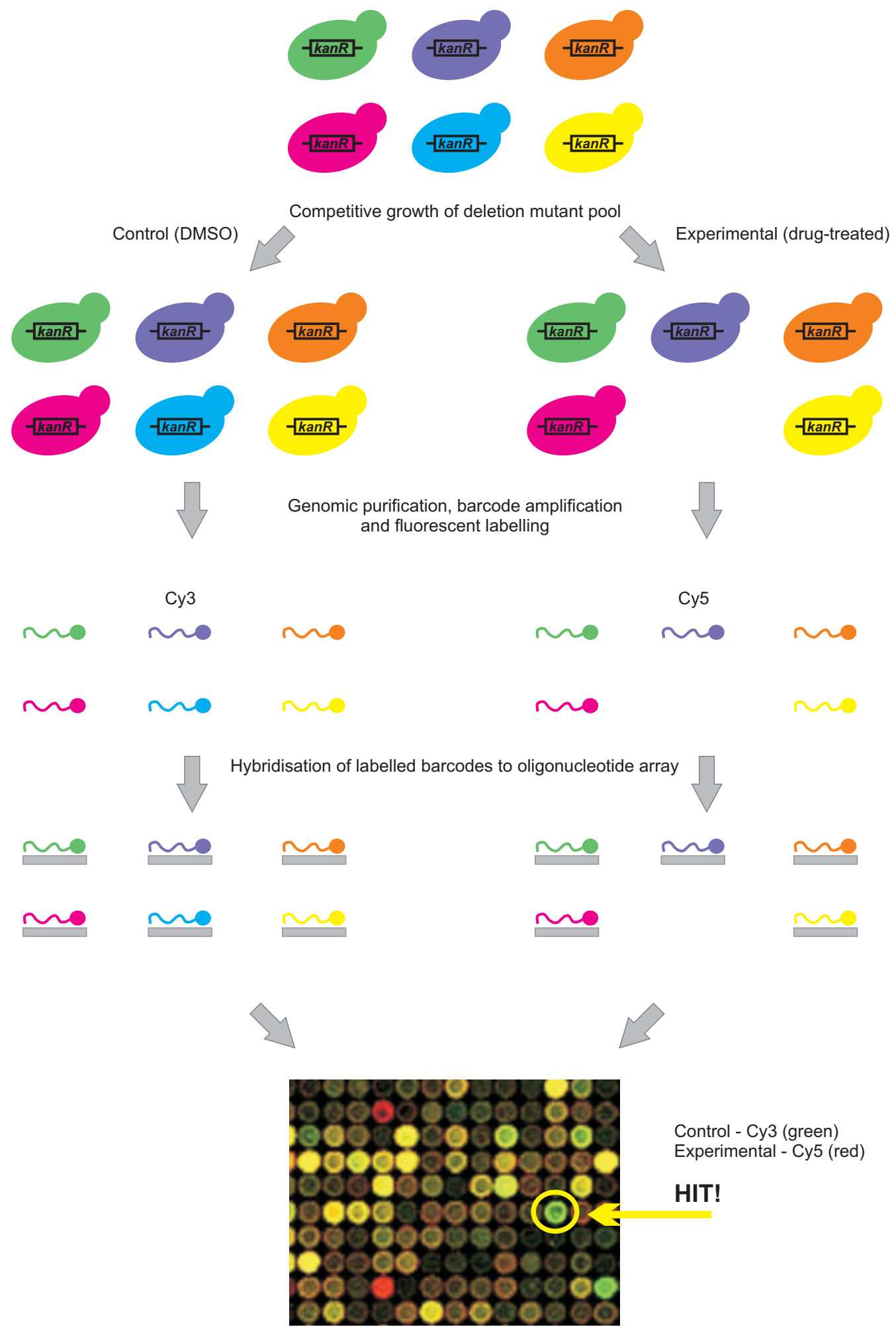

Figure 5.2 Chemical genetic profiling revealing the importance of a deleted gene to strain fitness under assay conditions, adapted from Boone et al. ${ }^{75}$ The deletion strains compete for growth in the assay pool, and those lacking genes important for growth under experimental conditions are out-competed and removed from the assay pool, as shown by the blue strain. This diminishes barcode intensity of the experimental population ( $\mathrm{Cy} 5$ ) relative to the control population $(\mathrm{Cy} 3)$ on the microarray slide. Strains unaffected by either condition appear yellow, while those detrimentally affected appear green. 


\subsubsection{Homozygous profiling}

Interactions uncovered by homozygous profiling (HOP) assays cannot reveal the primary cellular target of a bioactive molecule. Instead, synthetically sick or lethal interactions identify "friends of the target", genes which are related to the target of a small molecule in either cellular function or pathway and genetically buffer its absence. These genetic interactions point back to the unknown target in a "guilt by association" manner.

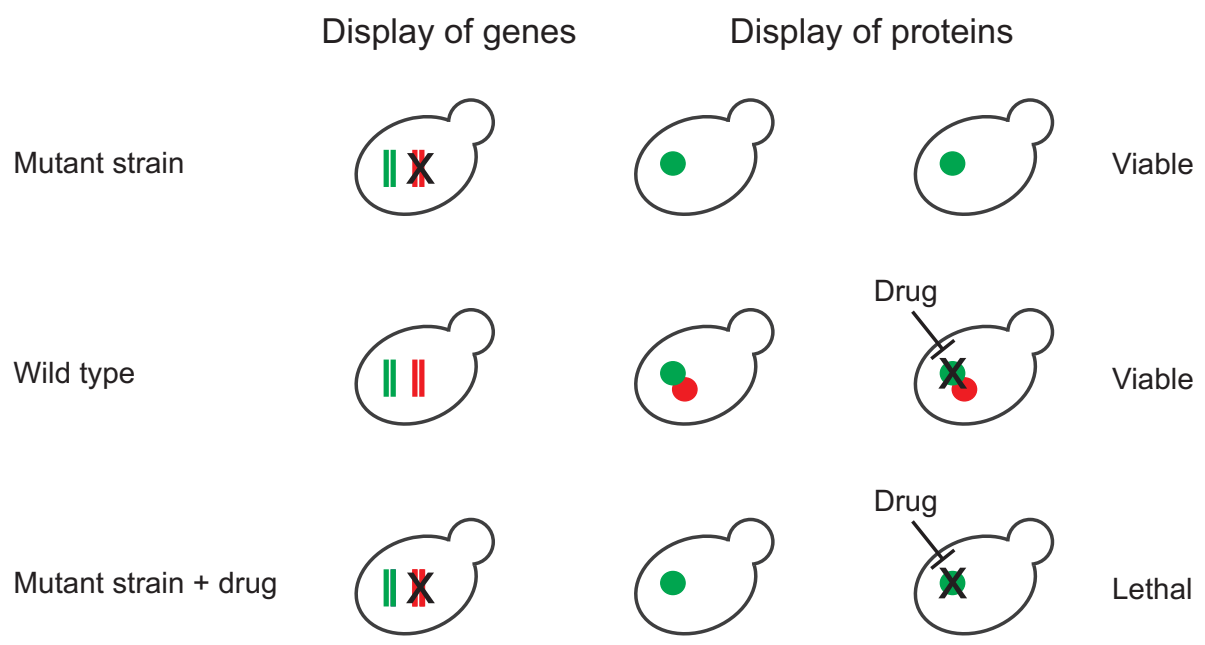

Figure 5.3 Synthetic genetic interactions uncovered by the homozygous profiling assay. A deletion mutant strain is viable except in the presence of a xenobiotic whose primary target is encoded by a gene buffering the initial deletion. These interactions identify genes related to the target of a small molecule in either cellular function or pathway.

\subsubsection{Haploinsufficiency profiling}

Concurrent assessment of both essential and nonessential gene interactions has the potential to identify a cellular target directly by the mechanism of drug-induced haploinsufficiency. ${ }^{212,213}$ This is achieved by screening the heterozygous diploid deletion mutant library, a near complete collection that omits only a few hundred (ca. $3 \%$ ) haploinsufficient genes. ${ }^{213}$

Haploinsufficiency profiling is based on gene dosage dependence. A heterozygous diploid mutant is deleted for one of two gene copies, halving the quantity of a given protein that can be produced by the cell. Under normal growth conditions, this reduced quantity of protein is sufficient to maintain proper function of the mutant strain. ${ }^{212,213}$ However, in the presence of a small molecule which targets the protein encoded by the heterozygous deletion, the mutant strain will be sensitised in comparison to strains with a full complement of the protein. ${ }^{212,213}$ This phenomenon is known as drug-induced haploinsufficiency. Synthetically lethal interactions are 
also revealed by HIP, therefore using both homozygous and heterozygous mutant screens is a complementary approach for identifying a cellular target. ${ }^{214}$

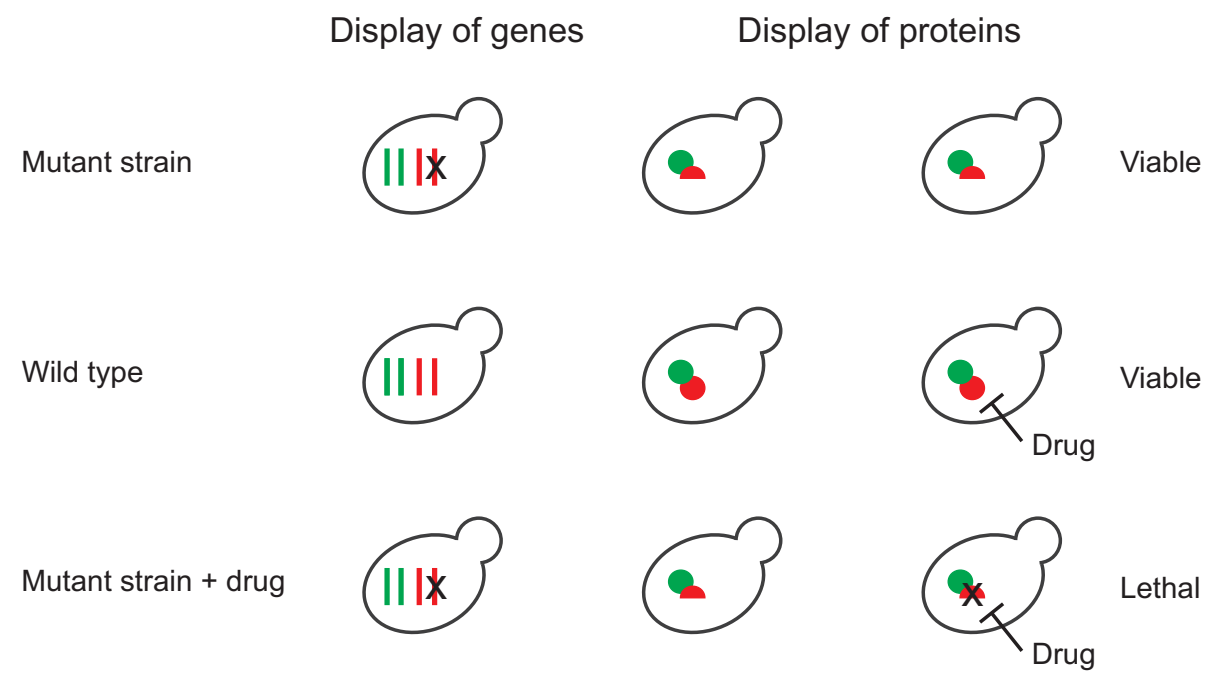

Figure 5.4 Depiction of drug-induced haploinsufficiency. A heterozygous deletion mutant can only produce half the quantity of a given protein in comparison to wild type yeast. However, the mutant remains viable except in the presence of a small molecule that targets the protein encoded by the heterozygous deletion. This sensitises the deletion strain relative to the wild type.

It is thought that the HIP assay is not applicable to compounds whose primary cellular target is encoded by an essential gene that is under dosage control. ${ }^{212,213}$ Upregulation of single gene copies is thought to circumvent the haploinsufficiency mechanism, ${ }^{213}$ potentially rendering important drug - gene-product interactions insignificant. It now appears however, that in general, protein levels in $S$. cerevisiae are not directly monitored and adjusted to a desired level. ${ }^{215}$

\subsection{Preliminary studies in S. cerevisiae}

As stated in Chapter 4, epiplakinic acid I (95) inhibited both haploid WT and $\Delta p d r$ strains, with $\mathrm{IC}_{50}$ values of 3.36 and $3.50 \mu \mathrm{mol} \mathrm{L} \mathrm{L}^{-1}$ respectively and a minimum inhibitory concentration (MIC) of $10 \mu \mathrm{mol} \mathrm{L}{ }^{-1}$ (Figure H.1 A*). Plakortolide X (97) was found to have no effect on the haploid WT strain but inhibited the haploid $\Delta p d r$ strain with an $\mathrm{IC}_{50}$ value of $4.45 \mu \mathrm{mol} \mathrm{L}{ }^{-1}$, and a $\mathrm{MIC}$ of $10 \mu \mathrm{mol} \mathrm{L}-1$ (Figure H.1 B). These results indicate that 95 is not subject to PDR efflux, whereas $\mathbf{9 7}$ may be a substrate for one of the PDR pumps. The primary structural difference between 95 and $\mathbf{9 7}$ is the absence of the free acid in $\mathbf{9 7}$, and it is fascinating that this results in such a difference in biological activity in yeast.

Owing to the robust nature of yeast cells, compounds that affect the growth of yeast are frequently cytostatic and inhibit their growth rather than killing

\footnotetext{
* Growth curves for all dose response assays can be found in Appendix $\mathrm{H}$
} 
them (cytotoxic). ${ }^{216}$ Some are truly cytotoxic, however, and induce a cell death process analogous to mammalian apoptosis. ${ }^{216,217}$ Colony-forming assays showed that following exposure to $\mathbf{9 5}$ for $17 \mathrm{~h}$ at its MIC, the drug-treated cells were unable to reproliferate on solid growth medium on the absence of 95. This is consistent with 95 causing irreversible cytostatic inhibition of yeast cells or being cytotoxic. Time-course assessment of the growth effects of $\mathbf{9 5}$ in WT yeast, indicated that $\mathbf{9 5}$ caused its effects within two hours of treatment at the MIC (Figure 5.5 A).

As cyclic peroxides are thought to affect $\mathrm{Ca}^{2+}$ modulation, inhibition assays were conducted in high and low $\mathrm{Ca}^{2+}$ environments. Consistent with 95 and 97 influencing intracellular $\mathrm{Ca}^{2+}$ levels, phenotypic enhancement was observed following co-treatment of WT and $\Delta p d r$ cells with a non-inhibitory concentration of $\mathrm{CaCl}_{2}$ and 95 or 97 (Table 5.1 and Figure H.2). Ethyleneglycol tetraacetic acid (EGTA) is a chelating ligand with a much higher affinity for $\mathrm{Ca}^{2+}$ over $\mathrm{Mg}^{2+}$ ions, and the ligand was used to produce a low $\mathrm{Ca}^{2+}$ environment. ${ }^{218}$ No recovery was observed upon co-treatment of WT cultures with 95 and EGTA (Figures 5.5 B and H.3).

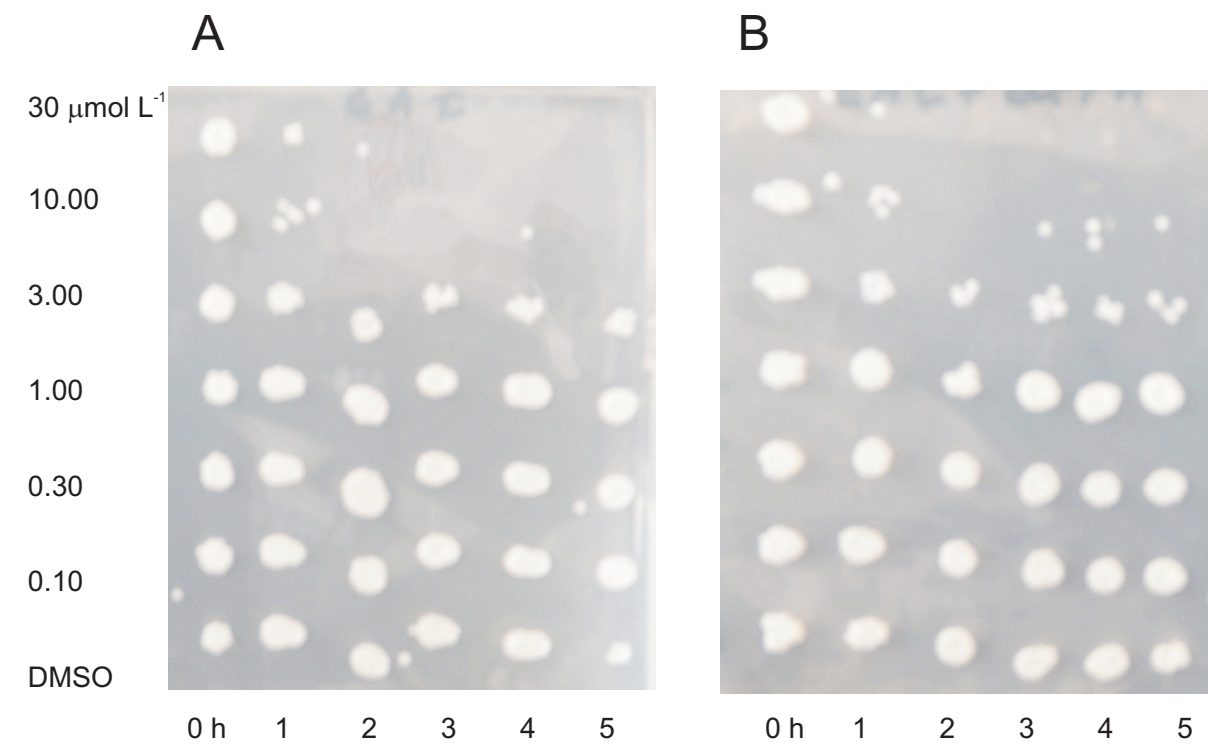

Figure 5.5 The kinetics of epiplakinic acid I (95) inhibiting S. cerevisiae cells are unchanged by the presence of EGTA. Plates were incubated for $72 \mathrm{~h}$ following inoculation.

A: $95\left(10 \mu \mathrm{mol} \mathrm{L}^{-1}\right)$ for $1 \mathrm{~h} \quad$ B: $\quad \operatorname{EGTA}\left(50 \mathrm{mmol} \mathrm{L}^{-1}\right)$ and $95\left(10 \mu \mathrm{mol} \mathrm{L}^{-1}\right)$

The observation of a significant increase in growth inhibition during co-treatment of cells with 95 and 97 and non-inhibitory concentration of $\mathrm{Ca}^{2+}$ is consistent with 95 and 97 affecting intracellular $\mathrm{Ca}^{2+}$ levels. However, the absence of phenotype recovery during co-treatment with EGTA is unexpected if the cyclic peroxides do affect their toxicity by modulating levels of the $\mathrm{Ca}^{2+}$ ion. However, the activities of 95 and 97 may also arise from release of $\mathrm{Ca}^{2+}$ from intracellular stores, in addition to influx of the ion from external sources. As such, an apparent lack of phenotype recovery may reflect an inability for the ligand to traverse the cell membrane and internally chelate cytosolic calcium. 
Table 5.1 Synergistic Inhibitory Effects of Cyclic Peroxides 95 and $\mathbf{9 7}$ and $\mathrm{CaCl}_{2}$.

\begin{tabular}{cccccc}
\hline & & \multicolumn{2}{c}{ Concentration } & & \\
\cline { 3 - 4 } Agent & Strain & $\begin{array}{c}\mathbf{9 5} \text { and } \mathbf{9 7} \\
\left(\mu \mathrm{molL}^{-1}\right)\end{array}$ & $\begin{array}{c}\mathrm{CaCl}_{2} \\
\left(\mathrm{mmolL}^{-1}\right)\end{array}$ & Residual Growth (\%) & Error $( \pm \%)$ \\
\hline \hline $\mathbf{9 5}$ & WT & 3.33 & & 90.4 & 0.5 \\
$\mathbf{9 5}$ & WT & 3.33 & 50 & 13.8 & 0.5 \\
$\mathbf{9 7}$ & $\Delta p d r$ & 6.35 & & 104.3 & 2.5 \\
$\mathbf{9 7}$ & $\Delta p d r$ & 6.35 & 50 & 4.6 & 9.0 \\
\hline
\end{tabular}

\subsubsection{Phenotypic profiling of the cyclic peroxides}

\section{Cellular Morphology}

Changes in cellular morphology were also analysed during assessment of epiplakinic acid I (95) and plakortolide X (97). Both compounds were found to decrease cell size and bud formation, with a similar decrease in cell size observed in response to $\mathrm{CaCl}_{2}$. Analysis by light microscopy showed a ca. $25 \%$ decrease in cell size in drugtreated populations (Table 5.2 and Figure 5.6). ${ }^{219,220}$ This may be symptomatic of cellular starvation. This was also observed using a GFP-tagged plasma membrane reporter strain (Figure H.5).
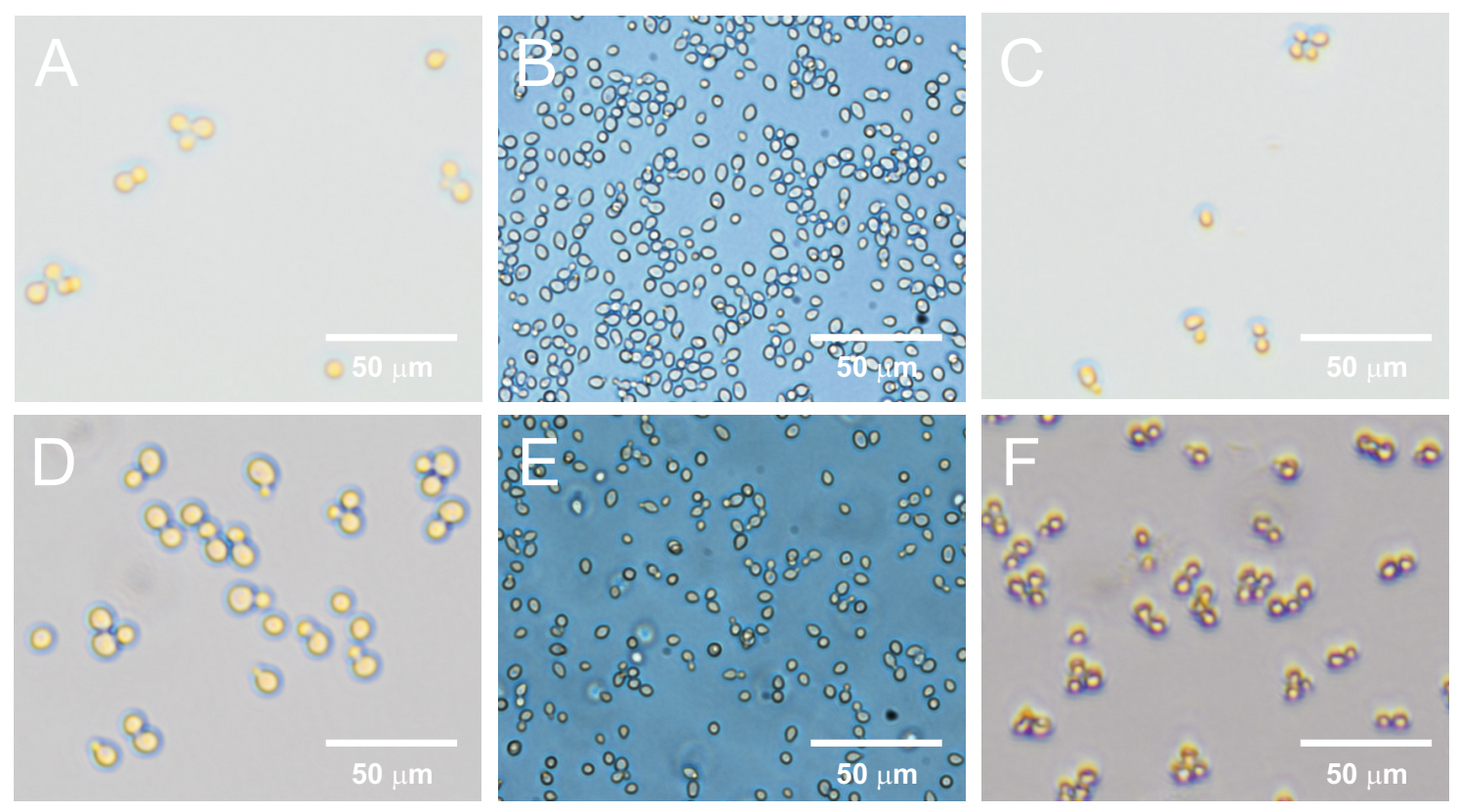

Figure 5.6 Epiplakinic acid I (95), plakortolide $\mathrm{X}(\mathbf{9 7})$ and $\mathrm{CaCl}_{2}$ cause a similar decrease in cell size in WT and $\Delta p d r S$. cerevisiae strains, respectively.
WT A: DMSO, $1 \%, 2 \mathrm{~h}$
B: $\mathrm{CaCl}_{2}, 50 \mathrm{mmol} \mathrm{L}^{-1}, 2 \mathrm{~h}$
C: $95,10 \mu \mathrm{mol} \mathrm{L}-1,2 \mathrm{~h}$
$\Delta p d r$
D: DMSO, $1 \%, 2 \mathrm{~h}$
$\mathrm{E}: \mathrm{CaCl}_{2}, 50 \mathrm{mmol} \mathrm{L}^{-1}, 2 \mathrm{~h}$
F: $\quad \mathbf{9 7}, 10 \mu \mathrm{mol} \mathrm{L}^{-1}, 2 \mathrm{~h}$ 
Table 5.2 Decrease in Cell Size Caused by 95, 97 and $\mathrm{CaCl}_{2}$ after $2 \mathrm{~h}$.

\begin{tabular}{ccccc}
\hline Agent & Strain & Concentration & Cell Size $(\mu \mathrm{m})$ & Error $( \pm \mu \mathrm{m})$ \\
\hline \hline $\mathbf{9 5}$ & WT & $10 \mu \mathrm{mol} \mathrm{L}^{-1}$ & 5.6 & 1.1 \\
$\mathrm{DMSO}$ & $\mathrm{WT}$ & $1 \%$ & 7.2 & 0.6 \\
$\mathrm{CaCl}_{2}$ & $\mathrm{WT}$ & $50 \mathrm{mmol} \mathrm{L}^{-1}$ & 4.9 & 0.6 \\
$\mathbf{9 7}$ & $\Delta p d r$ & $10 \mu \mathrm{mol} \mathrm{L}^{-1}$ & 3.9 & 1.6 \\
$\mathrm{DMSO}$ & $\Delta p d r$ & $1 \%$ & 8.6 & 1.1 \\
$\mathrm{CaCl}_{2}$ & $\Delta p d r$ & $50 \mathrm{mmol} \mathrm{L}^{-1}$ & 4.9 & 0.6 \\
\hline
\end{tabular}

\section{Cell cycle analyses}

The eukaryotic cell cycle consists of four distinct phases: $\mathrm{G}_{1}$-phase, S-phase, $\mathrm{G}_{2^{-}}$ phase (collectively known as interphase), and M-phase (comprising mitosis, in which the cell's duplicated chromosomes are divided between the two daughter cells, and cytokinesis, when the cytoplasm is divided in half, forming distinct cells). Initiation of each phase relies upon proper progression and completion of the previous phase, which is ensured by the phase-boundary checkpoints. A fifth phase, $\mathrm{G}_{0}$ or stationary phase, is entered when cells have temporarily stopped dividing. In yeast, this phase is entered in response to periods of environmental hardship or cellular starvation. ${ }^{221}$

As depicted in Figure 5.7, progression through the mitotic cell cycle of $S$. cerevisiae can be observed through bud analysis. The $\mathrm{G}_{1}$-S-phase transition is characterised by the formation of the bud site. ${ }^{222}$ The bud develops throughout S-phase, concurrent with DNA replication, as polarised actin migrates within the cell to the bud site, outwardly distorting the cell membrane and directing growth of the bud in the direction of polarisation. This continues into $\mathrm{G}_{2}$-phase. Budding yeast differ from mammalian cells systems in that the replicated genetic material only migrates into the bud/daughter cell during the $\mathrm{G}_{2}-\mathrm{M}$ transition. At this point the bud/daughter cell is fully formed, and nearly the same size as the mother cell, mitosis occurs followed by cytokinesis and the daughter cells are released.

An increase in small budded cells was observed in WT and $\Delta p d r$ cells in response to 95 and 97, confirmed by bud index (Figure 5.8). This indicated cell cycle delay during either the $\mathrm{G}_{1-\mathrm{S}} \mathrm{S}$ transition, or early S-phase, leading to a decrease of the $\mathrm{G}_{2}$ population. Flow cytometry analysis of WT populations treated with $\mathbf{9 5}$ supported this hypothesis, revealing a $50 \%$ increase S-phase populations in cells treated with 95 at the $\mathrm{IC}_{50}$, while treatment at the MIC appeared to inhibit during $\mathrm{G}_{2}$ (Table 5.3 and Figure H.4). These different results may be due to difficulties in distinguishing between the two phases in yeast via flow cytometry. ${ }^{216}$ 


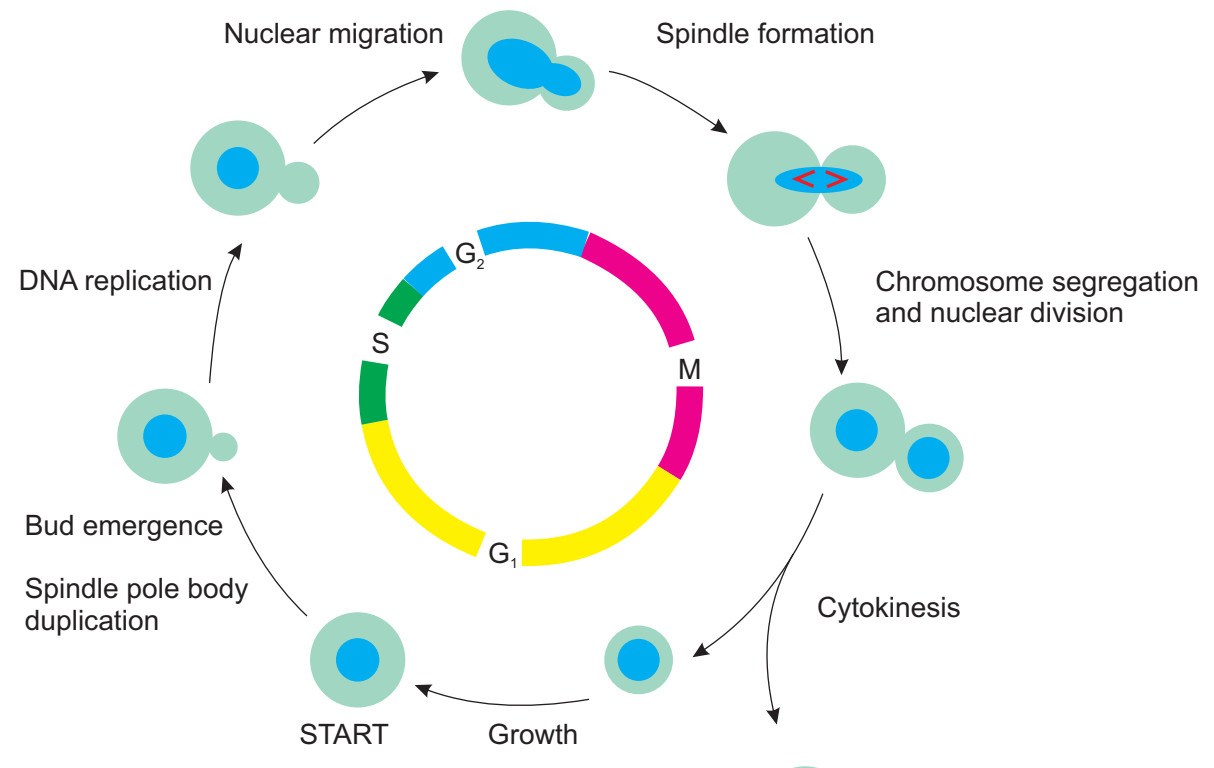

Figure 5.7 Cell cycle of $S$. cerevisiae after Calzone et al. ${ }^{223}$

Table 5.3 Effects of $\mathbf{9 5}$ on the S. cerevisiae Cell Cycle.

\begin{tabular}{ccccc}
\hline & & \multicolumn{3}{c}{ Phase population (\%) } \\
\cline { 3 - 5 } Agent & Concentration & $\mathrm{G}_{1}$ & $\mathrm{~S}$ & $\mathrm{G}_{2}$ \\
\hline \hline $\mathbf{9 5}$ & $3.4 \mu \mathrm{mol} \mathrm{L}^{-1}\left(\mathrm{IC}_{50}\right)$ & 30.5 & 40.9 & 58.9 \\
$\mathbf{9 5}$ & $7.0 \mu \mathrm{mol} \mathrm{L}^{-1}$ & 31.0 & 18.8 & 67.1 \\
$\mathbf{9 5}$ & $10 \mu \mathrm{mol} \mathrm{L}^{-1}(\mathrm{MIC})$ & 26.9 & 29.9 & 52.3 \\
DMSO & $1 \%$ & 40.4 & 18.4 & 55.6 \\
\hline
\end{tabular}

A

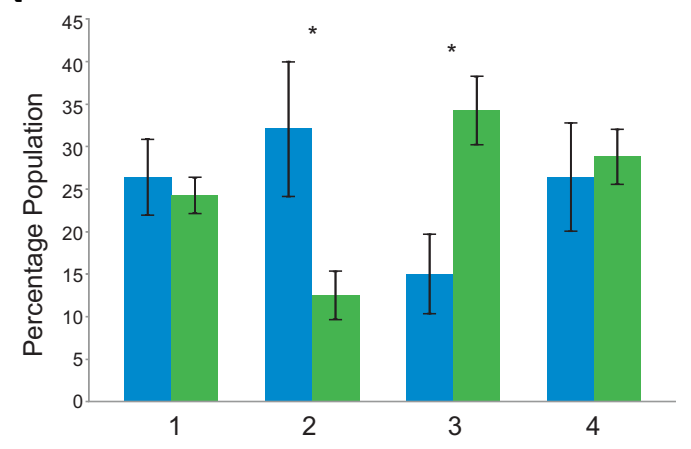

B

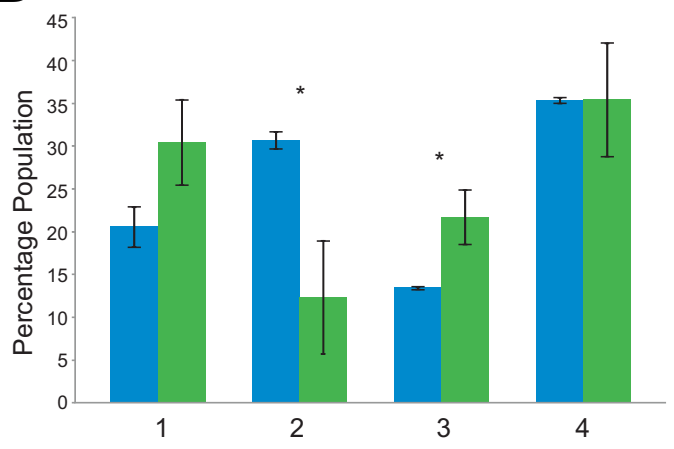

Figure 5.8 S. cerevisiae WT and $\Delta p d r$ cell populations displayed a small bud phenotype when treated with $\mathbf{9 5}$ and $\mathbf{9 7}$, correlating to an increase in S-phase populations at the expense of the $\mathrm{G}_{2}$ populations. $1=$ no bud, $2=$ small bud, $3=$ medium, $4=$ equal size.

* Indicates statistical significance as determined by T-test.

A: WT cells treated with $\mathbf{9 5}, 10 \mu \mathrm{mol} \mathrm{L^{-1 }}$ and DMSO, $1 \%$

B: $\Delta p d r$ cells treated with $\mathbf{9 7}, 10 \mu \mathrm{mol} \mathrm{L}^{-1}$ and DMSO, $1 \%$ 


\subsection{Chemical genetic profile of epiplakinic acid I}

Cyclic peroxides epiplakinic acid I (95) and plakortolide X (97) were both subjected to genome-wide homozygous profiling. The homozygous diploid deletion set was used to assess for chemical genetic interactions with 95, while Coorey assessed 97 in a hemizygous screen using the haploid deletion set ( $\Delta p d r$ background). ${ }^{224}$ Subsequent analysis for haploinsufficiency in the presence of $\mathbf{9 5}$ was undertaken with the heterozygous diploid deletion set. A full tabulation of the HOP and HIP profiles of 95 can be found in Appendix I.

\subsubsection{Homozygous profiling of epiplakinic acid I}

Functional annotation of the $S$. cerevisiae genome can be used to establish functional and physical relationships between the hits found in a chemogenomic screen. ${ }^{216}$ Grouping hits according to their gene ontology term annotation with on-line data analysis software such as FunSpec ${ }^{225}$ or YeastMine ${ }^{226}$ has significant advantages as all of the data used has been experimentally defined.

The removal of strains implicated in multi-drug resistance (MDR) has become common-place prior to gene ontology-based cluster analysis, owing to the observation that the inclusion of such genes places a bias on clusters formed within the dataset. ${ }^{210}$ Conversely, exclusion of MDR hits may lose information salient to the action of a specific compound. For example genes involved in vesicular mediated transport are frequently removed as their role in drug efflux recycling is thought to be compoundnon-specific. However, exclusion of these genes from the profile of a compound which directly affected these processes would severely devalue the chemical genetic screen. Hence, removal of MDR genes should be done with caution. ${ }^{216}$

The HOP profile of $\mathbf{9 5}$ identified 48 genes whose deletion caused increased sensitivity to 95. The removal of 19 genes which frequently occur in chemical genetic screens and five genes encoding dubious open reading frames left 21 genes displaying chemical genetic interactions in the presence of $\mathbf{9 5}{ }^{227}$ After removal of MDR genes, the profile of $\mathbf{9 5}$ showed functional clustering in the areas of cytoskeleton binding, vacuolar protein sorting, ribosome biogenesis, metabolism under stress conditions, response to stress, membrane organisation, Golgi sorting and mitochondrial function and repair, as classified by FunSpec ${ }^{225}$ and YeastMine. ${ }^{226}$ The unfiltered profile included a number of genes involved in vacuolar protein sorting (Table 5.4). The HOP profile of $\mathbf{9 5}$ did not identify any genes involved in upholding the PDR response, consistent with 95 not being subject to PDR efflux, as indicated by its similar activity in both the WT and $\Delta p d r$ strains. 
Table 5.4 Functional Categories of Deletion Strains Sensitive to Epiplakinic Acid I (95, $\left.3 \mu \mathrm{mol} \mathrm{L}^{-1}\right)$.

\begin{tabular}{|c|c|c|}
\hline GO Molecular Functions & $\mathrm{p}$-value & Gene deletion strain \\
\hline $\begin{array}{l}\text { Cytoskeletal actin polarisation } \\
\text { and cytoskeleton binding }\end{array}$ & $8.844 \mathrm{E}-06$ & 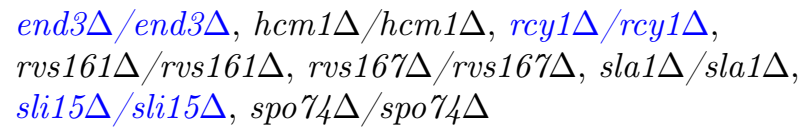 \\
\hline Golgi sorting & 0.002195 & $\begin{array}{l}\text { apl2 } \Delta / \operatorname{apl} 2 \Delta, \operatorname{chs} 5 \Delta / \operatorname{chs} 5 \Delta, \text { gos } 1 \Delta / \operatorname{gos} 1 \Delta, \\
\text { och } 1 \Delta / \text { och } 1 \Delta, \operatorname{rgp} 1 \Delta / \operatorname{rpg} 1 \Delta\end{array}$ \\
\hline Membrane organisation & 0.004122 & 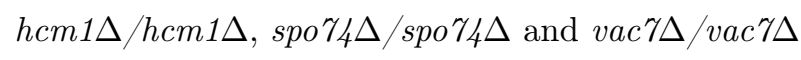 \\
\hline Metabolism under stress & 0.001258 & $\begin{array}{l}\text { aro } 7 \Delta / \operatorname{aro} 7 \Delta, \operatorname{crz} 1 \Delta / \operatorname{crz} 1 \Delta, \operatorname{csg} 2 \Delta / \operatorname{csg} 2 \Delta, \\
\text { hog2 } 2 / \text { hog2 } \Delta, \operatorname{grr} 1 \Delta / \operatorname{grr} 1 \Delta, \text { pfk2 } \Delta / \text { pfk2 } \Delta, \\
\text { vac7 } / \text { vac7 }\end{array}$ \\
\hline Ribosome biogenesis & 0.00048 & $\begin{array}{l}d b p 7 \Delta / d b p 7 \Delta, \text { rsa } 1 \Delta / \text { rsa } 1 \Delta, \text { rps } 9 b \Delta / \text { rps } 9 \Delta, \\
\text { sfp } 1 \Delta / \operatorname{sfp} 1 \Delta, \text { tod } 6 \Delta / \operatorname{tod} 6 \Delta, \text { yor019w } \Delta / \text { yor019w }\end{array}$ \\
\hline $\begin{array}{l}\text { Divalent cation and heavy } \\
\text { metals sensitivity }\end{array}$ & 0.0009385 & $\begin{array}{l}\operatorname{csg} 2 \Delta / \operatorname{csg} 2 \Delta, \quad \text { vps } 4 \Delta / \text { vps } 4 \Delta, \quad \operatorname{crz} 1 \Delta / \operatorname{crz} 1 \Delta \text { and } \\
\operatorname{sod} 1 \Delta / \operatorname{sod} 1 \Delta\end{array}$ \\
\hline $\begin{array}{l}\text { Vacuolar protein sorting } \\
\text { and transport }\end{array}$ & $3.25 \mathrm{E}-06$ & 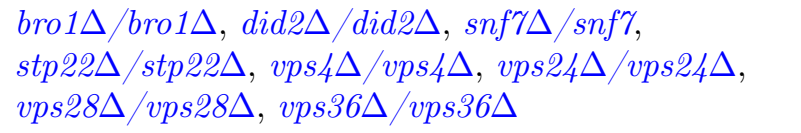 \\
\hline
\end{tabular}

* Genes implicated in MDR appear in blue.

Consistent with the small-cell and small-bud phenotype, the HOP profile included the mutant strains of cytoskeletal genes involved in bud formation and endocytosis; SLA1, RSA1, RVS161 and RVS16\%. Sla1p is a cytoskeletal binding protein, required for assembly of the cortical actin cytoskeleton. Sla1p plays an integral role in both bud formation and endocytosis through its interactions with proteins regulating actin dynamics. End3p forms a complex with Sla1p and Pan1p, which is involved in endocytosis, actin cytoskeletal organisation and cell wall morphogenesis. ${ }^{228}$ Rvs161p and Rvs167p interact to regulate polarisation of the actin cytoskeleton and are directly involved in endocytosis and exocytosis. They also play a role in maintaining cell polarity and viability in the event of osmotic stress or starvation. Hence, disruption or impairment of these genes and their functions can be detrimental to bud formation and endocytosis, consistent with the observed phenotype of $\mathbf{9 5}$. Furthermore, as 95 appears to affect cell size and budding, the deletion of actinrelated genes may result in a synergistic increase in sensitivity to the compound, down-stream of an initial chemical genetic interaction with 95. Hypersensitivity of the deletion strains of SLA1, RSA1, RVS161 and RVS167 to 95 was validated by a dose-response assay (Table 5.5 and Figure H.6), indicating that 95 may affect related cellular processes, such as actin coordination and endocytosis.

Although implicated in multi-drug resistance, SFP1 is integral to ribosome biogenesis in yeast, and genetic deficiency of SFP1 imparts translation-specific defects, significantly reducing the cell's ability to synthesise ribosomes, contributing to the 
observed starvation response. ${ }^{229}$ Hypersensitivity of the deletion strain to 95 may imply interaction of $\mathbf{9 5}$ with the target of rapamycin (TOR) pathway, Section 5.5.7.

Analysis was also undertaken on genes, the deletion of which appeared to confer resistance to 95 (z-score $>2$ ). The 44 resistant strains were submitted for gene ontology analysis (FunSpec ${ }^{225}$ ), however, time constraints prevented more in depth analysis of trends within the data. Full tabulation of the analysis can be found in Table I.4.

Table 5.5 Growth Inhibitory Effects of $\mathbf{9 5}$ on Cytoskeletal Deletion Mutant Strains.

\begin{tabular}{|c|c|c|c|}
\hline Strain & Concentration $\left(\mu \mathrm{mol} \mathrm{L}{ }^{-1}\right)$ & Residual Growth (\%) & Error $( \pm \%)$ \\
\hline "BY4743 WT & $\begin{array}{l}3.3\left(\mathrm{IC}_{50}\right) \\
\end{array}$ & $\begin{array}{ll}100.3 \\
\end{array}$ & 2.0 \\
\hline $\operatorname{sla} 1 \Delta / \operatorname{sla} 1 \Delta$ & 3.3 & 15.1 & 3.0 \\
\hline $\operatorname{sfp} 1 \Delta / \operatorname{sfp} 1 \Delta$ & 3.3 & 4.8 & 0.5 \\
\hline $\operatorname{rsa} 1 \Delta / \operatorname{rsa} 1 \Delta$ & 3.3 & 46.3 & 3.0 \\
\hline 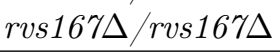 & 3.3 & 46.3 & 5.5 \\
\hline
\end{tabular}

\subsubsection{Haploinsufficiency profiling of epiplakinic acid I}

The HIP assay identified 124 genes which displayed haploinsufficiency in the presence of 95, eight of which are classified as essential according to the Saccharomyces Genome Database, ${ }^{226}$ (Table 5.6). Eleven genes implicated in multi-drug resistance, ${ }^{227}$ and 21 genes encoding genes with an unknown function were excluded from the profile. FunSpec ${ }^{225}$ cluster analysis of the homozygous gene profile, and the essential genes indicated by the heterozygous array displayed functional overlap in ribosome biogenesis $(\mathrm{p}$-value $=0.00048)$. This may further indicate interaction of $\mathbf{9 5}$ with the TOR starvation response pathway. The HIP profile also contained many genes functioning to mitochondrial repair, cytoskeletal polarisation and binding, ribosome generation, and plasma membrane organisation. ${ }^{226}$ The combined HOP and HIP profiles displayed functional clustering in response to $\mathrm{Zn}^{2+}$ ions $(\mathrm{p}$-value $=0.000192)$, and $\mathrm{Zn}^{2+}$ homeostasis $(\mathrm{p}$-value $=0.009733)$ as determined by FunSpec. ${ }^{225}$ The 99 strains deemed to display resistance to 95 (zscore $>2$ ) were similarly assessed by FunSpec cluster analysis, a full tabulation of which can be found in Table I.5. Significant insight into the biological mechanisms of small molecules can be gained through analysis of resistant deletion strains, and is worth undertaking. However, complete analysis and investigation of the resistant deletion strains was prevented by the time constraints on this project. 
Table 5.6 Functional Categories of Deletion Strains Haploinsufficient to Epiplakinic Acid I $\left(\mathbf{9 5}, 2 \mu \mathrm{mol} \mathrm{L}{ }^{-1}\right)$.

\begin{tabular}{|c|c|}
\hline GO Molecular Functions & Gene deletion strain $^{*}$ \\
\hline Metal ion homeostasis & $\begin{array}{l}d d p 1 \Delta / d d p 1 \Delta, \text { inp52 } \Delta / \text { inp 52 } \Delta, \text { irc7 } / \text { irc7 } \Delta, \\
i z h 1 \Delta / i z h 1 \Delta, \text { izh2 } \Delta / i z h 2 \Delta, \text { ybr016w } \Delta / \text { ybr016w } \Delta\end{array}$ \\
\hline $\begin{array}{l}\text { Cytoskeletal polarisation } \\
\text { and binding }\end{array}$ & $\begin{array}{l}\operatorname{abp} 140 \Delta / \operatorname{abp} 140 \Delta, \operatorname{cik} 1 \Delta / \operatorname{cik} 1 \Delta, \text { ede1 } \Delta / \operatorname{ede} 1 \Delta, \\
\operatorname{kin} 4 \Delta / \operatorname{kin} 4 \Delta, \operatorname{mad} 3 \Delta / \operatorname{mad} 3 \Delta, \operatorname{nap} 1 \Delta / \operatorname{nap} 1 \Delta, \\
\operatorname{pcl} 1 \Delta / \operatorname{pcl} 1 \Delta, \operatorname{rax} 1 \Delta / \operatorname{rax} \Delta 1\end{array}$ \\
\hline Ribosomal subunit proteins & 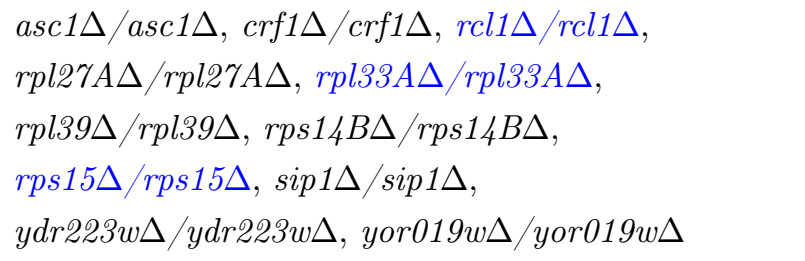 \\
\hline RNA binding proteins & $\begin{array}{l}\operatorname{prp} 43 \Delta / \operatorname{prp} 43 \Delta, \operatorname{rpc} 1 \Delta / r p c 10 \Delta, \tan 1 \Delta / \tan 1 \Delta, \\
\text { ygl232w } \Delta / \text { ygl232 } w \Delta, \text { yra2 } \Delta / \text { yra2 } \Delta\end{array}$ \\
\hline $\begin{array}{l}\text { Mitochondrial and oxidative } \\
\text { stress conditions }\end{array}$ & $\begin{array}{l}\operatorname{aim} 2 \Delta / \operatorname{aim} 2 \Delta, \operatorname{aim} 14 \Delta / \operatorname{aim} 14, \operatorname{crc} 1 \Delta / \operatorname{crc} 1 \Delta, \\
\operatorname{cox} 17 \Delta / \operatorname{cox} 17 \Delta, \operatorname{maf1} \Delta / \operatorname{maf1} \Delta, \operatorname{mne} 1 \Delta / \operatorname{mne} 1 \Delta, \\
\operatorname{mrm2} \Delta / \operatorname{mrm} 2 \Delta, \operatorname{mrpl11} / \operatorname{mrpl11} \Delta, \\
\operatorname{mrpl16} \Delta / \operatorname{mrpl16} \Delta, \operatorname{mrs} 1 \Delta / \operatorname{mrs} 1 \Delta, \operatorname{pxr} 1 \Delta / \operatorname{pxr} 1 \Delta, \\
\operatorname{rps} 7 A \Delta / \operatorname{rps} 7 A \Delta, \text { yir003w } \Delta / \text { yir003w } \Delta\end{array}$ \\
\hline Golgi sorting & $\begin{array}{l}\text { gga1 } \Delta / \text { gga1 } \Delta, \quad \text { gmh1 } \Delta / \text { gmh1 } \Delta, \quad \text { ste } 13 \Delta / \text { ste13 } \Delta, \\
\text { tvp23 } \Delta / \text { tvp23 } \Delta\end{array}$ \\
\hline $\begin{array}{l}\text { Utilisation of alternative carbon } \\
\text { sources }\end{array}$ & 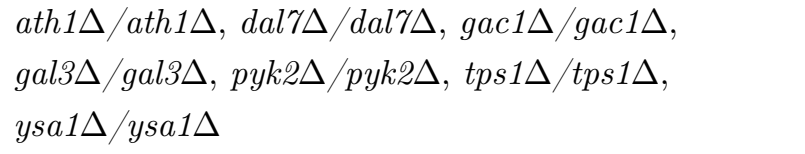 \\
\hline Amino acid biosynthesis & 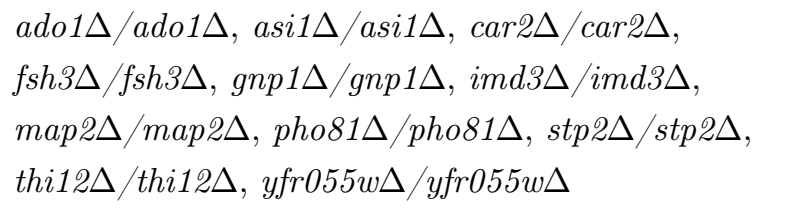 \\
\hline Fatty acid biosynthesis & fox2 $\Delta /$ fox $2 \Delta$, frm $2 \Delta /$ frm $2 \Delta$, ylr $400 w \Delta / y l r 400 w \Delta$ \\
\hline Plasma membrane organisation & $\begin{array}{l}\text { ast1 } \Delta / \operatorname{ast} 1 \Delta, \text { azr } 1 \Delta / \operatorname{azr} 1 \Delta, \operatorname{erv} 46 \Delta / \operatorname{erv} 46 \Delta, \\
\operatorname{rrt} 8 \Delta / \operatorname{rrt} 8 \Delta, \text { sur2 } \Delta / \operatorname{sur} 2 \Delta, \operatorname{pmt} 6 \Delta / \operatorname{pmt} 6 \Delta, \\
\operatorname{pst} 1 \Delta / \text { pst1 } \Delta, \text { yeh1 } \Delta / \text { yeh1 } \Delta\end{array}$ \\
\hline Meiosis & $\begin{array}{l}\text { mum3 } \Delta / \text { mum3 } \Delta, \text { ndt80 } \Delta / \text { ndt80 } \Delta, \text { osw1 } \Delta / \text { osw1 } \Delta, \\
\text { slz1 } \Delta / \text { siz1 } \Delta, \text { ssp2 } \Delta / \text { ssp2 } \Delta, \text { vhs1 } \Delta / \text { vhs1 } \Delta\end{array}$ \\
\hline DNA assembly and repair & $\begin{array}{l}\text { hht1 } \Delta / h h t 1 \Delta, \operatorname{rad} 4 \Delta / \operatorname{rad} 4 \Delta, \operatorname{rts} 2 \Delta / \operatorname{rts} 2 \Delta, \\
\operatorname{shu} 1 \Delta / \operatorname{shu} 1 \Delta, \text { ubr2 } \Delta / \text { ubr2 } \Delta\end{array}$ \\
\hline Transcription & $r \operatorname{tr} 1 \Delta / r \operatorname{tr} 1 \Delta, r \operatorname{tr} 2 \Delta / r \operatorname{tr} 2 \Delta$, ynl187w $/$ ynl187w $\Delta$ \\
\hline Protein synthesis & $\begin{array}{l}\text { eug } 1 \Delta / \text { eug } 1 \Delta, \text { ost } 5 \Delta / \text { ost } 5 \Delta, \text { ptp } 1 \Delta / \text { ptp } 1 \Delta, \\
\text { ssa3 } \Delta / \text { ssa3 } \Delta\end{array}$ \\
\hline Proteosome degradation & pci1 $\Delta /$ pci1 $\Delta$, yuh1 $1 /$ yuh1 $\Delta$ \\
\hline
\end{tabular}

* Essential genes appear in blue. 


\subsubsection{Plakortide $\mathrm{F}$ acid}

Comparison of the gene expression profile of plakortide F acid (122) ${ }^{169}$ and the homozygous profile of $\mathbf{9 5}$ proved informative. Plakortide $\mathrm{F}$ acid (122) induces upregulation of genes associated with response to stress, protein transport and sorting, carbohydrate metabolism, organelle organisation, amino acid metabolism, membrane organisation, cell wall organisation and sporulation. Functions pertaining to the activity of 95, namely cell cycle and ribosome biogenesis experienced downregulation in the presence of $\mathbf{1 2 2}$. Their study also showed an overall increase in intracellular $\mathrm{Ca}^{2+}$ and $\mathrm{Zn}^{2+}$-levels by ICP-MS. ${ }^{169}$

Only one direct overlap was observed between the RNA-expression profile of $\mathbf{1 2 2}^{169}$ and the homozygous profile of $\mathbf{9 5}$ - the $\mathrm{Ca}^{2+}$-sensitive stress response transcription factor Crz1p, ${ }^{\dagger}$ which is activated in response to elevated $\mathrm{Ca}^{2+}$ levels (Sections 5.5.5 and 5.5.6). Other calcium-responsive genes to experience upregulation in the presence of 122 included those encoding membrane bound ion pumps and ion channels. Calcium influx through the plasma membrane is mediated by the $\mathrm{Ca}^{2+}$ channels Cch1p and Mid1p, as shown in Figure 5.9. Intracellular levels are modulated by the pumps Pmr1p and Pmc1p, with Pmr1p sequestering $\mathrm{Ca}^{2+}$ and $\mathrm{Mg}^{2+}$ within the Golgi bodies. Pmclp and the $\mathrm{H}^{+} / \mathrm{Ca}^{2+}$ exchanger Vcxlp affect $\mathrm{Ca}^{2+}$ detoxification by increasing vacuolar storage, while Vcx1p will also release vacuolar $\mathrm{Ca}^{2+}$ stores into the cytoplasm in response to hyperosmotic stress. ${ }^{169,226,230}$

Table 5.7 Functional Categories of Genes Differentially Regulated in the Presence of Plakortide F Acid (122).

\begin{tabular}{ll}
\hline Status & Functional category \\
\hline \hline Upregulated & Response to stress \\
& Transport \\
& Carbohydrate metabolism \\
& Transcription \\
& Protein modification \\
& Organelle organisation \\
& Amino acid metabolism \\
& Membrane organisation \\
& Cell wall organisation \\
& Sporulation \\
& \\
Downregulated & Cell cycle \\
& Ribosome biogenesis \\
& Conjugation Translation \\
& Amino acid metabolism \\
& Signal transduction \\
& Meiosis \\
\hline
\end{tabular}

Sensitivity of the ion channel and ATP-pump deletion strains to plakortide F acid

\footnotetext{
$\dagger$ This is not unexpected: $7 \%$ is the highest reported overlap between the two techniques, observed when galactose is provided as a carbon source as opposed to glucose. ${ }^{76}$
} 


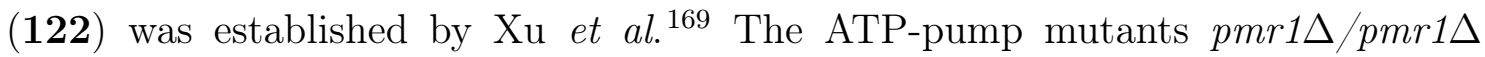
and $p m c 1 \Delta /$ pmc1s and the ion channel mutants $\operatorname{cch} 1 \Delta / \operatorname{cch} 1 \Delta$ and mid1s $/$ mid1s displayed hypersensitivity to epiplakinic acid I (95) relative to the WT control. As Cch1p and Mid1p are required for passage of $\mathrm{Ca}^{2+}$ into the cells, deletion of the genes should confer resistance. Hence, sensitivity of the deletion mutants to the cyclic peroxides is counterintuitive. However, one can imagine that the channels also allow exit of ions from within the cell, mitigating intracellular toxicity. Xu et al. also postulate that " $\mathrm{Ca}^{2+}$ deprivation in these mutants might result in a compensatory induction in intracellular $\mathrm{Ca}^{2+}$ levels, causing 122 (and 95) to be more toxic under these conditions". ${ }^{169}$

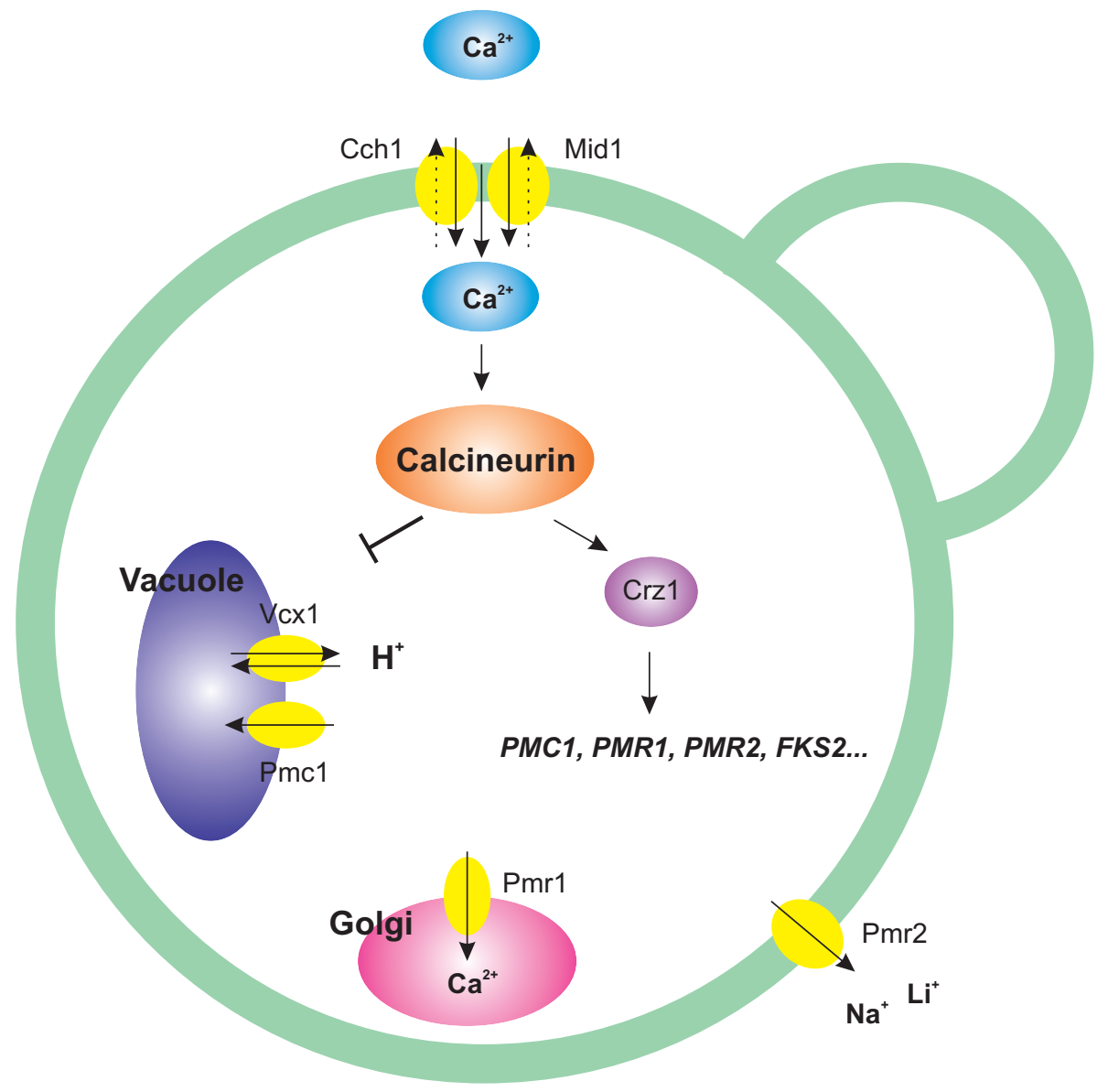

Figure 5.9 Depiction of the ion-channel and ATP-pump system for $\mathrm{Ca}^{2+}$ homeostasis in S. cerevisiae, adapted from Miyakawa et al. ${ }^{230}$

Another $\mathrm{Ca}^{2+}$-modulating agent, amiodarone, ${ }^{231}$ is thought to interact with Mid1p. ${ }^{232}$ Courchesne et al. observed some resistance of the mid1 $\Delta$, cch1 $\Delta$ double deletion to amiodarone. Furthermore, the $\operatorname{mid} 1 \Delta / \operatorname{mid} 1 \Delta$ strain did not show the same growth inhibition as the $\operatorname{cch} 1 \Delta / \operatorname{cch} 1 \Delta$ strain in response to amiodarone. Sensitivity of the Mid1p and Cch1p deletion strains to $\mathbf{1 2 2}$ and $\mathbf{9 5}$ indicates that neither are the cellular target of the cyclic peroxides $\mathbf{1 2 2}$ and 95. However, the compounds could potentially interact with the membrane-bound ion channels of another divalent ion, such the $\mathrm{Fe}^{2+}$ transporter Fet3p. The Mid1p and Cch1p channels has been shown 
to be essential to iron toxicity tolerance, ${ }^{233}$ as such it is not unreasonable that a reciprocal interaction may be occurring.

Consistent with plakortide $\mathrm{F}$ acid (122) increasing intracellular levels of $\mathrm{Ca}^{2+}$ and causing $\mathrm{Ca}^{2+}$ stress, $\mathrm{Xu}$ et al. also observed hypersensitivity in the deletion strain of genes essential to the function of calcineurin, a $\mathrm{Ca}^{2+} /$ calmodulin-dependent protein phosphatase. ${ }^{\ddagger} C N B 1$ encodes the regulatory subunit of the calcineurin heterodimer in yeast and the catalytic subunit of calcineurin is encoded by the functionally redundant genes CNA1 and CNA2. ${ }^{218}$ Mutation of both CNA1 and $C N A 2$, and the regulatory gene $C N B 1$, or exposure to calcineurin inhibitors such as FK506 and cyclosporin A compromises activity of the calcineurin phosphatase. CRZ1 encodes a $\mathrm{Ca}^{2+}$-responsive transcription factor that directs calcineurindependent transcription genes to mitigate $\mathrm{Ca}^{2+}$ toxicity. Hypersensitivity to $\mathbf{1 2 2}$ was displayed by the strains $\operatorname{crz} 1 \Delta / \operatorname{crz} 1 \Delta, c n b 1 \Delta / \operatorname{cnb} 1 \Delta$, cna $1 \Delta / \operatorname{cna} 1 \Delta$ and cna2 $\Delta /$ cna2 $\Delta .{ }^{169}$ The deletion strains $\operatorname{crz} 1 \Delta / \operatorname{crz} 1 \Delta$, and cna2 $\Delta /$ cna2 $\Delta$ displayed similar hypersensitivity to epiplakinic acid I (95). Slight sensitivity was observed in the $c n b 1 \Delta / c n b 1 \Delta$ strain, although assessment over a more restricted concentration range is required for definitive conclusion. Unexpectedly, the cna1 $/$ cna1s strain did not display sensitivity to 95 (Tables 5.8, 5.9 and Figure H.7).

Table 5.8 Growth Inhibitory Effects of 95 on $\mathrm{Ca}^{2+}$-Responsive Deletion Strains.

\begin{tabular}{|c|c|c|c|}
\hline Strain & Concentration $\left(\mu \mathrm{mol} \mathrm{L}{ }^{-1}\right)$ & Residual Growth (\%) & Error $( \pm \%)$ \\
\hline BY4743 WT & (7.6 & 99.0 & 5.0 \\
\hline $\operatorname{cna} 1 \Delta / \operatorname{cna} 1 \Delta$ & 7.6 & 70.5 & 3.0 \\
\hline cna2s / cna2s & 7.6 & 7.1 & 2.5 \\
\hline$c n b 1 \Delta / c n b 1 \Delta$ & 7.6 & 11.5 & 4.5 \\
\hline $\operatorname{crz} 1 \Delta / \operatorname{crz} 1 \Delta$ & 7.6 & 4.7 & 3.5 \\
\hline$h c m 1 \Delta / h c m 1 \Delta$ & 7.6 & 42.3 & 0.5 \\
\hline
\end{tabular}

Table 5.9 Growth Inhibitory Effects of $\mathbf{9 5}$ on $\mathrm{Ca}^{2+}-\mathrm{Pump} /$ Channel Deletion Strains.

\begin{tabular}{|c|c|c|c|}
\hline Strain & Concentration $\left(\mu \mathrm{mol} \mathrm{L}{ }^{-1}\right)$ & Residual Growth (\%) & Error $( \pm \%)$ \\
\hline$\overline{\text { BY4743 WT }}$ & 3.3 & (113.3 & 2.0 \\
\hline $\operatorname{cch} 1 \Delta / \operatorname{cch} 1 \Delta$ & 3.3 & 56.9 & 1.2 \\
\hline $\operatorname{mid} 1 \Delta / \operatorname{mid} 1 \Delta$ & 3.3 & 4.5 & 0.5 \\
\hline$p m c 1 \Delta / p m c 1 \Delta$ & 3.3 & 69.3 & 5.2 \\
\hline$p m r 1 \Delta / p m r 1 \Delta$ & 3.3 & 56.2 & 1.1 \\
\hline ygl16rws/ygl16rws & 3.3 & 3.5 & 0.5 \\
\hline
\end{tabular}

\footnotetext{
\$The calcium-modulated protein, calmodulin is a calcium-binding protein conserved in all eukaryotic systems. It can bind up to four calcium ions, and once activated it binds to and regulates many different protein targets affecting a myriad of cellular responses.
} 


\subsubsection{The $\mathrm{Ca}^{2+}$ second messenger system}

Calcium ions are integral to many physiologically important functions, including cytoskeletal dynamics, and as key regulators of the protein calmodulin, the ions play a pivotal role in muscle contraction in higher eukaryotes. The ion is an important second messenger, relaying signals from cell surface receptors to target molecules within the cell (in the cytoplasm or nucleus), inducing changes in cellular activity, and amplifying the strength of the initial signal through signal transduction cascades. ${ }^{\S}$ The $\mathrm{Ca}^{2+}$ second messenger system acts through cellular traffic of the metal ions via membrane ion channels and membrane-bound ion pumps, carefully monitoring cytoplasmic and organelle-located stores of the ion. The second messenger must be tightly controlled as disruption to the system has far-reaching effects, and as such, foreign molecules which in any way influence this system can be extensively detrimental to cellular function. While $\mathrm{Ca}^{2+}$-overload can trigger apoptosis in mammalian cells, ${ }^{234}$ decreased viability of WT $S$. cerevisiae has not been reported in response to disruption of $\mathrm{Ca}^{2+}$ levels alone. ${ }^{235}$ As such, a rise in intracellular $\mathrm{Zn}^{2+}$ levels could also contribute to the cellular starvation phenotype induced by $\mathbf{9 5}$ and $\mathbf{9 7}$. Translocation studies in the Zrt1p-GFP strain do not necessarily support this, however. In response to increased intracellular $\mathrm{Zn}^{2+}$,

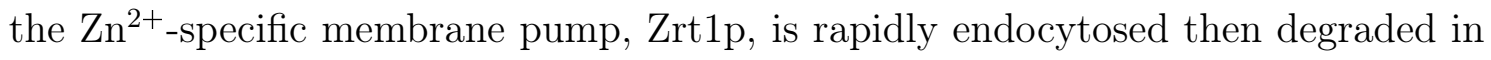
the vacuole. ${ }^{236}$ This response was not apparent upon exposure of the Zrt1p-GFP strain to 95 for $40 \mathrm{~min}$ (Figure H.8). This may indicate that 95 does not increase intracellular levels of $\mathrm{Zn}^{2+}$. However, if $\mathbf{9 5}$ does inhibit actin coordination, the lack of Zrt1p endocytosis may be accounted for.

\subsubsection{Epiplakinic acid I induces a $\mathrm{Ca}^{2+}$-related response}

Translocation studies were conducted in the Crz1p-GFP strain showing nuclear relocalisation of the GFP signal upon exposure to $\mathrm{CaCl}_{2}$ or epiplakinic acid I (95). This indicates that 95 induces the same cellular response as a spike in intracellular $\mathrm{Ca}^{2+}$. Coorey observed similar relocation in the Crz1p-GFP- $\Delta p d r$ strain in response to plakortolide $\mathrm{X}(\mathbf{9 7}),{ }^{224}$ consistent with 95 and $\mathbf{9 7}$ inducing a spike in intracellular $\mathrm{Ca}^{2+}$. Illustrating the $\mathrm{Ca}^{2+}$-specificity of the Crz1p response, the Crz1p-GFP displayed weak and transient nuclear relocalisation upon exposure to $\mathrm{Mg}^{2+}, \mathrm{Zn}^{2+}$ and $\mathrm{Fe}^{2+}$ (data not shown). The calcium-modulator amiodarone also induces translocation in the Crz1p-GFP strain, ${ }^{237}$ indicating the two compounds

\footnotetext{
§There are three main classes: hydrophilic molecules such as cAMP, cGMP, IP3, and $\mathrm{Ca}^{2+}$ located in the cytosol; hydrophobic molecules, including diacylglycerol, and phosphatidylinositols are associated with cellular membranes and diffuse from the plasma membrane into the intermembrane space where they can affect membrane-associated effector proteins; gases, such as $\mathrm{NO}, \mathrm{CO}$ and $\mathrm{H}_{2} \mathrm{~S}$ form the third division, and are capable of diffusing both through the cytosol and across cellular membranes.
} 
may have similar cellular effects. Amiodarone also induces a starvation response and decreases cell size, while flow cytometry studies with amiodarone have indicated cell cycle delays at both $\mathrm{S}$-phase and the $\mathrm{G}_{2}$-M transition as seen with $\mathbf{9 5}{ }^{237}$
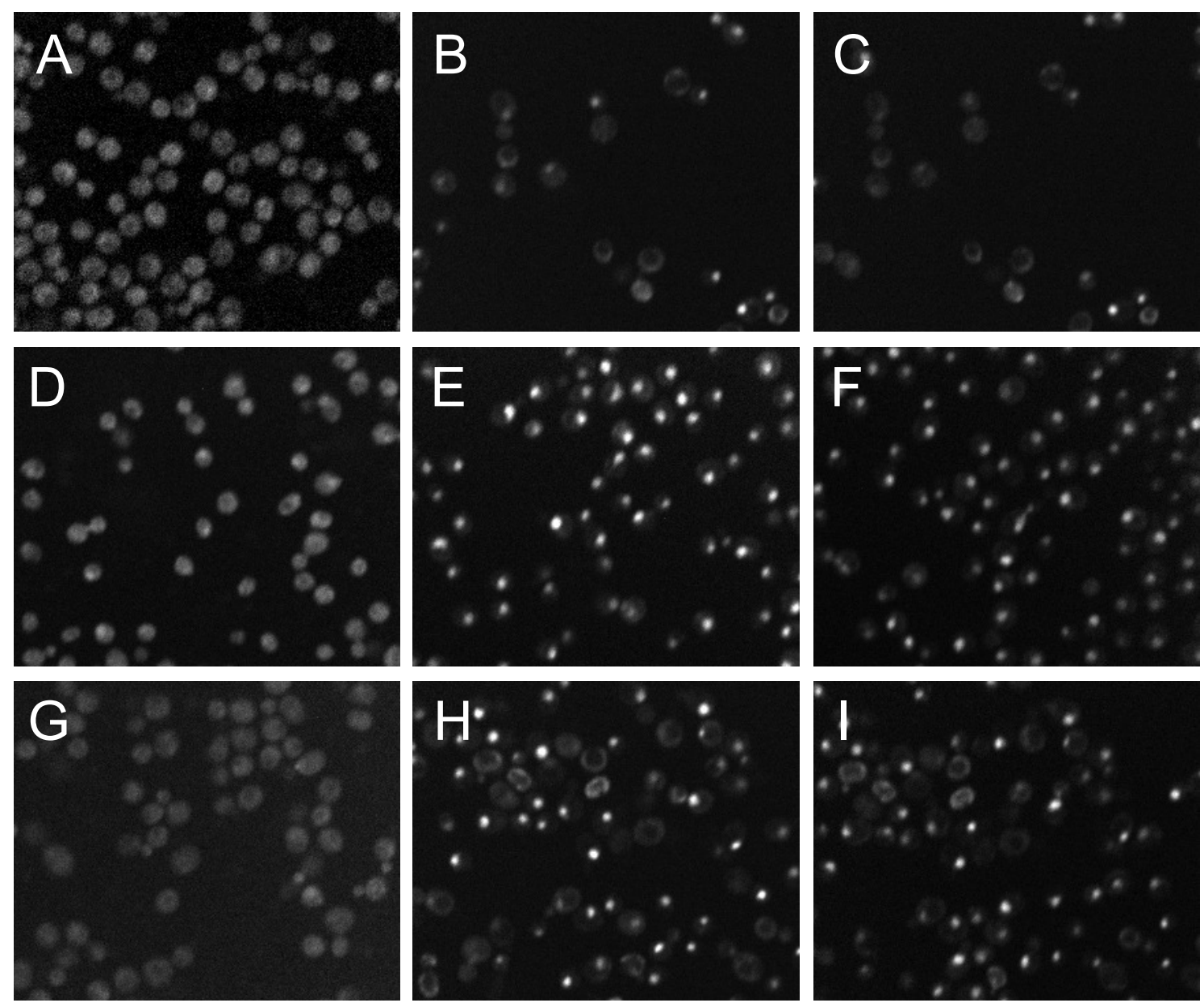

Figure 5.10 Translocation studies in the Crz1p-GFP strain indicating a similar response to a rise in intracellular $\mathrm{Ca}^{2+}$ and exposure to epiplakinic acid I (95).
A: $\operatorname{DMSO}(1 \%), 0$ min $\quad B:$
B: $95\left(10 \mu \mathrm{mol} \mathrm{L}^{-1}\right), 0 \mathrm{~min}$
$\mathrm{C}: \mathrm{CaCl}_{2}\left(50 \mathrm{mmol} \mathrm{L}^{-1}\right), 0 \mathrm{~min}$
D: $\quad \mathrm{DMSO}, \mathrm{t}=5 \mathrm{~min}$
E: $95, \mathrm{t}=5 \mathrm{~min}$
F: $\mathrm{CaCl}_{2}, \mathrm{t}=5 \mathrm{~min}$
G: $\quad$ DMSO, $\mathrm{t}=40 \mathrm{~min}$
$\mathrm{H}: \quad \mathbf{9 5}, \mathrm{t}=40 \mathrm{~min}$
I: $\quad \mathrm{CaCl}_{2}, \mathrm{t}=40 \mathrm{~min}$

Qualitative staining of WT cells with Calcium Orange fluorescent dye also indicated an increase in cellular $\mathrm{Ca}^{2+}$ levels in response to epiplakinic acid I (95). Consistent with 95 causing an increase in intracellular $\mathrm{Ca}^{2+}$ levels, the fluorescence intensity of the Calcium Orange probe was increased in WT cells treated separately with $\mathbf{9 5}$ and $\mathrm{CaCl}_{2}$, relative to the DMSO control (Figure 5.11). This is in agreement with $\mathrm{Xu}$ et al. who observed an increase in the intracellular levels of $\mathrm{Ca}^{2+}$ and $\mathrm{Zn}^{2+}$ in WT cells exposed to plakortide F (122) by ICP-MS analysis. ${ }^{169}$

Increased $\mathrm{Ca}^{2+}$ signaling is known to contribute to formation of reactive oxygen species (ROS), and associated cellular toxicity. ${ }^{238}$ Although a significant number of genes implicated in mitochondrial damage and response to oxidative stress displayed sensitivity to $\mathbf{9 5}$ in the HOP and HIP assays, no evidence for formation of ROS was found. To test for the formation of ROS in the presence on 95, translocation studies 
were conducted in a GFP-tagged reported strain with a red fluorescent protein (RFP) nuclear membrane localisation marker, Yap1p-GFP-NLS-mCherry. Yap1p is a stress response transcription factor, which normally resides in the cytoplasm and translocates to the nucleus in response to oxidative stress. Co-localisation of the GFP and RFP signals was not observed in response to 95, indicating that Yap1p did not undergo nuclear relocalisation (data not shown). This suggests that $\mathbf{9 5}$ does not cause the formation of ROS.

Flow cytometry analysis was also carried out in haploid WT cells using with dichlorofluorescein diacetate (DCF-DA) and propidium iodide (PI) to study the formation of ROS and cell death (Figure 5.12). Although PI staining in the upper left quadrant indicated increased cell death in presence of 95, the assay did not indicate that formation of ROS was responsible for the increase. However, PI staining is indicative of increased cell wall permeabilisation as a consequence of cell death, and as such this may indicate that $\mathbf{9 5}$ (and presumably $\mathbf{9 7}$ ) is truly cytotoxic to yeast.

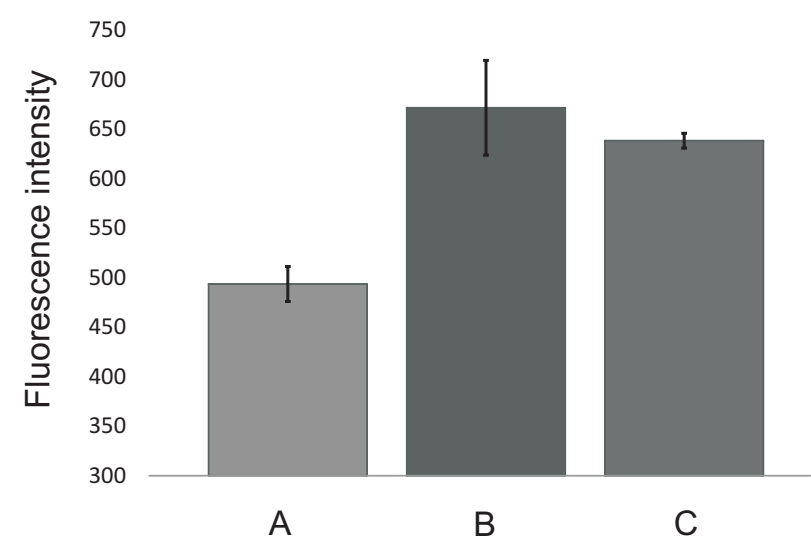

Figure 5.11 Qualitative staining of WT cells with Calcium Orange dye. A: $\quad$ DMSO, $1 \%$ B: $\quad \mathrm{CaCl}_{2}, 50 \mathrm{mmol} \mathrm{L}{ }^{-1} \quad \mathrm{C}: \quad$ Epiplakinic acid I, $10 \mu \mathrm{mol} \mathrm{L}^{-1}$ Increased fluorescence intensity is indicative of $\mathrm{Ca}^{2+}$-coordination by the fluorophore, as observed in the presence of $\mathrm{CaCl}_{2}$ (B) and 95 (C), relative to the DMSO control (A).

\subsubsection{Calcineurin signaling}

In $S$. cerevisiae calcineurin is required to maintain cell viability, becoming activated in response to environmental conditions, including exposure to high intracellular levels of $\mathrm{Ca}^{2+}$ and $\mathrm{Na}^{+}$, and to elevated temperature. In yeast (and mammals), Crz1p is the best-characterised substrate for activated calcineurin. ${ }^{239,240}$ The transcription factor Crz1p resides in the cytoplasm when calcineurin signaling is low, and is dephosphorylated upon activation of calcineurin, rapidly translocating to the nucleus where it affects cellular responses to mitigate $\mathrm{Ca}^{2+}$ toxicity. In addition to its role in yeast ion homeostasis, calcineurin is involved in recovery from $\mathrm{G}_{1}$ arrest 
caused by mating pheromones, such as $\alpha$-factor, ${ }^{240}$ and is essential to the function of deletion strains with defective cell walls. ${ }^{239,241}$

A

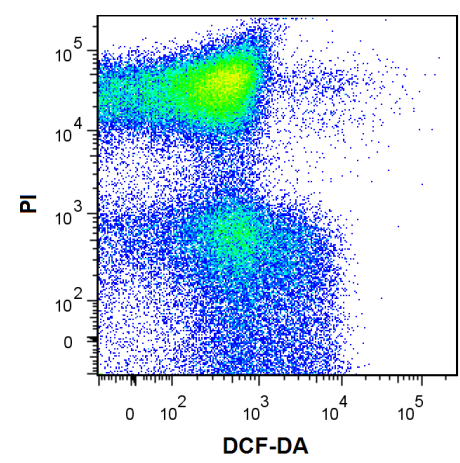

B

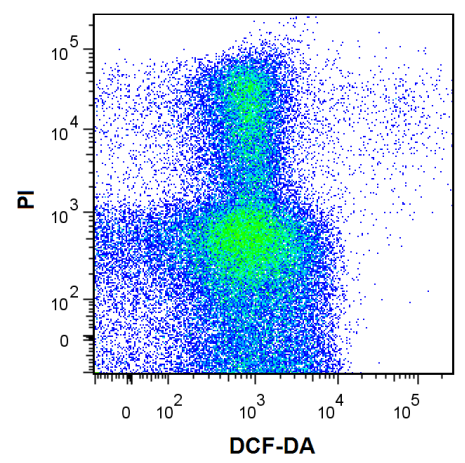

C

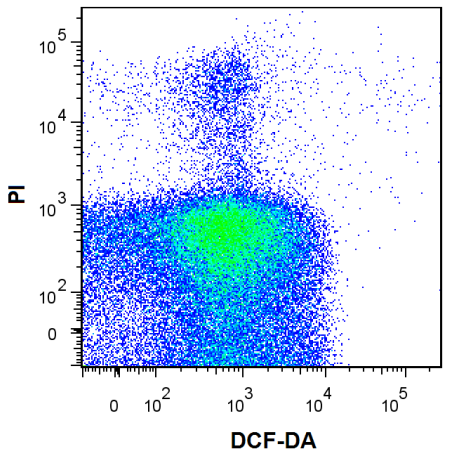

Figure 5.12 Flow cytometry imaging ROS formation with DCF-DA and PI in haploid WT cells.

A: Epiplakinic acid I, $10 \mu \mathrm{mol} \mathrm{L}-1 \quad$ B: $\quad \mathrm{H}_{2} \mathrm{O}_{2}, 10 \mu \mathrm{mol} \mathrm{L}-1 \quad \mathrm{C}: \quad$ DMSO, $1 \%$

The lower quadrants represent live cells stained with DCF-DA and cell death is indicated by PI staining of the populations in the top left quadrant. As indicated by the controls, the majority of the live cell population predominantly occupies the left quadrant, while a shift to the right indicates the presence of ROS. The increased cell death observed in the presence of $\mathbf{9 5}$ does not appear to be caused by the generation of ROS.

Upon activation of calcineurin, a suite of genes required for ion homeostasis is upregulated in a calcineurin/Crz1p-dependent manner. Following nuclear relocalisation, Crz1p binds specifically to a region of 24 base pairs within the promoter regions of the upregulated genes, termed the calcineurin dependent response element (CDRE). In this way, Crz1p functions downstream of calcineurin to affect calcineurin-dependent responses that mitigate $\mathrm{Ca}^{2+}$-related toxicity. Overproduction of Crz1p in WT cells also increases tolerance to $\mathrm{Mn}^{2+}$ and $\mathrm{Li}^{+}$, and is known to suppress the phenotypes of calcineurin mutants. ${ }^{242}$

The ion homeostasis genes which experience calcineurin/Crz1p-dependent regulation include the vacuolar and Golgi-bound $\mathrm{Ca}^{2+}$ and $\mathrm{Ca}^{2+} / \mathrm{Mn}^{2+}$-ATPases encoded by PMC1 and PMR1 (Figure 5.9). ${ }^{218,242}$ The ion-pumps are integral to intracellular ion homeostasis, and their Crz1p-mediated transcription is required for growth under high $\mathrm{Ca}^{2+}$ conditions. PMR2 encodes a plasma membrane $\mathrm{Na}^{+} / \mathrm{Li}^{+}$-ATPase required for growth in the presence of high concentration of the two ions. Expression of the pumps is induced by (high) $\mathrm{Ca}^{2+}$ and $\mathrm{Na}^{+}$in a calcineurin/Crz1p-dependent manner, and in response to high osmotic stress by the high osmolarity glycerol (HOG1) pathway. Deletion of these genes confers sensitivity to 95 (Figure H.7) and 122.

Independent of Crz1p, increased calcineurin signaling also causes depolarisation of the actin cytoskeleton (Section 5.5.7). Mulet et al. observed formation of small actin balls about the cellular peripheries upon brief exposure to $0.2 \mathrm{~mol} \mathrm{~L}^{-1}$ 


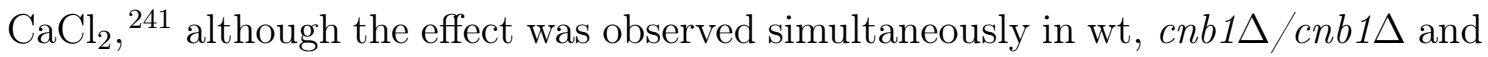
$\operatorname{crz} 1 \Delta / \operatorname{crz} 1 \Delta$ mutants, indicating actin depolarisation is independent of Crz1p.

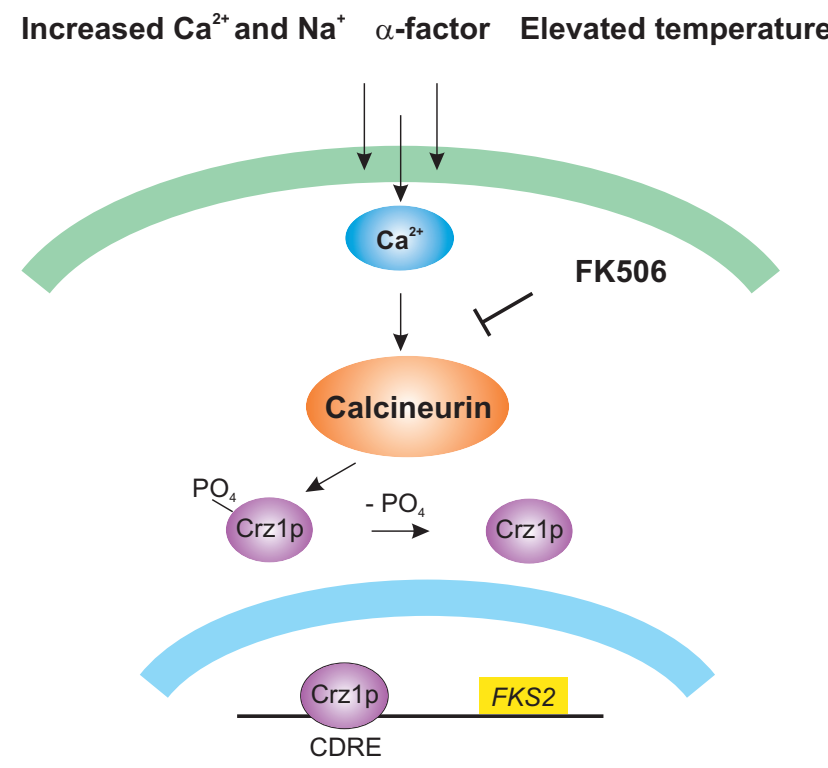

Figure 5.13 Calcineurin signaling pathway in S. cerevisiae and activation of Crz1, adapted from Yoshimoto et al. ${ }^{243}$ Calcineurin is activated by external stimuli, including elevated temperature and levels of $\mathrm{Na}^{+}$and $\mathrm{Ca}^{2+}$, and presence of the mating pheromone $\alpha$-factor. Calcineurin dephosphorylates Crz1, which undergoes nuclear translocation and initiates $\mathrm{Ca}^{2+}$-dependent transcription of genes, such as FKS2, containing the CDRE in their promoter regions. The calcineurin signaling cascade is rapidly and specifically inhibited by the presence of immunosuppressive drug FK506.

\subsubsection{Interactions between calcineurin signaling and TOR}

The TOR pathway is conserved among eukaryotes, controlling cell growth in response to nutrient availability and environmental stress. The TOR protein kinase comprises two functionally and structurally distinct protein complexes, TORC1 (TOR complex 1) and TORC2. In yeast, TORC1 contains either TOR1 or TOR2, whereas TORC2 contains exclusively TOR2. TORC1 is rapamycin-sensitive and controls growth-related processes such as transcription, translation, ribosome biogenesis, nutrient transport and autophagy in response to nutrient levels. ${ }^{220}$ Inactivation of TORC1 leads to a rapid downregulation of genes involved in ribosome biogenesis, with the carryon effect of decreased protein synthesis and cell growth. ${ }^{245}$ TORC2 is rapamycin-insensitive and controls polarisation of the actin cytoskeleton, acting to inhibit calcineurin-mediated depolarisation of the actin cytoskeleton. In this way, TORC2 allows a cell to resume growth after a stress has been overcome. ${ }^{241}$

The TORC2-calcineurin relationship is mutually antagonistic, and a downstream effect of increased $\mathrm{Ca}^{2+}$ levels and calcineurin signaling is inhibition of the TOR

\footnotetext{
"A similar phenotype is seen upon exposure to latrunculin A, known to stabilise monomeric actin by coordinating to thymosin $\beta .^{244}$
} 
pathway (Figure 5.14). ${ }^{246}$ Calcineurin prevents TOR-mediated growth under stress conditions in one way by inhibiting signaling between TORC2 and the proteins Slm1p and Slm2p, which act to regulate the actin cytoskeleton organisation in response to stress. ${ }^{247}$ Increased $\mathrm{Ca}^{2+}$ levels and calcineurin signaling promote dephosphorylation of the SLM proteins disrupting their function in actin regulation. ${ }^{241}$ Hence, calcineurin-activating agents, such as $\mathbf{1 2 2}$ and potentially 95 can initiate depolarisation of the actin cytoskeleton leading to the phenotype observed in this study. Similarly, TORC2 phosphorylation of Slm1p and Slm2p inhibits calcineurin-mediated depolarisation of the actin cytoskeleton and Crz1p-dependent gene transcription.

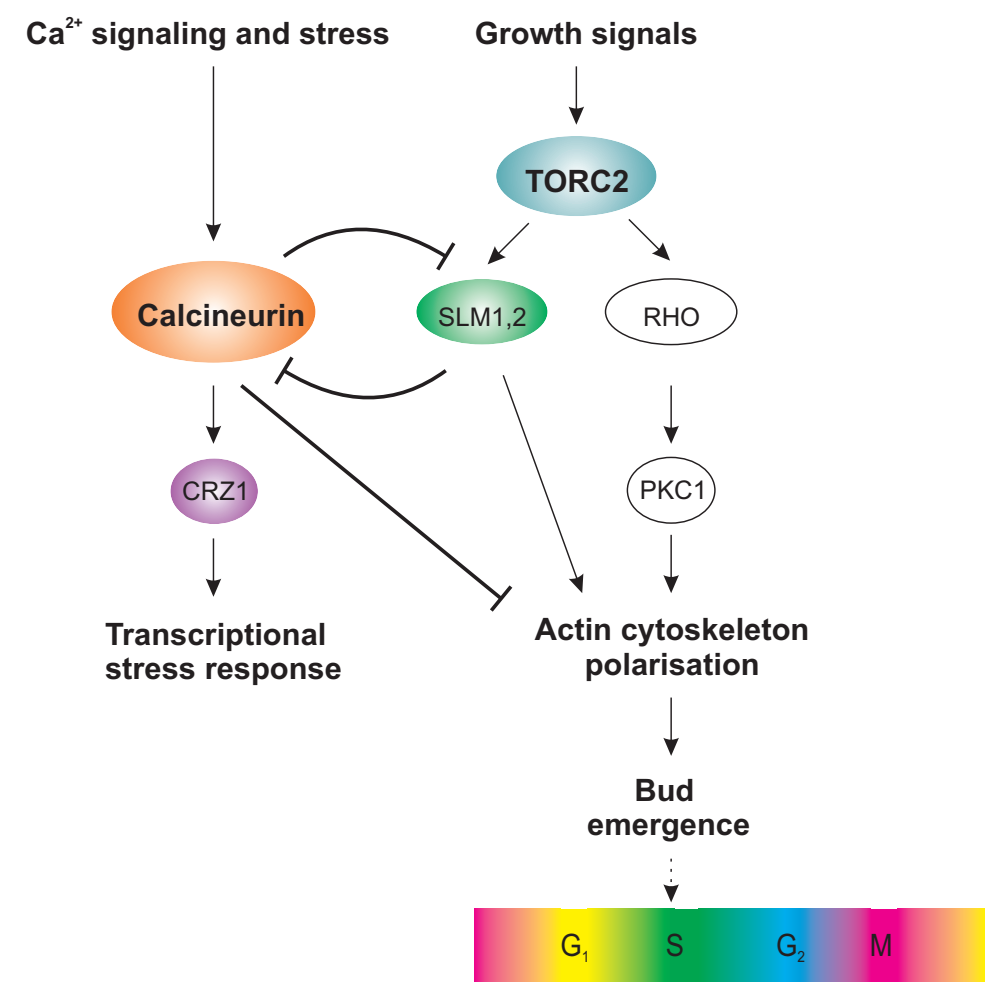

Figure 5.14 Antagonistic interactions between the calcineurin and TORC2 signaling pathways after Mulet et al. ${ }^{241}$

Inactivation of the TOR pathway, or treatment with rapamycin, produces a phenotype similar to that of epiplakinic acid I (95) and plakortolide X (97). Rapamycin inhibits cell cycle progression, initiating arrest at the $\mathrm{G}_{1}-\mathrm{S}$ phase boundary, and induces physiological changes consistent cellular starvation, including reduced cell size. ${ }^{219} \mathrm{Xu}$ et al. observed that co-treatment of cells with plakortide $\mathrm{F}$ acid (122) and non-inhibitory concentrations of calcineurin-specific inhibitors FK506 and cyclosporin A greatly enhanced the potency of 122. ${ }^{169}$ From this one can infer that 122, and presumably compounds $\mathbf{9 5}$ and $\mathbf{9 7}$, influence a cellular process closely related to that of FK506 and cyclosporin A, i.e. calcineurin signaling. Additionally, cyclosporin A has a similar effect as plakinid cyclic peroxides on $\mathrm{Ca}^{2+}$ pumping in the mammalian cardiac sarcoplasmic reticulum. ${ }^{120,197,248}$ 


\subsection{Mechanistic insights from phenotypic and chemical genetic profiling}

The phenotypic profiling assays carried out during this study, in combination with the expression profile of 122, suggests that 95 disrupts intracellular $\mathrm{Ca}^{2+}$ homeostasis. Translocation of the $\mathrm{Ca}^{2+}$-responsive transcription factor Crz1p was observed in the GFP strain following exposure to $\mathbf{9 5}$ and the same response was observed following addition of exogenous $\mathrm{Ca}^{2+}$ to growth media (Figure 5.10). This alone indicates that 95 causes increases in intracellular $\mathrm{Ca}^{2+}$. An overall increase in cellular $\mathrm{Ca}^{2+}$ following exposure to 95 was also shown by Calcium Orange staining (Figure 5.11). Furthermore, the deletion strains of CCH1, CNA2, CNB1, CRZ1, MID1, PMC1 and PMR1, which are required to mitigate $\mathrm{Ca}^{2+}$ toxicity, displayed hypersensitivity to 95 (Tables 5.8 and 5.9). Disruption of $\mathrm{Ca}^{2+}$ homeostasis could account for the phenotype caused by $\mathbf{9 5}$; reduced cell size and an increase in cells with small buds. The same decrease in cell size was observed following the addition of exogenous $\mathrm{CaCl}_{2}$ to yeast growth media (Table 5.2 and Figure 5.6).

Although the deletion strains of several genes integral to cell viability in environments of high $\mathrm{Ca}^{2+}$ were revealed in the HOP and HIP profiles of 95, significant enrichment of these functions was not indicated via FunSpec cluster analysis. It may be that when a small molecule inhibits a process so fundamental to cellular function, such as $\mathrm{Ca}^{2+}$ signaling, that too many pathways are affected to allow statistically significant enrichment of any one particular process. Such a phenomenon has also been hypothesised in the case of protein synthesis inhibitors, such as pateamine A. ${ }^{216}$ The combined HOP and HIP profiles of 95 did display enrichment in $\mathrm{Zn}^{2+}$ homeostasis ( $\mathrm{p}$-value $=0.009733$ ) and response to $\mathrm{Zn}^{2+}$ ions ( $\mathrm{p}$-value $=$ 0.000192), although the p-values associated with the enrichment does not appear particularly significant if disruption of ion homeostasis is the primary mechanism of the compound. Enrichment in $\mathrm{Zn}^{2+}$ regulation is, however, consistent with $\mathrm{Xu}$ et al. who observed increases in intracellular $\mathrm{Zn}^{2+}$ and $\mathrm{Ca}^{2+}$ in response to 122. In light of this dual increase in levels of the ions, it may be that the cyclic peroxides are causing an increase in intracellular levels of either $\mathrm{Zn}^{2+}$ or $\mathrm{Ca}^{2+}$, with a down stream effect of interfering with homeostasis of the other. Hence, both $\mathrm{Zn}^{2+}$ and $\mathrm{Ca}^{2+}$ may be responsible for inhibition of yeast cells by $\mathbf{9 5}$ and $\mathbf{9 7}$.

\subsubsection{Future directions}

The mechanism by which the cyclic peroxides 95, 97 and 122 raise intracellular ion levels requires addressing. Membrane hyperpolarisation has been hypothesised in the case of amiodarone; the amphiphilic molecule and has been shown to associate with 
the plasma membrane of yeast cells, hyperpolarising the membrane, which may allow influx of the ion. ${ }^{237}$ Epiplakinic acid I (95), plakortolide X (97) and plakortide F acid (122) could also behave in this manner given their polar cyclic peroxide moieties and aliphatic backbones. The aliphatic portions of the three compounds, particularly the $\mathrm{C}_{12}$ and $\mathrm{C}_{10}$ carbon chains of $\mathbf{9 5}$ and $\mathbf{9 7}$, could potentially incorporate into the plasma membrane. This could induce membrane curvature allowing both the passage of the compounds into the cell, and influx of $\mathrm{Ca}^{2+}$ and/or $\mathrm{Zn}^{2+}$ into the cytoplasm.

Interaction of 95 and 97 with membrane-bound ion pumps and channels is a likely mechanism by which cytosolic ion levels could rise. Efforts were made during this study towards the construction of a suite of strains carrying ion pump deletions and a corresponding GFP reporter (Table 5.10). Once compiled, the suite of GFP/deletion strains will be assessed for response to $\mathrm{Ca}^{2+}$ and $\mathrm{Zn}^{2+}$-induced stress. ZAP1 encodes a $\mathrm{Zn}^{2+}$-responsive transcription factor, which resides in both the nucleus and cytoplasm. Unlike Crz1p, Zap1p does not undergo nuclear translocation, however, application of FRET dyes to Zap1p can allow in vivo observation of $\mathrm{Zn}^{2+}$ traffic. ${ }^{249}$ Analysis of both transcription factors in the absence of membrane-pumps should provide insight into the mechanism of these cyclic peroxides.

Table 5.10 Proposed Ion Pump Deletion-GFP Strains

\begin{tabular}{|c|c|c|}
\hline \multirow[b]{2}{*}{ Target metal ion } & \multicolumn{2}{|c|}{ Target genes } \\
\hline & Deletion & GFP-reporter \\
\hline $\mathrm{Ca}^{2+}$ & $\operatorname{mid} 1 \Delta$ & Crz1p-GFP \\
\hline $\mathrm{Ca}^{2+}$ & $\operatorname{cch} 1 \Delta$ & Crz1p-GFP \\
\hline $\mathrm{Ca}^{2+}$ & pmc1s & Crz1p-GFP \\
\hline $\mathrm{Ca}^{2+}$ & $p m r 1 \Delta$ & Crz1p-GFP \\
\hline $\mathrm{Ca}^{2+}$ & $\operatorname{cch} 1 \Delta, \operatorname{mid} 1 \Delta$ & Crz1p-GFP \\
\hline $\mathrm{Ca}^{2+}$ and $\mathrm{Zn}^{2+}$ & $z r t 1 \Delta, z r t 2 \Delta$ & Crz1p-GFP \\
\hline $\mathrm{Ca}^{2+}$ and $\mathrm{Zn}^{2+}$ & $\operatorname{cch} 1 \Delta, \operatorname{mid} 1 \Delta$ & Zap1p-GFP \\
\hline $\mathrm{Zn}^{2+}$ & $z r t 1 \Delta, z r t 2 \Delta$ & Zap1p-GFP \\
\hline
\end{tabular}

Imaging studies will also be undertaken to further characterise the cellular effects of 95, including actin labeling with a fluorescent phalloidin conjugate, and visualisation of the endocytotic pathway with the fluorescent dye FM4-64FX. The effects of increased $\mathrm{Zn}^{2+}$ on cell size and the impact of exogenous $\mathrm{Ca}^{2+}$ and $\mathrm{Zn}^{2+}$ on bud formation will also be assessed. 


\subsubsection{Concluding remarks}

In summary, phenotypic and chemical genetic assessment of epiplakinic acid I (95) suggests that the growth inhibitory effects of $\mathbf{9 5}$ are caused by increased intracellular $\mathrm{Ca}^{2+}$ levels, leading to an increase in small budded cells and reduced cell size. This verifies the work of $\mathrm{Xu}$ et al. ${ }^{169}$ which attributes $\mathrm{Ca}^{2+}$-modulatory behaviour to the structurally related plakortide $\mathrm{F}$ acid (122). It may also be that increased levels of intracellular $\mathrm{Zn}^{2+}$ contribute to the activity of $\mathbf{9 5}$ and 122 in yeast.

Increases in cytosolic levels of $\mathrm{Ca}^{2+}$ and potentially $\mathrm{Zn}^{2+}$ may occur through interaction with the cyclic peroxide and either a membrane-bound ion pump or channel, or with the membrane itself. As such, generation of the ion pump deletionGFP strains is integral to elucidation of this mechanism, and future research will focus on the generation of these strains. Analysis of the double deletion mutants in particular should grant insight into the mechanism behind the activity of this class of compounds.

Unfortunately, further characterisation may be prevented owing to insufficient quantities of the cyclic peroxides available for analysis. This thesis has benefited from the assessment of specimens with a small sample size, however, this is a limiting factor for this particular arm of research. Complete characterisation of these compounds may be postponed until recollection of the same organism, or one containing similar metabolites. 


\section{Chapter 6}

\section{Concluding Remarks}

Natural products chemistry continues to play an important role in the identification of new pharmaceutical leads for the development of new therapeutic agents. Using a spectroscopy-guided isolation approach, the metabolite profiles of nine Tongan sponge specimens were investigated during this research. Analysis of HMBC experiments acquired from semi-purified fractions from each organism was integral to their selection for study. In order to maximise the probability of uncovering structural novelty in a well-explored environment, only specimens collected in small quantities and those with sparse distribution were chosen for investigation. While this particular facet presented challenges and restricted creative freedom during chromatography, it was also rewarding with seventeen new metabolites expediently isolated from five different organisms over the course of this study. These include two hydroxybutenolide sesterterpenes $\mathbf{2 3}$ and $\mathbf{2 4}$, and the alkaloid 14-bromohomofascaplysin (29). As five of the four sponges that afforded new metabolites were collected from 'Eua island, further investigation of organisms from this location in particular may be worthwhile.

Three labdane diterpenes, luakuliides $\mathrm{A}-\mathrm{C}(\mathbf{3 3 - 3 6 )}$ were isolated from an unidentified dictyoceratid sponge. The gem-dimethyl pair, characteristic of the labdane skeleton was evident in the HMBC experiment of the screening fraction, prompting further purification of the fractions. The luakuliide skeleton also features a hemiacetal moiety bridging the B-ring of the labdane bicycle. Luakuliide A (33) and its acetal derivative (34) were found to modulate NO and TNF- $\alpha$ levels in macrophages, demonstrating their immunomodulatory potential.

Investigation of two plakinid sponge specimens yielded the $\alpha$-pyrones lehualides $\mathrm{E}-\mathrm{K}$ (69-75), and a series of cyclic peroxides (95-98). In both cases the observation of heavily oxygenated sub-structures prompted further investigation of the organisms. The non-protonated nature of the major functional groups in both the lehualides and the cyclic peroxides makes it unlikely that either of these structural classes would have been revealed during spectroscopic organism screening without analysis of the HMBC experiment. 
Structural elucidation of the fully-substituted $\alpha$-pyrone system of lehualides $\mathrm{E}-\mathrm{K}$ (69-75) presented a challenge. Similarly unprotonated systems have previously been proposed on the basis of chemical shift alone, however, the substitution pattern of the compounds found in this study allowed for the unequivocal establishment of the $\alpha$-pyrone substructure. Lehualides $\mathrm{H}-\mathrm{K}(\mathbf{7 2}-\mathbf{7 5})$ also demonstrate several different sulfur-containing functional groups. The presence of the NMR-silent ${ }^{32} \mathrm{~S}$ nuclei was determined by analysis of ${ }^{1} J_{\mathrm{CH}}$ couplings of adjacent methylene and methyl centres. However, unequivocal identification of the functional groups required synthetic modeling of the proposed structural fragments and analysis of their ${ }^{1} \mathrm{H}$ and ${ }^{13} \mathrm{C}$ NMR spectra. ${ }^{134}$ Although NMR spectroscopy is an important tool to structural elucidation, the technique has its limits. As such it is important to provide further evidence of a structure wherever possible, be it through chemical derivatisation, $\mathrm{X}$-ray analysis of an isolated compound, or synthesis.

A variety of cyclic peroxides have been uncovered from plakinid sponges, however, the isolation of multiple classes from one organism is less frequently observed. In this case, three different polyketide cyclic peroxide classes, including the rare 1-hydroxy1,2-dioxolane metabolite 98, were encountered alongside two $5 \alpha, 8 \alpha$-epidioxysterols. Cyclic peroxides 95-98 all displayed demonstrated potent inhibition of the HL-60 cell line, with the more heavily oxygenated compounds affecting greater inhibition. As such, the significantly decreased sensitivity of HL-60 cells to $\mathbf{9 7}$ compared with $\mathbf{9 5}$ and $\mathbf{9 8}$ may be due to the absence of the free carboxylic acid moiety.

Isolation of secondary metabolites without knowledge of their biological potential requires a technique capable of delivering an unbiased assessment of biological activity. Genome-wide profiling assays were conducted in an attempt to biologically characterise epiplakinic acid I (95), using the Saccharomyces cerevisiae homozygous and heterozygous diploid deletion sets. This study required the development of new laboratory techniques, and an understanding of foreign experimental concepts. Although no definitive cellular target was determined, this study verified the widely held opinion that plakinid cyclic peroxides disrupt cellular calcium homeostasis.

While in this instance chemical genetic screening was not successful in identifying the precise mechanism of action of a small molecule, it has indicated specific assays that could pinpoint the precise molecular target of the molecule. Future research into the elucidation of this mechanism is planned, although the realities of isolation from rare organisms may prevent these characterisation studies. While the investigation of uncommon organisms from geographically isolated locations may yield structural novelty, recollection difficulties can hinder the development of new bioactives. 


\section{Chapter 7}

\section{General Experimental Procedures}

Optical rotations were performed on a Autopol II Automatic Polarimeter while UV spectra were acquired on an Agilent 8453 UV/Visible Spectrometer and IR spectra were recorded using a Perkin Elmer Spectrum One FT-IR spectrometer. NMR spectra were obtained using a $600 \mathrm{MHz}$ Varian DirectDrive spectrometer equipped with a triple resonance $\mathrm{HCN}$ cryogenic probe, operating at 600, 150 and $60 \mathrm{MHz}$ for ${ }^{1} \mathrm{H},{ }^{13} \mathrm{C}$ and ${ }^{15} \mathrm{~N}$ nuclei, respectively. All HSQC spectra were acquired without carbon-decoupling. Spectra were recorded in $\mathrm{CD}_{3} \mathrm{OD}$ (Cambridge Isotopes), $\mathrm{CDCl}_{3}$ (Merck), $\left(\mathrm{CD}_{3}\right)_{2} \mathrm{SO}$ (Cambridge Isotopes) and $\mathrm{C}_{6} \mathrm{D}_{6}$ (Cambridge Isotopes), with chemical shifts $\delta(\mathrm{ppm})$ referenced to the residual solvent peak [ $\left(\mathrm{CD}_{3} \mathrm{OD}: \delta_{\mathrm{C}} 49.00\right.$, $\left.\delta_{\mathrm{H}} 3.31\right),\left(\mathrm{CDCl}_{3}: \delta_{\mathrm{C}} 77.16, \delta_{\mathrm{H}} 7.26\right),\left(\left(\mathrm{CD}_{3}\right)_{2} \mathrm{SO}: \delta_{\mathrm{C}} 39.52, \delta_{\mathrm{H}} 2.50\right)$ and $\left(\mathrm{C}_{6} \mathrm{D}_{6}\right.$ : $\left.\left.\delta_{\mathrm{C}} 128.06, \delta_{\mathrm{H}} 7.16\right)\right] .{ }^{250}$ HRESIMS measurements were obtained using a Waters QTOF Premier ${ }^{\mathrm{TM}}$ Tandem Mass Spectrometer.

GFP and RFP cell studies were performed on a Perkin Elmer EvoTec OPERA. GFP and RFP were excited using $488 \mathrm{~nm}$ and $561 \mathrm{~nm}$ with emission fluorescence collected through 520/35 and 600/40 bandpass filters respectively. Cells were imaged using an Olympus IXF1 optical microscope operating with a CC12 Soft Imaging System. Cell growth was quantified using a using a Wallac EnVision 2102 Multilabel Plate Reader (Perkin Elmer) while cell population studies were performed on a FACS Canto ${ }^{\mathrm{TM}} \mathrm{II}$ Flow Cytometer (BD HTS) and manipulated on FlowJo version 7.6.1 flow cytometry analysis software (Tree Star Inc., Ashland, OR, USA). Cultures were concentrated using an Eppendorf 5810 Centrifuge and a 5415 R Microcentrifuge and suspended with a TallBoys Standard Vortex Mixer and an Eppendorf MixMate plate mixer.

2,3-Dihydroxypropoxypropyl-derivatised silica gel (DIOL) was used for all normalphased column chromatography while Supelco dianion HP20 and HP20SS polystyrene (divinylbenzene) resins were used for reversed-phase column chromatography unless otherwise stated. Size exclusion chromatography was performed using Sephadex LH20. HPLC-grade $\mathrm{CH}_{2} \mathrm{Cl}_{2}$ (Merck) and MeCN (Fluka) were used for general puposes and for HPLC purification, all other solvents (including $\mathrm{H}_{2} \mathrm{O}$ ) were distilled from glass immediately prior to use. TLC analyses were performed using Machery- 
Nagel Alugram Sil G/UV254 plates. The plates were analysed by fluorescence quenching under UV light $(\lambda=254 \mathrm{~nm})$ followed by dipping in $5 \% \mathrm{H}_{2} \mathrm{SO}_{4}$ in $\mathrm{MeOH}$ then dipping in $0.1 \%$ vanillin in $\mathrm{EtOH}$ and heating. Chemical reagents were purchased from Sigma-Aldrich and Penta International Corporation, unless otherwise specified, and used without further purification.

\subsection{Isolation of new diterpenes from PTN3 $45 \mathrm{E}$}

\subsubsection{Animal material}

A garlic-odoured specimen, PTN3_45E (96 g) was collected from the vertical rock sides of Tu'ungasika Island in the Vava'u group, at a depth of $12-15 \mathrm{~m}$ in November 2009. The specimen was frozen immediately and maintained at $-18^{\circ} \mathrm{C}$ until extraction. A voucher specimen (PTN3_45E) has been deposited at the School of Chemical and Physical Sciences, VUW, Wellington.

\subsubsection{Extraction of PTN3 45E}

A frozen $24 \mathrm{~g}$ sample was extracted twice in $100 \mathrm{~mL} \mathrm{MeOH}$ overnight. The filtered extracts were passed through a column of $40 \mathrm{~mL}$ HP20 resin in reverse order and the combined eluents were diluted 1:1 with $200 \mathrm{~mL} \mathrm{H}_{2} \mathrm{O}$. The diluted eluent was passed through the column again, further diluted with $400 \mathrm{~mL} \mathrm{H}_{2} \mathrm{O}$ to $25 \% \mathrm{MeOH}$ and passed through the column once more. The column was washed with $\mathrm{H}_{2} \mathrm{O}$ then eluted generating three $100 \mathrm{~mL}$ fractions of $30 \%, 75 \%$ and $100 \% \mathrm{Me}_{2} \mathrm{CO}$ in $\mathrm{H}_{2} \mathrm{O}$ of $10.5 \mathrm{mg}, 70.7 \mathrm{mg}$ and $121.5 \mathrm{mg}$ respectively.

Further reversed-phase purification of the $75 \% \quad \mathrm{Me}_{2} \mathrm{CO}$ in $\mathrm{H}_{2} \mathrm{O}$ fraction was undertaken on HP20SS, generating $\mathrm{Me}_{2} \mathrm{CO}$ in $\mathrm{H}_{2} \mathrm{O}$ fractions of $30-100 \% \mathrm{Me}_{2} \mathrm{CO}$. The $80 \% \mathrm{Me}_{2} \mathrm{CO}$ in $\mathrm{H}_{2} \mathrm{O}$ HP20SS fraction was further partitioned over HP20SS resin with $70-80 \% \mathrm{Me}_{2} \mathrm{CO}$ in $\mathrm{H}_{2} \mathrm{O}$ in $2 \%$ intervals. Analysis by TLC $(5 \% \mathrm{MeOH}$ in $\mathrm{CH}_{2} \mathrm{Cl}_{2}$ ) indicated two metabolites of similar polarity were present in the $70 \%$ and $72 \% \mathrm{Me}_{2} \mathrm{CO}$ in $\mathrm{H}_{2} \mathrm{O}$ fractions (rf. 0.32 and 0.35 ). Resolution of the metabolites was achieved by reversed-phase HPLC $\left(\mathrm{C}_{18}, 85 \% \mathrm{MeCN}\right.$ in $\left.\mathrm{H}_{2} \mathrm{O}\right)$ with $23(0.0007 \mathrm{~g})$ and $24(0.0015 \mathrm{~g})$ eluting at 10 and $12 \mathrm{~min}$ respectively.

iso-Secothorectolide (23): Clear oil, $0.7 \mathrm{mg} ;{ }^{1} \mathrm{H}$ and ${ }^{13} \mathrm{C}$ NMR data, see Table C.1; HRESIMS $m / z 439.2458[\mathrm{M}+\mathrm{Na}]^{+}$(calcd for $\mathrm{C}_{25} \mathrm{H}_{36} \mathrm{O}_{5} \mathrm{Na}, 439.2460 ; \Delta-0.5$ ppm); $m / z 415.2487[\mathrm{M}-\mathrm{H}]^{-}$(calcd for $\mathrm{C}_{25} \mathrm{H}_{35} \mathrm{O}_{5}, 415.2484 ; \Delta 0.3 \mathrm{ppm}$ ). 
1-Hydroxyluffarin Q (24): Clear oil, $1.5 \mathrm{mg} ;{ }^{1} \mathrm{H}$ and ${ }^{13} \mathrm{C}$ NMR data, see Table C.2; HRESIMS $m / z 439.2458[\mathrm{M}+\mathrm{Na}]^{+}$(calcd for $\mathrm{C}_{25} \mathrm{H}_{36} \mathrm{O}_{5} \mathrm{Na}, 439.2460 ; \Delta-0.5$ ppm); m/z 415.2476 [M - H] $]^{-}$(calcd for $\mathrm{C}_{25} \mathrm{H}_{35} \mathrm{O}_{5}, 415.2484 ; \Delta-0.8 \mathrm{ppm}$ ).

\subsection{Isolation of known terpenes from PTN4_10B}

\subsubsection{Animal material}

The odorous sponge specimen PTN4_10B (125 g) was collected using SCUBA from a vertical rock face at a depth of ca. $15 \mathrm{~m}$ on Fakalotulā island in the Vava'u group in November 2009. The specimen was frozen immediately and maintained at $-18^{\circ} \mathrm{C}$ until extraction. A voucher specimen is deposited at the School of Chemical and Physical Sciences, VUW, Wellington.

\subsubsection{Initial extraction of PTN4 10B}

A frozen $16.0 \mathrm{~g}$ sample was extracted twice for $20 \mathrm{~h}$ in $100 \mathrm{~mL} \mathrm{MeOH}$ at room temperature. In reverse order the extracts were passed through a column of 40 mL HP20 resin, the combined eluents were diluted 1:1 with $200 \mathrm{~mL} \mathrm{H}_{2} \mathrm{O}$, passed through the column again, further diluted with $400 \mathrm{~mL} \mathrm{H}_{2} \mathrm{O}$ to $25 \% \mathrm{MeOH}$ and passed through the column once more. The column was washed with $\mathrm{H}_{2} \mathrm{O}$ then eluted, generating three $100 \mathrm{~mL}$ fractions of $30 \%, 75 \%$ and $100 \% \mathrm{Me}_{2} \mathrm{CO}$ in $\mathrm{H}_{2} \mathrm{O}$. The $\mathrm{Me}_{2} \mathrm{CO}$ in $\mathrm{H}_{2} \mathrm{O}$ fractions (39.3 $\mathrm{mg}, 65.8 \mathrm{mg}$ and $96.2 \mathrm{mg}$ respectively) were evaporated to dryness under reduced pressure and subjected to $1 \mathrm{D}$ and $2 \mathrm{D}$ NMR analysis.

The crude NMR spectra of the $100 \% \mathrm{Me}_{2} \mathrm{CO}$ in $\mathrm{H}_{2} \mathrm{O}$ fraction was dominated by two compounds identified as ambliol B (25) and luffariellolide (26). Bench-top column chromatography of the lipophilic fraction (DIOL, $\mathrm{CH}_{2} \mathrm{Cl}_{2} / 20 \% \mathrm{MeOH}$ ) yielded $\mathrm{CH}_{2} \mathrm{Cl}_{2}$ fractions rich in ambliol $\mathrm{B}(\mathbf{2 5})$ and spectroscopically pure luffariellolide (26, $11.3 \mathrm{mg})$. Further bench-top purification afforded ambliol B (7.3 mg, 75\% hexane in $\mathrm{CH}_{2} \mathrm{Cl}_{2}$ ).

Ambliol B (25): Clear oil, $7.3 \mathrm{mg} ;[\alpha]_{D}^{17.5}+67.6^{\circ}\left(c 0.06, \mathrm{CHCl}_{3}\right) ;{ }^{1} \mathrm{H}$ and ${ }^{13} \mathrm{C}$ NMR data, see Table C.3.

Luffariellolide (26): Clear oil, $11.3 \mathrm{mg} ;[\alpha]_{D}^{17.5}+4.9^{\circ}\left(c 0.1, \mathrm{CHCl}_{3}\right) ;{ }^{1} \mathrm{H}$ and ${ }^{13} \mathrm{C}$ NMR data, see Table C.4. 


\subsection{Isolation of 14-bromohomofascaplysin}

\subsubsection{Animal material}

The slimy green sponge, PTN3_20B (40 g) was collected from the interior of a large cave at a depth of 12-15 m on 'Eua Island in November 2008. The specimen was frozen immediately then stored at $-18^{\circ} \mathrm{C}$ until extraction. A voucher specimen is deposited at the School of Chemical and Physical Sciences, VUW, Wellington.

\subsubsection{Extraction of PTN3 20B}

A frozen $20.5 \mathrm{~g}$ screening sample of PTN3_20B was extracted twice overnight for $18 \mathrm{~h}$ with $100 \mathrm{~mL} \mathrm{MeOH}$ at room temperature. The filtered extracts were passed through a column of $40 \mathrm{~mL}$ HP20 resin in reverse order and the combined eluents were diluted 1:1 with $200 \mathrm{~mL} \mathrm{H}_{2} \mathrm{O}$. The diluted eluent was passed through the column again, further diluted with $400 \mathrm{~mL} \mathrm{H}_{2} \mathrm{O}$ to $25 \% \mathrm{MeOH}$ and passed through the column once more. The column was washed with $\mathrm{H}_{2} \mathrm{O}$ then eluted generating three $100 \mathrm{~mL}$ fractions of $30 \%, 75 \%$ and $100 \% \mathrm{Me}_{2} \mathrm{CO}$ in $\mathrm{H}_{2} \mathrm{O}(13.0 \mathrm{mg}, 98.6 \mathrm{mg}$ and $90.0 \mathrm{mg}$ respectively).

The $75 \%$ and $100 \% \mathrm{Me}_{2} \mathrm{CO}$ in $\mathrm{H}_{2} \mathrm{O}$ fractions were further partitioned over HP20SS and the fractions $\left(30 \%, 40 \%, 50 \%, 60 \%, 70 \% 80 \%\right.$ and $100 \% \mathrm{Me}_{2} \mathrm{CO}$ in $\left.\mathrm{H}_{2} \mathrm{O}\right)$ analysed by ${ }^{1} \mathrm{H}$ NMR. The $50 \%$ and $60 \% \mathrm{Me}_{2} \mathrm{CO}$ in $\mathrm{H}_{2} \mathrm{O}$ HP20SS fractions were further purified by bench-top chromatography (DIOL, hexane $/ \mathrm{CH}_{2} \mathrm{Cl}_{2} / \mathrm{EtOAc} / \mathrm{MeOH} / \mathrm{H}_{2} \mathrm{O}$ ) with small quantities of the charged 14-bromohomofascaplysin (29) eluting slowly from the column in the $10 \% \mathrm{MeOH}$ in $\mathrm{H}_{2} \mathrm{O}$ fraction.

14-bromohomofascaplysin (29): Clear oil, ca. $700 \mu \mathrm{g} ;{ }^{1} \mathrm{H}$ and ${ }^{13} \mathrm{C}$ NMR data, see Table C.7; HRESIMS $m / z$ 407.0395 $[\mathrm{M}+\mathrm{H}]^{+}$(calcd for $\mathrm{C}_{21} \mathrm{H}_{16} \mathrm{~N}_{2} \mathrm{O}_{2} \mathrm{Br}$, 407.0395; $\Delta 0.0 \mathrm{ppm})$.

\subsection{3 $\mathrm{Me}_{2} \mathrm{CO}$-free extraction of PTN3 20B}

The remaining $19.5 \mathrm{~g}$ of PTN3 20B was extracted twice overnight for $18 \mathrm{~h}$ in $100 \mathrm{~mL}$ $\mathrm{MeOH}$, and the extracts were partitioned over HP20 resin. The column was eluted with eight fractions ranging from $30-100 \% \mathrm{MeOH}$ in $\mathrm{H}_{2} \mathrm{O}$, which were analysed by ${ }^{1} \mathrm{H}$ NMR. Further 2D analysis was performed in an attempt to identify either 14-bromohomofascaplysin or 14-bromofascaplysin, although neither compound was identified by these methods. 


\subsection{Isolation of halenaquinone}

\subsubsection{Animal material}

A red liver-coloured sponge specimen, PTN3_21D (50 g) was collected using SCUBA from the horizontal ceiling of a large cave at a depth of 12-15 m on 'Eua Island in November 2008. The specimen was frozen immediately and maintained at $-18^{\circ} \mathrm{C}$ until extraction. A voucher specimen is deposited at the School of Chemical and Physical Sciences, VUW, Wellington.

\subsubsection{Extraction of PTN3_21D}

The frozen sample $(22.0 \mathrm{~g})$ was extracted twice for $20 \mathrm{~h}$ in $100 \mathrm{~mL} \mathrm{MeOH}$ at room temperature. In reverse order the filtered extracts were passed through a column of $40 \mathrm{~mL} \mathrm{HP} 20$ resin, the combined eluents were diluted 1:1 with $200 \mathrm{~mL} \mathrm{H}_{2} \mathrm{O}$, passed through the column again, further diluted with $400 \mathrm{~mL} \mathrm{H}_{2} \mathrm{O}$ to $25 \% \mathrm{MeOH}$ and passed through the column once more. The column was washed with $\mathrm{H}_{2} \mathrm{O}$ then eluted generating three $100 \mathrm{~mL}$ fractions of $30 \%, 75 \%$ and $100 \% \mathrm{Me}_{2} \mathrm{CO}$ in $\mathrm{H}_{2} \mathrm{O}$ (41.5 mg, $95.4 \mathrm{mg}$ and $53.5 \mathrm{mg}$ respectively). The $75 \%$ and $100 \% \mathrm{Me}_{2} \mathrm{CO}$ in $\mathrm{H}_{2} \mathrm{O}$ fractions were evaporated to dryness under reduced pressure and subjected to $1 \mathrm{D}$ and 2D NMR analysis. Normal-phase chromatography of the $75 \% \mathrm{Me}_{2} \mathrm{CO}$ in $\mathrm{H}_{2} \mathrm{O}$ HP20 fraction (DIOL, hexane $/ \mathrm{CH}_{2} \mathrm{Cl}_{2} / \mathrm{EtOAc} / \mathrm{MeOH}$ ) and subsequent NMR analysis showed concentration of the signals of interest in the $75 \%$ hexane in $\mathrm{CH}_{2} \mathrm{Cl}_{2}$ fraction. Subsequent reversed-phase $\mathrm{HPLC}\left(\mathrm{C}_{18}, 90 \% \mathrm{MeCN}\right.$ in $\left.\mathrm{H}_{2} \mathrm{O}\right)$ yielded halenaquinone (31) (10.1 mg) as a red solid.

Halenaquinone (31): red solid, $10.1 \mathrm{mg} ;{ }^{1} \mathrm{H}$ and ${ }^{13} \mathrm{C}$ NMR data, see Table C.8.

\subsection{Isolation of luakuliides $\mathrm{A}-\mathrm{C}$}

\subsubsection{Animal material}

The dictyoceratid sponge sample PTN3_21E (90 g) was collected at 12-15 m from an unlit cave on 'Eua Island in November 2008. The specimen was frozen immediately and maintained at $-18^{\circ} \mathrm{C}$ until extraction. A second collection PTN3_50B (42 g) was made from a similar cave location at Shark's Tooth, Kitu's in the Vava'u Island group in November 2009. A voucher specimen is deposited at the School of Chemical and Physical Sciences, VUW, Wellington. 


\subsubsection{Screening extraction of PTN3 21E}

A $23 \mathrm{~g}$ sample was extracted while frozen in $\mathrm{MeOH}$ and partitioned over HP20 according to laboratory protocol (Appendix A). 1D and 2D NMR analysis of the $100 \%$ and $75 \% \mathrm{Me}_{2} \mathrm{CO}$ in $\mathrm{H}_{2} \mathrm{O}$ fractions (105.2 mg, $120.8 \mathrm{mg}$ respectively) revealed the presence of two closely related metabolites. Further reversed-phase fractionation of both fractions was undertaken over HP20SS resin producing fractions of $\mathrm{Me}_{2} \mathrm{CO}$ in $\mathrm{H}_{2} \mathrm{O}\left(30-100 \%, 10 \%\right.$ intervals). ${ }^{1} \mathrm{H} \mathrm{NMR}$ analysis of the $60 \%$ and $70 \% \mathrm{Me}_{2} \mathrm{CO}$ in $\mathrm{H}_{2} \mathrm{O}$ HP20SS fractions revealed luakuliide A (33, $\left.92.7 \mathrm{mg}\right)$ in exceptionally high spectroscopic purity.

The $100 \% \mathrm{Me}_{2} \mathrm{CO}$ fraction was further partitioned (DIOL, hexane/ $\mathrm{CH}_{2} \mathrm{Cl}_{2} / \mathrm{EtOAc}$ ) generating a steroid rich fraction ( $4.7 \mathrm{mg}, 50 \%$ hexane in $\mathrm{CH}_{2} \mathrm{Cl}_{2}$ ). Bench-top chromatography of the $90 \% \mathrm{Me}_{2} \mathrm{CO}$ in $\mathrm{H}_{2} \mathrm{O}$ fraction (DIOL, $\mathrm{CH}_{2} \mathrm{Cl}_{2} /$ EtOAc) yielded methyl-acetal analogue of luakuliide A, $34(16.1 \mathrm{mg})$, while the $80 \% \mathrm{Me}_{2} \mathrm{CO}$ in $\mathrm{H}_{2} \mathrm{O}$ HP20SS fraction contained a mixture of the two compounds.

\section{Ethanol extraction of PTN3 21E}

The remaining $67 \mathrm{~g}$ of PTN3_21E were extracted twice overnight in $200 \mathrm{~mL}$ EtOH and the extracts were partitioned over HP20 generating 30\%, $75 \%$ and $100 \% \mathrm{Me}_{2} \mathrm{CO}$ in $\mathrm{H}_{2} \mathrm{O}$ fractions. Compound $\mathbf{3 3}$ was clearly present within the extracts, however, the methyl-acetal analogue $\mathbf{3 4}$ was absent, indicating it was an artifact of $\mathrm{MeOH}$ extraction.

Derivatisation of luakuliide A (33): Luakuliide A (33, $5.0 \mathrm{mg})$ was dissolved in $5 \mathrm{~mL} 10 \% \mathrm{CDCl}_{3}$ in $\mathrm{MeOH}$ and maintained at $4{ }^{\circ} \mathrm{C}$ for $72 \mathrm{~h} .{ }^{1} \mathrm{H} \mathrm{NMR}$ analysis showed quantitative conversion to the methyl-acetal analogue $\mathbf{3 4}$.

\subsubsection{Extraction of PTN3 50B}

The $42 \mathrm{~g}$ sample was extracted twice overnight in $200 \mathrm{~mL} \mathrm{MeOH}$ and partitioned over $40 \mathrm{~mL}$ HP20 according as above. The $100 \%$ and $75 \% \mathrm{Me}_{2} \mathrm{CO}$ in $\mathrm{H}_{2} \mathrm{O}$ fractions were partitioned over HP20SS with $30-100 \% \mathrm{Me}_{2} \mathrm{CO}$ in $\mathrm{H}_{2} \mathrm{O}$ mixtures (10\% intervals). As previously observed, the $80-100 \% \mathrm{Me}_{2} \mathrm{CO}$ in $\mathrm{H}_{2} \mathrm{O}$ fractions contained compounds 33 and 34, while the $70 \%$ and $60 \% \mathrm{Me}_{2} \mathrm{CO}$ in $\mathrm{H}_{2} \mathrm{O}$ HP20SS fractions appeared to contain previously unencountered resonances, and was further partitioned over DIOL (hexane $/ \mathrm{CH}_{2} \mathrm{Cl}_{2} /$ EtOAc). The $33 \%$ EtOAc in $\mathrm{CH}_{2} \mathrm{Cl}_{2}$ fraction was then 
partitioned by HPLC $\left(\mathrm{C}_{18}, 70 \% \mathrm{MeCN}\right.$ in $\left.\mathrm{H}_{2} \mathrm{O}\right)$ with the butenolide diterpenes 35 and 36 eluting between 4.0-4.7 $\mathrm{min}$.

The $100 \% \mathrm{Me}_{2} \mathrm{CO}$ HP20SS fraction was devoid of both $\mathbf{3 3}$ and $\mathbf{3 4}$, and subjected to further normal-phase chromatography (DIOL, hexane $/ \mathrm{CH}_{2} \mathrm{Cl}_{2} / \mathrm{EtOAc}$ ). The minor terpenoid metabolites eluted in the $20 \% \mathrm{EtOAc} / \mathrm{CH}_{2} \mathrm{Cl}_{2}$ fraction, $\mathrm{HPLC}$ purification of which $\left(\mathrm{C}_{18}, 70 \% \mathrm{MeCN}\right.$ in $\left.\mathrm{H}_{2} \mathrm{O}\right)$ yielded 35 and 36, eluting closely at 4.2 and 4.5 min. Fractions from different collections of the two butenolide metabolites were combined and subjected to further chromatographic purification by $\operatorname{HPLC}\left(\mathrm{C}_{18}\right.$, $70 \% \mathrm{MeCN}$ in $\mathrm{H}_{2} \mathrm{O}$ ) generating ca. $600 \mu \mathrm{g}$ of both $\mathbf{3 5}$ and $\mathbf{3 6}$.

Normal-phase chromatography of the $70 \% \mathrm{Me}_{2} \mathrm{CO}$ in $\mathrm{H}_{2} \mathrm{O}$ HP20SS fraction (DIOL, $\mathrm{CH}_{2} \mathrm{Cl}_{2} /$ EtOAc) yielded an uncharacterised labdane diterpene in the $2.5 \%$ EtOAc in $\mathrm{CH}_{2} \mathrm{Cl}_{2}$ fraction, which was also identified within previously acquired spectra from the 'Eua specimen.

Luakuliide A (33): Clear oil, $92.7 \mathrm{mg} ;[\alpha]_{D}^{25.4}-10.8^{\circ}$ ( c 18.5, $\mathrm{CHCl}_{3}$ ), UV $(\mathrm{MeOH}) \lambda_{\max }(\log \epsilon), 213$ (2.90); IR (film) $\nu_{\max } 3385,2923,2851,1727,1458,1376$, 1161, $1027 \mathrm{~cm}^{-1} ;{ }^{1} \mathrm{H}$ and ${ }^{13} \mathrm{C}$ NMR data see Table 3.2; HRESIMS $m / z 341.2097$ $[\mathrm{M}+\mathrm{Na}]^{+}$(calcd for $\left.\mathrm{C}_{20} \mathrm{H}_{30} \mathrm{O}_{3} \mathrm{Na}, 341.2093 ; \Delta 1.2 \mathrm{ppm}\right)$.

Luakuliide A methyl-acetal (34): Clear oil, $16.1 \mathrm{mg} ;[\alpha]_{D}^{25.4}-75.0^{\circ}$ ( 0 0.14, $\left.\mathrm{CHCl}_{3}\right), \mathrm{UV}(\mathrm{MeOH}) \lambda_{\max }(\log \epsilon), 215$ (3.89) nm; IR (film) $\nu_{\max } 2954,2926,2860$, 1727, 1460, 1377, 1274, 1073, $999 \mathrm{~cm}^{-1} ;{ }^{1} \mathrm{H}$ and ${ }^{13} \mathrm{C}$ NMR data see Table 3.3; HRESIMS $m / z$ 355.2257 $[\mathrm{M}+\mathrm{Na}]^{+}$(calcd for $\mathrm{C}_{21} \mathrm{H}_{32} \mathrm{O}_{3} \mathrm{Na}, 355.2249 ; \Delta 2.3 \mathrm{ppm}$ ).

Luakuliide B (35): Clear oil, ca. $600 \mu \mathrm{g}$; UV (MeOH) ) $\lambda_{\max }(\log \epsilon), 235$ (3.80), 295 (3.73); IR (film) $\nu_{\max } 3250,2928,2853,1687,1647,1561,1440,1221,1094 \mathrm{~cm}^{-1}$; ${ }^{1} \mathrm{H}$ and ${ }^{13} \mathrm{C}$ NMR data see Table 3.4; HRESIMS $m / z 373.1996[\mathrm{M}+\mathrm{Na}]^{+}$(calcd for $\left.\mathrm{C}_{20} \mathrm{H}_{30} \mathrm{O}_{5} \mathrm{Na}, 373.1991 ; \Delta 1.3 \mathrm{ppm}\right)$.

Luakuliide C (36): Clear oil, ca. $600 \mu \mathrm{g}$; UV (MeOH) $\lambda_{\max }(\log \epsilon), 235$ (3.80), 295 (3.73); IR (film) $\nu_{\max } 3250,2928,2853,1687,1647,1561,1440,1221,1094 \mathrm{~cm}^{-1}$; ${ }^{1} \mathrm{H}$ and ${ }^{13} \mathrm{C}$ NMR data see Table 3.2; HRESIMS $m / z 373.1990[\mathrm{M}+\mathrm{Na}]^{+}$(calcd for $\mathrm{C}_{20} \mathrm{H}_{30} \mathrm{O}_{5} \mathrm{Na}, 373.1991 ; \Delta-0.3 \mathrm{ppm}$ ). 


\subsection{Isolation of lehualides $\mathrm{E}-\mathrm{K}$}

\subsubsection{Animal material}

The Plakortis sponge PTN3_20A (36.0 g) collected was using SCUBA from the horizontal ceiling of a large cave at a depth of 12-15 m on 'Eua Island in November 2008. The specimen was frozen immediately and kept at $-18^{\circ} \mathrm{C}$ until extraction. The surface of the corky specimen is covered in wide shallow nodules ca. $3 \mathrm{~mm}$ high, and the organism forms small oval pendant encrustations. Diod spicules of 180-250 $\mu \mathrm{m}$ in length were recovered following $\mathrm{HNO}_{3}$ digest of the organic material. A voucher specimen (PTN3_20A) has been deposited in the collection of Dr James Bell at the School of Biological Sciences, VUW, Wellington.

\subsubsection{Initial extraction of Plakortis sp. PTN3 20A}

The frozen sample $(21.0 \mathrm{~g})$ was extracted twice for $14 \mathrm{~h}$ in $100 \mathrm{~mL} \mathrm{MeOH}$ at room temperature. The second extract, followed by the first, were passed through a column of $40 \mathrm{~mL}$ HP20 resin, the eluents combined and diluted with $200 \mathrm{~mL} \mathrm{H}_{2} \mathrm{O}$. The diluted eluent was passed through the column again, further diluted to $25 \%$ $\mathrm{MeOH}$ and passed through the column once more. The column was washed with $\mathrm{H}_{2} \mathrm{O}$ then eluted generating three $100 \mathrm{~mL}$ fractions of $30 \%, 75 \%$ and $100 \% \mathrm{Me}_{2} \mathrm{CO}$ in $\mathrm{H}_{2} \mathrm{O}$. The $75 \%$ and $100 \% \mathrm{Me}_{2} \mathrm{CO}$ in $\mathrm{H}_{2} \mathrm{O}$ fractions were evaporated to dryness under reduced pressure then analysed by $1 \mathrm{D}$ and $2 \mathrm{D}$ NMR.

Sub-samples of the $75 \% \mathrm{Me}_{2} \mathrm{CO}$ in $\mathrm{H}_{2} \mathrm{O}$ fraction (ca. $40 \mathrm{mg}$ ) were further partitioned over $3 \mathrm{~mL}$ reversed-phase resin (HP20SS) with $\mathrm{MeOH}$ and $\mathrm{H}_{2} \mathrm{O}$. The columns were washed with $20 \mathrm{~mL} \mathrm{H} \mathrm{H}_{2} \mathrm{O}$ then eluted generating $10 \mathrm{~mL}$ fractions of $\mathrm{Me}_{2} \mathrm{CO}$ in $\mathrm{H}_{2} \mathrm{O}$ (30-100\%, 10\% intervals). The $50-100 \% \mathrm{Me}_{2} \mathrm{CO}$ in $\mathrm{H}_{2} \mathrm{O}$ fractions were assessed by ${ }^{1} \mathrm{H} \mathrm{NMR}\left(\mathrm{CDCl}_{3}\right)$. The $80 \% \mathrm{Me}_{2} \mathrm{CO}$ in $\mathrm{H}_{2} \mathrm{O}$ HP20SS fractions were evaporated to dryness then further partitioned over DIOL $\left(\mathrm{CH}_{2} \mathrm{Cl}_{2} / \mathrm{EtOAc} / \mathrm{MeOH}\right)$. The $100 \%$ $\mathrm{CH}_{2} \mathrm{Cl}_{2}$ fractions of the DIOL column was partitioned on reversed-phase $\operatorname{HPLC}\left(\mathrm{C}_{18}\right.$, $85 \% \mathrm{MeCN}$ in $\mathrm{H}_{2} \mathrm{O}$ ) with lehualides $\mathrm{F}$ and $\mathrm{G}$ (70 and 71) eluting at 6.5 and 10.5 min respectively $(2.8 \mathrm{mg}$, and $14.5 \mathrm{mg}$ total).

Sub-samples of the $100 \% \mathrm{Me}_{2} \mathrm{CO}$ fraction (ca. $40 \mathrm{mg}$ ) were partitioned over 3 $\mathrm{mL}$ HP20SS resin with $\mathrm{MeOH}$ and $\mathrm{H}_{2} \mathrm{O}$, the columns were washed with $20 \mathrm{~mL}$ $\mathrm{H}_{2} \mathrm{O}$ then eluted generating $10 \mathrm{~mL}$ fractions of $\mathrm{Me}_{2} \mathrm{CO}$ in $\mathrm{H}_{2} \mathrm{O}(30-100 \%, 10 \%$ intervals). The $80 \% \mathrm{Me}_{2} \mathrm{CO}$ in $\mathrm{H}_{2} \mathrm{O}$ fractions from the HP20SS columns were evaporated to dryness then further purified with bench-top DIOL chromatography (hexane/ $\mathrm{CH}_{2} \mathrm{Cl}_{2} /$ EtOAC). Bench-top $\mathrm{SiO}_{2}$ chromatography $\left(\mathrm{CH}_{2} \mathrm{Cl}_{2} /\right.$ EtOAc) of the 
$\mathrm{CH}_{2} \mathrm{Cl}_{2}$ DIOL fraction, followed by normal-phase HPLC (DIOL, $4 \%$ IPA in hexane) of the $2.5 \%$ EtOAc in $\mathrm{CH}_{2} \mathrm{Cl}_{2} \mathrm{SiO}_{2}$ fraction yielded lehualide $\mathrm{H}(\mathbf{7 2}, 700 \mu \mathrm{g})$ eluting at $16.1 \mathrm{~min}$.

\subsubsection{Second extraction of Plakortis sp. PTN3_20A}

The remaining $15 \mathrm{~g}$ of Plakortis sp. was extracted from frozen with $100 \mathrm{~mL} \mathrm{MeOH}$ for $14 \mathrm{~h}$. The extract was filtered and the sponge tissue extracted for a further 14 h with $100 \mathrm{~mL} \mathrm{MeOH}$. The second extract was filtered and loaded onto $40 \mathrm{~mL}$ of equilibrated HP20 resin beads followed by the first extract. The combined eluents diluted $50 \%$ with $\mathrm{H}_{2} \mathrm{O}$ and re-loaded onto the column, the eluent was further diluted $50 \%$ with $\mathrm{H}_{2} \mathrm{O}$ and passed through the column once again. The column was washed with $100 \mathrm{~mL} \mathrm{H}_{2} \mathrm{O}$, and eluted with $100 \mathrm{~mL}$ fractions of $30 \%, 75 \%$ and $100 \% \mathrm{Me}_{2} \mathrm{CO}$ in $\mathrm{H}_{2} \mathrm{O}$. Further reversed-phase partitioning of the $100 \% \mathrm{Me}_{2} \mathrm{CO} \mathrm{HP} 20$ fraction over HP20SS generated eight $\mathrm{Me}_{2} \mathrm{CO}$ in $\mathrm{H}_{2} \mathrm{O}$ fractions, $30-100 \%$ in $10 \% \mathrm{Me}_{2} \mathrm{CO}$ increments.

Normal-phase bench-top chromatography (DIOL, $\mathrm{CH}_{2} \mathrm{Cl}_{2} / \mathrm{EtOAc} / \mathrm{MeOH}$ ) of the combined $80 \% \mathrm{Me}_{2} \mathrm{CO}$ in $\mathrm{H}_{2} \mathrm{O}$ HP20SS fractions followed. The $100 \% \mathrm{CH}_{2} \mathrm{Cl}_{2}$ fractions were evaporated to dryness and subsequent NMR analysis showed that the signals of interest were concentrated within the fractions. Subsequent reversed-phase $\operatorname{HPLC}\left(\mathrm{C}_{18}, 85 \% \mathrm{MeCN}\right.$ in $\left.\mathrm{H}_{2} \mathrm{O}\right)$ of the $100 \% \mathrm{CH}_{2} \mathrm{Cl}_{2}$ fraction yielded lehualides $\mathrm{J}(\mathbf{7 4}, 500 \mu \mathrm{g}), \mathrm{H}(\mathbf{7 2}, 800 \mu \mathrm{g})$, I $(\mathbf{7 3}, 646 \mu \mathrm{g})$ and $\mathrm{E}(\mathbf{6 9}, 540 \mu \mathrm{g})$. Two related metabolites bearing terminal $\alpha$-pyrone and thioacetate moieties were also recovered, although not fully characterised.

The $100 \% \mathrm{Me}_{2} \mathrm{CO}$ HP20SS fractions were further partitioned over DIOL $\left(\mathrm{CH}_{2} \mathrm{Cl}_{2} /\right.$ EtOAc/MeOH) with lehualide $\mathrm{K}(75)$ eluting late in the $100 \% \mathrm{CH}_{2} \mathrm{Cl}_{2}$ fractions. Iterative cycles of bench-top chromatography (DIOL, hexane $/ \mathrm{CH}_{2} \mathrm{Cl}_{2}$ ) yielded lehualide $\mathrm{K}$ in ca. $85 \%$ purity (2.12 mg). Partitioning of the $60 \%$ and $70 \% \mathrm{Me}_{2} \mathrm{CO}$ in $\mathrm{H}_{2} \mathrm{O}$ HP20SS by reversed-phase $\mathrm{HPLC}\left(\mathrm{C}_{18}, 75 \%\right.$ MeCN in $\left.\mathrm{H}_{2} \mathrm{O}\right)$ afforded lehualide $\mathrm{J}(\mathbf{7 4}, 1.95 \mathrm{mg}$ total$)$.

Lehualide E (69): Clear oil, $540 \mu \mathrm{g}$; UV (MeOH) $\lambda_{\max }(\log \epsilon), 235$ (3.80), 295 (3.73); IR (film) $\nu_{\max } 3250,2928,2853,1687,1647,1561,1440,1221,1094 \mathrm{~cm}^{-1}$; ${ }^{1} \mathrm{H}$ and ${ }^{13} \mathrm{C}$ NMR data, see Table 4.1; HRESIMS $m / z 385.2399[\mathrm{M}+\mathrm{Na}]^{+}$(calcd for $\left.\mathrm{C}_{24} \mathrm{H}_{32} \mathrm{O}_{4} \mathrm{Na}, 385.2398 ; \Delta 0.2 \mathrm{ppm}\right)$.

Lehualide F (70): White solid, $2.8 \mathrm{mg} ; \mathrm{UV}(\mathrm{MeOH}) \lambda_{\max }(\log \epsilon), 235$ (3.81), 282 (3.70) nm; IR (film) $\nu_{\max }$ 3250, 2929, 2854, 1686, 1648, 1560, 1439, 1220, 1094 
$\mathrm{cm}^{-1} ;{ }^{1} \mathrm{H}$ and ${ }^{13} \mathrm{C}$ NMR data, see Table 4.2; HRESIMS $m / z 393.2042[\mathrm{M}+\mathrm{Na}]^{+}$ (calcd for $\mathrm{C}_{23} \mathrm{H}_{30} \mathrm{O}_{4} \mathrm{Na}, 393.2042 ; \Delta 0.0 \mathrm{ppm}$ ).

Lehualide G (71): White solid, $18.3 \mathrm{mg}$; UV (MeOH) $\lambda_{\max }(\log \epsilon), 235$ (3.44), 291 (3.55) nm; IR (film) $\nu_{\max }$ 3250, 2926, 2854, 1686, 1649, 1563, 1439, 1276, 1094 $\mathrm{cm}^{-1} ;{ }^{1} \mathrm{H}$ and ${ }^{13} \mathrm{C}$ NMR data, see Table 4.3; HRESIMS $m / z$ 421.2355 $[\mathrm{M}+\mathrm{Na}]^{+}$ (calcd for $\mathrm{C}_{25} \mathrm{H}_{34} \mathrm{O}_{4} \mathrm{Na}, 421.2355 ; \Delta 0.0 \mathrm{ppm}$ ).

Lehualide H (72): White solid, $1.5 \mathrm{mg} ; \mathrm{UV}(\mathrm{MeOH}) \lambda_{\max }(\log \epsilon), 255$ (3.68), 297 (4.29) nm; IR (film) $\nu_{\max }$ 2925, 2853, 1709, 1690, 1651, 1569, 1458, 1227, 670 $\mathrm{cm}^{-1} ;{ }^{1} \mathrm{H}$ and ${ }^{13} \mathrm{C}$ NMR data, see Table 4.4; HRESIMS $m / z 407.1864[\mathrm{M}+\mathrm{Na}]^{+}$ (calcd for $\mathrm{C}_{20} \mathrm{H}_{32} \mathrm{O}_{5} \mathrm{SNa}, 407.1868 ; \Delta-1.0 \mathrm{ppm}$ ).

Lehualide I (73): Clear oil, $646 \mu \mathrm{g}$; UV (MeOH) $\lambda_{\max }(\log \epsilon), 249$ (3.12), 297 (3.96) nm; IR (film) $\nu_{\max } 2926,2854,1712,1652,1570,1460,1370,1229,699 \mathrm{~cm}^{-1}$; ${ }^{1} \mathrm{H}$ and ${ }^{13} \mathrm{C}$ NMR data, see Table 4.5; HRESIMS $m / z 379.1920[\mathrm{M}+\mathrm{Na}]^{+}$(calcd for $\mathrm{C}_{19} \mathrm{H}_{32} \mathrm{O}_{4} \mathrm{SNa}$, 379.1919; $\left.\Delta 0.3 \mathrm{ppm}\right)$.

Lehualide J (74): Clear oil, $2.0 \mathrm{mg} ;[\alpha]_{D}^{19.1} 0.0^{\circ}\left(\right.$ c $\left.3.69 \times 10^{-3}, \mathrm{CHCl}_{3}\right)$, UV $(\mathrm{MeOH}) \lambda_{\max }(\log \epsilon), 249$ (2.88), 288 (3.52) nm; IR (film) $\nu_{\max }$ 2925, 2855, 1713, 1652, 1569, 1458, 1371, 1228, 1098, $699 \mathrm{~cm}^{-1}$; ${ }^{1} \mathrm{H}$ and ${ }^{13} \mathrm{C}$ NMR data, see Table 4.6; HRESIMS $m / z$ 395.1868 $[\mathrm{M}+\mathrm{Na}]^{+}$(calcd for $\mathrm{C}_{19} \mathrm{H}_{32} \mathrm{O}_{5} \mathrm{SNa}, 395.1868 ; \Delta 0.0$ $\mathrm{ppm})$.

Lehualide K (75): Clear oil, $2.1 \mathrm{mg}$ (impure); UV (MeOH) $\lambda_{\max }(\log \epsilon), 240$ (3.41), 296 (3.60) nm; IR (film) $\nu_{\max }$ 2926, 2852, 1690, 1651, 1569, 1458, 1227, 672 $\mathrm{cm}^{-1} ;{ }^{1} \mathrm{H}$ and ${ }^{13} \mathrm{C}$ NMR data, see Table 4.8; HRESIMS $m / z 705.3477[\mathrm{M}+\mathrm{Na}]^{+}$ (calcd for $\mathrm{C}_{36} \mathrm{H}_{58} \mathrm{O}_{8} \mathrm{~S}_{2} \mathrm{Na}, 705.3471 ; \Delta 0.9 \mathrm{ppm}$ ).

\subsubsection{Preparation of spectroscopic model compounds}

Preparation of octanethiol: 1-bromooctane $(5.95 \mathrm{~mL}, 34.20 \mathrm{mmol})$ was stirred in $30 \mathrm{~mL} \mathrm{MeOH}$ under ambient conditions. Thiourea (3.91 g, $0.05 \mathrm{~mol}$ ) was dissolved in $30 \mathrm{~mL} \mathrm{H}_{2} \mathrm{O}$ then added to the 1-bromooctane solution. The reaction was heated to reflux for $3.5 \mathrm{~h}$, then allowed to cool to room temperature. $\mathrm{KOH}(4.83 \mathrm{~g}, 0.09$ mol) was added and the reaction heated at reflux for a further hour to hydrolyse the isothiouronium salt. The reaction was cooled to room temperature, extracted 
twice with $30 \mathrm{~mL} \mathrm{CH}_{2} \mathrm{Cl}_{2}$, the aqueous phase washed with $3 \mathrm{~mL}$ concentrated $\mathrm{HCl}$ and re-extracted with $30 \mathrm{~mL} \mathrm{CH}_{2} \mathrm{Cl}_{2}$. The combined organic fractions were dried with $\mathrm{MgSO}_{4}$, filtered and evaporated under reduced pressure. The crude product was distilled under reduced pressure affording octanethiol in high purity as judged by ${ }^{1} \mathrm{H}$ NMR.

Preparation of octylthioacetate: Octanethiol (0.89 g, $6.10 \mathrm{mmol})$ was stirred under ambient conditions, treated with a molar excess of acetic anhydride (15.00 $\mathrm{mL}, 159.00 \mathrm{mmol}$ ) then heated with stirring to $90^{\circ} \mathrm{C}$ overnight. The reaction was cooled to room temperature, placed on ice and quenched with $20 \mathrm{~mL} \mathrm{H}_{2} \mathrm{O}$. Addition of $\mathrm{H}_{2} \mathrm{O}$ induced separation of the alkyl product and the aqueous phase was further extracted twice with $20 \mathrm{~mL} \mathrm{Et}_{2} \mathrm{O}$. The organic phase was dried over $\mathrm{MgSO}_{4}$, filtered and evaporated under reduced pressure. The crude product was then distilled under reduced pressure affording octylthioacetate in an $80 \%$ yield (0.92 g, $4.90 \mathrm{mmol})$.

Octylthioacetate: Colourless liquid, IR (film) $\nu_{\max } 698 \mathrm{~cm}^{-1} ;{ }^{1} \mathrm{H} \mathrm{NMR}\left(\mathrm{CDCl}_{3}\right.$, $600 \mathrm{MHz}) \delta 2.86(2 \mathrm{H}, \mathrm{t}, J=7.5 \mathrm{~Hz}, \mathrm{H}-1), 2.32\left(3 \mathrm{H}, \mathrm{s}, \mathrm{SC}(\mathrm{O}) \mathbf{C H}_{3}\right), 1.55(2 \mathrm{H}$, quin, $J$ $=7.6 \mathrm{~Hz}, \mathrm{H}-2), 1.33(2 \mathrm{H}$, quin, $J=7.4 \mathrm{~Hz}, \mathrm{H}-3), 1.23-1.30\left(8 \mathrm{H}, \mathrm{m}, \mathrm{H}_{2}-4-\mathrm{H}_{2}-7\right), 0.87$ $(3 \mathrm{H}, \mathrm{t}, J=6.5 \mathrm{~Hz}, \mathrm{H}-8) ;{ }^{13} \mathrm{C} \mathrm{NMR}\left(\mathrm{CDCl}_{3}, 150 \mathrm{MHz}\right) \delta 196.3\left(\mathrm{SC}(\mathrm{O}) \mathrm{CH}_{3}\right), 30.9$ $\left(\mathrm{SC}(\mathrm{O}) \mathbf{C H}_{3}\right.$ ), 31.9 (C-1), 30.8 (C-2), 29.6 (C-4-C-7 ), 29.30, 29.29, 29.22, 29.0 (C3), $22.8(\mathrm{C}-5), 14.3(\mathrm{C}-8)$; HRESIMS $[\mathrm{M}+\mathrm{Na}]^{+} \mathrm{m} / z 199.1133$ (calcd for $\mathrm{C}_{9} \mathrm{H}_{20} \mathrm{OS}$, 199.1138; $\Delta-2.0 \mathrm{ppm})$.

Preparation of dimethyloctylsulfide: Octanethiol $(0.42 \mathrm{~g}, 2.88 \mathrm{mmol})$ was stirred under ambient conditions in $20 \mathrm{~mL} \mathrm{MeOH}$. 1.2 equivalents of MeI were added and the reaction was heated at reflux for $30 \mathrm{~min}$. The reaction was cooled to room temperature, quenched with $20 \mathrm{~mL} \mathrm{H}_{2} \mathrm{O}$, and the reaction mixture was extracted twice with $20 \mathrm{~mL} \mathrm{Et}_{2} \mathrm{O}$. The organic phase was dried over $\mathrm{MgSO}_{4}$, filtered and evaporated under reduced pressure affording the dimethylation product in a $90 \%$ yield.

Dimethyloctylsulfide: Colourless liquid, ${ }^{1} \mathrm{H} \mathrm{NMR}\left(\mathrm{CDCl}_{3}, 600 \mathrm{MHz}\right) \delta 3.72(2 \mathrm{H}$, t, $J=7.8 \mathrm{~Hz}, \mathrm{H}-1), 3.33\left(6 \mathrm{H}, \mathrm{s}, \mathrm{S}\left(\mathrm{CH}_{3}\right)_{2}\right), 1.78(2 \mathrm{H}$, quin, $J=6.6 \mathrm{~Hz}, \mathrm{H}-2), 1.44$ $(2 \mathrm{H}$, quin, $J=6.6 \mathrm{~Hz}, \mathrm{H}-3), 1.30(2 \mathrm{H}$, quin, $J=6.6 \mathrm{~Hz}, \mathrm{H}-4), 1.20-1.28(6 \mathrm{H}, \mathrm{m}$, $\mathrm{H}-5-\mathrm{H}-7), 0.81$ (3H, s, H-8); ${ }^{13} \mathrm{C} \mathrm{NMR}\left(\mathrm{CDCl}_{3}, 150 \mathrm{MHz}\right) \delta 43.6$ (C-1), 31.7 (C-2), 29.0 (C-3), 28.9 (C-4), 28.3 (C-5), $25.6\left(\mathrm{~S}\left(\mathrm{CH}_{3}\right)_{2}\right.$ ), 24.3 (C-6), 22.7 (C-7), 14.2 (C-8); HRESIMS $[\mathrm{M}+\mathrm{H}]^{+} m / z 175.1523$ (calcd for $\mathrm{C}_{10} \mathrm{H}_{23} \mathrm{~S}, 175.1520 ; \Delta 1.7 \mathrm{ppm}$ ) 
Preparation of methyloctylsulfide: Octanethiol $(0.5 \mathrm{~mL}, \mathrm{mmol})$ was stirred under ambient conditions in $20 \mathrm{~mL} \mathrm{MeOH}$. One equivalent of MeI was added, and the reaction was heated at reflux for $20 \mathrm{~min}$. The reaction was cooled to room temperature, quenched with $20 \mathrm{~mL} \mathrm{H}_{2} \mathrm{O}$, and the reaction mixture was extracted twice with $20 \mathrm{~mL} \mathrm{Et}{ }_{2} \mathrm{O}$. The organic phase was dried over $\mathrm{MgSO}_{4}$, filtered and evaporated under reduced pressure affording the mono-methylation product in a $40 \%$ yield.

Methyloctylsulfide: Colourless liquid, IR (film) $\nu_{\max } 698 \mathrm{~cm}^{-1} ;{ }^{1} \mathrm{H}$ NMR $\left(\mathrm{CDCl}_{3}, 600 \mathrm{MHz}\right) \delta 2.48(2 \mathrm{H}, \mathrm{t}, J=7.2 \mathrm{~Hz}, \mathrm{H}-2), 2.09(3 \mathrm{H}, \mathrm{s}, \mathrm{H}-1), 1.58(2 \mathrm{H}$, quin, $J=7.2 \mathrm{~Hz}, \mathrm{H}-3), 1.37$ (2H, quin, $J=7.2 \mathrm{~Hz}, \mathrm{H}-4), 1.23-1.30$ (8H, m, H-5-H8), $0.88(3 \mathrm{H}, \mathrm{t}, J=7.2 \mathrm{~Hz}, \mathrm{H}-9) ;{ }^{13} \mathrm{C} \mathrm{NMR}\left(\mathrm{CDCl}_{3}, 150 \mathrm{MHz}\right) \delta 34.2(\mathrm{C}-2), 31.8$ (C-8), 29.15 (C-5), 29.13 (C-7), 29.1 (C-3), 28.8 (C-4), 22.6 (C-6), 15.5 (C-1), 14.0 (C-9).

Preparation of methyloctylsulfoxide A: Methyloctylsulfide $(0.5 \mathrm{~mL}, 2.6$ $\mathrm{mmol}$ ) was stirred in $30 \mathrm{~mL} \mathrm{MeOH}$, after which 1.5 equivalents (4.0 mmol) $\mathrm{KIO}_{4}$ in $3 \mathrm{~mL} \mathrm{H}_{2} \mathrm{O}$ were added and the cloudy solution stirred at room temperature for $12 \mathrm{~h}$. Methyloctylsulfoxide was produced in $100 \%$ yield. No purification was performed.

Preparation of methyloctylsulfoxide B: Methyloctylsulfide $(0.5 \mathrm{~mL}, 2.6$ mmol) was stirred in $30 \mathrm{~mL} \mathrm{MeOH}$, and treated with 1.5 equivalents (4.0 mmol) $30 \%$ $\mathrm{H}_{2} \mathrm{O}_{2}$ aqueous solution. The reaction was stirred for $12 \mathrm{~h}$ under ambient conditions affording the sulfoxide product in $100 \%$ yield. No purification was performed.

Methyloctylsulfoxide: White crystalline solid; IR (film) $\nu_{\max } 1027,748 \mathrm{~cm}^{-1}$; ${ }^{1} \mathrm{H} \mathrm{NMR}\left(\mathrm{CDCl}_{3}, 600 \mathrm{MHz}\right) \delta 2.73(1 \mathrm{H}, \mathrm{ddd}, J=14.9,9.8,5.8 \mathrm{~Hz}, \mathrm{H}-2 \mathrm{a}), 2.64(1 \mathrm{H}$, ddd, $J=16.2,9.3,5.8 \mathrm{~Hz}, \mathrm{H}-2 \mathrm{~b}), 2.56$ (3H, s, H-1), 1.75 (2H, quin, $J=7.0 \mathrm{~Hz}$, H-3), 1.44 (2H, sept, $J=8.4 \mathrm{~Hz}, \mathrm{H}-4), 1.22-1.34$ (8H, m, H-5-H-8), 0.88 (3H, t, $J$ $=7.1 \mathrm{~Hz}, \mathrm{H}-9) ;{ }^{13} \mathrm{C} \mathrm{NMR}\left(\mathrm{CDCl}_{3}, 150 \mathrm{MHz}\right) \delta 54.9(\mathrm{C}-2), 38.7$ (C-1), $31.9(\mathrm{C}-8)$, 29.3 (C-5), 29.2 (C-7), 28.9 (C-4), 22.8 (C-6), 22.7 (C-3), 14.2 (C-9); HRESIMS $[\mathrm{M}+\mathrm{Na}]^{+} m / z 199.1129$ (calcd for $\mathrm{C}_{9} \mathrm{H}_{20} \mathrm{OS}, 199.1133 ; \Delta-2.0 \mathrm{ppm}$ ).

Preparation of methyloctylsulfone: Methyloctylsulfide (0.5 mL, $2.6 \mathrm{mmol})$ was stirred in $25 \mathrm{~mL} \mathrm{CH}_{2} \mathrm{Cl}_{2}$ with excess of $3: 1$ activated $\mathrm{MnO}_{2}: \mathrm{KMnO}_{4}$. The suspension was stirred for $96 \mathrm{~h}$, filtered, and the residue washed with a 1:1 mixture of $\mathrm{MeOH}$ and $\mathrm{CH}_{2} \mathrm{Cl}_{2}$. The filtrate was evaporated affording a white crystalline solid in $100 \%$ yield. No further purification was deemed necessary. 
Methyloctylsulfone: White crystalline solid; IR (film) $\nu_{\max } 1273,1141,1128$, 1117, 765, $749 \mathrm{~cm}^{-1} ;{ }^{1} \mathrm{H} \mathrm{NMR}\left(\mathrm{CDCl}_{3}, 600 \mathrm{MHz}\right) \delta 3.00(2 \mathrm{H}, \mathrm{t}, J=8.4 \mathrm{~Hz}, \mathrm{H}-2)$, $2.89(3 \mathrm{H}, \mathrm{s}, \mathrm{H}-1), 1.85$ (2H, quin, $J=7.8 \mathrm{~Hz}, \mathrm{H}-3), 1.44(2 \mathrm{H}$, quin, $J=7.2 \mathrm{~Hz}$, $\mathrm{H}-4), 1.24-1.34$ (8H, m, H-5-H-8), 0.88 (3H, t, $J=7.2 \mathrm{~Hz}, \mathrm{H}-9) ;{ }^{13} \mathrm{C}$ NMR $\left(\mathrm{CDCl}_{3}\right.$, $150 \mathrm{MHz}) \delta 55.0$ (C-2), 40.5 (C-1), 31.8 (C-8), 29.14 (C-5), 29.05 (C-6), 28.5 (C-4), 22.7 (C-7), 22.6 (C-3), 14.2 (C-9).

\subsection{Isolation of new cyclic peroxides}

\subsubsection{Animal material}

The sponge PTN3_19D was collected from the horizontal ceiling of a large cave at a depth of 12-15 $\mathrm{m}$ on 'Eua Island and frozen immediately. Identified as a plakinid sponge species (order Homosclerophorida), the specimen forms thick, dense lobule encrustations $0.5-1.5 \mathrm{~cm}$ thick, appearing smooth but with a rough reticulated surface covered in wide shallow nodules ca. $3 \mathrm{~mm}$ high in some places. The texture is dense and cork-like, and the black-grey exterior contrasts against a tan-pink interior, which turns grey in alcohol. The abundant diods are $40-60 \mu \mathrm{m} \times 1-1.5 \mu \mathrm{m}$, triods were not observed. A voucher specimen is deposited in the School of Chemical and Physical Sciences, VUW, Wellington.

\subsubsection{Initial extraction of PTN3 19D}

A 26 g sample of PTN3_19D was extracted twice for $14 \mathrm{~h}$ in $100 \mathrm{~mL} \mathrm{MeOH}$ at room temperature then filtered. The second extract, followed by the first were passed through a column of $40 \mathrm{~mL}$ HP20 resin, the eluents combined and diluted with 200 $\mathrm{mL} \mathrm{H}_{2} \mathrm{O}$. The eluent was passed through the column again, further diluted to $25 \%$ $\mathrm{MeOH}$ with $400 \mathrm{~mL} \mathrm{H}_{2} \mathrm{O}$ and passed through the column once more. The column was washed with $\mathrm{H}_{2} \mathrm{O}$ then eluted, generating three $100 \mathrm{~mL}$ fractions of $30 \%, 75 \%$ and $100 \% \mathrm{Me}_{2} \mathrm{CO}$ in $\mathrm{H}_{2} \mathrm{O}$. The $75 \%$ and $100 \% \mathrm{Me}_{2} \mathrm{CO}$ in $\mathrm{H}_{2} \mathrm{O}$ fractions $(88.4 \mathrm{mg}$ and $155.3 \mathrm{mg}$ respectively) were evaporated under reduced pressure and analysed by 1D and 2D NMR. Sub-samples of the $75 \%$ and $100 \% \mathrm{Me}_{2} \mathrm{CO}$ in $\mathrm{H}_{2} \mathrm{O}$ fractions (ca. $40 \mathrm{mg}$ ) were further partitioned over $3 \mathrm{~mL}$ reversed-phase resin (HP20SS) resin with $\mathrm{MeOH} / \mathrm{H}_{2} \mathrm{O}$. The columns were washed with $20 \mathrm{~mL} \mathrm{H} \mathrm{H}_{2} \mathrm{O}$ then eluted generating 10 $\mathrm{mL}$ fractions of $\mathrm{Me}_{2} \mathrm{CO}$ in $\mathrm{H}_{2} \mathrm{O}\left(30-100 \% \mathrm{Me}_{2} \mathrm{CO}\right.$, in $10 \%$ increments). Further purification of the $70-100 \% \mathrm{Me}_{2} \mathrm{CO}$ in $\mathrm{H}_{2} \mathrm{O}$ fractions by DIOL oil chromatography (hexane $/ \mathrm{CH}_{2} \mathrm{Cl}_{2} / \mathrm{MeOH}$ ) gave a mixture of cyclic peroxides and long-chain fats within the different $\mathrm{CH}_{2} \mathrm{Cl}_{2}$ fractions. 
NMR spectra of the $50 \% \mathrm{CH}_{2} \mathrm{Cl}_{2}$ in $\mathrm{MeOH}$ DIOL fraction indicated steroidal content and the fraction was purified further on DIOL (hexane $/ \mathrm{CH}_{2} \mathrm{Cl}_{2}$ ). The $40 \%$ hexane in $\mathrm{CH}_{2} \mathrm{Cl}_{2}$ fraction was again partitioned over DIOL and purification of the $20 \%$ hexane in $\mathrm{CH}_{2} \mathrm{Cl}_{2}$ fraction by reversed-phase $\mathrm{HPLC}\left(\mathrm{C}_{18}, 90 \% \mathrm{MeCN}\right.$ in $\left.\mathrm{H}_{2} \mathrm{O}\right)$ yielded $5 \alpha, 8 \alpha$ epidioxy sterols $\mathbf{2 7}(652 \mu \mathrm{g})$ and $\mathbf{2 8}(537 \mu \mathrm{g})$ with retention times of $29 \mathrm{~min}$ and 39 min respectively.

The $25 \%$ hexane in $\mathrm{CH}_{2} \mathrm{Cl}_{2}$ DIOL fraction was found to contain epiplakinic acid I (95) in high spectroscopic purity. Analysis of the $2.5 \% \mathrm{MeOH}$ in $\mathrm{CH}_{2} \mathrm{Cl}_{2}$ fraction revealed compound $\mathbf{9 8}$ in reasonable purity. Attempts were made to further purify both compounds, however, the lipophilic nature of the long-chains induced co-elution on reversed phase. Further partitioning over DIOL (hexane $/ \mathrm{CH}_{2} \mathrm{Cl}_{2}$ ) at 0.5 drop $\mathrm{sec}^{-1}$ collecting $2 \mathrm{~mL}$ fractions was not observed to increase spectroscopic purity.

The non-polar $60 \%$ hexane in $\mathrm{CH}_{2} \mathrm{Cl}_{2}$ fractions generated by the initial DIOL column were partitioned by $\mathrm{HPLC}\left(\mathrm{C}_{18}, 95 \% \mathrm{MeCN}\right.$ in $\left.\mathrm{H}_{2} \mathrm{O}\right)$. Fractions collected in between chromophore peaks were rich in the cyclic peroxide metabolites. Size-exclusion chromatography (LH20, $1.5 \mathrm{~m}, \mathrm{MeOH}$ ) was utilised to remove the residual longchain fat contamination from the HPLC dregs, 170 fractions were collected with the material of interest eluting in fractions 37-41. The combined LH20 fractions were partitioned over DIOL (hexane $/ \mathrm{CH}_{2} \mathrm{Cl}_{2}$ ) with epiplakinic acid I methyl ester (96) eluting early in $\mathrm{CH}_{2} \mathrm{Cl}_{2}$ and plakortolide $\mathrm{X}(\mathbf{9 7})$ eluting in the $75 \%$ hexane in $\mathrm{CH}_{2} \mathrm{Cl}_{2}$ fractions.

\subsubsection{Second extraction of PTN3_19D}

Following accidental defrost, the remaining $75 \mathrm{~g}$ of PTN3_19D was extracted twice in $\mathrm{MeOH}$ and partitioned over HP20 as above. Analysis of the $100 \%$ and $75 \% \mathrm{Me}_{2} \mathrm{CO}$ in $\mathrm{H}_{2} \mathrm{O}$ fractions (69.3 $\mathrm{mg}$ and $252.4 \mathrm{mg}$ ) by ${ }^{1} \mathrm{H}$ and HMBC NMR confirmed the presence of the cyclic peroxides epiplakinic acid I (95) and plakortolide X (97) although in lower concentration than observed in the initial screening evaluation. One step of normal-phase purification was performed on the $100 \% \mathrm{Me}_{2} \mathrm{CO}$ fraction (DIOL, hexane $/ \mathrm{CH}_{2} \mathrm{Cl}_{2} / 10 \% \mathrm{MeOH}$ ).

The $75 \% \mathrm{Me}_{2} \mathrm{CO}$ in $\mathrm{H}_{2} \mathrm{O}$ HP20 fraction was partitioned over HP20SS generating ten fractions of $30-100 \% \mathrm{Me}_{2} \mathrm{CO}$ in $\mathrm{H}_{2} \mathrm{O}$. The $85 \%$ and $90 \% \mathrm{Me}_{2} \mathrm{CO}$ in $\mathrm{H}_{2} \mathrm{O}$ fractions were combined and partitioned again on HP20SS over a smaller window generating fractions of $75 \%, 80 \%, 82 \%, 84 \%, 86 \%, 88 \%$ and $90 \% \mathrm{Me}_{2} \mathrm{CO}$ in $\mathrm{H}_{2} \mathrm{O}$. The metabolites were all found to co-elute within the $75 \%$ and $80 \% \mathrm{Me}_{2} \mathrm{CO}$ in $\mathrm{H}_{2} \mathrm{O}$ fractions, which were subsequently partitioned on normal-phase. Epiplakinic acid I (95, ca. $2 \mathrm{mg}$ ) eluted in $100 \% \mathrm{CH}_{2} \mathrm{Cl}_{2}$. 
5 $\alpha \mathbf{8} \alpha$-epidioxysterol 27: Clear oil, $652 \mu \mathrm{g} ;[\alpha]_{D}^{24.6}-0.1^{\circ}\left(c 0.044, \mathrm{CHCl}_{3}\right)$; UV $(\mathrm{MeOH}) \lambda_{\max }(\log \epsilon), 222$ (3.73), $275(2.94) \mathrm{nm}$; IR (film) $\nu_{\max } 3391,2957,2929$, 2871, 1734, 1460, 1381, 1278, 1126, 969, $766 \mathrm{~cm}^{-1} ;{ }^{1} \mathrm{H}$ and ${ }^{13} \mathrm{C}$ NMR data, see Table C.5; HRESIMS $m / z 465.3345[\mathrm{M}+\mathrm{Na}]^{+}$(calcd for $\mathrm{C}_{21} \mathrm{H}_{40} \mathrm{O}_{5} \mathrm{Na}, 465.3379 ; \Delta 0.9$ ppm), $m / z 477.3135[\mathrm{M}+\mathrm{Cl}]^{-}$(calcd for $\mathrm{C}_{29} \mathrm{H}_{46} \mathrm{O}_{3} \mathrm{Cl}, 477.3145 ; \Delta 2.1 \mathrm{ppm}$ ).

5 $\alpha \mathbf{8} \alpha$-epidioxysterol 28: Clear oil, $537 \mu \mathrm{g} ;[\alpha]_{D}^{24.6}-0.4^{\circ}\left(c 0.036, \mathrm{CHCl}_{3}\right)$; UV $(\mathrm{MeOH}) \lambda_{\max }(\log \epsilon), 228(2.84) \mathrm{nm}$; IR (film) $\nu_{\max } 3366,2929,2870,2362,1717$, 1629, 1467, 1381, 1270, 1047, 936, $748 \mathrm{~cm}^{-1} ;{ }^{1} \mathrm{H}$ and ${ }^{13} \mathrm{C}$ NMR data, see Table C.6; HRESIMS $m / z 439.3188[\mathrm{M}+\mathrm{Na}]^{+}$(calcd for $\mathrm{C}_{21} \mathrm{H}_{40} \mathrm{O}_{5} \mathrm{Na}, 439.3192 ; \Delta 0.9$ ppm), m/z $451.2979[\mathrm{M}+\mathrm{Cl}]^{-}$(calcd for $\mathrm{C}_{27} \mathrm{H}_{44} \mathrm{O}_{3} \mathrm{Cl}, 451.2976 ; \Delta-0.7 \mathrm{ppm}$ ).

Epiplakinic acid I (95): Clear oil, ca. $3.5 \mathrm{mg} ;[\alpha]_{D}^{25.2}+1.9^{\circ}\left(c 0.068, \mathrm{CHCl}_{3}\right)$; UV $(\mathrm{MeOH}) \lambda_{\max }(\log \epsilon), 273(2.70) \mathrm{nm}$; IR (film) $\nu_{\max } 3388,2928,2920,2854,1717$, 1464, 1374, 1220, $1024 \mathrm{~cm}^{-1} ;{ }^{1} \mathrm{H}$ and ${ }^{13} \mathrm{C}$ NMR data, see Table 4.10; HRESIMS $\mathrm{m} / z$ $365.2668[\mathrm{M}+\mathrm{Na}]^{+}$(calcd for $\left.\mathrm{C}_{20} \mathrm{H}_{38} \mathrm{O}_{4} \mathrm{Na}, 365.2664 ; \Delta-1.1 \mathrm{ppm}\right), \mathrm{m} / z 341.2692$ $[\mathrm{M}-\mathrm{H}]^{-}$(calcd for $\left.\mathrm{C}_{20} \mathrm{H}_{37} \mathrm{O}_{4}, 365.2692 ; \Delta-2.3 \mathrm{ppm}\right)$.

Epiplakinic acid I methyl ester (96): Clear oil, ca. $500 \mu \mathrm{g}$; UV (MeOH) $\lambda_{\max }$ $(\log \epsilon), 273$ (2.70) nm; IR (film) $\nu_{\max } 3388,2928,2920,2854,1717,1464,1374$, 1220, $1024 \mathrm{~cm}^{-1} ;{ }^{1} \mathrm{H}$ and ${ }^{13} \mathrm{C}$ NMR data, see Table 4.11; HRESIMS $m / z 371.2790$ $[\mathrm{M}+\mathrm{Na}]^{+}$(calcd for $\mathrm{C}_{21} \mathrm{H}_{39} \mathrm{O}_{5} \mathrm{Na}, 371.2797 ; \Delta-1.9 \mathrm{ppm}$ ).

Plakortolide X (97): Clear oil, $1.21 \mathrm{mg} ;[\alpha]_{D}^{24.6} 0.0^{\circ}\left(c 0.08, \mathrm{CHCl}_{3}\right)$; UV (MeOH) $\lambda_{\max }(\log \epsilon), 275$ (2.80), 282 (2.57) nm; IR (film) $\nu_{\max } 2928,2921,2854,1782$, 1717, 1463, 1381, 1270, 1167, $1073 \mathrm{~cm}^{-1} ;{ }^{1} \mathrm{H}$ and ${ }^{13} \mathrm{C}$ NMR data, see Table 4.13; HRESIMS $m / z 349.2355[\mathrm{M}+\mathrm{H}]^{+}$(calcd for $\mathrm{C}_{19} \mathrm{H}_{34} \mathrm{O}_{4} \mathrm{Na}, 349.2355 ; \Delta-0.6 \mathrm{ppm}$ ), $m / z 325.2379[\mathrm{M}-\mathrm{H}]^{-}$(calcd for $\mathrm{C}_{20} \mathrm{H}_{37} \mathrm{O}_{4}, 325.2374 ; \Delta-1.5 \mathrm{ppm}$ ).

Compound 98: Clear oil, $856 \mu \mathrm{g} ;[\alpha]_{D}^{24.6}+4.1^{\circ}\left(c\right.$ 0.03, $\left.\mathrm{CHCl}_{3}\right)$; UV $(\mathrm{MeOH})$ $\lambda_{\max }(\log \epsilon), 280(3.05) \mathrm{nm}$; IR (film) $\nu_{\max } 3374,2928,2920,2854,1717,1464$, 1374, 1220, $11431024 \mathrm{~cm}^{-1} ;{ }^{1} \mathrm{H}$ and ${ }^{13} \mathrm{C}$ NMR data, see Table 4.15; HRESIMS $\mathrm{m} / z$ $395.2771[\mathrm{M}+\mathrm{Na}]^{+}$(calcd for $\mathrm{C}_{21} \mathrm{H}_{40} \mathrm{O}_{5} \mathrm{Na}, 395.2773 ; \Delta-0.8 \mathrm{ppm}$ ), m/z 371.2790 $[\mathrm{M}-\mathrm{H}]^{-}$(calcd for $\left.\mathrm{C}_{21} \mathrm{H}_{39} \mathrm{O}_{5}, 371.2797 ; \Delta-1.9 \mathrm{ppm}\right)$. 


\subsection{Biological evaluation of cyclic peroxides}

\subsubsection{Yeast strains}

All strains were derived from a $15 \%$ glycerol stock stored at $-80^{\circ} \mathrm{C}$. The deletion strains (Table 7.1) were streaked out onto $10 \mathrm{~cm}$ diameter agar plates containing synthetic complete (SC) medium or synthetic deficient in uracil medium (SDura), supplemented with the appropriate antibiotics to ensure selective growth; $200 \mu \mathrm{g} \mathrm{mL}{ }^{-1}$ Geneticin antibiotic (G418, Gibco, Invitrogen) and/or $100 \mu \mathrm{g} \mathrm{mL}^{-1}$ mourseothricin antibiotic (Nat, Werner BioAgents). The diploid BY4743 strain was streaked onto yeast extract peptone dextrose (YPD) agar and the diploid deletion strains were grown on YPD agar supplemented with G418 antibiotic (200 $\left.\mu \mathrm{g} \mathrm{mL}{ }^{-1}\right)$. The GFP-localised strains were streaked out onto $10 \mathrm{~cm}$ diameter agar plates containing SD-histidine medium. The freshly plated strains were incubated at $30^{\circ} \mathrm{C}$ for $48 \mathrm{~h}$ to allow the formation of single colonies then stored at $4^{\circ} \mathrm{C}$.

\section{Growth media}

Y7092 (wt) was cultured in SC medium; homozygous deletion strains were cultured in $5 \mathrm{~mL} \mathrm{SC}+$ with $200 \mu \mathrm{g} \mathrm{mL}^{-1} \mathrm{G} 418$ antibiotic; $\Delta p d r$ was cultured in $5 \mathrm{~mL}$ SD-ura +Nat $100 \mu \mathrm{g} \mathrm{mL}{ }^{-1}$; haploid strains with GFP-localisation markers were cultured immediately prior to use by serial-dilution in SD-his; and BY4743 was cultured in $5 \mathrm{~mL}$ SC. YPD was used during assessment of $\mathbf{2 9}$ as a growth inhibitor and during transformation procedures (refer Appendix B for growth media recipies).

\subsubsection{Preliminary assay protocols}

\section{Liquid-phase dose-reponse assays}

Three $5 \mathrm{~mL}$ aliquots of SC, SD-ura or YPD growth media were inoculated with different single colonies of a given yeast strain and cultures were grown to saturation overnight in a rotating drum at $30^{\circ} \mathrm{C} .{ }^{251}$ The saturated cultures were diluted to $5 \times 10^{5}$ cells $\mathrm{mL}^{-1}$ in fresh media (SC, SD-ura or YPD), as determined by haemocytomer, and $99 \mu \mathrm{L}$ aliquots were added to 96 -well tissue culture plates in a triplicate format. Seven point, half-log serial dilutions of compounds 29, 95 and $\mathbf{9 7}$ (10.00-0.10 $\mathrm{mmol} \mathrm{L}^{-1}$ ) were prepared from $10.00 \mathrm{mmol} \mathrm{L}^{-1}$ working stocks dissolved in DMSO. A $1 \mu \mathrm{L}$ volume of diluent was added per well, giving a final concentration range of $100.00-0.01 \mu \mathrm{mol} \mathrm{L}{ }^{-1}$. DMSO (1\% final concentration) was added to one well as a control. 
Table 7.1 Yeast Strains Used During this Study.

\begin{tabular}{|c|c|c|c|}
\hline Strain & Ploidy & Genotype & Origin \\
\hline yCG 117 & haploid & $\begin{array}{l}\text { MATQ can } 1 \Delta 1:: S T E 2 p r- \\
\text { Sp_HIS5; lyp } 1 \Delta ; \\
\text { ura3 } \Delta O:: N A T ; \\
\text { leu2 } \Delta 0 ; \text { his } 3 \Delta ; \\
\text { met } 1 \Delta 50 ; \text { LYS2+ }\end{array}$ & $\begin{array}{l}\text { Starting lab } \\
\text { strain }\end{array}$ \\
\hline $8025-\alpha$ & haploid & 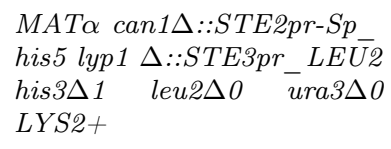 & yCG 112 \\
\hline$\Delta p d r$ & haploid & 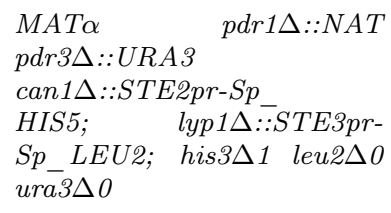 & yCG 198 \\
\hline BY4743 (wt) & diploid & 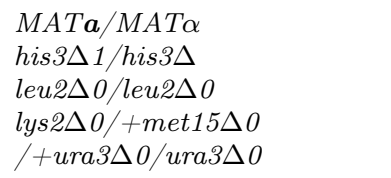 & $\begin{array}{l}\text { Starting lab } \\
\text { strain }\end{array}$ \\
\hline 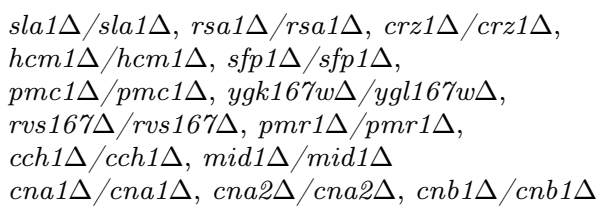 & diploid & $\begin{array}{l}\text { Homozygous deletion strains } \\
\text { with G418 resistance }\end{array}$ & $\begin{array}{l}\text { Homozygous } \\
\text { deletion set } \\
\text { (Open } \\
\text { Biosystems) }\end{array}$ \\
\hline Crz1p-GFP & diploid & 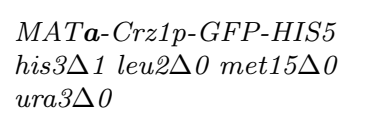 & $\begin{array}{l}\text { MATa-GFP } \\
\text { Library } \\
\text { (Invitrogen) }\end{array}$ \\
\hline Zrtp-GFP & diploid & $\begin{array}{l}\text { MATa-Zrt1p-GFP-HIS5 } \\
\text { his } 3 \Delta 1 \text { leu2 } \Delta O \text { met } 15 \Delta O \\
\text { ura } 3 \Delta O\end{array}$ & $\begin{array}{l}\text { MATa-GFP } \\
\text { Library } \\
\text { (Invitrogen) }\end{array}$ \\
\hline Zap1p-GFP & diploid & $\begin{array}{l}\text { MATa-Zap1p-GFP-HIS5 } \\
\text { his } 3 \Delta 1 \text { leu2 } \Delta O \text { met } 15 \Delta O \\
\text { ura3 } \Delta O\end{array}$ & $\begin{array}{l}\text { MATa-GFP } \\
\text { Library } \\
\text { (Invitrogen) }\end{array}$ \\
\hline Yap1p-GFP mCherry & haploid & 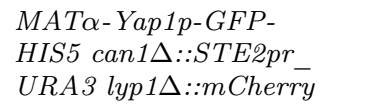 & yCG 379 \\
\hline Mrh1p-GFP + mCherry/NLS-RS2 & haploid & $\begin{array}{l}M A T \alpha-M r h 1 p-G F P- \\
H I S 5 \text { can1 } 1: \text { STE2pr } \\
\text { URA3 lyp } 1 \Delta:: m \text { Cherry/ } \\
\text { NLS-RS2 }\end{array}$ & yCG 321 \\
\hline
\end{tabular}

Plates were mixed by vortexing for 1 min at $950 \mathrm{rpm}$ and incubated at $30^{\circ} \mathrm{C}$ for a maximum of $18 \mathrm{~h}$. After incubation, cells were resuspended by vortexing, and their growth was quantified by measuring optical density (OD) at $590 \mathrm{~nm}$. Residual growth $(\%)$ was calculated for each concentration using the formula ((OD $D_{\exp }-$ $\left.\mathrm{OD}_{\text {blank }} / \mathrm{OD}_{\mathrm{DMSO}}-\mathrm{OD}_{\text {blank }}\right)^{* 100)}$. Results are reported as the mean \pm standard error for two independent experiments performed in triplicate. The $\mathrm{IC}_{50}$ values were determined using SigmaPlot and the MIC values were inferred from the plots. 


\section{Cytotoxicity assays}

Colony forming assay: Stationary-phase cultures of WT cells and $\Delta p d r$ were diluted to $5 \times 10^{5}$ cells $\mathrm{mL}^{-1}$ in fresh $\mathrm{SC}$ or SD-ura, then treated with either $\mathbf{9 5}$ (wt) or $97(\Delta p d r)$ at final concentrations of 10 and $100 \mu \mathrm{mol} \mathrm{L} \mathrm{L}^{-1}$. Rapamycin $\left(2.00 \mathrm{nmol} \mathrm{L}^{-1}\right)$ and cycloheximide $\left(3.35 \mu \mathrm{mol} \mathrm{L}{ }^{-1}\right)$ served as positive controls for cytotoxic and cytostatic activity respectively, with DMSO acting as the negative control. The drug-treated cells were vortexed then incubated at $30^{\circ} \mathrm{C}$ for $18 \mathrm{~h}$. The cells were washed with fresh media and inoculated onto solid agar plates of SC or $\mathrm{SD}$-ura medium then incubated for $48 \mathrm{~h}$ at $30^{\circ} \mathrm{C}$. The plates were inspected visually for colony growth and compared to the controls. Colony growth indicated cytostatic inhibition by the experimental compound and the absence of growth was indicative of cytotoxicity or irreversible inhibition.

Kill curve: The effective rate of cytotoxicity was measured by removing $1 \mu \mathrm{L}$ aliquots from each dilution point within a dose-response assay and spotting them onto a $10 \times 15 \mathrm{~cm}$ rectangular YPD plate hourly from 0 to $6 \mathrm{~h}$ and once more after $24 \mathrm{~h}$. As there was a set volume rather than a set cell count spotted, the DMSO control is expected to show increased growth with time relative to the drug-treated cultures. The plate was incubated for $24 \mathrm{~h}$ and inspected visually for colony growth. The assay was performed for $\mathbf{9 5}$ and $\mathbf{9 7}$ with WT and $\Delta p d r$ cultures respectively.

Inhibition in the presence and absence of $\mathbf{C a}^{2+}$ : To determine the effect of $\mathrm{Ca}^{2+}$ concentration upon the cytotoxicity of $\mathbf{9 5}$ and $\mathbf{9 7}$, inhibition assays in high and low environments of $\mathrm{Ca}^{2+}\left(50 \mathrm{mmol} \mathrm{L}^{-1} \mathrm{CaCl}_{2}\right.$ and $100 \mathrm{mmol} \mathrm{L}{ }^{-1}$ EGTA). Doseresponse assays were performed in triplicate over a narrow concentration range (30-1 $\mu \mathrm{mol} \mathrm{L}{ }^{-1}$ final concentration) against the WT and $\Delta p d r$ strains in the presence of 95 and 97. Three growth conditions were tested: inhibitory agent; agent +50 mmol L-1 $\mathrm{CaCl}_{2}$; and agent $+100 \mathrm{mmol} \mathrm{L}{ }^{-1}$ EGTA. As with the kill curve assay, $1 \mu \mathrm{L}$ aliquots from each dilution point within the assays were removed and spotted onto a $10 \times 15 \mathrm{~cm}$ rectangular YPD plate hourly at $\mathrm{t}=0-6$ and $24 \mathrm{~h}$. The plate was incubated for $24 \mathrm{~h}$ and inspected visually for colony growth. The assay was performed for $\mathbf{9 5}$ and $\mathbf{9 7}$ with WT and $\Delta p d r$ cultures respectively. Residual growth of the dose-response assays was calculated after $17 \mathrm{~h}$ incubation and the $\mathrm{IC}_{50}$ values calculated for the different conditions (Figures H.2 and H.3). Dose-response assays were performed against the WT and $\Delta p d r$ strains with 95 and 97 in high and low $\mathrm{Ca}^{2+}$ environments. 


\section{Cell cycle inhibition studies}

Analysis by bud index: Fresh overnight cultures of haploid WT and $\Delta p d r$ cells were diluted to $5 \times 10^{5}$ cells $\mathrm{mL}^{-1}$ in fresh SC or SD-ura, then treated with either 95 (wt) or $97(\Delta p d r)$ at their MIC $\left(10 \mu \mathrm{mol} \mathrm{L}{ }^{-1}\right.$ final concentration). DMSO served as a negative control. At 2, 4 and $6 \mathrm{~h}$ time-points, $10 \mu \mathrm{L}$ aliquots were removed, diluted 1:100 in $\mathrm{H}_{2} \mathrm{O}$ and $10 \mu \mathrm{L}$ samples of the populations were assessed by haemocytometer. Cell counts were made at each time-point, and the individual cells were scored according to bud size corresponding to their stage in the cell cycle. Cells were visually assessed and imaged at $\mathrm{t}=2$ and $4 \mathrm{~h}$ and the assays were repeated periodically to assess potential degradation of the inhibitory agents $\mathbf{9 5}$ and $\mathbf{9 7 .}$

Cell cycle analysis by flow cytometry: Three fresh overnight cultures of haploid WT cells were diluted to $5 \times 10^{6}$ cells $\mathrm{mL}^{-1}$ in fresh SC and cultured for a further $4 \mathrm{~h}$ to mid-log growth phase. Aliquots were taken and diluted to give $1 \mathrm{~mL}$ cultures $1 \times 10^{7}$ cells $\mathrm{mL}^{-1}$. Cells were treated with DMSO and 95 at 10, 7 and $3.4 \mu \mathrm{mol} \mathrm{L}{ }^{-1}$ and incubated at $30^{\circ} \mathrm{C}$ for $4 \mathrm{~h}$. Cells were pelleted and washed with $5 \mathrm{~mL}$ Tris $\mathrm{HCl}$ buffer $\left(50 \mathrm{mmol} \mathrm{L}^{-1}\right)$ and resuspended in $1.5 \mathrm{~mL} \mathrm{H}_{2} \mathrm{O}$. To each $1.5 \mathrm{~mL}$ sample, $3.5 \mathrm{~mL}$ absolute $\mathrm{EtOH}$ was added as a fixative $(70 \% \mathrm{EtOH}$ final concentration) and the cells were incubated at $4{ }^{\circ} \mathrm{C}$ overnight. Cells were pelleted, EtOH removed and the cells were washed twice with $2 \mathrm{~mL}$ Tris $\mathrm{HCl}$ buffer $(50 \mathrm{mmol}$ $\mathrm{L}^{-1}$ ), resuspending each time by sonication. Cells were treated with $1.25 \mathrm{~mL}$ RNase solution (diluted 10-fold in $50 \mathrm{mmol} \mathrm{\textrm {L } ^ { - 1 }}$ Tris $\mathrm{HCl}$ buffer) and incubated for $2 \mathrm{~h}$ at $37^{\circ} \mathrm{C}$ with shaking. The cells were again pelleted, the RNase solution was removed, and then treated with $2 \mathrm{~mL}$ of fresh pepsin solution for $5 \mathrm{~min}$ at room temperature. $100 \mu \mathrm{L}$ aliquots were then added to $2 \mathrm{~mL}$ solutions of SytoxGreen $\left(1 \mu \mathrm{mol} \mathrm{L}{ }^{-1}\right.$ in Tris $\mathrm{HCl}$ buffer), placed on ice and imaged immediately. A FACScan flow cytometer was used to visualise cells and CellQuest Pro software (BD Biosciences) was used to generate contour plots for each sample. Flow cytometry cell population data was manipulated with FlowJo version 7.6.1 flow cytometry analysis software. Populations were manually gated according to the untreated control and recorded as percentage of total population within the sample. ${ }^{252}$

RNase stock solution, 10x:

$\begin{array}{ll}\text { RNase } & 100 \mathrm{mg} \\ \mathrm{NaOAc}\left(3 \mathrm{~mol} \mathrm{~L}^{-1}\right) & 333 \mu \mathrm{L} \\ \mathrm{H}_{2} \mathrm{O} & 9.7 \mathrm{~mL}\end{array}$

\section{Pepsin solution:}

$\begin{array}{ll}\text { Pepsin } & 50 \mathrm{mg} \\ \mathrm{HCl}\left(1 \mathrm{~mol} \mathrm{~L}^{-1}\right) & 550 \mu \mathrm{L} \\ \mathrm{H}_{2} \mathrm{O} & 9.45 \mathrm{~mL}\end{array}$


Analysis of reactive oxygen species formation by flow cytometry Overnight

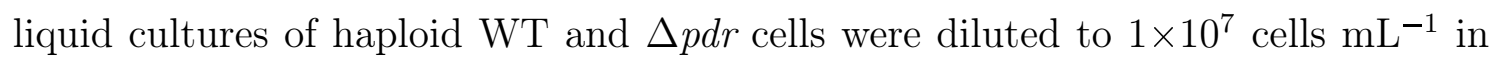
fresh SC medium. In duplicate, $100 \mu \mathrm{L}$ aliquots of WT and $\Delta p d r$ culture were treated with experimental and control agents for $1 \mathrm{~h}$ in microcentrifuge tubes. Wildtype and $\Delta p d r$ cells were treated by 95 and $\mathbf{9 7}$ respectively and positive controls of both strains were treated with $1 \mu \mathrm{L}$ aliquots of $1 \mathrm{mmol} \mathrm{L}^{-1} \mathrm{H}_{2} \mathrm{O}_{2}$ and DMSO then incubated for $30 \mathrm{~min}$ at $30^{\circ} \mathrm{C}$. DCF-DA was added $\left(50 \mu \mathrm{mol} \mathrm{L}{ }^{-1}\right.$ final concentration) and the cells were incubated for a further $15 \mathrm{~min}$. The cultures were placed on ice, diluted with $400 \mu \mathrm{L} \mathrm{H}_{2} \mathrm{O}$, and co-stained with $6 \mathrm{mg} \mathrm{mL}^{-1}$ propidium iodide prior to visualisation. Cells were visualised on the FACScan flow cytometer and CellQuest Pro software (BD Biosciences) was used to generate contour plots for each sample. Cell population data obtained from the flow cytometer was modelled on FlowJo version 7.6.1 flow cytometry analysis software. Populations were manually gated according to the untreated control and recorded as percentage of total population within the sample.

\subsubsection{Chemical genetic profiling of epiplakinic acid I}

\section{Homozygous profiling by microarray}

A $0.5 \mathrm{~mL}$ aliquot of the homozygous deletion set pool was inoculated into $10 \mathrm{~mL}$ $\mathrm{SC}$ and supplemented with $10 \mu \mathrm{L}$ G418 $\left(200 \mathrm{mg} \mathrm{mL}^{-1}\right.$ in a $50 \mathrm{~mL}$ falcon tube. The culture was incubated overnight at $30^{\circ} \mathrm{C}$ in a rotating drum. Five parallel $10 \mathrm{~mL}$ cultures SC were seeded with $5 \times 10^{6}$ cells from the fresh culture of the YKO pool. Four of the cultures were treated with 95 to final concentrations of 4, 3, 2 and 1 $\mu \mathrm{mol} \mathrm{L}{ }^{-1}$ and a $100 \mu \mathrm{L}$ aliquot of DMSO served as control in the fifth culture. The cultures were incubated for 10 generations ca. $15 \mathrm{~h}$ at $30^{\circ} \mathrm{C}$ in a rotating drum. It was determined that $3 \mu \mathrm{mol} \mathrm{L}{ }^{-1}$ of $\mathbf{9 5}$ inhibited cellular growth by $30 \%\left(\mathrm{IC}_{30}\right)$ relative to the DMSO control. The cultures treated with DMSO and $3 \mu \mathrm{mol} \mathrm{L} \mathrm{L}^{-1}$ $\mathbf{9 5}$ were then diluted to $5 \times 10^{6}$ cells in $10 \mathrm{~mL}$ fresh $\mathrm{SC}$, treated again DMSO or $\mathbf{9 5}$ and cultured for a further 10 generations.

Cell populations were determined by haemocytometer and duplicate $1.5 \mathrm{~mL}$ aliquots of both control and 95-treated cultures were pelleted for $2 \mathrm{~min}$ at 10,000 rpm in microcentrifuge tubes and the supernatant discarded. Genomic DNA was then extracted from the cell pellets in accordance with the Master Pure ${ }^{\mathrm{TM}}$ Yeast DNA Purification Kit. The cell pellets were resuspended in $300 \mu \mathrm{L}$ of yeast cell lysis solution by vortexing then incubated at $65^{\circ} \mathrm{C}$ for $15 \mathrm{~min}$ then placed on ice for 5 min. A $150 \mu \mathrm{L}$ aliquot of MPC protein precipitation reagent was added to each lysed sample, mixed by vortex for ca. $10 \mathrm{~s}$ and the cellular debris pelleted by centrifugation 
at $10,000 \mathrm{rpm}$ for $10 \mathrm{~min}$.

The supernatant was transferred into a clean microcentrifuge tube and the genomic DNA was precipitated out by the addition of $500 \mu \mathrm{L}$ IPA. This was mixed by inverting the tube and the DNA pelleted by centrifugation at 10,000 rpm for 10 min. The DNA was washed twice with $70 \%$ EtOH solution then dried briefly at room temperature. The DNA was dissolved in $35 \mu \mathrm{L}$ Tris EDTA buffer and incubated at $65^{\circ} \mathrm{C}$ for $10 \mathrm{~min}$. A $1 \mu \mathrm{L}$ aliquot of RNase $(5 \mu \mathrm{g} \mathrm{mL}-1)$ was added to each microcentrifuge tube and the samples were incubated at $37^{\circ} \mathrm{C}$ with shaking for 30 $\min$.

The duplicate samples were combined and $\mathrm{H}_{2} \mathrm{O}$ was added to a final volume of 0.5 $\mathrm{mL}$. The DNA was extracted with $500 \mu \mathrm{L}$ of phenol $/ \mathrm{CHCl}_{3} /$ isoamyl alcohol mix $(25: 24: 1)$. The phases were emulsified by vortex and separated by centrifugation for $10 \mathrm{~min}$ at 16,000 $\mathrm{rpm}$. The organic layer was removed and the aqueous phase washed with $500 \mu \mathrm{L}$ of $\mathrm{CHCl}_{3}$. $1 \mathrm{~mL}$ EtOH and $40 \mu \mathrm{L} \mathrm{NaOAc}$ were added to the aqueous layer and shaken gently. The DNA was precipitated during incubation for $25 \mathrm{~min}$ at $4{ }^{\circ} \mathrm{C}$, and the pellets recovered following centrifugation (10 min, 13,000 rpm). The DNA pellets were washed twice with $70 \%$ EtOH then dissolved in $35 \mu \mathrm{L}$ Tris EDTA $\left(10 \mathrm{~min}, 60^{\circ} \mathrm{C}\right)$ and maintained at $4{ }^{\circ} \mathrm{C}$ until further use.

The DNA was quantified using a Hoechst 33258 dye and calf thymus as the DNA standard. Four separate PCR reactions were conducted with $100 \mathrm{ng}$ of starting DNA template. These consisted of the Up and Dn tags for both the control and experimental DNA samples. The Up tags were labeled with Cy3-dye markers and Dn tags with Cy5. The experimental conditions and primer sequences are shown below.

\section{Primer sequences:}

D1

U1

D2comp-Cy3 or -Cy5

U2comp-Cy3 or - $\mathrm{Cy} 5$
5/CGGTGTCGGTCTCGTAG-/3

5/GATGTCCACGAGGTCTCT-»3

5/-Cy [3/5]-CGAGCTCGAATTCATCGAT-ı

5/-Cy [3/5-GTCGACCTGCAGCGTACG-ı3

\section{PCR primer combinations:}

Control DNA (no compound) Up tag PCR Primers U1 and U2comp-Cy3 Dn tag PCR Primers D1 and D2comp-Cy3

\section{Experimental (compound)} Up tag PCR Primers U1 and U2comp-Cy5 Dn tag PCR Primers D1 and D2comp-Cy5

Each PCR reaction was performed in $60 \mu \mathrm{L}$ final volume, containing $1.5 \mathrm{mmol} \mathrm{L}^{-1}$ $\mathrm{MgCl}_{2}, 0.2 \mathrm{mmol} \mathrm{L}{ }^{-1} \mathrm{dNTPs}$, and $1 \mu \mathrm{mol} \mathrm{L}{ }^{-1}$ primers. PCR Master Mix was made up as follows. Four negative controls were also prepared with $4.0 \mu \mathrm{L} \mathrm{H}_{2} \mathrm{O}$ in place 
of DNA.

Table 7.2 PCR Master Mix

\begin{tabular}{ccc}
\hline & \multicolumn{2}{c}{ Volume $(\mu \mathrm{L})$} \\
\cline { 2 - 3 } Component & $1 \times$ & $9 \times(1$ array $)$ \\
\hline \hline $10 x$ Platinum taq buffer & 6.0 & 54.0 \\
$50 \mathrm{mmol} \mathrm{L}^{-1} \mathrm{MgCl}_{2}$ & 1.8 & 6.2 \\
$5 \mathrm{mmol} \mathrm{L}^{-1}($ each $) \mathrm{dNTPs}$ & 2.4 & 21.6 \\
Platinum taq $\left(5\right.$ units $\left.\mu \mathrm{L}^{-1}\right)$ & 0.2 & 1.8 \\
$\mathrm{H}_{2} \mathrm{O}$ & 40.8 & 367.2 \\
\hline Total volume & $\mathbf{5 1 . 2}$ & $\mathbf{4 6 0 . 8}$ \\
\hline
\end{tabular}

PCR reaction components, $8 \times 0.2 \mathrm{~mL}$ tubes:

Labelled primer $\left(25 \mu \mathrm{mol} \mathrm{L}{ }^{-1}\right) \quad 2.4 \mu \mathrm{L}$

Unlabelled primer $\left(25 \mu \mathrm{mol} \mathrm{L}{ }^{-1}\right) \quad 2.4 \mu \mathrm{L}$

Master mix

$51.2 \mu \mathrm{L}$

DNA $\left(25 \mathrm{ng} \mu \mathrm{L}^{-1}\right)$ or $\mathrm{H}_{2} \mathrm{O}$

$4.0 \mu \mathrm{L}$

\section{PCR conditions:}

$94{ }^{\circ} \mathrm{C} \quad 3 \mathrm{~m}$

$94^{\circ} \mathrm{C} \quad 30 \mathrm{~s}$

$50^{\circ} \mathrm{C} \quad 30 \mathrm{~s} \quad 38$ cycles

$72{ }^{\circ} \mathrm{C} \quad 30 \mathrm{~s}$

$72^{\circ} \mathrm{C} \quad 5 \mathrm{~m}$

$10^{\circ} \mathrm{C}$ hold

A $5 \mu \mathrm{L}$ aliquot of each PCR condition was combined with $1 \mu \mathrm{L}$ loading dye and run on a high resolution 4\% MetaPhor Agarose gel with 1.5 $\mu \mathrm{L}$ 10-bp ladder (Invitrogen) checking for both contamination and amplification of the 56-bp Up and Dn tags.

A $20 \mu \mathrm{L}$ aliquot of blocking primer mix $\left(12.5 \mu \mathrm{L}, 100 \mu \mathrm{mol} \mathrm{L} \mathrm{L}^{-1} \mathrm{U} 1\right.$ primer; 12.5 $\mu \mathrm{L}, 100 \mu \mathrm{mol} \mathrm{L}{ }^{-1} \mathrm{D} 1$ primer; $12.5 \mu \mathrm{L}, 100 \mu \mathrm{mol} \mathrm{L}{ }^{-1} \mathrm{U} 2$ block; $12.5 \mu \mathrm{L}, 100$ $\mu$ mol L ${ }^{-1}$ D2 block; $50 \mu \mathrm{L} \mathrm{H}_{2} \mathrm{O}$ ) was combined with $55 \mu \mathrm{L}$ each PCR product. The complimentary oligonucleotide sequences bind with the priming regions of the PCR products preventing them from binding with each other and allowing them to hybridise to the microarray. The barcode tags were precipitated by adding $24 \mu \mathrm{L}$ NaOAc $\left(3 \mathrm{~mol} \mathrm{~L}^{-1}\right.$, pH 5.2), $600 \mu \mathrm{L}$ EtOH and $1 \mu \mathrm{L} 5 \mathrm{mg} \mathrm{mL}^{-1}$ linear acrylamide to initiate precipitation. Solutions were mixed by vortex and the PCR products precipitated at $-20^{\circ} \mathrm{C}$ over $2 \mathrm{~h}$.

\section{Blocking primer sequences:}

D2block 5/ATCGATGAATTCGAGCTCG

U2block 5/-CGTACGCTGCAGGTCGAC 
The PCR products were recovered by centrifugation at $13,000 \mathrm{rpm}$ at $4{ }^{\circ} \mathrm{C}$ for 30 $\mathrm{m}$ and at washed at $4{ }^{\circ} \mathrm{C}$ with $70 \%$ EtOH. The EtOH was removed and the PCR products and washed were dried briefly in the dark, at room temperature, then redissolved $50 \mu \mathrm{L} \mathrm{H}_{2} \mathrm{O}$ and maintained at $-20^{\circ} \mathrm{C}$ until hybridisation.

DNA hybridisation to microarray slide: The $50 \mu \mathrm{L}$ PCR products were combined with $50 \mu \mathrm{L}$ filter-sterilised $2 x$ hybridisation buffer $\left(2 \mathrm{~mol} \mathrm{~L}{ }^{-1} \mathrm{NaCl}, 20\right.$ mmol L ${ }^{-1}$ Tris $\mathrm{HCl}$ pH 7.5, $1 \%$ Triton X-100. DTT ( 1 mmol L ${ }^{-1}$ final concentration) was added to the hybridisation buffer immediately prior to use. The buffer and PCR products were pipetted gently to mix, avoiding bubbles, and the hybridisation mix was denatured at $95^{\circ} \mathrm{C}$ for 2 min.

A $100 \mu \mathrm{L}$ aliquot of hybridisation mix was applied to the gasket slide avoiding bubble formation. The microarray slide was placed active side down, on to gasket slide, the chamber was assembled and rotated gently to ensure wetting of the entire slide and continuous movement of the hybridsation mix. The slide was then rotated at a moderate speed for $4 \mathrm{~h}$ at $42^{\circ} \mathrm{C}$ in a hybridisation oven. After baking, the gasket slide was removed, rinsed 5 times $\left(210 \mathrm{~mL} \mathrm{H}_{2} \mathrm{O}, 90 \mathrm{~mL}\right.$ SSPE and $0.15 \mathrm{~mL}$ Triton X), centrifuged to remove residual $\mathrm{H}_{2} \mathrm{O}$ and dried in the dark with $\mathrm{SiO}_{2}$ dessicant. The microarray slide was sent to Les McNoe at Otago Genomics Facility, Department of Biochemistry, University of Otago.

Microarray data analysis: GenePix 6.0 (Molecular Devices, Sunnyvale, CA, USA) was used to align the microarray image and extract data from the image file. The GenePix results file (.gpr file) was opened in a spreadsheet program and the control spots, spots flagged as poor quality, and those corresponding to essential gene deletions were removed. The Up and Dn tags were separated into separate worksheets and saved as .txt files. Each .txt file was uploaded into the web-based SNOMAD software (standardisation and normalisation of microarray data) and normalised according to the median F635 (Cy5) and median F532 (Cy3) data columns (as the data types "ONEintensities" and "TWOintensities", respectively). Transformation parameters are as follows; Perform and Graph the transformation in Steps 3, Log transformation (Log base 2), 4, calculation of mean log (intensities) and $\log$ (ratios), and 5, local mean normalization across element signal intensity, (Span 0.3, Trim 0.1). ${ }^{253}$ The data was submitted for processing and the results were copied into a fresh worksheet. Data spots with low $(<500)$ median F532 scores were removed to reduce the level of noise in the data, and replicates were removed using a "replicate removal" script. ${ }^{254}$ The normalised log ratios for tag replicates were averaged, and $z$-scores ( $x$-mean/standard deviation) were calculated. The data was sorted by $z$-scores, with $z$-scores $<-3.0$ denoted as a probable significant hit. 
Database searches: Gene hits were categorised according to their molecular and biological functions and their cellular components using the web-based program FunSpec $^{225}$. The Saccharomyces Genome Database ${ }^{226}$ was used to define the molecular and biological functions of gene hits and to analyse the synthetic lethal interactions of each genetic hit.

\section{Haploinsufficiency profiling by microarray}

The essential and nonessential gene cassettes of $S$. cerevisiae were assessed simultaneously for haploinsufficiency in the presence of 95. All practical proceedings were the same as for the HOP, however $2 \mu \mathrm{mol} \mathrm{L}{ }^{-1} \mathbf{9 5}$ was found to elicit a $30 \%$ growth reduction of the heterozygous deletion pool relative to the DMSO control. Data analysis differed in that values corresponding to essential genes were included during processing.

Microarray validation by dose-response: The sensitivity of homozygous dele-

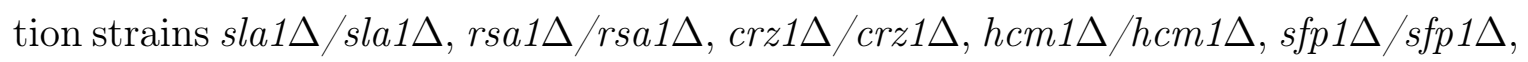

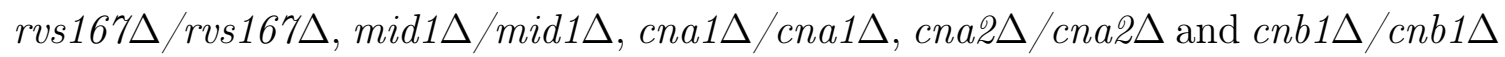
to epiplakinic acid I (95) was assessed by dose-response relative to the diploid wildtype starting strain BY4743. The same general protocol as above was followed during these validation assays with half-log dilutions from $100-0.1 \mu \mathrm{mol} \mathrm{L^{-1 }}$ final

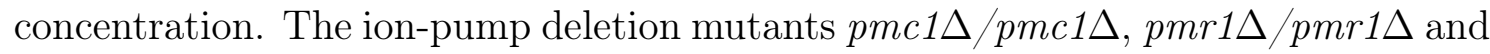
$\operatorname{cch} 1 \Delta / \operatorname{cch} 1 \Delta$ were also assessed relative to the diploid WT strain with an eightpoint dilution across two orders of magnitude; 30, 20, 10, 3, 2, 1 and $0.03 \mu \mathrm{mol} \mathrm{L}{ }^{-1}$ final concentration.

Microarray validation by single-concentration growth inhibition: Slow

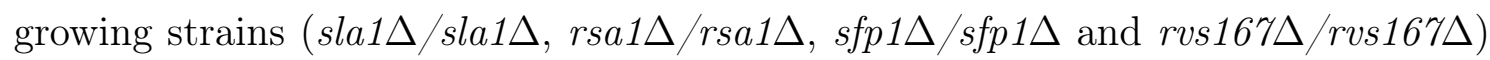
were assessed for inhibition relative to the WT at the $\mathrm{IC}_{30}$ value used for the

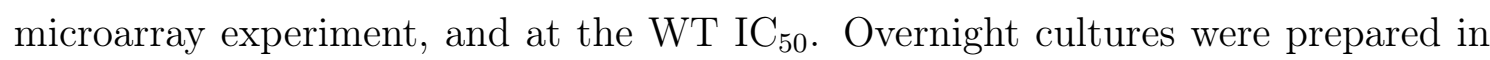
triplicate from different single colonies and incubated for $18 \mathrm{~h}$ at $30^{\circ} \mathrm{C}$ in a rotating drum. The strains were diluted to $5 \times 10^{4}$ cells $\mathrm{mL}^{-1}$ in $2 \mathrm{~mL}$ fresh $\mathrm{SC}$ supplemented with G418 antibiotic $\left(200 \mu \mathrm{g} \mathrm{mL}^{-1}\right)$. Nine $99 \mu \mathrm{L}$ aliquots of each culture were added to a 96-well tissue culture plate and treated in triplicate at 3.5 and $3.0 \mu \mathrm{mol} \mathrm{L}{ }^{-1}$. DMSO, $1 \mu \mathrm{L}$, served as control. Optical density measurements were taken hourly ( $\mathrm{t}$ $=0-8 \mathrm{~h}$ ) and again at $20 \mathrm{~h}$ to quantify growth relative to the BY4743 WT strain. 


\subsubsection{Imaging studies}

Prior to all imaging studies, GFP-labelled strains were cultured overnight on solid agar plates (SD-his). Cells were transferred into 384-well clear bottomed microtitre plates (Perkin Elmer Cell Carrier) by pipette and four $50 \mu \mathrm{L}$ liquid cultures (SC) were made by serial dilution. Cultures were inoculated with control and experimental agents and incubated for a given period at $30^{\circ} \mathrm{C}$ imaging with the OPERA microscope at specified time points.

Assessment of the nuclear-cytoplasmic ratio: The plasma-membrane protein GFP strain Mrh1p-GFP with nuclear membrane marker NLS-RS2/mCherry was cultured overnight on solid nutrient medium (SC-his). Cells of the GFP/mCherry strain were transferred into a 384-well clear bottomed microtitre plate (Perkin Elmer Cell Carrier) by pipette and a four-point serial dilution of $50 \mu \mathrm{L}$ liquid cultures (SC) was made in duplicate. Cultures were inoculated with $1 \%$ DMSO control and 95 at the MIC concentration then incubated for $4 \mathrm{~h}$, imaging at 2 and $4 \mathrm{~h}$.

Translocalisation of Crz1p-GFP in response to $\mathrm{Ca}^{2+}$ : Freshly cultured Crz1p-GFP cells were transferred from solid medium into a 384-well clear-bottomed microtitre plate as above. Parallel serial dilutions (50 $\mu \mathrm{L}$ liquid SC) were prepared over four points and cultured for $2 \mathrm{~h}$ prior to exposure to $1 \%$ DMSO, $50 \mathrm{mmol} \mathrm{L}^{-1}$ $\mathrm{CaCl}_{2}$ and $10 \mu \mathrm{mol} \mathrm{L}{ }^{-1} \mathbf{9 5}$. Cells were imaged at $5 \mathrm{~min}$ intervals from $\mathrm{t}=0-40$ min.

Specificity of Crz1p-GFP translocation to $\mathrm{Ca}^{2+}$ : Freshly cultured Crz1pGFP cells were transferred from solid medium into a 384-well clear-bottomed microtitre plate as above. Parallel serial dilutions (50 $\mu \mathrm{L}$ liquid SC) were prepared over four points and cultured for $2 \mathrm{~h}$ prior to exposure to $1 \%$ DMSO, $50 \mathrm{mmol} \mathrm{L}^{-1}$ of the following metal salts: $\mathrm{CaCl}_{2}, \mathrm{MgCl}_{2}, \mathrm{ZnSO}_{4}, \mathrm{CuSO}_{4}$ and $\mathrm{KCl}$, and $10 \mu \mathrm{mol}$ $\mathrm{L}^{-1}$ 95. Cells were imaged at $5 \mathrm{~min}$ intervals from $\mathrm{t}=0-40 \mathrm{~min}$.

ROS induction with $\mathrm{H}_{2} \mathrm{O}_{2}$ in the Yap1p-GFP/mCherry strain: Freshly cultured Yap1p-GFP/mCherry cells were transferred into a 384-well clear-bottomed microtitre plate as above. Four, parallel serial dilutions $(50 \mu \mathrm{L}$ liquid $\mathrm{SC})$ were prepared over four points and cultured for $2 \mathrm{~h}$ prior to exposure to 1\% DMSO, 50 mmol L ${ }^{-1} \mathrm{CaCl}_{2}, 10 \mu \mathrm{mol} \mathrm{L}{ }^{-1} \mathbf{9 5}$, and $1 \mathrm{mmol} \mathrm{L}^{-1} \mathrm{H}_{2} \mathrm{O}_{2}$. Cells were imaged at 5 min intervals from $\mathrm{t}=0-40 \mathrm{~min}$. 
Effect of epiplakinic acid I upon membrane-bound ion pumps: Cultures of the Zrt1-GFP, Zap1-GFP and Crz1-GFP strains were prepared as stated above and transferred into 4-point serial dilutions of liquid SC in a clear-bottomed 384-well microtitre plate. The three GFP strains were simultaneously exposed to $1 \%$ DMSO control, $50 \mathrm{mmol} \mathrm{L}{ }^{-1} \mathrm{CaCl}_{2}, \mathrm{MgCl}_{2}$ and $\mathrm{ZnSO}_{4}$ and $10 \mu \mathrm{mol} \mathrm{L}{ }^{-1}$ 95. Response to these species was assessed at 5 min intervals from $\mathrm{t}=0-40 \mathrm{~min}$.

Quantification of intracellular $\mathbf{C a}^{2+}$ levels: Calcium Orange (Molecular Probes, Invitrogen) was used to quantify changes in the level of intra-cellular $\mathrm{Ca}^{2+}$ upon exposure to 95 relative to the DMSO control. Thee $5 \mathrm{~mL}$ SC cultures of the diploid WT BY4743 strain were incubated at $30^{\circ} \mathrm{C}$ in a rotating drum for $18 \mathrm{~h}$ then diluted to $5 \times 10^{5}$ cells $\mathrm{mL}^{-1}$ in fresh SC. Cultures were treated with $\mathbf{9 5}$ at the MIC $\left(10 \mu \mathrm{mol} \mathrm{L}{ }^{-1}\right)$, and DMSO served as positive control. As per the methods of use protocol, following $4 \mathrm{~h}$ exposure to 95 and DMSO control, cells were resuspended by vortexing and three $300 \mu \mathrm{L}$ aliquots of each experimental condition were were individually transferred into fresh microcentrifuge tubes, and incubated $30 \mathrm{~min}$ in the presence of Calcium Orange $\left(5 \mu \mathrm{mol} \mathrm{L}{ }^{-1}\right)$. Emission intensity was measured in two ways: Cells were washed twice with $\mathrm{H}_{2} \mathrm{O}$ to remove free ligand and resuspended in $100 \mu \mathrm{L}$ and the emission intensity of the probe measured (excitation $549 \mathrm{~nm}$, emission $576 \mathrm{~nm}$ ). Emission intensity of the cell/dye suspension was also measured to determine the effects of cell loss during the washing process.

\subsubsection{Transformation of 8025- $\alpha$ strain}

Plasmid purification: Cultures of bacteria expressing the pRS-316 plasmid were grown overnight in $2 \mathrm{~mL} \mathrm{LB}$ supplemented with $2 \mu \mathrm{L}$ ampicillin. The cells were harvested and subjected to RNA digest and the extracted plasmids were purified with the Invitrogen Plasmid Preparation kit. Purity of the extracted plasmid was determined by gel electrophoresis ( $1 \%$ agarose). The plasmid was stored at $-20{ }^{\circ} \mathrm{C}$ prior to use in transformation.

Preparation of yeast cell cultures for transformation: Cells from a fresh overnight culture of the yeast strain 8025 - $\alpha$ were diluted to $2.5 \times 10^{8}$ in $150 \mathrm{~mL}$ prewarmed YPD and incubated with shaking at $30^{\circ} \mathrm{C}$ for $4 \mathrm{~h}$ in $250 \mathrm{~mL}$ conical flasks to achieve mid-log growth of the culture. After $4 \mathrm{~h}$ growth the $\mathrm{OD}_{590}$ was measured and duplicate $25 \mathrm{~mL}$ aliquots of the culture $\left(1.75 \times 10^{8}\right.$ cells $)$ were washed twice with $25 \mathrm{~mL} \mathrm{H}_{2} \mathrm{O}$. The cells were harvested by centrifuge and resuspended in $1 \mathrm{~mL} \mathrm{H}_{2} \mathrm{O}$, transferred into microcentrifuge tubes, washed again with $\mathrm{H}_{2} \mathrm{O}$ and $570 \mu \mathrm{L}$ aliquots of cell concentrate $\left(10^{8}\right.$ cells) were further diluted in $\mathrm{H}_{2} \mathrm{O}$ to a total volume of $1 \mathrm{~mL}$ 
then harvested by centrifuge. To each microcentrifuge tube $360 \mu \mathrm{L}$ transformation mix (including plasmid DNA) was added and the yeast cells resuspended by vortex.

Transformation mix:

$\begin{array}{lll}\text { PEG 3350 } & 50 \% \mathrm{w} / \mathrm{w} & 240 \mu \mathrm{L} \\ \text { LiOAc } & 1 \mathrm{~mol} \mathrm{~L}^{-1} & 36 \mu \mathrm{L} \\ \text { single-stranded carrier DNA } & 2.0 \mathrm{mg} \mathrm{mL}^{-1} & 50 \mu \mathrm{L} \\ \text { Plasmid DNA in } \mathrm{H}_{2} \mathrm{O} & & 34 \mu \mathrm{L}\end{array}$

Heat-shock was applied by placing the microcentrifuge tubes in a $42{ }^{\circ} \mathrm{C}$ water-bath for $40 \mathrm{~min}$, after which the tubes were centrifuged at 10,000 rpm for $30 \mathrm{~s}$ and the supernatant was removed by pipette. The cell pellet was resuspended in $1 \mathrm{~mL}$ YPD and incubated for $3 \mathrm{~h}$ at $30^{\circ} \mathrm{C}$ with shaking. A $150 \mu \mathrm{L}$ aliquot of transformed cell culture were plated onto YPD agar, and the remainder harvested and resuspended in $150 \mu \mathrm{L}$ YPD and plated similarly. Plates were incubated for $36 \mathrm{~h}$ at $30^{\circ} \mathrm{C}$ and four transformants were randomly selected then streaked again on YPD then incubated for $24 \mathrm{~h}$ at $30^{\circ} \mathrm{C}$.

\subsubsection{Attempted transformation of $z r t 1 \Delta / z r t 1 \Delta$ and $\operatorname{cch} 1 \Delta / \operatorname{cch} 1 \Delta$ strains}

Plasmid purification: As above, bacteria expressing the pMT 2932 and 2916 plasmids encoding the URA3 MX cassette were cultured overnight in $2 \mathrm{~mL} \mathrm{LB}$ supplemented with $2 \mu \mathrm{L}$ ampicillin. The cells were harvested and the plasmids extracted and purified as per the Invitrogen Plasmid preparation kit. Restriction digests were performed on both plasmids.

$\begin{array}{ll}\text { Restriction digest as performed in microcentrifuge tubes: } \\ \text { Plasmid DNA in } \mathrm{H}_{2} \mathrm{O} & 50 \mu \mathrm{L} \\ 10 x \text { Buffer } 2 & 5 \mu \mathrm{L} \\ \text { EcoR1 enzyme } & 1 \mu \mathrm{L} \\ \text { Bam } \mathrm{H} 1 \text { enzyme } & 1 \mu \mathrm{L} \\ \text { Bovine serum albumin } & 0.5 \mu \mathrm{L}\end{array}$




\section{Appendix A}

\section{Sponge Screening and Cyclic Loading Protocol}

\section{A.1 Cyclic loading and backloading}

Cyclic loading is a technique developed by Northcote and West ${ }^{255}$ and was frequently employed during this research. The technique was used to fractionate crude extracts of organisms during the screening process, separating the undesirable nonpolar fats and highly polar salts and carbohydrates from the intermediate polarity compounds of interest, i.e. secondary metabolites. This laboratory employs PSDVB (poly(styrene-divinylbenzene)) cross-linked polymeric resin beads as the stationary phase. PSDVB is a macro-porous, rigid resin which is chemically inert and stable across a large $\mathrm{pH}$ range, and can be reused a number of times without decreased performance. The beads are devoid of polar functional groups so irreversible binding or degradation of polar substrates has not been observed.

As depicted in Figure A.1 the crude $(\mathrm{MeOH})$ extract is passed through a PSDVB column, allowing adhesion of non-polar metabolites within the extract to the column. The eluent is diluted 1:1 with $\mathrm{H}_{2} \mathrm{O}$ and re-cycled through the column iteratively, diluted 1:1 with $\mathrm{H}_{2} \mathrm{O}$ at each stage. Increased eluent polarity facilitates adsorption of increasing polar metabolites to the stationary phase, in effect the opposite of conventional chromatography. After adhesion of the desired material to the column, it is eluted with mixtures of $\mathrm{H}_{2} \mathrm{O}$ and an organic modifier of decreasing polarity, commonly $30 \%, 75 \%$ and $100 \% \mathrm{Me}_{2} \mathrm{CO}$ in $\mathrm{H}_{2} \mathrm{O}$.

The $30 \%$ and $75 \% \mathrm{Me}_{2} \mathrm{CO}$ in $\mathrm{H}_{2} \mathrm{O}$ fractions are then backloaded onto the screening column using the same cyclic technique, the column is air dried and then eluted with organic solvent. This generates fractions devoid of $\mathrm{H}_{2} \mathrm{O}$ which can be easily evaporated under reduced pressure.

This laboratory uses water-miscible solvents such as $\mathrm{MeOH}$ and $\mathrm{Me}_{2} \mathrm{CO}$, which are 


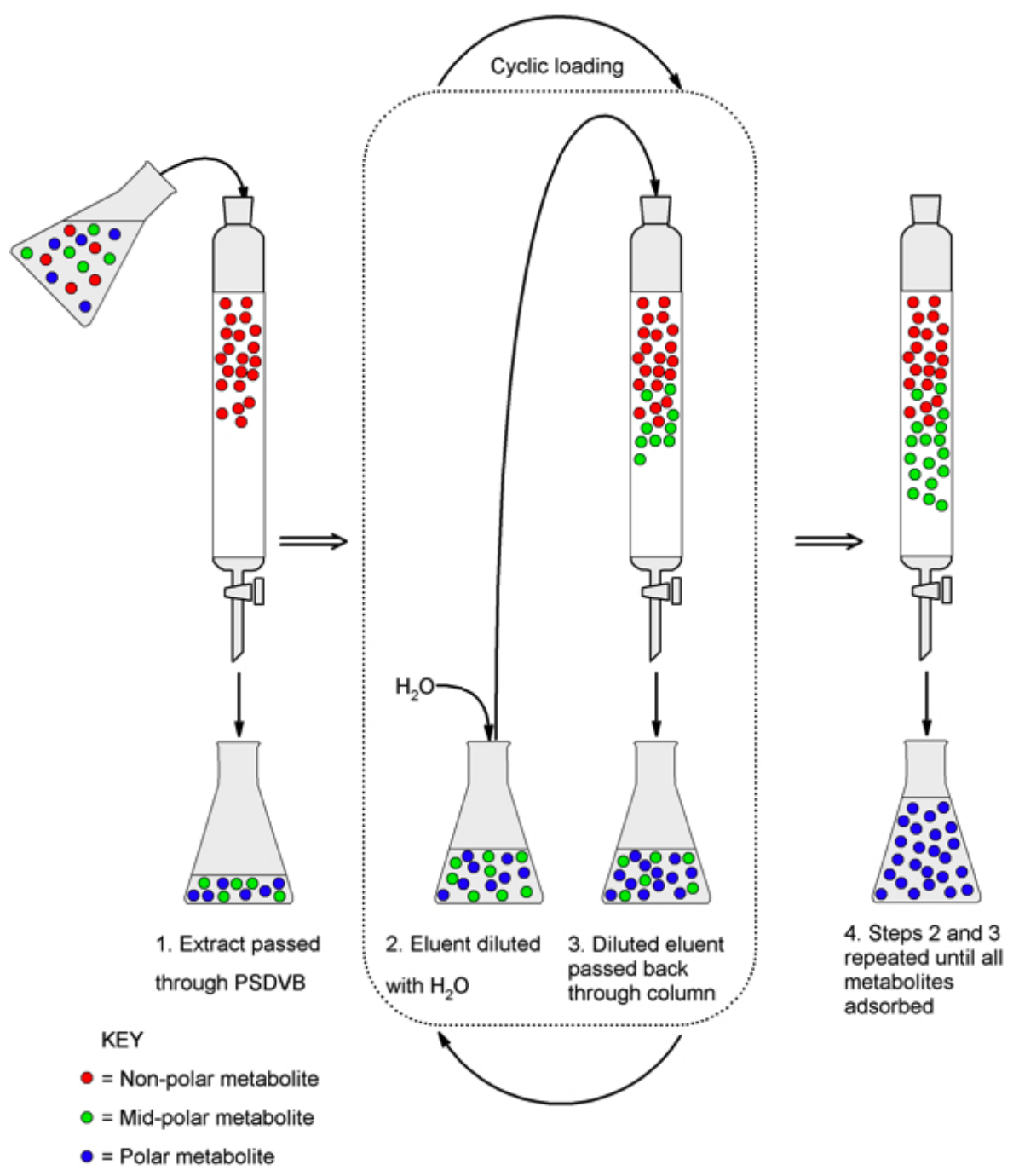

Figure A.1 A depiction of the cyclic loading process.

considerably more environmentally friendly than the halogenated solvents frequently used in other approaches such as liquid-liquid partitioning. Large quantities of eluent are generated during the procedure - the major draw-back of the technique. For a 20 $\mathrm{g}$ screening sample of sponge, $4.1 \mathrm{~L}$ of eluent is produced. However, an automated set-up is is available for large-scale extractions using an HPLC pump system.

\section{A.2 Screening protocol}

\section{Equipment required (per screen)}

$1 \times$ screening column loaded with $40 \mathrm{~mL}$ of HP20 resin beads equilibrated in MeOH.

\section{Standard Preparation}

Prepare an NMR quantification standard of $100 \mu \mathrm{L}$ nitromethane in $5 \mathrm{~mL} \mathrm{CDCl}_{3}$. 


\section{Voucher sample preparation}

Take a voucher specimen of ca. $10 \mathrm{~g}$ of the crude sponge material ensuring that both the ectoderm and the endoderm are represented. Label and store the voucher sample in $75 \%$ IPA in $\mathrm{H}_{2} \mathrm{O}$.

\section{Extraction}

- Extract $\sim 20 \mathrm{~g}$ of crude sponge material in $100 \mathrm{~mL} \mathrm{MeOH}$ overnight.

- Filter the first extract and set aside.

- Re-extract the sponge material (and any filter paper/celite as necessary) in $100 \mathrm{~mL} \mathrm{MeOH}$ overnight.

- Filter the second extract. Keep all sponge material (and any filter paper/celite as necessary) until the screen is complete at which time it may be discarded.

\section{A.3 Cyclic loading}

- Pass the second extract through the screen column with a flow rate of ca. 10 $\mathrm{mL} \min ^{-1}$.

- Pass the first extract through the screen column with a flow rate of ca. $10 \mathrm{~mL}$ $\min ^{-1}$. Combine both eluents.

- Dilute the combined eluents with $200 \mathrm{~mL} \mathrm{H}_{2} \mathrm{O}$. Pass the diluted eluents back through the screen column at a flow rate of ca. $10 \mathrm{~mL} \mathrm{~min}^{-1}$.

- Dilute the eluent with $400 \mathrm{~mL} \mathrm{H}_{2} \mathrm{O}$. Pass the diluted eluent back through the screen column at a flow rate of ca. $10 \mathrm{~mL} \mathrm{~min}^{-1}$. The eluent should be kept until the screen is complete at which time it may be discarded.

\section{Elution}

- Elute the screen column with $100 \mathrm{~mL}$ of $\mathrm{H}_{2} \mathrm{O}$ at a flow rate of ca. $10 \mathrm{~mL}$ $\min ^{-1}$. The $\mathrm{H}_{2} \mathrm{O}$ eluent can be discarded immediately.

- Elute the screen column with $100 \mathrm{~mL}$ of $30 \% \mathrm{Me}_{2} \mathrm{CO}$ in $\mathrm{H}_{2} \mathrm{O}$ at a flow rate of ca. $10 \mathrm{~mL} \mathrm{~min}^{-1}$.

- Elute the screen column with $100 \mathrm{~mL}$ of $75 \% \mathrm{Me}_{2} \mathrm{CO}$ in $\mathrm{H}_{2} \mathrm{O}$ at a flow rate of ca. $10 \mathrm{~mL} \mathrm{~min}^{-1}$. 
- Elute the screen column with $100 \mathrm{~mL}$ of $\mathrm{Me}_{2} \mathrm{CO}$ at a flow rate of ca. $10 \mathrm{~mL}$ $\min ^{-1}$.

\section{Backloading the $75 \% \mathrm{Me}_{2} \mathrm{CO}$ Fraction}

- Dilute the $75 \% \mathrm{Me}_{2} \mathrm{CO}$ fraction with $100 \mathrm{~mL} \mathrm{H}_{2} \mathrm{O}$. Pass the diluted eluent through the backloading column at a flow rate of ca. $8 \mathrm{~mL} \mathrm{~min}^{-1}$.

- Dilute the eluent with $200 \mathrm{~mL}$ of $\mathrm{H}_{2} \mathrm{O}$. Pass the diluted eluent back through the backloading column at a flow rate of ca. $8 \mathrm{~mL} \mathrm{~min}{ }^{-1}$. The eluent should be kept until the screen is complete at which time it may be discarded.

- Elute the backloading column with $100 \mathrm{~mL}$ of $\mathrm{Me}_{2} \mathrm{CO}$.

\section{Backloading the $30 \% \mathrm{Me}_{2} \mathrm{CO}$ Fraction}

- Dilute the $30 \% \mathrm{Me}_{2} \mathrm{CO}$ fraction with $100 \mathrm{~mL}$ of $\mathrm{H}_{2} \mathrm{O}$. Pass the diluted eluent through the backloading column at a flow rate of ca. $8 \mathrm{~mL} \mathrm{~min}{ }^{-1}$.

- Dilute the eluent with $200 \mathrm{~mL}$ of $\mathrm{H}_{2} \mathrm{O}$. Pass the diluted eluent back through the backloading column at a flow rate of ca. $8 \mathrm{~mL} \mathrm{~min}{ }^{-1}$. The eluent should be kept until the screen is complete at which time it may be discarded.

- Elute the backloading column with $100 \mathrm{~mL}$ of $\mathrm{Me}_{2} \mathrm{CO}$.

\section{A.4 Processing and NMR analysis}

\section{Processing the $75 \% \mathrm{Me}_{2} \mathrm{CO}$ Fraction}

- Rotary-evaporate the $\mathrm{Me}_{2} \mathrm{CO}$ eluent of the backloading column to dryness and transfer to a pre-weighed sample vial. Evaporate to dryness and record the mass.

- Prepare an NMR sample of the sample in $500 \mu \mathrm{L} \mathrm{CD}_{3} \mathrm{OD}$ in a $5 \mathrm{~mm}$ NMR tube with $10 \mu \mathrm{L}$ of the nitromethane standard.

\section{NMR Analysis of the $75 \% \mathrm{Me}_{2} \mathrm{CO}$ Fraction}

- Run a ${ }^{1} \mathrm{H}$ spectrum of the sample using the standard ${ }^{1} \mathrm{H}$ experiment with 64 transients (ca. $4 \mathrm{~min}$ ) 
- Run a COSY spectrum of the sample using the standard gCOSY parameter set. (ca. $20 \mathrm{~min}$ )

- Run a fully-coupled HSQC spectrum of the sample using the standard Hcoupled HSQCad parameter set. (ca. $51 / 2$ h)

- Run a fully-coupled HMBC spectrum of the sample using the standard Hcoupled gHMBC parameter set. (ca. $81 / 4$ h)

\section{Processing the $30 \%$ Fraction}

- Rotary-evaporate the $\mathrm{Me}_{2} \mathrm{CO}$ eluent of the backloading column to dryness and transfer to a pre-weighed sample vial. Evaporate to dryness and record the mass.

\section{Processing the $100 \%$ Fraction}

- Rotary-evaporate the $\mathrm{Me}_{2} \mathrm{CO}$ eluent of the screen column to dryness and transfer to a pre-weighed sample vial. Evaporate to dryness and record the mass.

- Prepare an NMR sample of the sample in $700 \mu \mathrm{L}$ of $\mathrm{CDCl}_{3}$ in a $5 \mathrm{~mm} \mathrm{NMR}$ tube with $10 \mu \mathrm{L}$ of the nitromethane standard.

\section{NMR Analysis of the $100 \%$ Fraction}

- Run a ${ }^{1} \mathrm{H}$ spectrum of the sample using the standard ${ }^{1} \mathrm{H}$ experiment with 64 transients (ca. $4 \mathrm{~min}$ ).

- Run a COSY spectrum of the sample using the standard gCOSY parameter set. (ca. $20 \mathrm{~min}$ ).

- Run a fully-coupled HSQC spectrum of the sample using the standard Hcoupled HSQCad parameter set. (ca. $51 / 2$ h).

- Run a fully-coupled HMBC spectrum of the sample using the standard Hcoupled gHMBC parameter set. (ca. $81 / 4 \mathrm{~h}$ ). 


\section{A.5 Spicule analysis}

- Submerge a $1 \mathrm{~cm}^{3}$ piece of extracted sponge in $3 \mathrm{~mL} \mathrm{HNO}_{3}$ in a glass test tube. Ensure that both ecto- and endo-derms are represented in the tissue sample.

- Monitor decomposition of the organic tissue (ca. $24 \mathrm{~h}$ ).

- When the organic matter is dissolved, dilute the acid with $5 \mathrm{~mL} \mathrm{H}_{2} \mathrm{O}$.

- Centrifuge for 5 min to pallet the spicules.

- Remove the aqueous solution and wash the recovered spicules with $5 \mathrm{~mL} \mathrm{H}_{2} \mathrm{O}$. Repeat the washing procedure twice more.

- Air dry the spicules for $24 \mathrm{~h}$ then analyse by SEM. 


\section{Appendix B}

\section{Yeast Growth Media}

\section{YPD liquid media}

\begin{tabular}{lll}
\hline Liquid volume (total) & $1000 \mathrm{~mL}$ & $800 \mathrm{~mL}$ \\
\hline \hline Yeast extract & $10 \mathrm{~g}$ & $8 \mathrm{~g}$ \\
Peptone & $20 \mathrm{~g}$ & $16 \mathrm{~g}$ \\
Adenine & $0.12 \mathrm{~g}$ & $0.096 \mathrm{~g}$ \\
Agar & $20 \mathrm{~g}$ & $16 \mathrm{~g}$ \\
\hline
\end{tabular}

Autoclave and cool to $65^{\circ} \mathrm{C}$, add $40 \%$ glucose solution to a final concentration of $2 \$$ and antibiotics as required.

\section{SD liquid media}

\begin{tabular}{lll}
\hline Liquid volume (total) & $1000 \mathrm{~mL}$ & $800 \mathrm{~mL}$ \\
\hline \hline $\mathbf{2 x}$ Agar solution & & \\
Agar & $20 \mathrm{~g}$ & $16 \mathrm{~g}$ \\
$\mathrm{H}_{2} \mathrm{O}$ & $500 \mathrm{~mL}$ & $400 \mathrm{~mL}$
\end{tabular}

\section{$2 x$ SD solution}

\begin{tabular}{lll}
$\mathrm{H}_{2} \mathrm{O}$ & $500 \mathrm{~mL}$ & $400 \mathrm{~mL}$ \\
Yeast nitrogen base & $1.7 \mathrm{~g}$ & $1.36 \mathrm{~g}$ \\
Monosodium glutamate & $1 \mathrm{~g}$ & $0.8 \mathrm{~g}$ \\
Required amino acids & $2 \mathrm{~g}$ & $1.6 \mathrm{~g}$ \\
\hline
\end{tabular}

Autoclave and cool the $2 x$ agar and $2 x \mathrm{SD}$ solutions to $65^{\circ} \mathrm{C}$, and combine the solutions while stirring, add $40 \%$ glucose solution ( $5 \%$ final concentration) and supplements as required. 


\section{Appendix C}

\section{NMR Data for Known Compounds}

Table C.1 ${ }^{1} \mathrm{H}$ and ${ }^{13} \mathrm{C}$ NMR Data $\left(600 \mathrm{MHz}, \mathrm{CDCl}_{3}\right)$ for iso-Secothorectolide (23).

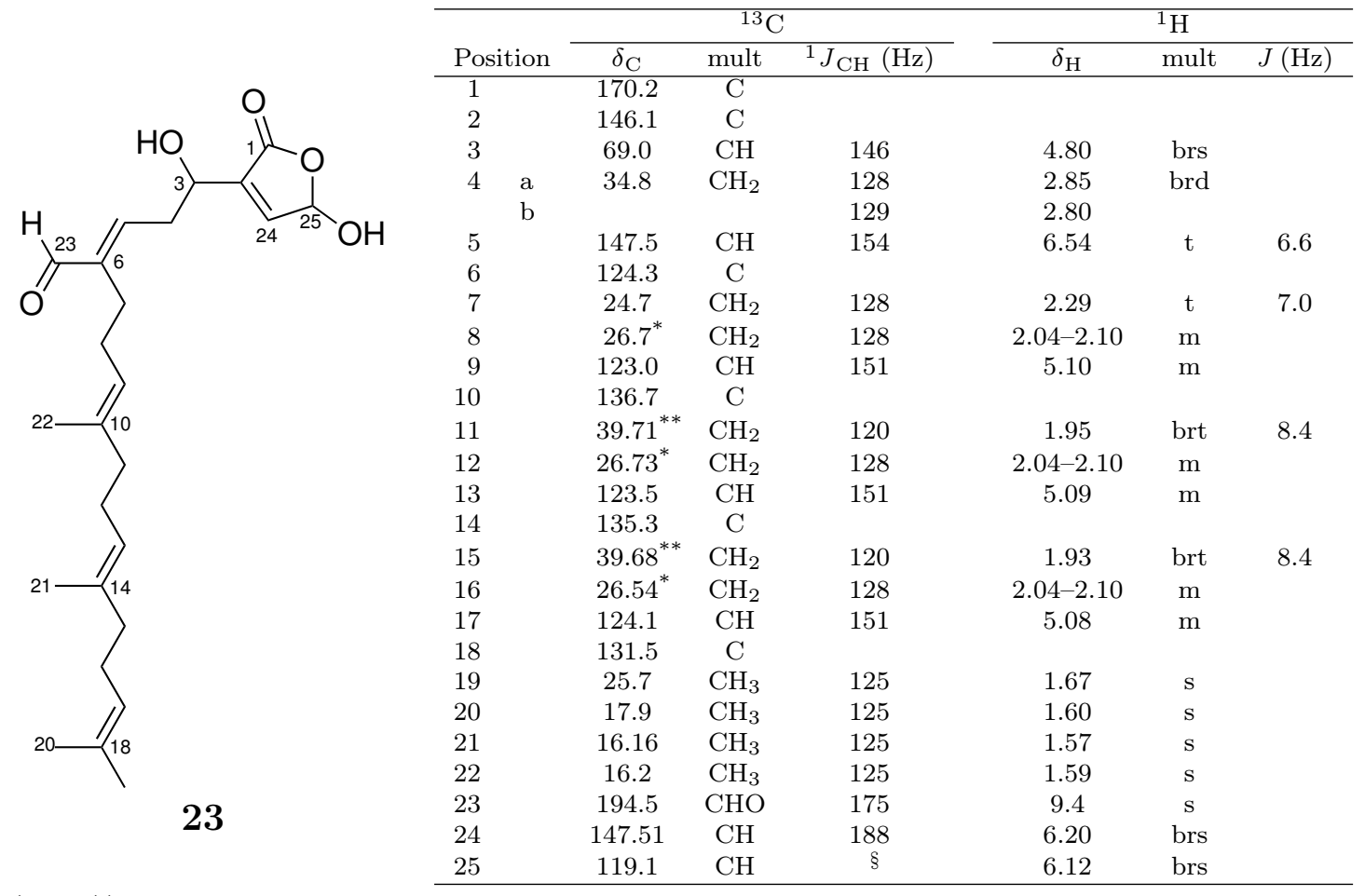

* and **Assignment interchangeable

$\S$ Signal unresolved and immeasurable 
Table C.2 ${ }^{1} \mathrm{H}$ and ${ }^{13} \mathrm{C}$ NMR Data $\left(600 \mathrm{MHz}, \mathrm{CDCl}_{3}\right)$ for 1-Hydroxyluffarin Q (24).

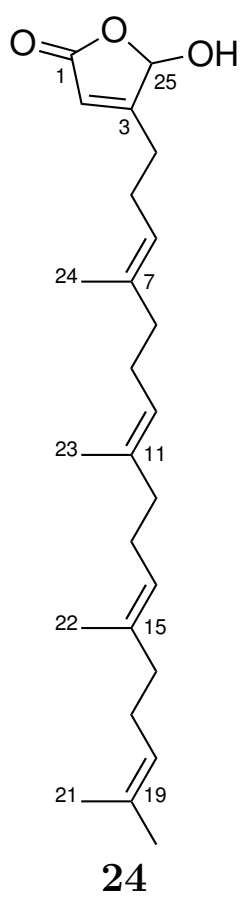

\begin{tabular}{|c|c|c|c|c|c|c|}
\hline \multirow[b]{2}{*}{ Position } & \multicolumn{3}{|c|}{${ }^{13} \mathrm{C}$} & \multicolumn{3}{|c|}{${ }^{1} \mathrm{H}$} \\
\hline & $\delta_{\mathrm{C}}$ & mult & ${ }^{1} J_{\mathrm{CH}}(\mathrm{Hz})$ & $\delta_{\mathrm{H}}$ & mult & $J(\mathrm{~Hz})$ \\
\hline 1 & 171.5 & $\mathrm{C}$ & & & & \\
\hline 2 & 117.8 & $\mathrm{CH}$ & 184 & 6.09 & $\mathrm{~s}$ & \\
\hline 3 & 168.3 & $\mathrm{C}$ & & & & \\
\hline 4 & 26.1 & $\mathrm{CH}_{2}$ & 122 & 2.16 & $\mathrm{~m}$ & \\
\hline 5 & 26.8 & $\mathrm{CH}_{2}$ & 120 & 2.06 & quin & 7.5 \\
\hline 6 & 120.9 & $\mathrm{CH}$ & 161 & 5.70 & brd & 5.1 \\
\hline 7 & 135.21 & $\mathrm{C}$ & & & & \\
\hline 8 & $27.0^{* *}$ & $\mathrm{CH}_{2}$ & & $2.05-2.11$ & $\mathrm{~m}$ & \\
\hline 9 & 32.7 & $\mathrm{CH}_{2}$ & 128 & 2.12 & $\mathrm{~m}$ & \\
\hline 10 & 124.2 & $\mathrm{CH}$ & 151 & 5.10 & brt & 7.6 \\
\hline 11 & 137.5 & C & & & & \\
\hline 12 & 39.9 & $\mathrm{CH}_{2}$ & 123 & 1.97 & $\mathrm{~m}$ & \\
\hline 13 & $26.9^{* *}$ & $\mathrm{CH}_{2}$ & & $2.05-2.11$ & $\mathrm{~m}$ & \\
\hline 14 & 123.5 & $\mathrm{CH}$ & 152 & 5.12 & brt & 6.8 \\
\hline 15 & 136.1 & $\mathrm{C}$ & & & & \\
\hline 16 & 39.8 & $\mathrm{CH}_{2}$ & 123 & 1.97 & $\mathrm{~m}$ & \\
\hline 17 & $26.95^{* *}$ & $\mathrm{CH}_{2}$ & & $2.05-2.11$ & $\mathrm{~m}$ & \\
\hline 18 & 124.5 & $\mathrm{CH}$ & 151 & 5.08 & brt & 7.6 \\
\hline 19 & 131.5 & C & & & & \\
\hline 20 & 25.9 & $\mathrm{CH}_{3}$ & 124 & 1.67 & $\mathrm{~s}$ & \\
\hline 21 & 17.8 & $\mathrm{CH}_{3}$ & 124 & 1.59 & s & \\
\hline 22 & $16.24^{*}$ & $\mathrm{CH}_{3}$ & 124 & 1.60 & $\mathrm{~s}$ & \\
\hline 23 & $16.21^{*}$ & $\mathrm{CH}_{3}$ & 124 & 1.60 & $\mathrm{~s}$ & \\
\hline 24 & 16.1 & $\mathrm{CH}_{3}$ & 125 & 1.59 & $\mathrm{~s}$ & \\
\hline 25 & 91.4 & $\mathrm{CH}$ & 168 & 5.31 & $\mathrm{~s}$ & \\
\hline
\end{tabular}

* and **Assignment interchangeable

Table C.3 ${ }^{1} \mathrm{H}$ and ${ }^{13} \mathrm{C}$ NMR Data $\left(600 \mathrm{MHz}, \mathrm{CDCl}_{3}\right)$ for ambliol B (25).

\begin{tabular}{|c|c|c|c|c|c|c|c|c|}
\hline & & & ${ }^{13} \mathrm{C}$ & & & ${ }^{1} \mathrm{H}$ & & \\
\hline & Po: & ion & $\delta_{\mathrm{C}}$ & mult & ${ }^{1} J_{\mathrm{CH}}(\mathrm{Hz})$ & $\delta_{\mathrm{H}}$ & mult & $J(\mathrm{~Hz})$ \\
\hline & 1 & $\mathrm{a}$ & 21.4 & $\mathrm{CH}_{2}$ & 125 & 1.59 & brd & \\
\hline & & b & & & 126 & 1.34 & $\mathrm{td}$ & $14.0,4.4$ \\
\hline & 2 & $\mathrm{a}$ & 22.4 & $\mathrm{CH}_{2}$ & 128 & 1.56 & & \\
\hline & & b & & & 126 & 1.46 & & \\
\hline & 3 & $\mathrm{a}$ & 36.8 & $\mathrm{CH}_{2}$ & 124 & 1.64 & $\mathrm{td}$ & \\
\hline & 4 & b & & $\mathrm{C}$ & 128 & 1.09 & brd & 13.4 \\
\hline & 5 & & 76.4 & C & & & & \\
\hline & 6 & $\mathrm{a}$ & 25.5 & $\mathrm{CH}_{2}$ & 122 & 1.71 & $\mathrm{td}$ & $14.0,4.2$ \\
\hline & & b & & & 126 & 1.41 & $\mathrm{dt}$ & \\
\hline & 7 & $\mathrm{a}$ & 24.6 & $\mathrm{CH}_{2}$ & 126 & 2.08 & $\mathrm{tt}$ & $17.8,4.0$ \\
\hline & & b & & & 127 & 1.25 & $\mathrm{~m}$ & \\
\hline & 8 & & 40.5 & $\mathrm{CH}$ & 120 & 1.58 & d & 7.1 \\
\hline & 9 & & 37.5 & $\mathrm{C}$ & & & & \\
\hline & 10 & & 35.1 & $\mathrm{CH}$ & 127 & 1.70 & quin & 4.5 \\
\hline 20 & 11 & $\mathrm{a}$ & 40.2 & $\mathrm{CH}_{2}$ & 125 & 1.64 & $\mathrm{~m}$ & \\
\hline 25 & & b & & & 126 & 1.29 & $\mathrm{~m}$ & \\
\hline & 12 & & 18.1 & $\mathrm{CH}_{2}$ & 125 & 2.36 & $\mathrm{dt}$ & $9.8,8.0$ \\
\hline & 13 & & 126.2 & C & & & & \\
\hline & 14 & & 138.6 & $\mathrm{CH}$ & 202 & 7.2 & $\mathrm{~s}$ & \\
\hline & 15 & & 111.1 & $\mathrm{CH}$ & 174 & $6.25, \mathrm{~s}$ & & \\
\hline & 16 & & 142.8 & $\mathrm{CH}$ & 202 & $7.35, \mathrm{~s}$ & & \\
\hline & 17 & & 23.7 & $\mathrm{CH}_{3}$ & 123 & 1.01 & $\mathrm{~s}$ & \\
\hline & 18 & & 24.6 & $\mathrm{CH}_{3}$ & 124 & 0.86 & $\mathrm{~s}$ & \\
\hline & 19 & & 14.3 & $\mathrm{CH}_{3}$ & 123 & 0.95 & d & \\
\hline & 20 & & 21.0 & $\mathrm{CH}_{3}$ & 124 & 1.13 & $\mathrm{~s}$ & \\
\hline
\end{tabular}


Table C.4 ${ }^{1} \mathrm{H}$ and ${ }^{13} \mathrm{C}$ NMR Data $\left(600 \mathrm{MHz}, \mathrm{CDCl}_{3}\right)$ for Luffariellolide (26).

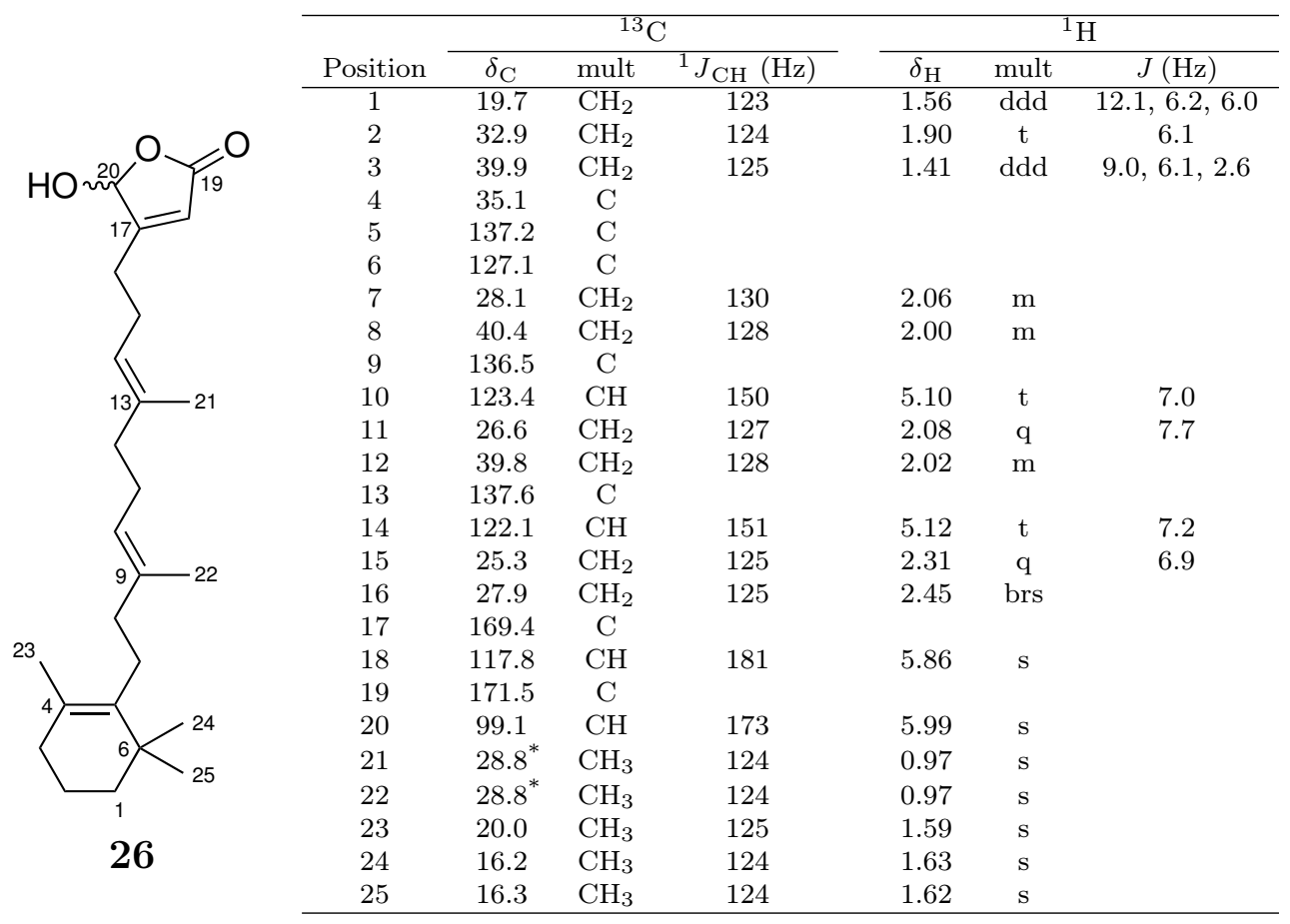

*Indistinguishable 
Table C.5 ${ }^{1} \mathrm{H}$ and ${ }^{13} \mathrm{C}$ NMR Data $\left(600 \mathrm{MHz}, \mathrm{CDCl}_{3}\right.$ ) for $5 \alpha, 8 \alpha$-Epidioxysterol (27).

\begin{tabular}{|c|c|c|c|c|c|c|c|}
\hline \multirow{2}{*}{\multicolumn{2}{|c|}{ Position }} & \multicolumn{3}{|c|}{${ }^{13} \mathrm{C}$} & \multicolumn{3}{|c|}{${ }^{1} \mathrm{H}$} \\
\hline & & $\delta_{\mathrm{C}}$ & mult & ${ }^{1} J_{\mathrm{CH}}(\mathrm{Hz})$ & $\delta_{\mathrm{H}}$ & mult & $J(\mathrm{~Hz})$ \\
\hline \multirow[t]{2}{*}{1} & $\mathrm{a}$ & 34.8 & $\mathrm{CH}_{2}$ & 128 & 1.95 & $\mathrm{td}$ & $13.5,3.4$ \\
\hline & b & & & 129 & 1.69 & $\mathrm{dtd}$ & $13.2,3.4$ \\
\hline \multirow[t]{2}{*}{2} & $\mathrm{a}$ & 30.2 & $\mathrm{CH}_{2}$ & 125 & 1.84 & dd & $12.9,3.9$ \\
\hline & b & & & 125 & 1.53 & $\mathrm{q}$ & $14.0,3.9$ \\
\hline 3 & & 66.6 & $\mathrm{CH}$ & 144 & 3.81 & $\mathrm{~s}$ & \\
\hline \multirow[t]{2}{*}{4} & $\mathrm{a}$ & 37.1 & $\mathrm{CH}_{2}$ & 141 & 2.11 & ddd & $13.9,5.3,1.9$ \\
\hline & $\mathrm{b}$ & & & 125 & 1.94 & dd & $13.1,11.3$ \\
\hline 5 & & 82.3 & $\mathrm{C}$ & & & & \\
\hline 6 & & 135.5 & $\mathrm{CH}$ & 168 & 6.14 & d & 8.5 \\
\hline 7 & & 130.9 & $\mathrm{CH}$ & 170 & 6.51 & d & 8.7 \\
\hline 8 & & 79.6 & $\mathrm{C}$ & & & & \\
\hline 9 & & 51.2 & $\mathrm{CH}$ & 130 & 1.49 & $\mathrm{t}$ & 5.1 \\
\hline 10 & & 37.0 & $\mathrm{C}$ & & & & \\
\hline \multirow[t]{2}{*}{11} & $\mathrm{a}$ & 23.5 & $\mathrm{CH}_{2}$ & 127 & 1.50 & $\mathrm{dd}$ & $10.1,7.0$ \\
\hline & $\mathrm{b}$ & & & 127 & 1.21 & dd & $18.6,3.2$ \\
\hline \multirow[t]{2}{*}{12} & $\mathrm{a}$ & 39.5 & $\mathrm{CH}_{2}$ & 127 & 1.96 & $\mathrm{~d}$ & 14.4 \\
\hline & b & & & 124 & 1.23 & brd & 5.8 \\
\hline 13 & & 44.7 & $\mathrm{C}$ & & 1.23 & brd & 6.0 \\
\hline 14 & & 51.8 & $\mathrm{CH}$ & 123 & 1.56 & $\mathrm{~m}$ & \\
\hline \multirow[t]{2}{*}{15} & $\mathrm{a}$ & 20.8 & $\mathrm{CH}_{2}$ & 125 & 1.77 & $\mathrm{dtd}$ & $7.4,12.2,3.2$ \\
\hline & b & & & 116 & 1.4 & dd & $11.9,6.1$ \\
\hline \multirow[t]{2}{*}{16} & $\mathrm{a}$ & 29.1 & $\mathrm{CH}_{2}$ & 126 & 1.69 & $\mathrm{dt}$ & $13.7,3.5$ \\
\hline & b & & & 127 & 1.34 & dd & $22.4,9.8$ \\
\hline 17 & & 56.2 & $\mathrm{CH}$ & 123 & 1.23 & $\mathrm{~m}$ & \\
\hline 18 & & 13.0 & $\mathrm{CH}_{3}$ & 125 & 0.82 & $\mathrm{~s}$ & \\
\hline 19 & & 18.3 & $\mathrm{CH}_{3}$ & 125 & 0.88 & $\mathrm{~s}$ & \\
\hline 20 & & 40.1 & $\mathrm{CH}$ & 126 & 2.03 & $\mathrm{dtd}$ & $23.6,7.9,2.7$ \\
\hline 21 & & 21.3 & $\mathrm{CH}_{3}$ & 125 & 1.01 & $\mathrm{~d}$ & 6.4 \\
\hline 22 & & 137.8 & $\mathrm{CH}$ & 148 & 5.14 & dd & $15.0,8.3$ \\
\hline 23 & & 130.0 & $\mathrm{CH}$ & 152 & 5.04 & dd & $15.3,8.7$ \\
\hline \multirow[t]{2}{*}{$24(1)$} & a & 25.5 & $\mathrm{CH}_{2}$ & 124 & 1.41 & $\mathrm{~m}$ & \\
\hline & b & & & 124 & 1.17 & $\mathrm{~d}$ & 10.1 \\
\hline $24(2)$ & & 12.6 & $\mathrm{CH}_{3}$ & 128 & 0.80 & $\mathrm{t}$ & 7.5 \\
\hline 25 & & 31.2 & $\mathrm{CH}$ & 127 & 1.52 & $\mathrm{~m}$ & \\
\hline 26 & & 19.1 & $\mathrm{CH}_{3}$ & 122 & 0.79 & d & 6.7 \\
\hline 27 & & 21.1 & $\mathrm{CH}_{3}$ & 124 & 0.84 & d & 6.4 \\
\hline
\end{tabular}

†Selected correlations.

*Weak correlations.

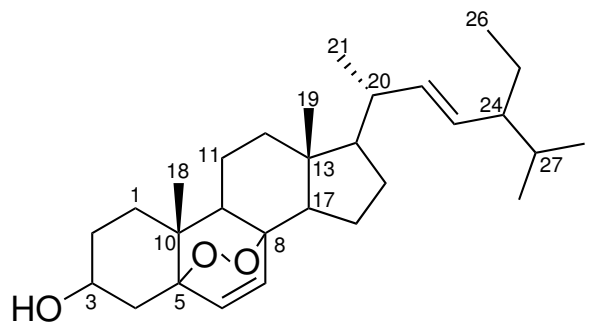

27 
Table C.6 ${ }^{1} \mathrm{H}$ and ${ }^{13} \mathrm{C}$ NMR Data $\left(600 \mathrm{MHz}, \mathrm{CDCl}_{3}\right)$ for $5 \alpha, 8 \alpha$-Epidioxysterol (28).

\begin{tabular}{|c|c|c|c|c|c|c|c|}
\hline \multirow{2}{*}{\multicolumn{2}{|c|}{ Position }} & \multicolumn{3}{|c|}{${ }^{13} \mathrm{C}$} & \multicolumn{3}{|c|}{${ }^{1} \mathrm{H}$} \\
\hline & & $\delta_{\mathrm{C}}$ & mult & ${ }^{1} J_{\mathrm{CH}}(\mathrm{Hz})$ & $\delta_{\mathrm{H}}$ & mult & $J(\mathrm{~Hz})$ \\
\hline \multirow[t]{2}{*}{1} & $\mathrm{a}$ & 34.8 & $\mathrm{CH}_{2}$ & 127 & 1.95 & $\mathrm{td}$ & $13.3,3.6$ \\
\hline & $\mathrm{b}$ & & $\mathrm{CH}_{2}$ & 129 & 1.69 & $\mathrm{dt}$ & $13.7,3.4$ \\
\hline \multirow[t]{2}{*}{2} & $\mathrm{a}$ & 30.2 & $\mathrm{CH}_{2}$ & 133 & 1.83 & brd & 12.6 \\
\hline & b & & & 125 & 1.54 & $\mathrm{~m}$ & \\
\hline 3 & & 66.6 & $\mathrm{CH}$ & 144 & 3.97 & $\mathrm{~m}$ & \\
\hline \multirow[t]{2}{*}{4} & $\mathrm{a}$ & 37.1 & $\mathrm{CH}_{2}$ & 126 & 2.11 & ddd & $13.8,5.1,2.0$ \\
\hline & $\mathrm{b}$ & & $\mathrm{CH}_{2}$ & 127 & 1.91 & dd & $11.7,13.6$ \\
\hline 5 & & 82.3 & $\mathrm{C}-\mathrm{O}$ & & & & \\
\hline 6 & & 135.5 & $\mathrm{CH}$ & 166 & 6.24 & d & 9.1 \\
\hline 7 & & 130.9 & $\mathrm{CH}$ & 168 & 6.51 & $\mathrm{~d}$ & 9.1 \\
\hline 8 & & 79.6 & $\mathrm{C}-\mathrm{O}$ & & & & \\
\hline 9 & & 51.2 & $\mathrm{CH}$ & 128 & 1.48 & $\mathrm{~m}$ & \\
\hline 10 & & 37.0 & $\mathrm{C}$ & & & & \\
\hline \multirow[t]{2}{*}{11} & $\mathrm{a}$ & 23.5 & $\mathrm{CH}_{2}$ & 127 & 1.50 & $\mathrm{~m}$ & \\
\hline & b & & $\mathrm{CH}_{2}$ & 126 & 1.21 & $\mathrm{~m}$ & \\
\hline \multirow[t]{2}{*}{12} & $\mathrm{a}$ & 39.5 & $\mathrm{CH}_{2}$ & 128 & 1.98 & $\mathrm{~d}$ & 9.4 \\
\hline & b & & & 126 & 1.22 & $\mathrm{~m}$ & \\
\hline 13 & & 44.9 & & & & & \\
\hline 14 & & 51.7 & $\mathrm{CH}$ & 121 & 1.54 & $\mathrm{~m}$ & \\
\hline \multirow[t]{2}{*}{16} & $\mathrm{a}$ & 28.4 & $\mathrm{CH}_{2}$ & 125 & 1.91 & $\mathrm{~m}$ & \\
\hline & $\mathrm{b}$ & & & 127 & 1.36 & $\mathrm{~m}$ & \\
\hline 17 & & 56.5 & $\mathrm{CH}$ & 124 & 1.17 & quin & 8.0 \\
\hline 18 & & 12.8 & $\mathrm{CH}_{3}$ & 122 & 0.80 & $\mathrm{~s}$ & \\
\hline 19 & & 18.3 & $\mathrm{CH}_{3}$ & 126 & 0.88 & $\mathrm{~s}$ & \\
\hline 20 & & 35.4 & $\mathrm{CH}$ & 123 & 1.36 & quin & 2.7 \\
\hline 21 & & 18.7 & $\mathrm{CH}_{3}$ & 124 & 0.89 & $\mathrm{~d}$ & 6.7 \\
\hline \multirow[t]{2}{*}{22} & $\mathrm{a}$ & 36.1 & $\mathrm{CH}_{2}$ & 124 & 1.34 & $\mathrm{t}$ & 12.7 \\
\hline & $\mathrm{b}$ & & $\mathrm{CH}_{2}$ & 124 & 1.00 & d & 6.9 \\
\hline \multirow[t]{2}{*}{23} & $\mathrm{a}$ & 23.9 & $\mathrm{CH}_{2}$ & & 1.32 & $\mathrm{~m}$ & \\
\hline & b & & & & 1.14 & $\mathrm{~m}$ & \\
\hline 24 & & 39.6 & $\mathrm{CH}_{2}$ & & 1.1 & & \\
\hline 25 & & 28.1 & $\mathrm{CH}_{2}$ & 125 & 1.51 & $\mathrm{~m}$ & \\
\hline 26 & & 22.7 & $\mathrm{CH}_{3}$ & 125 & 0.86 & $\mathrm{~d}$ & 6.6 \\
\hline 27 & & 23.0 & $\mathrm{CH}_{3}$ & 124 & 0.87 & d & 6.6 \\
\hline 28 & & 33.2 & $\mathrm{CH}$ & 124 & 1.46 & qd & $11.7,6.1$ \\
\hline
\end{tabular}

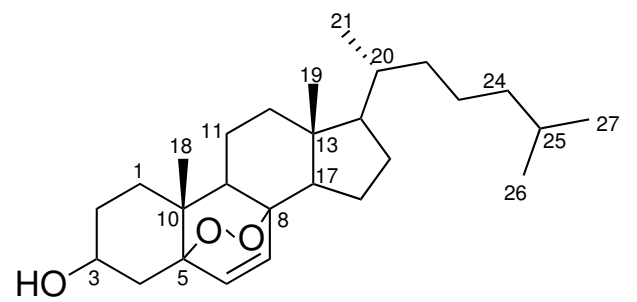

28 
Table C.7 ${ }^{1} \mathrm{H}$ and ${ }^{13} \mathrm{C}$ NMR Data $\left(600 \mathrm{MHz}, \mathrm{CDCl}_{3}\right)$ of 14-Bromohomofascaplysin (29).<smiles>O=C(O)CC1(O)c2ccccc2-[n+]2ccc3c([nH]c4cc(Br)ccc43)c21</smiles>

\begin{tabular}{|c|c|c|c|c|c|c|}
\hline \multirow[b]{2}{*}{ Position } & \multicolumn{3}{|c|}{${ }^{13} \mathrm{C}$} & \multicolumn{3}{|c|}{${ }^{1} \mathrm{H}$} \\
\hline & $\delta_{\mathrm{C}}$ & mult & ${ }^{1} J_{\mathrm{CH}}(\mathrm{Hz})$ & $\delta_{\mathrm{H}}$ & mult & $J(\mathrm{~Hz})$ \\
\hline 1 & 137.21 & $\mathrm{C}$ & & & & \\
\hline 2 & 124.4 & $\mathrm{CH}$ & 165 & 7.90 & $\mathrm{dd}$ & $7.7,0.9$ \\
\hline 3 & 130.6 & $\mathrm{CH}$ & 164 & 7.70 & $\mathrm{td}$ & $7.4,0.7$ \\
\hline 4 & 130.9 & $\mathrm{CH}$ & 165 & 7.8 & d & 7.9 \\
\hline 5 & 113.8 & $\mathrm{CH}$ & 167 & 8.28 & d & 8.5 \\
\hline \multirow{2}{*}{\multicolumn{7}{|c|}{$\begin{array}{c}0 \\
\mathrm{~N}^{+}-1\end{array}$}} \\
\hline & & & & & & \\
\hline 7 & 124.0 & $\mathrm{CH}$ & 192 & 9.38 & d & 8.6 \\
\hline 8 & 117.2 & $\mathrm{CH}$ & 175 & 8.83 & d & 8.6 \\
\hline 9 & 135.0 & $\mathrm{C}$ & & & & \\
\hline 10 & 119.1 & $\mathrm{C}$ & & & & \\
\hline 11 & 124.5 & $\mathrm{CH}$ & 167 & 8.4 & $\mathrm{~d}$ & 8.5 \\
\hline 12 & 125.8 & $\mathrm{CH}$ & 171 & 7.66 & $\mathrm{dd}$ & $8.48,1.66$ \\
\hline 13 & 126.7 & $\mathrm{C}$ & & & & \\
\hline 14 & 115.6 & $\mathrm{CH}$ & 172 & 8.02 & d & 1.37 \\
\hline \multirow{2}{*}{\multicolumn{7}{|c|}{ NH-2 }} \\
\hline & & & & & & \\
\hline 16 & 131.0 & C & & & & \\
\hline 17 & 143.95 & C & & & & \\
\hline 18 & 77.81 & $\mathrm{C}$ & & & & \\
\hline 19 & 50.1 & $\mathrm{CH}_{2}$ & 130 & 4.20 & $\mathrm{dd}$ & $24.1,18.6$ \\
\hline 20 & 205.1 & C & & & & \\
\hline 21 & 28.0 & $\mathrm{CH}_{3}$ & 128 & 1.98 & $\mathrm{~s}$ & \\
\hline
\end{tabular}

Table C.8 ${ }^{1} \mathrm{H}$ and ${ }^{13} \mathrm{C}$ NMR Data $\left(600 \mathrm{MHz}, d_{3}-\mathrm{MeCN}\right)$ of Halenaquinone (31).<smiles>C[C@]12CCC(=O)c3coc(c31)C(=O)c1cc3c(cc12)C(=O)C=CC3=O</smiles>

\begin{tabular}{|c|c|c|c|c|c|c|c|}
\hline \multirow{2}{*}{\multicolumn{2}{|c|}{ Position }} & \multicolumn{3}{|c|}{${ }^{13} \mathrm{C}$} & \multicolumn{3}{|c|}{${ }^{1} \mathrm{H}$} \\
\hline & & $\delta_{\mathrm{C}}$ & mult & ${ }^{1} J_{\mathrm{CH}}(\mathrm{Hz})$ & $\delta_{\mathrm{H}}$ & mult & $J(\mathrm{~Hz})$ \\
\hline 1 & & 149.7 & $\mathrm{CH}$ & 211 & 8.37 & $\mathrm{~s}$ & \\
\hline 2 & & 122.7 & $\mathrm{C}$ & & & & \\
\hline 3 & & 191.5 & $\mathrm{C}$ & & & & \\
\hline \multirow[t]{2}{*}{4} & $\mathrm{a}$ & 36.2 & $\mathrm{CH}_{2}$ & 125 & 3.05 & ddd & $18.7,13.3,5.4$ \\
\hline & $\mathrm{b}$ & & & 132 & 2.72 & ddd & $18.6,4.7,1.9$ \\
\hline \multirow[t]{2}{*}{5} & b & 32.7 & $\mathrm{CH}_{2}$ & 134 & 2.89 & ddd & $13.1,5.4,1.9$ \\
\hline & $\mathrm{b}$ & & & 131 & 2.25 & $\mathrm{td}$ & $13.2,4.8$ \\
\hline 6 & & 36.9 & $\mathrm{C}$ & & & & \\
\hline 7 & & 148.4 & $\mathrm{C}$ & & & & \\
\hline 8 & & 144.7 & $\mathrm{C}$ & & & & \\
\hline 9 & & 170.5 & $\mathrm{C}$ & & & & \\
\hline 10 & & 137.0 & $\mathrm{C}$ & & & & \\
\hline 11 & & 125.6 & $\mathrm{CH}$ & 167 & 9.08 & $\mathrm{~s}$ & \\
\hline 12 & & 130.7 & $\mathrm{C}$ & & & & \\
\hline 13 & & 184.1 & $\mathrm{C}$ & & & & \\
\hline 14 & & 139.0 & $\mathrm{CH}$ & 170 & 7.06 & d & 11.4 \\
\hline 15 & & 139.6 & $\mathrm{CH}$ & 170 & 7.08 & d & 11.1 \\
\hline 16 & & 184.5 & $\mathrm{C}$ & & & & \\
\hline 17 & & 134.0 & $\mathrm{C}$ & & & & \\
\hline 18 & & 123.8 & $\mathrm{CH}$ & 163 & 8.30 & $\mathrm{~s}$ & \\
\hline 19 & & 154.7 & $\mathrm{C}$ & & & & \\
\hline 20 & & 29.4 & $\mathrm{CH}_{3}$ & 130 & 1.66 & $\mathrm{~s}$ & \\
\hline
\end{tabular}




\section{Appendix D \\ ${ }^{1} \mathrm{H}$ NMR Spectra of Known \\ Compounds}




\section{iso-Secothorectolide}

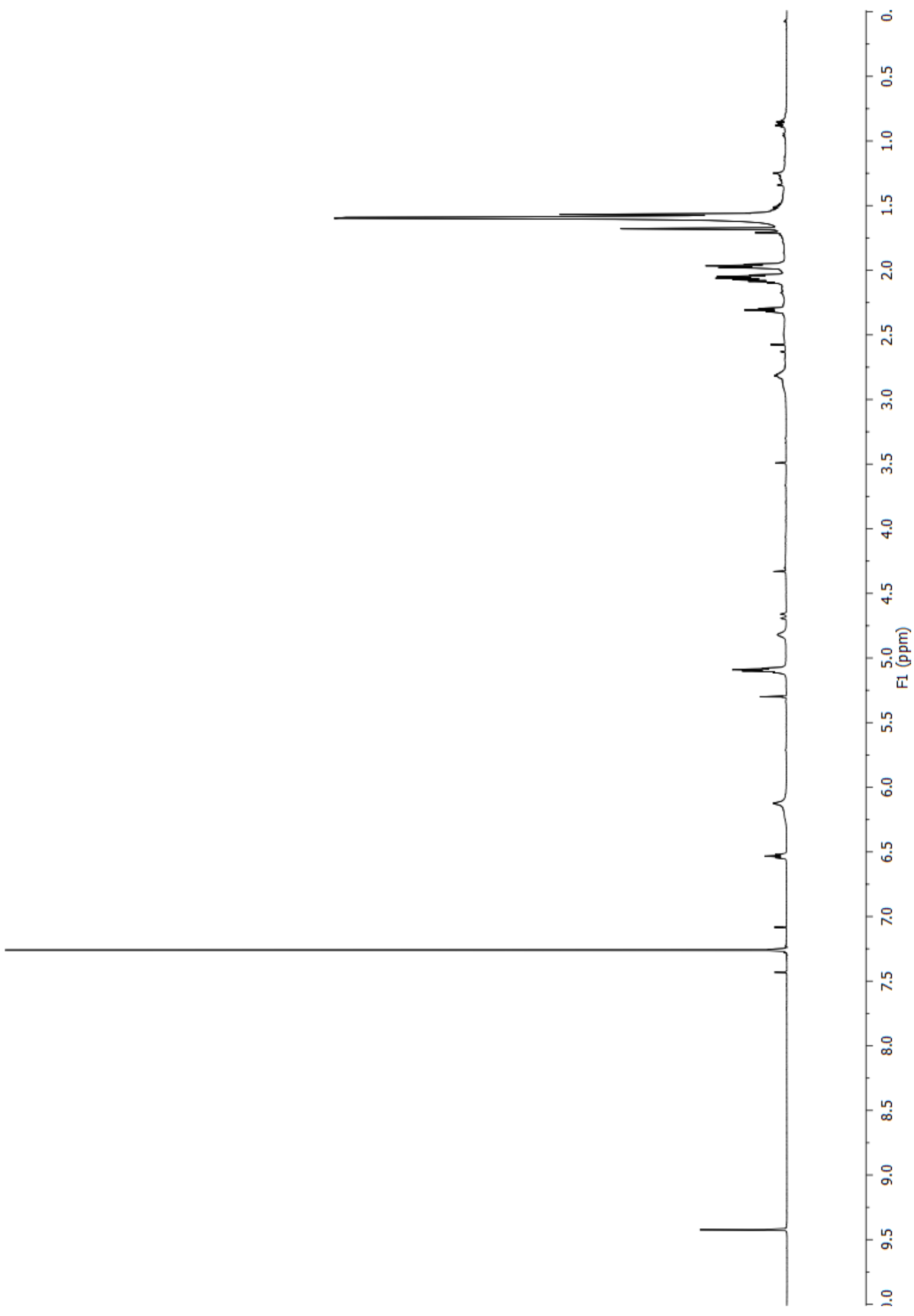

${ }^{1} \mathrm{H}$ NMR spectrum of iso-secothorectolide (23) (600 $\left.\mathrm{MHz}, \mathrm{CDCl}_{3}\right)$. 


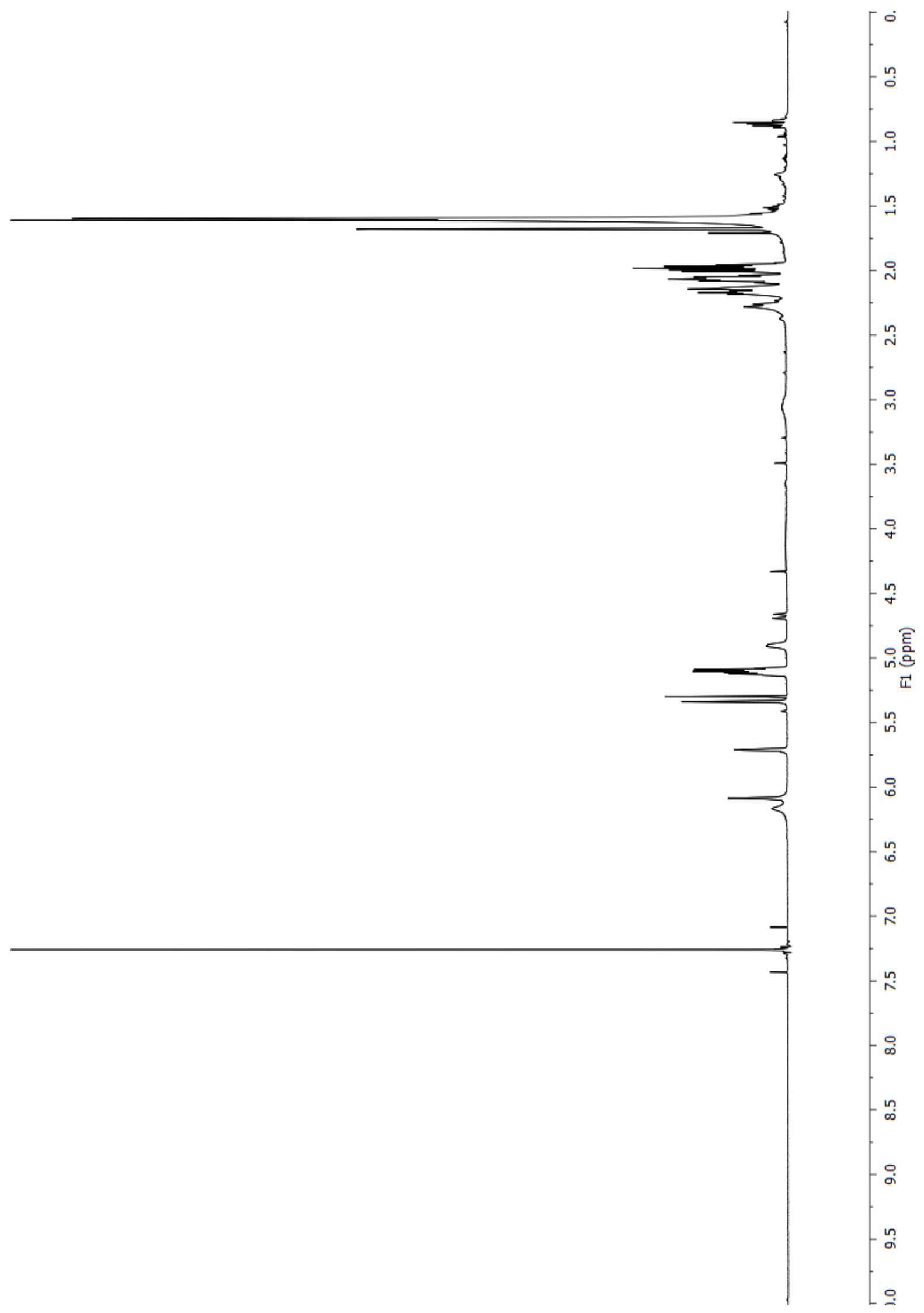

${ }^{1} \mathrm{H}$ NMR spectrum of 1-hydroxyluffarin Q (24) (600 MHz, $\mathrm{CDCl}_{3}$ ). 
Ambliol B

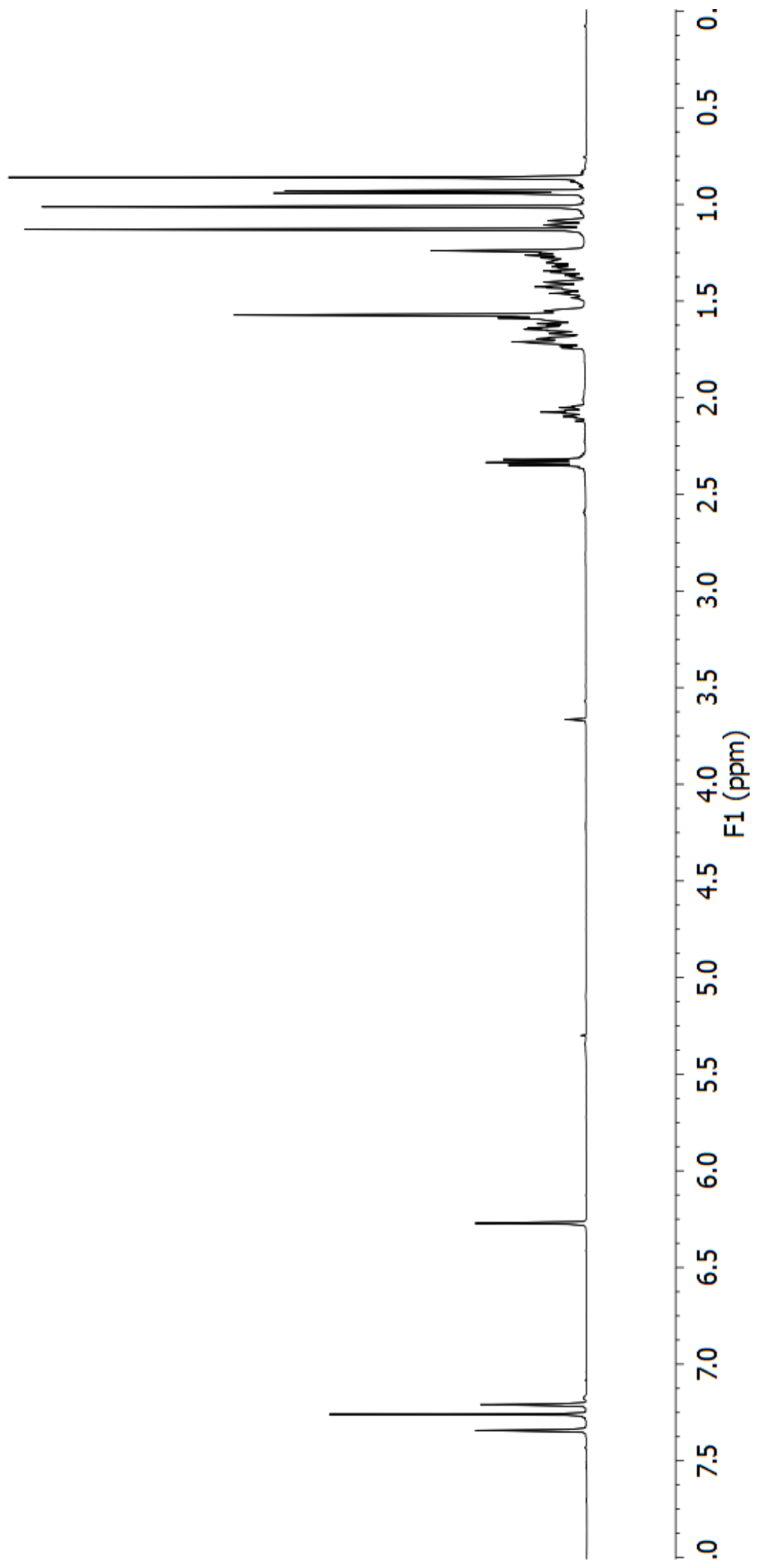

${ }^{1} \mathrm{H}$ NMR spectrum of ambliol B (25) (600 $\mathrm{MHz}, \mathrm{CDCl}_{3}$ ). 


\section{Luffarielolide}

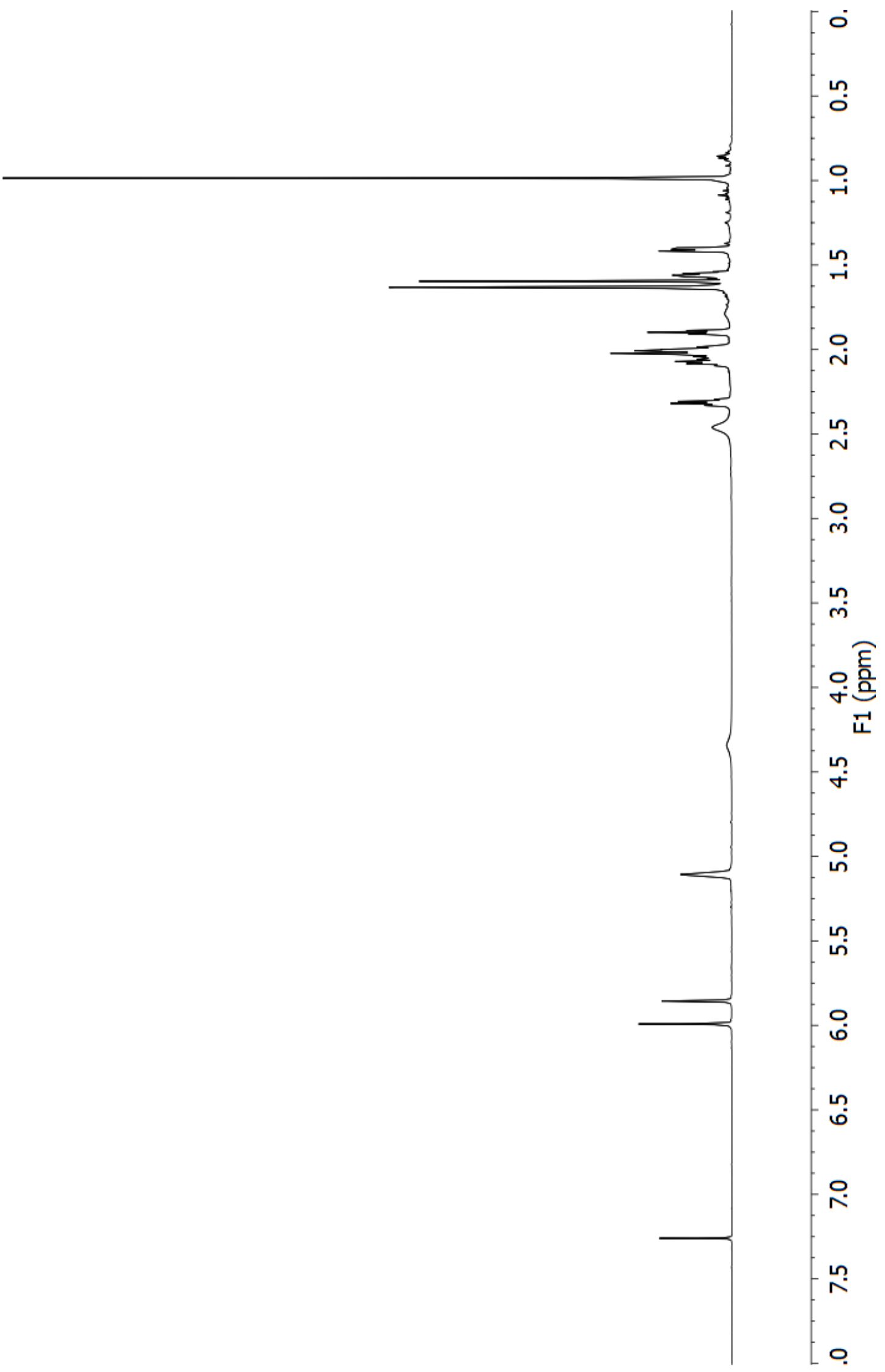

${ }^{1} \mathrm{H}$ NMR spectrum of luffarielolide (26) (600 $\left.\mathrm{MHz}, \mathrm{CDCl}_{3}\right)$. 


\section{4-Bromohomofascaplysin}

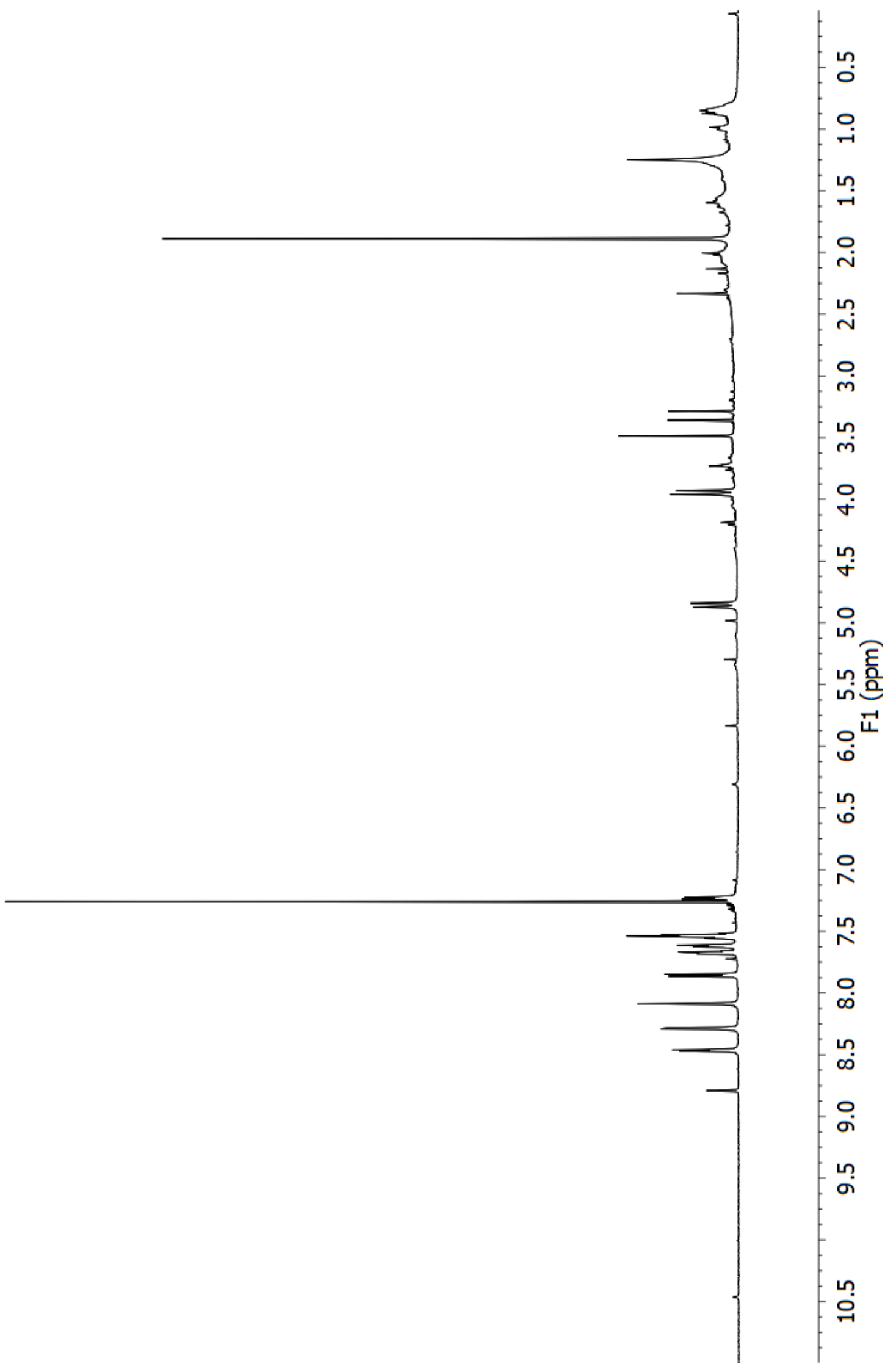

${ }^{1} \mathrm{H}$ NMR spectrum of 14-bromohomofascaplysin (29) (600 MHz, $\mathrm{CDCl}_{3}$ ). 
$5 \alpha, 8 \alpha$-Epidioxysterol 27

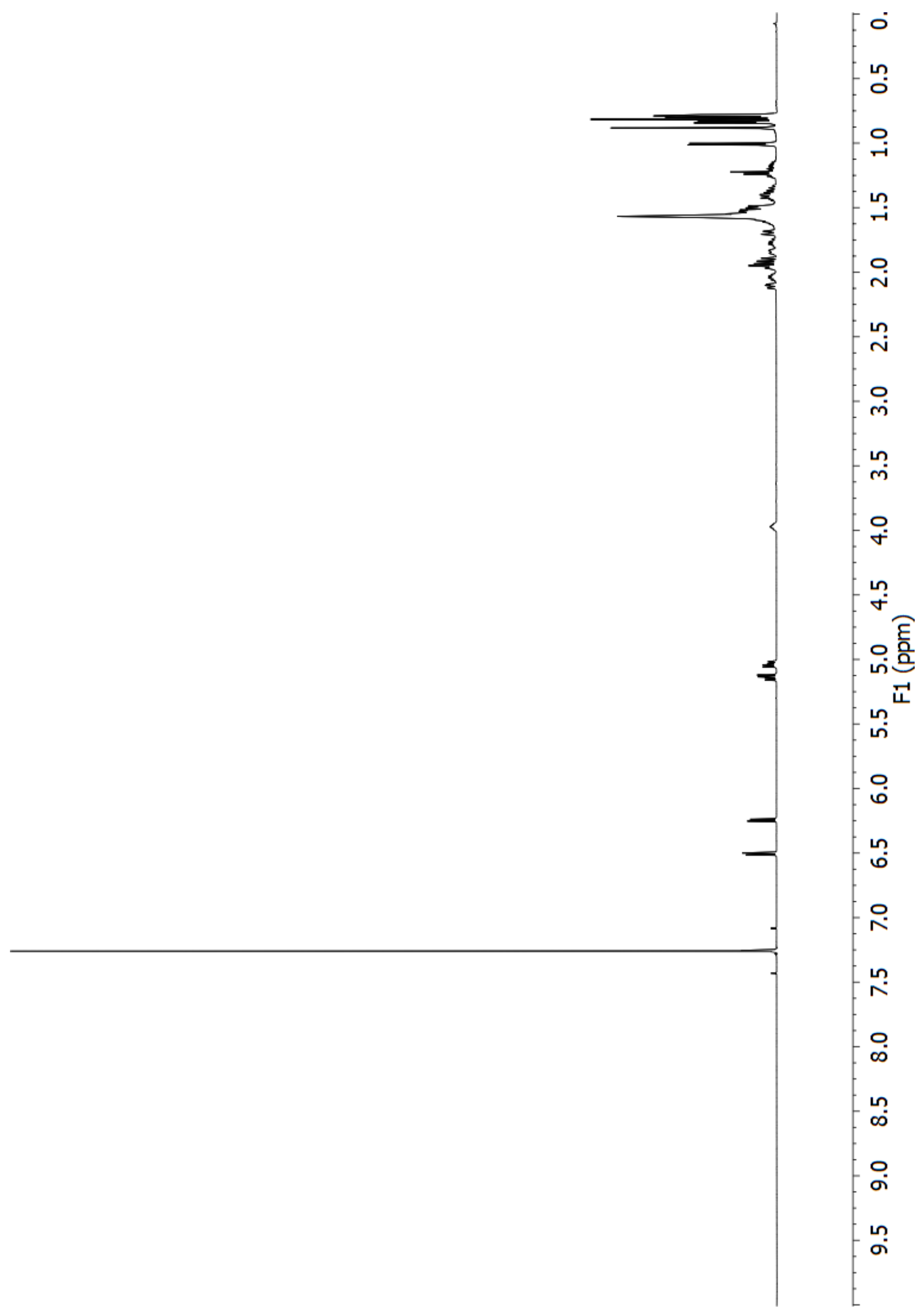

${ }^{1} \mathrm{H}$ NMR spectrum of $5 \alpha, 8 \alpha$-epidioxysterol 27 (600 $\mathrm{MHz}, \mathrm{CDCl}_{3}$ ). 
$5 \alpha, 8 \alpha$-Epidioxysterol 28

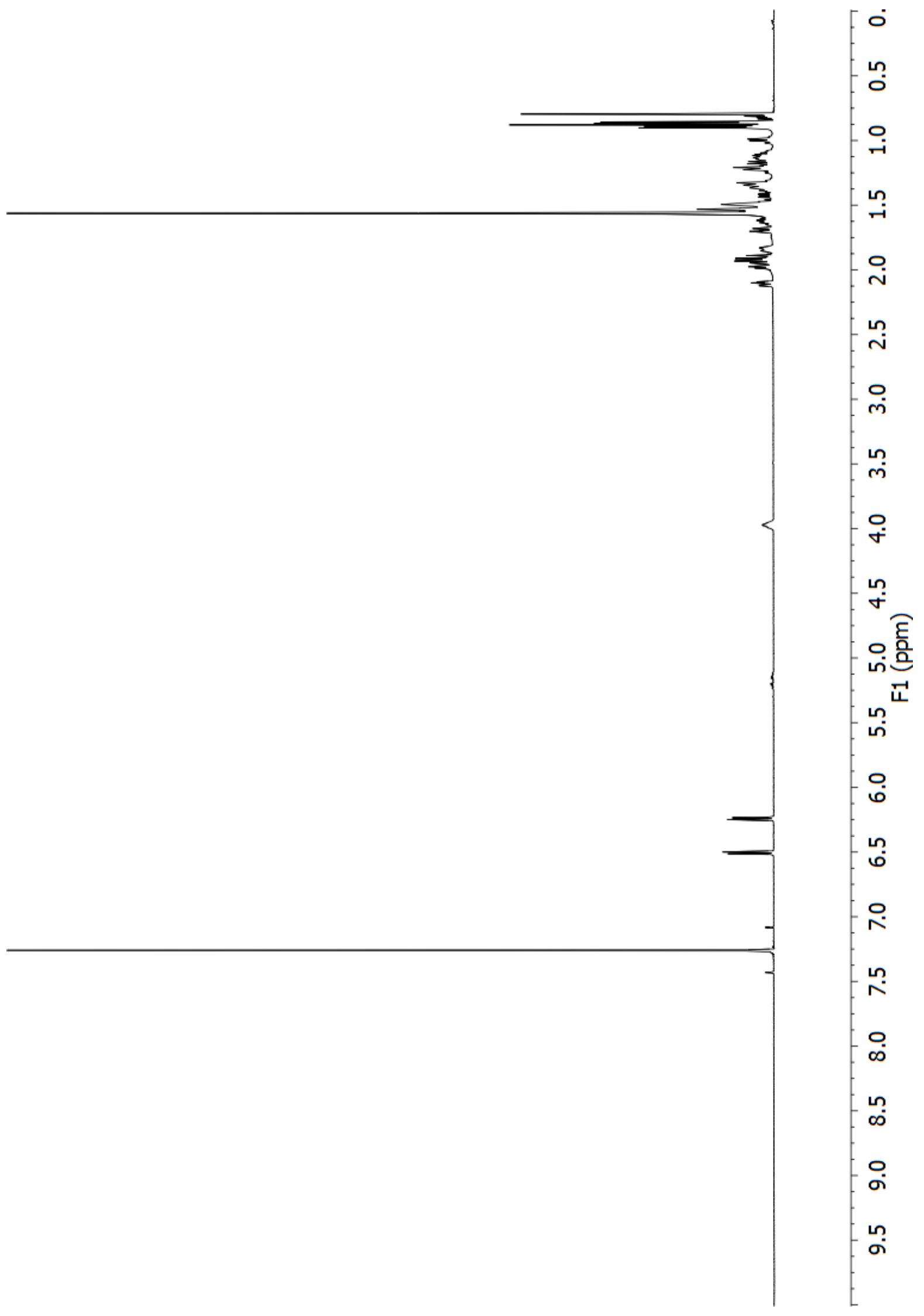

${ }^{1} \mathrm{H}$ NMR spectrum of $5 \alpha, 8 \alpha$-epidioxysterol $28\left(600 \mathrm{MHz}, \mathrm{CDCl}_{3}\right)$. 


\section{Halenaquinone}

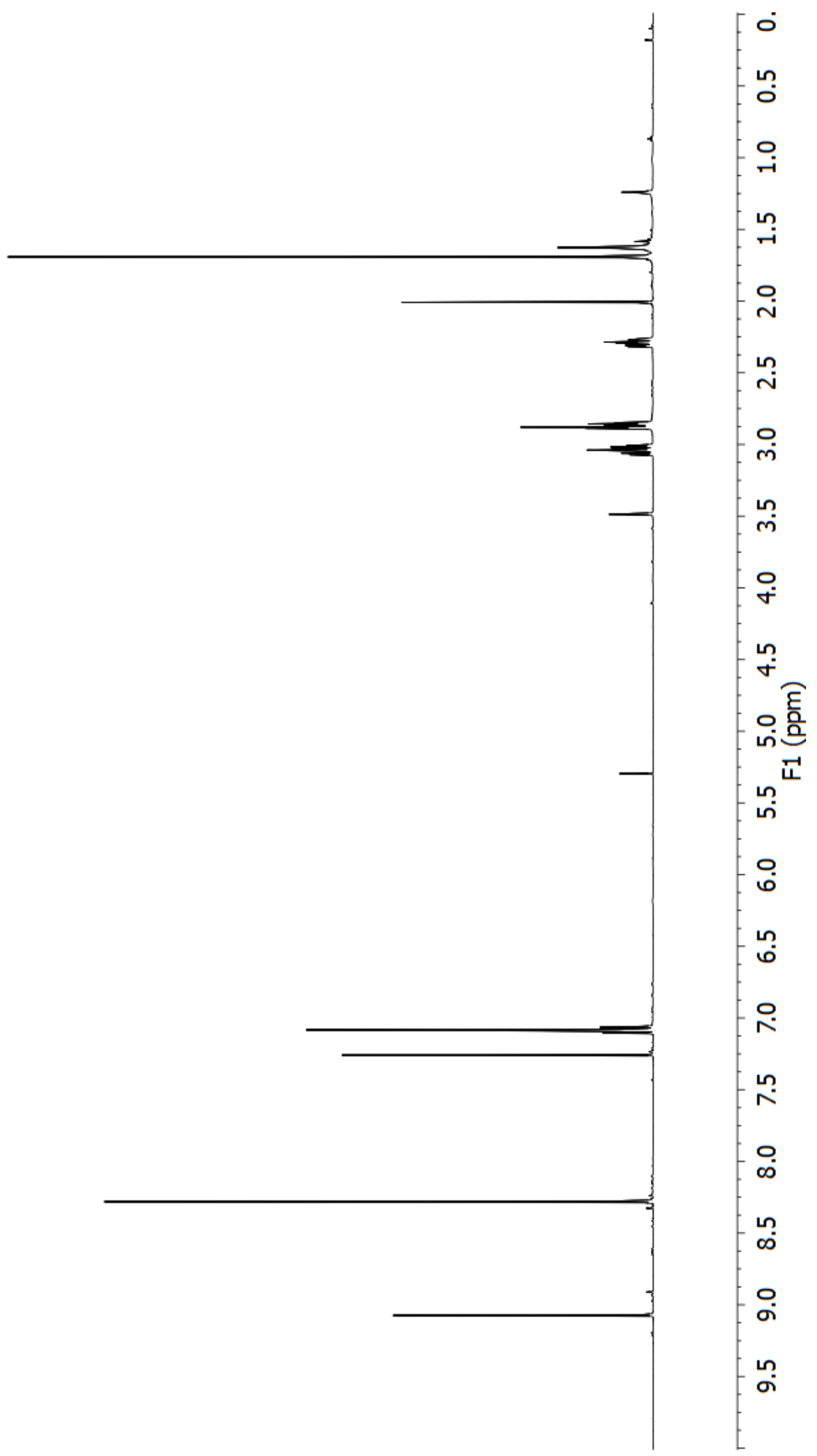

${ }^{1} \mathrm{H}$ NMR spectrum of halenaquinone (31) (600 $\left.\mathrm{MHz}, \mathrm{CDCl}_{3}\right)$. 
Appendix E

NMR Spectra of Luakuliides A-C 
Luakuliide A

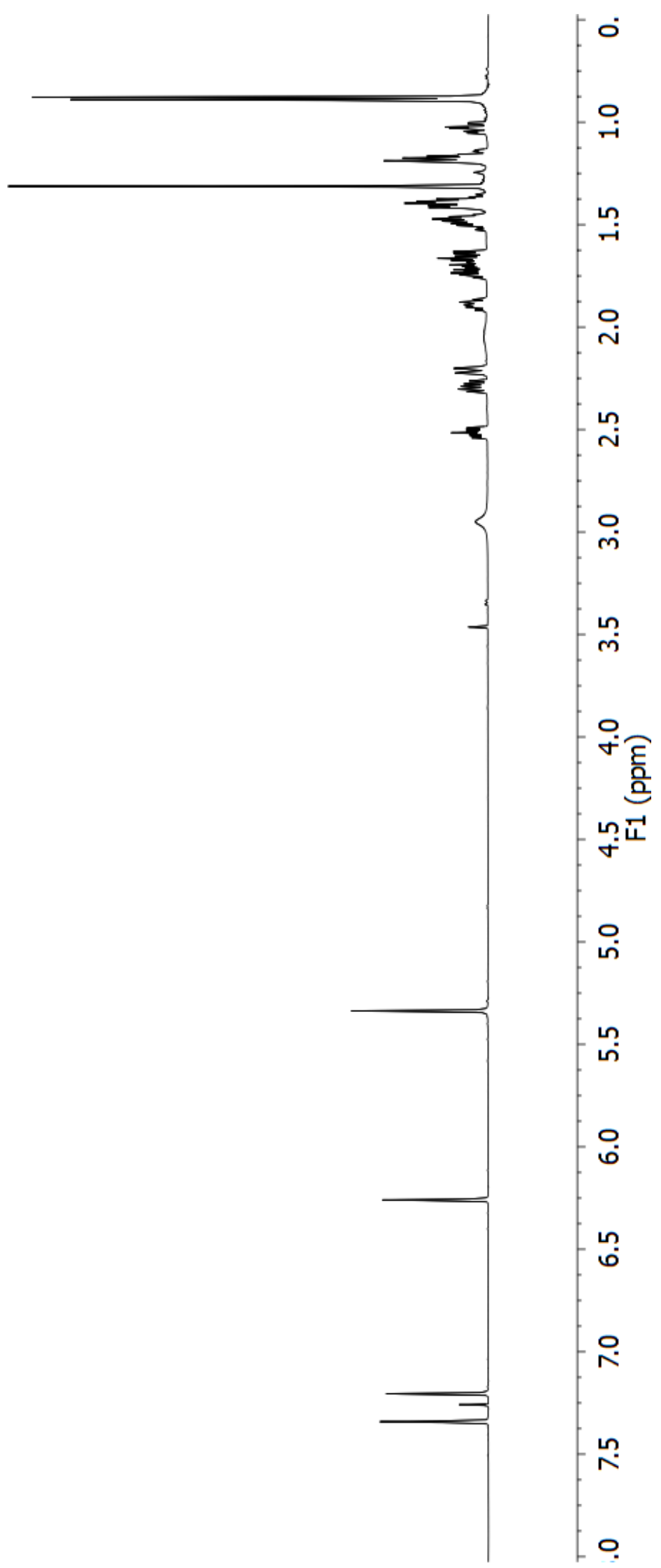

${ }^{1} \mathrm{H}$ NMR spectrum of luakuliide A (33) $\left(600 \mathrm{MHz}^{\mathrm{CDCl}}{ }_{3}\right)$. 


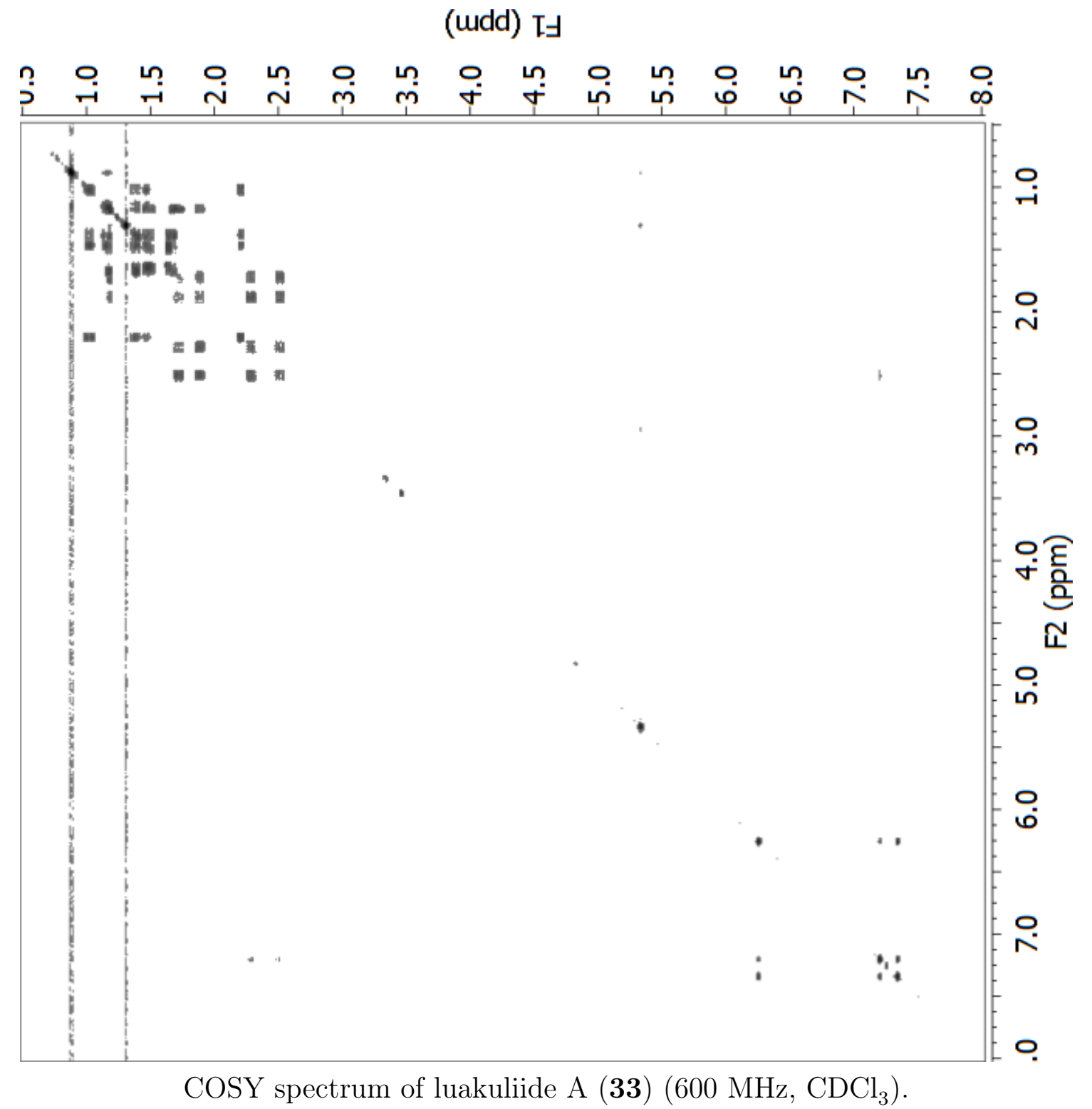




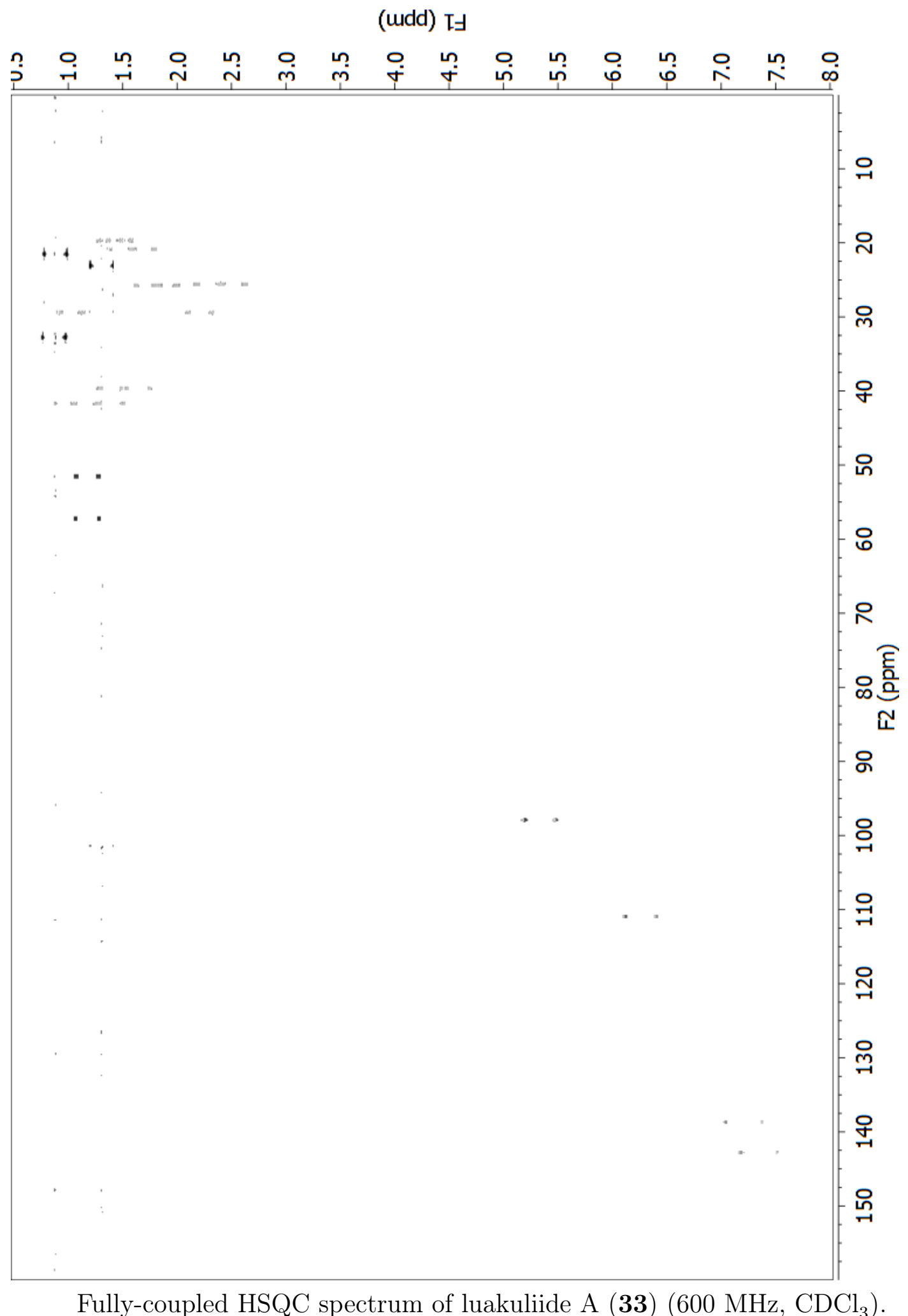


(udd) 더

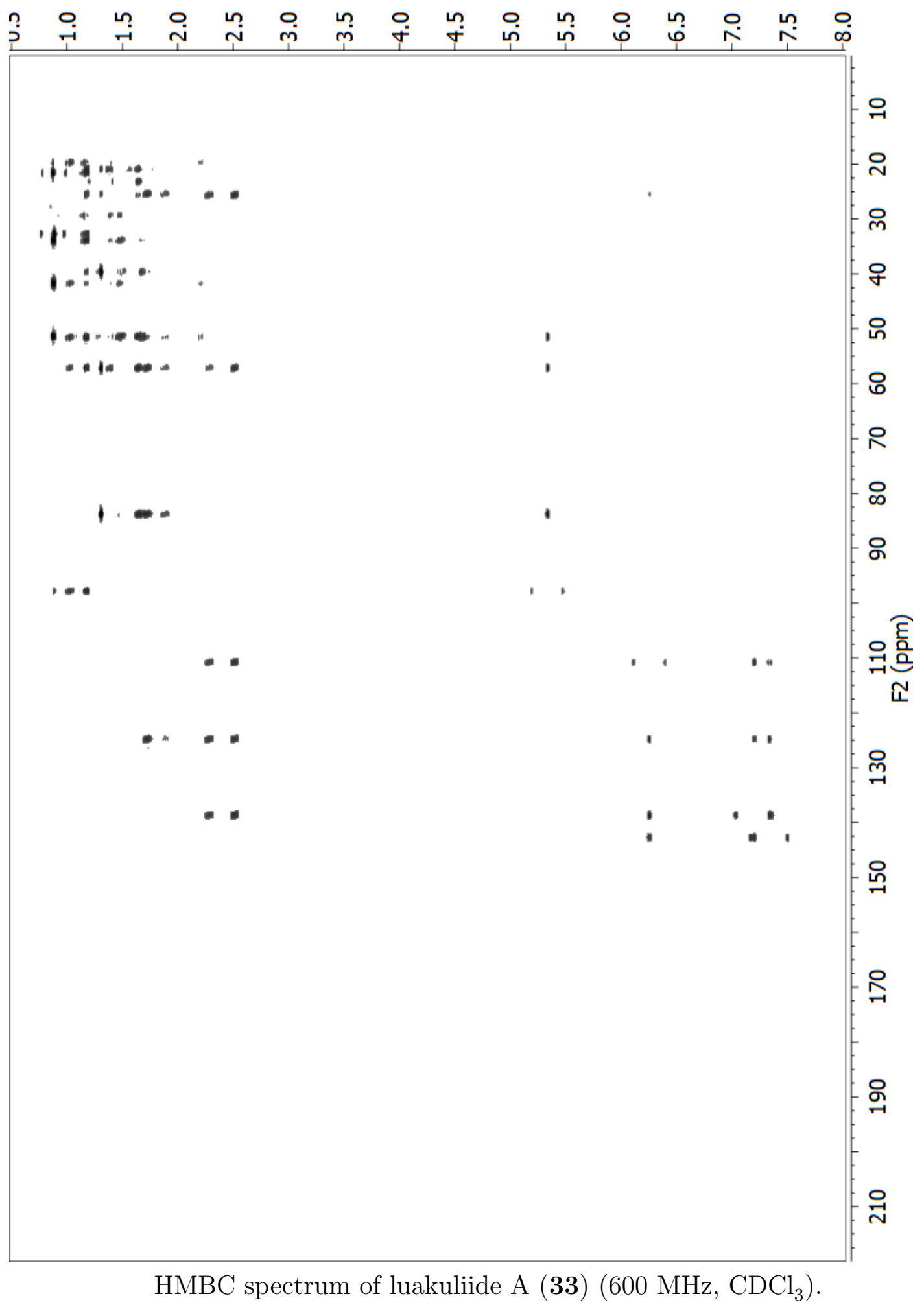




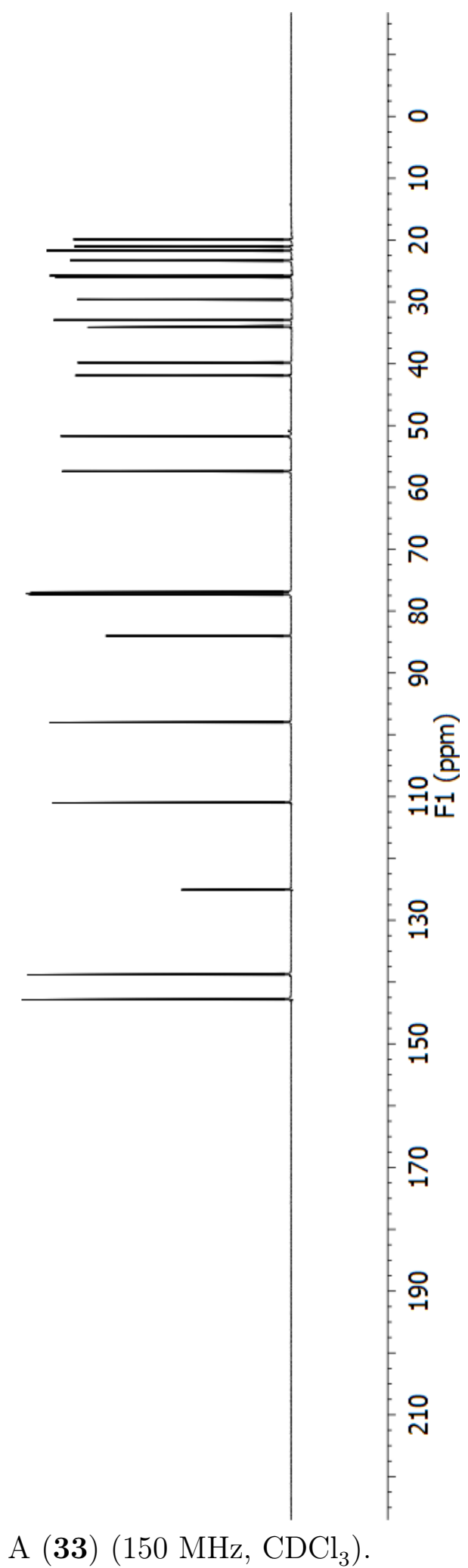

${ }^{13} \mathrm{C}$ NMR spectrum of luakuliide A (33) $\left(150 \mathrm{MHz}, \mathrm{CDCl}_{3}\right)$. 


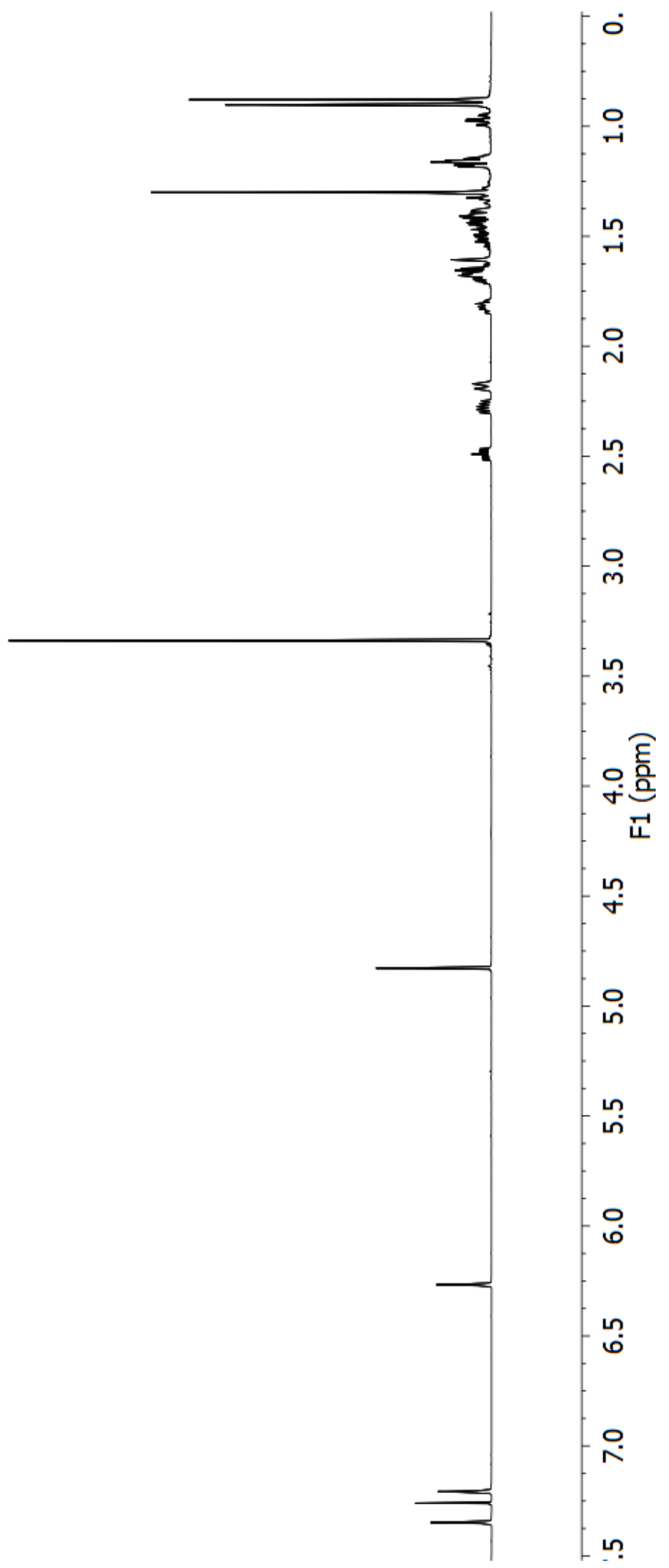

${ }^{1} \mathrm{H}$ NMR spectrum of luakuliide A methyl acetal (34) $\left(600 \mathrm{MHz}, \mathrm{CDCl}_{3}\right)$. 
(udd) 더

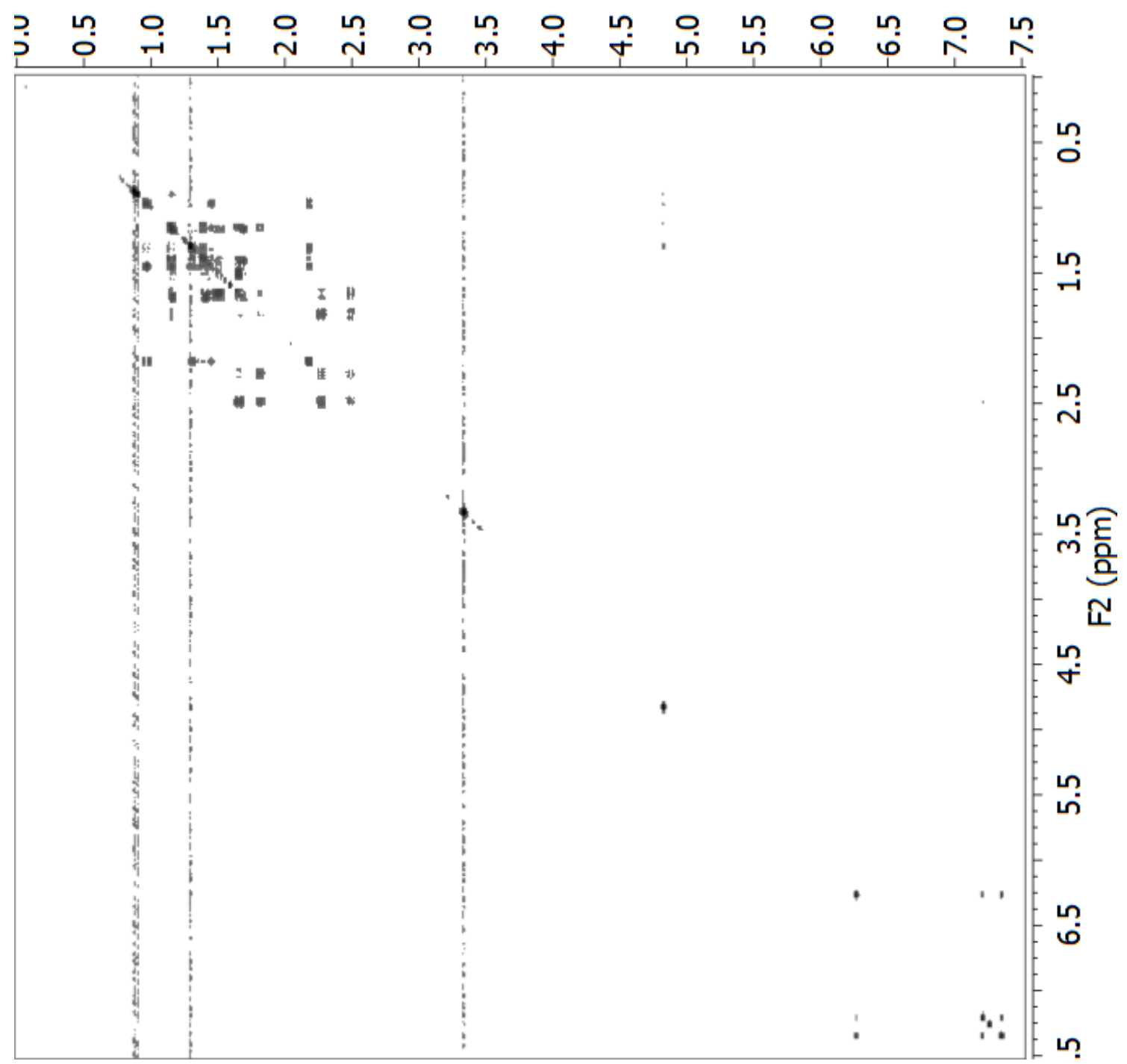

COSY spectrum of luakuliide A methyl acetal (34) $\left(600 \mathrm{MHz}, \mathrm{CDCl}_{3}\right)$. 
(udd) 더

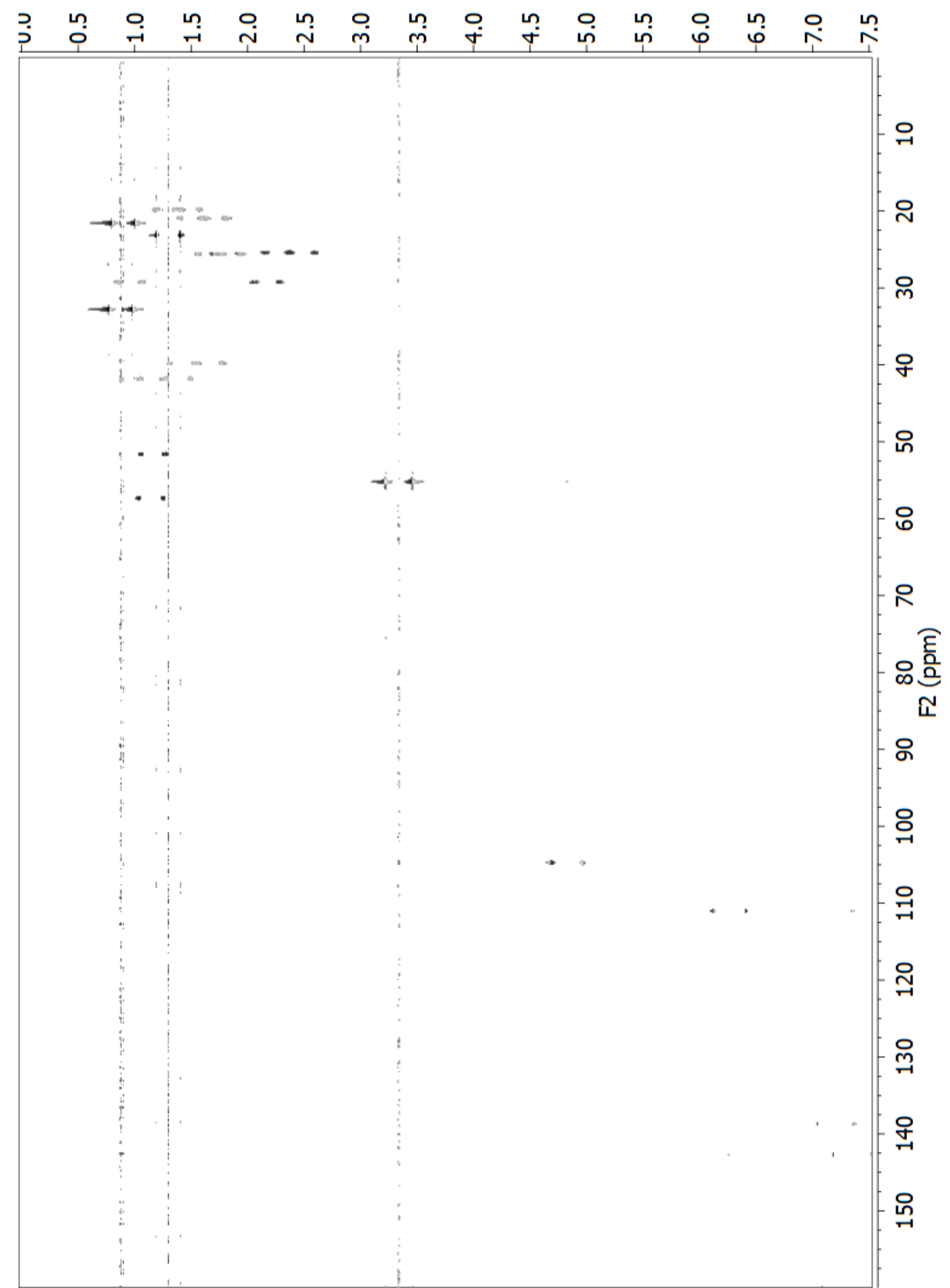

Fully-coupled HSQC spectrum of luakuliide A methyl acetal (34) $\left(600 \mathrm{MHz}, \mathrm{CDCl}_{3}\right)$. 


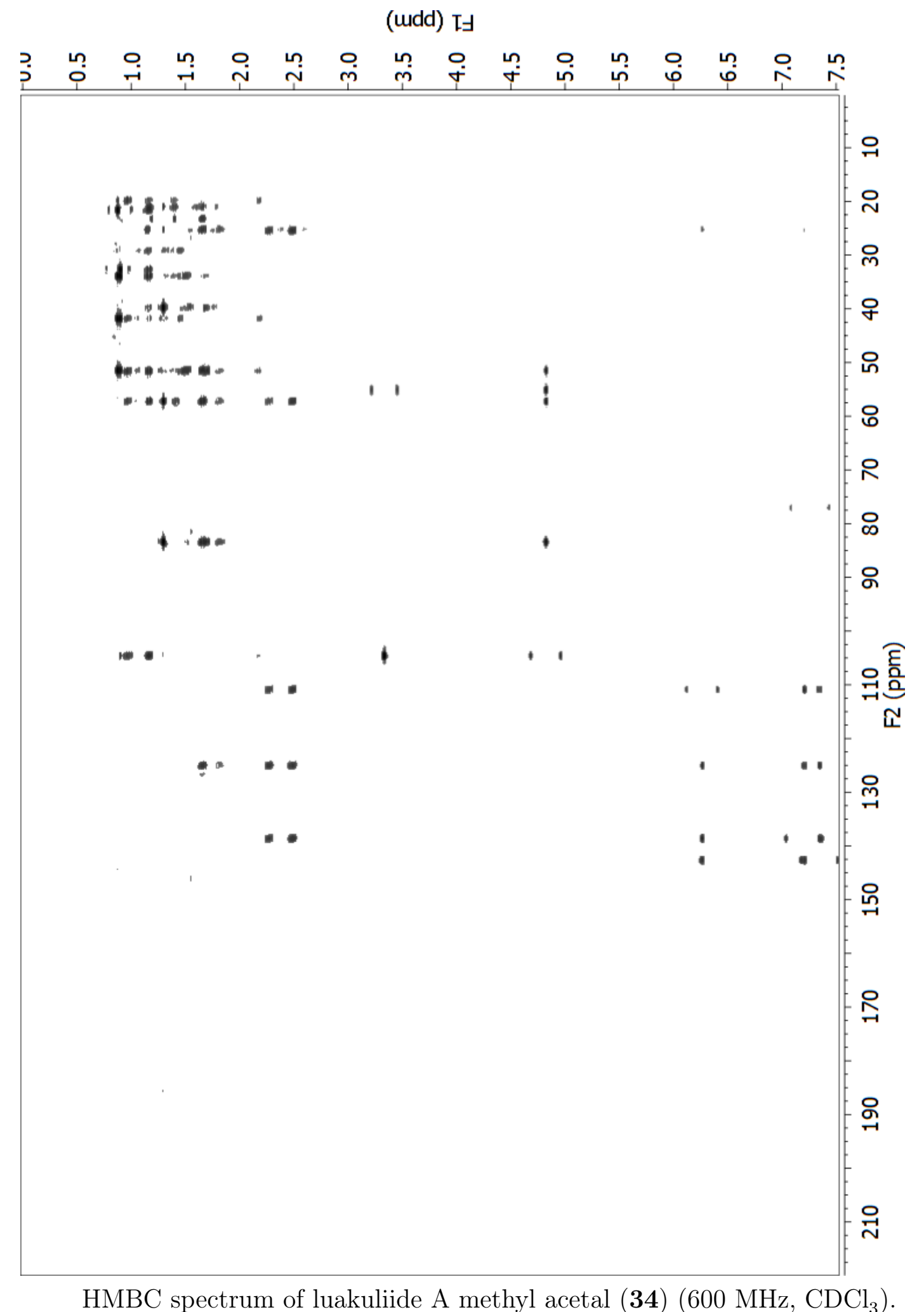




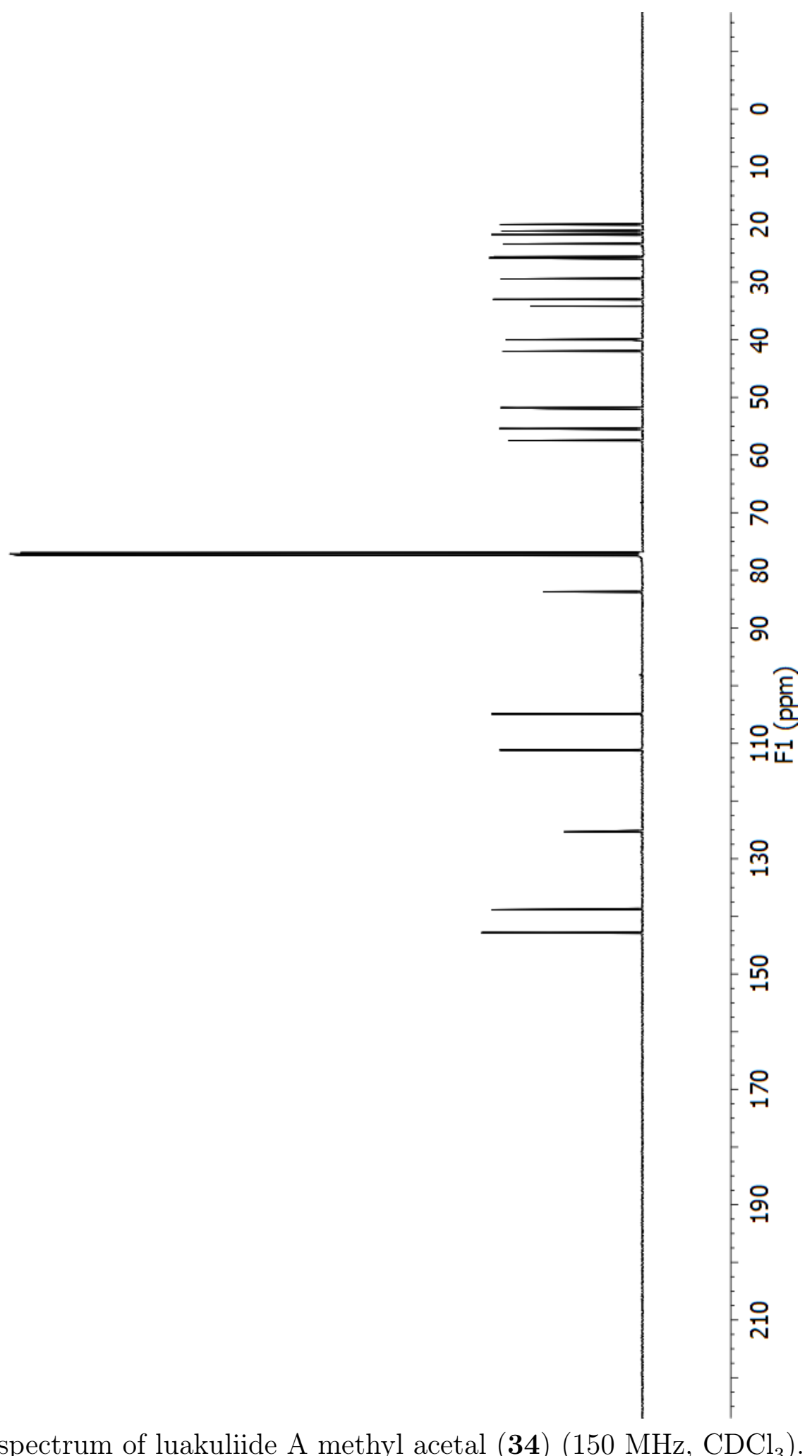

${ }^{13} \mathrm{C}$ NMR spectrum of luakuliide A methyl acetal $(34)\left(150 \mathrm{MHz}, \mathrm{CDCl}_{3}\right)$. 


\section{Luakuliide B}

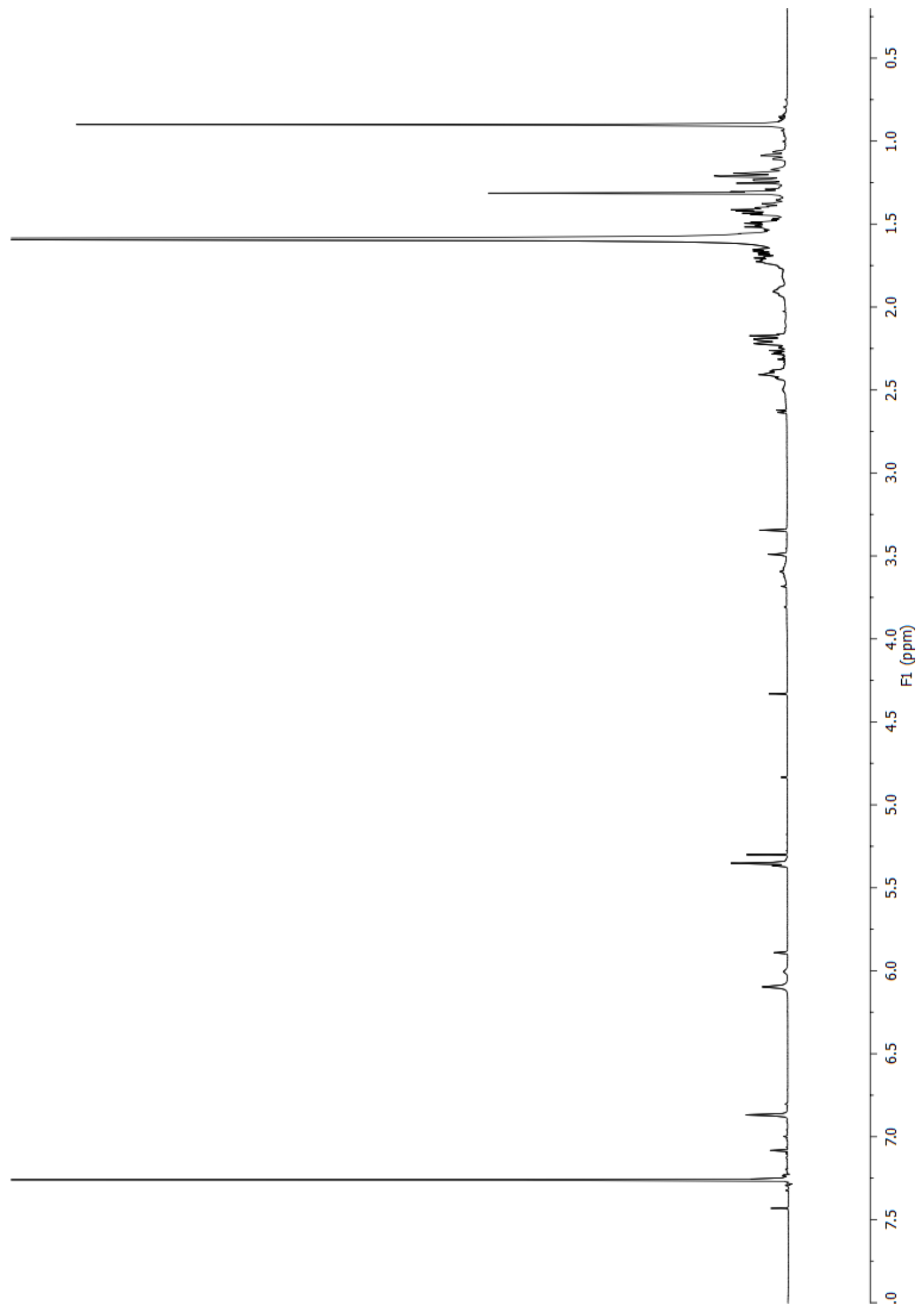

${ }^{1} \mathrm{H}$ NMR spectrum of luakuliide B (35) (600 MHz, $\mathrm{CDCl}_{3}$ ). 


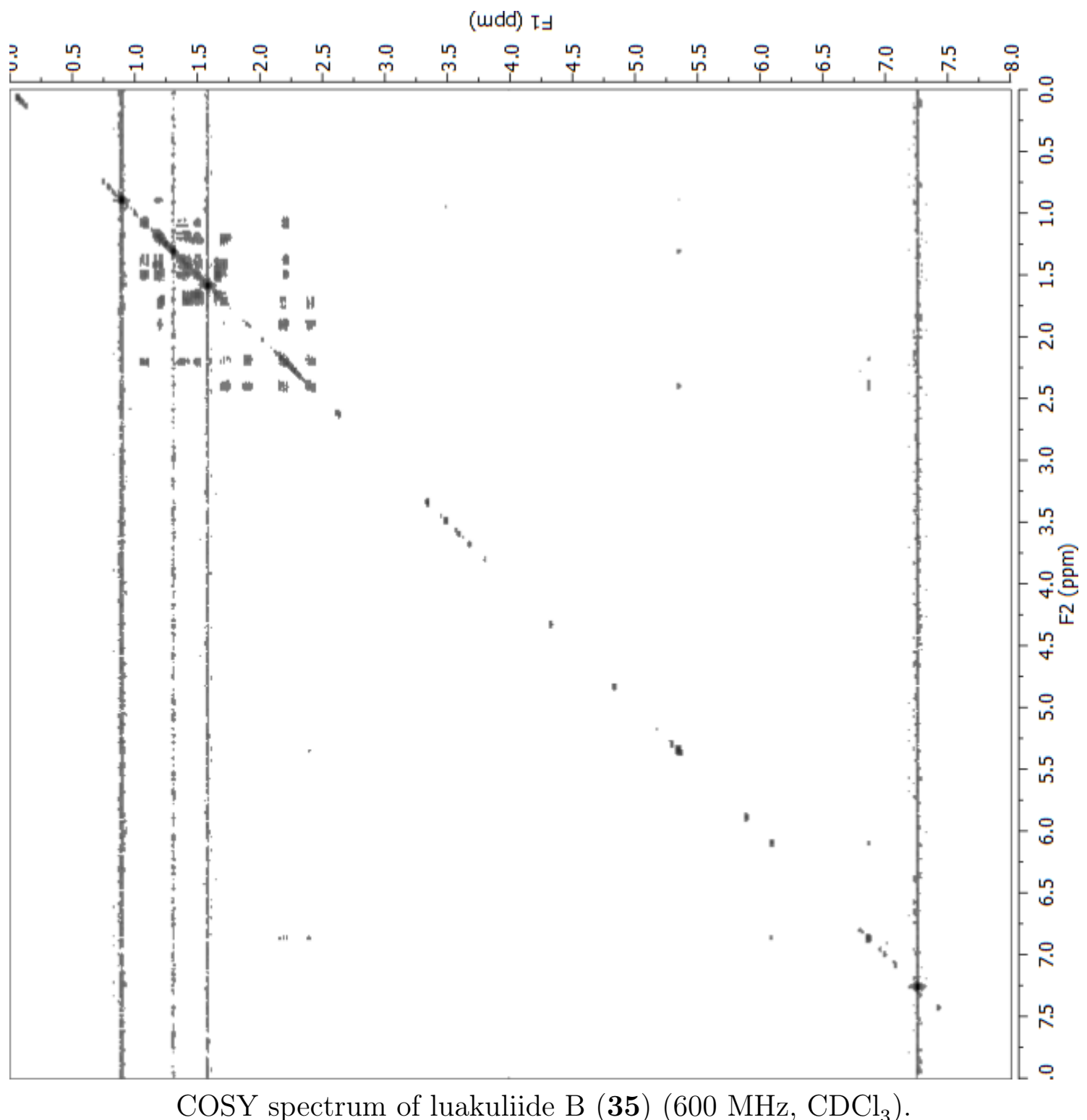

229 


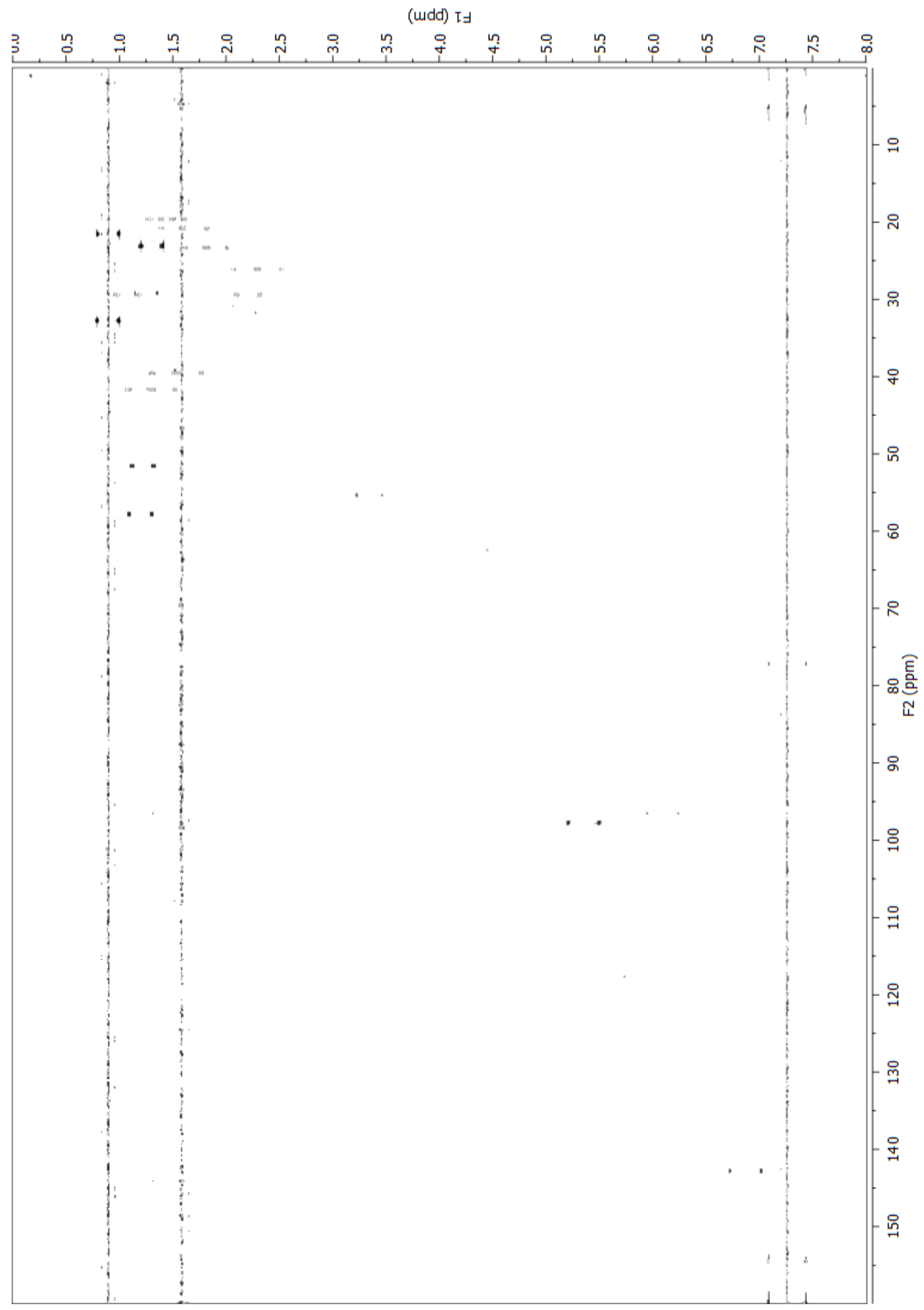

Fully-coupled HSQC spectrum of luakuliide B (35) (600 MHz, $\mathrm{CDCl}_{3}$ ). 


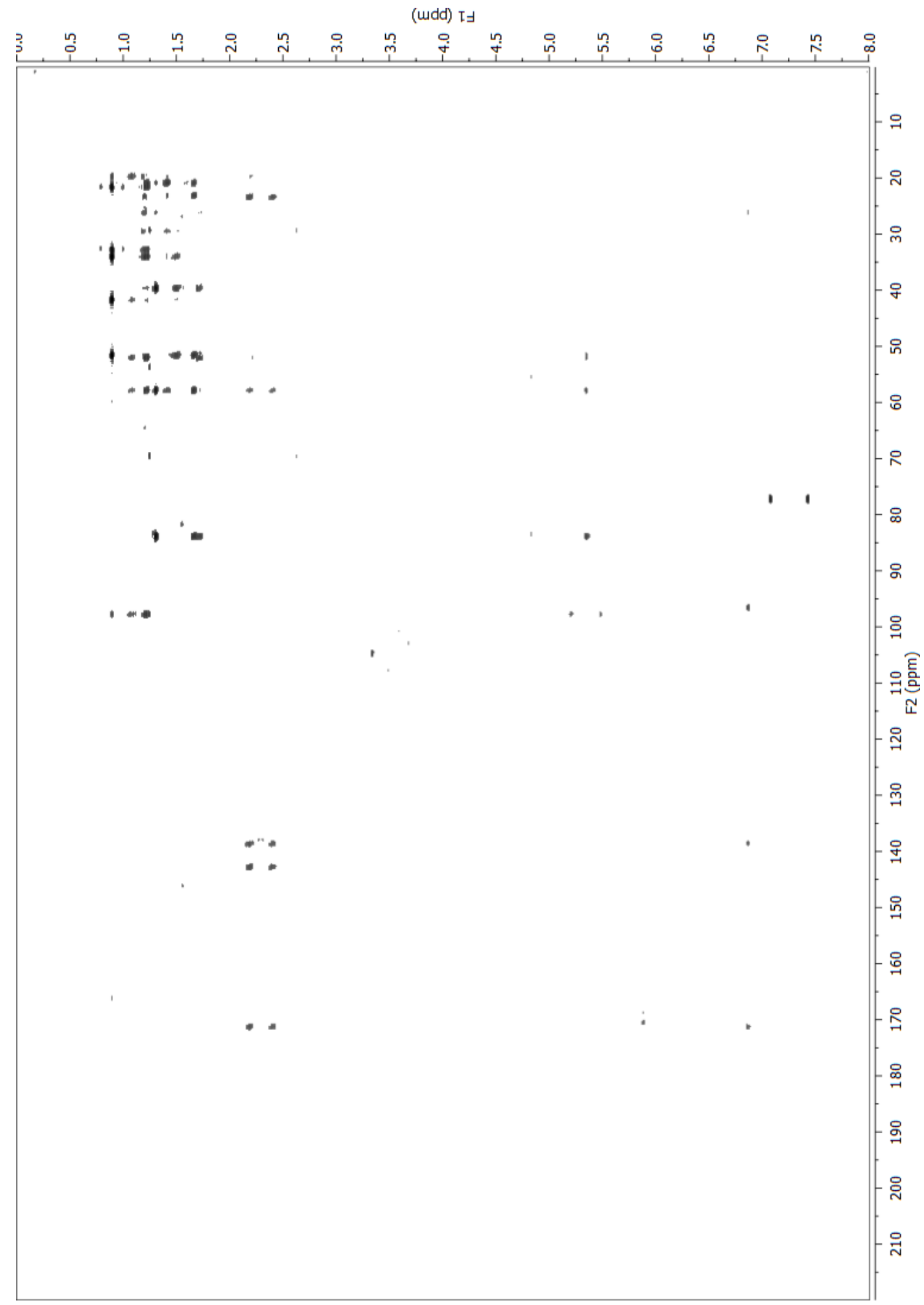

HMBC spectrum of luakuliide B (35) (600 MHz, $\left.\mathrm{CDCl}_{3}\right)$. 


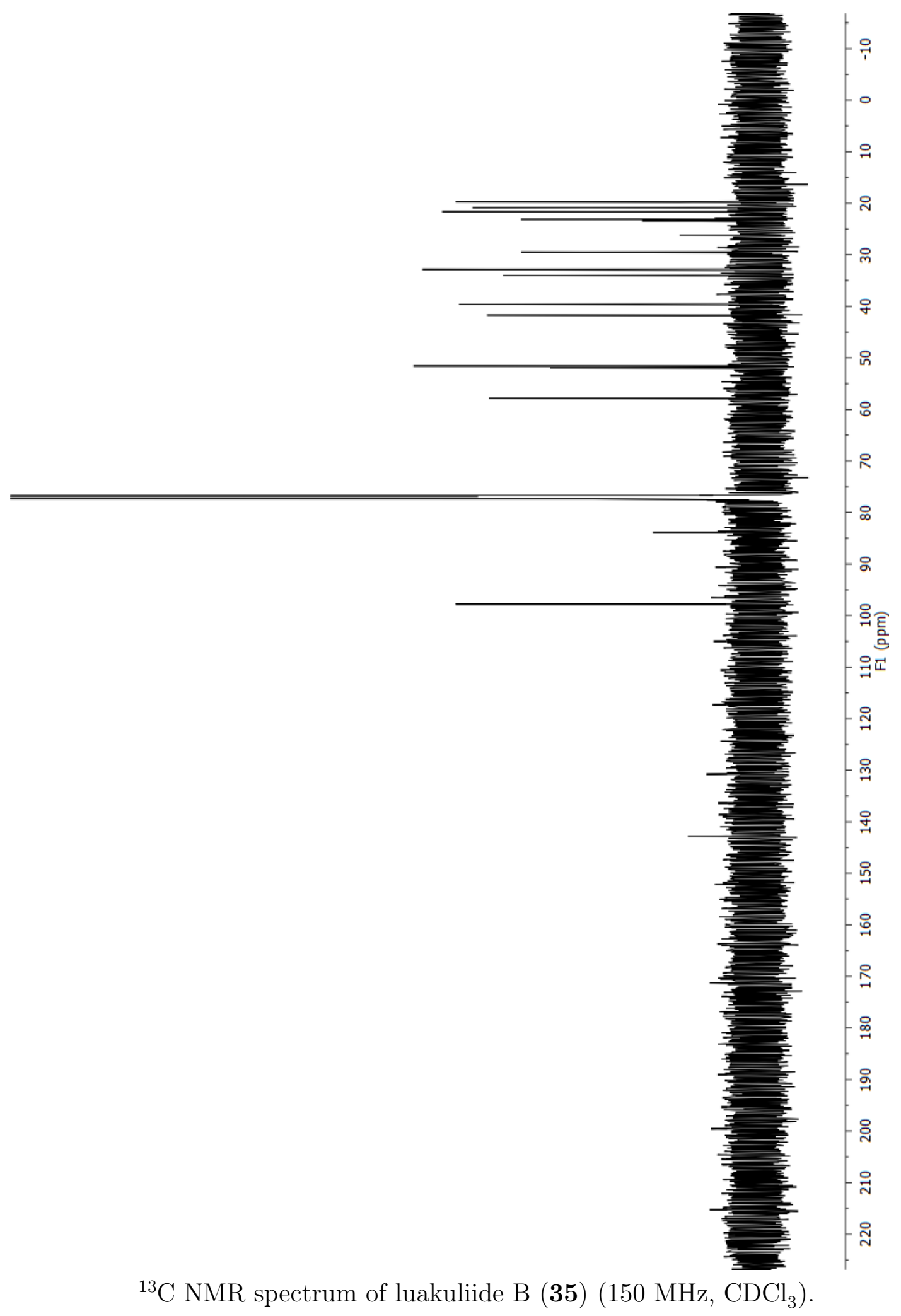




\section{Luakuliide B}

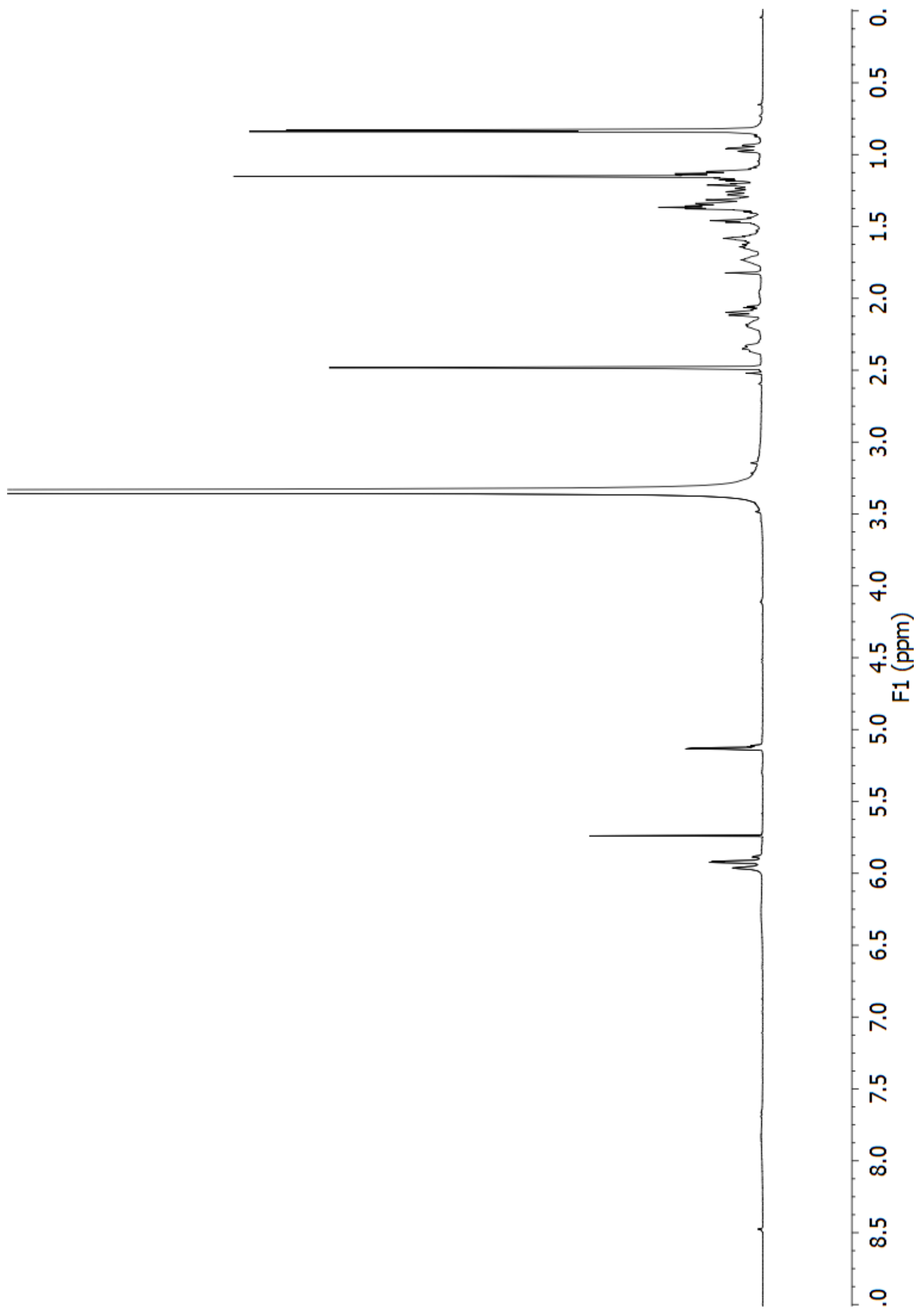

${ }^{1} \mathrm{H}$ NMR spectrum of luakuliide B (35) (600 MHz, DMSO) 


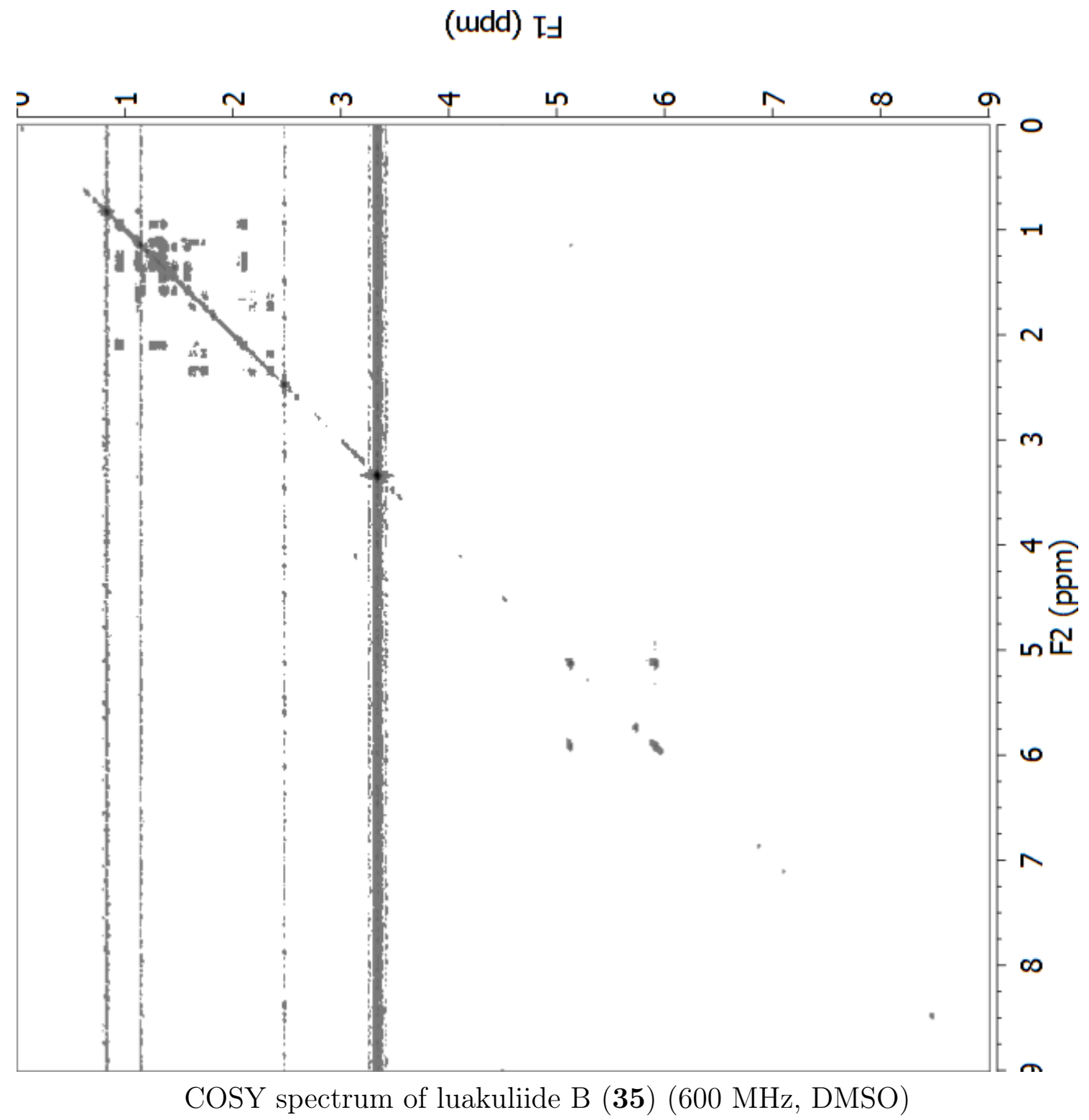




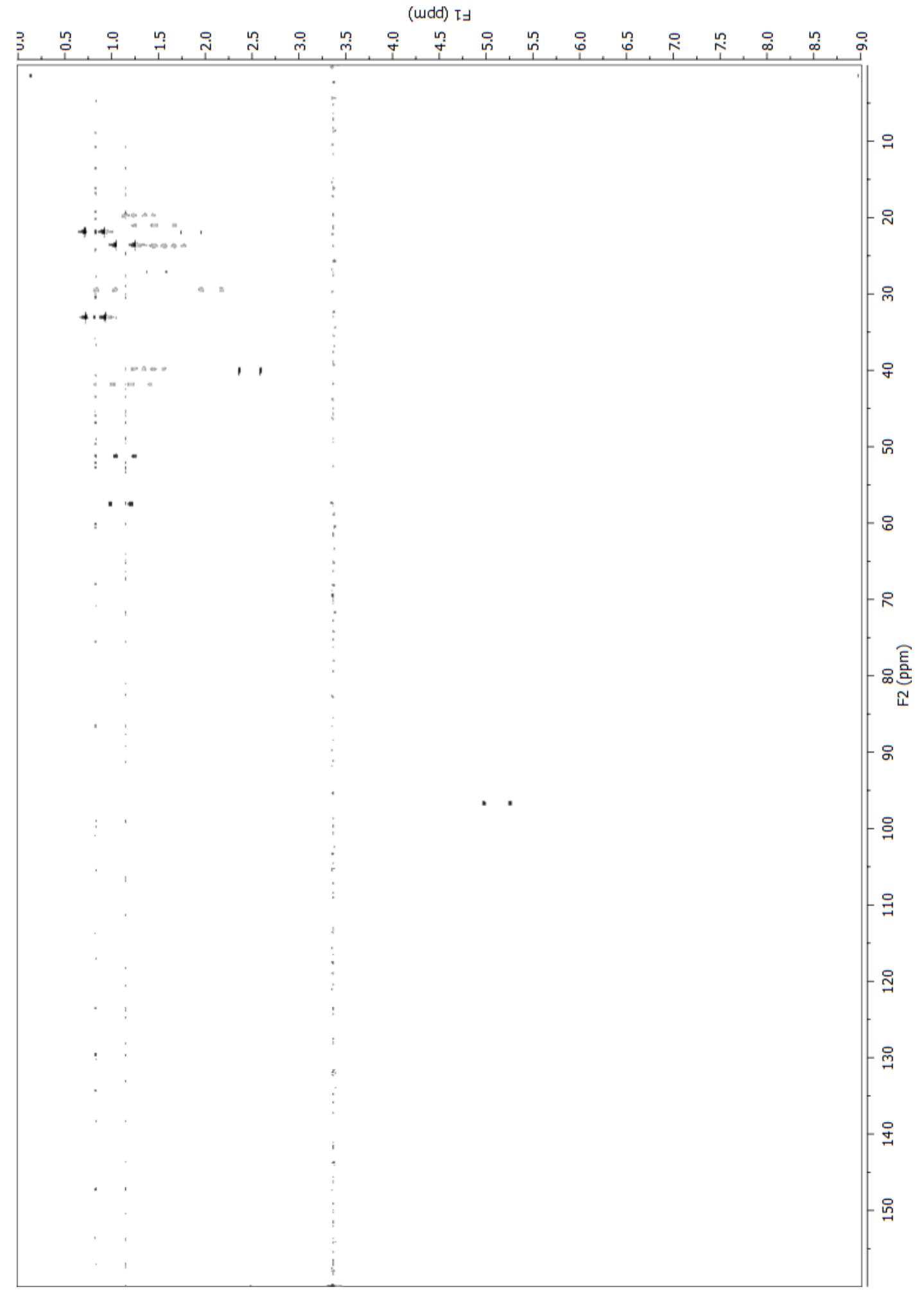

Fully-coupled HSQC spectrum of luakuliide B (35) (600 MHz, DMSO) 


$$
\text { (udd) 더 }
$$

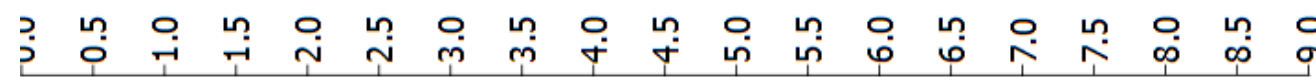

$$
\text { ii, }
$$$$
\text { (1) }
$$

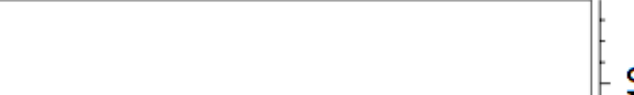

으

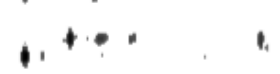

- 으

으

- 우
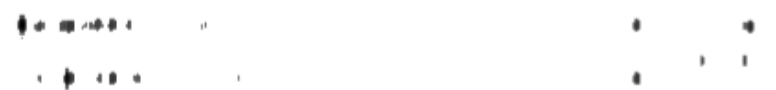

으

8

ㅇ

ஃ

오

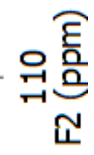

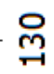

육

$\stackrel{4}{\circ}$

윽

은

HMBC spectrum of luakuliide B (35) (600 MHz, DMSO) 


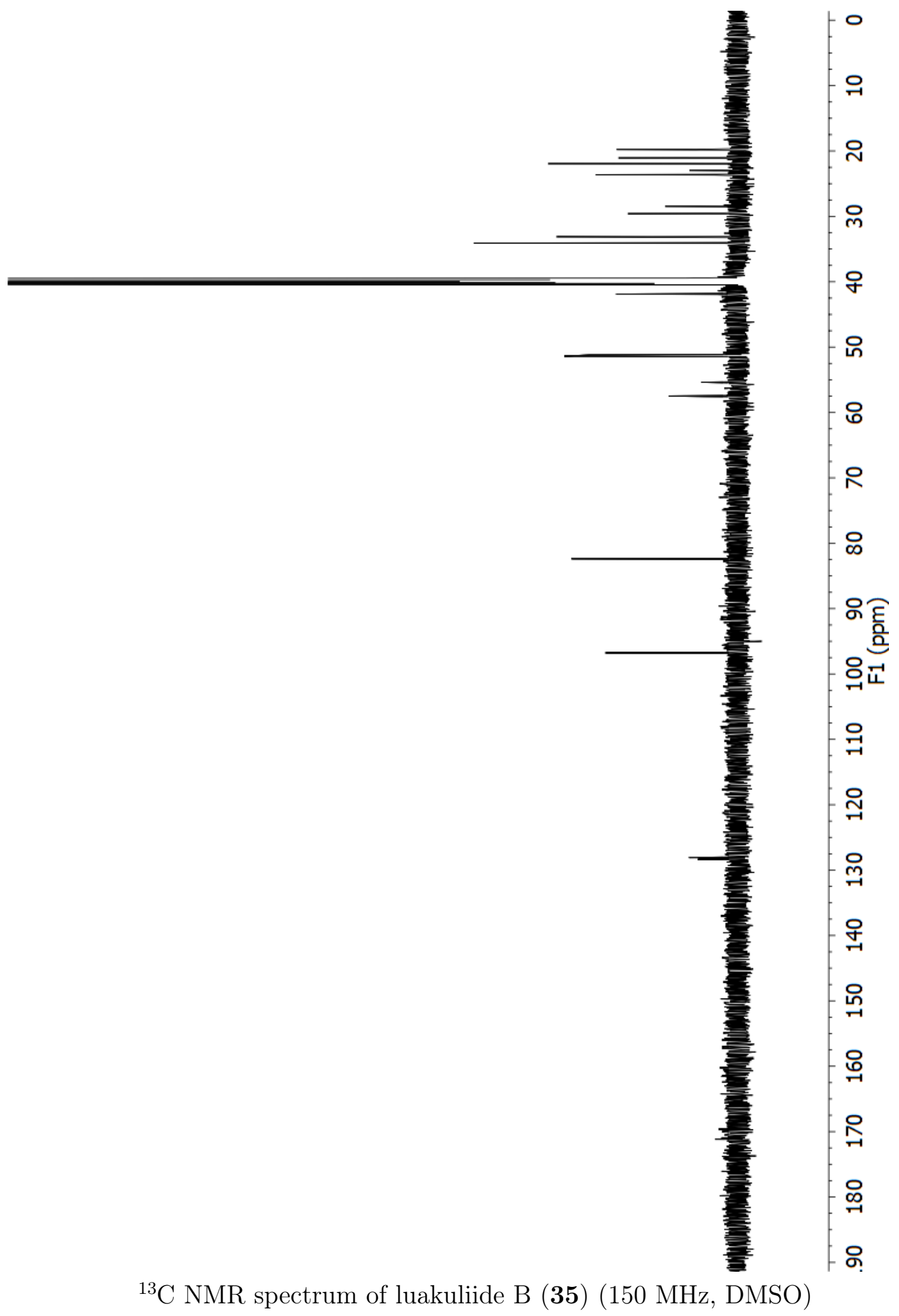




\section{Luakuliide C}

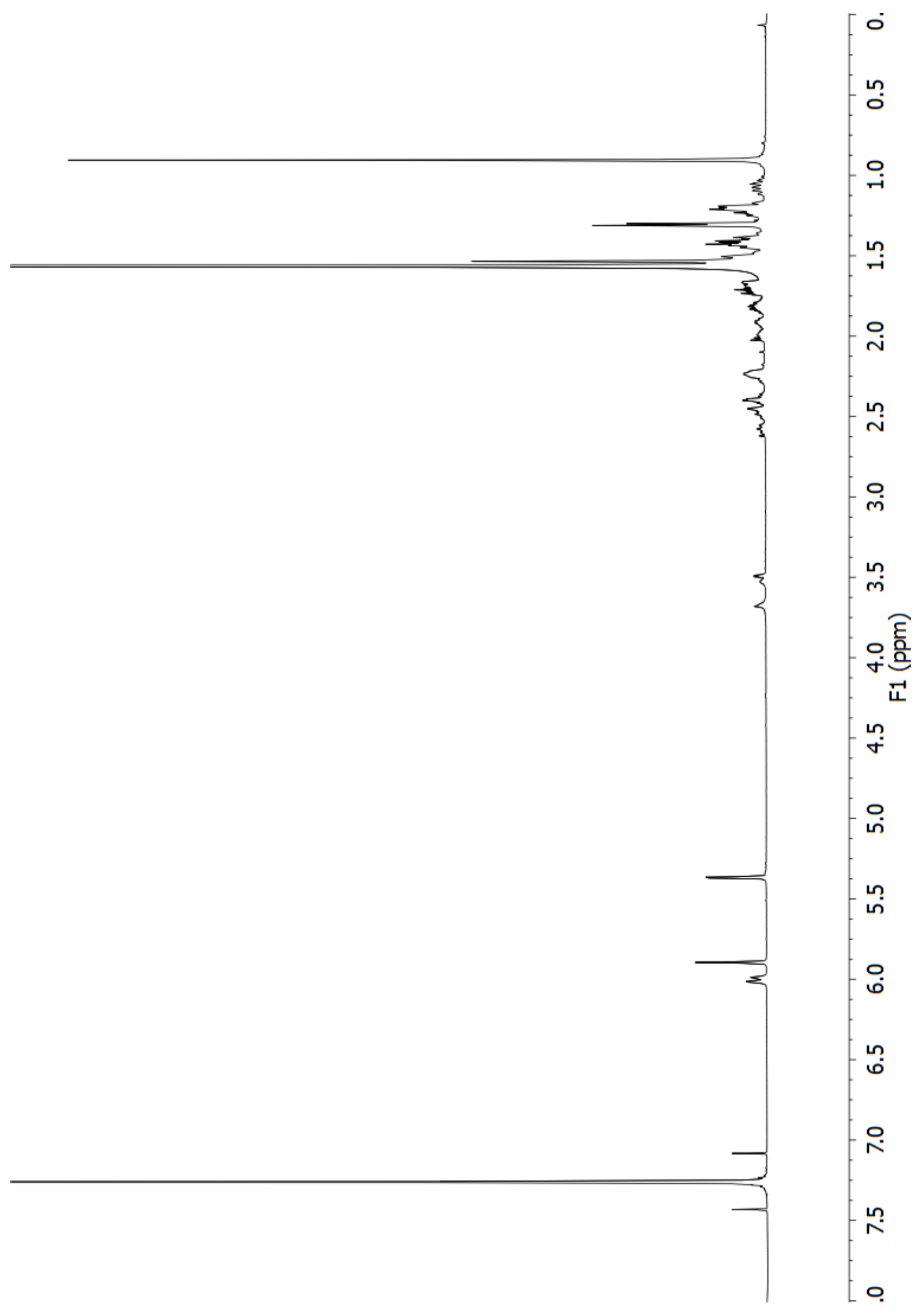

${ }^{1} \mathrm{H}$ NMR spectrum of luakuliide $\mathrm{C}(\mathbf{3 6})\left(600 \mathrm{MHz}, \mathrm{CDCl}_{3}\right)$. 


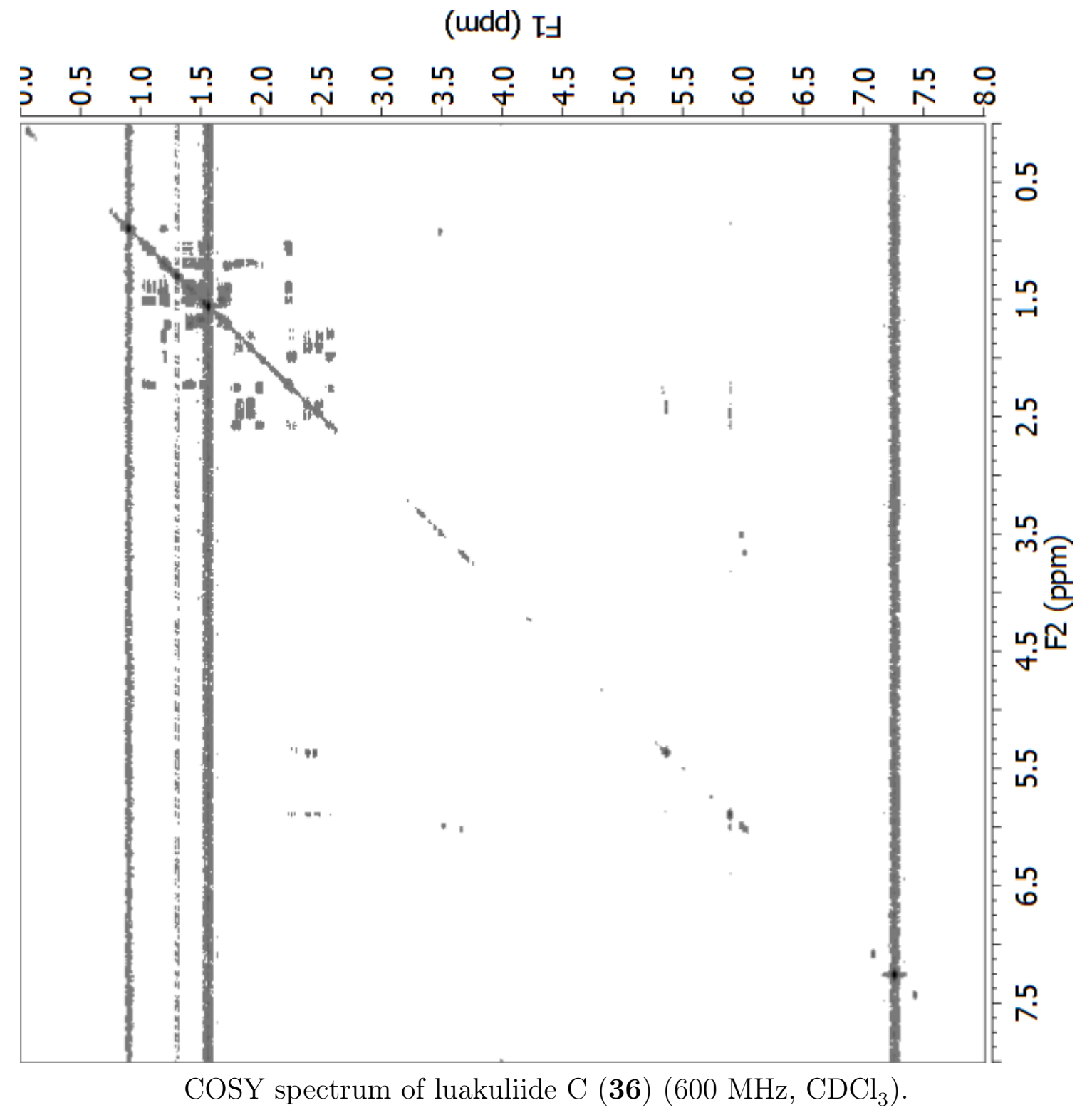




$$
\text { (udd) 더 }
$$

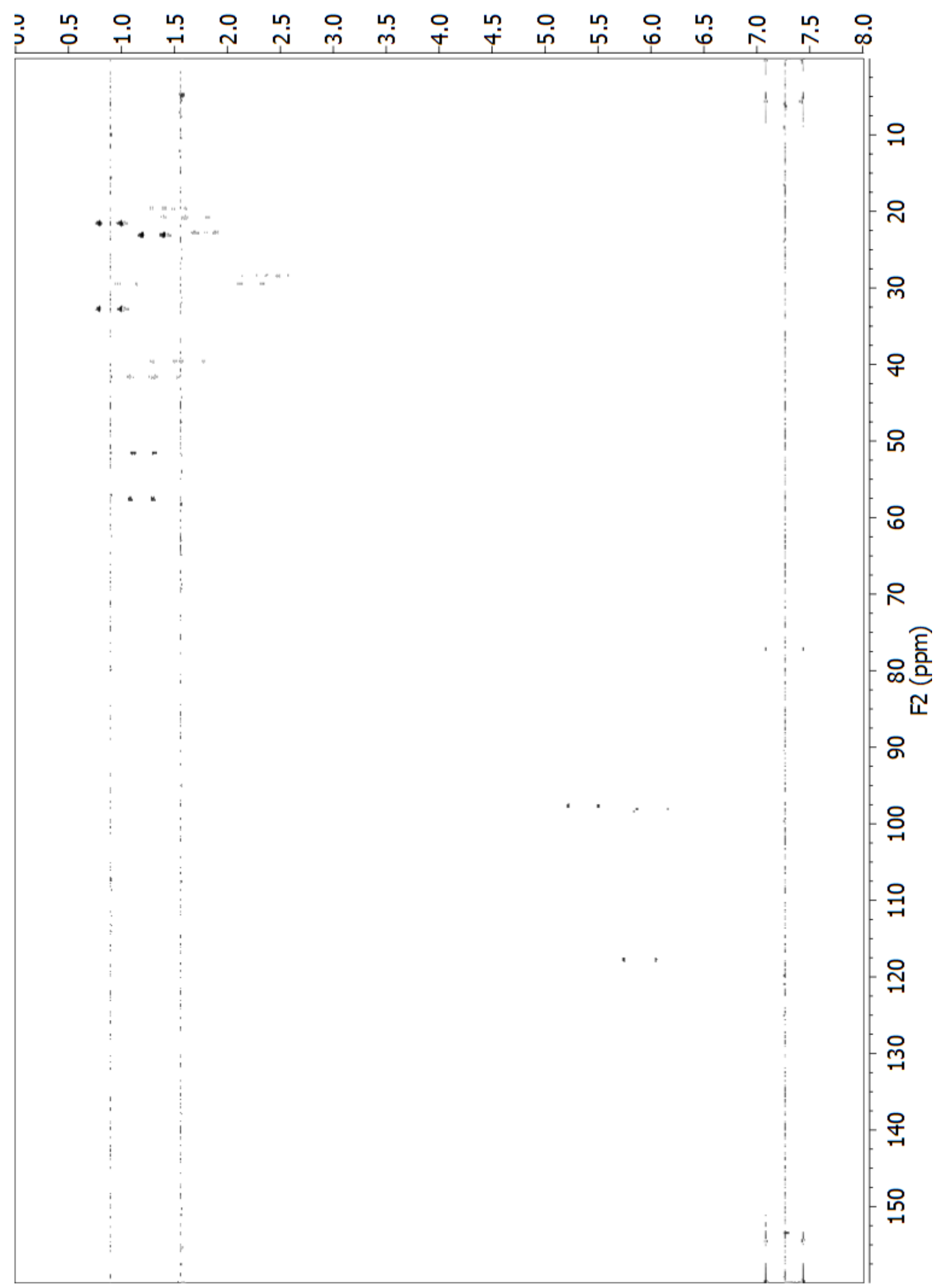

Fully-coupled HSQC spectrum of luakuliide C (36) $\left(600 \mathrm{MHz}, \mathrm{CDCl}_{3}\right)$. 
(udd) It

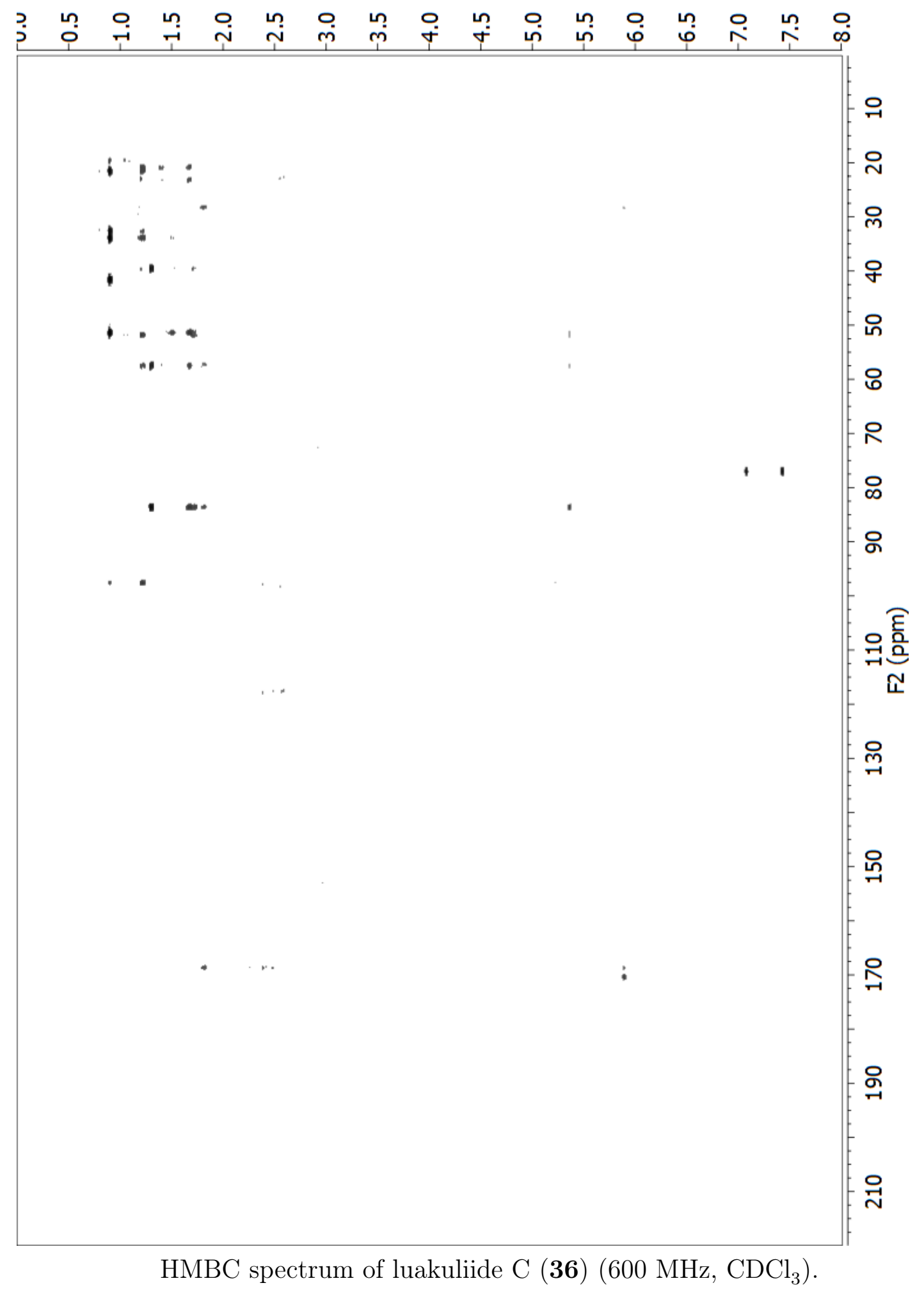




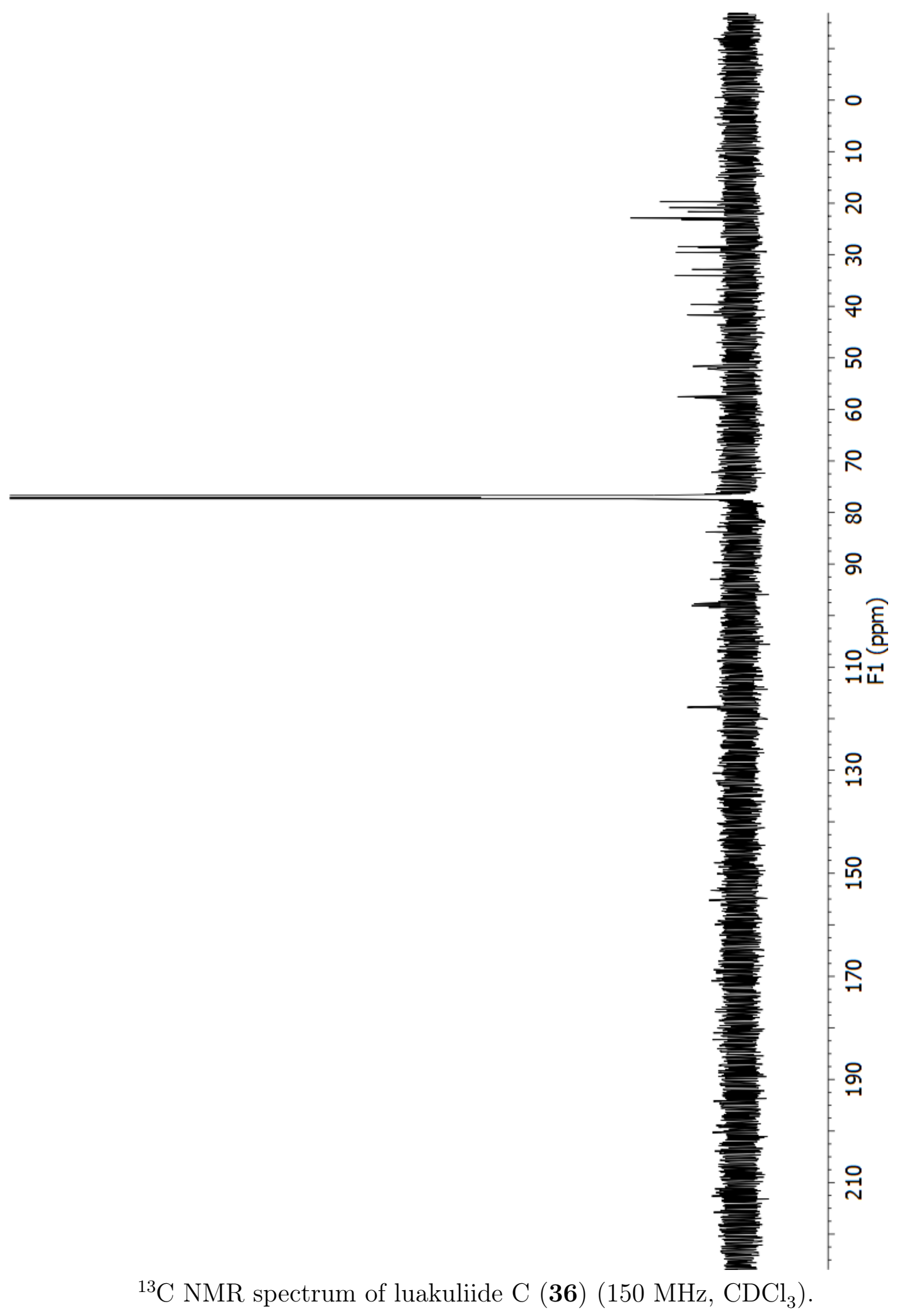




\section{Luakuliide C}

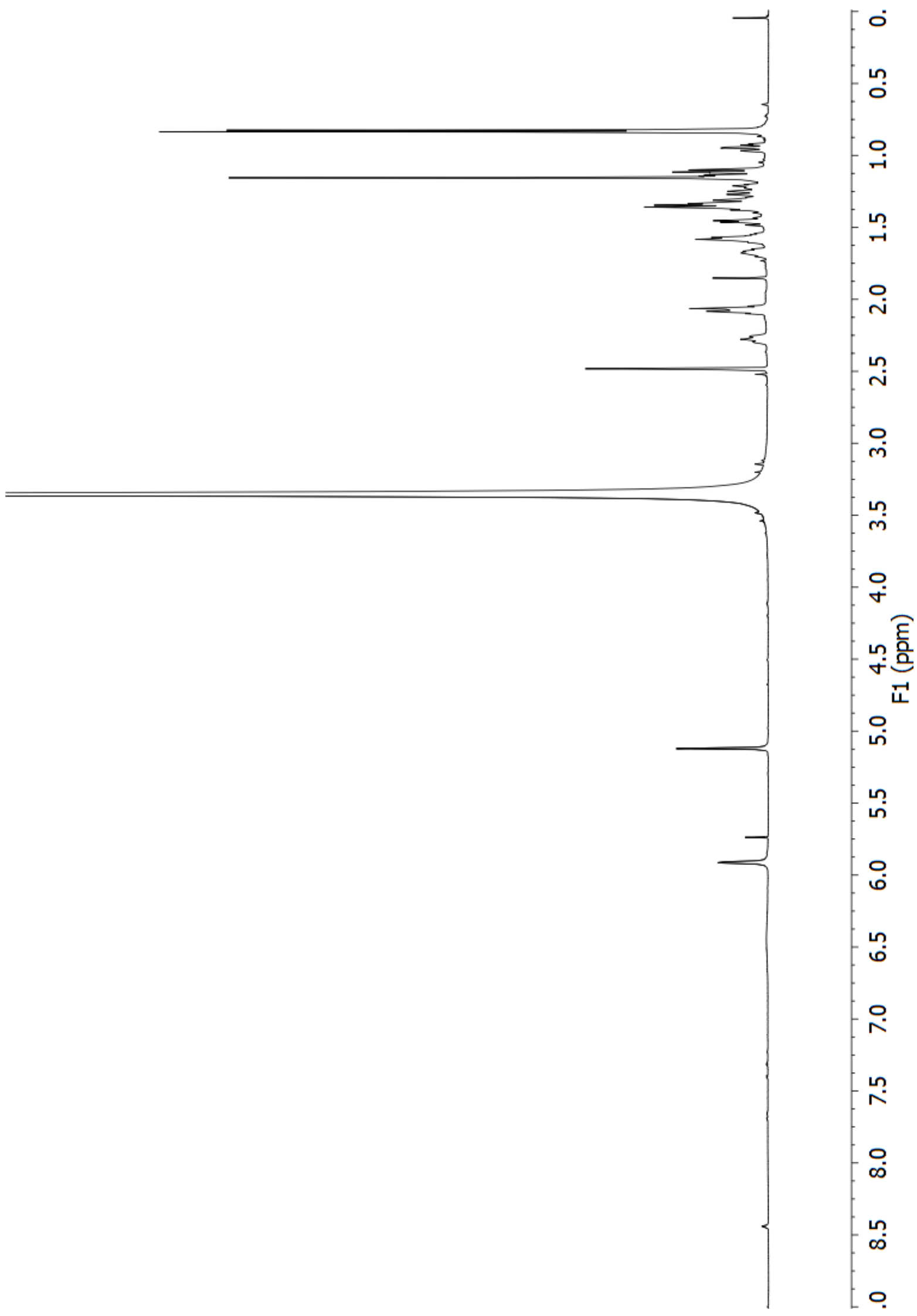

${ }^{1} \mathrm{H}$ NMR spectrum of luakuliide C (36) (600 MHz, DMSO) 


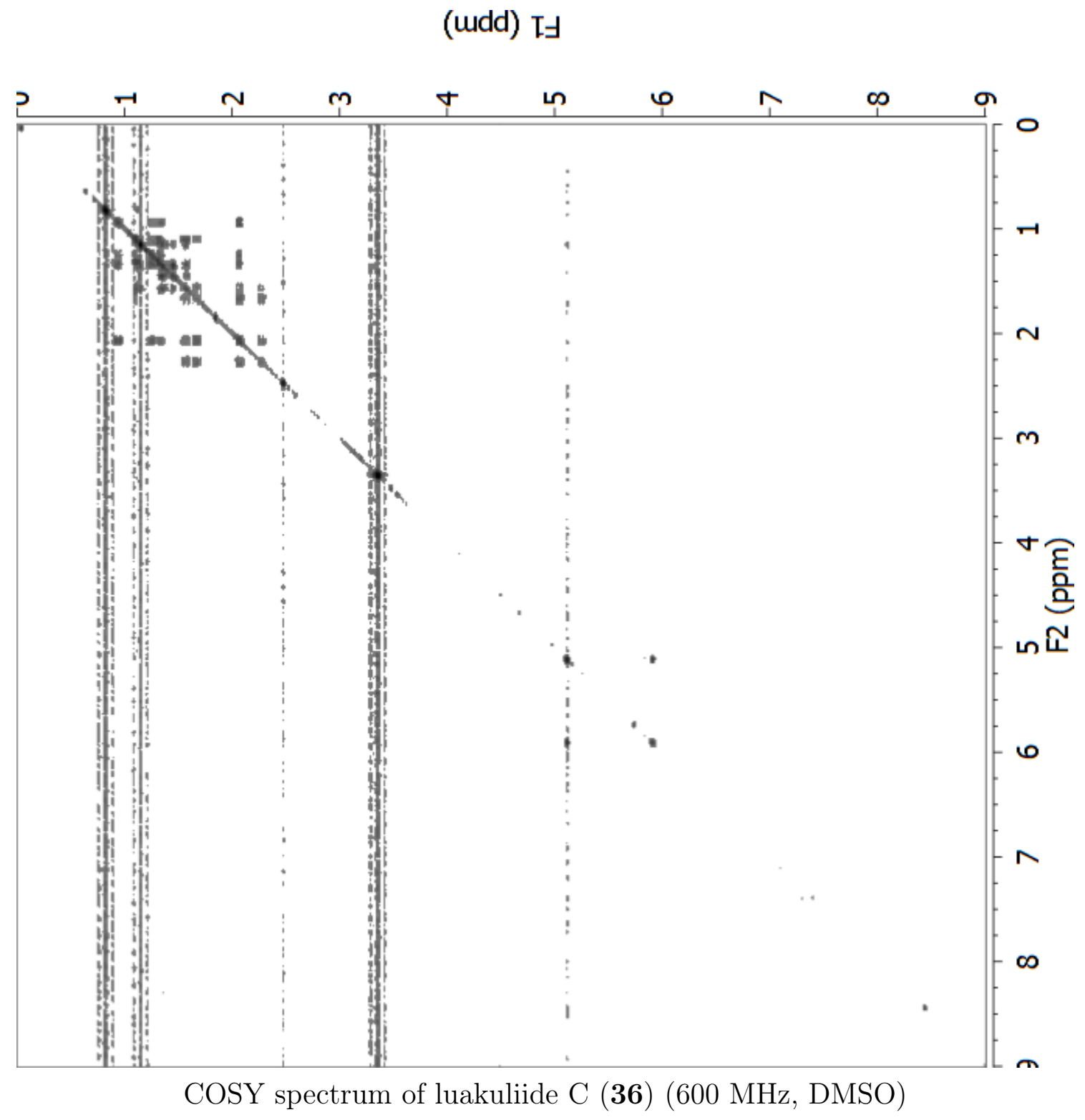




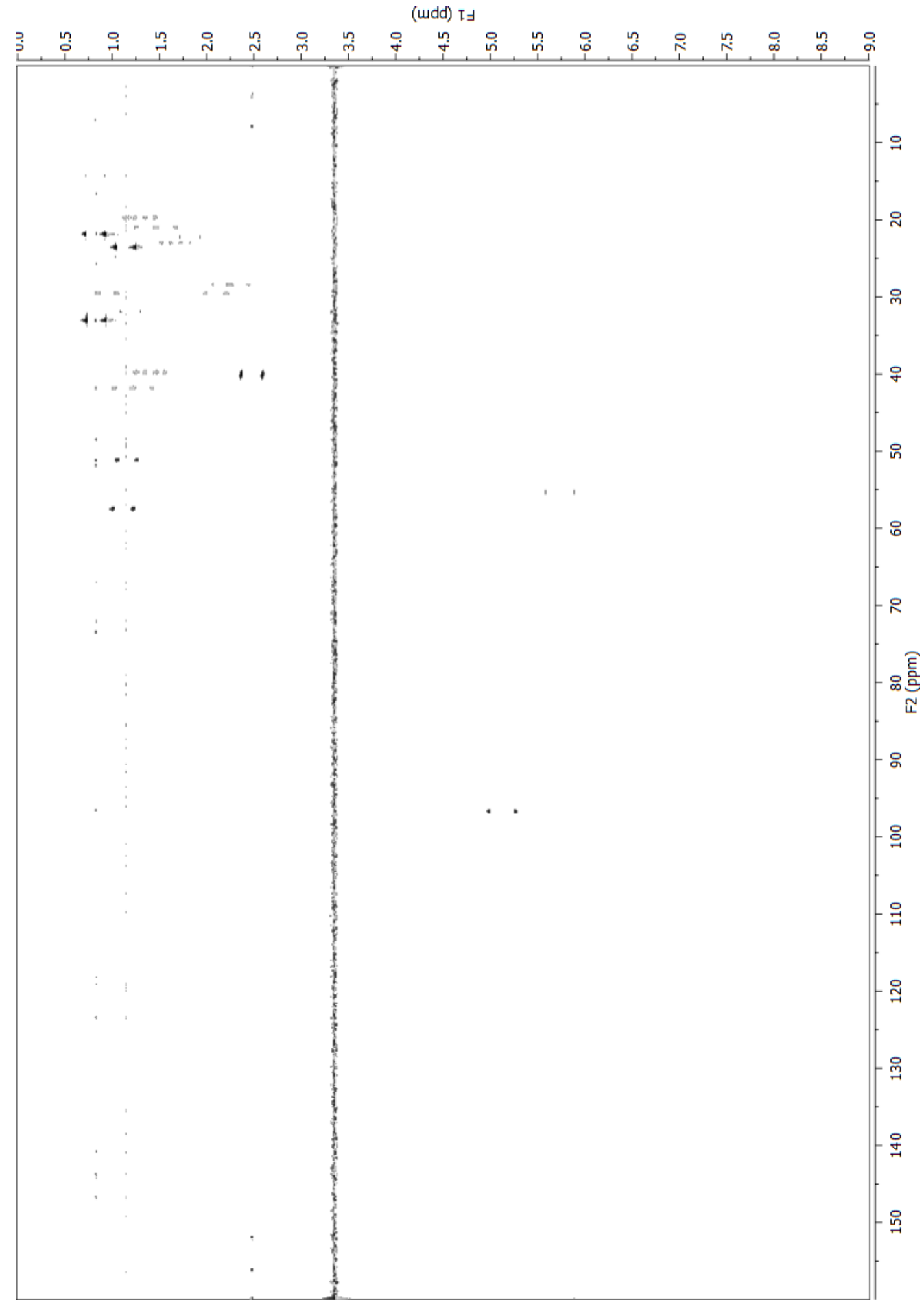

Fully-coupled HSQC spectrum of luakuliide C (36) (600 MHz, DMSO) 


$$
\text { (udd) 더 }
$$

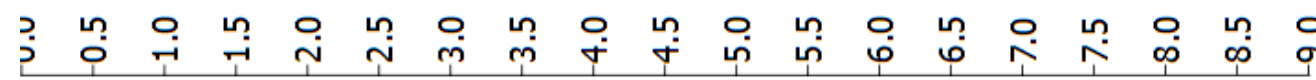

ifin...

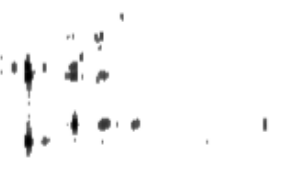

으

은

오

- 우

$1+\cdots \cdots+$

( 1, . .

으

은

ㅇ

오

요

음음
든

$\stackrel{9}{\rightarrow-1}$

육

$\stackrel{4}{\circ}$

윽

읏

HMBC spectrum of luakuliide C (36) (600 MHz, DMSO) 


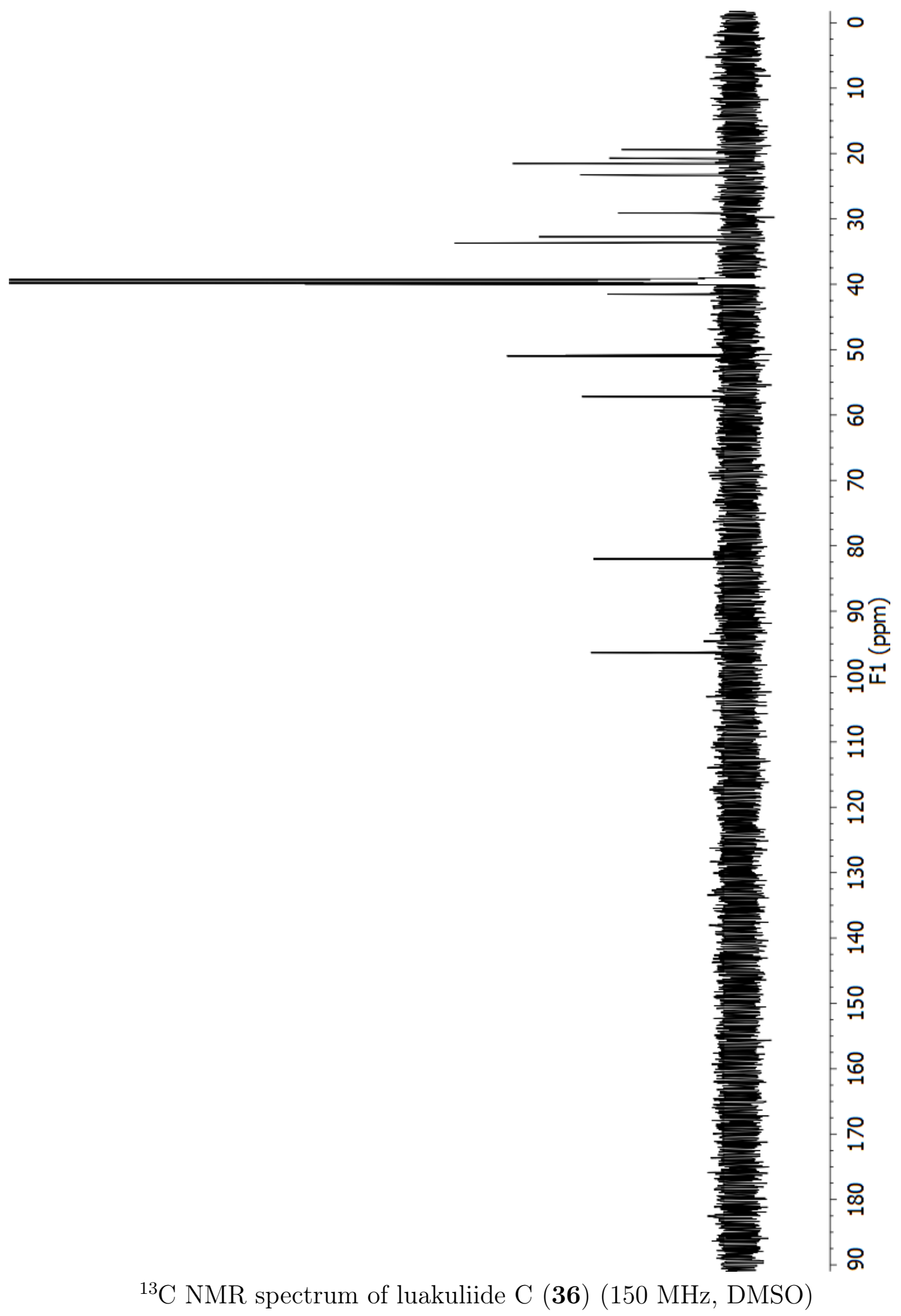


Appendix F

NMR Spectra of Lehualides E-K 


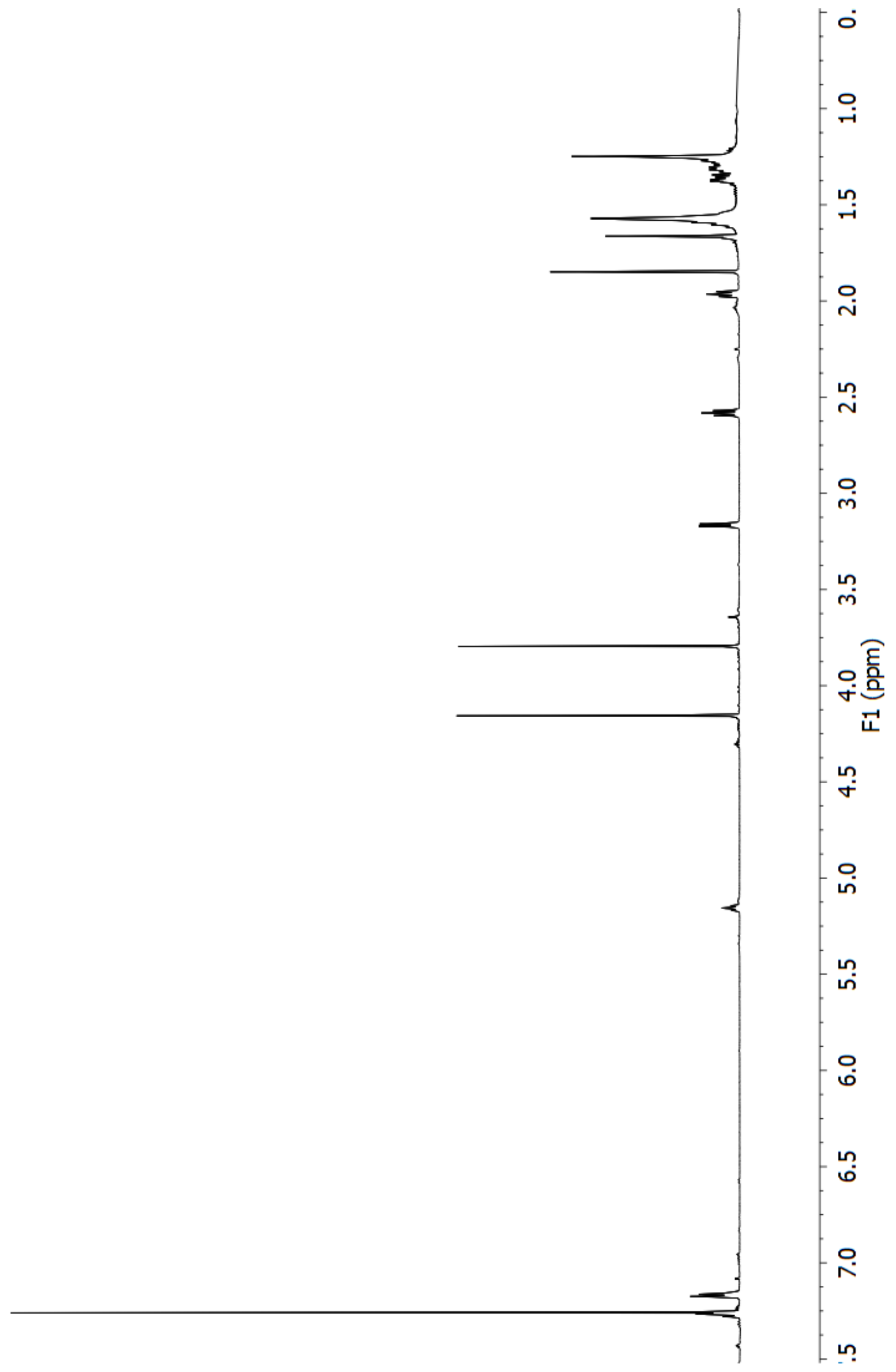

${ }^{1} \mathrm{H}$ NMR spectrum of lehualide E (69) (600 $\mathrm{MHz}, \mathrm{CDCl}_{3}$ ). 


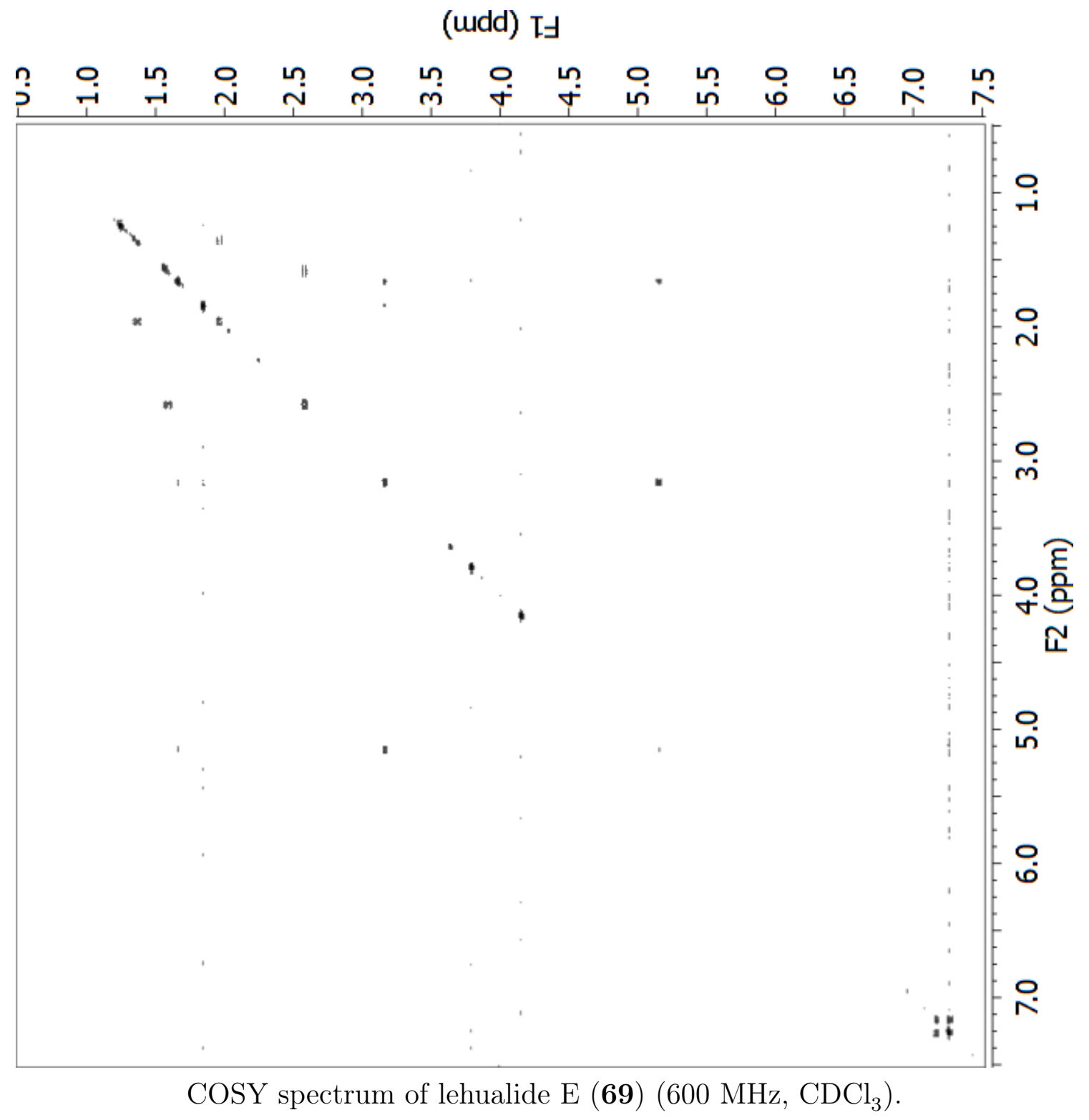


(udd) I与

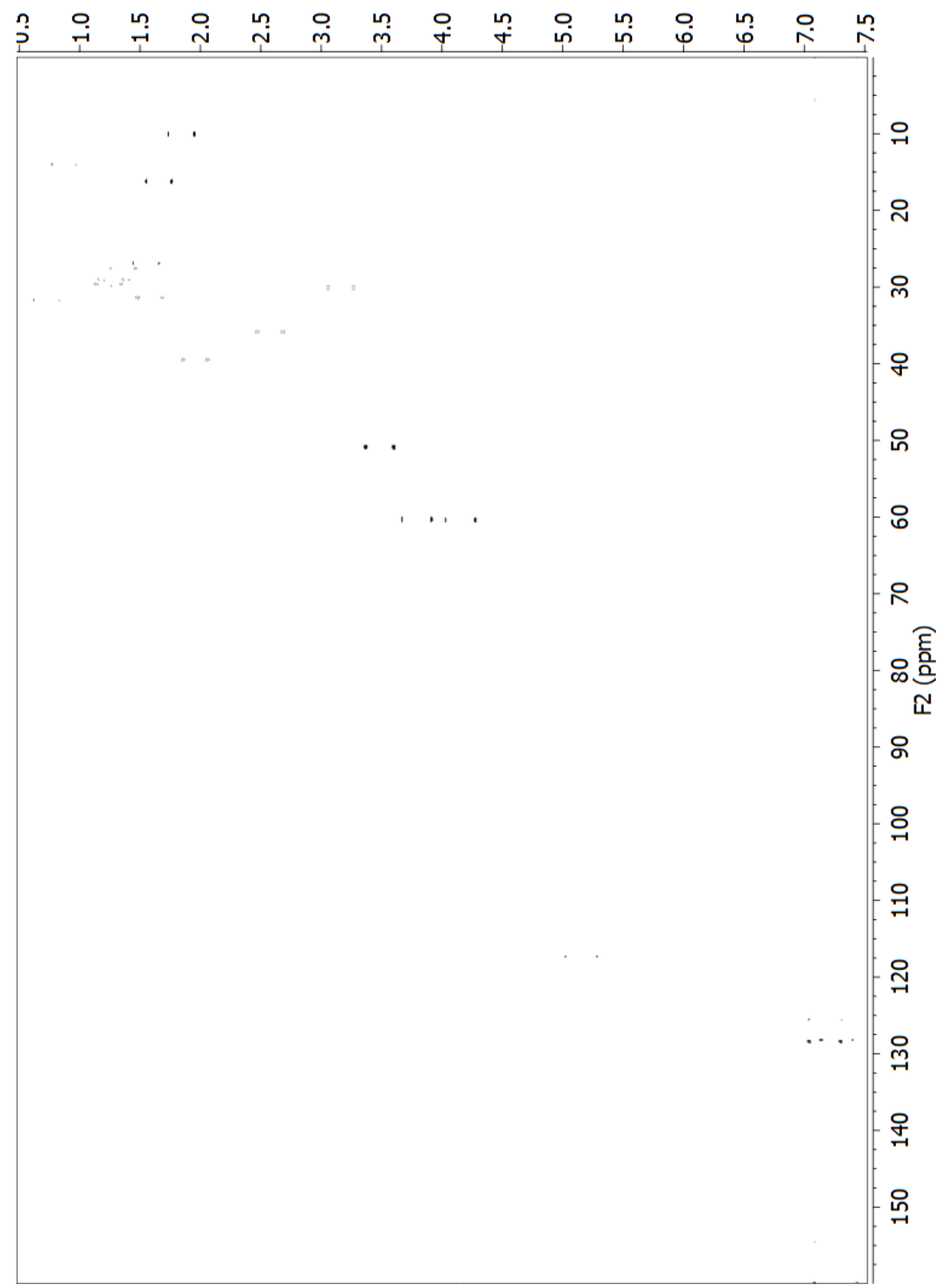

Fully-coupled HSQC spectrum of lehualide E (69) (600 MHz, $\mathrm{CDCl}_{3}$ ). 


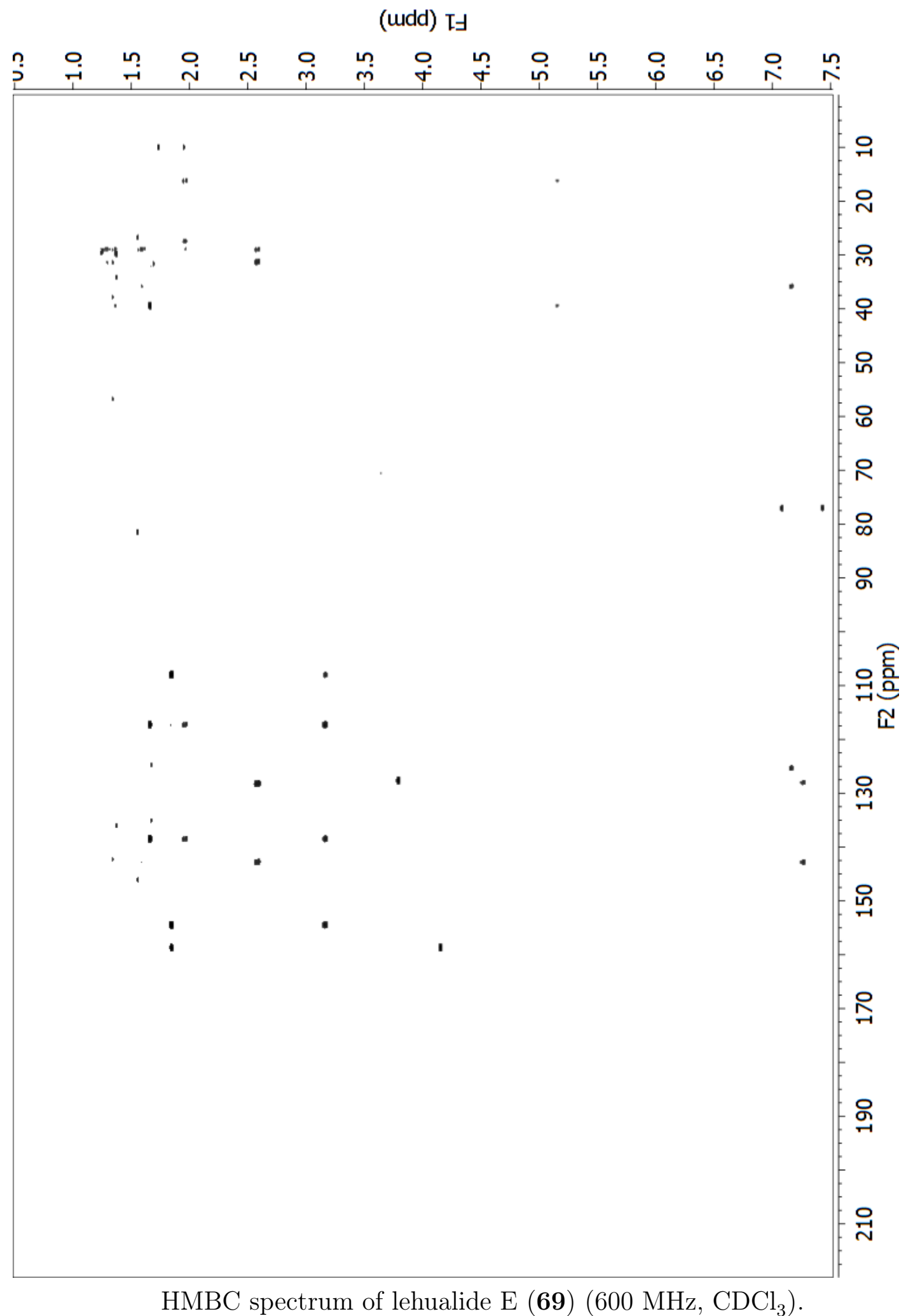

HMBC spectrum of lehualide E (69) (600 MHz, $\mathrm{CDCl}_{3}$ ).

252 


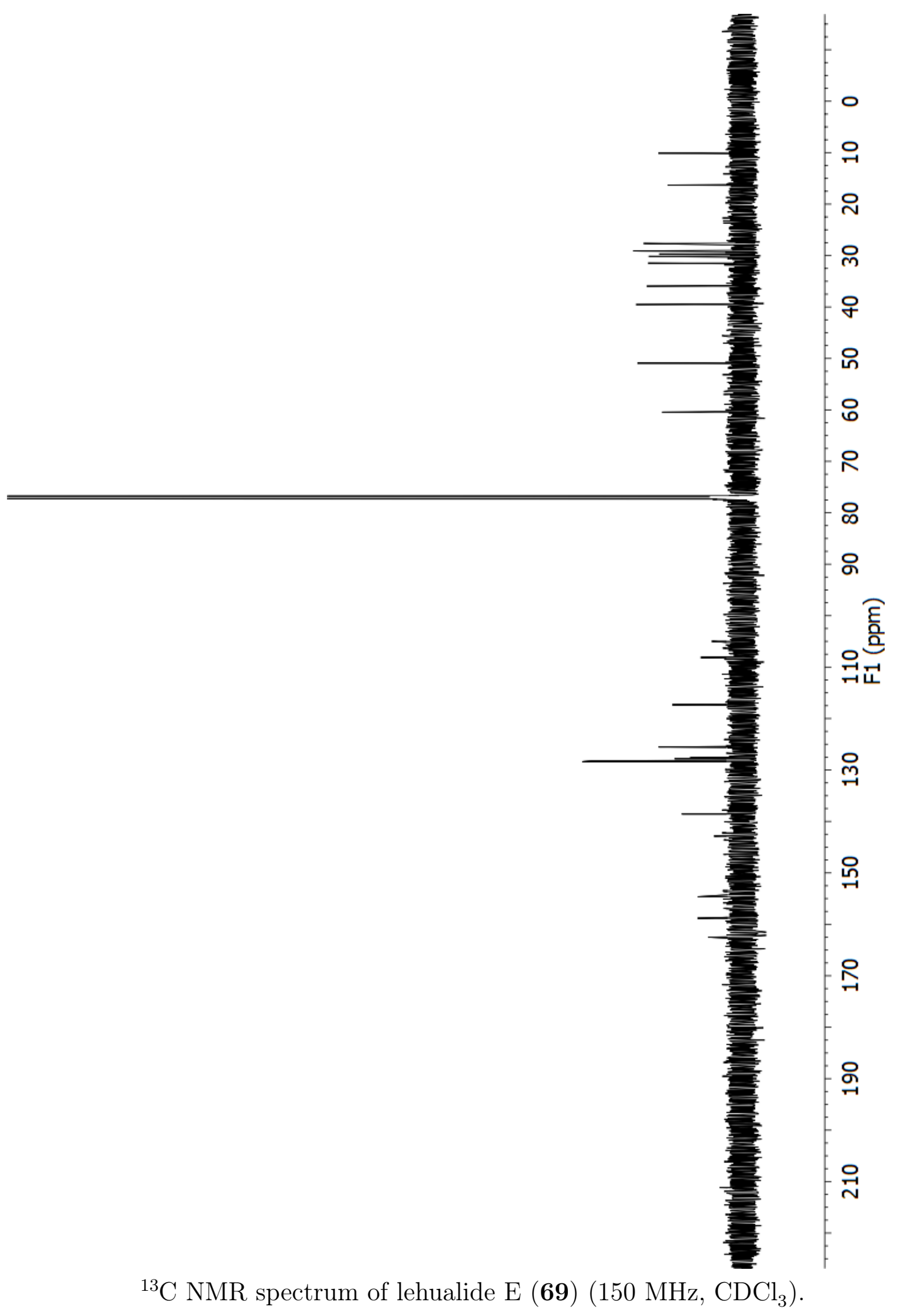




\section{Lehualide F}

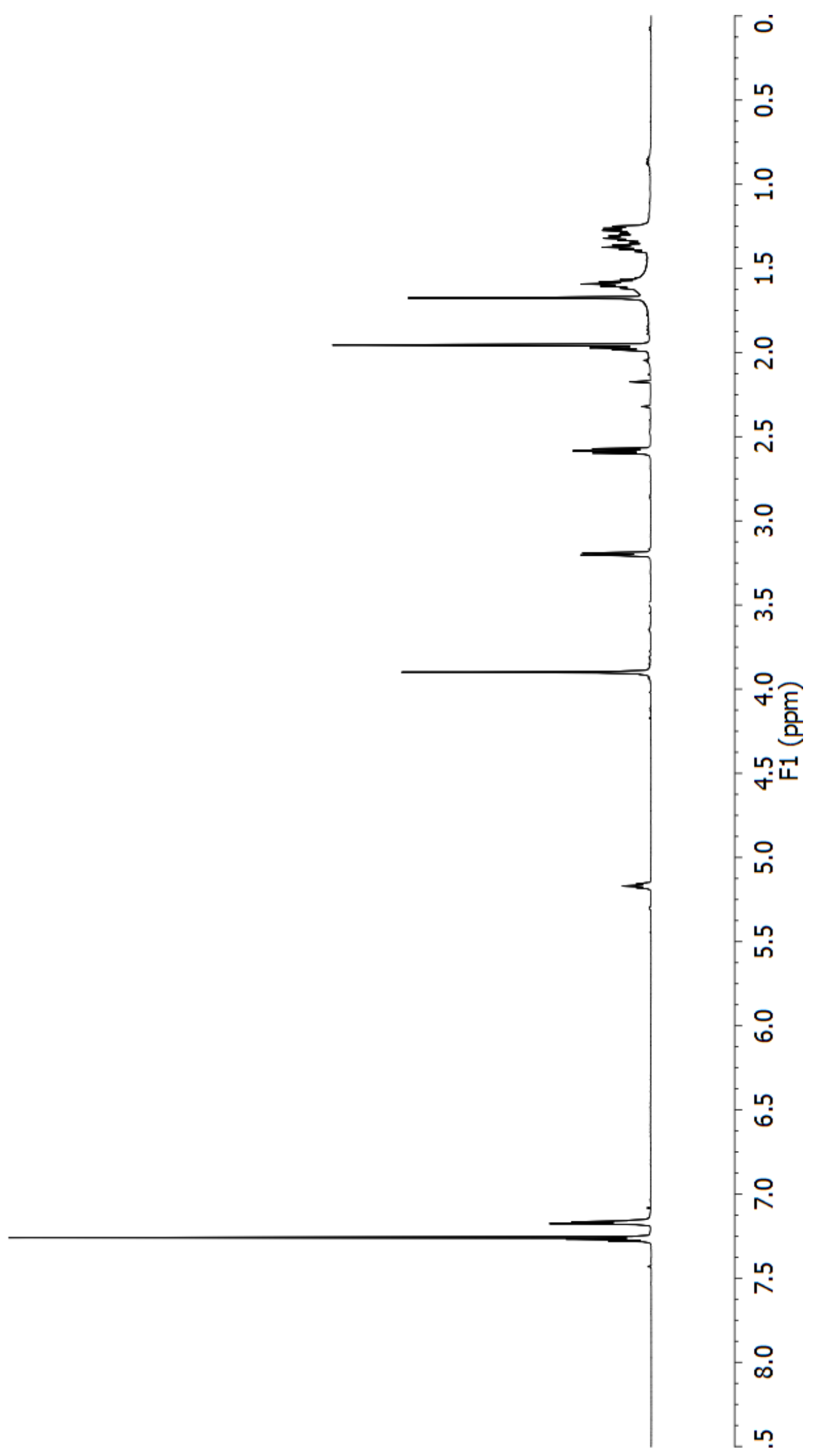

${ }^{1} \mathrm{H}$ NMR spectrum of lehualide F (70) (600 MHz, $\mathrm{CDCl}_{3}$ ). 


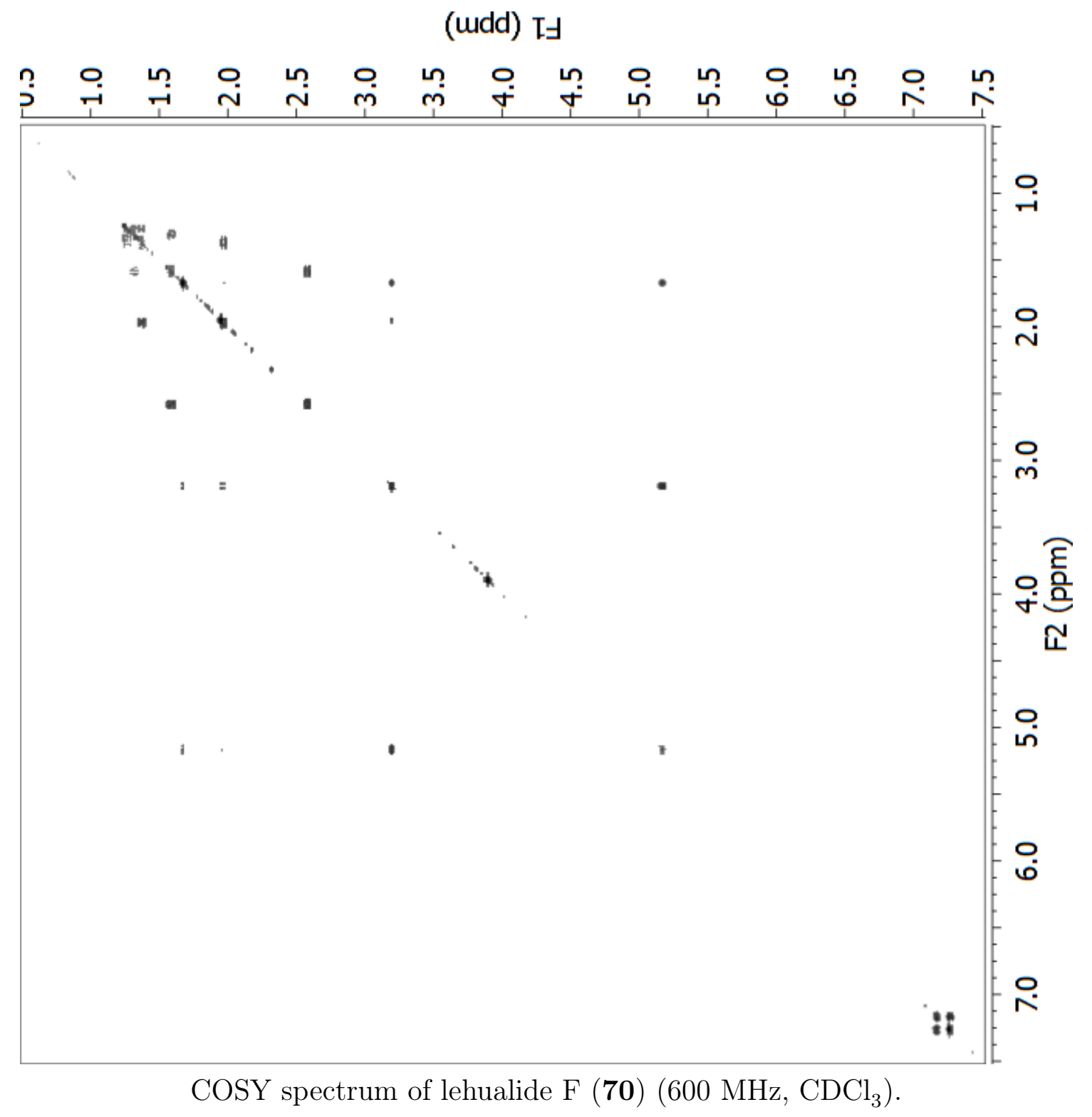




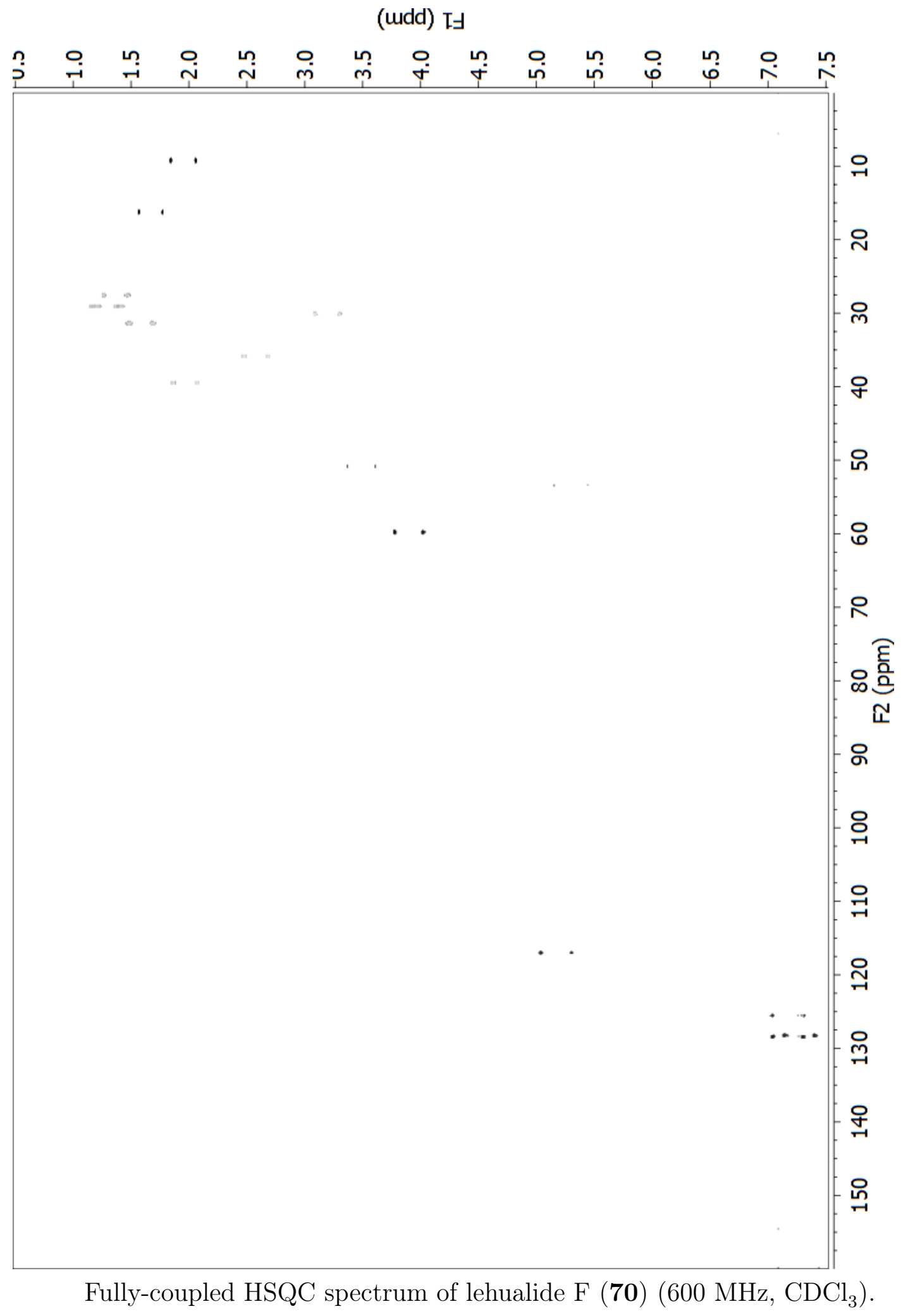

256 
(udd) I与

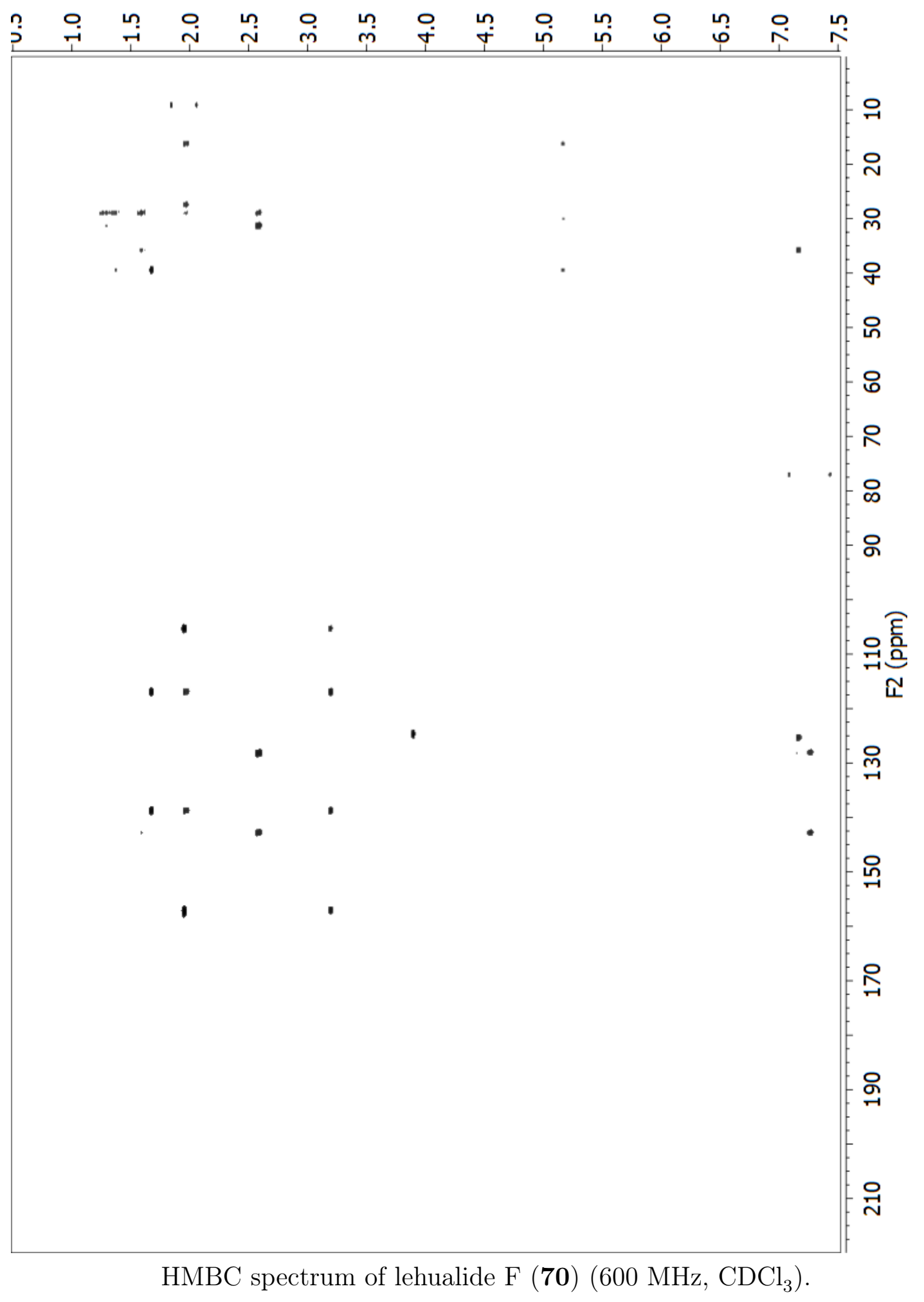




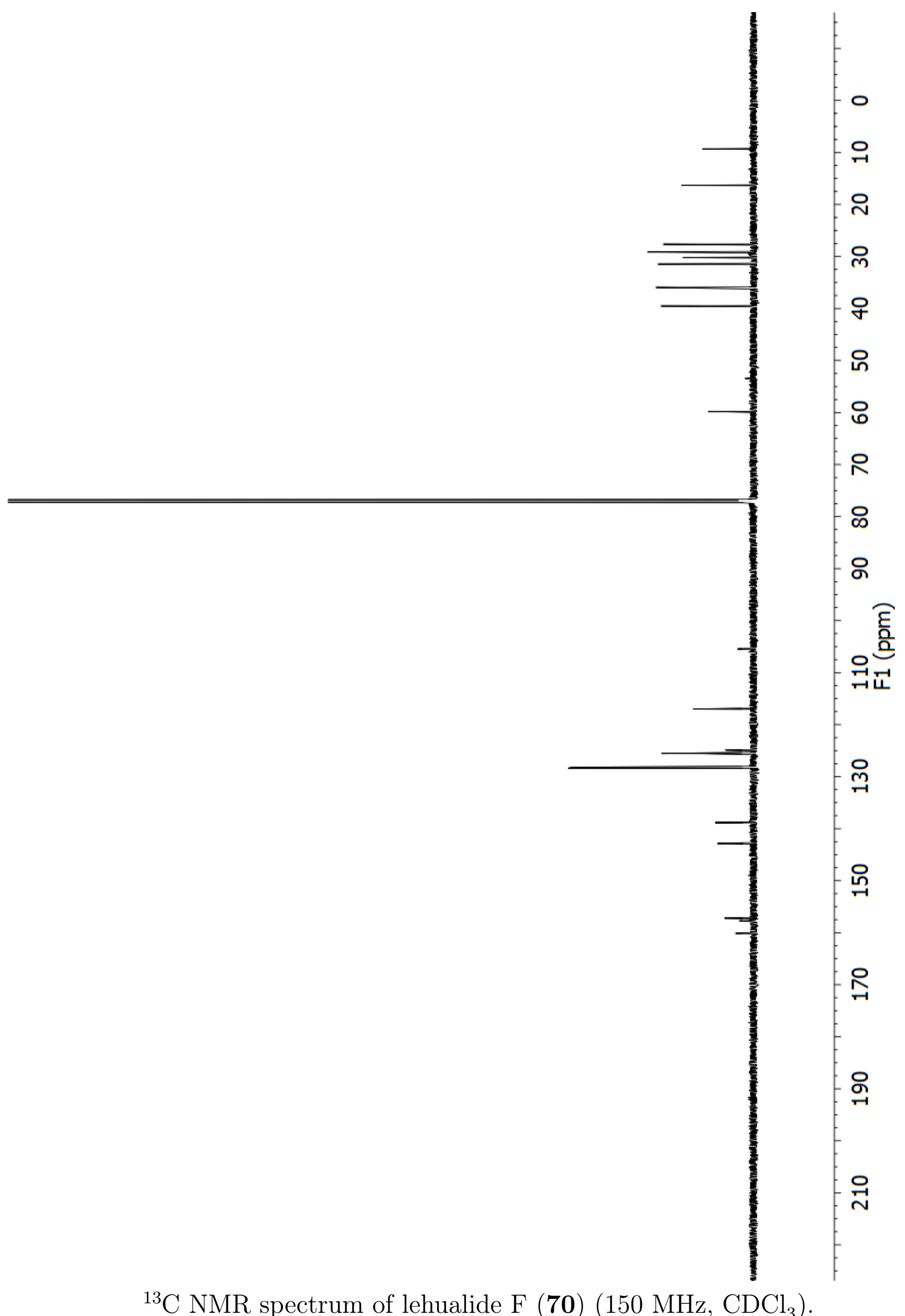

${ }^{13} \mathrm{C}$ NMR spectrum of lehualide F (70) $\left(150 \mathrm{MHz}, \mathrm{CDCl}_{3}\right)$. 
Lehualide G

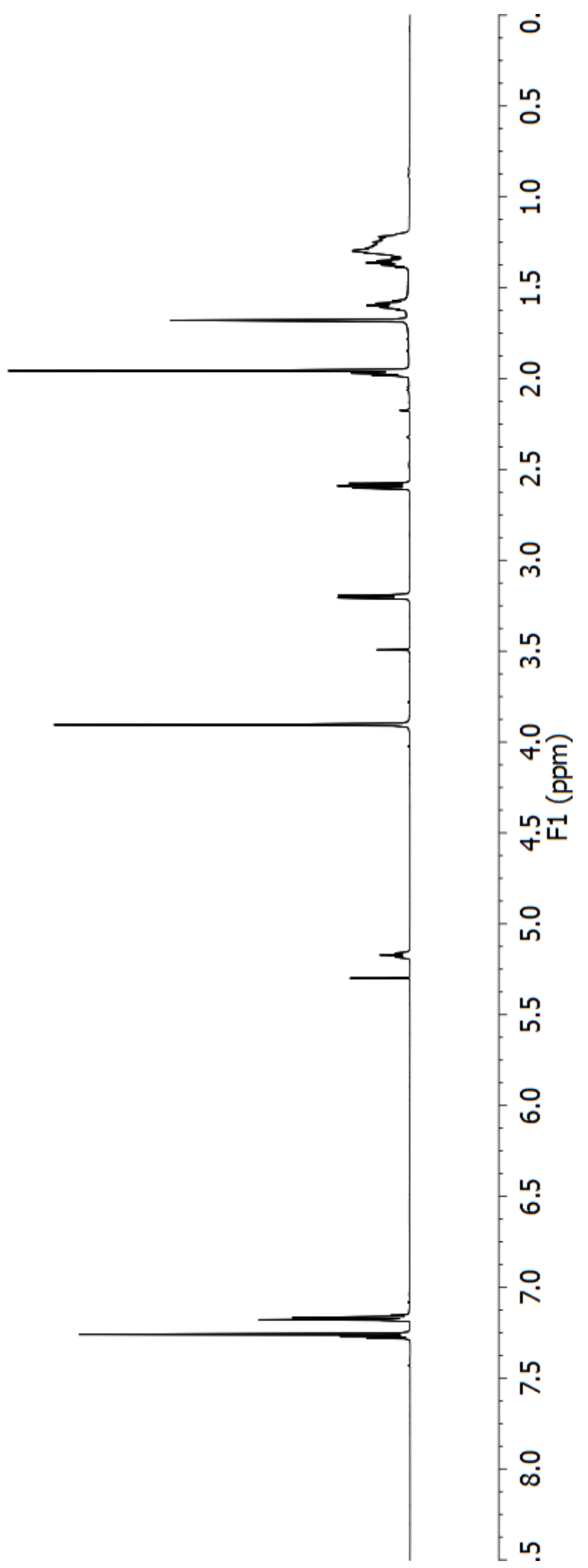

${ }^{1} \mathrm{H}$ NMR spectrum of lehualide $\mathrm{G}(\mathbf{7 1})\left(600 \mathrm{MHz}, \mathrm{CDCl}_{3}\right)$. 


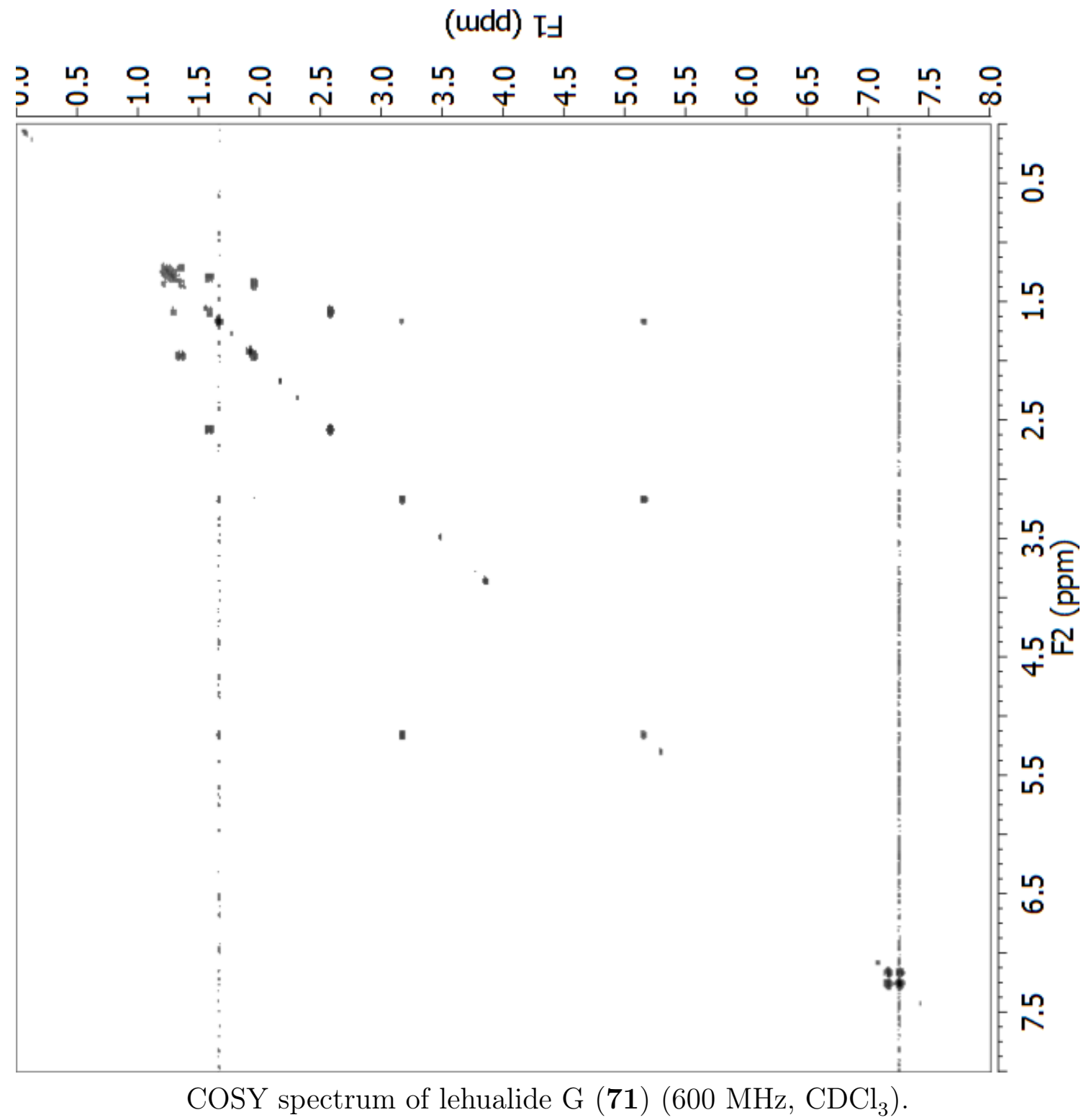


(udd) I与

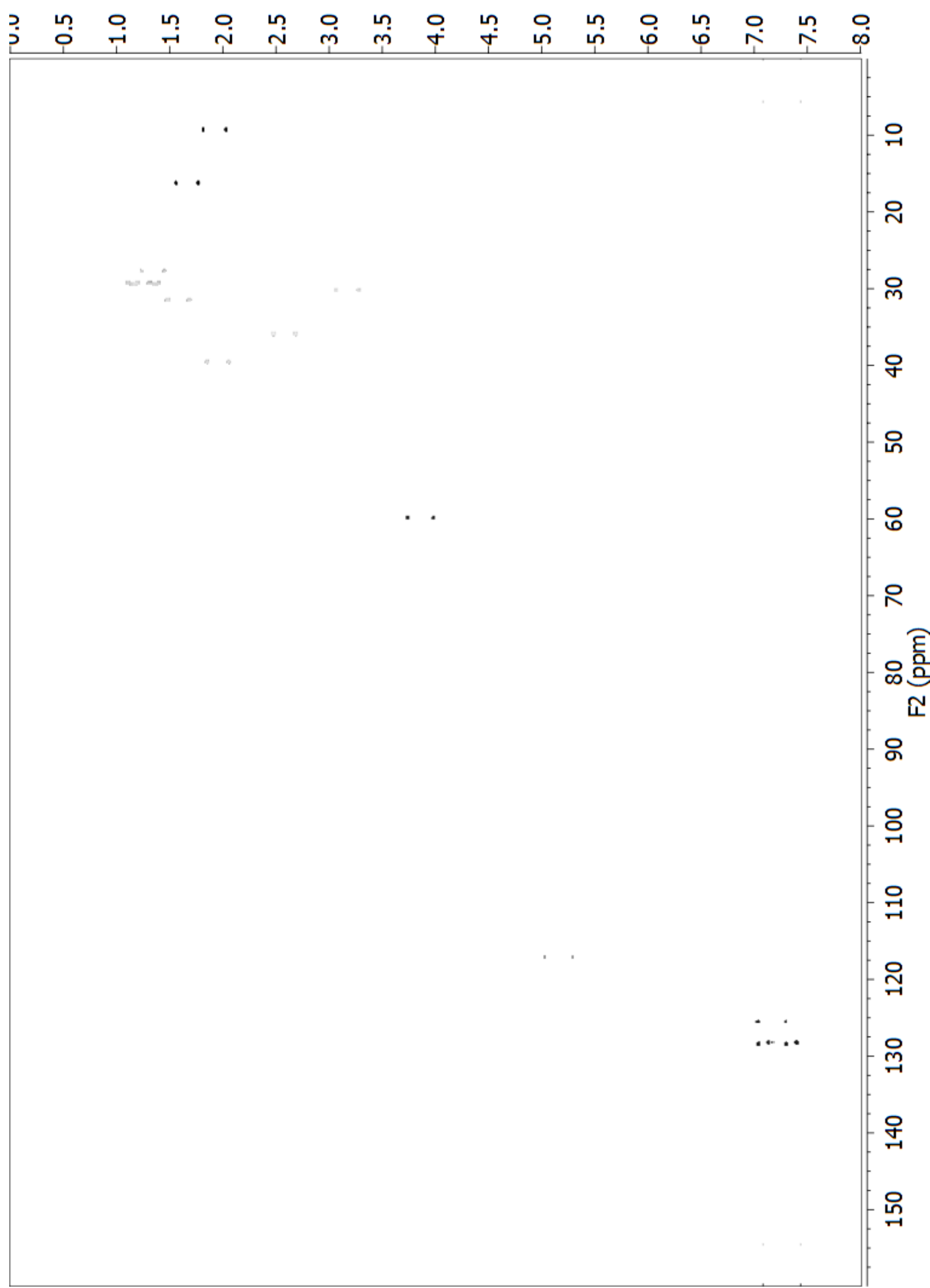

Fully-coupled HSQC spectrum of lehualide G (71) (600 MHz, $\mathrm{CDCl}_{3}$ ). 


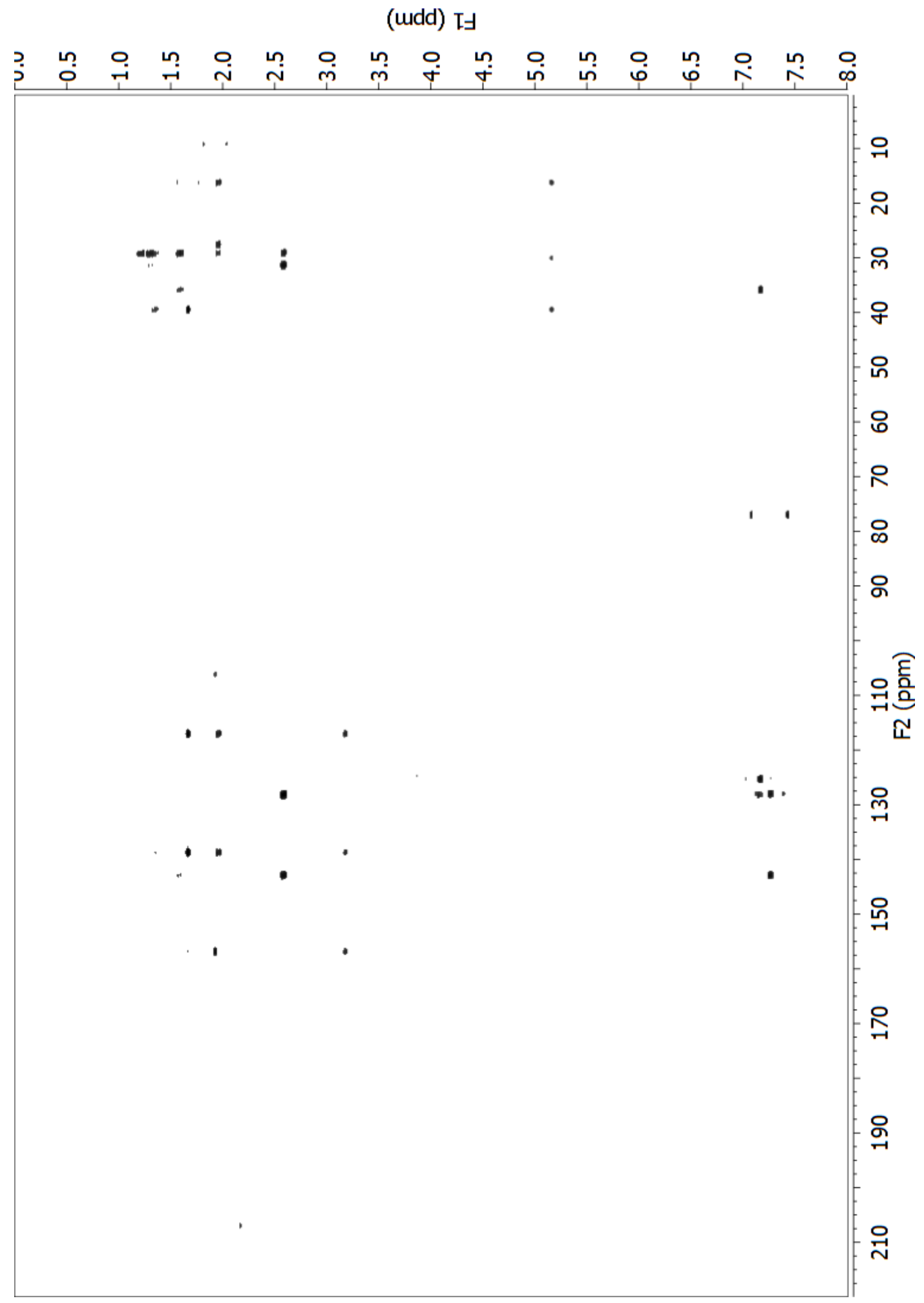

HMBC spectrum of lehualide G (71) (600 $\mathrm{MHz}^{\mathrm{CDCl}} \mathrm{CDC}_{3}$ ). 


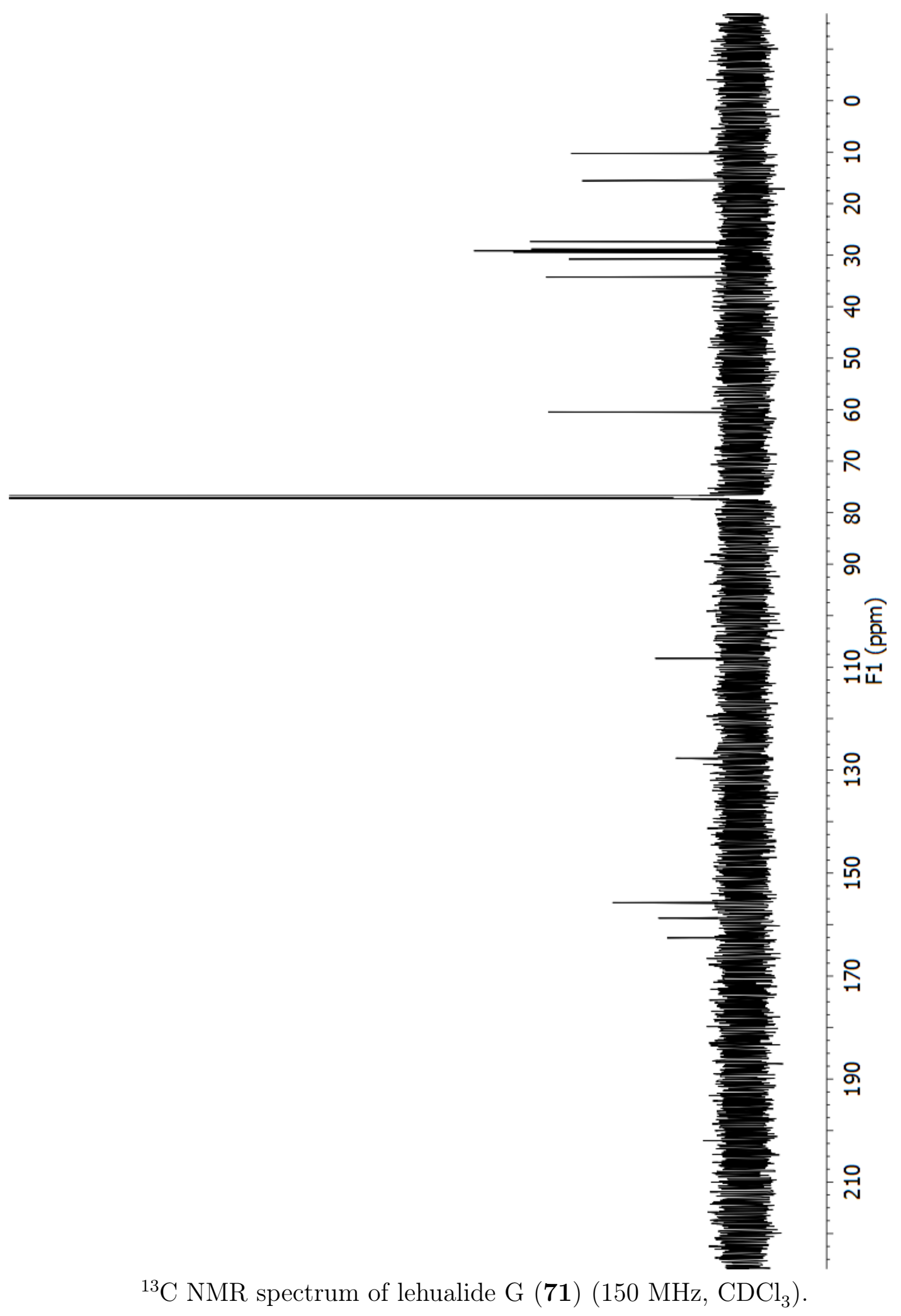




\section{Lehualide $\mathbf{H}$}

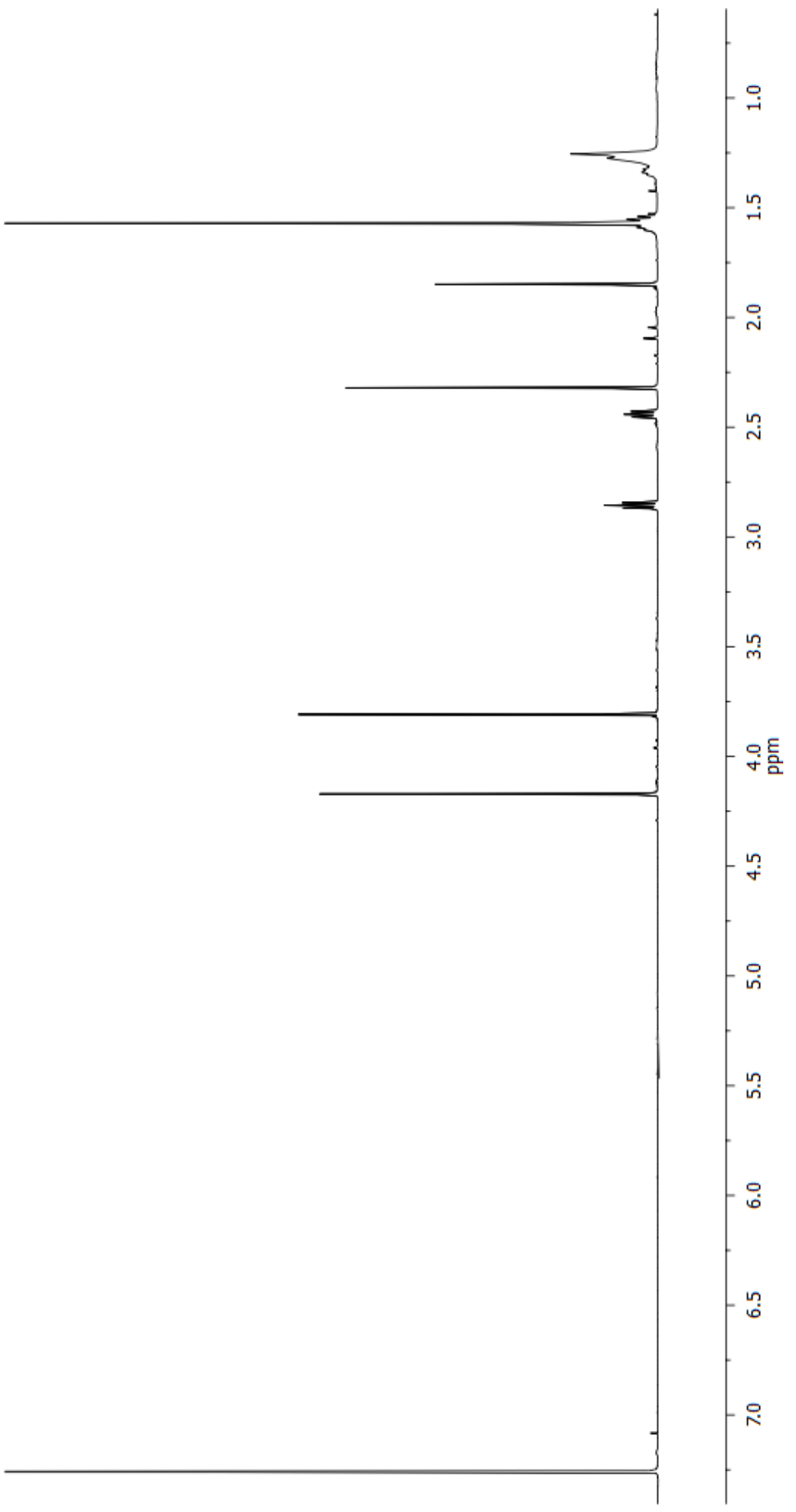

${ }^{1} \mathrm{H}$ NMR spectrum of lehualide $\mathrm{H}(\mathbf{7 2})\left(600 \mathrm{MHz}, \mathrm{CDCl}_{3}\right)$. 


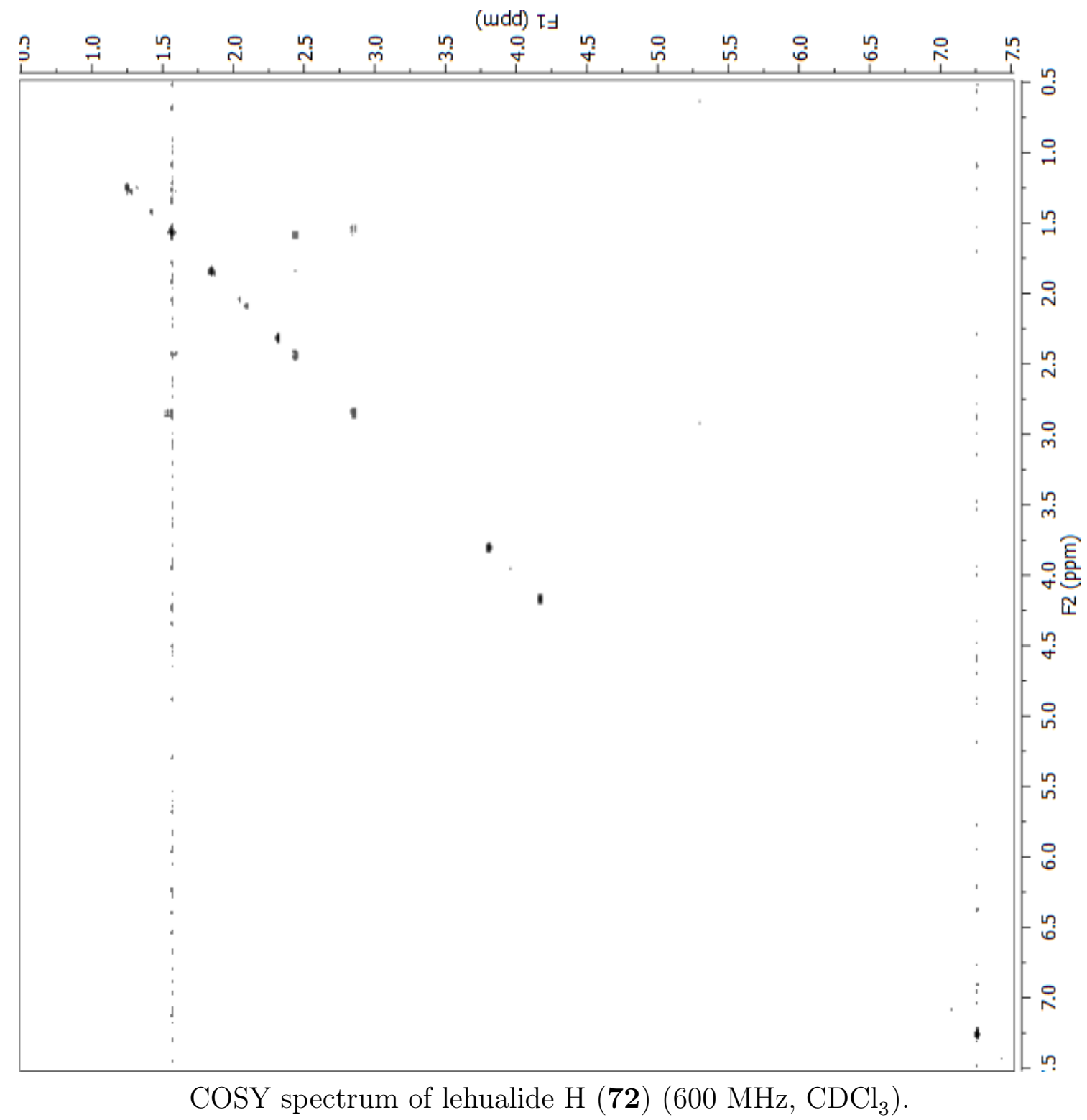




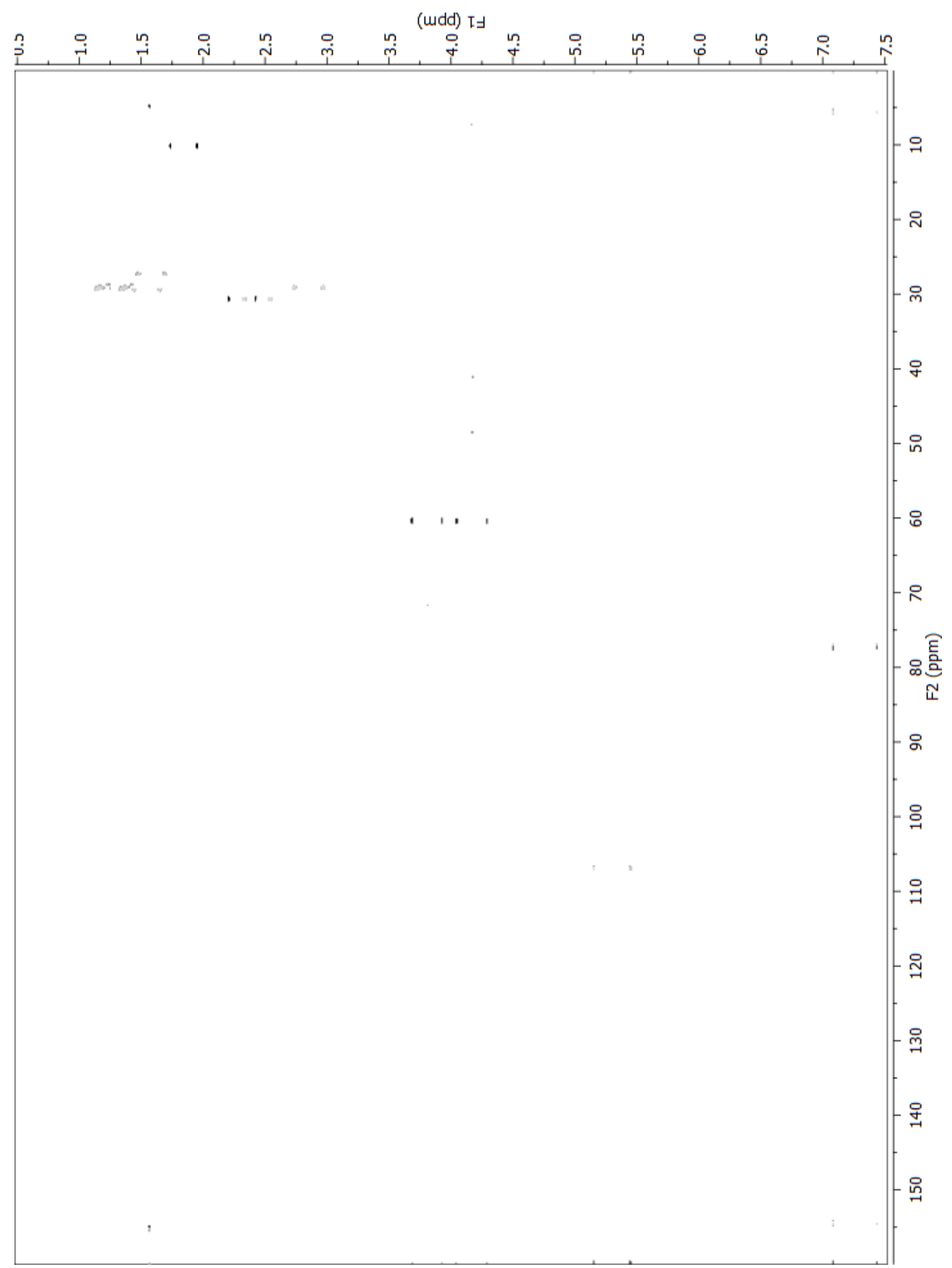

Fully-coupled HSQC spectrum of lehualide $\mathrm{H}(\mathbf{7 2})\left(600 \mathrm{MHz}, \mathrm{CDCl}_{3}\right.$ ). 


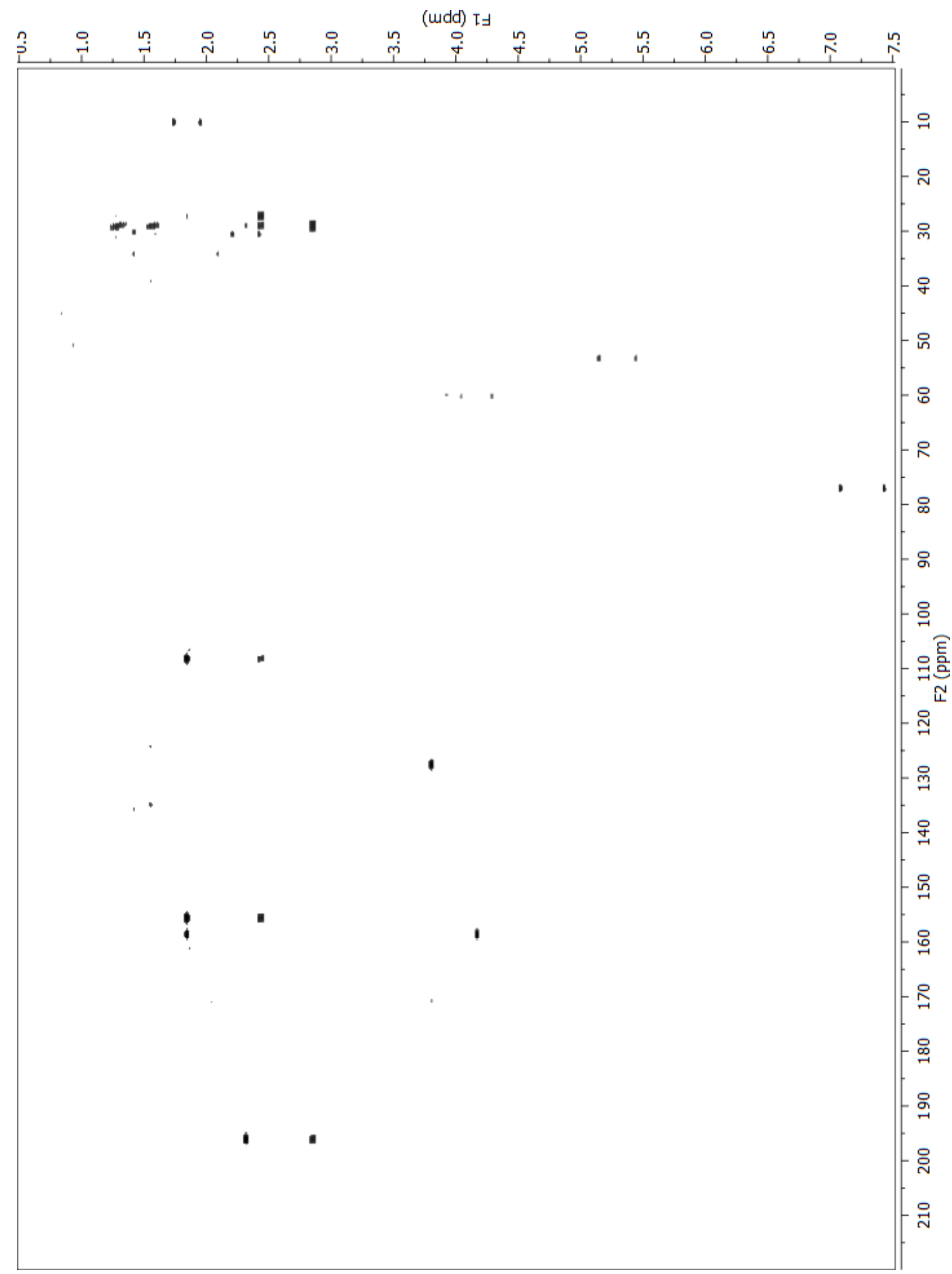

HMBC spectrum of lehualide $\mathrm{H}(\mathbf{7 2})\left(600 \mathrm{MHz}, \mathrm{CDCl}_{3}\right)$. 


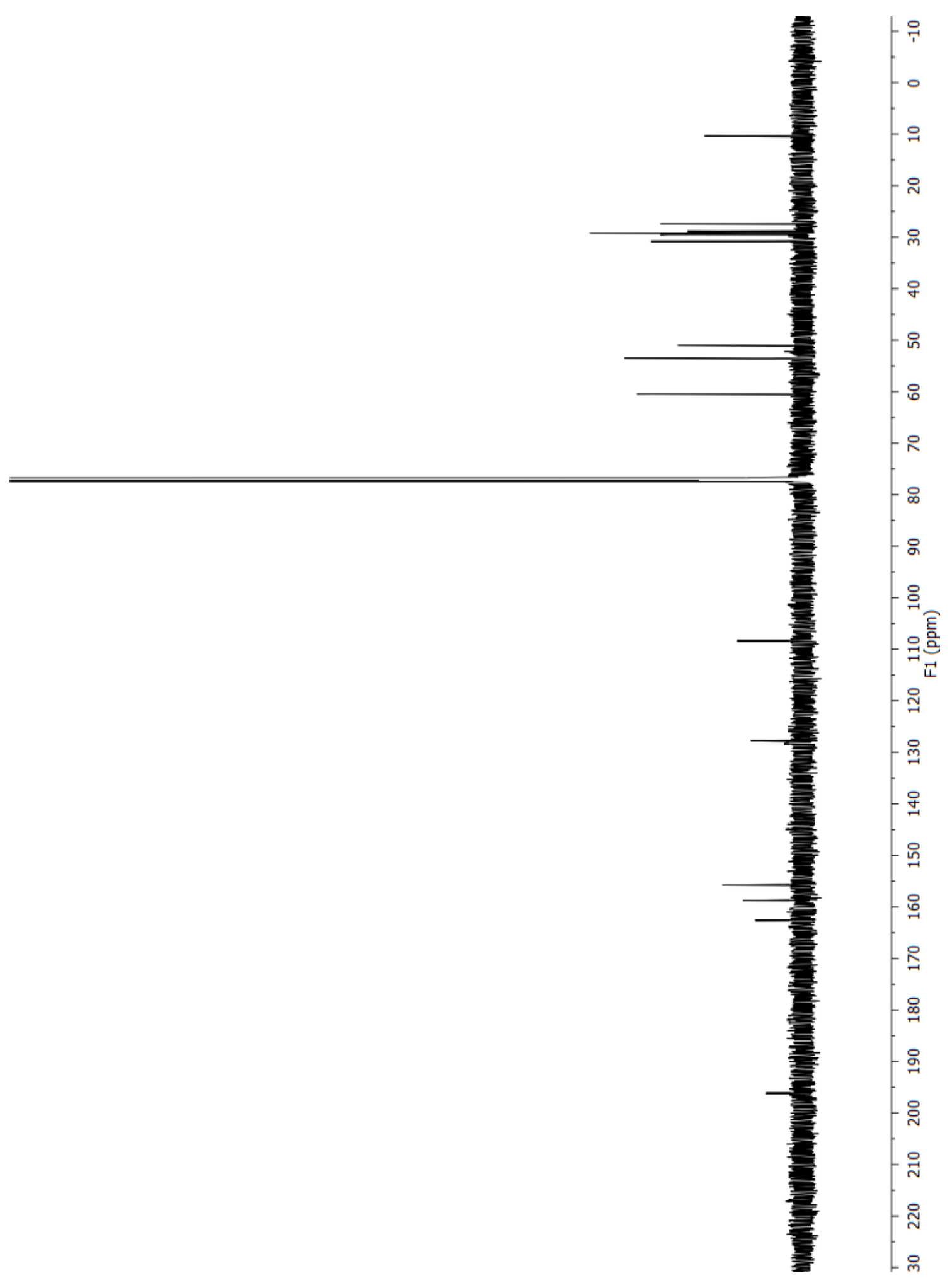

${ }^{13} \mathrm{C}$ NMR spectrum of lehualide $\mathrm{H}(\mathbf{7 2})\left(150 \mathrm{MHz}, \mathrm{CDCl}_{3}\right)$. 
Lehualide I

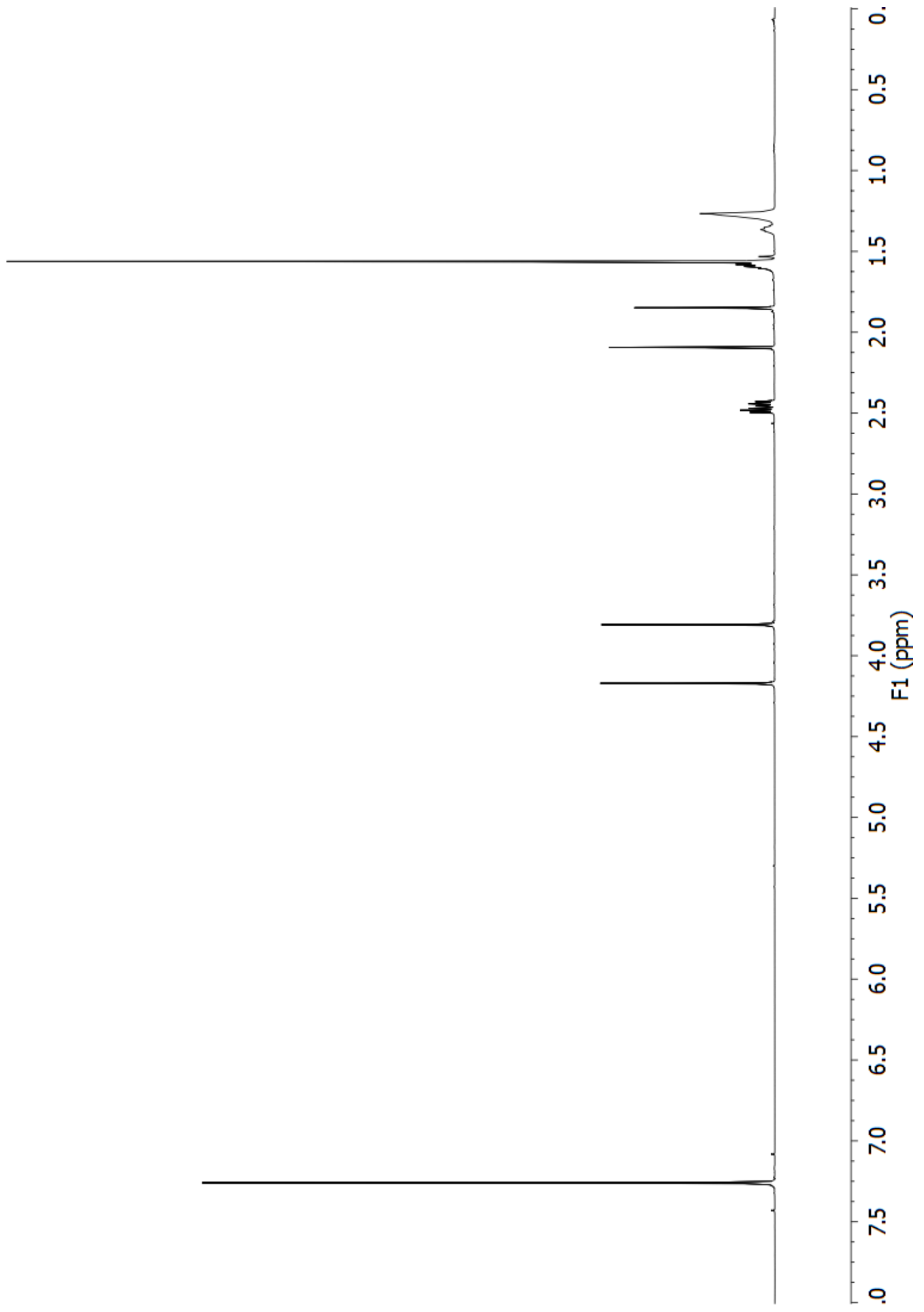

${ }^{1} \mathrm{H}$ NMR spectrum of lehualide I (73) $\left(600 \mathrm{MHz}, \mathrm{CDCl}_{3}\right)$. 


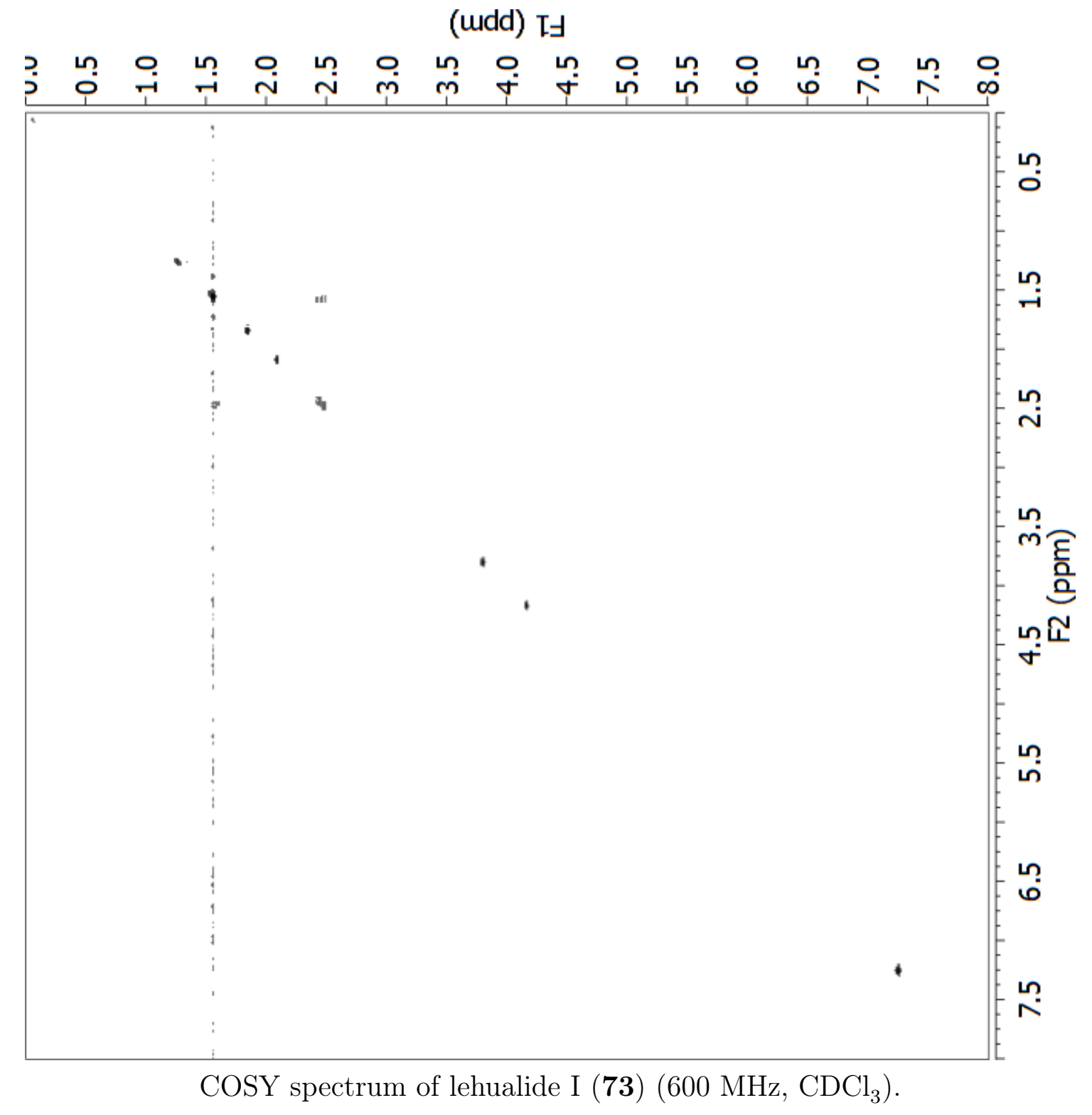


(udd) 더

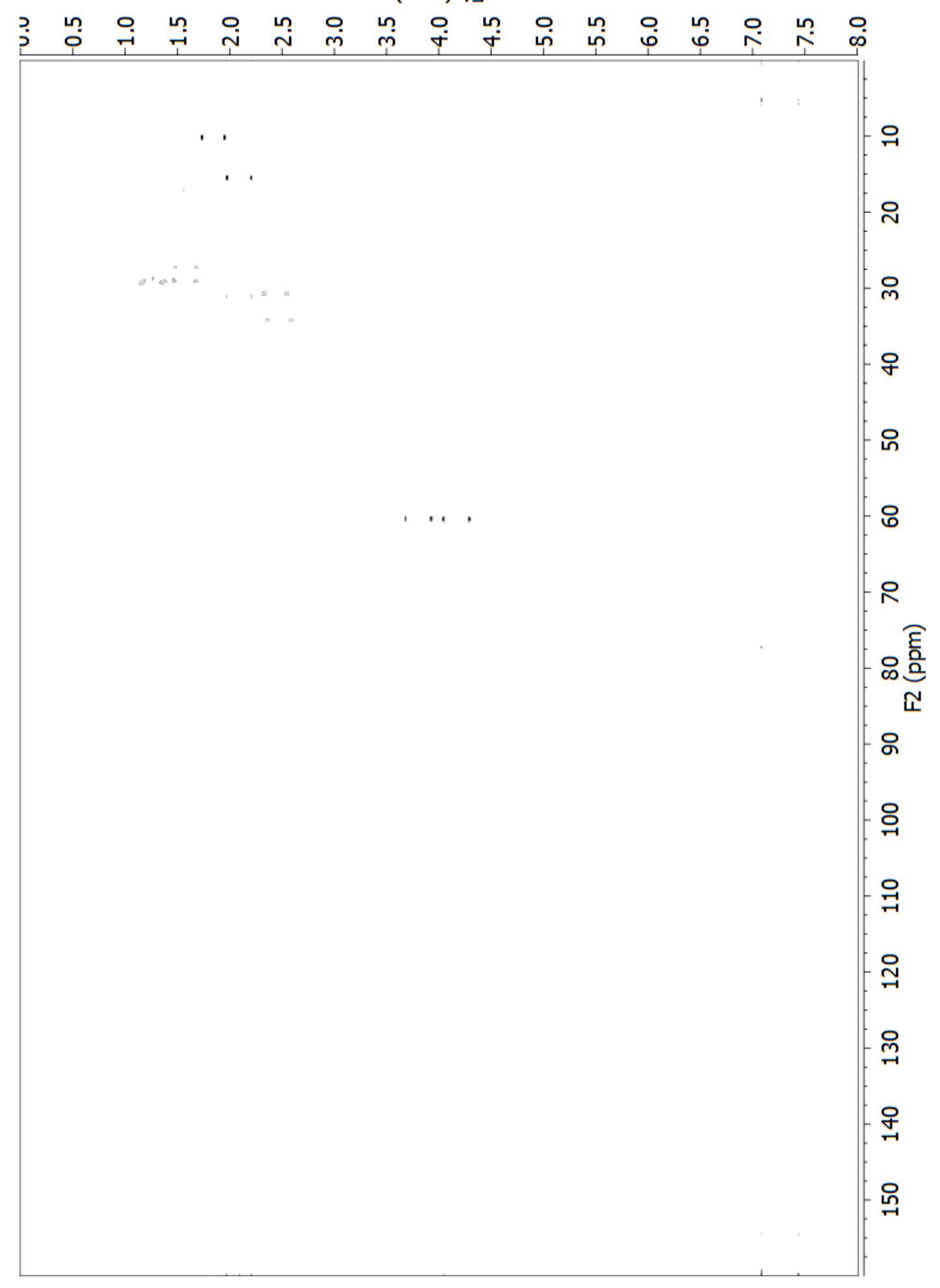

Fully-coupled HSQC spectrum of lehualide I (73) (600 MHz, $\mathrm{CDCl}_{3}$ ). 


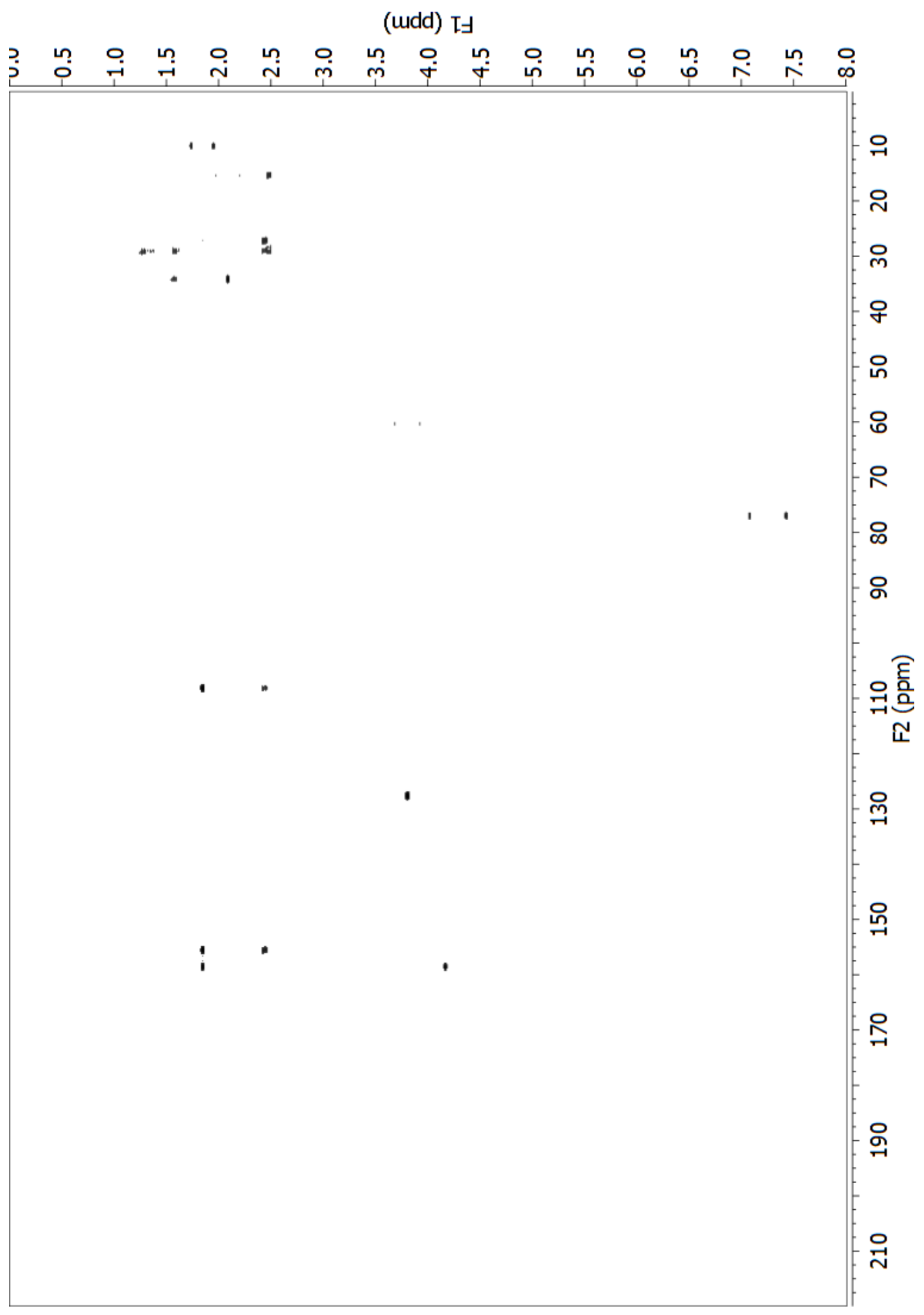

HMBC spectrum of lehualide I (73) $\left(600 \mathrm{MHz}, \mathrm{CDCl}_{3}\right)$.

272 


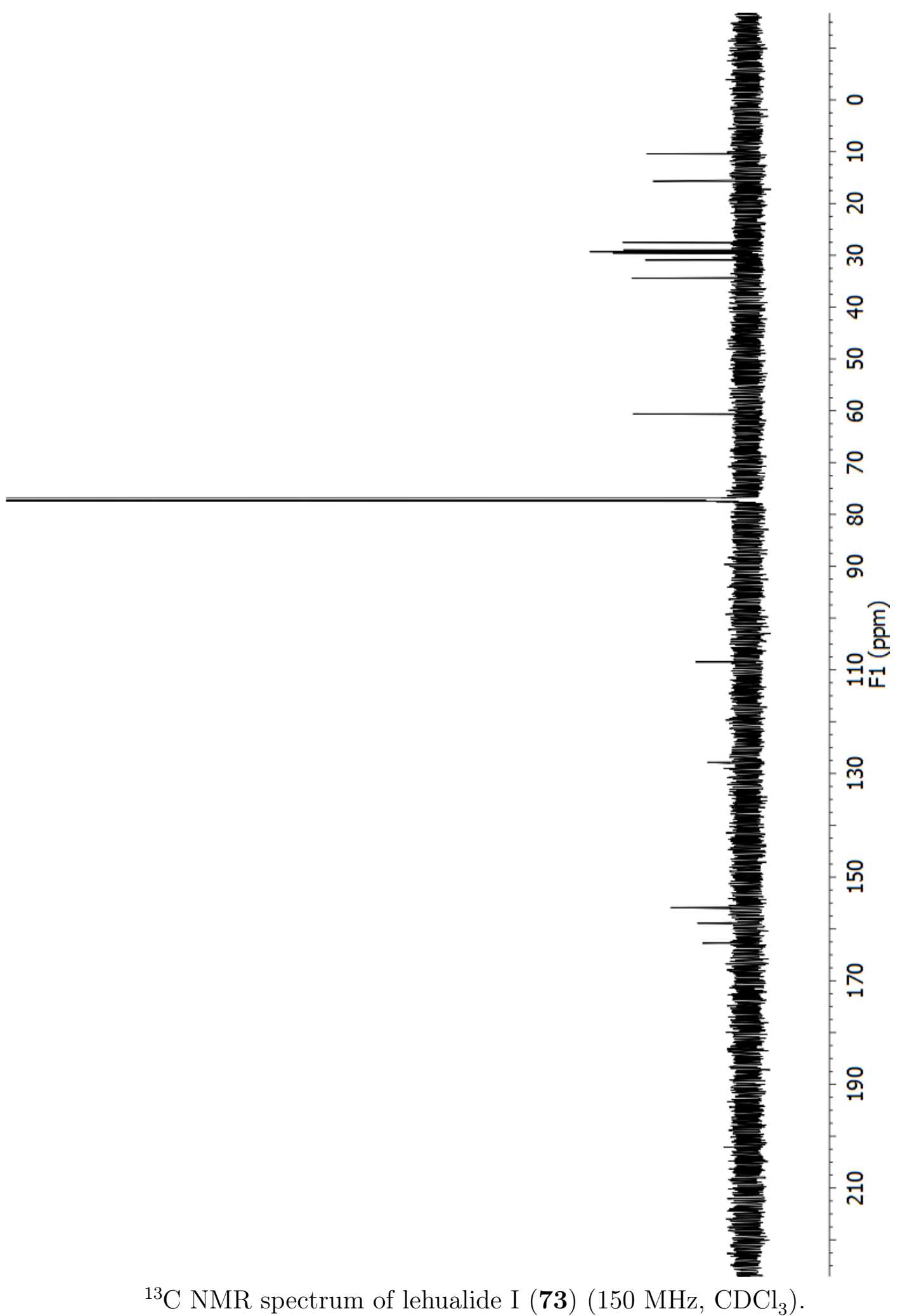


Lehualide J

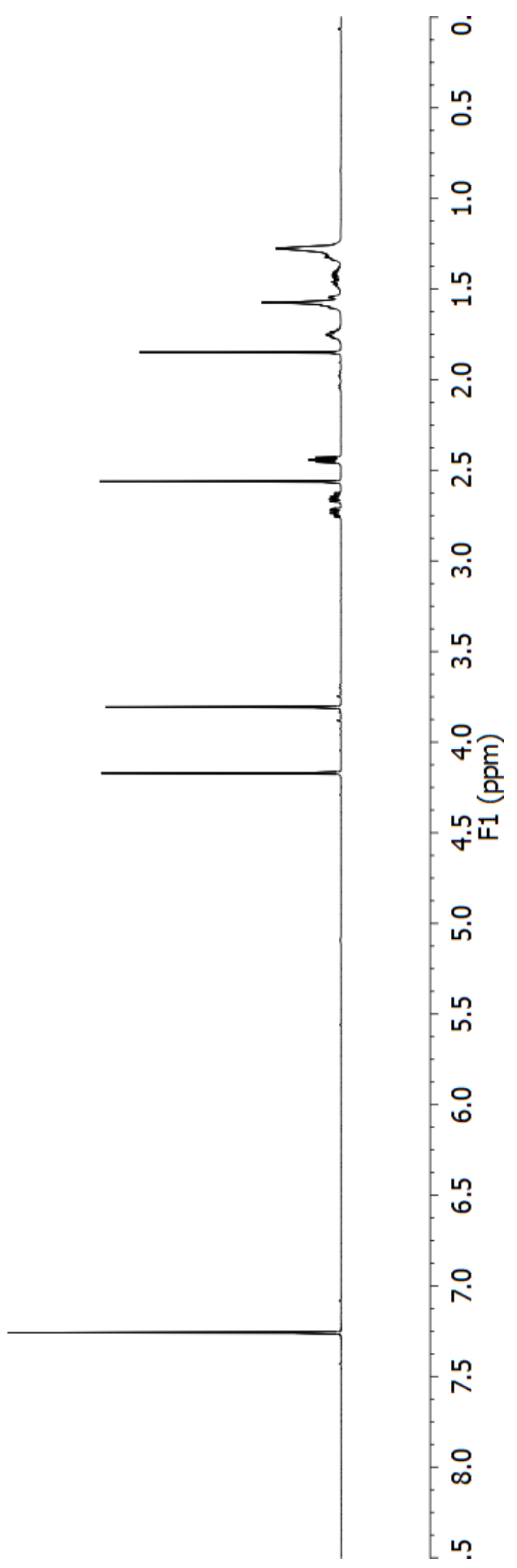

${ }^{1} \mathrm{H}$ NMR spectrum of lehualide $\mathrm{J}(\mathbf{7 4})\left(600 \mathrm{MHz}, \mathrm{CDCl}_{3}\right)$. 
(udd) It

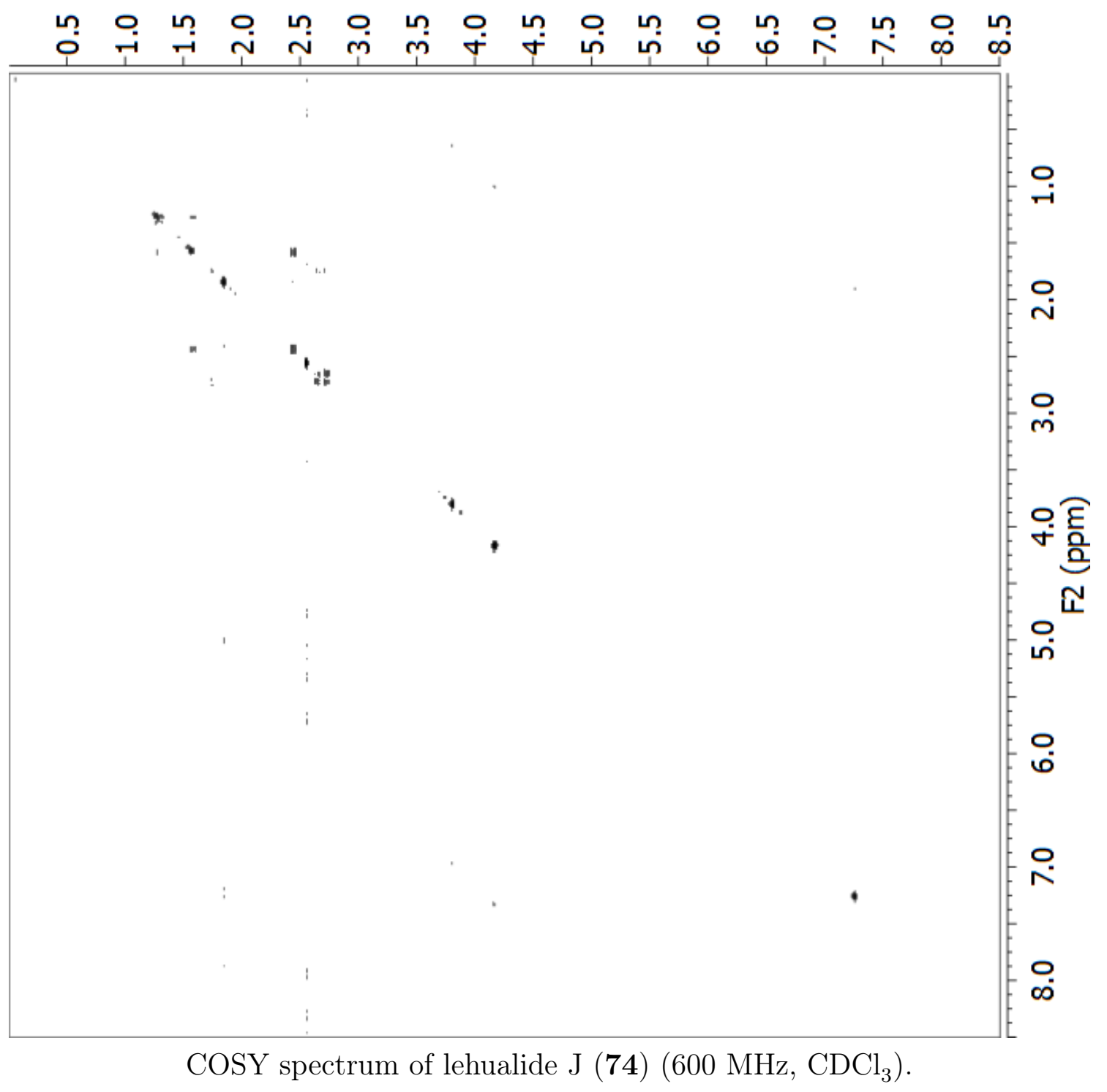


(udd) 더

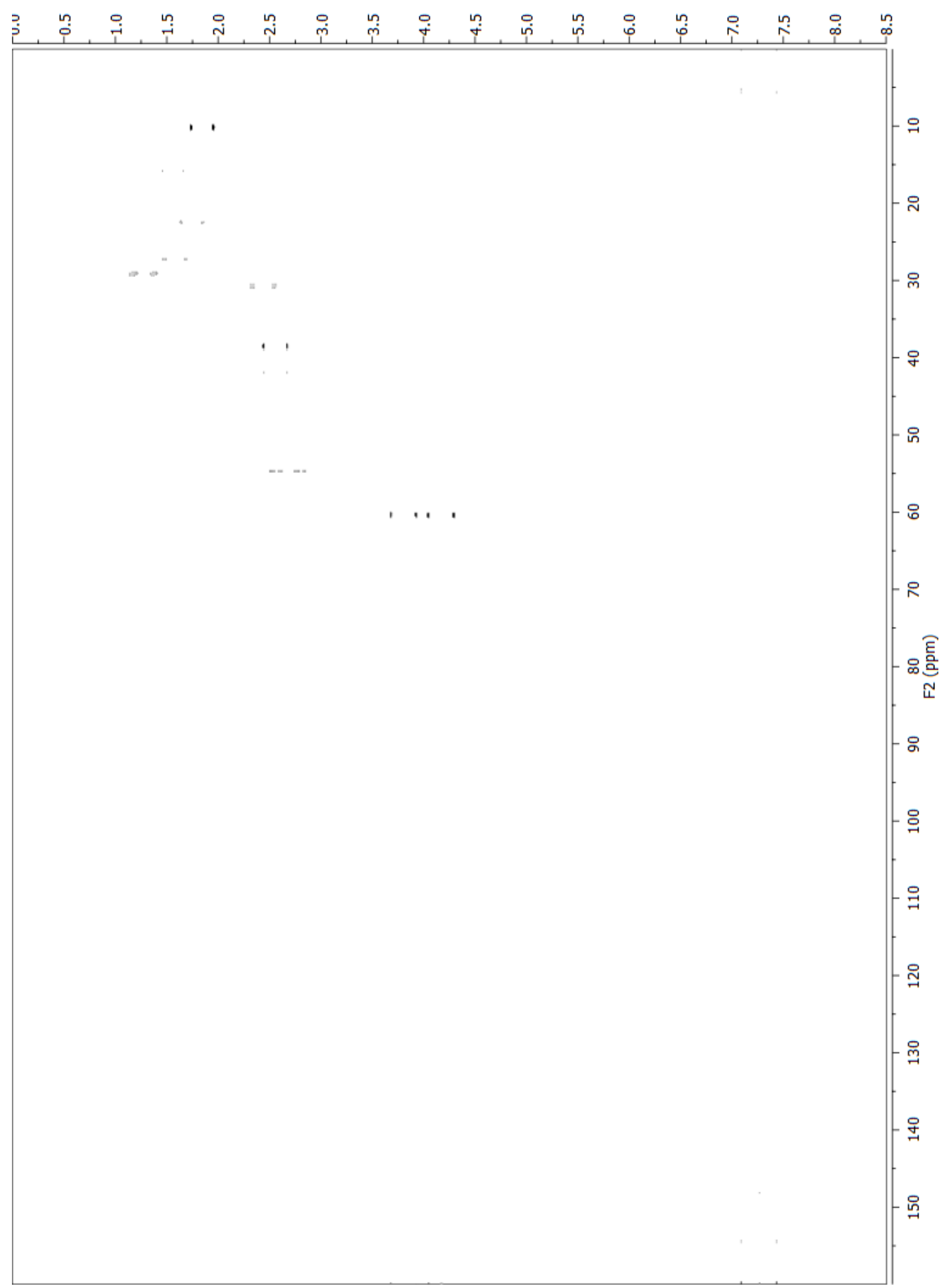

Fully-coupled HSQC spectrum of lehualide $\mathrm{J}(\mathbf{7 4})\left(600 \mathrm{MHz}, \mathrm{CDCl}_{3}\right)$. 
(udd) 더

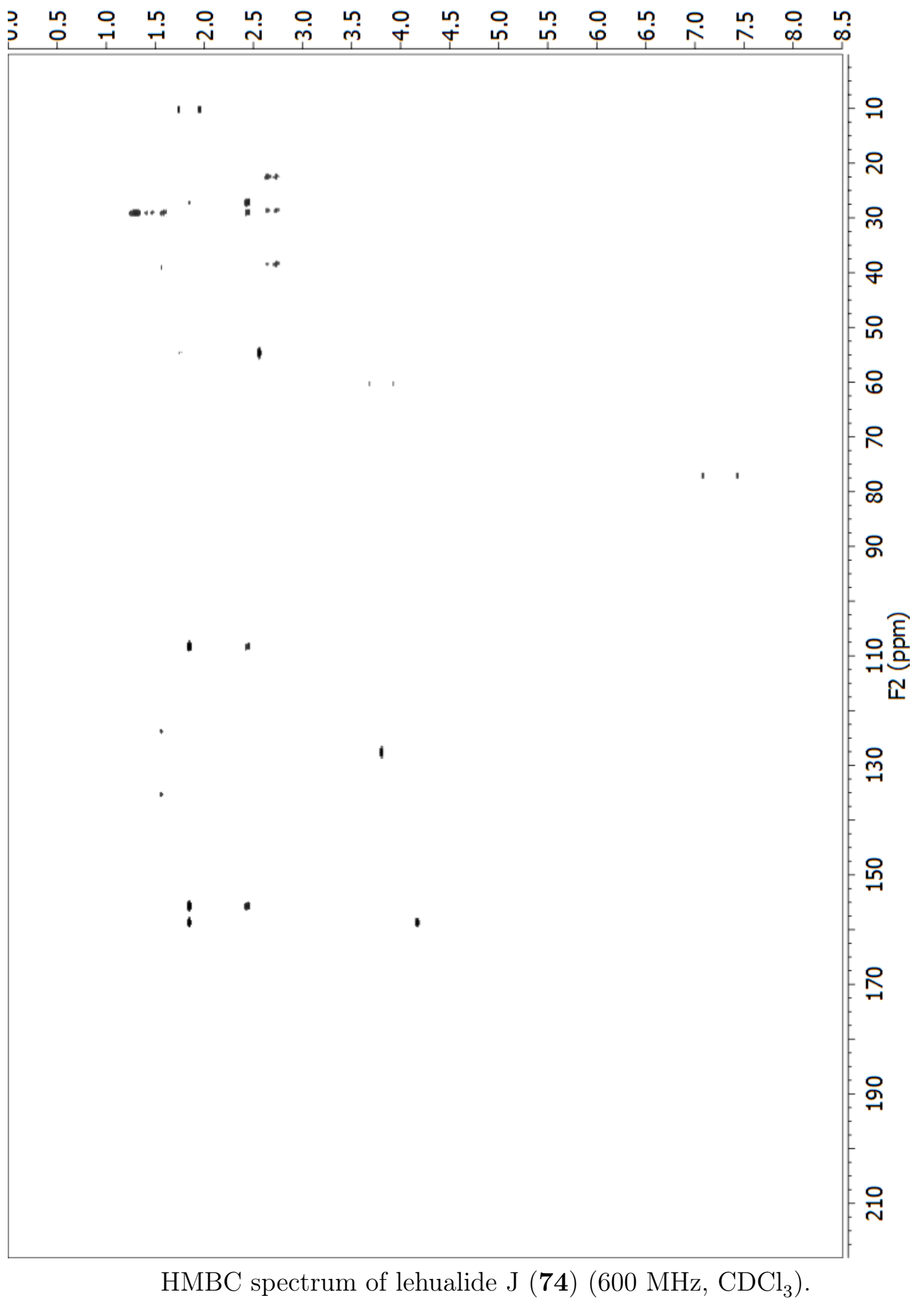




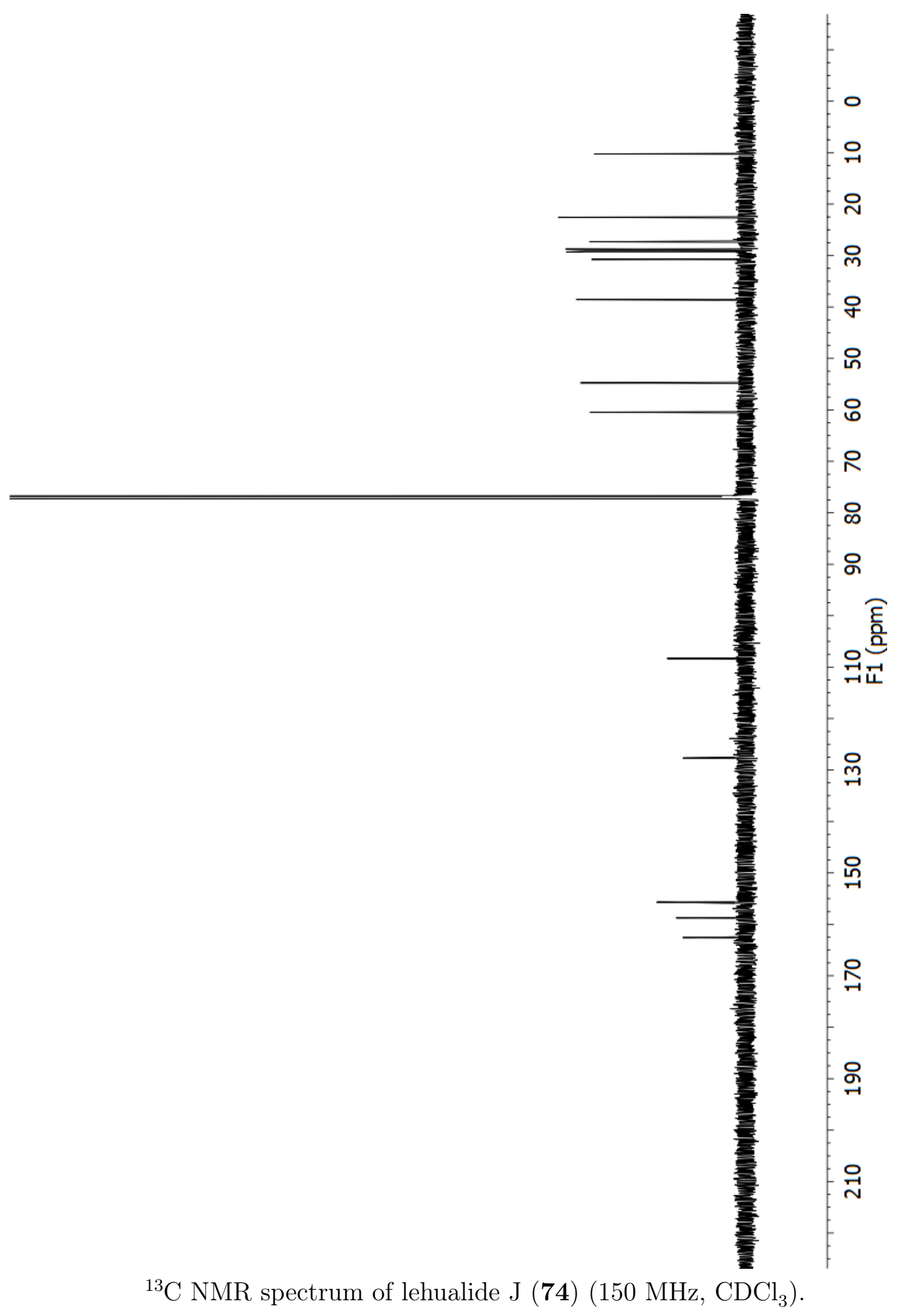




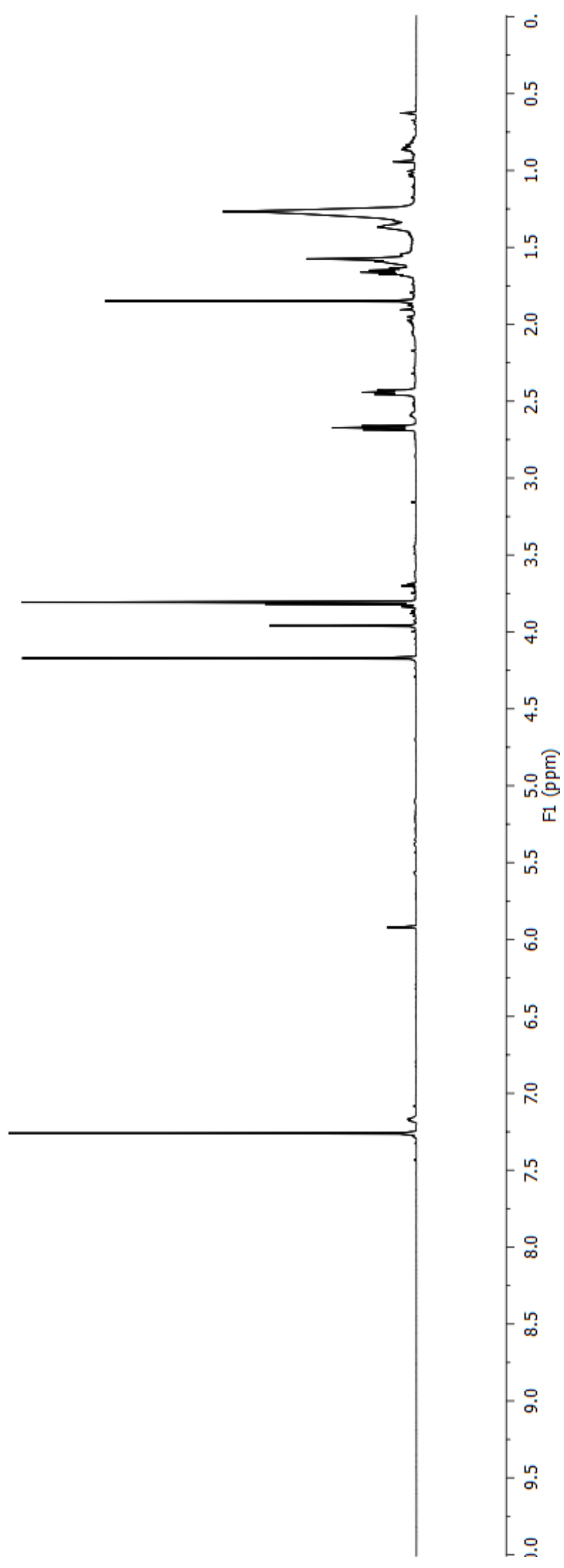

${ }^{1} \mathrm{H}$ NMR spectrum of lehualide $\mathrm{K}(\mathbf{7 5})\left(600 \mathrm{MHz}, \mathrm{CDCl}_{3}\right)$. 
(wdd) $\mathrm{I}\lrcorner$

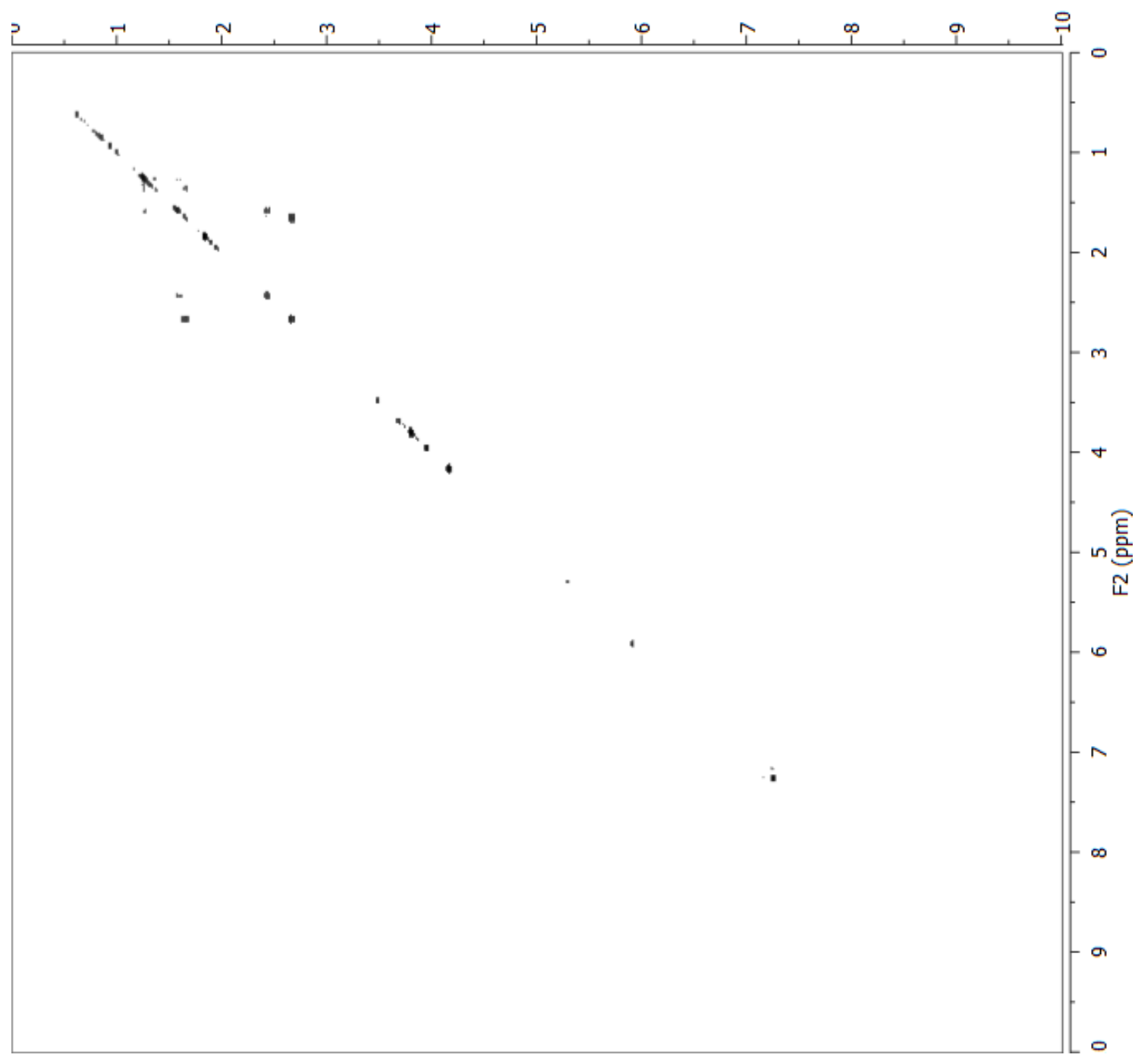

COSY spectrum of lehualide $\mathrm{K}(\mathbf{7 5})\left(600 \mathrm{MHz}, \mathrm{CDCl}_{3}\right)$. 
(udd) $\mathrm{I}\lrcorner$

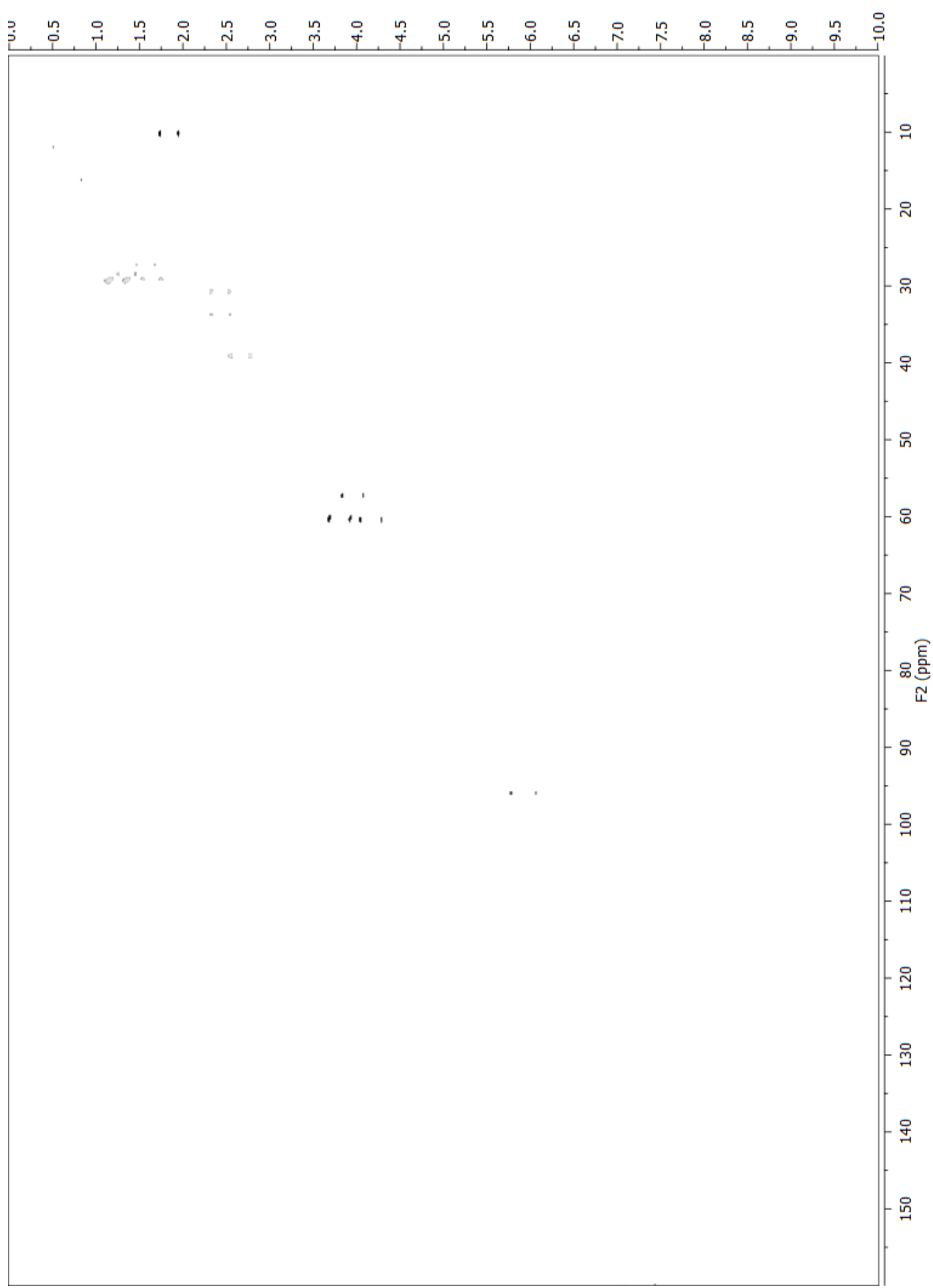

Fully-coupled HSQC spectrum of lehualide $\mathrm{K}(\mathbf{7 5})\left(600 \mathrm{MHz}, \mathrm{CDCl}_{3}\right)$. 


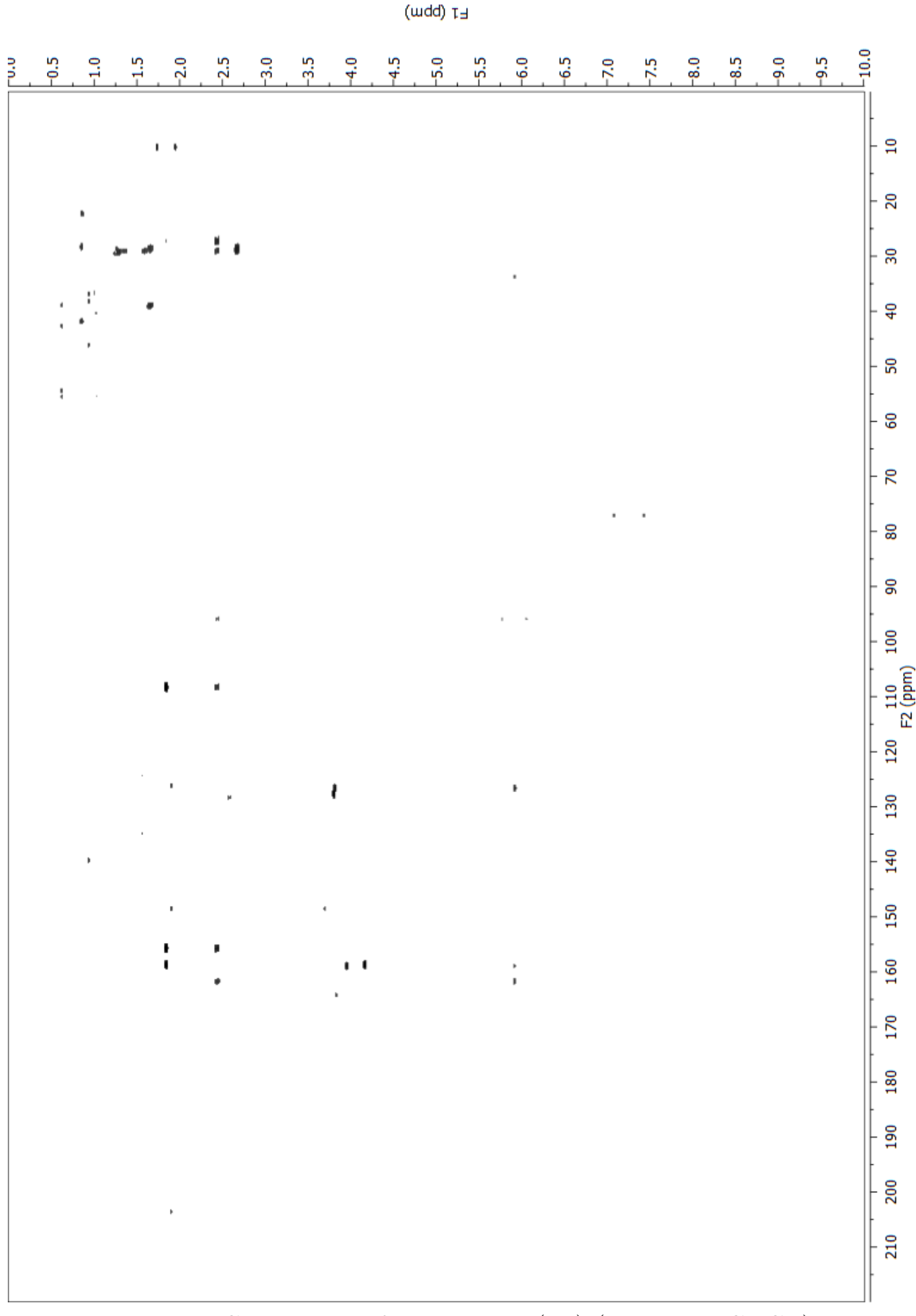

HMBC spectrum of lehualide $\mathrm{K}(\mathbf{7 5})\left(600 \mathrm{MHz}, \mathrm{CDCl}_{3}\right)$.

282 


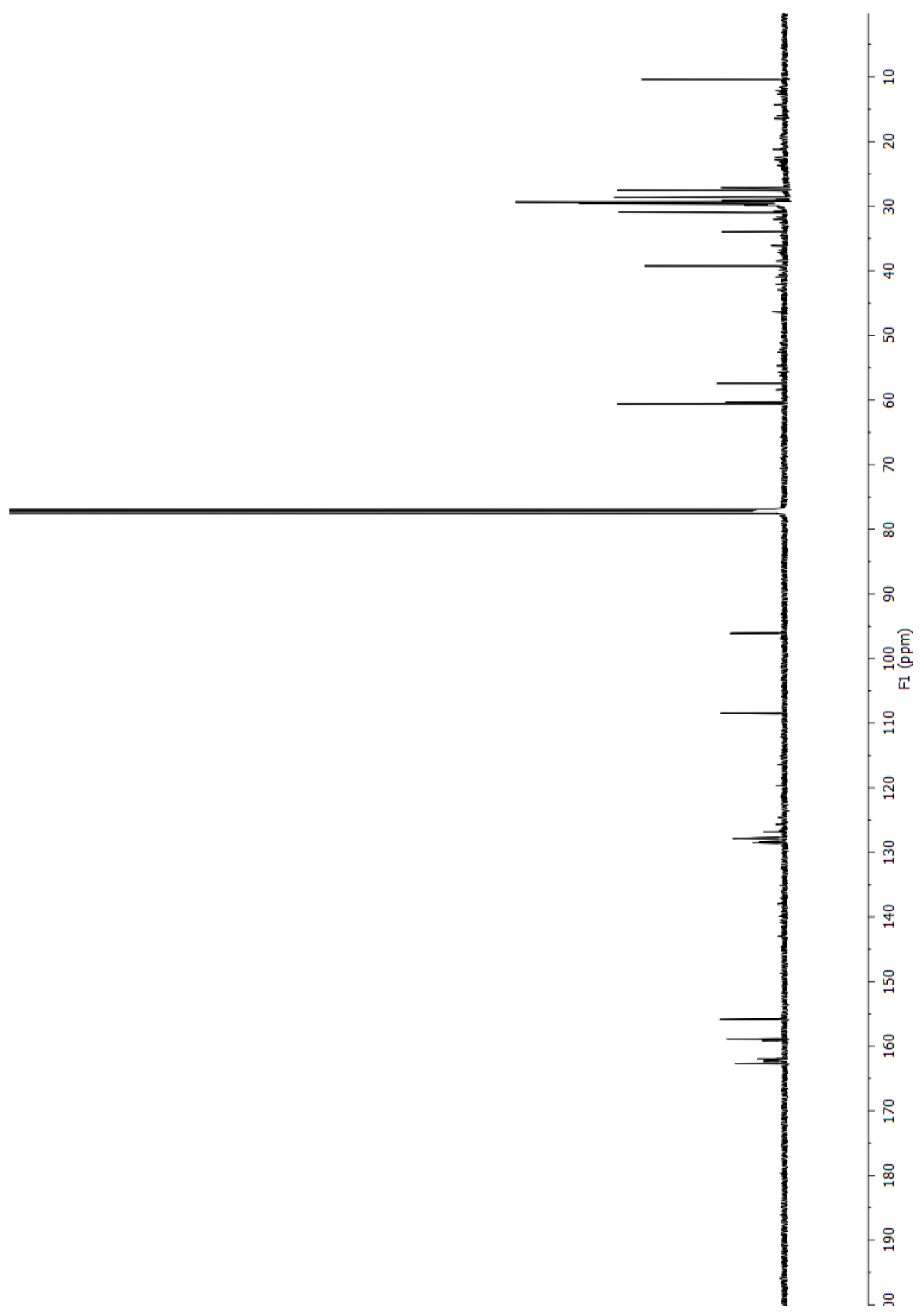

${ }^{13} \mathrm{C}$ NMR spectrum of lehualide $\mathrm{K}(\mathbf{7 5})\left(150 \mathrm{MHz}^{\mathrm{CDCl}}{ }_{3}\right)$. 
Appendix G

NMR Spectra of New Cyclic

Peroxides 
Epiplakinic Acid I

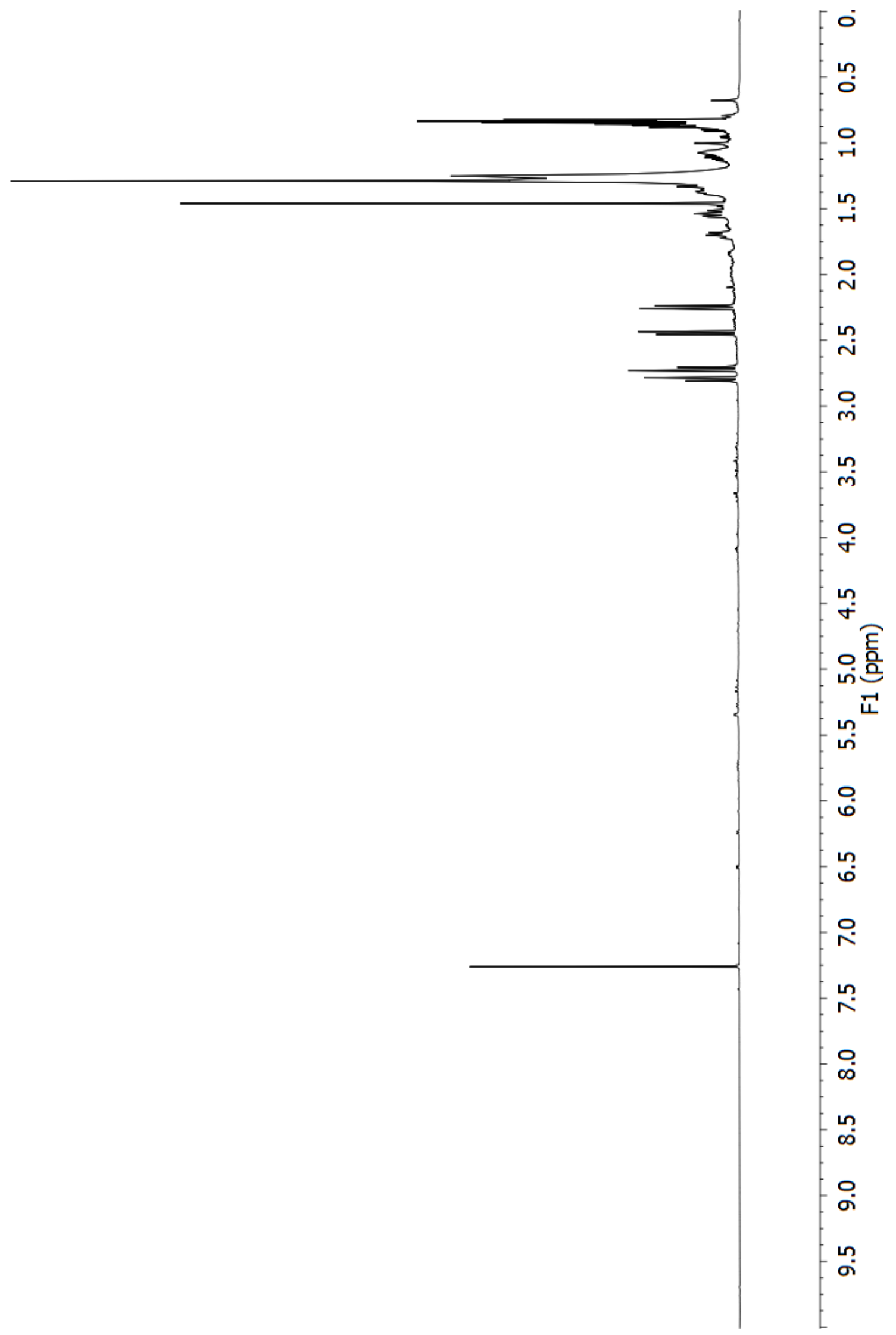

${ }^{1} \mathrm{H}$ NMR spectrum of epiplakinic acid I (95) (600 MHz, $\mathrm{CDCl}_{3}$ ). 
(udd) IJ

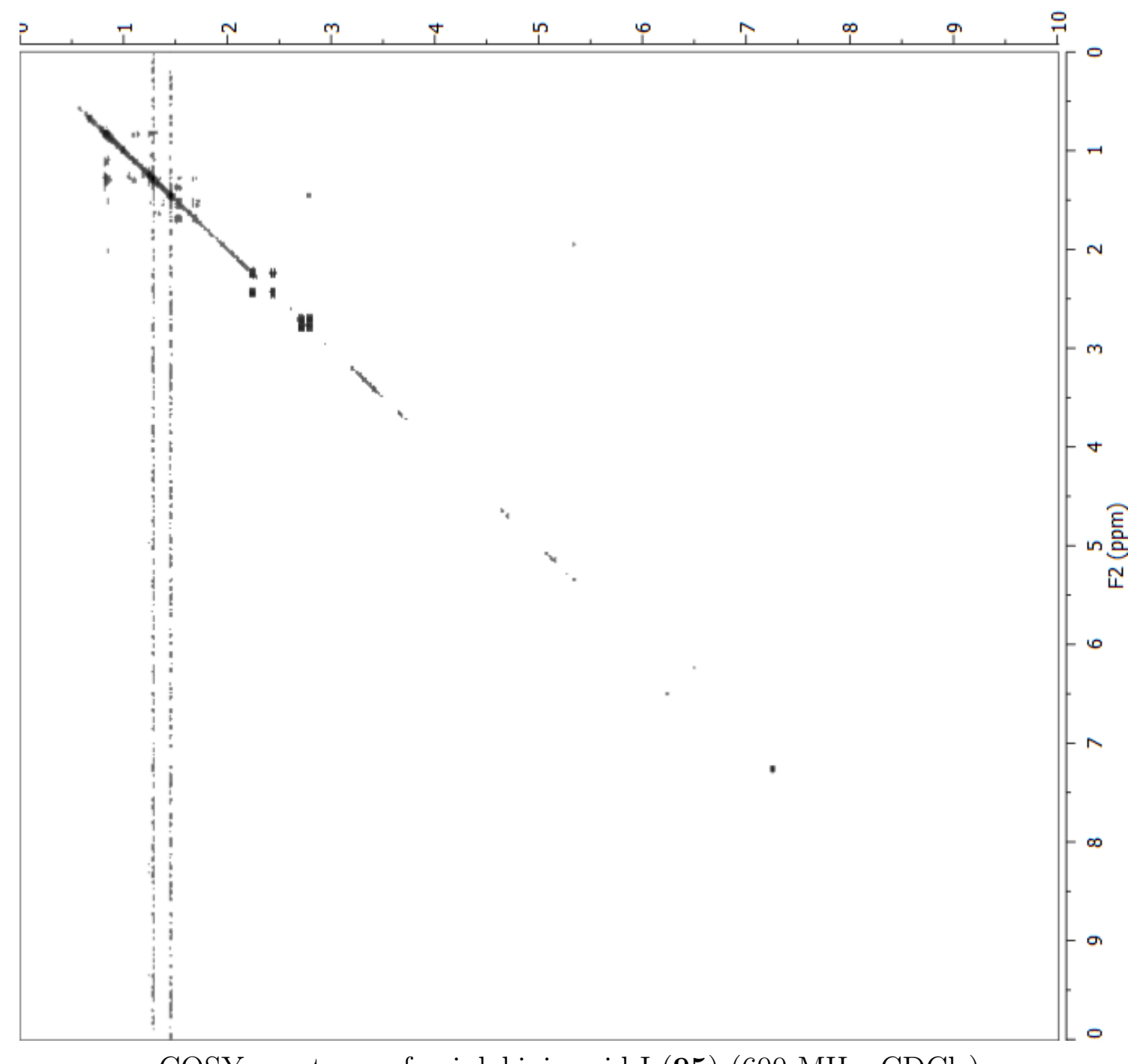

COSY spectrum of epiplakinic acid I (95) $\left(600 \mathrm{MHz}, \mathrm{CDCl}_{3}\right)$. 
(udd) $\mathrm{I}\lrcorner$

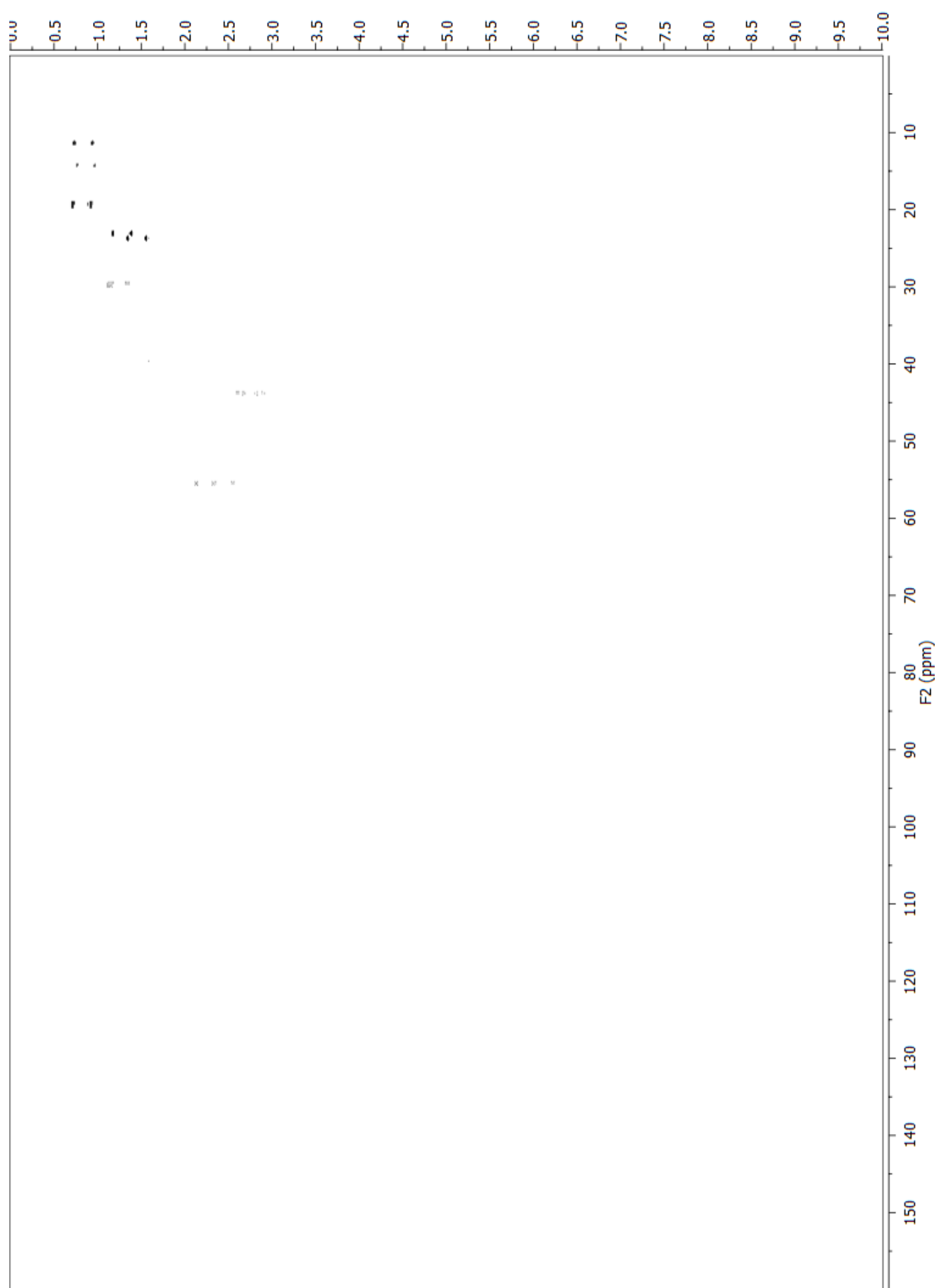

Fully-coupled HSQC spectrum of epiplakinic acid I (95) $\left(600 \mathrm{MHz}, \mathrm{CDCl}_{3}\right)$. 


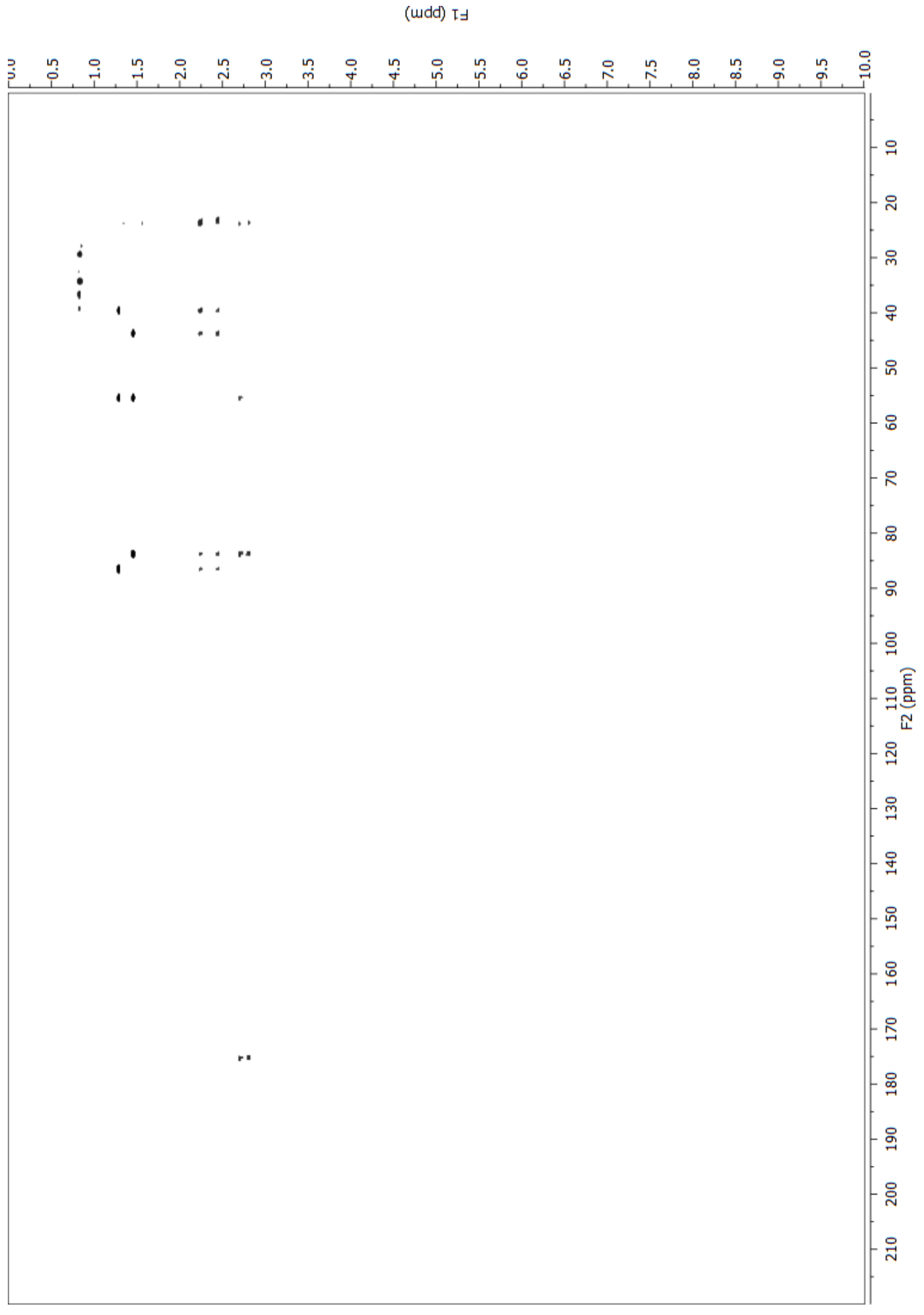

HMBC spectrum of epiplakinic acid I (95) $\left(600 \mathrm{MHz}, \mathrm{CDCl}_{3}\right)$.

288 


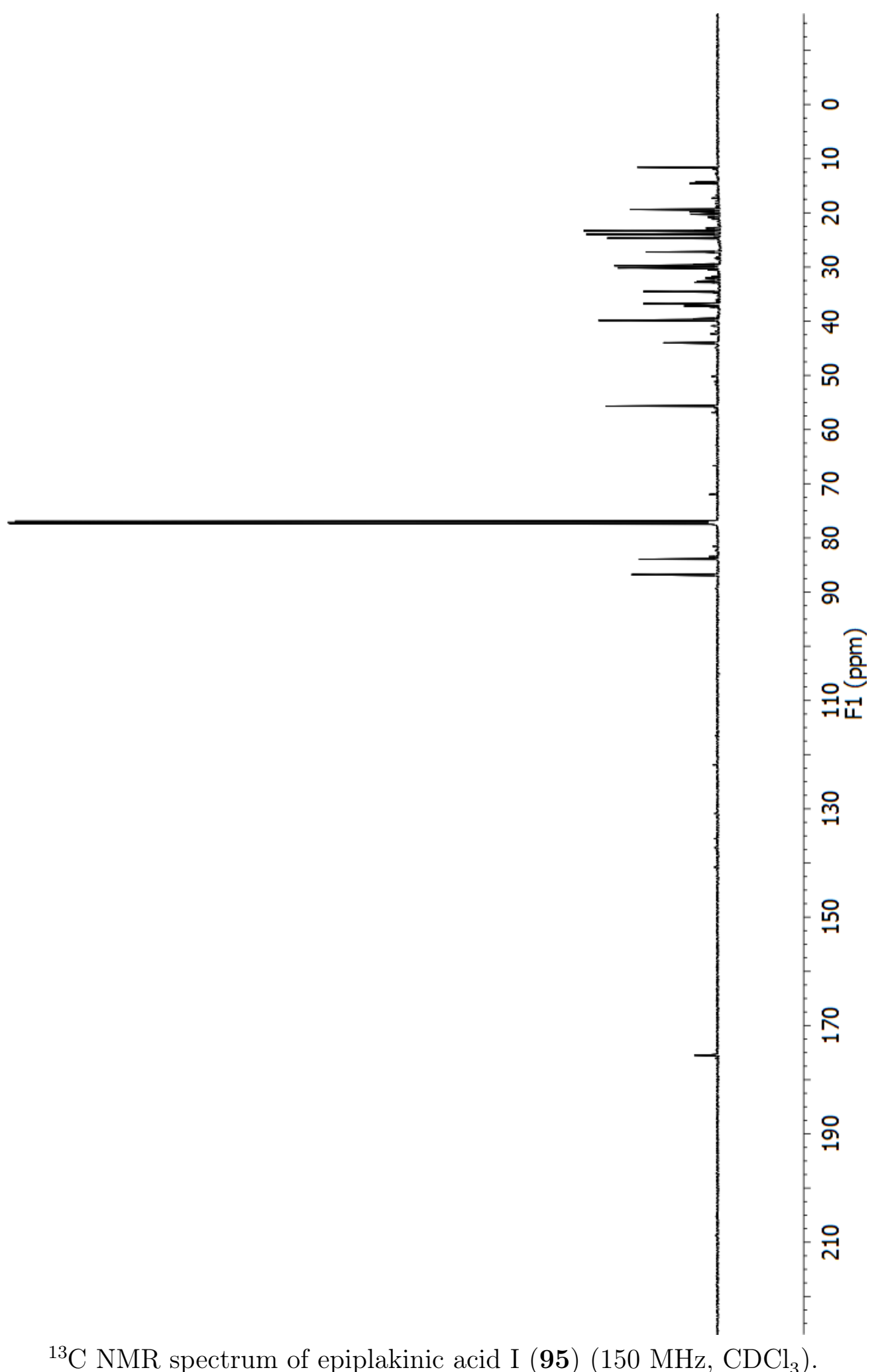

${ }^{13} \mathrm{C}$ NMR spectrum of epiplakinic acid I (95) (150 MHz, $\mathrm{CDCl}_{3}$ ). 


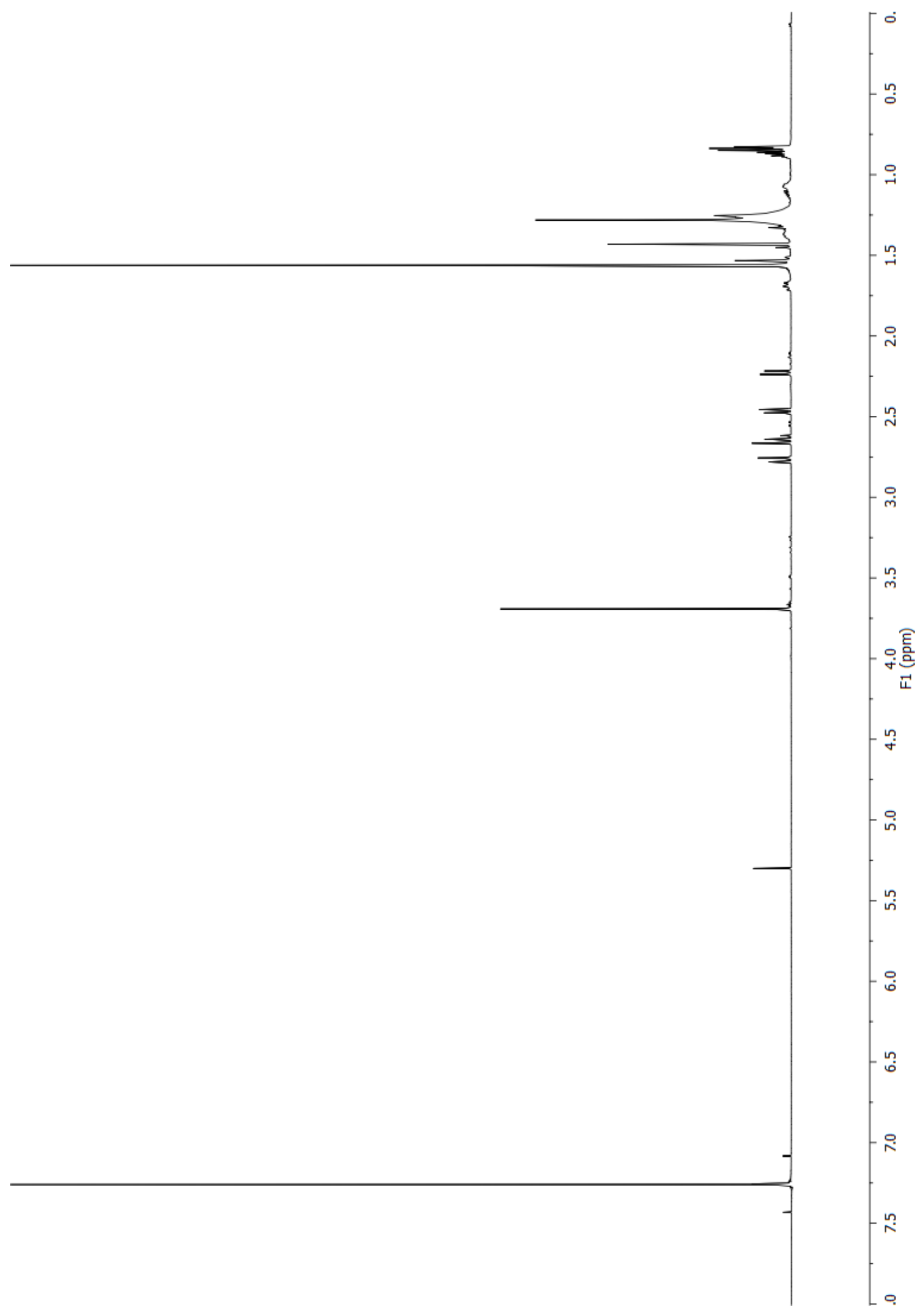

${ }^{1} \mathrm{H}$ NMR spectrum of epiplakinic acid I methyl ester (96) (600 MHz, $\mathrm{CDCl}_{3}$ ). 
(udd) $T\lrcorner$

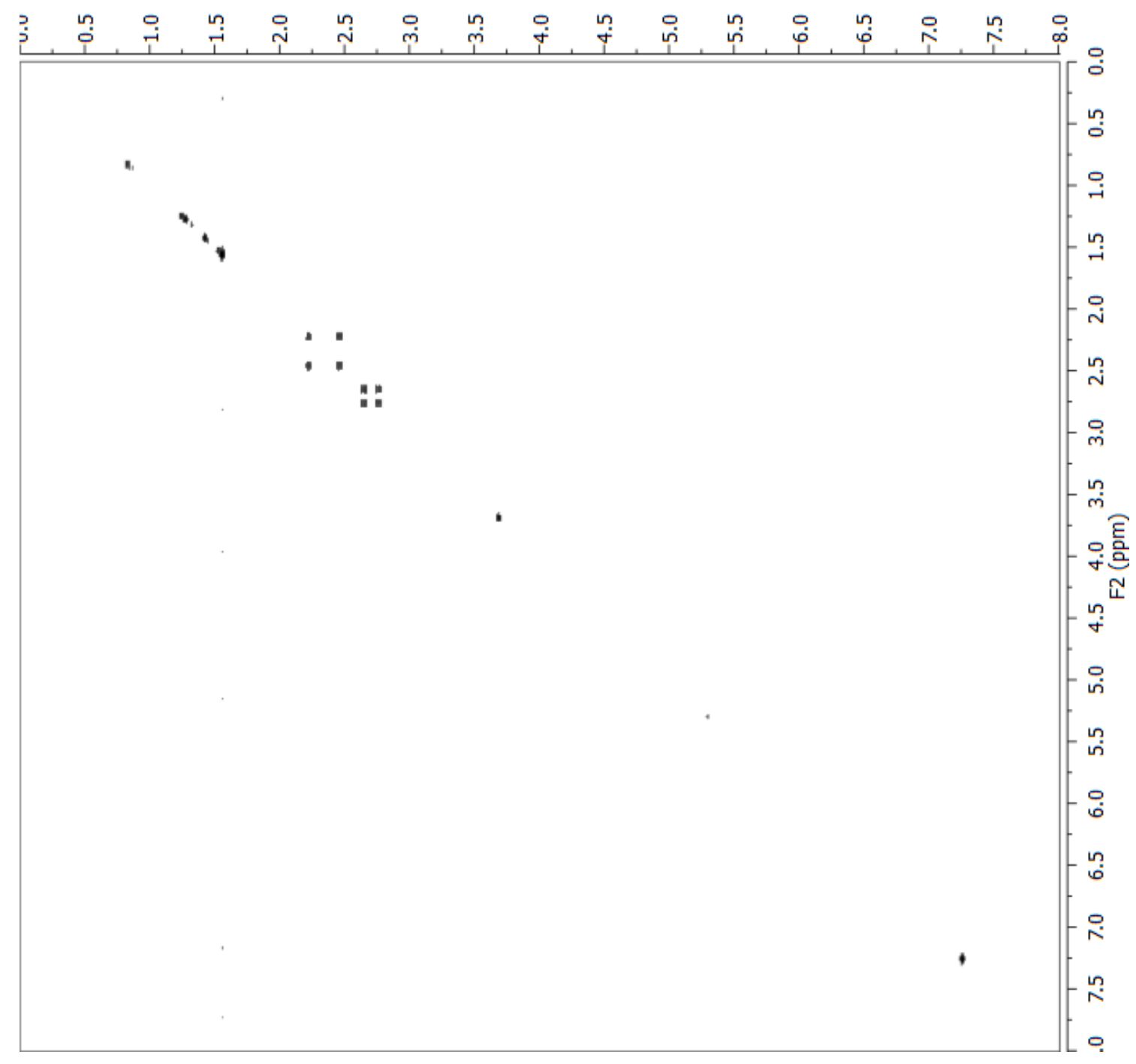

COSY spectrum of epiplakinic acid I methyl ester (96) $\left(600 \mathrm{MHz}, \mathrm{CDCl}_{3}\right.$ ). 
(udd) $\mathrm{I}\}$

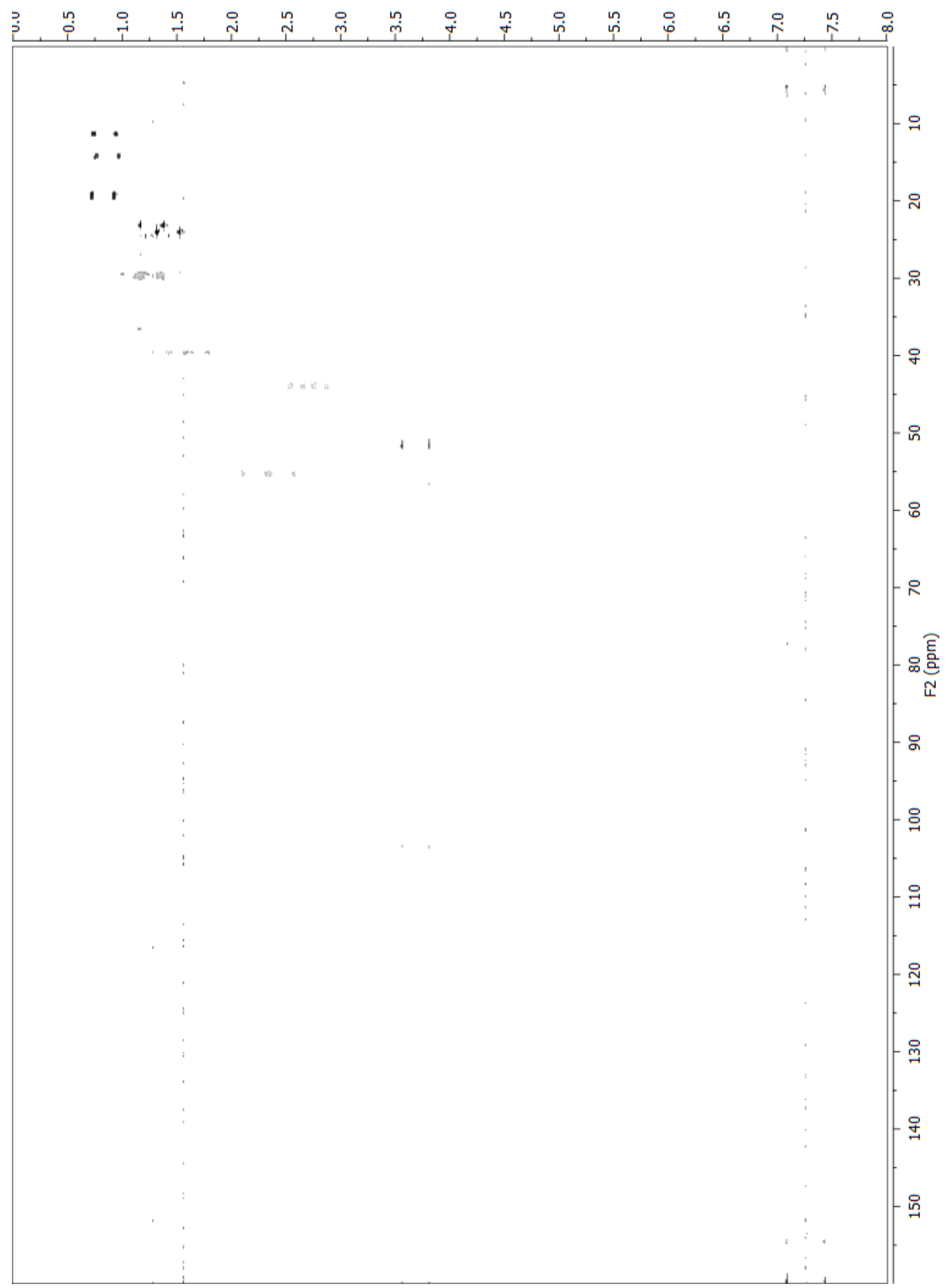

Fully-coupled HSQC spectrum of epiplakinic acid I methyl ester (96) $\left(600 \mathrm{MHz}, \mathrm{CDCl}_{3}\right)$. 
(udd) $\tau\lrcorner$

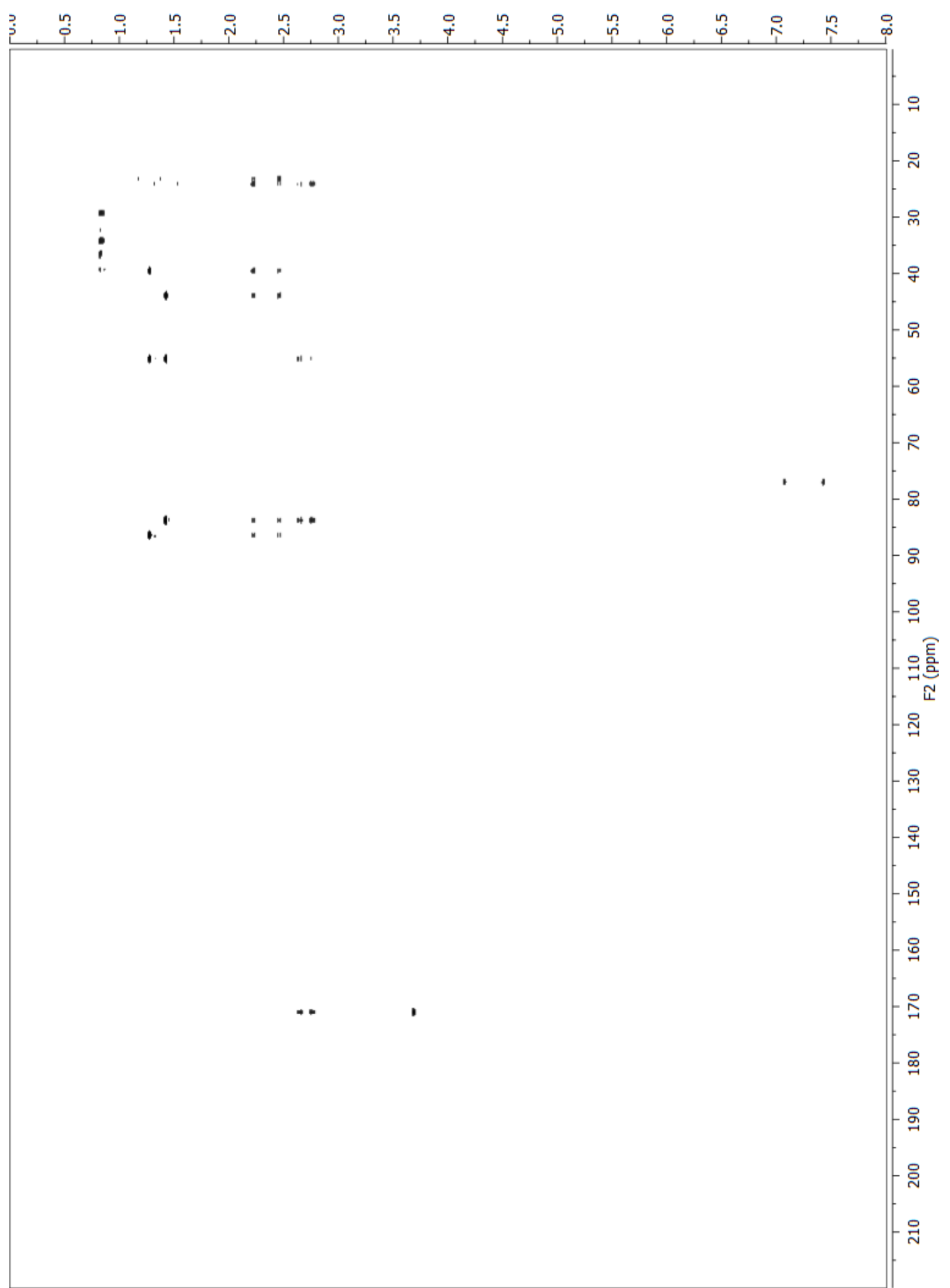

HMBC spectrum of epiplakinic acid I methyl ester (96) (600 MHz, $\mathrm{CDCl}_{3}$ ). 


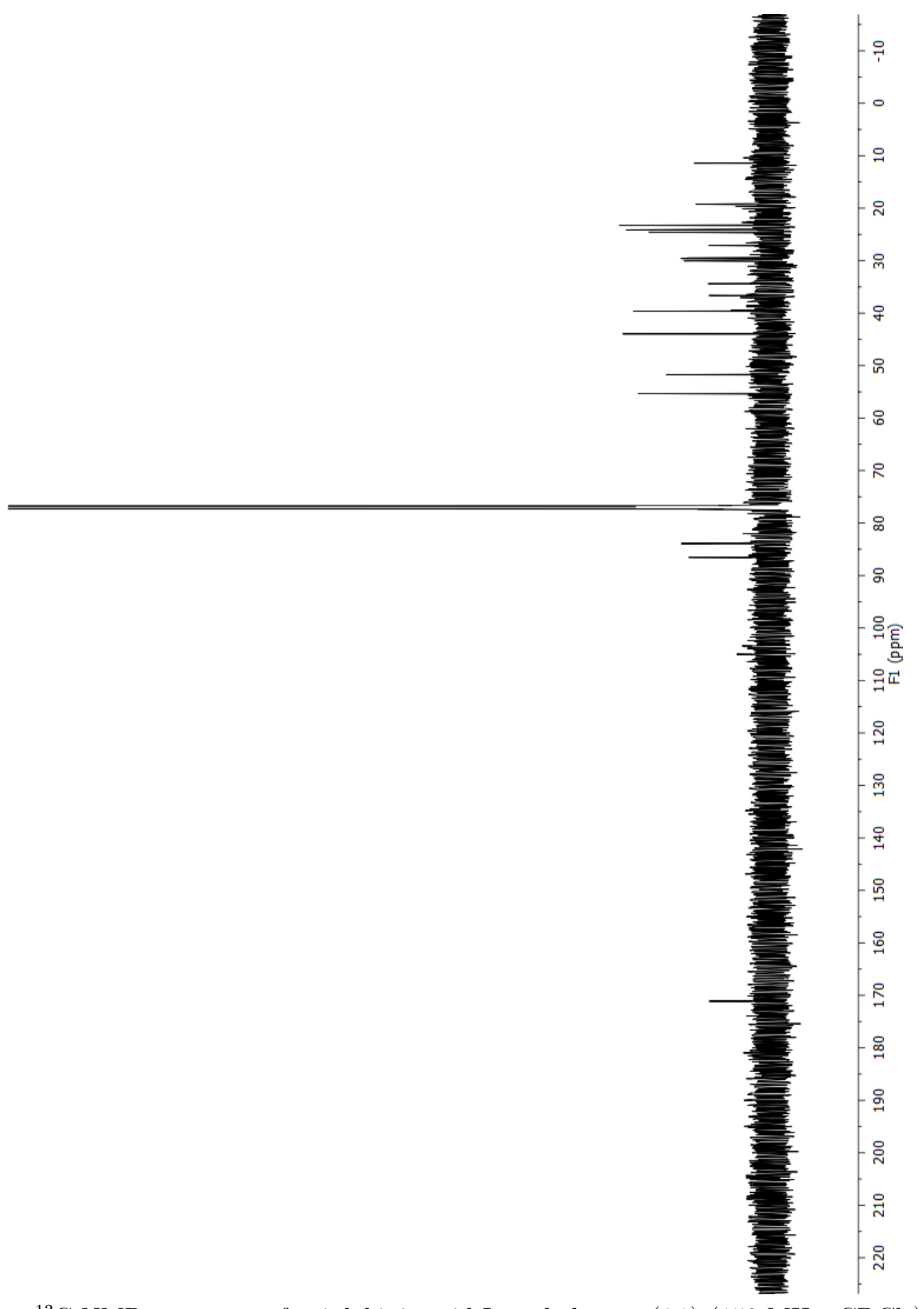

${ }^{13} \mathrm{C}$ NMR spectrum of epiplakinic acid I methyl ester (96) $\left(150 \mathrm{MHz}, \mathrm{CDCl}_{3}\right)$. 


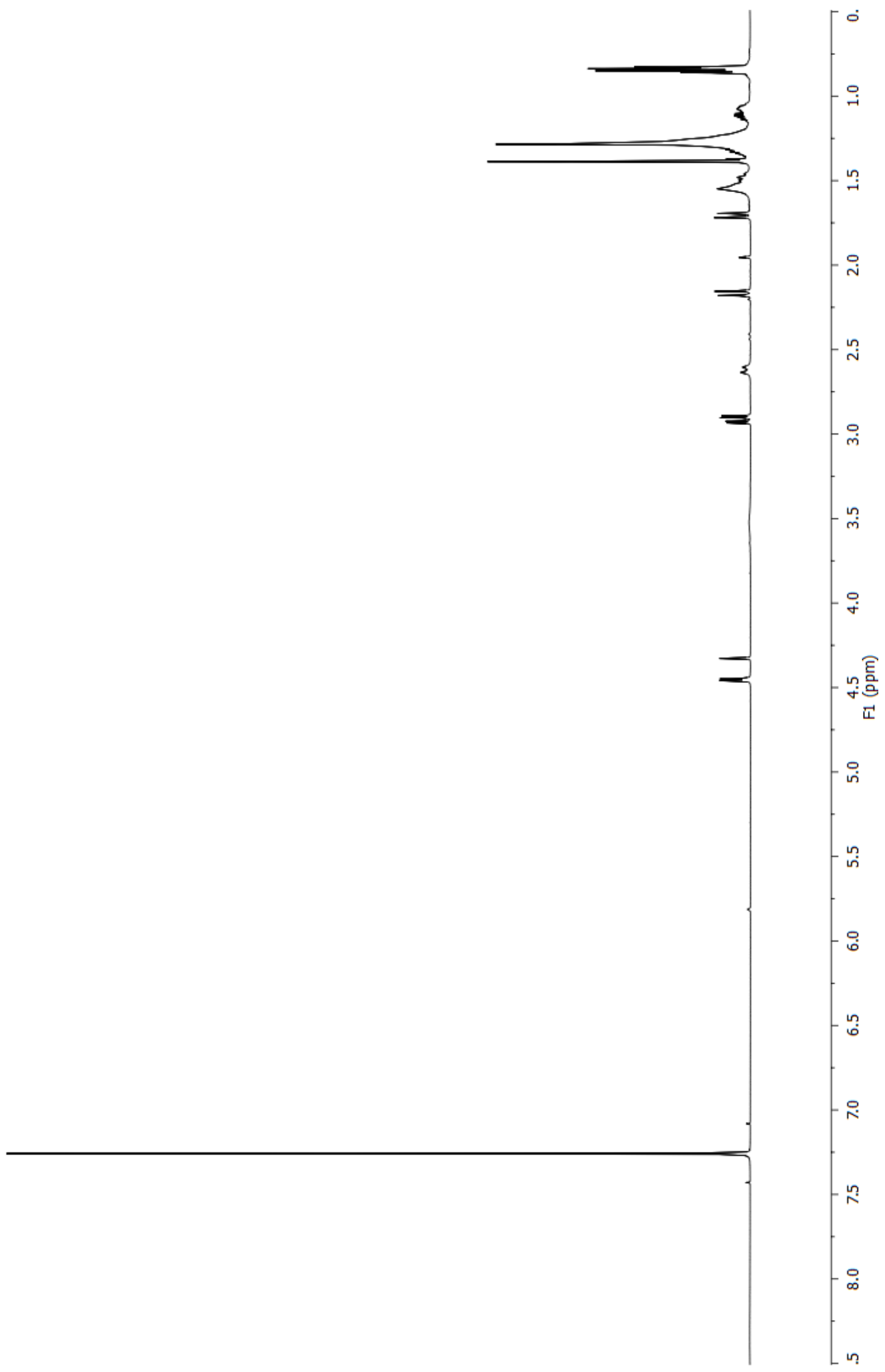

${ }^{1} \mathrm{H}$ NMR spectrum of plakortolide X (97) (600 $\mathrm{MHz}, \mathrm{CDCl}_{3}$ ). 
(udd) 더

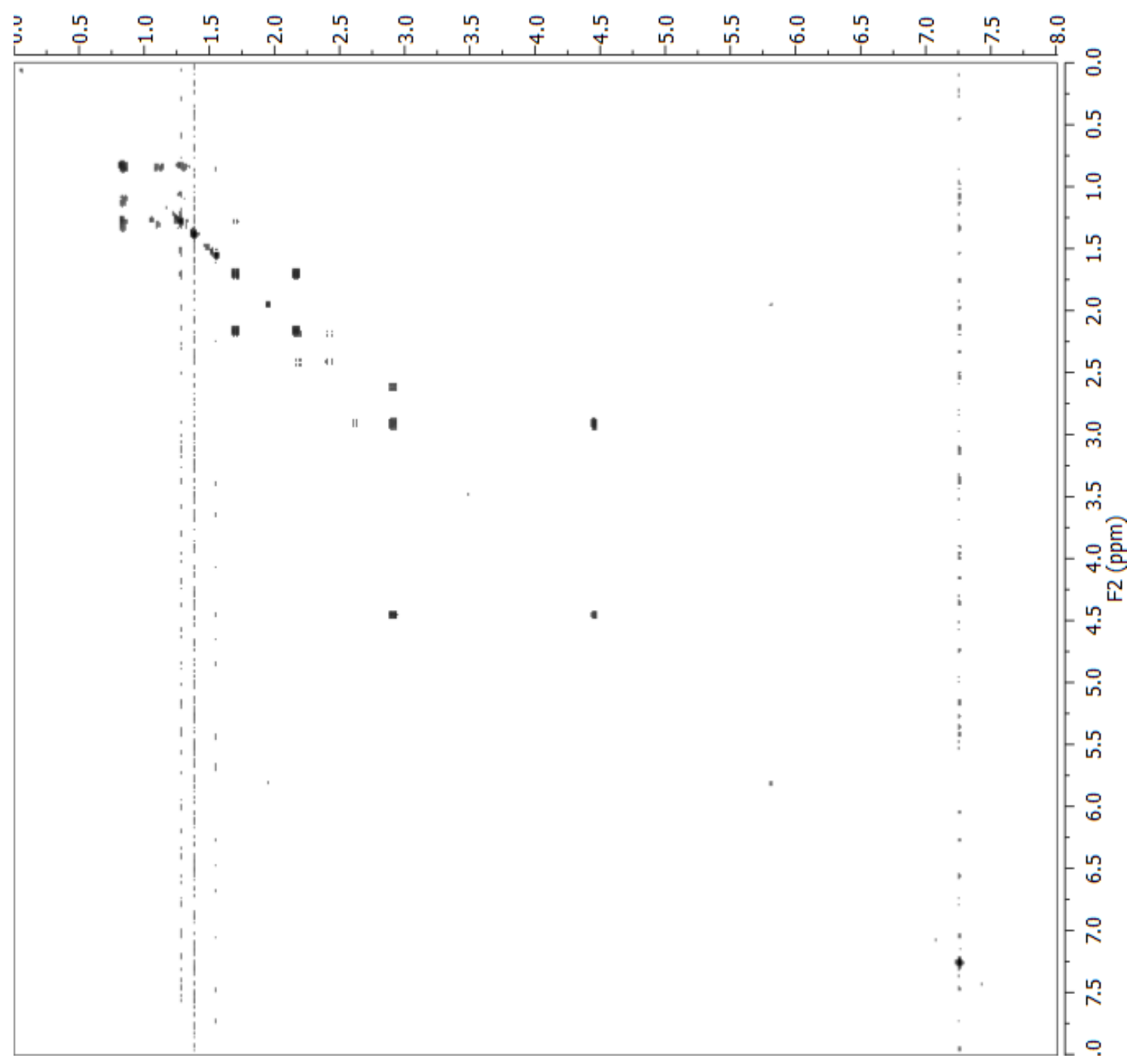

COSY spectrum of plakortolide X (97) $\left(600 \mathrm{MHz}^{\mathrm{C}} \mathrm{CDCl}_{3}\right)$. 
(udd) $\mathrm{I}\lrcorner$

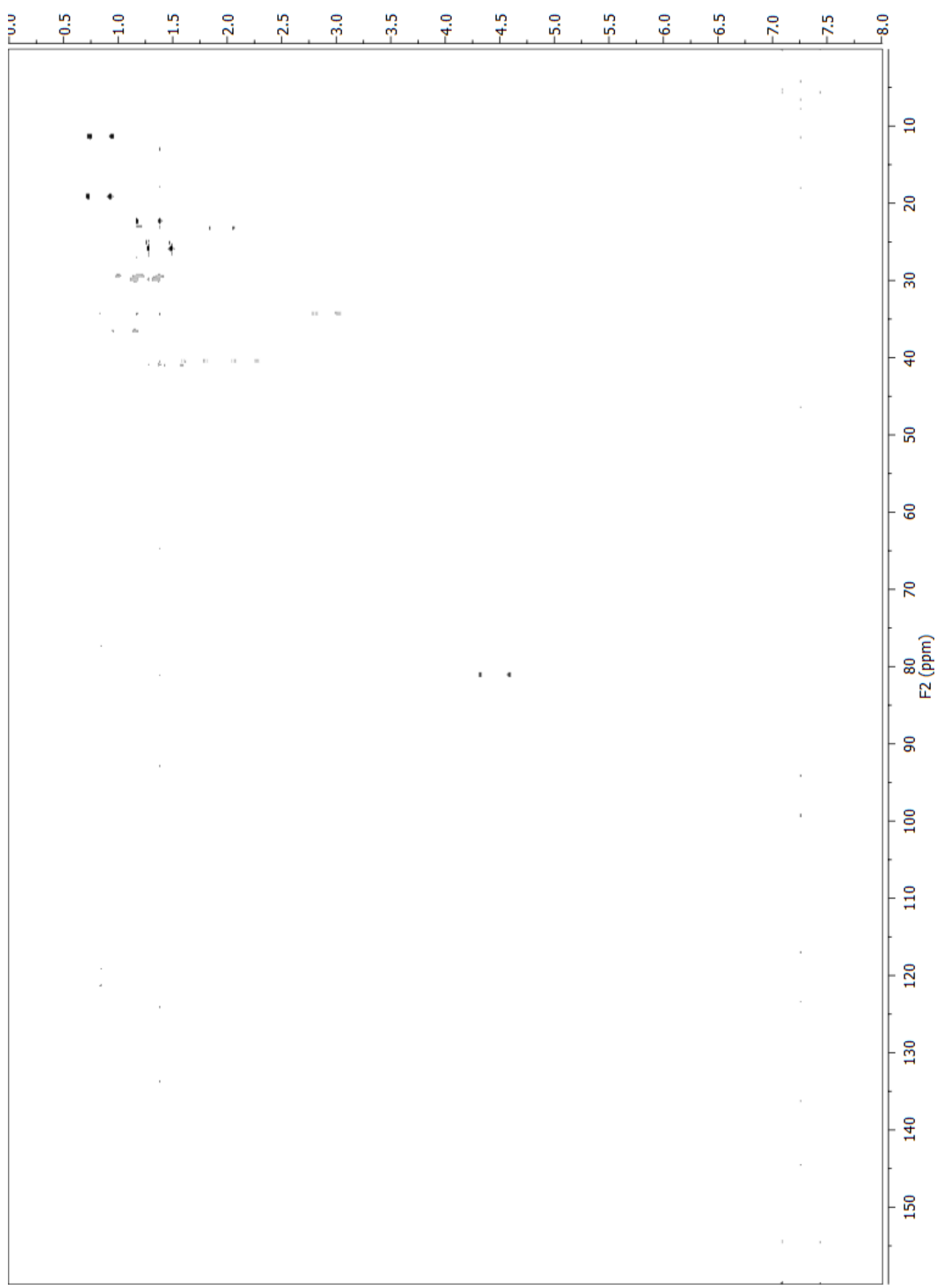

Fully-coupled HSQC spectrum of plakortolide X (97) $\left(600 \mathrm{MHz}, \mathrm{CDCl}_{3}\right)$. 
(udd) T-

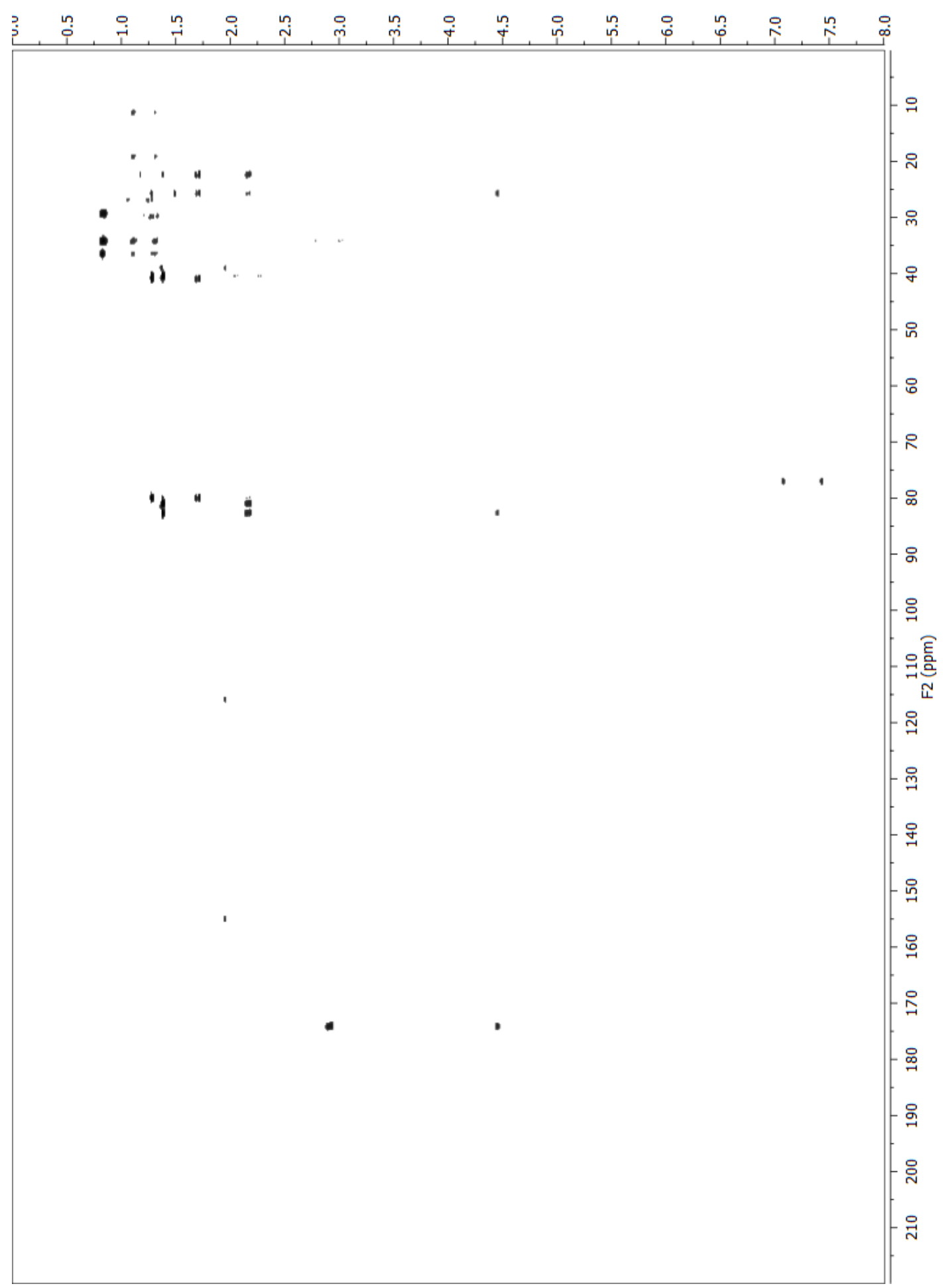

HMBC spectrum of plakortolide X (97) $\left(600 \mathrm{MHz}, \mathrm{CDCl}_{3}\right)$. 


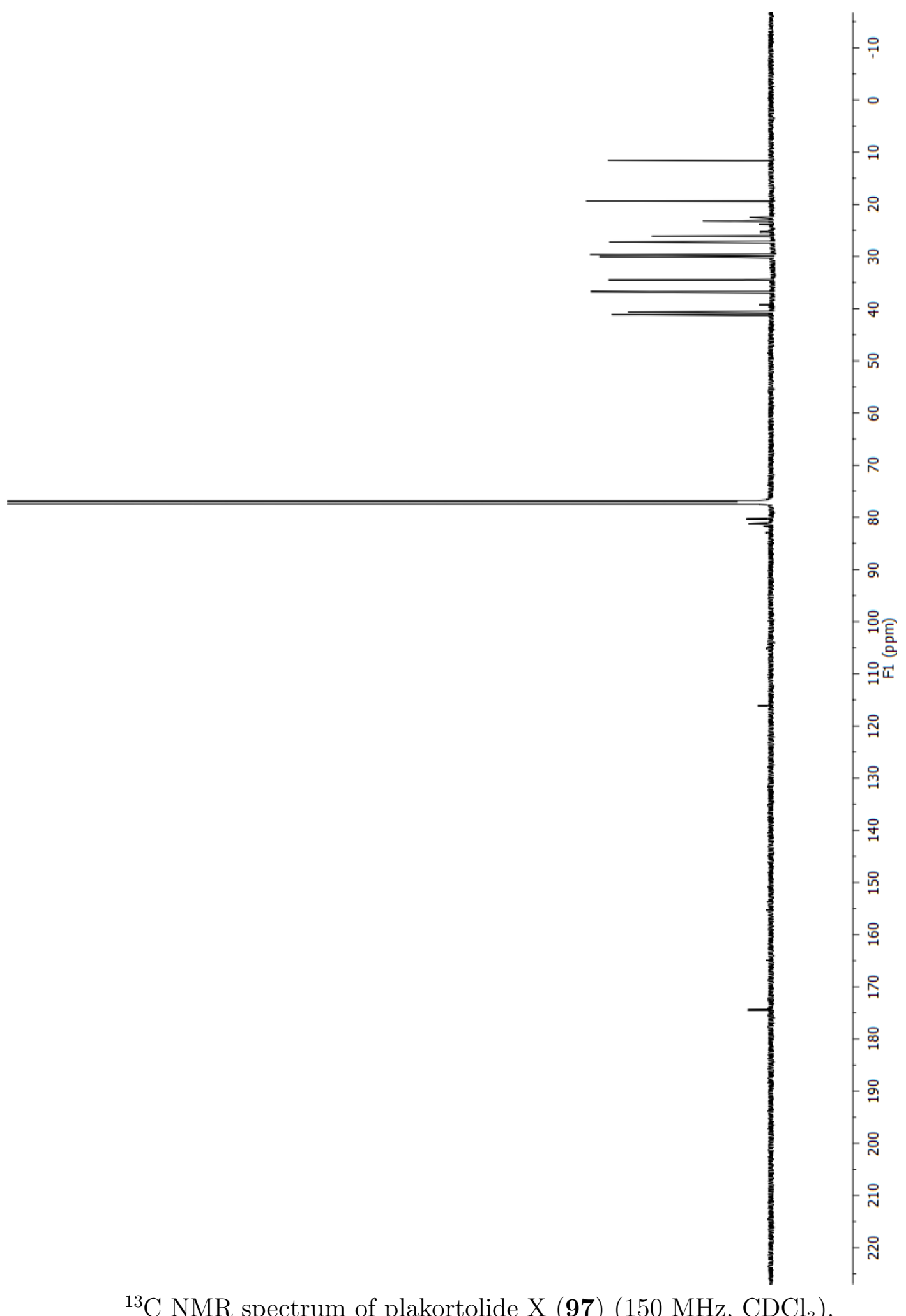

${ }^{13} \mathrm{C}$ NMR spectrum of plakortolide X (97) (150 MHz, $\mathrm{CDCl}_{3}$ ). 
Cyclic peroxide 98

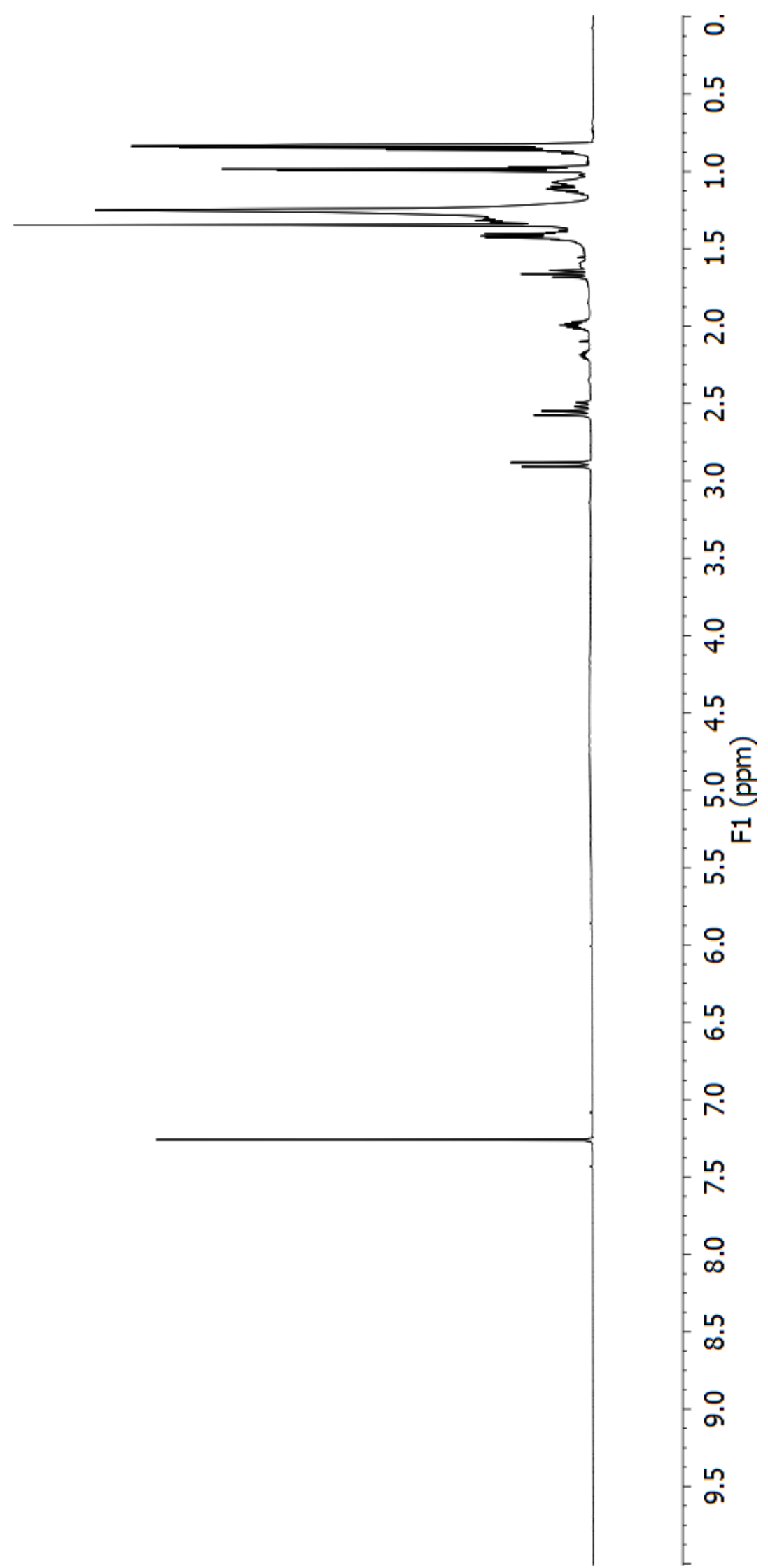

${ }^{1} \mathrm{H}$ NMR spectrum of cyclic peroxide $98\left(600 \mathrm{MHz}, \mathrm{CDCl}_{3}\right)$. 


\section{(udd) IJ}

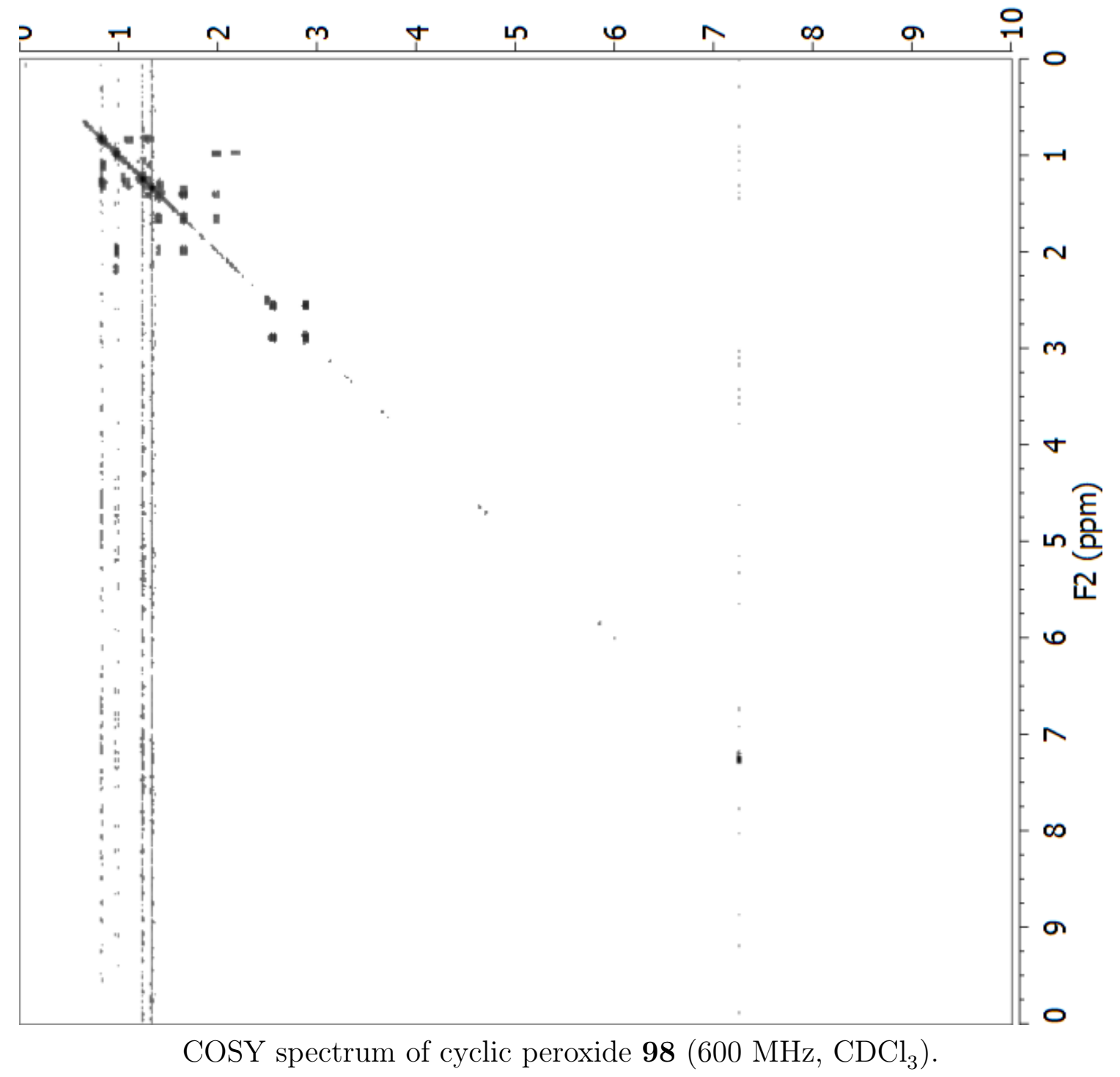




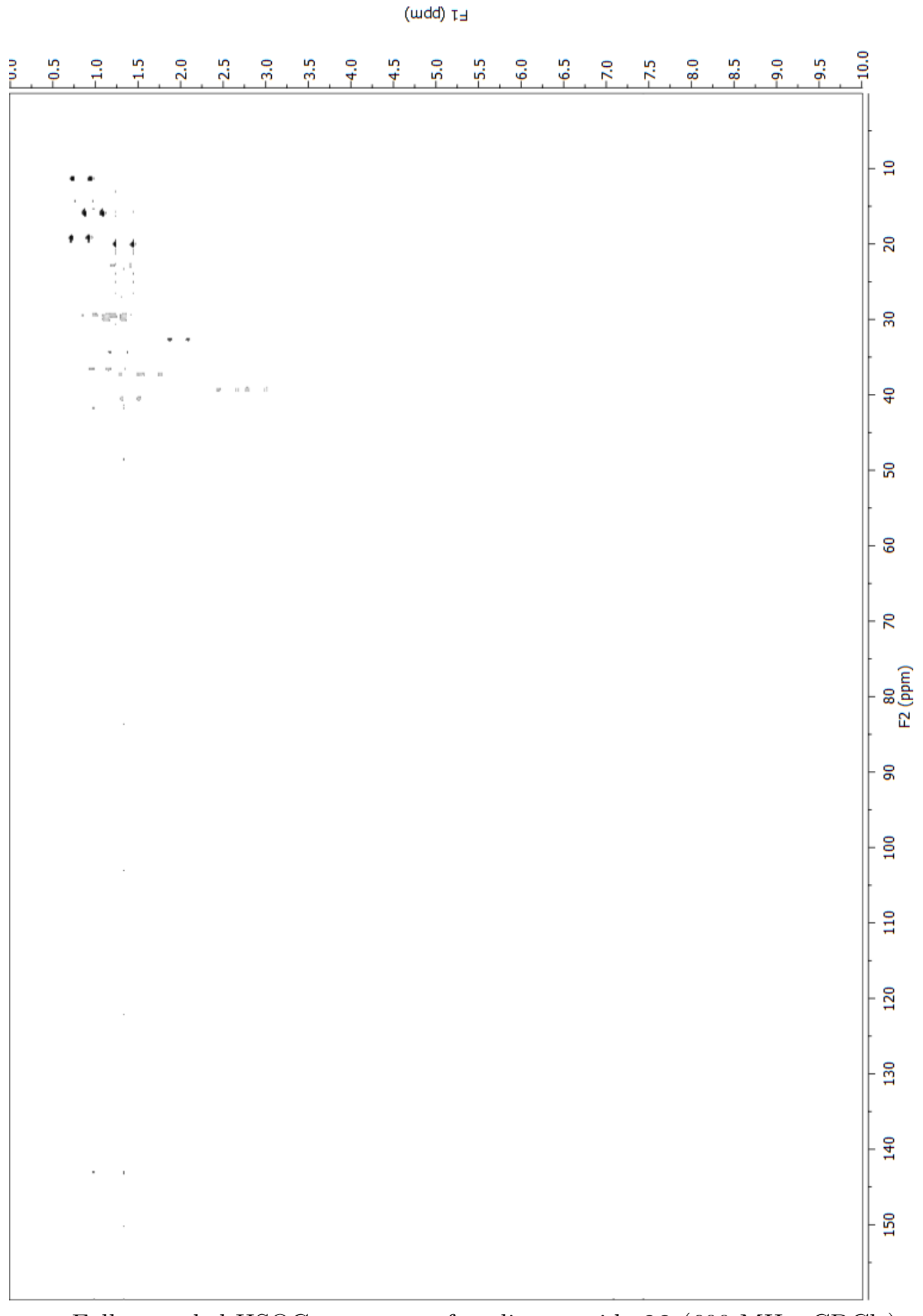

Fully-coupled HSQC spectrum of cyclic peroxide $\mathbf{9 8}\left(600 \mathrm{MHz}, \mathrm{CDCl}_{3}\right)$.

302 
(udd) 더

ว

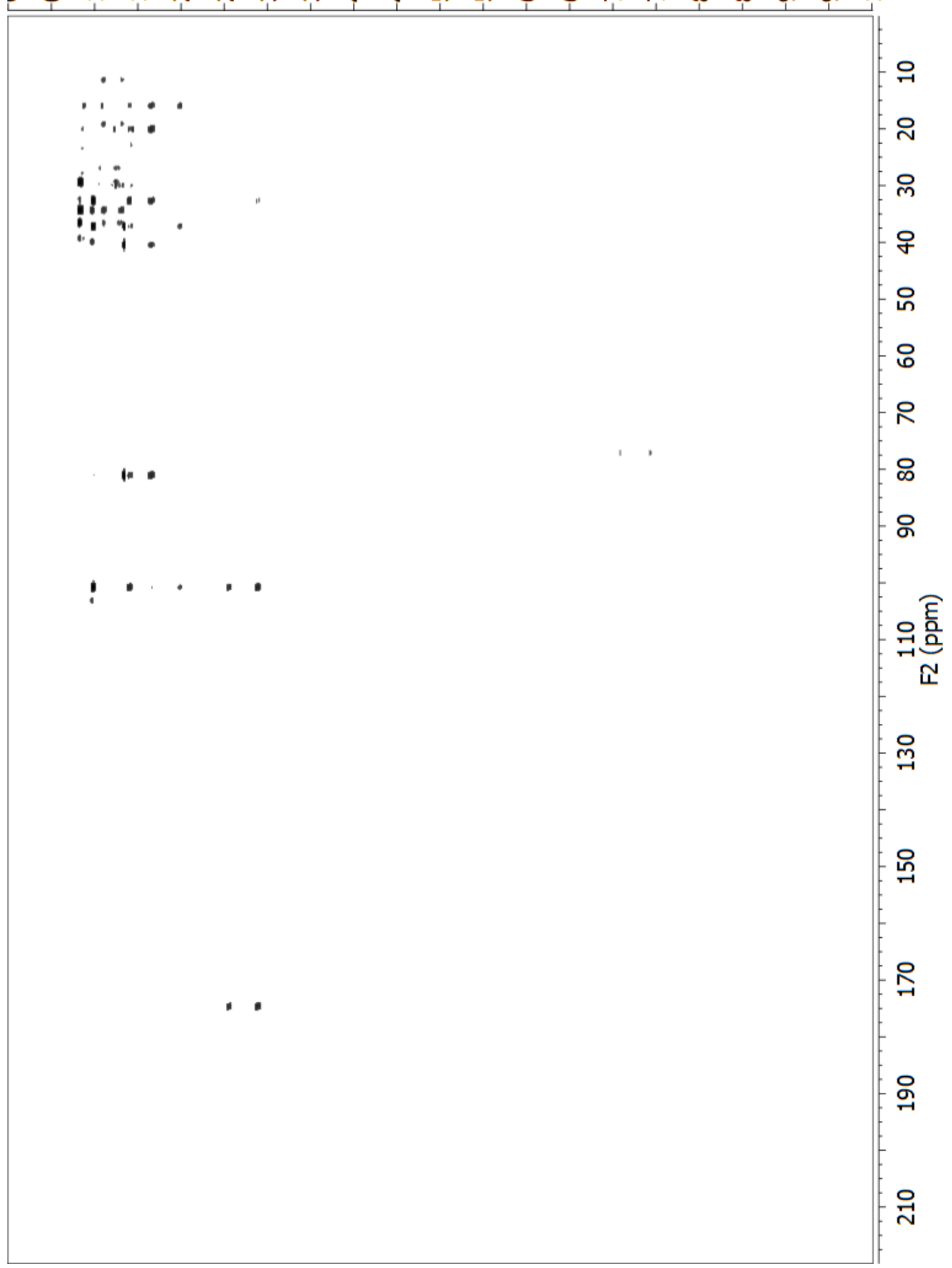

HMBC spectrum of cyclic peroxide $98\left(600 \mathrm{MHz}, \mathrm{CDCl}_{3}\right)$. 


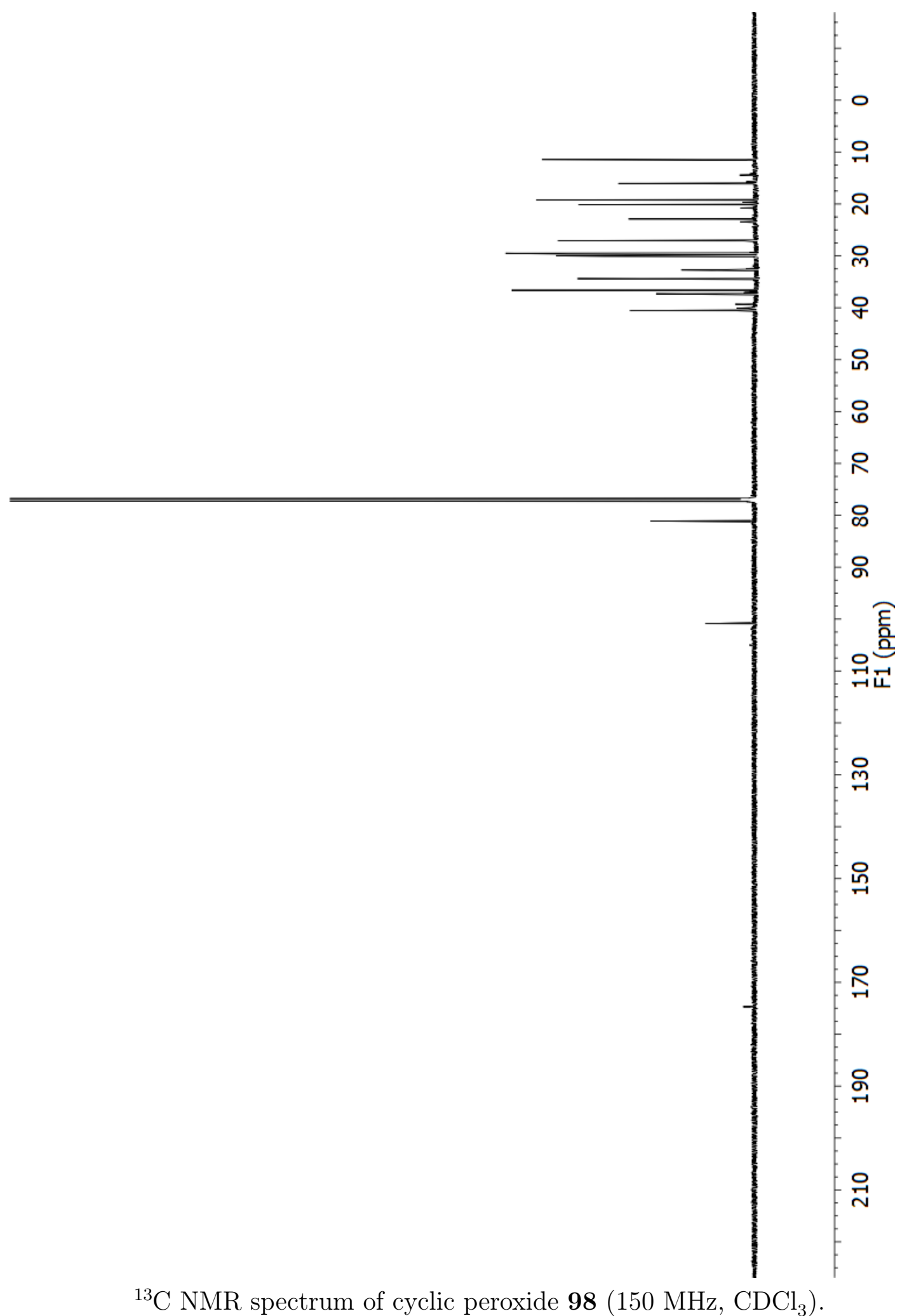




\section{Appendix $\mathrm{H}$}

\section{Phenotypic Profile of 95}

A

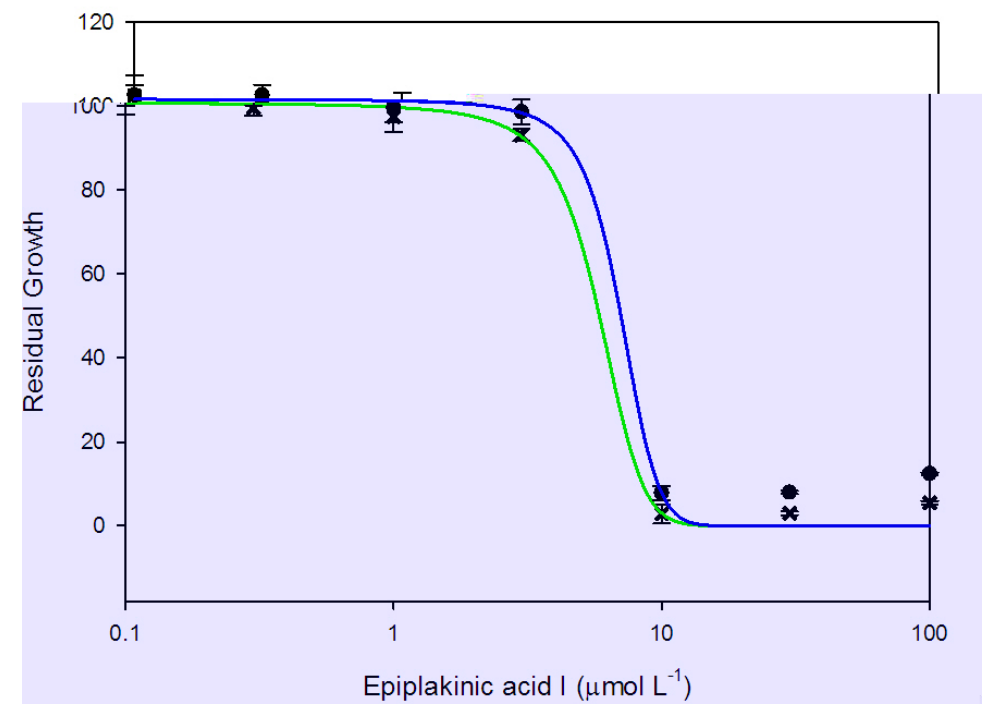

B

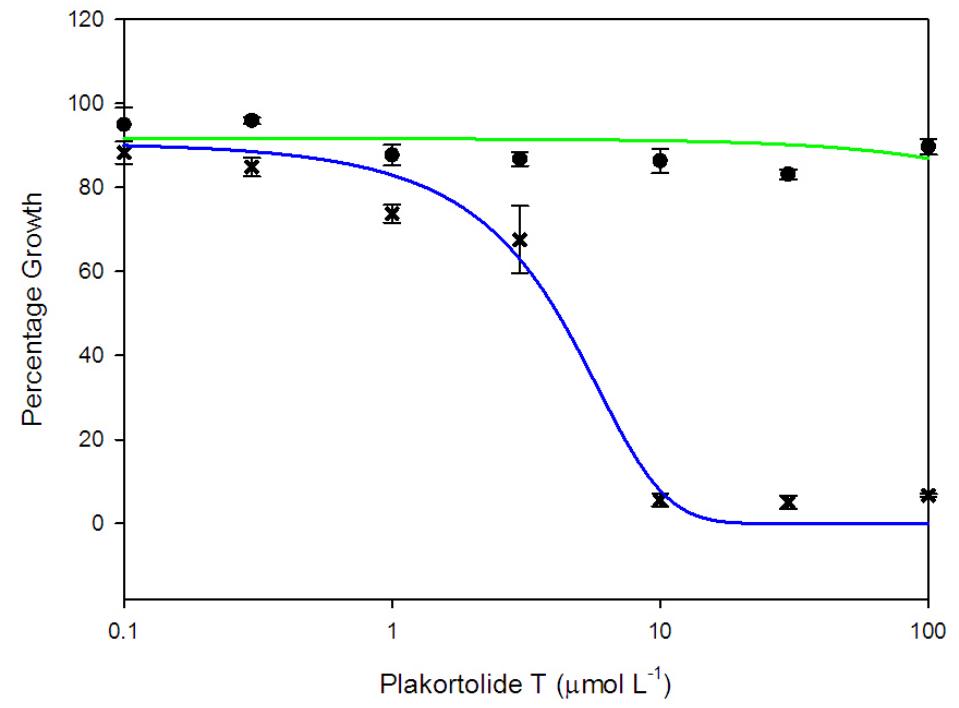

Figure H.1 Dose-response curves for epiplakinic acid I (95) and plakortolide X (97). 95 displays similar inhibition of both strains, while $\mathbf{9 7}$ only inhibits the $\Delta p d r$ strain.
A: $\quad$ 95, WT $(-\bullet)$ and $\Delta p d r(-\times-)$
B: $\quad$ 97, WT $(-\bullet)$ and $\Delta p d r(-\times-)$ 
A

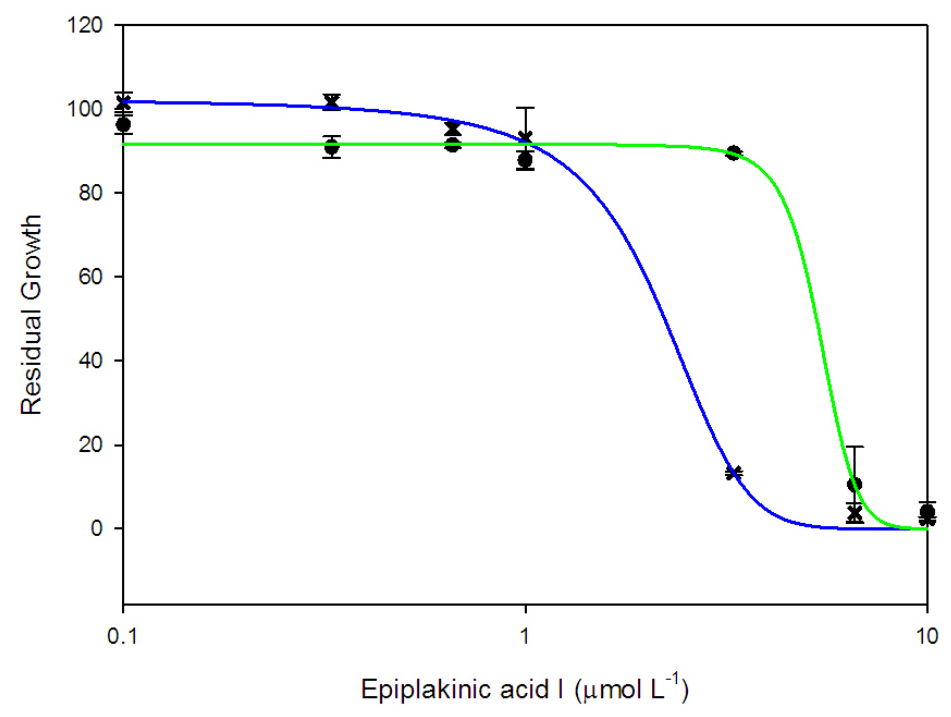

B

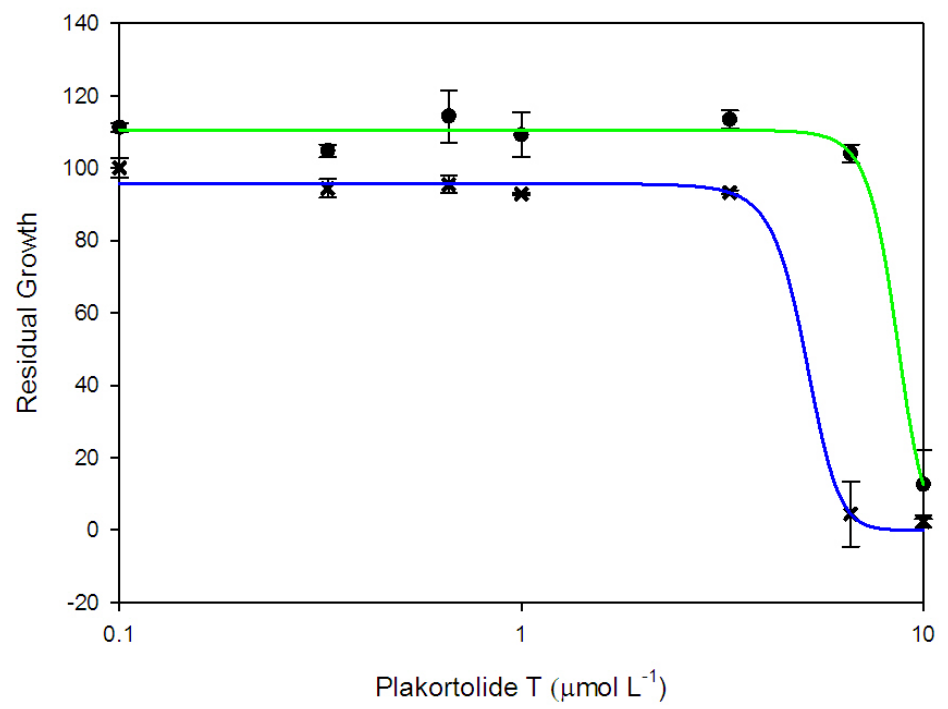

Figure H.2 Activity of epiplakinic acid I (95) and plakortolide X (97) is enhanced by high $\mathrm{Ca}^{2+}$ levels.
A: $95(--) \quad \mathrm{IC}_{50} 4.98 \mu \mathrm{mol} \mathrm{L}-1 \quad 95$ and $50 \mathrm{mmol} \mathrm{L}^{-1} \mathrm{CaCl}_{2}(-\times-) \quad \mathrm{IC}_{50} 1.88 \mu \mathrm{mol} \mathrm{L}^{-1}$
B: $97(\bullet-) \quad \mathrm{IC}_{50} 7.38 \mu \mathrm{mol} \mathrm{L}-1 \quad 97$ and $50 \mathrm{mmol} \mathrm{L}^{-1} \mathrm{CaCl}_{2}(-\times-) \quad \mathrm{IC}_{50} 4.68 \mu \mathrm{mol} \mathrm{L}^{-1}$ 


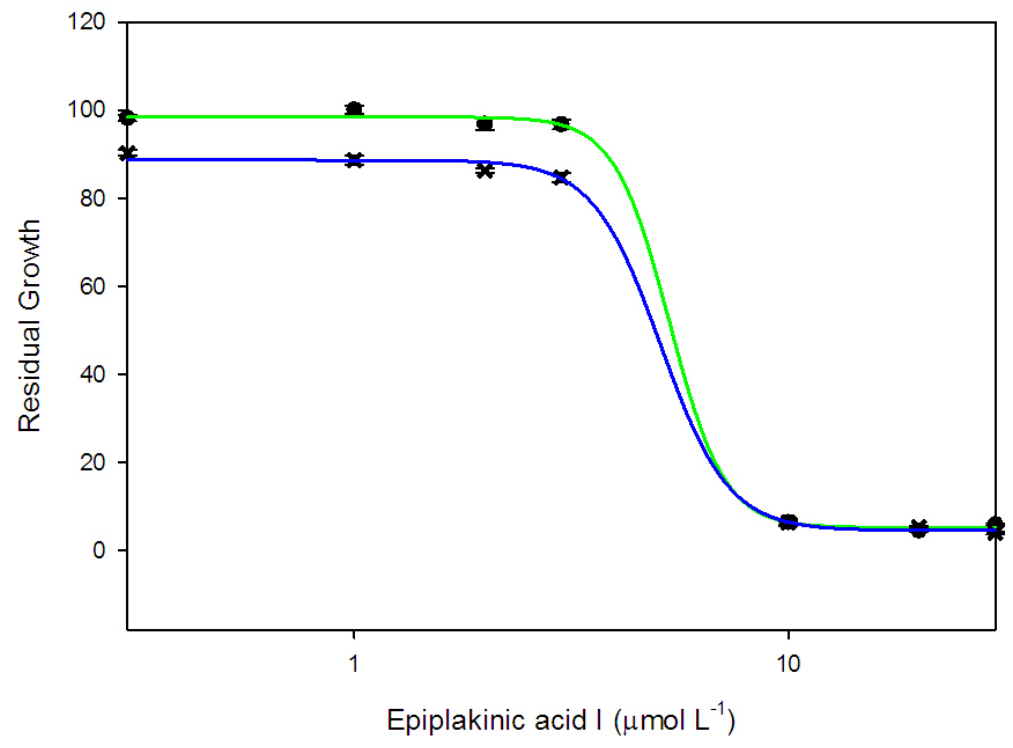

Figure H.3 Dose-response curves for epiplakinic acid I and plakortolide X in the presence of EGTA.
A: $95(-x-)$
95 and EGTA, $10 \mathrm{mmol} \mathrm{L}^{-1}$
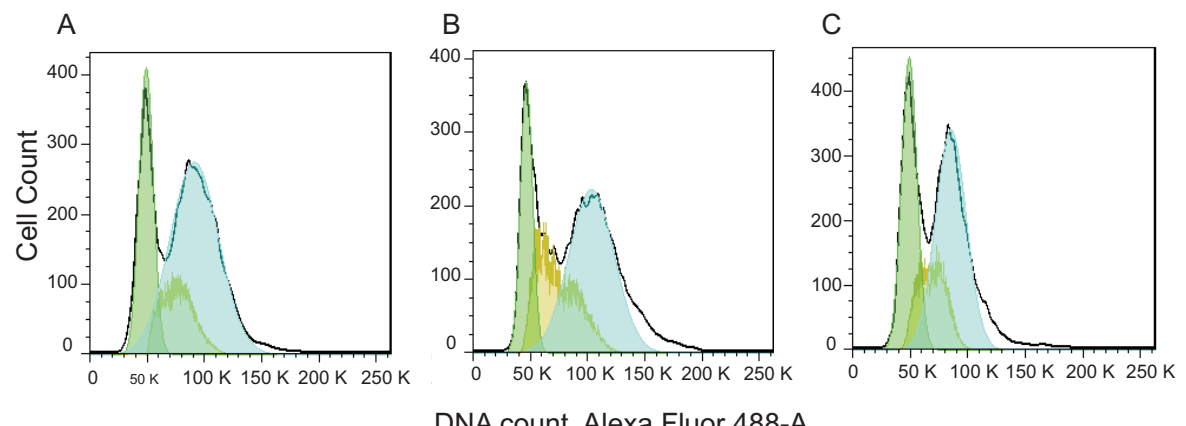

Figure H.4 Epiplakinic acid I (95) causes a 50\% increase in S-phase population of cells treated at the $\mathrm{IC}_{50}$, and increases the $\mathrm{G}_{2}$ population when administered at the MIC.
A: DMSO, $1 \%$
B: $\quad \mathbf{9 5}, \mathrm{IC}_{50}\left(3.45 \mu \mathrm{mol} \mathrm{L}^{-1}\right)$
C: $95, \mathrm{MIC}\left(10 \mu \mathrm{mol} \mathrm{L}^{-1}\right)$ 

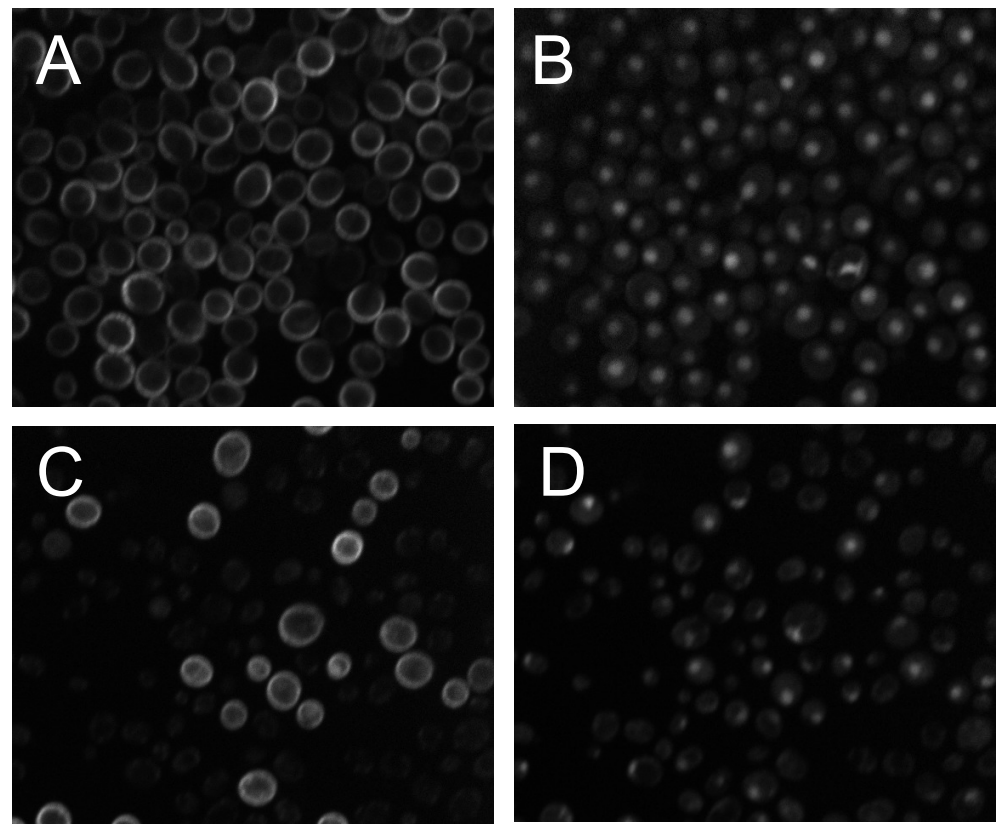

Figure H.5 Epiplakinic acid I (95) affects a decrease in cell size in S. cerevisiae. The red proteins mCherry and Red Star II localise to the cytoplasm and nucleus respectively, while the GFP-tagged Mrh1p resides in the plasma membrane.
A: $\operatorname{DMSO}(1 \%), 2 \mathrm{~h}$, Mrh1-GFP
B: $\quad$ DMSO (1\%), 2 h, mCherry/Red Star II
C: $95\left(10 \mu \mathrm{mol} \mathrm{L}^{-1}\right), 2 \mathrm{~h}, \mathrm{Mrh1}-\mathrm{GFP}$
D: $95\left(10 \mu \mathrm{mol} \mathrm{L}^{-1}\right), 2 \mathrm{~h}, \mathrm{mCherry} / \operatorname{Red}$ Star II

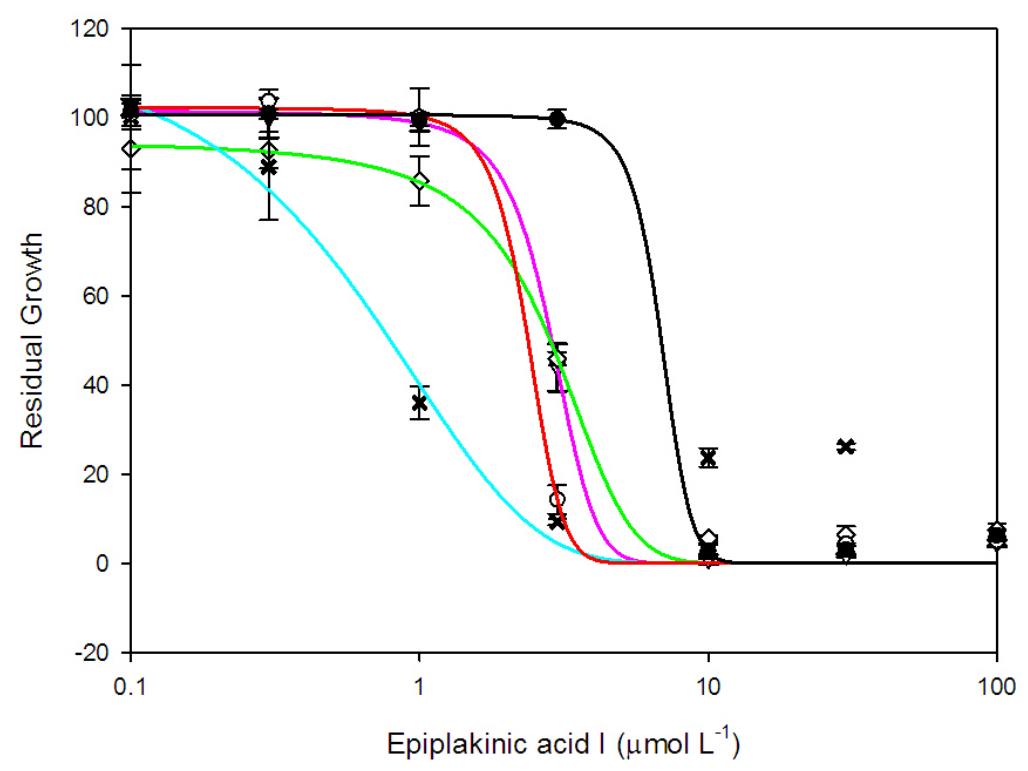

Figure H.6 Hypersensitivity of cytoskeletal mutants to epiplakinic acid I (95) relative to the BY4743 WT strain $(-\bullet-)$.
$\triangle R V S 167 \quad(-\nabla-)$
$\mathrm{IC}_{50} 1.91 \mu \mathrm{mol} \mathrm{L}-1$
$\triangle S L A 1 \quad(-\mathrm{O}-)$
$\mathrm{IC}_{50} 1.98 \mu \mathrm{mol} \mathrm{L}-1$
$\triangle S F P 1 \quad(-\times-) \quad \mathrm{IC}_{50} 2.80 \mu \mathrm{mol} \mathrm{L}^{-1}$
$\triangle R S A 1 \quad(\diamond-) \quad \mathrm{IC}_{50} 2.82 \mu \mathrm{mol} \mathrm{L}-1$ 
A

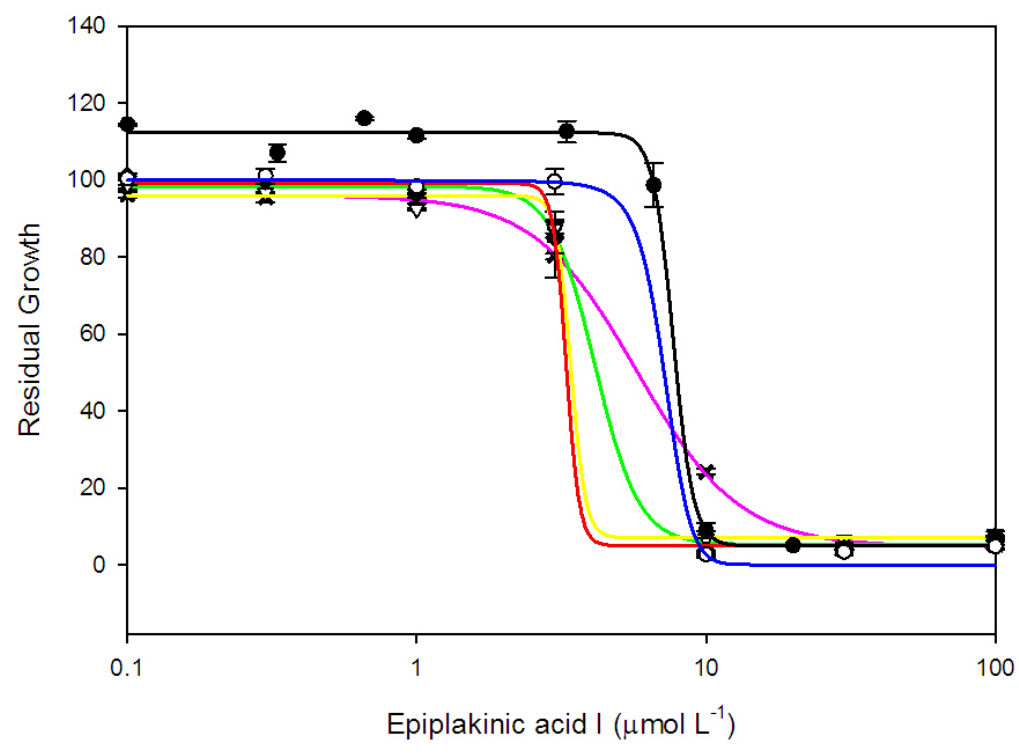

B

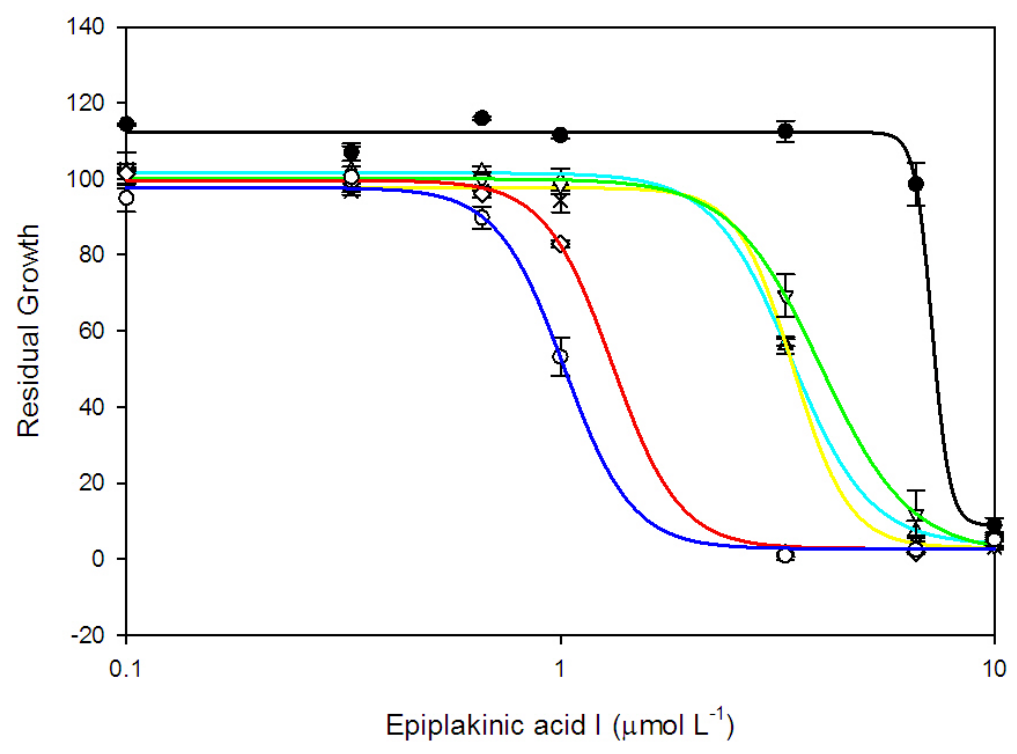

Figure H.7 Dose-response curves for epiplakinic acid I against $\mathrm{Ca}^{2+}$-responsive deletions strains relative to the WT control.
A: WT BY 4743
$\triangle C N A 2$
$(-\bullet-)$
$(\triangle) \quad \mathrm{IC}_{50} 3.56 \mu \mathrm{mol} \mathrm{L}^{-1}$
$\triangle C N A 1$
$(-\circ-) \quad \mathrm{IC}_{50} 3.77 \mu \mathrm{mol} \mathrm{L}^{-1}$
$\triangle C R Z 1$
$(-\diamond) \quad \mathrm{IC}_{50} 3.49 \mu \mathrm{mol} \mathrm{L}{ }^{-1}$
$\triangle C N B 1$
$\left({ }^{-} \nabla^{-}\right) \quad \mathrm{IC}_{50} 3.53 \mu \mathrm{mol} \mathrm{L}^{-1}$
$\triangle H C M 1$
$(-\times-) \quad \mathrm{IC}_{50} 6.31 \mu \mathrm{mol} \mathrm{L}^{-1}$
B: $\quad$ WT BY4743
$\triangle M I D 1$
$(-\bullet-)$
$(-\diamond)$
$\triangle C C H 1$
$\mathrm{IC}_{50} 3.28 \mu \mathrm{mol} \mathrm{L}-1$
$\triangle P M C 1$
$(\triangle) \quad \mathrm{IC}_{50} 3.60 \mu \mathrm{mol} \mathrm{L}-1$
$\triangle P M R 1$
$(-\times-) \quad \mathrm{IC}_{50} 3.36 \mu \mathrm{mol} \mathrm{L}-1$
$\triangle Y G L 167 W$
$\left(-\nabla^{-}\right) \quad \mathrm{IC}_{50} 5.25 \mu \mathrm{mol} \mathrm{L}-1$
$(-\circ-) \quad \mathrm{IC}_{50} 3.15 \mu \mathrm{mol} \mathrm{L}^{-1}$ 

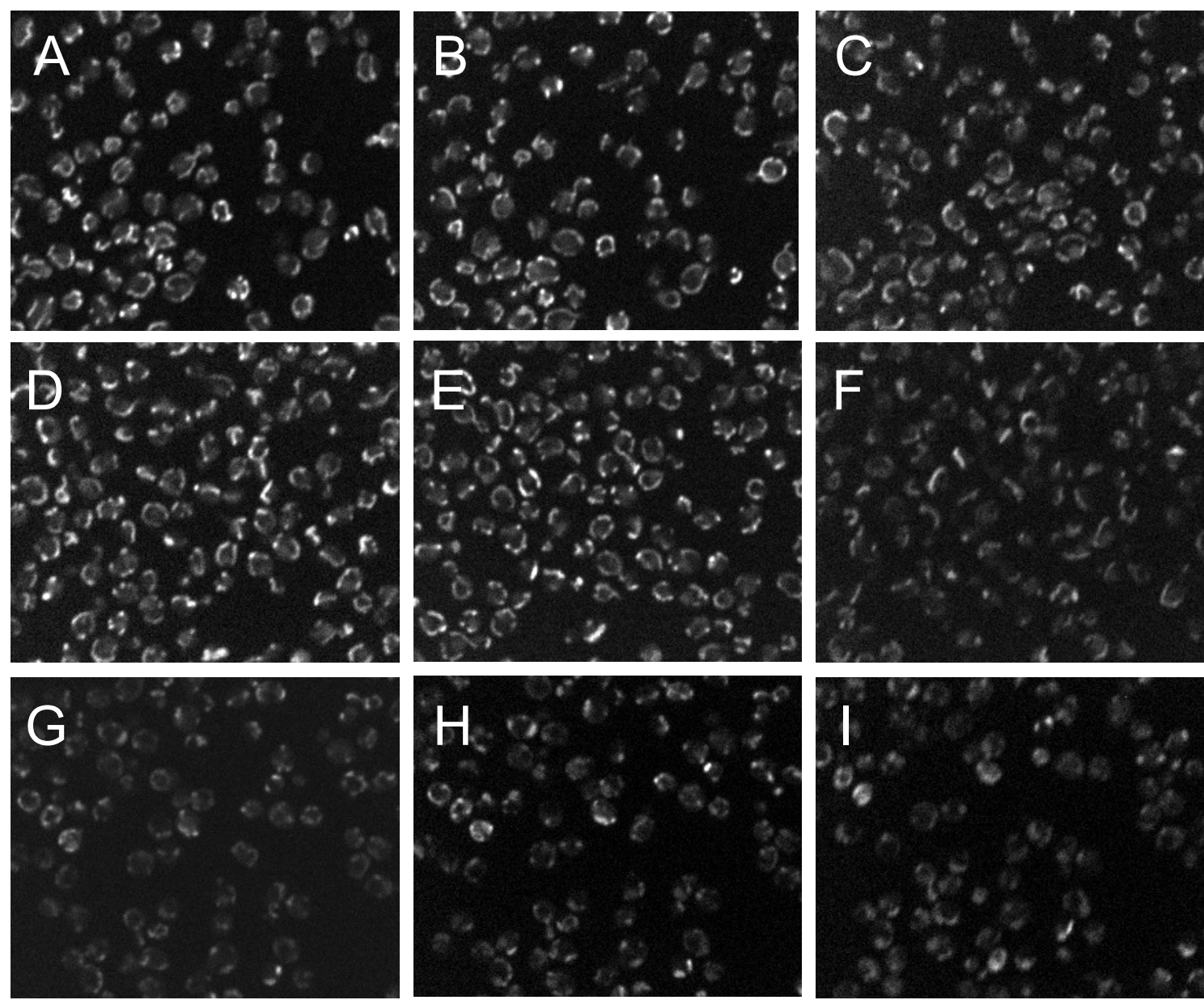

Figure H.8 Translocation studies in the Zrt1p-GFP, non-conclusive for endocytosis of the membrane pump in response to a rise in intracellular $\mathrm{Zn}^{2+}$ or exposure to $\mathbf{9 5}$.

A: DMSO, 0 min $\quad \mathrm{B}: \quad \mathrm{DMSO}, \mathrm{t}=10 \mathrm{~min} \quad \mathrm{C}: \quad \mathrm{DMSO}, \mathrm{t}=40 \mathrm{~min}$

D: $\quad 95,0 \mathrm{~min}$

E: $\quad 95, \mathrm{t}=10 \mathrm{~min}$

F: $\quad \mathbf{9 5}, \mathrm{t}=40 \mathrm{~min}$

G: $\mathrm{ZnCl}_{2}, 0$ min

$\mathrm{H}: \mathrm{ZnCl}_{2}, \mathrm{t}=10 \mathrm{~min} \quad \mathrm{I}: \mathrm{ZnCl}_{2}, \mathrm{t}=40 \mathrm{~min}$ 


\section{Appendix I}

\section{Chemical Genetic Profile of 95}

Table I.1 Homozygous Profile of Epiplakinic Acid I (95).

\begin{tabular}{|c|c|c|c|}
\hline \multicolumn{2}{|c|}{ Up Tag } & \multicolumn{2}{|c|}{ Dn Tag } \\
\hline Gene & z-score & Gene & z-score \\
\hline$\overline{\text { SLA1 }}$ & "-5.809413688 & $\overline{\text { SLA1 }}$ & -4.410560386 \\
\hline VPS24 & -4.972817922 & RPS9B & -4.36002436 \\
\hline VPS28 & -4.697856815 & END3 & -4.222755852 \\
\hline END3 & -4.436000379 & YER087W & -3.991511202 \\
\hline YIL157C & -4.334762811 & STP22 & -3.934688549 \\
\hline YOR019W & -4.161528418 & GOS1 & -3.911094923 \\
\hline CRZ1 & -4.125482084 & TPS2 & -3.834067429 \\
\hline YOR161C & -4.048027013 & VPS28 & -3.741074729 \\
\hline YNL120C & -4.025318625 & VPS36 & -3.685492132 \\
\hline RVS161 & -3.979063015 & RGP1 & -3.659586023 \\
\hline OCH1 & -3.791609729 & SNF7 & -3.576692859 \\
\hline SNF7 & -3.576648865 & DBP7 & -3.53354966 \\
\hline SLI15 & -3.572018966 & YBL054W & -3.48368878 \\
\hline BRO1 & -3.457644953 & SOD1 & -3.444793226 \\
\hline APL2 & -3.346409981 & VAC7 & -3.429958961 \\
\hline APT2 & -3.269609242 & GRR1 & -3.37594831 \\
\hline VPS4 & -3.205010706 & YPL005W & -3.362444783 \\
\hline HCM1 & -3.123723593 & SPO74 & -3.304200493 \\
\hline SFP1 & -3.110038414 & RCY1 & -3.290998502 \\
\hline PFK2 & -3.029516051 & RVS167 & -3.204320695 \\
\hline VPS24 & -4.972817922 & CSG2 & -3.171599027 \\
\hline & & DID2 & -3.16113872 \\
\hline & & CHS5 & -3.147043804 \\
\hline & & YLR338W & -3.093662482 \\
\hline & & RSA1 & -3.088253698 \\
\hline & & IRS4 & -3.057798815 \\
\hline & & ARO7 & -3.027509409 \\
\hline
\end{tabular}

Bold font indicates genes with tag overlap. 
Table I.2 Haploinsufficiency Profile of Epiplakinic Acid I (95).

\begin{tabular}{|c|c|c|c|}
\hline \multicolumn{2}{|c|}{ Up Tag } & \multicolumn{2}{|c|}{ Dn Tag } \\
\hline Gene & z-score & Gene & z-score \\
\hline YBL044W & -9.434195341 & 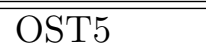 & -11.41086097 \\
\hline EDE1 & -8.904361216 & KIN4 & -10.08904761 \\
\hline YKL200C & -8.806319953 & YLR123C & -9.940118526 \\
\hline UBP13 & -8.521427816 & VHS1 & -8.40816393 \\
\hline ENT4 & -8.259266665 & INP52 & -7.877981485 \\
\hline UBR2 & -7.827602252 & CAR2 & -7.590762838 \\
\hline NDT80 & -7.110771208 & MRM2 & -7.488420803 \\
\hline YMR316C-A & -6.806339037 & RPS15 & -7.405188592 \\
\hline YFR043C & -6.655317155 & MRS1 & -7.323103591 \\
\hline ELA1 & -6.592775111 & EUG1 & -6.945223471 \\
\hline YOR298W & -6.477197383 & YLR241W & -6.693412231 \\
\hline YJL163C & -6.472217167 & SSP2 & -6.651131162 \\
\hline THI12 & -6.427515036 & YDL206W & -6.526193924 \\
\hline YER139C & -6.341261909 & COX17 & -6.402868993 \\
\hline YNL187W & -6.334975414 & YDR223W & -6.149852711 \\
\hline YLR202C & -6.334490923 & YLR269C & -5.860689213 \\
\hline MAP2 & -6.248179852 & PHO36 & -5.56226546 \\
\hline DDP1 & -6.153337929 & MAD3 & -5.413899915 \\
\hline PCI8 & -6.005399364 & GAL3 & -5.351678774 \\
\hline YBL083C & -5.881486314 & PMT6 & -5.279371881 \\
\hline AIM2 & -5.838454891 & HIS3 & -5.175504603 \\
\hline YIL077C & -5.791565696 & DAL7 & -5.166777412 \\
\hline YDR084C & -5.644580502 & FRM2 & -5.115838933 \\
\hline LST4 & -5.626825535 & RPS14B & -5.023021712 \\
\hline AST1 & -5.519434755 & YER181C & -5.019790686 \\
\hline EUG1 & -5.211588583 & MRPL11 & -5.003391004 \\
\hline ERV46 & -5.210483898 & YUH1 & -4.97076341 \\
\hline PST1 & -5.205556664 & YGL232W & -4.866882063 \\
\hline YOR019W & -5.148338476 & PTP1 & -4.618553681 \\
\hline AZR1 & -5.09881151 & SLZ1 & -4.617101441 \\
\hline PYK2 & -5.056831381 & RPS7A & -4.38453948 \\
\hline YIM1 & -5.041111789 & FOX2 & -4.344719978 \\
\hline YPL245W & -4.960736203 & RAX1 & -4.334933264 \\
\hline SSA3 & -4.867248834 & RPL27A & -4.236754744 \\
\hline YFR055W & -4.766419283 & MSN5 & -4.222920612 \\
\hline YMR210W & -4.766383731 & GGA1 & -4.205278815 \\
\hline YKR017C & -4.659871866 & PRP43 & -4.189182085 \\
\hline ATH1 & -4.642439614 & GMH1 & -3.90118805 \\
\hline PHO81 & -4.441089116 & RAD4 & -3.86603065 \\
\hline MAF1 & -4.410447644 & GAC1 & -3.745939683 \\
\hline YOR255W & -4.389717345 & YSA1 & -3.662090839 \\
\hline PCL1 & -4.362060286 & YGL082W & -3.599448702 \\
\hline STE13 & -4.272390889 & HHT1 & -3.59855679 \\
\hline ASC1 & -4.171738708 & PXR1 & -3.486747419 \\
\hline RPC10 & -4.036284207 & RPL39 & -3.447685757 \\
\hline ASI1 & -4.006071145 & RPL33A & -3.422225152 \\
\hline
\end{tabular}

Bold font indicates genes with tag overlap.

Essential genes appear in blue. 
Table I.3 Haploinsufficiency Profile of Epiplakinic Acid I Continued (95).

\begin{tabular}{lllll}
\hline \multicolumn{2}{c}{ Up Tag } & & \multicolumn{2}{c}{ Dn Tag } \\
\cline { 1 - 2 } \cline { 5 - 5 } Gene & z-score & & Gene & z-score \\
\hline \hline CIK1 & -3.741965011 & & YIL086C & -3.212591118 \\
RTS2 & -3.886488775 & & TPS1 & -3.410693771 \\
YFR046C & -3.826413009 & & DIP5 & -3.385823841 \\
PDR8 & -3.754045725 & & YGR107W & -3.132920087 \\
YDR066C & -3.677657055 & & IMD3 & -3.116253877 \\
GNP1 & -3.660006156 & & NAP1 & -3.110696791 \\
YJR015W & -3.656590627 & & YLL012W & -3.110245285 \\
YOL048C & -3.646448665 & & MRPL16 & -3.101164361 \\
YIR003W & -3.6222659 & & ADO1 & -3.086583503 \\
YOS9 & -3.599699728 & & MNE1 & -3.015526831 \\
RCL1 & -3.536428723 & & \\
STP2 & -3.501269521 & & \\
YLR400W & -3.420612012 & & \\
CRC1 & -3.409312476 & & \\
SHU1 & -3.28657986 & & \\
SIP1 & -3.285653011 & & \\
FSH3 & -3.2770514 & & \\
ABP140 & -3.235341139 & & \\
YGL160W & -3.114316827 & & \\
YDR492W & -3.096230308 & & \\
YBR016W & -3.095670033 & & \\
YRA2 & -3.020672642 & & \\
SUR2 & -3.000979236 & & \\
\hline
\end{tabular}

Bold font indicates genes with tag overlap.

Essential genes appear in blue. 
Table I.4 Functional Categories of Deletion Strains Resistant to Epiplakinic Acid I (95, $3 \mu \mathrm{mol} \mathrm{L}{ }^{-1}$, z-score $>2$ ).

\begin{tabular}{lll}
\hline GO Molecular Function & p-value & Gene deletion strain \\
\hline \hline $\begin{array}{l}\text { Phosphatidylinositol transporter } \\
\text { activity }\end{array}$ & 0.000371781 & SFH5 and CSR1 \\
$\begin{array}{l}\text { Phospholipase activity } \\
\text { FMN reductase activity }\end{array}$ & 0.000371781 & PLB3, YOR022C, CSR1 and PLB3 \\
$\begin{array}{l}\text { NAD(P)H dehydrogenase } \\
\text { (quinone) activity }\end{array}$ & 0.0062093 & LOT6 \\
$\begin{array}{l}\text { High affinity zinc ion uptake and } \\
\text { transmembrane transporter }\end{array}$ & 0.0062093 & LOT6 \\
$\begin{array}{l}\text { activity } \\
\text { bis(5'-Adenosyl)-triphosphatase } \\
\text { activity }\end{array}$ & 0.0062093 & HNT2 \\
$\begin{array}{l}\text { Phosphatidylserine catabolic } \\
\text { process }\end{array}$ & 0.0062093 & PLB3 \\
$\begin{array}{l}\text { Regulation of fatty acid } \\
\text { metabolic process }\end{array}$ & 0.0062093 & CSR1, PLB3 and YOR022C \\
\begin{tabular}{l} 
Lipid transport \\
\hline
\end{tabular} & 0.00733151 & SFH5 and CSR1 \\
\hline
\end{tabular}


Table I.5 Functional Categories of Heterozygous Deletion Strains Resistant to Epiplakinic Acid I $\left(\mathbf{9 5}, 2 \mu \mathrm{mol} \mathrm{L}{ }^{-1}\right.$, z-score $\left.>2\right)$.

\begin{tabular}{|c|c|c|}
\hline GO Molecular Functions & p-value & Gene deletion strain \\
\hline $\begin{array}{l}\text { Structural constituent of } \\
\text { ribosome }\end{array}$ & $9.07 \mathrm{E}-05$ & $\begin{array}{l}\text { RPS8A, RPS11A, RSM10, RPS13, } \\
\text { RPS18A, RPL27B, RPS2, RPS21B, } \\
\text { RSM22, RPS27A, RPL20A and RPS28A }\end{array}$ \\
\hline SSU rRNA binding & 0.00130933 & RPS13 and RPS2 \\
\hline $\begin{array}{l}\text { Inositol or phosphatidylinositol } \\
\text { phosphatase activity }\end{array}$ & 0.00587519 & INM2 and INP52 \\
\hline $\begin{array}{l}\text { S-adenosylmethionine- } \\
\text { dependent methyltransferase } \\
\text { activity }\end{array}$ & 0.00309695 & RSM22, NOP2 and YNL092W \\
\hline $\begin{array}{l}\text { Adenyl-nucleotide exchange } \\
\text { factor activity }\end{array}$ & 0.00320969 & LHS1 and SIL1 \\
\hline $\begin{array}{l}\text { Post-translational protein } \\
\text { targeting to membrane, translo- } \\
\text { cation }\end{array}$ & 0.00748032 & SBH1 and LHS1 \\
\hline $\begin{array}{l}\text { SRP-dependent cotranslational } \\
\text { protein targeting to membrane } \\
\text { and translocation }\end{array}$ & 0.00925952 & SBH1 and SIL1 \\
\hline $\begin{array}{l}\text { Structural constituent of nuclear } \\
\text { pore }\end{array}$ & 0.00444974 & NUP192 and NSP1 \\
\hline Translation & $6.78 \mathrm{E}-05$ & $\begin{array}{l}\text { FMT1, RPS8A, SLM5, RPS11A, RSM10, } \\
\text { RPS13, GCD6, RPS18A, RPL27B, RPS2, } \\
\text { RPS0A, RPS20, RPL40A, RPS21B, RPL39, } \\
\text { RSM22, RPS27A, MEF1, RPL37A, RPS30A, } \\
\text { RPL13B, RPL20A, YNL040W, RPL16B, } \\
\text { YNL122C, RPS28A, RPS6A, MSD1 } \\
\text { and RPL43A }\end{array}$ \\
\hline rRNA processing & 0.00059314 & $\begin{array}{l}\text { RPS8A, RPS11A, RSM10, RPS13, } \\
\text { GCD6, RPS18A, RPL27B, RPS2, } \\
\text { RPS21B, RSM22, RPS27A, MEF1, } \\
\text { RPL20A, YNL040W and RPS28A }\end{array}$ \\
\hline rRNA export from nucleus & 0.000640291 & NUP84, RPS18A, RPS2, and RPS28A \\
\hline
\end{tabular}




\section{Bibliography}

1. Newman, D. J.; Cragg, G. M.; Snader, K. M. Nat. Prod. Rep. 2000, 17, 215234.

2. National Cancer Institute, 2012. http://www.cancer.gov/aboutnci/ servingpeople/cancer-statistics/costof cancer.

3. New Zealand Cancer Society, 2012. http://www.cancernz.org.nz/ divisions/auckland/about/cancer-statistics/.

4. Newman, D. J.; Cragg, G. M. J. Nat. Prod. 2007, 70, 461-477.

5. Koehn, F. E.; Carter, G. T. Nat. Rev. Drug Discov. 2005, 4, 206-220.

6. Piggott, A.; Karuso, P. Mar. Drugs 2005, 3, 36-63.

7. Clardy, J.; Walsh, C. Nature 2004, 432, 829-837.

8. Oberlies, N. H.; Flora, S.; Weaver, A. L. Chemistry International 2003, 25, NA.

9. Thayer, A. Chem. Eng. News 2003, 18, 6.

10. Bentley, T. S.; Hanson, S. G. 2011 U.S. organ and tissue transplant cost estimates and discussion, 2011.

11. Dickschat, J. S. Beilstein J. Org. Chem. 2011, 7, 1620-1621.

12. Stone, M. J.; Williams, D. H. Mol. Microbiol. 1992, 6, 29-34.

13. Berenbaum, M. R. Proc. Natl. Acad. Sci. USA 1995, 92, 2-8.

14. Haefner, B. Drug Discov. Today 2003, 8, 536-544.

15. Verdine, G. L. Nature 1996, 384, 11-13.

16. Hruby, V. J.; Shenderovich, M.; Lam, K. S.; Lebl, M. Mol. Divers. 1996, 2, $46-56$.

17. Newman, D. J.; Cragg, G. M. J. Nat. Prod. 2012, 75, 311-335.

18. Lam, K. S. Trends Microbiol. 2007, 15, 279-289.

19. Paterson, I.; Anderson, E. A. Science 2005, 310, 451-453.

20. Feher, M.; Schmidt, J. M. J. Chem. Inf. Comp. Sci. 2002, 43, 218-227.

21. Lipinski, C. A.; Lombardo, F.; Dominy, B. W.; Feeney, P. J. Adv. Drug Deliv. Rev. 1997, 23, 3-25.

22. Stahura, F. L.; Xue, L.; Godden, J. W.; Bajorath, J. J. Mol. Model. 2000, 6, $550-562$.

23. Stahura, F. L.; Godden, J. W.; Xue, L.; Bajorath, J. J. Chem. Inf. Comp. Sci. 2000, 40, 1245-1252. 
24. Costantino, V.; Fattorusso, E.; Menna, M.; Taglialatela-Scafati, O. Curr. Med. Chem. 2004, 11, 1671-1692.

25. Dandapani, S.; Marcaurelle, L. A. Nature Chemical Biology 2010, 6, 861-863.

26. Rosén, J.; Gottfries, J.; Muresan, S.; Backlund, A.; ; Oprea, T. I. J. Med. Chem. 2009, 52, 1953-1962.

27. National Oceanic and Atmospheric Administration, 2012. http://www. noaa. gov/index.html.

28. Blunt, J. W.; Munro, M. H. G. MarinLit, Marine Literature Database, 2011.

29. Luch, A. Molecular, Clinical and Environmental Toxicology: Clinical Toxicology; Springer, 2010; pp 68-69.

30. Anderson, W. H. J. Ethnopharmacol. 1988, 23, 121-126.

31. Hwang, D. F.; Noguchi, T. Adv. Food Nutr. Res. 2007, 52, 141-236.

32. Chau, R.; Kalaitzis, J. A.; Neilan, B. A. Aquatic Toxicology 2011, 104, 61-72.

33. New World Encyclopaedia, 2012. http://www. newworldencyclopedia.org/.

34. Pomati, F.; Burns, B. P.; Neilan, B. A. App. Environmen. Microbiol. 2004, 70, 4711-4719.

35. Molinski, T. F.; Dalisay, D. S.; Lievens, S. L.; Saludes, J. P. Nat. Rev. Drug Discov. 2009, 8, 69-85.

36. Miljanich, G. P. Curr. Med. Chem. 2004, 11, 3029-3040.

37. Müller, W. E. G.; Müller, I. M. Integr. Comp. Biol. 2003, 43, 281-292.

38. Systema Porifera. A Guide to the Classification of Sponges; Hooper, J. N. A., van Soest, R. W. M., Eds.; Kluwer Academic/Plenum Publishers: New York, 2002; Vol. 1.

39. Northcote, P. Verbal communication, 2011.

40. Newman, D. J.; Cragg, G. M.; Snader, K. M. J. Nat. Prod. 2003, 66, 1022 1037.

41. Bergmann, W.; Feeney, R. J. J. Am. Chem. Soc. 1950, 72, 2809-2810.

42. Bergmann, W.; Feeney, R. J. J. Org. Chem. 1951, 16, 981-987.

43. Bergmann, W.; Burke, D. C. J. Org. Chem. 1955, 20, 1501-1507.

44. Sipkema, D.; Franssen, M. C. R.; Osinga, R.; Tramper, J.; Wijffels, R. H. Mar. Biotechnol. 2005, 7, 142-162.

45. Wright, K. Nature 1986, 323, 283.

46. Ledford, H. Nature 2010, 468, 608-609.

47. Hirata, Y.; Uemura, D. Pure Appl. Chem. 1986, 58, 701-710. 
48. Wojnar, J. M. Isolation of New Secondary Metabolites from New Zealand Marine Invertebrates, Ph.D. thesis, Victoria University of Wellington, 2008.

49. König, G. M.; Kehraus, S.; Seibert, S. F.; Abdel-Lateff, A.; Müller, D. ChemBioChem 2006, 7, 229-238.

50. Laroche, M.; Imperatore, C.; Grozdanov, L.; Costantino, V.; Mangoni, A.; Hentschel, U.; Fattorusso, E. Marine Biology 2007, 151, 1365-1373.

51. Faulkner, D. J.; Unson, M. D.; Bewley, C. A. Pure Appl. Chem. 1994, 66, 1983-1990.

52. Lee, O. O.; Wong, Y. H.; Qian, P.-Y. Appl. Environ. Microbiol. 2009, 75, 3513-3521.

53. Lee, O. O.; Yang, L. H.; Li, X.; Pawlik, J. R.; Qian, P.-Y. Mar. Ecol. Prog. Ser. 2007, 339, 25-40.

54. Lee, O. O.; Chui, P. Y.; Wong, Y. H.; Pawlik, J. R.; Qian, P.-Y. Appl. Environ. Microbiol. 2009, 75, $6147-5156$.

55. NationMaster, 2012. NationMaster.com.

56. The Official Site of Tonga Tourism, 2011. http://www.thekingdomoftonga. $\mathrm{com} /$.

57. Crews, P.; Kakou, Y.; Quinoa, E. J. Am. Chem. Soc. 1988, 110, 4365-4368.

58. Kakou, Y.; Crews, P.; Bakus, G. J. J. Nat. Prod. 1987, 50, 482-484.

59. Sashidhara, K. V.; White, K. N.; Crews, P. J. Nat. Prod. 2009, 72, 588-603.

60. Singh, A. J. The Structure-Directed Isolation of New Secondary Metabolites from South Pacific Marine Sponges, Ph.D. thesis, Victoria University of Wellington, 2012.

61. Tanaka, J.; Higa, T. Tetrahedron Lett. 1996, 37, 5535-5538.

62. Field, J. J.; Singh, A. J.; Kanakkanthara, A.; Halafihi, T.; Northcote, P. T.; Miller, J. H. J. Med. Chem. 2009, 52, 7328-7332.

63. Vuorela, P.; Leinonen, M.; Saikku, P.; Tammela, P.; Rauha, J. P.; Wennberg, T.; Vuorela, H. Natural Products in the Process of Finding New Drug Candidates; Bentham Science Publishers Ltd., 2004; Vol. 11, pp 13751389 .

64. Rollinger, J. M.; Langer, T.; Stuppner, H. Strategies for Efficient Lead Structure Discovery from Natural Products; Bentham Science Publishers Ltd., 2006; Vol. 13, pp 1491-1507.

65. Hilton, B.; Martin, G. J. Nat. Prod. 2010, 73, 1465-1469.

66. Popplewell, W. L. Isolation and Structural Elucidation of New Secondary Metabolites from New Zealand Red Algae, Ph.D. thesis, Victoria University of Wellington, 2008.

67. Bertelli, D.; Lolli, M.; Papotti, G.; Bortolotti, L.; Serra, G.; Plessi, M. J. Agric. Food. Chem. 2010, 58, 8495-8501. 
68. Košir, I. J.; Kidrič, J. J. Agric. Food. Chem. 2000, 49, 50-56.

69. Nilsson, M.; Duarte, I. F.; Almeida, C.; Delgadillo, I.; Goodfellow, B. J.; Gil, A. M.; Morris, G. A. J. Agric. Food. Chem. 2004, 52, 3736-3743.

70. Lambert, J. B.; Heckenbach, E. A.; Wu, Y.; Santiago-Blay, J. A. J. Nat. Prod. 2010, 73, 1643-1648.

71. Winssinger, N.; Barluenga, S. Chem. Commun. 2007, 22-36.

72. Roe, S. M.; Prodromou, C.; O’Brien, R. J. Med. Chem. 1999, 42, 260-266.

73. Daux, W. L. W.; Charles, M. Dev. Toxicol. Environ. Sci. 1980, 49, 153-160.

74. Miksicek, R. J. J. Steroid Biochem. Mol. Biol. 1994, 49, 153-160.

75. Boone, C.; Bussey, H.; Andrews, B. J. Nature Rev. Gen. 2007, 8, 437-449.

76. Giaever, G.; Flaherty, P.; Kumm, J.; Proctor, M.; Nislow, C.; Jaramillo, D. F.; Chu, A. M.; Jordan, M. I.; Arkin, A. P.; Davis, R. W. Nature 2002, 418, 387-391.

77. Adomas, A.; Heller, G.; Olson, A.; Osborne, J.; Karlsson, M.; Nahalkova, J.; Zyl, L. V.; Sederoff, R.; Stenlid, J.; Finlay, R.; Asiegbu, F. Tree Physiol. 2008, 28, 888-897.

78. Taufa, T. New Sesterterpenes from Marine Sponges from the Tropical Waters of the Kingdom of Tonga, M.Sc. thesis, Victoria University of Wellington, 2010.

79. Woolner, V. H. The Isolation and Structure Elucidation of Secondary Metabolites from Tongan Marine Organisms, M.Sc. thesis, Victoria University of Wellington, 2012.

80. Gauvin, A.; Smadja, J.; Aknin, M.; Faure, R.; Gaydou, E.-M. Can. J. Chem. 2000, 78, 986-992.

81. Horton, P. A.; Longley, R. E.; Kelly-Borges, M.; McConnell, O. J.; Ballas, L. M. J. Nat. Prod. 1994, 5\%, 1374-1381.

82. Gunatilaka, A. A. L.; Gopichand, Y.; Schmitz, F. J.; Djerassi, C. J. Org. Chem. 1980, 46, 3860-3886.

83. Ioannou, E.; Abdel-Razik, A. F.; Zervou, M.; Christofidis, D.; Alexi, X.; Vagias, C.; Alexis, M. N.; Roussis, V. Steroids 2009, 74, 73-80.

84. Chan, A. Unpublished PhD Research, 2011, 2011.

85. Segraves, N. L.; Robinson, S. J.; Garcia, D.; Said, S. A.; Fu, X.; Schmitz, F. J.; Pietraszkiewicz, H.; Valeriote, F. A.; Crews, P. J. Nat. Prod. 2004, 67, 783792.

86. Roll, D. M.; Scheuer, P. J.; Matsumoto, G. K.; Clardy, J. J. Am. Chem. Soc. 1983, 105, 6177-6178.

87. Harada, N.; Uda, H.; Kobayashi, M.; Shimizu, N.; Kitagawa, I. J. Am. Chem. Soc. 1989, 111, 5668-5674. 
88. Vacher, H. L.; Quinn, T. M. Geology and Hydrogeology of Carbonate Islands; Elsevier, 1997; p 948.

89. Zhi-Daz, M.; Ning, X.; Pei, Z.; Shou-Zun, Z.; Chong-Sh, W.; Qi-Tai, Z. Phytochemistry 1991, 30, 4175-4177.

90. Lee, I.-S.; Ma, X.; Chai, H.-B.; Madulid, D. A.; Lamont, B. R.; O’Neill, M. J.; Besterman, J. M.; Farnsworth, N. R.; Soejarto, D. D.; Cordell, G. A.; Pezzuto, J. M.; Kinghorn, D. A. Tetrahedron 1995, 51, 21-28.

91. Chinou, I. Curr. Med. Chem. 2005, 12, 1295-1317.

92. Williams, J. The cytotoxic and immunomodulatory properties of novel labdane diterpenes., 2010, January 2010.

93. Leahy, D. C. Inhibition of Eukaryotes, 2010-2011, 2010-2011.

94. London, C. A.; Abbas, A. K.; Kelso, A. Veterinary Immunology and Immunopathology 1998, 63, 37-44.

95. Mackay, I. R.; Leskovsek, N. V.; Rose, N. R. J. Autoimmun. 2008, 30, 5-11.

96. Javeed, A.; Ashraf, M.; Riaz, A.; Ghafoor, A.; Afzal, S.; Mukhtar, M. M. Eur. J. Pharm. Sci. 2009, 38, 283-290.

97. Kishore, R.; McMullen, M. R.; Cocuzzi, E.; Nagy, L. E. Comp. Hepatol. 2004, 3, S31.

98. Sosroseno, W.; Bird, P. S.; Seymour, G. J. Anaerobe 2009, 15, 95-98.

99. Girón, N.; Través, P. G.; Rodríguez, B.; López-Fontal, R.; Boscá, L.; Hortelano, S.; de las Heras, B. Toxicol. Appl. Pharmacol. 2008, 228, 179-189.

100. Zani, C. L.; Alves, T. M. A.; Queirá, R.; Fontes, E. S.; Shin, Y. G.; Cordell, G. A. Phytochemistry 2000, 53, 877-880.

101. Cocker, J. D.; Halsall, T. G.; Bowers, A. J. Chem. Soc. 1956, 4249-4262.

102. Tanaka, J.; Marriott, G.; Higa, T.; Higa, T. J. Nat. Prod. 2001, 64, 1468-1470.

103. Tasdemir, D.; Concepción, G. P.; Mangalindan, G. C.; Harper, M. K.; Hajdu, E.; Ireland, C. M. Tetrahedron 2000, 56, 9025-9030.

104. Peters, R. J. Nat. Prod. Rep. 2010, 27, 1521-1530.

105. San-Martin, A.; Quezada, E.; Soto, P.; Palacios, Y.; Rovirosa, J. Can. J. Chem. 1996, 74, 2471-2475.

106. Diaz-Marrero, A.; Issi, N.; Canales, V.; Chamy, C.; San-Martín, A.; Darias, J.; Rovirosa, J. Nat. Prod. Res. 2008, 22, 1516-1520.

107. Diaz-Marrero, A. R.; Dorta, E.; Cueto, M.; Rovirosa, J.; San-Martin, A.; Loyola, A.; Darias, J. Tetrahedron 2003, 59, 4805-4809.

108. Gray, C. A.; Davies-Coleman, M. T.; McQuaid, C. Nat. Prod. Lett. 1998, 12, $47-53$. 
109. van Wyk, A. W. W.; Gray, C. A.; Whibley, C. E.; Osoniyi, O.; Hendricks, D. T.; Caira, M. R.; Davies-Coleman, M. T. J. Nat. Prod. 2008, 71, 420-425.

110. Knass, W.; Reuter, B.; Zapp, J. J. Biochem. 1997, 326, 449-454.

111. Walker, R. P.; Faulkner, D. J. J. Org. Chem. 1981, 46, 1098-1102.

112. Meccia, G.; Rosquete, C.; Rojas, L. B.; Feliciano, A. S. Flavour Frag. J. 2006, 21, 559-561.

113. Diaz, M. C.; van Soest, R. W. M. The Plakinidae: a Sytematic Review. 4th International Porifera Conference, University of Amsterdam, 1994; pp 99-109.

114. Sandler, J. S.; Colin, P. L.; Hooper, J. N. A.; Faulkner, D. J. J. Nat. Prod. 2002, 65, 1258-1261.

115. Jimínez, C.; Quinoa, E.; Crews, P. Tetrahedron Lett. 1991, 32, 1843-1846.

116. Campagnuolo, C.; Fattorusso, E.; Taglialatela-Scafati, O. Eur. J. Org. Chem. 2003, 2003, 284-287.

117. Borrelli, F.; Campagnuolo, C.; Capasso, R.; Fattorusso, E.; TaglialatelaScafati, O. Eur. J. Org. Chem. 2004, 15, 3227-3232.

118. Campagnuolo, C.; Fattorusso, C.; Fattorusso, E.; Ianaro, A.; Pisano, B.; Taglialatela-Scafati, O. Org. Lett. 2003, 5, 673-676.

119. Fattorusso, E.; Romano, A.; Scala, F.; Taglialatela-Scafati, O. Molecules 2008, $13,1465-1471$.

120. Murayama, T.; Ohizumi, Y.; Nakamura, H.; Sasaki, T.; Kobayashi, J. Cell Mol. Life Sci. 1989, 45, 898-899.

121. Smith, S.; Tsai, S.-C. Nat. Prod. Rep. 2007, 24, 1041-1072.

122. Higgs, M.; Faulkner, D. J. J. Org. Chem. 1978, 43, 3454-3457.

123. Davidson, B. S. J. Org. Chem. 1991, 56, 6722-6724.

124. Yong, K. W. L.; de Voss, J. J.; Hooper, J. N. A.; Garson, M. J. J. Nat. Prod. 2011, 74, 194-207.

125. Gochfeld, D. J.; Hamann, M. T. J. Nat. Prod. 2001, 64, 1477-1479.

126. Campagnuolo, C.; Fattorusso, E.; Romano, A.; Taglialatela-Scafati, O.; Basilico, N.; Parapini, S.; Taramelli, D. Eur. J. Org. Chem. 2005, 2005, 50775083.

127. del Sol Jimínez, M.; Garzón, S. P.; Rodríguez, A. D. J. Nat. Prod. 2003, 66, 655-661.

128. Williams, D. E.; Allen, T. M.; van Soest, R.; Behrisch, H. W.; Andersen, R. J. J. Nat. Prod. 2001, 64, 281-285.

129. Rudi, A.; Afanii, R.; Gravalos, L. G.; Aknin, M.; Gaydou, E.; Vacelet, J.; Kashman, Y. J. Nat. Prod. 2003, 66, 682-685. 
130. Huang, X.-H.; van Soest, R.; Roberge, M.; Andersen, R. J. Org. Lett. 2003, 6, $75-78$.

131. Berrue, F.; Thomas, O. P.; Laville, R.; Prado, S.; Golebiowski, J.; Fernandez, R.; Amade, P. Tetrahedron 2007, 63, 2328-2334.

132. Sata, N.; Abinsay, H.; Yoshida, W. Y.; Horgen, F. D.; Sitachitta, N.; Kelly, M.; Scheuer, P. J. J. Nat. Prod. 2005, 68, 1400-1403.

133. Jeso, V.; Micalizio, G. C. J. Am. Chem. Soc. 2010, 132, 11422-11424.

134. Barber, J. M.; Quek, N. C.; Leahy, D. C.; Miller, J. H.; Bellows, D. S.; Northcote, P. T. J. Nat. Prod. 2011, 74, 809-815.

135. Silverstein, R. M.; Webster, F. X. Spectrometric Identification of Organic Compounds, 6th ed.; John Wiley and Sons, 1997.

136. Nakagawa, A.; Ohno, H.; Miyano, K.; Omura, S. J. Org. Chem. 1980, 45, 3268-3274.

137. Lacey, M. J.; MacDonald, C. G.; Pross, A.; Shannon, J. S. Aust. J. Chem. 1970, 23, 1421-1429.

138. Schumacher, R. W.; Davidson, B. S. Tetrahedron 1995, 51, 10125-10130.

139. Baldwin, J. E.; Hackler, R. E.; Scott, R. M. J. Chem. Soc. 1969, 1415-1416.

140. Kubec, R.; Kim, S.; McKeon, D. M.; Musah, R. A. J. Nat. Prod. 2002, 65, 960-964.

141. Ranu, B. C.; Dey, S. S.; Hajra, A. Green Chemistry 2003, 5, 44-46.

142. Kaczorowska, K.; Kolarska, Z.; Mitka, K.; Kowalski, P. Tetrahedron 2005, 61, 8315-8327.

143. Kowalski, P.; Mitka, K.; Ossowska, K.; Kolarska, Z. Tetrahedron 2005, 61, 1933-1953.

144. Shaabani, A.; Mirzaei, P.; Naderi, S.; Lee, D. G. Tetrahedron 2004, 60, 1141511420.

145. Barbarella, G.; Dembech, P.; Garbesi, A.; Fava, A. Org. Magn. Reson. 1976, 8, 108-114.

146. Davies, J. A. Adv. Inorg. Chem. Radiochem. 1961, 24, 115-187.

147. Marks, H. S.; Hilson, J. A.; Leichtweis, H. C.; Stoewsand, G. S. J. Agric. Food. Chem. 1992, 40, 2098-101.

148. Abdel-Sattar, E.; El-Mekkawy, S. Nat. Prod. Res. 2009, 23, 861-865.

149. Graham, S. K.; Lambert, L. K.; Pierens, G. K.; Hooper, J. N. A.; Garson, M. J. Aust. J. Chem. 2010, 63, 867-872.

150. Lake, R. J.; McCombs, J. D.; Blunt, J. W.; Munro, M. H. G.; Robinson, W. T. Tetrahedron Lett. 1988, 29, 4971-4972. 
151. Lam, H. W.; Cooke, P. A.; Pattenden, G.; Bandaranayake, W. M.; Wickramasinghe, W. A. J. Chem. Soc., Perkin Trans. 1 1999, 847-848.

152. Quinoa, E.; Crews, P. Tetrahedron Lett. 1987, 28, 3229-3232.

153. Schneemann, I.; Ohlendorf, B.; Zinecker, H.; Nagel, K.; Wiese, J.; Imhoff, J. F. J. Nat. Prod. 2010, 73, 1444-1447.

154. Zhang, Y.; Li, X.-M.; Feng, Y.; Wang, B.-G. Nat. Prod. Res. 2010, 24, 10361043.

155. Liu, D.; Li, X.-M.; Meng, L.; Li, C.-S.; Gao, S.-S.; Shang, Z.; Proksch, P.; Huang, C.-G.; Wang, B.-G. J. Nat. Prod. 2011, 74, 1787-1791.

156. Trisuwan, K.; Rukachaisirikul, V.; Sukpondma, Y.; Preedanon, S.; Phongpaichit, S.; Rungjindamai, N.; Sakayaroj, J. J. Nat. Prod. 2008, 71, 1323-1326.

157. Wang, C.-Y.; Wang, B.-G.; Brauers, G.; Guan, H.-S.; Proksch, P.; Ebel, R. J. Nat. Prod. 2002, 65, 772-775.

158. Cutignano, A.; Fontana, A.; Renzulli, L.; Cimino, G. J. Nat. Prod. 2003, 66, 1399-1401.

159. Ciavatta, M. L.; Manzo, E.; Nuzzo, G.; Villani, G.; Cimino, G.; Cervera, J. L.; Malaquias, M. A. E.; Gavagnin, M. Tetrahedron Lett. 2009, 50, 527-529.

160. Rovirosa, J.; San-Martín, A. Quìm. Nova 2006, 29, 52-53.

161. Hochlowski, J.; Coll, J.; Faulkner, D. J.; Clardy, J. J. Am. Chem. Soc. 1984, $106,6748-50$.

162. Kong, F.; Singh, M. P.; Carter, G. T. J. Nat. Prod. 2005, 68, 920-923.

163. Singh, M. P.; Kong, F.; Janso, J. E.; Arias, D. A.; Suarez, P. A.; Bernan, V. S.; Petersen, P. J.; Weiss, W. J.; Carter, G.; Greenstein, M. J. Antibiot. 2003, 56, 1033-1044.

164. Begley, T. P.; Xi, J.; Kinsland, C.; Taylor, S.; McLafferty, F. Current Opin. Chem. Biol. 1999, 3, 623-629.

165. White, P. J.; Millar, G.; Coggins, J. R. Biochem. J. 1988, 251, 313-322.

166. Yong, K. W. L.; Lambert, L. K.; Hayes, P. Y.; de Voss, J. J.; ; Garson, M. J. J. Nat. Prod. 2012, 75, 351-360.

167. Holland, H. L. Nat. Prod. Rep. 2001, 18, 171-181.

168. Motomasa Kondo Kobayashi, K.; Kitagawa, I. Chem. Pharm. Bull. 1993, 41, 1324-1326.

169. Xu, T.; Feng, Q.; Jacob, M. R.; Avula, B.; Mask, M. M.; Baerson, S. R.; Tripathi, S. K.; Mohammed, R.; Hamann, M. T.; Khan, I. A.; Walker, L. A.; Clark, A. M.; Agarwal, A. K. Antimicrob. Agents Chemother. 2011, 55, 16111621.

170. Qureshi, A.; Salvá, J.; Harper, M. K.; Faulkner, D. J. J. Nat. Prod. 1998, 61, $1539-1542$. 
171. Chen, Y.; Killday, K. B.; McCarthy, P. J.; Schimoler, R.; Chilson, K.; Selitrennikoff, C.; Pomponi, S. A.; Wright, A. E. J. Nat. Prod. 2001, 64, 262-264.

172. Jiménez-Romero, C.; Ortiz, I.; Vicente, J.; Vera, B.; Rodrìguez, A. D.; Nam, S.; Jove, R. J. Nat. Prod. 2010, 73, 1694-1700.

173. Manzo, E.; Ciavatta, M. L.; Melck, D.; Schupp, P.; de Voogd, N.; Gavagnin, M. J. Nat. Prod. 2009, 72, 1547-1551.

174. Jungwirth, H.; Kuchler, K. FEBS Letters 2006, 580, 1131-1138.

175. Rogers, B.; Decottignies, A.; Kolaczkowski, M.; Carvajal, E.; Balzi, E.; Goffeau, A. J. Mol. Microbiol. Biotechnol. 2001, 3, 207-214.

176. Sakemi, S.; Higa, T.; Anthoni, U.; Christophersen, C. Tetrahedron 1987, 43, 263-268.

177. Shen, Y.-C.; Prakash, C. V. S.; Kuo, Y.-H. J. Nat. Prod. 2001, 64, 324-327.

178. Wells, R. J. Tetrahedron Lett. 1976, 17, 2637-2638.

179. Toth, S. I.; Schmitz, F. J. J. Nat. Prod. 1994, 57, 123-127.

180. Patil, A. D.; Freyer, A. J.; Carte, B.; Johnson, R. K.; Lahouratate, P. J. Nat. Prod. 1996, 59, 219-223.

181. Tanaka, J.; Higa, T.; Suwanborirux, K.; Kokpol, U.; Bernardinelli, G.; Jefford, C. W. J. Org. Chem. 1993, 58, 2999-3002.

182. Butler, M. S.; Capon, R. J. Aust. J. Chem. 1993, 46, 1363-1374.

183. Fontana, A.; Cimino, G.; Gavagnin, M.; Lez, M. C. G.; Estornell, E. J. Med. Chem. 2001, 44, 2362-2365.

184. Reyes, F.; Rodríguez-Acebes, R.; Fernanández, R.; Bueno, S.; Francesch, A.; Cuevas, C. J. Nat. Prod. 2009, 73, 83-85.

185. Subrahmanyam, C.; Rao, C. V.; Anjaneyulu, V.; Satyanarayana, P.; Rao, P. V. S.; Ward, R. S.; Pelter, A. Tetrahedron 1992, 48, 3111-3120.

186. Alam, M.; Martin, G. E.; Zektzer, A. S.; Weinheimer, A. J.; Sanduja, R.; Ghuman, M. A. J. Nat. Prod. 1993, 56, 774-779.

187. Avery, M. A.; Fan, P.; Karle, J. M.; Bonk, J. D.; Miller, R.; Goins, D. K. J. Med. Chem. 1978, 39, 1885-1897.

188. Hommel, M. J. Biol. 2008, 7, 1-5.

189. Mayer, A. M. S.; Rodríguez, A. D.; Berlinck, R. G. S.; Hamann, M. T. Biochim. Biophys. Acta 2009, 1790, 283-308.

190. Kuria, K. A. M.; Chepkwony, H.; Govaerts, C.; Roets, E.; Busson, R.; de Witte, P.; Zupko, I.; Hoornaert, G.; Quirynen, L.; Maes, L.; Janssens, L.; Hoogmartens, J.; Laekeman, G. J. Nat. Prod. 2002, 65, 789-793.

191. Schneider, C.; Pratt, D. A.; Porter, N. A.; Brash, A. R. Chem. Biol. 2007, 14, 473-488. 
192. Capon, R. J.; Macleod, J. K. Tetrahedron 1985, 41, 3391-3404.

193. Compagnone, R. S.; Pińa, I. C.; Rangel, H. R.; Dagger, F.; Suárez, A. I.; Reddy, M. V. R.; Faulkner, D. J. Tetrahedron 1998, 54, 3057-3068.

194. Jung, M.; Ham, J.; Song, J. Org. Lett. 2002, 4, 2763-2765.

195. Ovenden, S. P. B.; Capon, R. J. J. Nat. Prod. 1999, 62, 214-218.

196. Capon, R. J.; MacLeod, J. K. J. Nat. Prod. 1987, 50, 225-229.

197. Patil, A. D.; Freyer, A. J.; Bean, M. F.; Carté, B. K.; Westley, J. W.; Johnson, R. K.; Lahouratate, P. ChemInform. 1996, 27, NA.

198. Carter, S.; Pitt, S. J.; Colyer, J.; Sitsapesan, R. J. Mem. Biol. 2011, 240, 21-33.

199. Paddon-Jones, G. C.; Hungerford, N. L.; Hayes, P.; Kitching, W. Org. Lett. 1999, 1, 1905-1907.

200. Mbaya, E.; Oules, B.; Caspersen, C.; Tacine, R.; Massinet, H.; Pennuto, M.; Chretien, D.; Munnich, A.; Rotig, A.; Rizzuto, R.; Rutter, G. A.; PaterliniBrechot, P.; Chami, M. Cell Death Differ. 2010, 17, 1855-1866.

201. Rafiee, M. A.; Hadipour, N. L.; Naderi-manesh, H. J. Chem. Inf. Model. 2005, 45, 366-370.

202. D'Ambrosio, M.; Guerriero, A.; Deharo, E.; Debitus, C.; Munoz, V.; Pietra, F. Helv. Chim. Acta 1998, 81, 1285-1292.

203. Brenner, C. Genome Biol. 2004, 5, 1-4.

204. Wolfger, H.; Mamnun, Y. M.; Kuchler, K. J. Biol. Chem. 2004, 279, 1159311599.

205. Hillenmeyer, M. E.; Fung, E.; Wildenhain, J.; Pierce, S. E.; Hoon, S.; Lee, W.; Proctor, M.; St.Onge, R. P.; Tyers, M.; Koller, D.; Altman, R. B.; Davis, R. W.; Nislow, C.; ; Giaever, G. Science 2008, 320, 362-365.

206. Goffeau, A. Yeast 1996, 12, 1603-1605.

207. Goffeau, A. et al. Science 1996, 274, 546, 563-7.

208. Yibmantasiri, P.; Leahy, D. C.; Busby, B. P.; Angermayr, S. A.; Sorgo, A. G.; Boeger, K.; Heathcott, R.; Barber, J. M.; Moraes, G.; Matthews, J. H.; Northcote, P. T.; Atkinson, P. H.; Bellows, D. S. Mol. BioSyst. 2012, 8, 902912 .

209. Lum, P. Y. et al. Cell 2004, 116, 121-137.

210. Parsons, A. B. et al. Cell 2006, 126, 611-625.

211. Baetz, K.; McHardy, L.; Gable, K.; Tarling, T.; Rebérioux, D.; Bryan, J.; Andersen, R. J.; Dunn, T.; Hieter, P.; Roberge, M. Proc. Natl. Acad. Sci. USA 2004, 101, 4525-4530.

212. Giaever, G.; Shoemaker, D. D.; Jones, T. W.; Liang, H.; Winzeler, E. A.; Astromoff, A.; Davis, R. W. Nat. Genet. 1999, 21, 278-283. 
213. Deutschbauer, A. M.; Jaramillo, D. F.; Proctor, M.; Kumm, J.; Hillenmeyer, M. E.; Davis, R. W.; Nislow, C.; Giaever, G. Genetics 2005, 169, 1915-1925.

214. Giaever, G.; Flaherty, P.; Kumm, J.; Proctor, M.; Nislow, C.; Jaramillo, D. F.; Chu, A. M.; Jordan, M. I.; Arkin, A. P.; Davis, R. W. Proc. Natl. Acad. Sci. USA 2004, 101, 793-798.

215. Springer, M.; Weissman, J. S.; Kirschner, M. W. Mol. Syst. Biol. 2010, 6, 1-6.

216. Matthews, J. H. The Molecular Pharmacology of Pateamine A, Ph.D. thesis, Victoria University of Wellington, 2010.

217. Almeida, B.; Silva, A.; Mesquita, A.; Sampaio-Marques, B.; Rodrigues, F.; Ludovico, P. BBA-Mol. Cell Res. 2008, 1783, 1436-1448.

218. Edlind, T.; Smith, L.; Henry, K.; Katiyar, S.; Nickels, J. Molecular Microbiology 2002, 46, 1365-2958.

219. Zaragoza, D.; Ghavidel, A.; Heitman, J.; Schultz, M. C. Mol. Cell Biol. 1998, $18,4463-4470$.

220. Mayer, C.; Grummt, I. Oncogene 2006, 25, 6384-6391.

221. Herman, P. K. Curr. Opin. Microbiol. 2002, 602-607.

222. Hartwell, L. H.; Culotti, J.; Pringle, J. R.; Reid, B. J. Science 1974, 183, 46-51.

223. Calzone, L. Temporal organization of the budding yeast cell cycle: General principles and detailed simulations, Ph.D. thesis, Virginia Polytechnic Institute and State University, 2003.

224. Coorey, N. Innovative tools to discover potential drug-candidates, M.Sc. thesis, Victoria University of Wellington, 2011, 2011.

225. Robinson, M. D.; Grigull, J.; Mohammad, N.; Hughes, T. R. BMC Bioinformatics 2002, 3, 1-5.

226. Saccharomyces Genome Database, 2012. http://www.yeastgenome.org/.

227. Hillenmeyer, M. E.; Ericson, E.; Davis, R. W.; Nislow, C.; Koller, D.; Giaever, G. Genome Biol. 2010, 11, 1-17.

228. Toret, C. P.; Drubin, D. G. J. Cell Sci. 2007, 119, 1501-1503.

229. Fingerman, I.; Nagaraj, V.; Norris, D.; Vershon, A. K. Eukaryotic Cell 2003, 2, 1061-1068.

230. Miyakawa, T.; Mizunuma, M. Biosci. Biotechnol. Biochem. 2007, 71, 633-645.

231. Gupta, S. S.; Ton, V.-K.; Beaudry, V.; Rulli, S.; Cunningham, K.; Rao, R. J. Biol. Chem. 2003, 278, 28831-28839.

232. Courchesne, W. E.; Ozturk, S. Molecular Microbiology 2003, 47, 223-234.

233. Peiter, E.; Fischer, M.; Sidaway, K.; Roberts, S. K.; Sanders, D. FEBS Lett. 2005, 579, 5697-5703. 
234. Wang, H. G.; Pathan, N.; Ethell, I. M.; Krajewski, S.; Yamaguchi, Y.; Shibasaki, F.; McKeon, F.; Bobo, T.; Franke, T. F.; Reed, J. Science 1999, 284, 339-343.

235. Tsuzi, D.; Maeta, K.; Takatsume, Y.; Izawa, S.; Inoue, Y. FEBS Lett. 2004, 569, 301-306.

236. Gitan, R. S.; Luo, H.; Rodgers, J.; Broderius, M.; Eide, D. J. Biol. Chem. 1998, 273, 28617-28624.

237. Zhang, Y.-Q.; Rao, R. J. Biol. Chem. 2007, 282, 37844-37853.

238. Decuypere, J.-P.; Monaco, G.; Missiaen, L.; Smedt, H. D.; Parys, J. B.; Bultynck, G. J. Aging Res. 2011, 2011, 1-20.

239. Cyert, M. S. Biochem. Biophys. Res. Commun. 2003, 311, 1143-1150.

240. Shitamukai, A.; Hirata, D.; Sonobe, S.; Miyakawa, T. J. Biol. Chem. 2004, 279, 3651-3661.

241. Mulet, J. M.; Martin, D. E.; Loewith, R.; Hall, M. N. J. Biol. Chem. 2006, 281, 33000-33007.

242. Stathopoulos, A. M.; Cyert, M. S. Genes Dev. 1997, 11, 3432-3444.

243. Yoshimoto, H.; Saltsman, K.; Gasch, A. P.; Li, H. X.; Ogawa, N.; Botstein, D.; Brown, P. O.; Cyert, M. S. J. Biol. Chem. 2002, 277, 31079-31088.

244. Yarmola, E. G.; Somasundaram, T.; Boring, T. A.; Spector, I.; Bubb, M. R. J. Biol. Chem. 2000, 275, 28120-28127.

245. Martin, D. E.; Powers, T.; Hall, M. N. Cell Metabolism 2006, 9, 259-260.

246. Gulati, P.; Gaspers, L. D.; Dann, S. G.; Joaquin, M.; Nobukuni, T.; Natt, F.; Kozma, S. C.; Thomas, A. P.; Thomas, G. Cell Metab. 2008, 7, 456-465.

247. Kamble, C.; Jain, S.; Murphy, E.; Kim, K. J. Biosci. 2011, 36, 79-96.

248. Park, K. S.; Kim, T. K.; Kim, D. H. Am. J. Physiol. 1999, 276, 865-872.

249. Qiao, W.; Mooney, M.; Bird, A. J.; Winge, D. R.; Eide, D. J. Biochemistry 2006, 106, 8674-8679.

250. Gottlieb, H. E.; Kotlyar, V.; Nudelman, A. J. Org. Chem. 1997, 62, 7512-7515.

251. Amberg, D. C.; Burke, D. J.; Strathern, J. N. Methods In Yeast Genetics, English ed.; Cold Spring Harbor Laboratory Press, 2005.

252. Haase, S.; Reed, S. Cell Cycle 2002, 1, 132-136.

253. Peyser, B. D.; Irizarry, R.; Spencer, F. A. Methods Mol. Biol. 2008, 416, 369381.

254. Jack, C. Replicate removal script; Victoria University of Wellington, 2012.

255. West, L. M. The isolation of secondary metabolites from New Zealand marine sponges, Ph.D. thesis, Victoria University of Wellington, 2001. 